\title{
It's All One Curriculum: Activities for a Unified Approach to Sexuality, Gender, HIV, and Human Rights Education [Bangla]
}

International Sexuality and HIV Curriculum Working Group

Nicole Haberland

Population Council

Deborah Rogow

Follow this and additional works at: https://knowledgecommons.popcouncil.org/departments_sbsr-pgy

Part of the Demography, Population, and Ecology Commons, Family, Life Course, and Society Commons, Gender and Sexuality Commons, International Public Health Commons, and the Medicine and Health Commons How does access to this work benefit you? Let us know!

\section{Recommended Citation}

International Sexuality and HIV Curriculum Working Group. 2011. "It's All One Curriculum: Activities for a Unified Approach to Sexuality, Gender, HIV, and Human Rights Education [Bangla]," edited by Nicole Haberland and Deborah Rogow. New York: Population Council. 


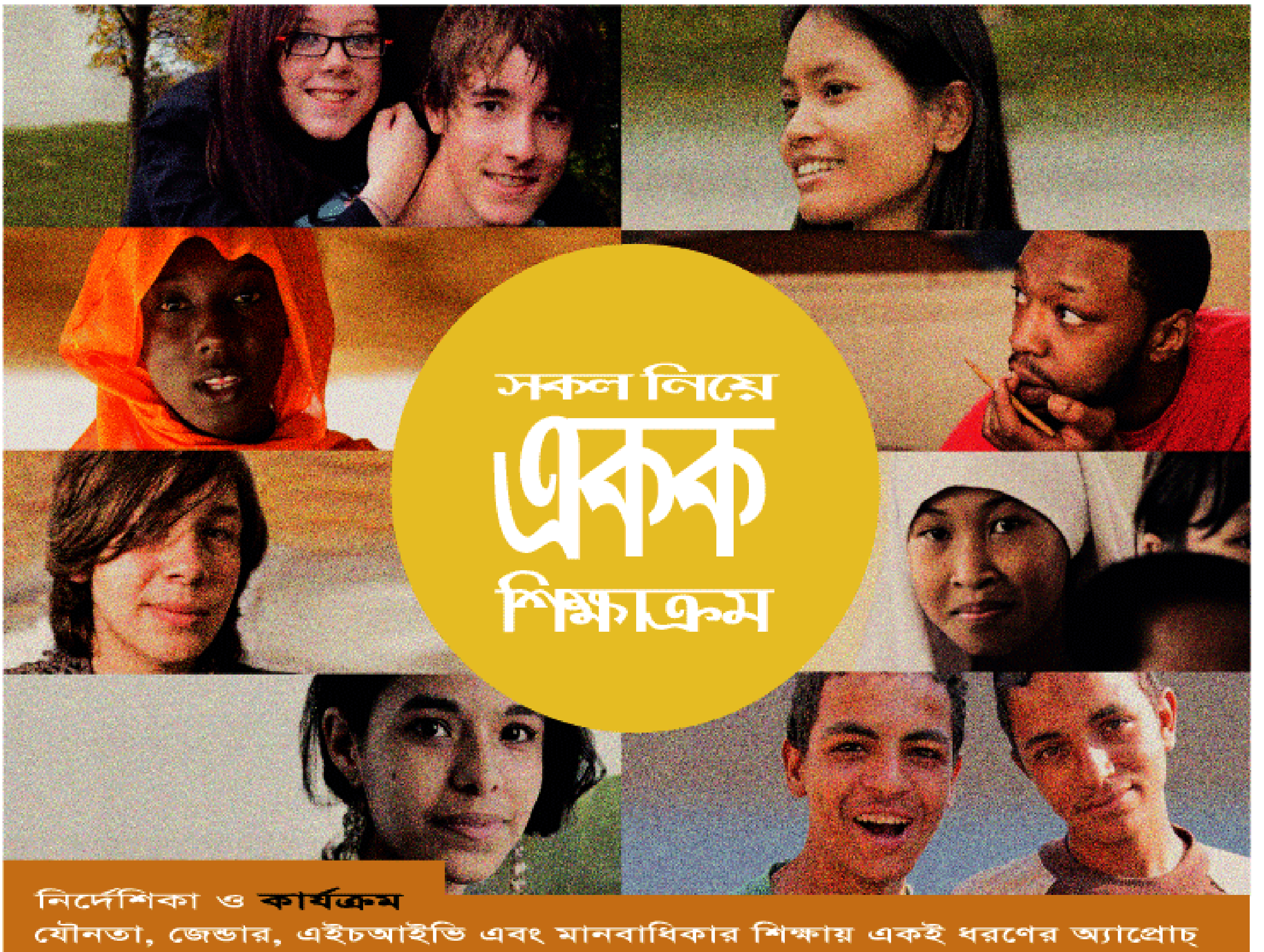





\section{मকल निश्ে 【্রক एकिकाक्रम}

যৌনতা, জেন্ডার, এইচআইভি এবং মানবাধিকার শিক্ষায় একই ধরনের অ্যাশ্রাচের জন্য নির্দেশিকা ও কার্য অ্রম 


\section{$(R-A$}

GIRIS' POWER WITHATIUE GPI) NIGERIA

IPPF

IPPF

O

INTERNATIONAL WOMEN'S

HEALTH COALITION

\section{Mexfam}

Salud para tu familia

1 Population Council
সকল নিয়ে একক শিক্ষাক্রম : যৌনতা, জেন্ডার, এইচআইভি এবং মানবাধিকার শিক্ষায় একই ধরনের অ্যাপ্রোচ্যর জন্য নির্দেশিকা ও কার্যক্রম তৈরি করা হয়েছে একটি আন্তর্জাতিক ওয়ার্কিং গ্রতপের মাধ্যমে যা নিয়প্রদত্ত সংস্থাগুলির (বর্ণমালা অনুসারে তালিকাবদ্ধ) প্রতিনিধিদের

দ্বারা গঠিত:

সিআরইএ (ইন্ডিয়া): কারোলিন এর্ল, সুনিতা কুজুর, গীতা মিশ্রা

গার্ল পাওয়ার ইনিসিয়েটিভ (নাইজেরিয়া): বেইন মাধুনাগু, গ্রেস ওসাকু

ইন্টারন্যাশনাল প্লানড প্যারেন্টহুড ফেডারেশন: ডোর্টজে ব্রাইকেন

আইপিপিএফ/ওয়ের্ট্টান হেমিসফেয়ার রিজিওন: জেসি কাইডি, ডেনিচ কোহন

ইন্টারন্যাশনাল উইমেন’স হেলথ কোয়ালিশন: কেলি ক্যাসটাগনারো, কোরেইন হুইটাকের

মেক্সফাম (মেক্সিকো): ওফেলিয়া আগুইলার

পপুলেশন কাউন্সিল: নিকোল হারবারল্যান্ড, ডেবোরাহ রোগাউ

ওয়ার্কিং গ্রুপটি বয়ঃসন্ধিকালীন যৌনতা/এইচআইভি সম্পর্কিত শিক্ষায় দৃষ্টিভঙ্গি ও কারিগরি দক্ষতার একটি পরিসীমা উল্লেখ করেছেন যেখানে অধিকার ও জেন্ডার, কর্মসূচি ব্যবস্থাপনা, গবেষণা এবং এ্যাডভোকেসির বিষয়গুলিও অন্তর্ভুক্ত। এন্ড্রিয়া ইরভিনও কয়েকটি অংশের খসড়া তৈরিতে সহায়তা করেছেন ।

\section{আন্তর্জাতিক যৌনতা ও এইচআইভি পাঠ্যক্রম ওয়ার্কিং গ্রুপ কর্তৃক লিখিত নিকোল হারবারল্যান্ড ও ডেবোরাহ রোগাউ কর্তৃক সম্পাদিত}

প্রকল্প সমন্বয়কারীগণ: মিশেল স্কায়ের ও জোনাহ স্টুয়ার্ট ব্রুন ডেজ

অলংকরণ: ইমামুয়েলা ফ্রিজারিও, হিউন অহু, সি এন্ড জি পার্টনারস, নিই ইয়র্ক সিটি।

মুদ্রণ : মাইক ভোসিকা, ক্রিসটিনা সে, মিশেল স্কায়ের, লুসান শাপিরো।

অনুলিপি সম্পাদক: কারেন টীডি-হোলমস

পঠন-স্তরের সম্পাদক: জিনা ডুকেয়ান

প্রচ্ছদের ছবিগুলির জন্য আলোকচিত্র স্বীকৃতি (উপরে বাম থেকে ঘড়ির কাটা অনুসারে): মৌরা ক্যারোল; রন অ্যালগমান, ইউজেইন মার্টিন; মানোচের কেঘাতি, আইআরআইএন-এর সৌজন্যে; মেলিসা মে; গাবে কুনি, আইপিপিএফ/ডাব্লিউএইচআর; মাইকেল নিউমান; নেইল থমাস, আইআরআইএন-এর সৌজন্যে। প্রচ্ছদে ছাড়ানো ছবির আলোকচিত্র স্বীকৃতি: কার্যকর শিক্ষণ পদ্ধতি, মার্ক টুসমান, ইউএনএফপিএ গুয়াতেমালা-এর সৌজন্যে; অধিবেশনগুলি, আন্দ্রিয়া লিনস, ইএমপাওয়ার এবং সিএএসএ ম্যাক্সিকো-এর লৌজন্যে; অতিরিক্ত সম্পদ, এ্যামি জয়সি. অন্যান্য ছবির আলোকচিত্র স্বীকৃতি ছবির পরই দেয়া আছে।

পপুলেশন কাউন্সিল, নিউ ইয়র্ক কর্তৃক প্রকাশিত

বিনামূল্যে যে কোন সময় ডাউনলোড করতে : www.popcouncil.org/publications/books/2010_ItsAllOne.asp

সত্ত্বাধিকারীৎ২০০৯ দ্যা পপুলেশন কাউন্সিল, আইএনসি

প্রকাশনা স্বীকৃতি ও বিনামূল্যে বিতরণ করা শর্তে এই বইয়ের যেকোন অংশ লেখক বা প্রকাশকের অনুমতি ছাড়া অনুলিপি করা যেতে পারে। যেকোন বাণিজ্যিক পুনর্মুদ্রণের জন্য পপুলেশন কাউন্সিলের লিখিত পূর্বানুমতি দরকার। নির্দিষ্ট অধিবেশনে উল্লিখিত যেকোন মূল উৎসকে স্বীকৃতি দিন।

আইএসবিএন: নির্দেশিকা: ৯৭b-০-b-৭b-৩৪-১১৭-৭; অধিবেশনগুলি: ৯৭b-০-b-৭b-৩৪-১১b--৪, লেট: ৯৭b-০-b-৭b-৩৪-১৯৯-১ 
সম্পাদনা

ফাতেমা সুলতানা শুভ্রা, জগন্নাথ বিশ্ববিদ্যালয়

নুর মোহাম্মাদ, ইউএনএফপিএ

ওবায়দুর রব, পপুলেশন কাউন্সিল

ভাষান্তর

শান্তা শ্যামলী মনীষা, পপুলেশন কাউন্সিল

অমর কৃষ্ণ বৈদ্য, পপুলেশন কাউন্সিল

কাজী তামান্না কেয়া, পপুলেশন কাউন্সিল

নার্গিস সুলতানা, পপুলেশন কাউন্সিল

সহয়েগীতা

ঈশিতা জাহান, নন্দিনী লোপা, মোঃ কামরুজ্জামান ভূইয়া, ঈশিতা ফারজানা হক

ভাষাগত সংশোধন

লোপামূদ্রা রহমান, জগন্নাথ বিশ্ববিদ্যালয়

অলংকরণ

কাজী রাজীবুল হক

প্রকাশকাল

জানুয়ারী, 2011

Copyright@2011, The Population Council, Inc.

This publication was made possible by a grant from UNFPA. The opinions expressed herein are those of the authors and do not reflect the views of the Population Council or UNFPA.

Suggested citation : Nicole Haberland, Deborah Rogow, Ofelia Aguilar, Doortie Breaken, Jessie Clyde, Caroline Earle, Denise Khon, Bene Madunagu, Grace Osakue, and Corinne Whitaker. Shanta Shyamolee Moneesha, Amar

K Baidya, Kazi Tamanna Keya, Nargis Sultana and Forhana Rahman (trans.) 2009, 2012. Shokol Nia Ekok

Shikhakrom: Jounota, grnder, HIV ebong Manobadhikar Shikkhai ek-i dhoroner Approach er jonno Karjokrom [Its

All One Curriculum: Activities for a unified Approach to Sexuality, Gender, HIV, and Human Rights

Education].New York: Population Council. 
বাংলাদেশের ১৬ কোটি মানুষের এক পঞ্চমাংশ কিশোর-কিশোরী যাদের বয়স ১০- ১৯ বছরের মধ্যে। তাদের অনেকেরই প্রজনন স্বাস্থ্য, জেণ্গার, এইচআইভি এবং মানবাধিকার নিয়ে সঠিক কোন ধারণা নেই। ভুল তথ্য, অসচেতনতামূলক পারিপার্শ্বিকতা ও জীবন দক্ষতার অভাব যুব সমাজের অনেককে অনিরাপদ যৌণ আচরণ, মাদকদ্রব্য সেবনসহ বিভিন্ন রকম ঝুঁকিপূর্ণ অভ্যাস ও অসামাজিক কার্যকলাপের দিকে ঠেলে দেয় । তাই কিশোর-কিকোরী ও যুব, যারা আমাদের বর্তমান ও ভবিষ্যত প্রজন্ম, তাদেরকে যৌন ও প্রজনন স্বাস্থ্য, এইচআইভি ও জেন্ডার বিষয়ক পর্যাপ্ত তথ্য দিয়ে সঠিক পথে পরিচালিত হতে সাহায্য করা খুবই প্রয়োজন । এই লক্ষ্যে ইউএনএফপিএ’র সহযোগিতায় পপুলেশন কাউন্গিল কিশোর-কিশোরী ও যুবদের জীবন দক্ষতা ভিত্তিক দিক নির্দেশনা পেতে 'All in One Curriculum' এর বাংলা সংকলনটি ওয়েব সাইটে প্রকাশ করে। এই শিক্ষাক্রমটি হলো তাদের জন্য একটি আদর্শ কারিকুলাম যারা ক্লিনিক কমিউনিটি এবং স্কুলের কারিকুলাম তৈরি এবং বিতরণের সাথে সম্পৃক্ত। এটি শৌন ও প্রজনন স্বাস্থ্য ও এর সুস্থ্যতাসহ সামাজিক ও ব্যক্তি কেন্দ্রিক নির্দেশকগুলো বুঝতে শিক্ষার্থীদের সক্ষম করে। ইতিমধ্যে যেসব কার্যক্রম চালু রয়েছে তার সাথে এই শিক্ষাক্রমটি একটি নতুন মাত্রা যোগ করে যুব সমাজকে যৌনতা, জেঞ্তার, এইচআইভি এবং মানবাধিকার শিক্ষায় সচেতন করে তাদের আরো সমৃদ্ধ জীবন যাপনে অগ্রণী ভূমিকা পালন করবে বনে আশা করি ।

\section{ড. ওবায়দুর রব}

কান্ট্রি ডিরেক্টর

পপুলেশন কাউন্সিল 


\section{সূচিপত্র}

बार्यकत्र শिकाদাन পब্ধতি

সকল হার্যक्্ম

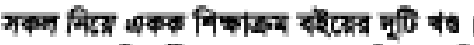

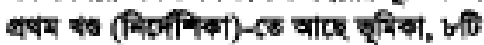

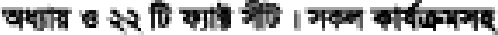

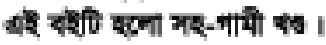

बৌन वाद्या

अषितिশन د-8

खिভাব্র

৩২

वधियেশन e-১৫

खৌनण

$\varangle 8$

अधिविশन ১৭-২৫

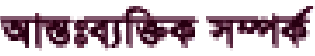

$৮ 8$

वधियেশन ২१-০

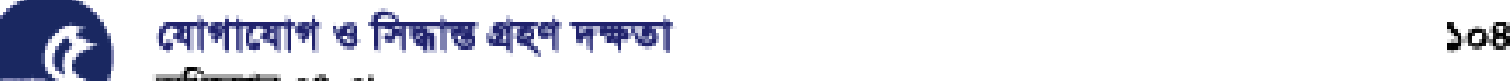

अधिक्यिশम $08-\infty$

ग२१

वधिবেশन 8०-8৫

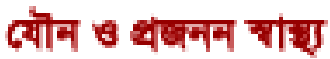

$28 \gtrsim$

अधिक्েশन 8b-৫२

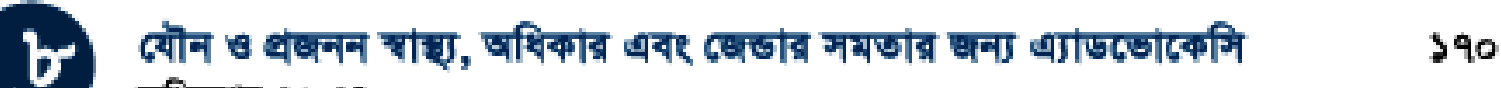
वधिखেশन ए०-०8 


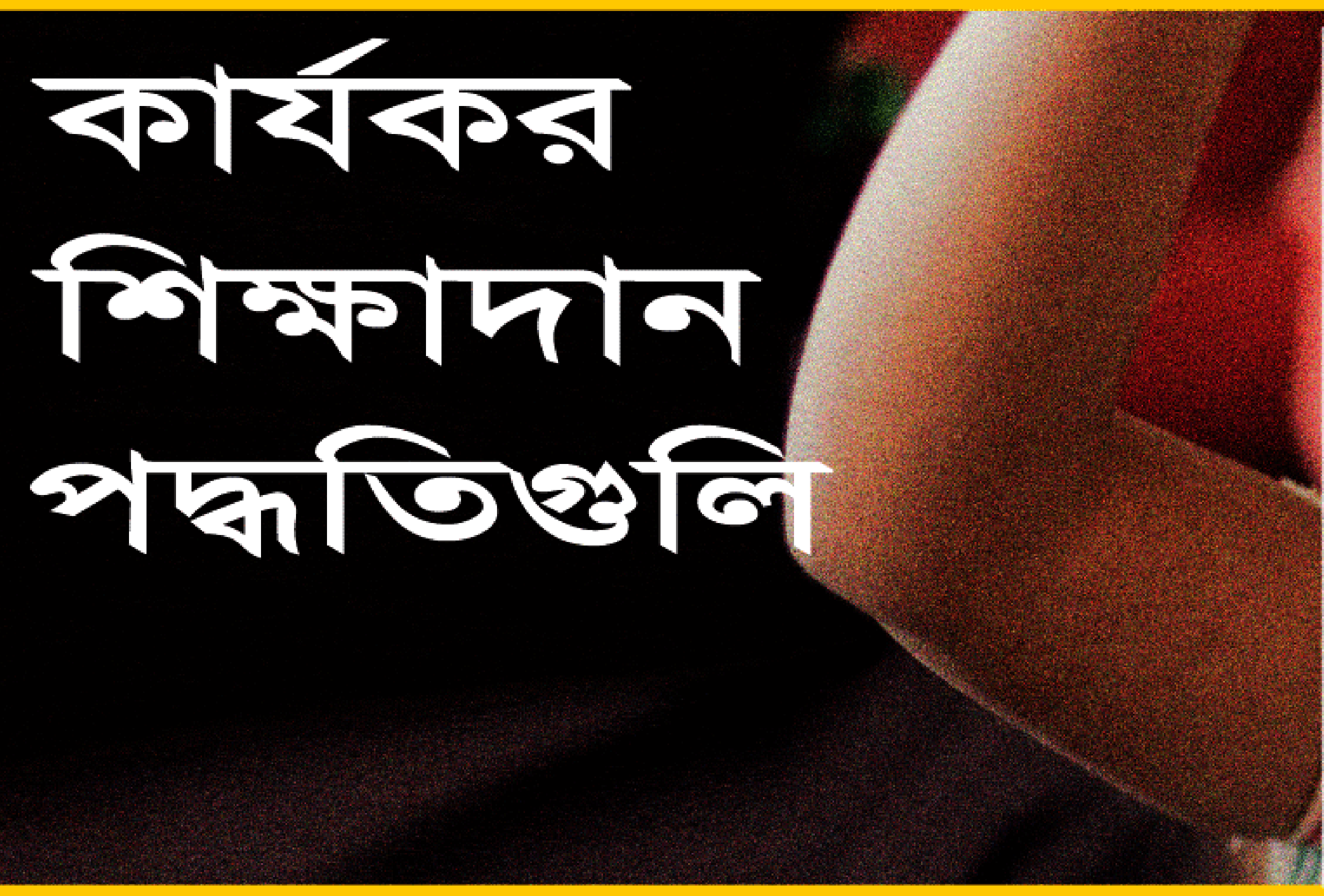




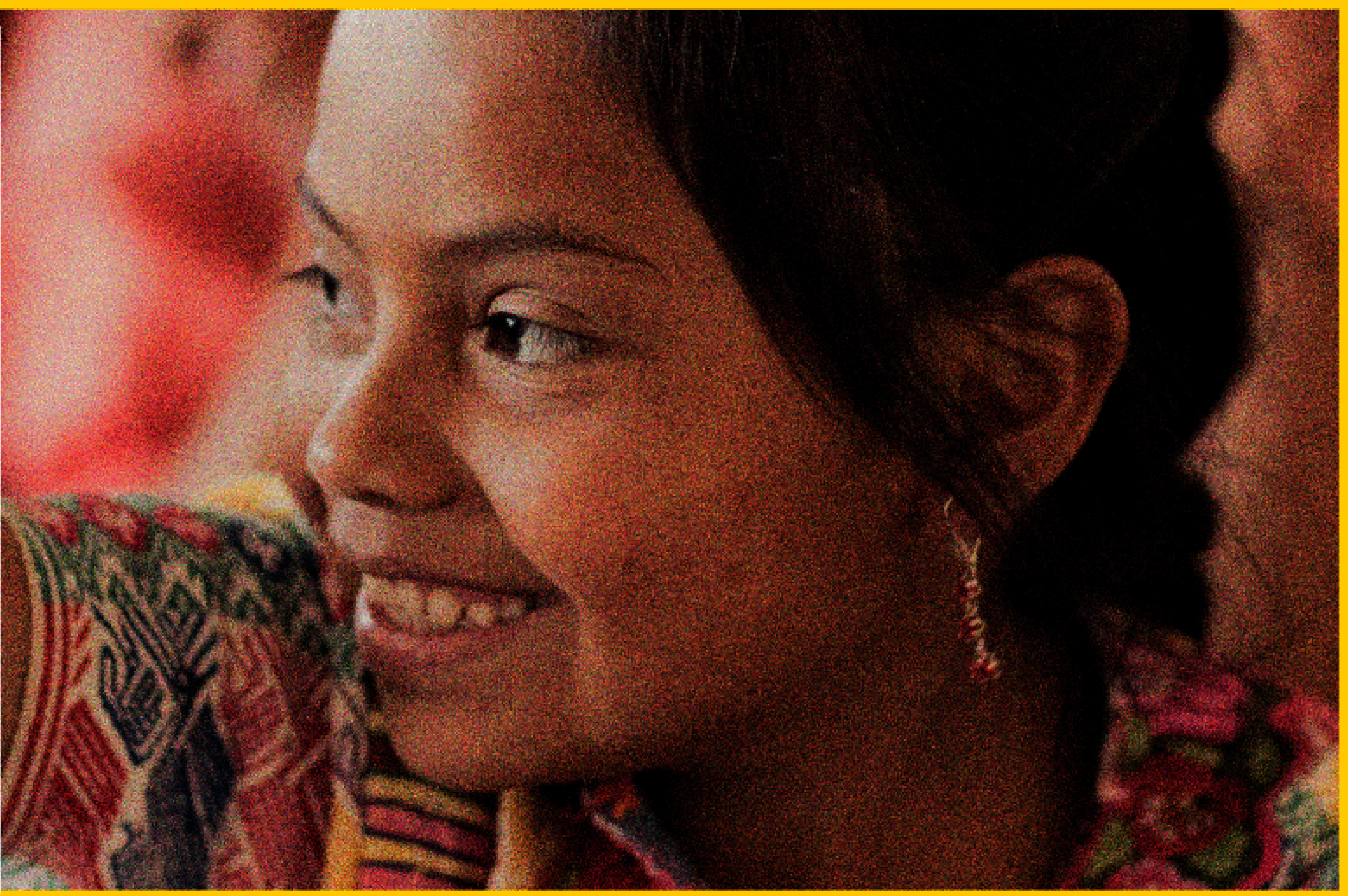




\section{শিক্ষক এবং পাঠ্যক্রম প্রণেতাদের জন্য কিছু কথা}

আমরা কী কেখাচ্ছি সেটি যেমন গুরুত্বপূর্ণ, তেমনি কীভাবে আমরা তা শেখাচ্ছি সেটিও সমান গুরুত্বপূর্ণ। টৌনতা, এইচআইভি, জেন্ডার এবং অধিকার নিয়ে পড়ানোর সবচচয়ে কার্যকরী পদ্ধতি হল অংশগ্রহুমূলক, শিক্ষার্থী-কেন্দ্রিক শিক্ষা ব্যবস্থা। কার্যত, এসব পদ্ধতিসমূহ যুব সমাজকে বেঁটে থাকার জন্য তৈরী হতে সহায়তা করে এবং তাদের সুস্বাস্থ্য ও সুখী জীবন-যাপনে সাহায্য করে।

সহজভাবে ও দক্ষতার সাথে অংশগ্রহণমূলক পাঠদান পদ্ধতি প্রয়োগ করার জন্য শিক্ষকদের প্রস্তুতি এবং সমর্থনের প্রয়োজন রয়েছে। এই খটেণ্ড শিক্ষার্থী-কেন্দ্রিক, পারস্পারিক সক্রিয় শিক্ষণ পদ্ধতির মূল নীতিগুলি সম্পর্ক্ক ধারণা দেয়া হয়েছে। বিশেষভাবে এইচআইভি এইডস এবং যৌনতা সম্পর্কিত পাঠ্যক্রমের ক্ষেত্রে এই সংকলনের কিছু সুনির্দিষ্ট পরামর্শ রয়েছে । সর্বোপরি, এতে বিদ্যালয় এবং মাঠ পর্যায় উভয়ক্ষেত্রেই পাঠ্যক্রমকে বাস্তবসম্মত ভাবে ব্যবহার করার জন্য প্রয়োজনীয় দিক-নির্দেশনা দেয়া হয়েছে।

‘সকল নিয়ে একক শিক্ষাক্রম’ (It'sAll in One Curriculum)- এ দুটো বই আছে । এই বইতে শ্রেণিকক্ষের (কার্যক্রমে) সম্পৃক্ত থাকার জন্য এই বইয়ে মোট ৫৪টি অধিবেশন রয়েছে। অন্যদিকে, এর সম্পূরক বইটিও (নির্দেশিকা) অংশগ্রহণমূলক, শিক্ষার্থী-কেন্দ্রিক পাঠদান পদ্ধতির জন্য সহায়ক হিসেবে কাজ করে। উদাহরণস্বরূপ, নির্দেশিকার অধ্যায়গুলি বিভিন্ন সময়ে 'অনুচিন্তার ক্ষেত্র (points of relflection)' তৈরী করে যা বাছাইকৃত আলোচ্য বিষয়গুলোর উপর জটিল চিন্তার উদ্রেগ করে, এবং অধ্যায়-৮- শিক্ষার্থীদের অভিজ্ঞতা থেকে শিক্ষা নেয়ার উপর জোর দেয়। 


\section{শিক্ষার্থী-কেন্দ্রিক, পারস্পরিকভাবে সক্রিয় শিক্ষাদান পদ্ধতির যৌক্তিকতা}

শিক্ষা কোন নিক্কিয়ভাবে গ্রহণ প্রক্রিয়া নয়। মানুষ বরং অবিরত তথ্য সংগ্রহ করতে থাকে এবং তারা যা কিছু শিখে তা নিজেদের জ্ঞান এবং অভিজ্ঞতার আলোকে পুর্ণ ব্যাখ্যা করতে থাকে। এই প্রক্রিয়াকে কার্যকরী পাশাপাশি সম্ভবপর করার জন্য শিক্ষকেরা সক্রিয়ভাবে শিক্ষার্থীদেরকে নিজ থেকে শিখে নেয়ায় নিযুক্ত রাখেন।*

বস্তুত, অনেক গবেষকই এই সিদ্ধান্তে উপনীত হয়েছেন যে, পারস্পারিকভাবে সক্রিয়, শিক্ষার্থী-কেন্দ্রিক শিক্ষা পদ্ধতি অন্যান্য শিক্ষা পদ্ধতিগুলোর চেয়ে অধিকতর ফলপ্রসূ । এরকম পদ্ধতি শিক্ষার্থীদের আকৃষ্ট করে এবং সমালোচনামূলক (Critical thinking) চিন্তাধারায় ত্বরান্বিত করে । যে সকল বিষয়গুলি শিক্ষার্থীদের জীবনে সরাসরি প্রভাব ফেনে এই ধরণের দৃষ্টিভঙ্গি (approach) সেই সকল বিষয়ের উপর তথ্য আত্যীকরণ ও নতুন নতুন দক্ষতা অর্জনে সহায়তা করে ${ }^{8}$

দীর্ঘ সময় ধরে, ৫ৌনতা ও এইচআইভি বিষয়ক অনেক শিক্ষা কার্যক্রনের মট্যে অংশগ্রহণমূলক শিখন দৃষ্টিভঙ্গি প্রধান অবনম্বন হয়ে এলেছে। আরো অনেক অনেক শিক্ষক এখন সমাজবিজ্ঞান, পৌরনীতি, বিজ্ঞান এবং সাহিত্যসহ বিভিন্ন বিষয়ের ক্ষেত্রে এই গতিশীল পদ্ধতি এবং বিভিন্ন রকম “হাতে কলন্ শিক্ষা” কর্মসূচি প্রয়োগ করেন । তারা লক্ষ্য করেন যে, শ্রেণিকক্ষে অংশগ্রহণ ও স্বাধীনভাবে মত প্রকাশ গণতান্ত্রিক মনোভাবকেও উৎসাহিত করে। বাস্তবিকই, বিভিন্ন দেলের জরিপ সূত্রে দেখা গেছে, যে সব শিক্ষার্থীরা এই ধরনের মুক্ত শিখন পদ্ধতিতে পড়াফ্ডনা করেছে তারা জেন্ডার সমতাকে বেশি সমর্থন করে। ৷ পরিশে৫ে, শিক্ষকেরা এই বিবরণ দেন যে, এই পদ্ধতিতে শিক্ষা দিতে গিয়ে তারা নিজেরাই অনেক কিছু শিখছেন ।

* ‘ছাত্র’, ‘শিক্ষার্থী’ এবং ‘অংশগ্রহণকারী’ শব্দগুলো এই বইয়ের সর্বত্র পারস্পরিক পরিবর্তনযোগ্যভাবে ব্যবহার করা হয়েছে । কারণ যৌনতা ও এইচআইভি সম্পর্কে বিদ্যালয়ে, সামাজিক গোষ্ঠী দ্বারা, এবং অন্যান্য ক্ষেত্রে শিক্ষা দেয়া হয়।

পারস্পরিকভাবে সক্রিয়, শিক্ষার্থী-কেন্দ্রিক শিক্ষাদান কী?

পারস্পরিকভাবে সক্রিয়, শিক্ষার্থী-কেন্দ্রিক শিক্ষাদান পদ্ধতি হলো পরস্পর সম্পর্কিত কিছু নীতিমালা এবং সর্বোত্তম চর্চা যা শিক্ষার্থীদেরকে তাদের নিজস্ব শিখন পদ্ধতির সাথে সক্রিয়ভাবে সম্পৃক্ত করে।

এই নীতিমালা এবং এর অনুশীলন চর্চা পরবর্তী পৃষ্ঠায় বর্ণনা করা হয়েছে, যাঢে গুরুত্বারোপ করা হয়েছে: শিক্ষার পরিবেশ, শিক্ষার পদ্ধতি; গভীর চিন্তার দক্ষতা এবং শিক্ষার্থী যা শিখল তা তাদের জীবনের সাথে যোগ করতে সাহায্য করা ।

পারস্পরিকভাবে সক্রিয় এবং শিক্ষার্থীকেন্দ্রিক শিক্ষাদানের জন্য কিছু নাম ব্যবহৃত হয়। বেশিরভাগ ক্ষেত্রেই একে বলা হয় (বা একই রকম মনে করা হয়) অংশগ্রহণমূলক, অভিজ্তणামূলক, বা হাতে-কলমে শিক্ষা । 
অধিকার, জেন্ডার, যৌনতা, এইচআইভি, এবং যৌনস্বাস্থ্য নিয়ে শিক্ষাদানের জন্য বিশেষভাবে লক্ষণীয়:

বিষয়গুলো স্পর্শকাতর হওয়ার কারণে অথবা তাদের ইতিহাস বা পারিবারিক অবস্থার কারণে শিক্ষার্থীরা অস্বস্তিবোধ করতে পারে। কীভাবে অপ্রয়োজনীয় সংকট এড়ানো যায় তার পরিকল্পনা করুন ।

শিক্ষার্থীরা আপনার সাথে অপরিকল্পিত গর্ভধারণ, নির্যাতনের অভিঞ্ঞতা, অথবা অন্যান্য ব্যক্তিগত ও পারিবারিক সমস্যা নিয়ে কথা বলতে পারে। যথার্থ দূরত্ত বজায় রেখে কীভাবে তাদেরকে সহায়তা করবেন তা ভাবুন। স্থানীয় কর্মসূচি/সেবাগুলি (যেমন, গৃহহীন কিশোর-কিশোরীদের জন্য একটি নিরাপদ স্থান অথবা নির্যাতিত হয়েছে এমন নারীদদর জন্য সেবা) সম্পর্কে জানুন। নির্যাতন বা বিপদের ক্ষেত্রে যথাযথ কর্তৃপক্ষকে জানানোর প্রয়োজন সম্পর্কে সঢেতন হোন ।

উপকরণের সাথে আপনার নিজস্ব স্বস্তির মাত্রা নিয়ে ভাবুন এবং প্রয়োজন অনুयায়ী সহযোগিতা ও উপদেশ চান।

সকল নিয়ে একক শিক্ষাক্রম (It'sAll OneCurriculum) সমতা, সম্মান, ও মানবাধিকারের মূল্যবোধকে সমর্থন করে। নিশ্চিত হোন যে, যেভাবে শিক্ষাক্রনে জেন্ডার ও যৌনতার মূল্যবোধকে সম্মান করা হয়েছে তা আপনি আপনার নিজস্ব ব্যক্তিগত বা ধর্মীয় মূল্যবোটে প্রতিফলিত করতে স্বস্তিবোধ করেন ।

\section{পারস্পরিকভাবে, শিক্ষার্থী-কেন্দ্রিক শিক্ষাদানের নীতিমালা}

\section{সমতা, সম্মান এবং মানবাধিকারের ভিত্তিতে শিক্ষার একটি পরিবেশ তৈরি করতন ।}

• শিক্ষার জন্য সহায়ক পরিবেশ তৈরি করুন । সকল শিক্ষার্থীদের নিজেদের অন্তর্ভুক্ত হওয়া, ওনতে পারা, স্বস্তিবোধ করা এবং হাস্যকর পরিস্থিতি থেকে মুক্ত মনে করা উচিত, বিশেষ করে যখন তারা কোন নতুন ধারণা বা চিন্তা সম্পর্কে জানছে। সকলের অংশগ্রহণকে উৎসাহিত করুন বিশেষ করে যারা নিঃসঙ্গ বা ভীত অনুভব করে। (বিভিন্ন অবস্থা এবং পরিস্থিতি এ ধরনের অনুভূতির সূত্রপাত করতে পারে। যেমন- জেন্ডার, সামাজিক শ্রেণি বা বয়সের সাথে সম্পৃক্ত সামাজিক ক্ষমতার পার্থক্য একটি কারণ হতে পারে। বিদ্যালয়ে যে ভাষায় কথা বলা হয় শিক্ষার্থী সেই ভাযায় কম সাবলীল হনে আলোচনায় অংশগ্রহণ করা থেকে বিরত থাকতে পারে। শারীরিক প্রতিবন্ধী এবং যার অন্য কোন বিশেষ চাহিদা আছে তারা লজ্জা পেতে পারে।) ৫ে সকল কর্মকাণ সম্মান বৃদ্ধি ও দল গঠনকে উন্নত করে এবং নেতৃত্বৈর সুয্যাগ প্রসারিত করে তা কাজে লাগিয়ে আপনি শ্রেণিকক্ষে অন্তর্ভুক্তি বৃদ্ধি করতে পারেন। অবশ্যই পারিপার্শ্বিক নিরাপত্তা ও গোপনীয়তা নিশ্চিত করাটাও জরুরী।

• সেশনের শুরুতেই শিক্ষার্থীদের নিজেদের জন্য কী করে একটি সম্মানজনক শ্রেণিকক্ষ অর্জন করা সম্ভব তার একটি তালিকা তৈরী করতে একত্রে কাজ করতে বলুন ।

• শিক্ষার্থীদেরকে অন্য মানুযের গোপনীয়তাকে সম্মান করতে বলুন। এবং তাদের মনে করিয়ে দিন যে সব তথ্য তারা গোপনীয় বলে মনে করে সেসব তথ্য তারা যেন অন্যদের কাছে প্রকাশ না করে। মনে রাখতে হবে, শ্রেণীতে আলোচিত সংবেদনশীল বিষয় নিয়ে পরবর্তীতে শ্রেণীপাঠ শেষে কিছু শিক্ষার্থী অন্যদেরকে ভয়ভীতি দেখাতে পারেন এমনকি অসম্মান বা হয়রানিও করতে পারেন । শিক্ষার্থীদের আশ্বস্ত করুন যে, আপনি, তাদের শিক্ষক হিলেবে সকল আলোচনা গোপন রাখবেন। এই ভাবে আপনি অন্যদের গোপনীয়তাকে সম্মান করার জন্য একচন অনুকরণীয় আদর্শ হয়ে উঠতে পারেন । ${ }^{\text {- }}$

• যে সব বিষয়ে আলোচনা করতে তারা অস্বস্তিবোধ করে সেসব বিষয়ে আলোচনা না করার অধিকার তাদের রয়েছে।

• যতটা সম্ভব বিনোদনমূলক কর্মকান্ডের মাধ্যনে শিখনকে আনন্দদায়ক করে তুলুন । 
২ শিক্ষার্থীরা ইতিমধ্যে একটি বিষয় সম্পর্কে যা জানে ও ভাবে তার সাথে তাদের অভিজ্ঞতা, নতুন তথ্য এবং ধারণা

যোগ করে একটি প্রক্রিয়া চালু করুন ।

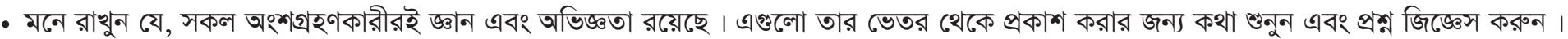

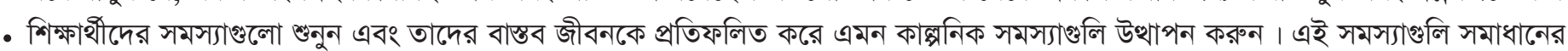
ক্ষেত্রে, তাদের নিজস্ব পছন্দ টৈরি করতে এবং একইতাবে তাদদর ভাবনা বিকাণের জন্য তাদদর সম্পত্ত করুন ।

• শিক্ষার্থীদদরকে তাদদর নিজজদের জীবনের কোন একটি সমস্যা বলতে কী বোঝায় তা জানতে উৎসাহিত করুন।

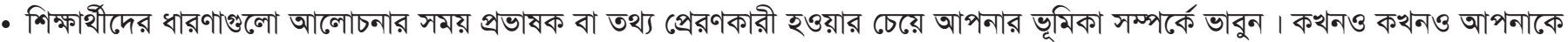
শিক্ষার্থীদদর বাস্তব ঘটনা সম্পর্কিত ভুল ধারণা সঠিক করতে অথবা কখন একটি মন্ত্য অসম্মানজনক হয় তা বুঝটে সহায়তা করতে হতে পারে।

• মাঝে মাঝে, আপনি আপনার নিজস্ব অভিজ্ঞতা থেকে পাঠটির সাথে সম্পৃক্ত একটি উদাহরণ দিতে পারেন। যাই হোক, শিক্ষার্থীদদর সাথে আপনার যথার্থ দূরত্ব বজায় রাখতে অতিমাত্রায় সতর্ক থাকুন।

৩ শিক্ষার্থীদেরকে সম্পৃক্ত করতে ভিনমাত্রার কৌশল ব্যবহার করুণ

• অনেকগুলো পদ্ধতি ও পথ টৈরি করুন যেগুলো আপনি আপনার শিক্ষার্থীদের শিখন চাহিদার জন্য গ্রহণ করতে পারবেন । [পারস্পরিকভাবে সক্রিয় শিক্ষাদান পদ্ধতির ওপর b-৯ নম্বর পৃষ্ঠাগুলি দেখুন।]

• আপনি যদি এমন কোন পাঠের বা অধিবেশনের পরিকল্পনা করেন যা আপনার কাছে নতুন তাহলে প্রথমে অধিবেশনটি এবং এ সম্পর্কিত পাঠ্যসূচি যা আপনি পড়াতে মনস্ত করেছেন তার শিখন উদ্দেশ্যগুলি সতর্কভাবে পর্যালোচনা করুন । পূর্বেই সকল উপকরণ সংগ্রহ করুন।

• নমনীয়তার সাথে আকষ্মিক কোন পরিস্থিতি মোকাবেলা করার জন্য একটি সহায়ক পরিকল্পনা গ্রহণ করুন (যেমন- সময় নির্ধারণ, আবহাওয়া বা শিক্ষার্থীদের প্রতিক্রিয়া)

• পরীক্ষামূলক শিখন পদ্ধতি ও পাঠের বাছাইকৃত গ্রন্থপঞ্জির তালিকার জন্য এই বইয়ের শেমের অতিরিক্ত তথ্য সম্পদ অংশ দেখুন।

• যতটা সম্ভব, বিনোদনমূলক কর্মকাত্ডের মাধ্যন্ শিখনকে আনন্দদায়ক করে তুলুন !
অধিকার, জেন্ডার, যৌনতা, এইচআইভি এবং যৌনস্বাস্থ্য বিষয়ে শিক্ষাদানের জন্য বিশেষভাবে লক্ষণীয়:

यদি আপনি একটি সহশিক্ষার পরিবেশে থাকেন তবে সম্মিলিতভাবে বোঝা ও খোলামেলা পরিবেশের জন্য কিছু কিছু অধिবেশন অথবা আলোচনার ক্ষেত্রে পরবর্তী আলোচনার জন্য দলটিকে পুনরায় একত্র করার পূর্ব্রে ছেলেদের থেকে মেয়েদের আলাদা করার চেষ্টা করুন । প্রয়োজনমতো একই কক্ষের পৃথক জায়গা ব্যবহার করুন। 


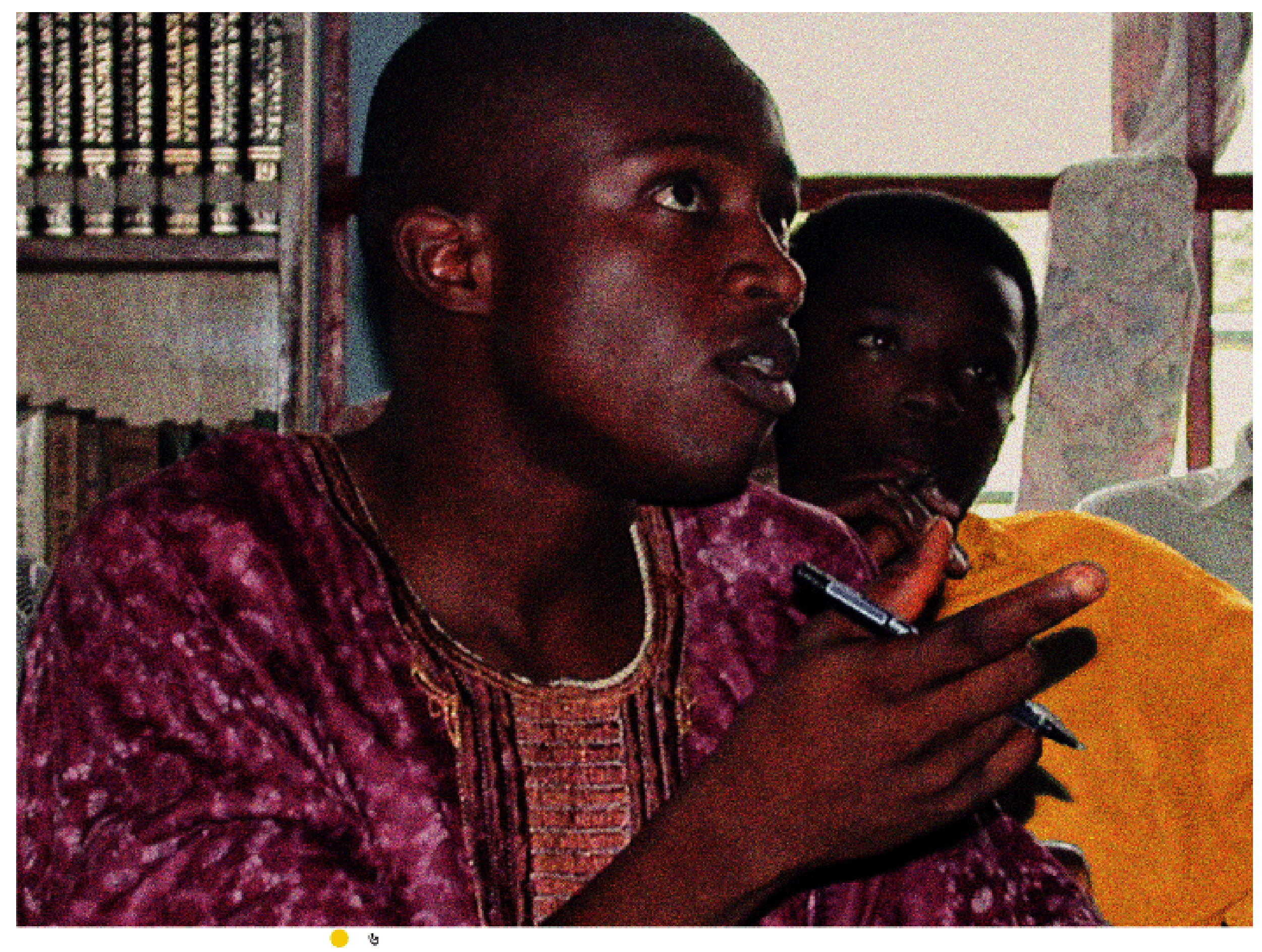


8 শিক্ষার্থীদের প্রকাশ ক্ষমতা, পড়ার ক্ষমতা, তাদের নিজেদের জীবন ও চারপাশের জগৎ সম্পর্কে গভীরভাবে চিন্তা করার ক্ষমতা এবং সমস্যা সমাধানের ক্ষমতার ওপর তৈরি।

• শিক্ষার্থীদেরকে গতানুগতিক জ্ঞান নিয়ে প্রশ্ন করতে উৎসাহিত করুন । তাদেরকে তাদের বিশ্বাস ও তাদদর সমাজের বৈশিষ্ট্য ও রীতি-নীতি সম্পর্কে ভাবতে বলুন । নিজেদের থেকে ভিন্ন মতাম কে সম্মানের সাথে প্রকাশ করতে বলুন।

• উনুক্ত প্রশ্নের মাধ্যমে আলোচনা শুরু করুন । ভেতরকার দ্বন্ধ ও গভীরতর সত্য জানার জন্য শিক্ষার্থীদের গ্রীক দার্শনিক পদ্ধতি অনুযায়ী ‘কেন’ প্রশ্ন করতে বলুন। নির্দেশিকার ১ থেকে ৭ পর্যন্ত অধ্যার়ে ‘অনুচিন্তার ক্ষেত্র’ আছে। এগুলো গভীর সমালোচনামূলক বিশেশবণ এবং আনোচনায় উৎসাহিত করে।

• সৃজনশীলতাকে উৎসাহিত করুন ।

• শিক্ষার্থীদেরকে তাদের ভাবনার বিষয়ে ঝুঁকি নিতে ও ভুল হুেে ভীত না হতে উৎসাহিত করুন। আপনি নিজে থেকে এমন আচরণ প্রদর্শন করতে আগ্রহী হোন।

• বিষয়সমূহ খেঁ|জার এবং দলীয় কার্যক্রনে সম্পৃক্ত করার জন্য ছোট দলে বিন্যস্ত করুন ।

• সমস্যা বিশেষণ ও সম্ভাব্য সমাধান সুপারিশ করার জন্য বিভিন্ন দৃষ্টিকোণ থেকে উৎসাহিত করুন ।

৫ শিক্ষার্থীরা যা শিখল তা তাদের জীবনে ও সমাজে যেন প্রয়োগ করতে পারে সে সামর্থ্যকে উৎসাহিত করুন । যা তাদেরকে সক্রিয় নাগরিক হতে সহায়তা করবে এবং ইতিবাচক পরিবর্তনে তাড়িত করবে।

• এমন পাঠ্যসূচি বাছাই করুন যা প্রাসঙ্গিক এবং এমন শিক্ষণ পদ্ধতি বাছাই করুন তেটি সমালোচনামূলক চিন্তাকে উৎসাহিত করে। শিক্ষার্থীদেরকে বারবার পাঠ্যসূচিগুলোকে নিজেদের জীবন ও জগতের সাথে সম্পৃক্ত করতে বলুন। উপকরণগুলো তাদের পক্ষপাতহীনভাবে ও সামাজিক ন্যায়বিচারের আলোকে দেখার জন্য উৎসাহিত করুন ।

• সেবা বা সমাজভিত্তিক গবেষণা ও কার্যক্রম অন্তর্ভুক্ত করে এমন শিখনের সুযোগ একত্রিত করুন । যেখানে আপনি কাজ করছেন সেখানকার সংস্কৃতি সম্পর্কে সচেতন হোন যেন আপনি আপনার শিক্ষার্থীদেরকে ক্ষতিকর কোন দিকে না নিয়ে যান ।

• আপনার প্রতিষ্ঠানের (এবং সম্ভবত কমিউনিটির) মধ্যে পরামর্শ দেয়ার প্রয়োজন আছে কিনা এবং কীভাবে তা যুবসমাজের কথা বলার সামর্থ্য ও অধিকার সম্পর্কে এবং তাদের নিজেদের মতামতের ওপর ভিত্তি করে কাজ করার জন্য প্রয়োজন তা ভেবে দেখুন।

• মনে রাখুন যে, যুবসমাজ প্রায়ই তাদদর শিক্ষক ও সমাজের নেতাদের কাছ থেকে উৎসাহ প্রায়।
অধিকার, জেন্ডার, যৌনতা, এইচআইভি এবং

যৌনস্বাস্থ্য বিষয়ে

শিক্ষাদানের জন্য

বিশেষভাবে লক্ষণীয়:

সচেতন হোন যে শিক্ষার্থীরা কিছু মৌলিক অনুমান পৃনর্বিবেচনা করতে পারে যা তারা সব সময়ের জন্যই স্বীকৃত হিলেবে জেনে এসেছে। যখন তারা শেখে কীভাবে বাড়িতে ও সমাজে তাদদর স্থায়িত্ব ও নিরাপত্তা রক্ষা করতে হয় তখন তাদের আদর্শ ও আকাজ্কারে সামঞ্য্য করতে সহায়তা করুন ।

এই প্রসজে পাঠদান করতে গেলে আপনি বাধার সম্মুখীন হতে পারেন। আপনার প্রতিষ্ঠানে ও সমাজে কে আপনার প্রধান সমর্থক/মিত্র তা জানুন । তাদের সম্পর্কেও সচেতন হোন যারা আপনার চেষ্টাকে বিরোধিতা করতে পারে। সমর্থন ও পরামর্শ 


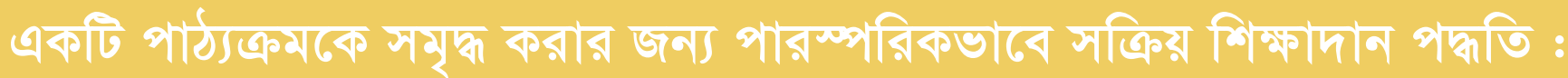

নানামুখী পদ্ধতির ব্যবহার আপনাকে আরো বেশি পরিসরের শিক্ষার্থীর কাছে পৌঁছাত সক্ষম ও শিক্ষার্থীদেরকে সম্পৃক্ত রাখতে সাহায্যে করে । এই পন্থায় শিক্ষার্থীরা বিভিন্ন বিষয়ের মাঝে সৃজনশীল যোগসূত্র তৈরি করতে সমর্থ হয়। শিক্ষার্থীদের আগ্রহ বৃদ্ধি করার জন্য মৌলিক নির্দেশনাসহ কিছু বহুল ব্যবহৃত পদ্ধতি ও এগুলোর ব্যবহার নিচে দেওয়া হল ।

উদ্গীপক - এগুলো হল কোন দল সম্পর্কে ইতিবাচক অনুভূতি বৃদ্ধির জন্য সাজানো সংক্ষিপ্ত দলীয় কর্মকাণ্ত। এগুনো দলীয় শক্তিকে গুরুত্ব দেয় এবং নতুন চিন্তা ধারা প্রকাশের জন্য ব্যবহার হয়। এ সকল কর্মকাণ্ড সৃজনশীল চিন্তা করতে সহায়তা করে। এগুনো বিলেষভাবে একটি সেশনের শুরুতে বা আলোচনার বিষয় পরিবর্তন করতে সাহায্য করে। কয়েকটি উদাহরণ হলः জড়তা ভশ, নাম নিয়ে খেলা, গান এবং শরীর চর্চা।

অালো|তনার মৃড়া - এ সকল কর্মকাণ্ড আলোচনার বিষয় উত্থাপন করে এবং পরবর্তীতে আরো জানার জন্য শিক্ষার্থীদের মাঝে প্রশ্ন তৈরি করে। এগুলো সাধারণত একটি পাঠের শরুতে সবতেয়ে বেশি কার্যকরী হয় । কয়েকটি উদাহরণ হলः চিন্তার ঝড়, কেসস্টাডি, দ্রংত লিখিত জবাবের জন্য ব্যবহৃত বিভিন্ন প্রশ্ন অথবা উক্তি,একমত/দ্বিমত অনুশীলন, সমস্যা চাপিয়ে দেয়া, এবং স্বল্পদৈর্ঘ্য চলচ্চিত্র ও পড়া।

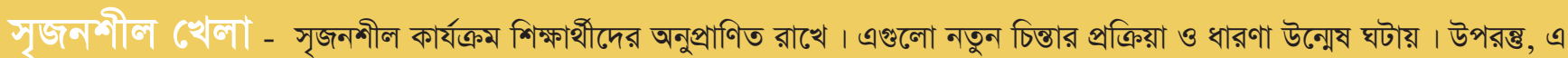
সকল কার্যক্র সেই সকল শিক্ষার্থীদের অর্থপূর্ণভাবে অংশ নিতে সহায়তা করে যারা এ ধরনের খেলায় শেখে ও বেশি যোগায়াগ করতে পারে। কয়েকটি উদাহরণ হলः খেলা, চিত্রকর্ম, অভিনয় ও নাটক, কবিতা ও সৃজনশীল লেখা। 
দলীয় অালো|তনা - শিক্ষার্থীদের নিজেদেরকে প্রকাশ করার, নিজের কথা শোনানোর এবং অন্যের কথা শোনার জন্য সুযোগের প্রয়োজন । এগুলো তাদের কথা বলার ও শোনার দক্ষতা বৃদ্ধি করে । এগুলো তাদের চিন্তার দক্ষতা পরিলোধন এবং তাদের ভাবনা ও জ্ঞানকেও বিস্তার করতে পারে । উপরন্তু আপনি প্রত্যেককে অংশগ্রহণ করার একটি সুযোগ দিয়ে এবং গণতান্ত্রিক মূল্যবোধ ও সংস্কৃতি উৎসাহিত করতে এই ধরনের অধিবেশন ব্যবহার করতে পারেন । কয়েকটি উদাহরণ হল: অনানুষ্ঠানিক কথোপকথন, নির্বাচিত বক্তাদের আলোচনা ও বির্তক ।

অং: কাজের জন্য আপনার শ্রেণির শিক্ষার্থীদেরকে ভাগ করে। কয়েকটি উদাহরণ হল: এলাকার মানচিত্র তৈরি, 'সমস্যা বৃক্ষ’, গবেষণা প্রকল্প এবং গণমাধ্যমের বার্তা বিশেষণ।

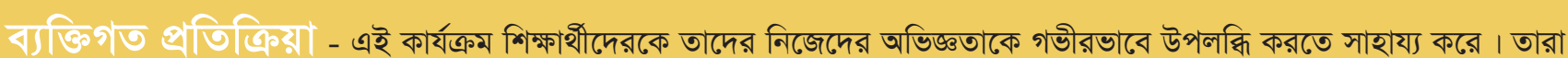
পরিপক্কতা ও বিচারবোধ লালন করে। তারা মনোভাব ও আচরণের নতুন দরজা খুনে দিতে পারে। কয়েকটি উদাহরণ হল: একটি পত্রিকা রাখা, স্মৃতিকে পরিচালিত করা, মূল্যবোধের স্পষ্টতা ও সৃজনশীল চিত্রকর্ম প্রকল্প ।

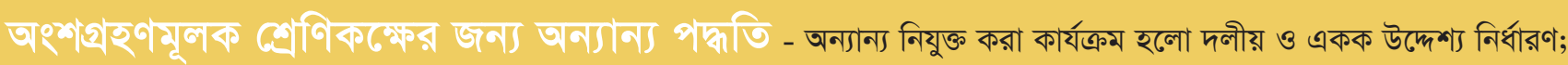
শ্রেণিকক্ষ ব্যবন্থাপনায় সহায়তা করতে অংশগ্রহণমূলক কাজ নির্দিষ্টকরণ (দিনের প্রতিটেদন লেখক, উক্তি পাঠক, হাজিরা লেখক, দিনের নেতা); এবং পাঠ ও যা শেখা হয়েছে তা পুনরায় পড়া। 


\section{একটি শিক্ষাক্রম তৈরি ও বাস্তবায়নের জন্য বিশেষ বিবেচ্যগুলি}

সকল নিয়ে একক শিক্ষাক্রমটি (It'sAll OneCurriculum) বিশ্বের সবার জন্যই উপযুক্ত। এটি সর্বজনীন নীতির ভিত্তিত টৈরি; সুতরাং আপনারা আপনার স্থানীয় ক্ষেত্রে বা মানুভের শিক্ষার জন্য এখান থেকে বিষয়বস্তু নিয়ে একটি কার্যকরী শিক্ষাক্রম বা শিক্ষার জন্য একটি অধ্যায় তৈরি করতে পারেন । এটি করার জন্য কিছু পরিসরের শিক্ষা ও কর্মসূচির বিষয়ে আপনার মনোযোগ প্রয়োজন যেখানে শিক্ষকদের দক্ষতা, শিক্ষার্থীদের সামর্থ্য, মা-বাবার প্রস্তুতি, সামাজিক মূল্যবোধ এবং বিষয়টির জন্য আপনার কাছে শ্রেণিকক্ষের পর্যাপ্ত সময় অন্তর্ভুক্ত। যখন আপনি একটি শিক্ষাক্রম তৈরি করবেন তখন এই বিষয়গুনো বিবেচনায় নেয়ার জন্য নিচের প্রশ্নগুলো আপনাকে নিদের্শনা দেবে। এগুনোর কিছু বিষয় অন্য মানুভের দায়িত্ব হতে পারে- যেমন, বাস্তবায়নকারী সংস্থার কর্মী বা সহায়ক সামাজিক ব্যক্তিবর্গ। যাই হোক, এই বিষয়গুলো এখানে অন্তর্ভুক্ত করা হয়েছে কারণ এই বিষয়গুলো যৌনতা বা এইচআইভি সংক্রমণ প্রতিরোধ নিয়ে সফল শিক্ষাক্রম প্রতিষ্ঠিত করার মূল কাজ হতে পারে। (যে প্রাসঙিক বই ও উপকরণের যোগসুত্র এই বিষয়গুনোর ওপর আরো বিস্তারিত নির্দেশনা দিয়েছে তার জন্য অতিরিক্ত তথ্য সম্পদের অংশ দেখুন।)

\section{নীতি বিষয়ক বিবেচ্যগুলি}

জাতীয় এবং স্থানীয় নীতি ও সম্পদগুলো কী কী, যা এই শিক্ষাক্রম প্রতিষ্ঠিত করতে আপনি কীভাবে সফল হবেন তাকে প্রভাবিত করতে পারে? যেমন, কী কী আইন বা নীতি বিদ্যালয়ে যৌনতা এবং/অথবা এইচআইভি প্রতিরোধ শিক্ষাকে নিয়ন্ত্রণ করে? প্রতিবন্ধী যুব সমাজের কাছে যৌন শিক্ষা পৌঁছানো নিশ্চিত করার জন্য কী কী উদ্দ্যোগ নেয়া হয়েছে? যে সকল গবেষণা কিশোর বয়সে গর্ভধারণ, যৌনবাহিত সংক্রমণেরে হার, জেন্ডার সমতা এবং মানবাধিকারের মধ্যে যোগসুত্র দেখায় সে সকল গবেষণা সম্পর্কে কর্মসূচি ব্যবস্থাপকরা কি সচেতন ? এই যোগসুত্রগুনো বিবেচনা করার জন্য তারা কি কর্মসূচিগুনো সাজিয়েছে? এখানে কি নানা ধরনের অর্থের উৎস আছে যেখান থেকে আপনার কর্মসূচি বা বিদ্যালয় অর্থ পেতে পারে ? আপনার কি সমাজের মূল নীতিনির্ধারক, আপনার সংস্থার কর্মী বা অন্য ব্যক্তিদের লক্ষ্য করে এ্যাডভোকেসি কার্যক্রম নেয়া উচিত? কারা আপনার প্রধান সমমনা দল/ব্যক্তি? 


\section{পরিকল্পনা বিষয়ক বিব্বে্যকুলি}

সমাজের বৃহত্তর শিক্ষাখাতের উদ্দেশ্য ও লক্ষ্যের সাথে এই পাঠ্যক্রম কি যথার্থ? পরিকল্পনা প্রণয়নের জন্য মূল ইস্যুগুলো কী কী? এই মূল বিবেচ্য বিষযগুনো কী আপনি কতটা সফলতার সাথে পাঠ্যক্রমটি বাস্তবায়িত করেছেন তার উপর প্রভাব ফেলবে? ওরুতেই কি ধরণের প্রাথমিক বিবেচনা করে নেয়া (বা অবশ্যই করা) প্রয়োজন? যেমন ধরুন আপনি কি নিশ্চিত হবেন যে, এই পাঠ্যক্রনের প্রধান কর্মকর্তাগণ এবং অংশীদাররা এই পাঠ্যক্রমের গুরুত্ব বুঝেছেন এবং এটাকে সফলভাবে সচল করবার জন্যও প্রতিশ্রুতিবদ্ধ? কীভাবে প্রকল্পের সংগঠকরা পিতামাতা এবং সমপ্রদায়ের সমর্থনকে জোরালো করতে পারে ? সন্তানের সাথে তাদের পিতা-মাতার যৌন স্বাস্থ্য নিয়ে যথাযথভাবে কথা বলার জন্য কী কী ব্যবস্থা বিদ্যামান রয়েছে? এই ধরণের সহায়তা প্রদানের অভিজ্ঞতা থেকে প্রকল্পের সংগঠকরা কীভাবে নানান বিষয় শিখতে পারে ? বিদ্যামান স্বাস্থ্য, পরামর্শ ও অন্যান্য রেফারেল সেবার সাথে যোগাযোগের জন্য তাদের সবচেয়ে ভালো উপায়গুলো কী ? এই প্রকল্পের উন্নয়নের জন্য নীতি নির্দেশক কমিটি বা কর্মী দলের সাথে কারা অংশগ্রহণ করতে আগ্রহী? যেমন ধরুন, সমাজের সদস্যরা, যুব সমাজ, পিতা-মাতা, শিক্ষক প্রতিনিধি, শিক্ষক ও এনজিও এর প্রতিনিধি এরা সকলেই গুরুত্বপূর্ণ মতামত দিতে পারেন । এই প্রক্রিয়া আপনাকে প্রয়োজনীয় জ্ঞান চিহ্নিত করতে ও আহরণ করতে সহায়তা করবে। এটি কর্মসূচীকে আরো শক্তিশালী ও স্থানীয়ভাবে আরো উপযুক্ত করবে এবং সমাজের সদস্যদের মধ্যে কর্মসূচীর জন্য মালিকানাবোধ তৈরি করবে। এই কর্মসূচী সম্পর্কে সমাজের সদস্যদের সাথে যোগাযোগ করার ও শিক্ষা দেয়ার জন্য কী সুয্যাগ সুবিধা বিদ্যামান রয়েছে? এই প্রকল্পের শুরুতে আপনি কিভাবে সর্বোত্তম ধারণা একত্রীকরণ করতে পারেন? যেমন- আপনি সমাজের নেতা, বিশেষভাবে বিবেচিত দলগুলির সাথে মুখোমুখি আলোচনার কথা ভাবতে পারেন, শিক্ষাক্রম সম্পর্কে সমাজের মানুযের যে বিশেষ উদ্বেগগুলো থাকতে পারে, তা আপনি কীভাবে সবচেয়ে ভাল করে টৈরি করতে পারেন ?

\section{সম্পদের চরম সীমাবদ্ধতা রয়েছে এমন ক্ষেত্রে ব্যবহার করার জন্য একটি শিক্ষাক্রম তৈরি করা}

একটি বিশেষ স্থানে আপনার কাছে কী কী সম্পদ পর্যাপ্ত আছে? দারিদ্র্য যেভাবে জেন্ডার, অধিকার, যৌনতা, ও যৌনস্বাস্থ্যের সাথে পারস্পরিক ক্রিয়া প্রতিক্রিয়া করে তা বোঝাতে শিক্ষাক্রমের পাঠ্যসূচি কীভাবে খাপ খাওয়ানো যায় ? স্বল্প সম্পদপূর্ণ স্থানে কীভাবে আপনি শিক্ষাক্রমটি খাপ খাওয়াতে পারেন ? যেমন- আপনি কি মৌখিক শিক্ষণ কৌশলগুলি অথবা পাঠদানের জন্য উনুক্ত স্থানের ওপর আস্থা রাখেন? বেসরকারি সংস্থা বা সমাজের সদস্যরা নগদ অর্থের বদলে অন্য কোনো প্রকার সাহায্য করতে পারে কি? 


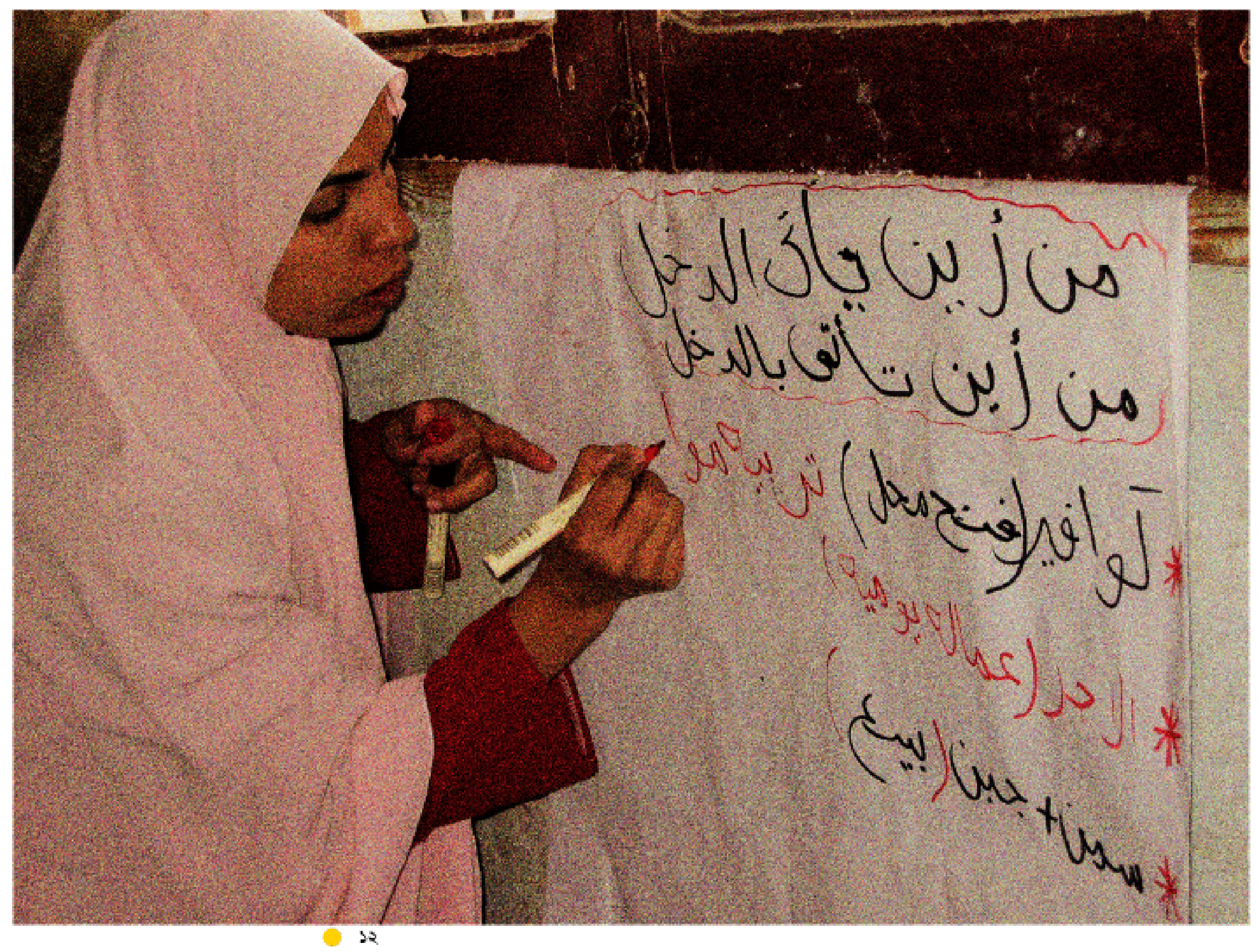




\section{আপনার শিক্ষাত্রমটিকে প্রাতিষ্ঠানিক শিক্ষণ মানের সাথে সংযুক্ত করা}

এই বইয়ের প্রতিটি অধিবেশনে সাধারণ প্রাতিষ্ঠানিক দক্ষতার সাথে সম্পর্কিত একটি করে উদ্দেশ্য আছে (যেমন- উনুক্ত লেখা, বর্ণনামূলক লেখা, সমস্যা সমাধান ও বিশেযণ, সাধারণ গবেষণা প্রকল্প পরিকল্পনা ও বাস্তবায়ন, বা জন সম্মুখে কথা বলা)। এই পাঠ্যক্রম স্বাস্থ্য শিক্ষা ও জীববিদ্যার জায়গায় যৌনতা ও এইচআইভি শিক্ষাকে অন্তর্ভুক্ত করার জন্য কাজ করে এবং কীভাবে এই কার্যক্রম শিক্ষক ও বিদ্যালয়ের শিক্ষা উদ্দেশ্য পূরণ করে তাও স্পষ্ট করে। বিদ্যালয়ের জন্য একটি শিক্ষাক্রম তৈরি করতে এই প্রশ্নগুলো বিবেচনা করুন: একটি নির্দিষ্ট বিষয়বস্তুর জন্য পূর্বে বিদ্যমান কোন শিক্ষার মানগুলি অথবা মূল্যায়নের মাপকাঠিগুলি অবশ্যই পূরণ করতে হবে? নির্দেশিকা বইয়ের কোন অধ্যায় বা অংশ আদর্শ শিক্ষার মানগুনোর সাথে সবচেয়ে ভাল যোগসুত্র তৈরি করে? নিয়লিখিত অধিবেশনগুনোর কোন কোন প্রাতিষ্ঠানিক উদ্দেশ্য ঐ মানগুনো অর্জনে সবচেয়ে বেশি সহায়তা করতে পারে? এইচআইভি প্রতিরোধ বা জেন্ডার সমতা বৃদ্ধি করতে বিভিন্ন শিক্ষা শাখার মধ্যে সমন্বয়ের জন্য কী কী সুয়াগ রয়েছে? তাছাড়া মূলধারার শিক্ষাক্রমের সাথে প্রতিযোগিতায় কীভাবে যৌন/এইচআইভি শিক্ষার একটি সৃজনশীল পদ্ধতি সার্বিকভাবে শিক্ষার মান বৃদ্ধি করে?

\section{বিশেষ জনগোষ্ঠীর জন্য প্রাসঙ্গিকভাবে খাপ খাওয়া|নো}

শিক্ষকেরা কীভাবে নির্দিষ্ট জনগোষ্ঠীর চাহিদার সাথে সামঞ্জস্যপৃর পাঠ্যসূচি ও কার্যক্রমগুলি নিশ্চিত করেন? যেমন- আপনি কি করে স্বল্প শিক্ষিত বা সংশোধিত গোষ্ঠীর জন্য প্রস্তাবিত কার্যক্রমগুলির পরিবর্তন করেন ? আপনি কি নিশিত করতে পারেন যে, এই বিষয় বস্তুগুলো কিছু কিছু গোষ্ঠীর জন্য বিশেষ গুরুত্ব বহনন করে যেমন- অল্পবয়সী অভিবাসী শ্রমিক, বিবাহিত কিণোরী মেয়ে, সৈনিক, বা এতিমদের বিশেষ উদ্বেগের বিষয়গুনোকে প্রতিফলিত করে? এই সকল জনগোষ্ঠী শিক্ষার ক্ষেত্রে যে বাস্তব বাধার মুখোমুখি হয় সেগুনো আপনি কীভাবে সমাধান করবেন? আপনি কি একটি নিরাপদ স্থানে প্রবেশের সুবিধা দিতে পারবেন? আপনি কি শ্রেণিকক্ষে পাঠদানকে একটি স্বল্প সময়ের ছকের উপযুক্ত করতে পারবেন? যে সকল শিক্ষার্থীর সামাজিক প্রতিষ্ঠানের সাথে অল্প যোগাযোগ আছে কীভাবে আপনি তাদের কাছে পৌঁছাবেন? কোন সামাজিক সম্পদ ও রেফারেলগুলি তারা সম্ভবত বেশি পেতে চায়?

\section{শিক্ষকদের প্রস্তুতি}

শিক্ষকরা কি পারস্পরিকভাবে সক্রিয়, শিক্ষার্থী-কেন্দ্রিক কৌশলগুলি গ্রহণ করতে প্রস্তুত? তাদের কি এ ধরনের দক্ষতা বৃদ্ধির সম্ভাবনা আছে? এই দক্ষতার সাথে শিক্ষাক্রমটি মানানসই করার সর্বোত্তম উপায় কী? কী ধরনের প্রশিক্ষণ শিক্ষকদেরকে থৌনতা ও জেন্ডার সম্পর্কে তাদের ব্যক্তিত ও ধর্মীয় বিশ্বাস পরীক্ষা করতে সক্ষম করবে যেন তারা আন্তর্জাতিক মানবাধিকার নীতির সাথে সংগতিপূর্ণ হয় সেই উপায়ে বিষয়গুলো পড়াতে আরো ভালোভাবে প্রস্তুতি নিতে পারে? চরম রক্ষণশীল সমাজে সবচেয়ে কার্যকরীভাবে কাজ করার জন্য কী শিক্ষকদেরকে সাহায্য করবে? 


\section{মূল্যায়ন বিষয়ক ভাবনা}

অনেক শিক্ষাবিদ তাঁদের যৌনতা ও এইচআইভি কর্মসূচিগুনোর প্রভাব নির্ণয় করতে চাইবে। কি ফলাফল বা মান নিয়ন্ত্রকগুলি কর্মসূচির উদ্দেশ্য অর্জনের অগ্রগতি সবচেয়ে ভালভাবে দেখাতে পারবে ? যেমন- শিক্ষার্থীদের কি জ্ঞান, দৃষ্টিভঙ্গি ও নির্দিষ্ট আচরণের পরিবর্তন হবে? কর্মসূচিটি কি ঘনিষ্ঠ সম্পর্কের মধ্যকার বাধ্যকরণ ও সহিংসতা কমাবে? এটি কি শিক্ষার্থীদেরকে বিদ্যালয় বা একটি শিখন কর্মসূচিতে রাখতে সাহায্য করবে? এটি কি নাগরিক অংশগ্রহণ বৃদ্ধি করবে? নতুন শিক্ষা কর্মসূচির কি একটি পরীক্ষামূলক অংশ থাকবে যা তাড়াতাড়ি শিক্ষা নেওয়া ও সমন্বয়সাধনে সাহায্য করতে পারে?

\section{সহায়তা খুঁজুন ও সক্রিয় থাকুন}

আপনি যৌনতা, জেন্ডার ও মানবাধিকারের ওপর একটি শিক্ষাক্রম তৈরি ও প্রতিষ্ঠিত করার জন্য কাজ করতে থাকবেন। অনেক স্থানে, এই কাজ সন্দেহ ও বিরোধিতার মুখোমুখি হতে পারে। এমন প্রতিক্রিয়া হতে উত্তরণে সাহায্যের জন্য পূর্বেই সমাজের সদস্য ও প্রতিষ্ঠানের সাথে সংযোগ স্থাপন করুন । আপনি কাজ ঋরুর পূর্বে মা-বাবাসহ সমাজের মূল সদস্যদের সাথে অর্থপূর্ণভাবে সম্পৃক্ততা নিশ্চিত করুন । এই মানুযদের সাথে খোলানেলা ও সৎ যোগাযোগ রক্ষা করুন । আপনার নিজেকে ও কর্মসূচি অংশীদারদেরকে নীতিবান রাখা গুরুত্বপূর্ণ। কর্মসূচির প্রতিক্রিয়া জানতে সময় নিন । এটি কীভাবে এগিয়ে চলছে? এটি কি তার উদ্দেশ্য পূরণ করছে? কোনো পাঠ্যসূচির সংশোধন কি প্রয়োজন ?

আপনার অর্জনকে শ্রুদ্ধা করুন এবং কখনো উদ্যম ছেড়ে দেবেন না। 


\section{প্রান্তটীকাগুলি}

১ অনুগ্রহপূর্বক লক্ষ্য করুন যে, এই বই শিক্ষার্থী কেন্দ্রিক, পারস্পরিকভাবে সক্রিয় শিক্ষা প্রদানের একটি সংক্ষিপ্ত ধারণা দেয়। এটি প্রশিক্ষণ কর্মশালা, প্রথম পাঠ্যপুস্তক, অথবা এই বিষয়ে সাবগ্তন্ত বা ম্যানুয়ালের অন্তর্ভূক্ত বা বিকল্প নয়। এই বইয়ের শেযে অতিরিক্ত তথ্য সম্পদের অংশ্শে এই বিষয়ের বাছাইকৃত গ্রন্থ ও উপকরণগুলি দেয়া আছে

2 Worldwide, learner-centered, interactive, teaching has an important foundation in Paulo Freire's theory of "critical pedagogy." For evidence related to outcomes including academic achievement, students' attitudes, students' retention, and self-esteem, see: Gross Davis, Barbara. 1993. Tools for Teaching San Francisco: Jossey-Bass Publishers: Manswell Butty, Jo-Anne. 2001 "Teacher instruction, student attitudes, and mathematics performance among 10th and 12 th grade Black and Hispanic students," Journal of Negro Eduction 70(1/2): 19-37; Prince, Michael. 2004."Does learning work? A review of the research," Journal of Engineering Education 93(3):223-231. Slavin Robert, 1980 "Co Engineering Education $93(3): 223-231$; Slavin, Robert. 1980 "Cooperative

In some settings, educators also refer to critical thinking skills as metacognition. For a discussion of metacognitive skills and . 2006. "What is metacognition?" Phi Delta Kappon 87(9):696-699.

4 Kirby, Doug. 2007. "Emerging Answers 2007: Research Findings on Programs to Reduce Teen Pregnancy and Sexually Transmitted Diseases." Washington, DC: National Campaign to Prevent Teen and Unplanned

Pregnancy.

5 Pettersson, T. 2003. "Basic Values and Civic Education: A Comparative Analysis of Adolescent Orientations Towards Gender Equality and Good Citizenship." World Values Survey.

<www.worldvaluessurvey.org/ library/>. Accessed 13 May 2009.
6 A simple opener, where participants generate the rules and guidelines for the class and for discussions, is important. Some teachers call this the "class bill of rights" or other similar titles. See for example, activities on "Making guiding rules" in Chapter 1 in any of the three HIV/AIDS Alliance's Our Future series (sexuality and life-skills education curriculum in Zambia):

$<$ www.aidsalliance.org/custom_asp/publications/view. asp?publication_id=211\&languageen>..Accessed 13 May 2009.

7 See, for example, Chapter 1 in any of the three HV/AI DS Alliance's Our Future series (sexuality and life-skills education curriculum in Zambia):

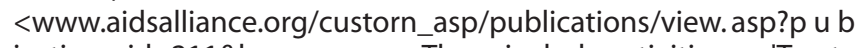
icatic $n_{-}$id $=211$ \&languageen $>$. These include activities on 'Trust and keeping secrets" and "Working together with respect."

8 As stated in the Teaching Tips for Unit 3 in the GUIDELINES volume, you should be aware that you may have legally mandated reporting requirements, for example, if a student discloses sexual abuse. Know what the requirements are in your location.

9 Scholars have documented the wide variation in people's learning strengths, styles, and needs. For example, the idea of multiple intelligences has challenged the concept of a single form of intelligence (Gardner, Howard. The Theory of Multiple Intelligences. 2nd revised edition. 2006. Basic Books. <books.google.com/books ?idr_vLmG9qEROgC\&dq=howard+gardner+multiple+intelilgences

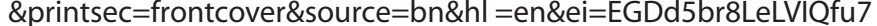
$\mathrm{KSBDg} \&$ saX\&oi=book result \& resnum $=5>$. 


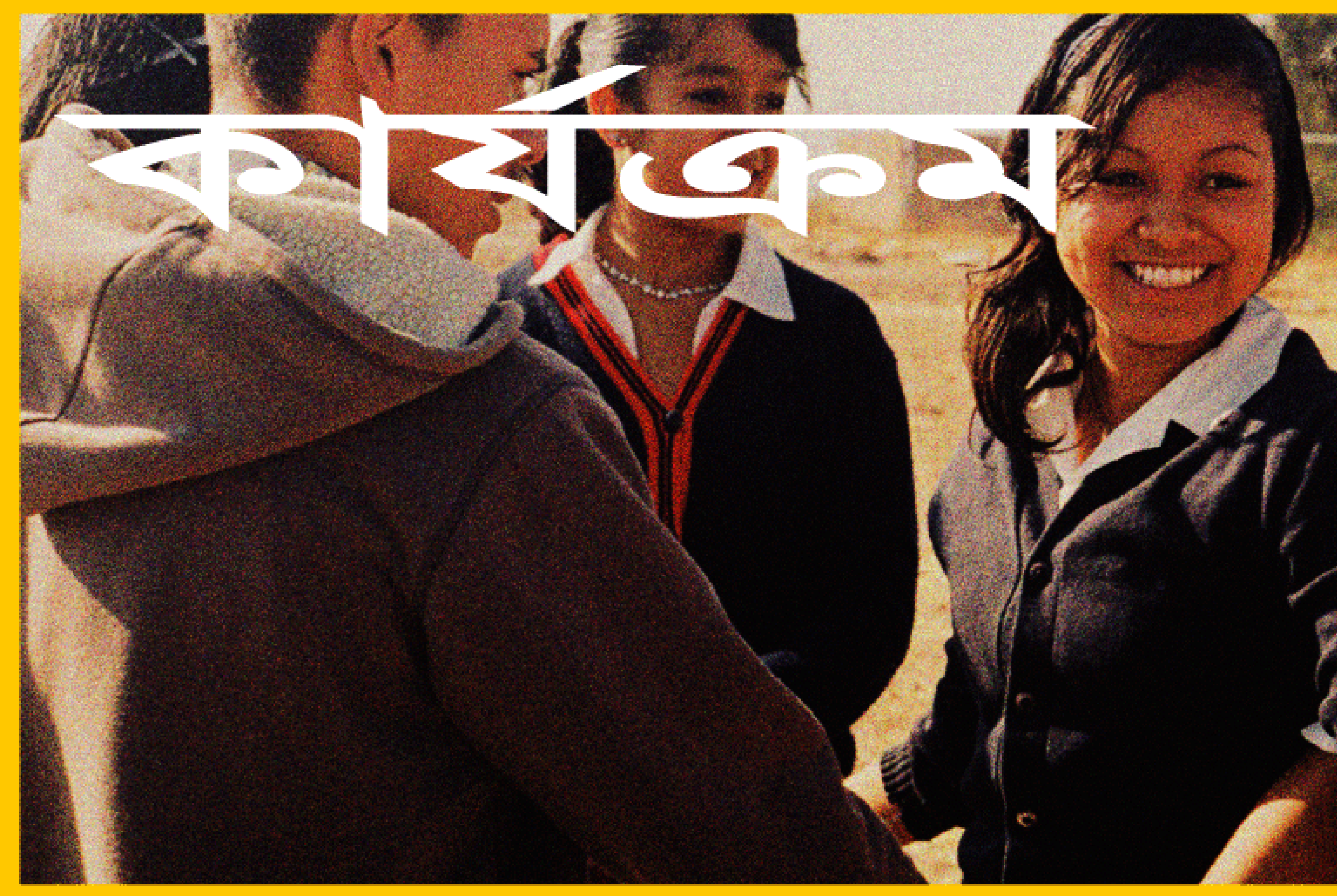




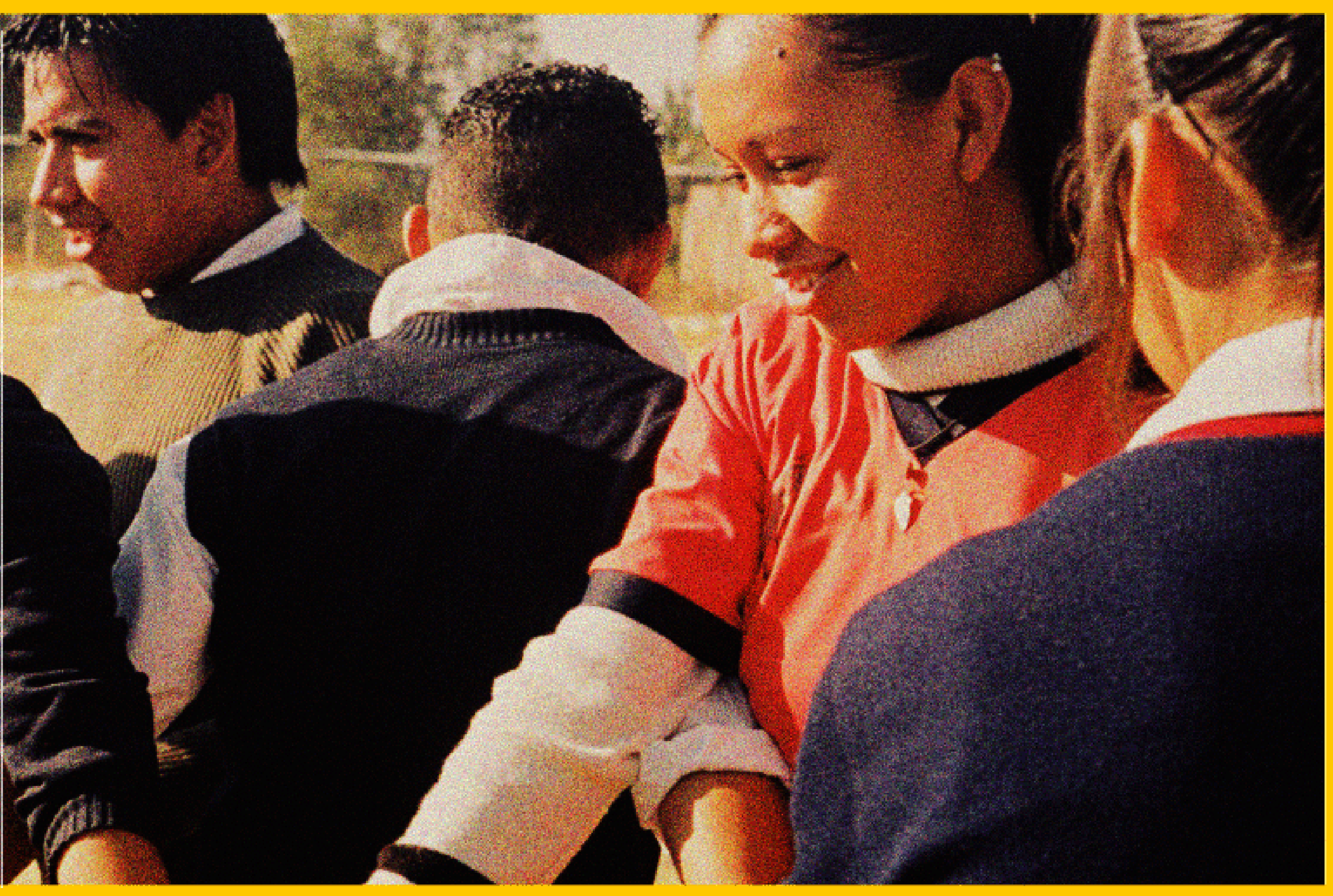


১৫ বছরের কম বয়সী শিক্ষার্থীদের জন্য

यদিও এই বইটি প্রাথমিকভাবে অপেক্ষাকৃত বয়স্ক কিশোর-কিলোরীদদর কথা মনে রেণে তৈরি করা হয়েছিল, এগুলোর মধ্যে অনেক অধिবেশন আপনার সমাজের ওপর ভিত্তি করে দশ বছর বয়সী বাচ্চাদের জন্য উপযুক্ত অথবা খাপ খাওয়ানোর যোগ্য ।

আপনি এই ছোট শিক্ষার্থীদদর জন্য যে ২১ টি কার্যক্রমে সবচেয়ে বেশী ব্যবহার উপযোগী হবে বলে দেখতে পারেন সেগুলো হল:

\begin{tabular}{|c|c|c|}
\hline ব্যায় ১ & কार्यक्र2 & $\partial$ \\
\hline অধ্যায় २ & কার্यক্রম & ৫-৬, ৮-১১,১৫ \\
\hline অধ্যায় ৩ & কার্यক্রম & ১৮, ২০ \\
\hline অধ্যায় 8 & কार्यক্রম & ২৭, ২৯, ৩২ \\
\hline অধ্যায় ৫ & কार्यক্রম & ৩৬ \\
\hline অধ্যায় ৬ & কार्यক্রম & $80-80$ \\
\hline ধ্যা & কার্যক্রম & ৪৯-৫০ \\
\hline ্যায় & (201 & (४) \\
\hline
\end{tabular}

\section{সকল নিয়ে একক শিক্ষাক্রম শিখন কার্যক্রমে স্বাগতম !}

\section{বাস্তবভিত্তিক ও কার্যকরী হওয়ার জন্য এই কার্যক্রমগুলি তৈরি হয়েছে।}

প্রতিটি কার্যক্রম সর্বোপরি শিক্ষা গ্রহণকে মনে রেখেই সাজানো হয়েছে। অন্তত: যৌন ও এইচআইভি শিক্ষা সার্বিক প্রাতিষ্ঠানিক শিক্ষাগত অর্জনকে শক্তিশালী করতে পারে।

ঐ উ দ্লেশ্যকে সহায়তা করার জন্য এই কার্যক্রমগুলো দুই ধরনের শিখন উড্লেশ্য নিয়ে টৈরি করা হয়েছে: (১) উপস্থিত বিষয় সম্পর্কিত শিক্ষা এবং (২) নির্দিষ্ট প্রাতিষ্ঠানিক দক্ষতা শক্তিশালী করা, যেমন- উচ্চতর বা সমালোচনামূলক চিন্তা, লেখা, গবেষণা, জনসমক্ষে বক্তৃতা, এবং সমন্বিত পরিকল্পনা ও সমস্যা সমাধান। উপরন্তু, এই অধিবেশনগুনো অর্থপূর্ণ এবং বেশির ভাগই আনন্দময় বলে বিদ্যালয়ের সাথে শিক্ষার্থীদের সম্পৃক্ততা ও সংত্যোগ বৃদ্ধি করে।

প্রতিটি কার্যক্রম নির্দেশিকা বইয়ের নির্দিট্ট বিষয়বস্তুর সাথে সংযুক্ত করা আছে।

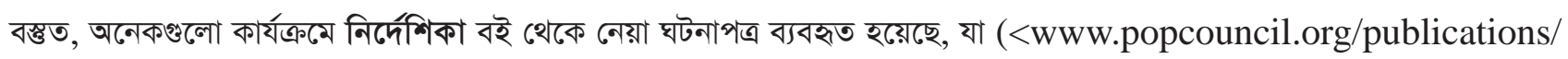
books/2011_ItsAllOne asps) এই ঠিকানায় পাওয়া যাবে।

এই সম্পূর্ণ কার্যক্রমগুলি যেমনি পনের বছর এবং এর বেশি বয়সের যুবক-যুবতীর জন্য উপযুক্ত, তেমনি অনেকগুলো একক কার্যক্রম অপেক্ষাকৃত ছোটদের জন্যও ব্যবহৃত হুতে পারে।

যে কার্যক্রমগুলো ১০-১৪ বছর বয়লের ছেলে-নেয়েদের জন্য সমানভাবে উপযুক্ত সেগুলোর জন্য বাম দিকের মার্জিনের পাকেরর তালিকা দেখুন।

কার্যক্রমগুনো শিক্ষার্থীদের জন্য হ্যান্ডআউট, অনুশীলনপত্র, শিক্ষকের উত্তরমালা ও শিক্ষকদের জন্য পূর্বতথ্যসহ তৈরি। এই সুবিধাজনক উপকরণসহ কার্যক্রমগুলো অত্যাবশ্যকীয়ভাবে ব্যবহারের-জন্য-প্রস্তত। দয়া করে পরবর্তী পৃষ্ঠার লক্ষণীয়গুনো পুনরায় পড়ে নিশ্চিত হোন যে, এই কার্যক্গুগুলো আপনার নিজস্ব শিক্ষার্থীদের যথাযথ প্রয়োজন মেটায়। 
বিদ্যালয়ের জন্য: বিদ্যালয়ে পড়ানোর ক্ষেত্রে এর যথাযথ প্রয়োণের জন্য অধিকাংশ কার্যক্রমগুলো যেন 80 মিনিটের একটি (বা মাঝে মাঝে দুই বা তিনটি) সেশনে শেষ হয় সেই ভাবে তৈরি করা হয়েছে। পূর্বের পৃষ্ঠায় উল্লেখ করা হয়েছে যে, এই কার্যক্রমগুলো প্রাতিষ্ঠানিক শিক্ষাগত উদ্দেশ্য অন্তর্ভুক্ত করে। এগুলো ব্যক্তিগতভাবে অর্থপূর্ণ ও উপভোগ্য হবার কারণে এই কার্যক্রমগুলি বিদ্যালয়ের সাথে শিক্ষার্থীদের সংতোগ আরো বৃদ্ধি করতে পারে।

সমাজভিত্তিক কর্মসূচির জন্যঃ এই ধারাবাহিক কার্যক্রমগুলি ‘অংশগ্রহণকারী’ ব্যবহার না করে ‘শিক্ষার্থী’ ব্যবহার করে। সহজ হওয়ার জন্য আমরা এই শব্দ ব্যবহার করেছি, কিন্তু শব্দটি শখ শ্রেণিকক্ষের শিক্ষার্থীদেরকে বোঝায় না। বিদ্যালয়ে পড়ি বা না পড়ি আমরা সকলেই শিক্ষার্থী

অল্প শিক্ষিত শিক্ষার্থীদের জন্য: যদিও অনেকগুলো কার্যক্রমে লেখার বিষয় অন্তর্ভুক্ত আছে তবুও যেখানে শিক্ষার্থীদের অল্প বা কোন অক্ষর জ্ঞান নেই সেখানে ব্যবহার করার জন্য বেশির ভাগই তৎক্ষণাৎভাবে মৌখিক কার্যক্রম হিসেবে খাপ খাওয়ানো যাবে। যেমন-এগুনোর অনেক কার্যক্রমে নির্দেশিত লিখিত আলোচনার বর্ণনা করতে ছবির কার্ড তৈরি করে খাপ খাওয়ানো যেতে পারে।

আপনার পরিকল্পনাকে সহজ করতে: প্রত্যেক কার্যক্রমে একটি সংক্ষিপ্ত কার্যক্রম সম্পর্কিত ধারণা, শিখন উদ্দেশ্য, কার্যক্রুমের জন্য প্রয়োজনীয় সময় (সময়কাল), আপনার প্রয়োজন হবে এমন উপকরণের তালিকা, এবং প্রস্তুতির জন্য প্রয়োজনীয় যে কোন ধাপ অন্তর্ভূত্ত আছে। আপনি কীভাবে পাঠটি উপস্থাপন করবেন তা পূর্বে নির্ধারণ করার জন্য নিজেকে সাহায্য করতে এটি উপস্থাপনের পূর্বে কার্যক্রমটি দুইবার ভালভাবে পড়ুন । বাছাইকৃত কার্যক্রমের প্রক্রিয়া ও ভাবের সাথে সহজ হওয়ার জন্য আপনি বিভিন্ন সহকর্মী বা বন্ধুর সাথে মহড়া দিলে এটি আপনার জন্য সহায়ক হতে পারে। অবশ্যই যে কোনো পাঠ পরিকল্পনার অংশ হিলেবে আপনার নিজেকে নির্দেশিকা বইয়ের (অথবা আপনার নিজের শিক্ষাক্রম) থেকে এ সম্পর্কিত বিষয়বস্তর সাথে পরিচিত হওয়ার প্রয়োজন হবে।
সাংস্কতিক ভিন্নতার ক্ষেত্রে: কার্যক্রমগুলি বিস্তত পরিসরে ব্যবহার উপযোগী হওয়ার জন্য সাজানো হয়েছে। যাই হোক, আপনার সমাজে কিছু কার্যক্রম অন্যগুলোর চেয়ে বেশি উপযুক্ত মনে হতে পারে। অনেকগুলো কার্যক্রমের নির্দেশনাও বলে যে, উদাহরণ হিসেবে দেয়া নাম ও পরিস্থিতি আপনি পরিবর্তন করতে পারেন যেন সেগুলো আপনার শিক্ষার্থীদের জন্য আরো বেশি বোধগম্য ও সহজ হয়

অধিবেশন বাছাই বিষয়ে লক্ষণীয়: এই কার্যক্রমগুলো কোনো একক শিক্ষাক্রম থেকে নেয়া হয়নি। বরং এগুলো হল আপনি যেভাবে আপনার নিজস্ব শিক্ষাক্রম বা পাঠ পরিকল্পনা তৈরি করবেন বা খাপ খাওয়াবেন তার নমুনা কার্যক্রম। এমন কার্যক্রম বাছাই করুন যা আপনার শেখানো বিষয়গুলোর অংশ হিসেবে বক্তৃতা, লিখিত উপকরণ, চলচ্চিত্র, বা অন উপাদানের জন্য সবচেয়ে ভাল পরিপূরক হয়। এই বইয়ের শেষে অতিরিক্ত তথ্য সম্পদের গ্রন্থপঞ্জির অতিরিক্ত শিক্ষাক্রমের যোগসূত্র দেয়া আছে

উপকরণ বিষয়ে একটি লক্ষণীয়: অনেক কার্যক্রমে উল্লেখ আছে যে, আপনার চকবোর্ড ও চকের প্রয়োজন হবে। অবশ্যই আপনি নিউজপ্রিন্ট কাগজ ও মার্কার, হোয়াইট বোর্ড, অথবা আপনার শিক্ষার্থীদের পড়ার জন্য যথেষ্ট বড় যে কোন পৃষ্ঠা ব্যবহার করতে পারেন।

সময় বিষয়ে লক্ষণীয়: আপনার দলের আকার ও বৈশিষ্ট্য এবং আপনি আলোচনার জন্য কতটা সময় দিতে চান তার ওপর নির্ভর কটে এই কার্যক্রমগুলির সময়কাল পবিবর্তনরোগ্য। যদি নির্দেশাবলিতে ইঙ্রিত দেয়া থাকে তাহলে শেষে আলোচনার জন্য সময় রাখা গুরুত্রপর্ণ। 


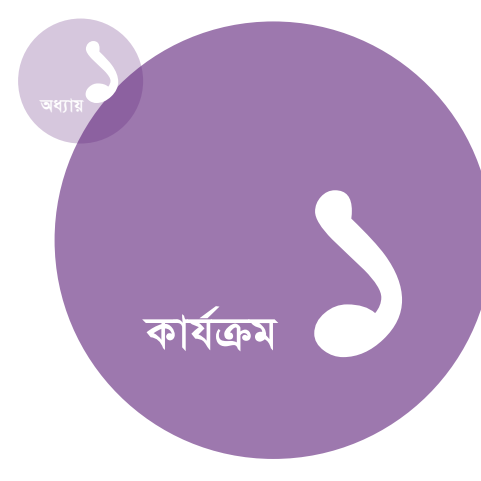

\section{সময়কাল :}

ধাপ ১-২: ১৫ মিনিট (৩নং ধাপের আগের দিন বাড়ির কাজ হিলেবে দেয়া যেতে পারে)

ধাপ ৩-৭: ৪० মিনিট

\section{উপকরণ:}

বোর্ড ও চক।

\section{প्रস্তুতি:}

উদ্ধৃতিগুলো পুনরায় পডূন। অনুলিপি করার সুযোগ থাকলে প্রত্যেক শিক্ষার্থীর জন্যে উদ্ধৃতিগুলোর একটি করে অনুলিপি তৈরি করুন । অন্যথায় পাঠদান শুরুর আগে বোর্ডে সবগুনো উদ্ধৃতি লিখুন। ধাপ-১ ও ধাপ-২ বাড়ির কাজ হিলেবে দিবেন কিনা তা ধাপ-৩ শুরুর আগে সিদ্ধান্ত নিন।

\section{কেন পক্ষপাতহীনতা সম্পর্কে জানা প্রয়োজন}

অধিবেশন সম্পর্কিত ধারণা: শিক্ষার্থীরা বিশিষ্ট ব্যক্তির উদ্ধৃতিগুলো অলোচনার মাধ্যনে ন্যায়পরায়ণতা বিষয়ে চিন্তা করবে।

উদ্দেশ্য: শিক্ষার্থীদের ন্যায়পরায়ণতার গুরতত্ব সম্পর্কে বুঝতে সাহায্য করা; ন্যায়পরায়ণতা ও মানবাধিকারের মধ্যে মিলগুলো খুঁজে পেতে সাহায্য করা; লেখা এবং বিশ্লেষণ ক্ষমতা শক্তিশালী করা।

\section{निर्দেশাবলি}

১ ব্যাখ্যা করুন যে, সারা বিশ্বের বিশিষ্ট ব্যক্তিদের থেকে এই উদ্ধৃতিগুলো নেয়া হয়েছে । শিক্ষার্থীদের সমস্বরে একটি একটি করে উদ্ধৃতিগুনো পড়তে দিন। এ সময় উদ্ধৃতিগুলো আলোচনা করবেন না।

পক্ষপাতহীনতা হচ্ছে প্রকৃত ন্যায়বিচার।

মহিলারা অনিরাময় যোগ্য অসুখে মারা যাচ্ছে না, বরং তারা মারা যাচ্ছে, সমাজ এখনও বুঝে উঠতে পারেনি যে তাদের জীবন মূল্যবান।

অন্যের সন্তানের প্রতি আমাদের পক্ষপাতহীনতাই আমাদের ভবিষ্যতকে গড়ে তুলবে

জীবনে চলার পথে যাদের সাথে মিশেছ তাদের প্রতি দয়াশীল হও, যারা জীবনের সাথে যুদ্ধ করছে।

ততক্ষণ পর্যন্ত অন্যদের প্রতি সৎ হও, যতক্ষণ পর্যন্ত তারা তোমার প্রতি সৎ না হয়

পক্ষপাতহীনতা হলো তারার মাঝে দীপ্তি।

এমনভাবে জীবন গড়ো যেন মানুষ পক্ষপাতহীনতা ও শ্রদ্ধার কথা ভাবলে তোমাকে মনে করে।

সকল মানুষকে আমরা আমাদের হৃদয় ও নীতিতে বর্ণ, জেন্ডার, বয়স, রাজনৈতিক বিশ্বাস বা ধর্মের উর্ধ্ব পক্ষপাতহীনতা ও শ্রদ্ধার সাথে বিবেচনা করব।
২ শিক্ষার্থীদের নিম্যোক্ত নির্দেশগুলো পড়ে শোনান:

• এ এমন একটি উদ্ধৃতি বেছে নাও যা তোমাকে অনুপ্রাণিত করে।

- তুমি এ সম্পর্কে কী মনে কর এবং কেন তোমার এটি ভাল লেগেছে বা উৎসাহিত করেছে তা নিয়ে কমপক্ষে এক পাতা লেখ ।

• তোমার নিজের জীবন থেকে অথবা তোমার সমাজ বা অন্য কোথাও ঘটে যাওয়া এমন একটা উদাহরণ দাও যেখানে এই বার্তাটি সহায়ক হতে পারে। এটি এমন একটা সময়ের উদাহরণ হতে পারে যখন তোমার সাথে কেউ খারাপ ব্যবহার করেছিল অথবা তুমি কারো সাথে ভাল ব্যবহার করনি, অথবা অন্যদের এমন কোন আচরণ করতে দেখেছ।

• উক্ত উদ্ধৃতির বার্তাটি কীভাবে সহায়ক হতে পারে ?

- পরবর্তীতে তুমি তোমার লেখাটি দলের অন্যদের মাঝে পড়ে শোনানোর একটি সুযোগ পেতে পার, কিন্তু তোমাকে এটা নাও করতে হতে পারে।

• এ সম্পর্কে কারো কোনো প্রশ্ন আছে কি? 
৩ পছন্দের উদ্ধৃতি সম্পর্কে লিখিত আলোচনা নিয়ে মত বিনিময় করতে ইচ্ছুক এমন শিক্ষার্থীদেরকে ডাকুন । (যদি আপনি ইতিমধ্যে লেখা/ উত্তরগুলো সংগ্রহ করে থাকেন ও পড়ে থাকেন তাহনে আপনি এদের মধ্যে থেকে এক বা দুই জনকে বলার জন্য নির্বাচন করতে পারেন)। আলোচনা/মতবিনিময়ের জন্য শিক্ষার্থীদেরকে ধন্যবাদ জানান।

8 यদি প্রথম দুই বা তিন জনের মধ্যে সবাই তাদের নিজেদের সাথে খারাপ ব্যবহার করা হয়েছে এমন ঘটনা লিখে থাকে তাহলে এমন কাউকে ডাকুন যে উদাহরণ হিসেবে সে অন্য কারো সাথে খারাপ ব্যবহার বা অন্য কেউ কারো সাথে খারাপ ব্যবহার করেছে এমন উল্লেখ করেছে ।

৫ আগ্রহী পাঠকদের ধন্যবাদ দিন এবং শিক্ষার্থীদেরকে প্রশ্ন করুন:

• পক্ষপাতহীন হওয়া কি সবসময় সহজ?

• শেষ উদ্ধৃতিটি দেখ (আমরা আমাদের মনে. . . . . .)

• আমরা কি সব পার্থক্যের উর্ধ্ব্ব থেকে সকল মানুষকে পক্ষপাতহীন ও মর্যাদার সাথে বিবেচনা/মূল্যায়ন করি ? আমাদের আইন কি সকলকে পক্ষপাতহীন ও মর্যাদার সাথে বিবেচনা করে? কেউ কি উদাহরণ দিতে পারবে ? [यদি সম্ভব হয়, শিক্ষার্থীদের নিজেদের সমাজের ঘটনা এবং অন্য দেশের কোন ঘটনা যা তারা শুনেছে এমন কিছু মনে করতে উৎসাহ দিन ।]

৬ শিক্ষার্থীদেরকে বলুন:

• পরস্পরের সাথে পক্ষপাতহীন ও মর্যাদার সাথে আচরণ করা বলতে কী বোঝায় বা কীভাবে একজন মানুষের মূল্যায়িত হবার অধিকার আছে সে সম্পর্কে মানুয সব সময় একমত নয়। ৫০ বছরেরও বেশি সময় ধরে বিশ্ব সমপ্রদায় একমত পোষণ করছে যে, প্রত্যেক মানুষের সম্মান ও মর্যাদার সাথে বিবেচিত হবার অধিকার রয়েছে।
• পক্ষপাতহীনতার ব্যাপারে প্রত্যেক ব্যক্তি কিছু নির্দিষ্ট রক্ষাব্যবস্থা ও অধিকারের দাবী রাখে। এই অধিকারগুলোকেই বলে মানবাধিকার। এগুলো নির্দেশ করে আমরা ব্যক্তি হিসেবে এবং সমাজের সদস্য হিসেবে পরস্পরের সাথে কীভাবে আচরণ করব, শ্যু এই কারণে যে আমরা মানুষ।

৭ জিজ্ঞাসা করুন এবং আলোচনার করার সুয়োগ দিন:

• তোমরা কি একটা মৌলিক অধিকারের কথা বলতে পারবে যা প্রত্যেক ব্যক্তির থাকা উচিত ? [এ ধরনের অধিকারগুনো জানার চেষ্টা করুন: খাদ্য, বাসস্থান, শিক্ষা ও নিরাপত্তা।]

- আসলেই কি প্রত্যেক ব্যক্তি এই অধিকারগুনো উপভোগ করে?

• যে উদ্ধৃতিগুলো সম্পর্কে তোমরা লিখেছিলে সেই মূল উদ্ধৃতিগুনো দেখ। পক্ষপাতহীনতা সম্পর্কে উক্ত উদ্ধৃতিগুনো কি মানবাধিকারের বৃহত্তর ধারণার সাথে প্রাসঙ্গিক ?

\section{শিক্ষকদের জন্য তথ্য}

এই কার্যক্রমে ব্যবহৃত উদ্ধৃতিগুলো নিয়লিখিত ব্যক্তিদের থেকে নেওয়া (ধাপ-১ এ উল্লিথিত ক্রমানুসারে): বিচারক পোটার স্টিওয়ার্ট; আন্তর্জাতিক চিকিৎসা সংক্রান্ত নেতা মোহাম্মদ ফাতাউল্লাহ; প্রাচীন গ্রীক দার্শনিক ফিনো; অভিনেতা এলান অলডা; নামহীন; লেখক এইচ. জ্যাকসন ব্রাউন, জুনিয়র; সাবেক মার্কিন রষ্ট্রপতি বিল ক্রিনটন। 


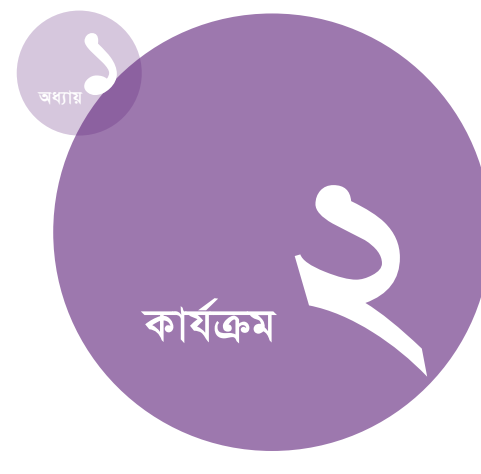

সময়কাল :

ধাপ -১-২: ৪० মিनिট

ধাপ -৩-৫: ৪৫ মিনিট

\section{উপকরণ}

বোর্ড ও চক; শিক্ষকের জন্য কমপক্ষে একটি সর্বজনীন মানবাধিকার ঘোষণাপত্রের অনুলিপি।

\section{প्रস্তুতি:}

শিক্ষার্থীরা মানবাধিকারের

মূল্যবোধগুলি যেমন- সমতা এবং বৈষম্যহীনতা সম্পর্কে বোঝার পরে এই কার্যক্রমটি শুরু করা উচিত সর্বজনীন মানবাধিকার ঘোষণাপত্রটি ভালভাবে পড়ন এবং নিশ্চিত হোন যে প্রত্যেকটি অধিকারের যথার্থ অর্থ আপনি বোঝেন।

\section{মানবাধিকার পরিচিতি}

অধিবেশন সম্পর্কিত ধারণা: শিক্ষার্থীরা নতুন কোন কাল্পনিক দেশের জন্য চায় এমন কিছু অধিকারের তালিকা তৈরি করবে এবং সেটা সর্বজনীন মানবাধিকারের সাথে তুলনা করবে। তারা মানবাধিকার সম্পর্কে বড়দের ধারণা যাচাই করার জন্য সাক্ষাৎকার निবে ।

উদ্দেশ্য: শিক্ষার্থীদেরকে মানবাধিকারগুলির ধারণা বর্ণনা করতে এবং তাদেরকে কিছু মৌলিক অধিকারের নাম উনেখ করতে সক্ষম করা; বিমূর্ত চিন্তার দক্ষতা শক্তিশালী করা।

\section{निर्দেশাবলि}

১ শিক্ষার্থীদের চার বা পাঁচ জনকে নিয়ে এক-একটি দনলে ভাগ করুন । তাদেরকে বলুন:

• তোমরা একটা নতুন দ্বীপের বাসিন্দা হবে যেখানে মানুষের বসবাস উপযোগী সব কিছুই আছে। পূর্বে এখানে কেউ বসবাস করেনি তাই সেখানে কোন আইন ও ইতিহাস নেই।

• তোমাদের অবশ্যই ১০টি মানবাধিকারের তালিকা তৈরি করতে হবে যেগুলি দ্বীপের সকলের ওপর স্বয়ংক্রিয়ভাবে আরোপিত হবে।

• তোমরা কেউ তোমাদের অবস্থান, জেন্ডার, জাতিগোষ্ঠী/বর্ণ, শ্রেণি বা যৌন পরিচয় জান না । তোমাদের তালিকা তৈরির জন্য ১০ মিনিট সময় দেওয়া হলো।

২ প্রত্যেক দলকে তাদের তালিকা উপস্থাপন করতে বলুন। সব দলের তালিকা সমন্বিত করে একটি “দলীয় তালিকা” তৈরি করুন, যাতে উলিখিত প্রত্যেকটি অধিকারই থাকবে।

৩ সর্বজনীন মানবাধিকারগুলি সম্পর্কে বলুন । বুঝিয়ে বলুন যে, এটা বিশ্বের সব মানুষের জন্য অধিকারের তালিকা। প্রত্যেককে উচ্চস্বরে একটি করে অনুচ্ছেদ পড়তে বলুন। শেষ অনুচ্ছেদটি পড়ার পর কারও কোনো প্রশ্ন আছে কি-না জিজ্ঞস করুন ।
8 ১০ মিনিটের জন্য শিক্ষার্থীদের তাদের ছোট দলে ফিরে যেতে বলুন বিবেচনা করতে বলুন:

• আমাদের তৈরি তালিকার সাথে সর্বজনস্বীকৃত মানবাধিকারগুলির को को মिল আছে?

• সর্বজনীন মানবাধিকার তালিকা থেকে কোন অধিকারগুলি নেওয়া रয়नि?

• আমরা কি আমাদের তালিকায় আরও কিছূ অধিকার যোগ করতে চাই?

• দলীয় তালিকায় এমন কোন অধিকার আছে কি যা সর্বজনীন মানবাধিকার তালিকায় নেই?

৫ ছোট দলগুলিকে পুনরায় একত্রিত করুন এবং শিক্ষার্থীরা 8 নং ধাপের প্রশ্নগুনোর যে উত্তর দিয়েছে তা পুনরায় পড়ন। প্রত্যেক ব্যক্তির ক্ষেত্রে প্রযোজ্য সর্বজনস্বীকৃত মানবাধিকারগুনো থাকা কতটুকু গুরুত্বপূর্ণ তা জিজ্ঞেস করুন ।

বাড়ির কাজ: দুইজন প্রাপ্তবয়স্ক ব্যক্তির সাক্ষাৎকার নেবে। তাদেরকে প্রশ্ন করবে: "মানবাধিকার বলতে আপনি কী বোঝেন ? আপনি শুনেছেন কমপক্ষে এমন একটি মানবাধিকার সম্পর্কে বলতে পারবেন?" তোমার সাথে ঐ ব্যক্তি কোন বিষয়টি আলোচনা করলো, তুমি কী শিখলে এবং বিষয়টি সমাধানের জন্য করা যেত এমন কিছু আছে যেটা তোমার মনে হয় তা লেখ। 


\section{সহজজ ভাষায় সার্বজনীন মানবাধিকার ঘোষণাগুলোর তালিকা}

প্রত্যেকটি মানবাধিকার সম্পর্কে নিম্নোক্ত তালিকাতে সহজভাবে বলা আছে ।

ইংরেজীতে দাগ্তরিক পাঠ্যবস্তুর জন্য <www.ohchr.org/EN/UDHR/Pages/Language.aspx?LangID=eng.> এই ঠিকানায় খোঁজ করুন ।

ধারা ১: সমস্ত মানুষ স্বাধীনভাবে সমান মর্যাদা এবং অধিকার নিয়ে জনুগ্রহণ করে। তাদের বিবেক এবং বুদ্ধি আছে; সুতরাং সকনেরই একে অপরের প্রতি ভ্রাতৃত্বসুলভ মনোভাব নিয়ে আচরণ করা উচিত।

ধারা ২: প্রত্যেকে নিন্নোক্ত অধিকারগুলি দাবি করতে পারে । যাই হোক না কেন তার-

• জেন্ডার

• গায়ের রং

• ভাষা

• রাজনৈতিক বিশ্বাস

- ধर्ग

• সম্পদ অথবা দারিদ্র

. সামাজিক দল

• জন্যুগ্রহণকারী দেশ ও

• দেশ স্বাধীন বা পরাধীন।

ধারা ৩: জীবন, স্বাধীনতা এবং নিরাপদভাবে বেঁচে থাকার অধিকার আছে।

ধারা 8: কারো অধিকার নেই তোমাকে তার ক্রীতদাস মনে করার এবং তোমারও অধিকার নেই কাউকে তোমার ক্রীতদাস বানানোর।

ধারা ৫: তোমাকে অত্যাচার করার অধিকার কারোর নেই।

ধারা ৬: তুমি সকলের মতো আইনগতভাবে সবখানে একইভাবে সুরক্ষিত হবে।

ধারা ৭: আইন সকলের জন্য সমান; এটি সবার উপর সমানভাবে প্রয়োগ করতে হবে ।

ধারা ৮: তোমার দেশ তোমাকে যে অধিকারগুলি দিয়েছে সেগুলি না পেলে তুমি আইনগত প্রতিকার চাইতে পারবে।

ধারা ৯: তোমাকে কারাগারে নেওয়া বা রাখার এবং অন্যায়ভাবে বা সুনির্দিষ্ট কারণ ছাড়া তোমাকে তোমার দেশ থেকে বিতাড়িত করার অধিকার কারো নেই ।

ধারা ১০: যদি তোমাকে অবশ্যই বিচারের সম্মুখীন হতে হয় তাহলে সেটা জনসম্মুখে হওয়া উচিত। যারা তোমাকে বিচার করবে তাদের অন্যদের দ্বারা প্রভাবিত হওয়া উচিত নয়। 


\section{সহজজ ভাষায় সর্বজনীন মানবাধিকার ঘোষণাগুলোর তালিকা}

ধারা ১১: যতক্ষণ পর্যন্ত না তুমি দোষী সাব্যস্ত হও ততক্ষণ তোমাকে নির্দোষ মনে করা উচিত। যদি তুমি কোন অপরাধে অভিযুক্ত হও, তোমার সর্বদাই আত্নপক্ষ সমর্থন করার অধিকার থাকা উচিত। যেটা তুমি করনি তার জন্য তোমাকে অভিযুক্ত করা বা শাস্তি দেওয়ার অধিকার কারোর নেই।

ধারা ১২: যদি কেউ তোমার সুনাম ক্ষুণ্ন করতে, তোমার বাসায় ঢুকতে, তোমার চিঠি খুলতে চেষ্টা করে অথবা তোমাকে বা তোমার পরিবারকে সুনির্দিষ্ট কারণ ছাড়া বিরক্ত করে তবে এগুনো থেকে রক্ষা পাওয়ার অধিকার তোমার আছে।

ধারা ১৩: তোমার দেশের যেকোন স্থানে যাতায়াত করার অধিকার তোমার আছে। তোমার দেশ ত্যাগ করে অন্যদেশে যাওয়ার; এবং যদি তুমি চাও তাহলে নিজ দেশে ফিরে আসার অধিকার আছে।

ধারা ১8: কেউ যদি তোমাকে আঘাত করে তাহলে তোমার অন্য দেশে আশ্রয় চাইবার অধিকার আছে। তুমি যদি মারাত্নক কোন মানবাধিকার লজ্ঘন কর তবে এই অধিকার হারাতে পার।

ধারা ১৫: তোমার একটি দেশে বসবাস করার অধিকার আছে এবং তোমার যদি অন্য কোন দেশে বাস করতে ইচ্ছা হয় তাহুে কোন নির্দিষ্ট কারণ ছাড়া কেউ তোমাকে বাধা দিতে পারবে না।

ধারা ১৬: এএজন ব্যক্তি তখনই বিয়ে করতে ও পরিবার গঠন করতে পারবে যখন তাকে আইনগতভাবে সে অধিকার দেওয়া হবে। এ বিষয়ে তোমার গায়ের রং, দেশ বা ধর্ম কোন বাধা হবে না । যখন পুরুষ্য ও মহিনারা বিয়ে করবে এবং বিচ্ছিন্ন হবে তখন এ বিষয়ে তারা একই অধিকার পাবে। জোরপূর্বক কাউকে বিয়ে দেওয়া যাবে না । তোমার দেশের সরকার তোমার পরিবার ও এর সদস্যদের রক্ষা করবে।

ধারা ১৭: তোমার কোন কিছু অর্জন করার অধিকার আছে এবং কোন সুনির্দিষ্ট কারণ ছাড়া সেটা নিয়ে নেয়ার অধিকার কারও নেই ।

ধারা ১৮: স্বাধীনভাবে নিজ ধর্মে বিশ্বাস করা, তা পরিবর্তন করা এবং নিজে বা অন্যদের সাথে এটি পালন করার অধিকার তোমার আছে।

ধারা ১৯: তোমার ইচ্ছামত চিন্তা করার ও কথা বলার অধিকার আছে এবং কেউ তোমাকে এ বিষয়ে বাধা দিতে পারবে না । তুমি অন্য যেকোন দেশের মানুষসহ যে কারও সাথে তোমার ভাবনা প্রকাশ করতে পার।

ধারা ২০: তোমার শান্তিপূর্ণভাবে সভা-সমাবেশ করার ও তাতে অংশগ্রহণ করার অধিকার আছে। কেউ তোমাকে জোরপূর্বক কোন দনে অন্তর্ভুক্ত করতে পারবে না ।

ধারা ২১: অন্য সবার মতো দেশের রাজনৈতিক বিষয়ে অংশগ্রহণ করার অধিকার তোমার আছে। এটা তুমি নিজে সরকারের অন্তর্ভুক্ত হয়ে বা তোমার পছন্দের রাজনৈতিক নেতা নির্বাচনের মাধ্যমেও করতে পার। নিয়মিত ও গোপন ভোটের মাধ্যমে সরকার নির্বাচন হবে। তোমার ভোটাধিকার থাকবে ও সকল ভোট সমানভাবে গণনা করা হবে। 


\section{সহজ ভাষায় সর্বজনীন মানবাধিকার ঘোষণাগুলোর তালিকা}

ধারা ২২: তোমার ও অন্যান্য সকলের জন্য দেশ যেসকল সুবিধা (সংস্কৃতি, কাজকর্ম, সমাজ কল্যাণ) প্রদান করে সে সকল সুবিধা তৈরি ও গ্রহণের জন্য তুমি যে সমাজে বসবাস করছো সে সমাজ সহযোগিতা করবে।

ধারা ২৩: তোমার জীবনयাপন ও পরিবারকে সহায়তা করার জন্য কাজ করার, স্বাধীনভাবে কাজ বেছে নেবার এবং বেতন পাবার অধিকার আছে। যদি নারী ও পুরুষ্ব একই কাজ করে তাহলে তারা একই বেতন পাবে। সকল কর্মীর তাদের স্বার্থের পক্ষে কথা বলা ও রক্ষার জন্য একত্রিত হওয়ার অধিকার আছে।

ধারা ২৪: কর্মদিবস বেশি দীর্ঘ হওয়া উচিত নয়। সকলেরই বিশ্রাম ও নিয়মিত বেতনসহ ছুটি পাবার অধিকার আছে।

ধারা ২৫: তুমি ও তোমার পরিবার যেন অসুস্থ না হও; ক্ষুধার্ত না হও; বস্ত্র এবং বাসস্থান থাকে; তুমি যদি কর্মহীন, অসুস্থ, বৃদ্ধ, স্বামী বা স্ত্রী মৃত্যুবরণ বা অন্য কোন কারণে জীবন-যাপনে অক্ষম হও তাহলে তুমি যা চাও সেটি পাওয়ার অধিকার তোমার আছে। সম্ভাব্য মা এবং তার সন্তান উভয়েরই বিলেষ সাহায্য পাবে। বিবাহিত বা অবিবাহিত যে মায়ের সন্তান হোক না কেন প্রত্যেক সন্তানেরই অন্যদের মত একই অধিকার আছে।

ধারা ২৬: তোমার শিক্ষালাভের অধিকার আছে; এবং প্রত্যেকেই শিক্ষা লাভের সুযোগ পাবে। প্রাথমিক শিক্ষা অবৈতনিক হবে। যে কোন পেশা বা দক্ষতা বা তুমি যে পর্যন্ত শিক্ষালাভ করতে চাও তা তুমি করতে পারবে। বিদ্যালয়ে তোমার সকল মেধা বিকশিত করতে পারবে। জাতিগোষ্ঠী, ধর্ম বা পূর্বাবস্থা যাই হোক না কেন তুমি অন্যদের সাথে একত্রে শিক্ষা নিতে পারবে। তুমি কী শিখবে এবং কীভাবে শিখবে তা তোমার পিতা-মাতার বেছে নেবার অধিকার আছে ।

ধারা ২৭: সমাজের শিল্প, বিজ্ঞান ও অন্য যেকোন সুফলে অংশগ্রহণ করার অধিকার তোমার আছে। শিল্পী, লেখক বা বৈজ্ঞানিক হিলেবে তোমার কাজ সংরক্ষিত হবার ও এগুলি থেকে তোমার উপকৃত হবার অধিকার আছে।

ধারা ২৮: তোমার অধিকারের প্রতি শ্রদ্ধাবোধ নিশ্চিত করা উচিত এবং এটি রক্ষার জন্য একটি আদেশ প্রতিষ্ঠিত হওয়া উচিত; এবং এটি স্থানীয় ও বিশ্বব্যাপী হওয়া উচিত।

ধারা ২৯: সমাজের প্রতি তোমার দায়িত্ব আছে। আইন তোমার মানবাধিকার নিশ্চিত করবে। এটি সবাইকে সম্মান দেবার ও নিজে সম্মান পাবার অনুমতি দেয়।

ধারা ৩০: পৃথিবীর কোন অংশের সমাজ বা ব্যক্তি এমন কিছু করবে না যাতে এই তালিকার কোন অধিকার ক্ষুন্ন হয় ।

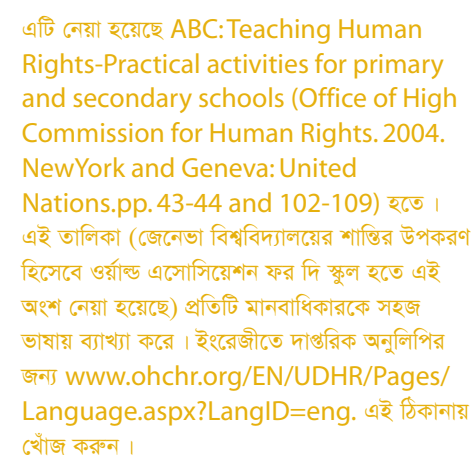

এটি নেয়া হয়েছে $A B C:$ Teaching Human Rights-Practical activities for primary and secondary schools (Office of High Commission for Human Rights. 2004. NewYork and Geneva: United Nations.pp. 43-44 and 102-109) হতে এই তালিকা (জেনেভা বিশ্ববিদ্যালয়ের শান্তির উপকরণ হিলেবে ওর্য়াল্ড এসোসিয়েশন ফর দি স্কুল হতে এই অংশ নেয়া হয়েছেছ) প্রতিটি মানবাধिকারকে সহজ ভাযায় ব্যাখ্যা করে। ইংরেজীতে দাগ্তরিক অনুলিপির জন্য www.ohchr.org/EN/UDHR/Pages/ Language.aspx?Lang|D=eng. এই ঠিকানায় খোঁজ করুন 


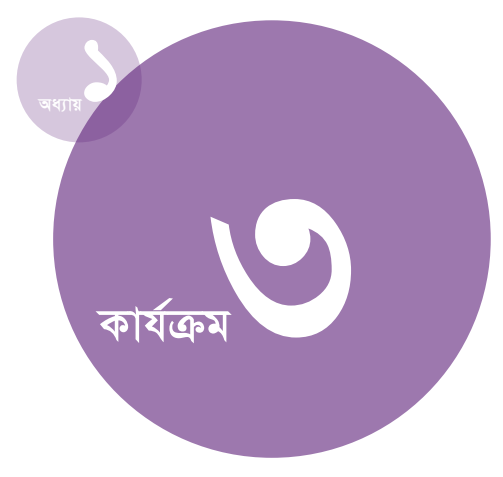

\section{সময়কাল :}

8৫ मिनिট

\section{উপকরণ:}

বোর্ড ও চক

\section{প্রস্তুতি:}

আপনার সমাজের সাথে মিলে যায় এমন যে কোন জনগোষ্ঠী ডান পাশের তালিকায় যোগ করুন । কার্যক্রম শুরুর আগে ১ নং ধাপ শেষ করুন ।

\section{ক্ষমতা, প্রাধান্য এবং সমতা}

অধিবেশন সম্পর্কিত ধারণা: শিক্ষার্থীরা সামাজিক ক্ষমতা ও বিশেষ সুবিধা কিংবা বৈষম্যের অভিজ্ঞতার মধ্যে সম্পর্ক চিহ্নিত করতে এবং এর প্রতিফলন ঘটাতে পারবে

উদ্দেশ্য: শিক্ষার্থীদেরকে সমাজে ক্ষমতার বন্টন সম্পর্কে আলোচনা করতে সক্ষম করা; ক্ষমতা কীভাবে বিশেষ সুবিধা, বৈষম্য ও নিপীড়নকে সম্পৃক্ত করে তা শনাক্ত করা এবং এই বিষয়গুলিকে তাদদর নিজেদের জীবনের সাথে মিলিয়ে দেখা; বিশেযণ এবং সমস্যা সমাধানের দক্ষতা বৃদ্ধি করা।

\section{निर्দেশাবলি}

১ বোর্ড দুইটি কলারের একটি চার্ট আঁকুন। থ্রথম কলারের নাম দিন 'অধिকতর ক্ষমতা/সুবিধা' এবং দ্বিতীয় কলামের নাম দিন 'কম ক্ষমতা/সুবিধা’ । ব্যাখ্যা করুন যে, শিক্ষার্থীরা নিজজদের জীবনে ও সমাজে তাদের ক্ষমতা, বিকেষ সুবিধা ও সমতা নিয়ে আলোচনা করবে।

২ “সমাজে যে সকল দলের/গোষ্ঠীর অসম ক্ষমতা থাকার প্রবণতা রয়েছে” এই শিরোনাম থেকে প্রথম উদাহরণটি পডুন (ধনী ও গরীব)

৩ জিজ্ঞেস করুন কোন দল ‘অধিকতর ক্ষমতার’ কলানে এবং কোন দল ‘কম ক্ষমতার’ কলামে যাবে। শিক্ষার্থীদের মনে করিয়ে দিন যে এই শ্রেণিবিন্যাস সর্বজনীনভাবে সত্য বা চূড়ান্ত নয়।

8 শিক্ষার্থীদের উত্তর সঠিক ঘরে লিখুন। প্রত্যেক কলামে তাদের উত্তর লিখে তালিকার বাকি অংশ পড়ন । (বিভিন্ন নৃ-বগাষ্ঠী, বর্ণগোষ্ঠী ও ধর্মগোষ্ঠীর ক্ষেত্রে আপনি নিজের এলাকার নির্দিষ্ট দলের নাম উলেখ করে দিতে পারেন।)

\section{সমাজের যে সকল দলের/গোষ্ঠীর অসম ক্ষমতা থাকার প্রবণতা রয়েছে}

ধনী/গরীব

পুরুষ্ব/মহিলা

ভিন্ন লিঙ্গের মানুভের প্রতি যৌনাকাজ্ষী/সমকামী

কর্মকর্তা/কর্মচারী

রাজনীতিবিদ/এলাকার সদস্য

নাগরিক/শরণার্থী

স্বাভাবিক ব্যক্তি/প্রতিবন্ধী ব্যক্তি

সংখ্যাগুরু নৃ-গোষ্ঠী/সংখ্যালঘু নৃ-গোষ্ঠী

সংখ্যাগুরু ধর্মীয় শ্রেণি/সংখ্যালঘু ধর্মীয় শ্রেণি

দেখতে আকর্ষণীয় এমন মানুষ/দেখতে আকর্ষণীয় নয় এমন মানুষ 


\section{(c) জিজ্ঞস করুন:}

• তালিকার দলগুনোর মধ্যে কাদের বেশি সুবিধা থাকার সম্ভাবনা আছে তা দেখ । এই তালিকার কোনো দলের সাথে কি তোমার ব্যক্তি জীবনের মিল খুঁজে পাও? তুমি কি একমত যে, এই দল সমাজে সাধারণত বেশি ক্ষমতা ভোগ করে ? তোমার নিজের অভিজ্ঞতা থেকে অথবা তোমার শোনা ক্ষমতার এমন পার্থক্য নিয়ে কয়েকটি বাক্য লেখ। [ শিক্ষাথ্থীদের লেখার জন্য ৩-৫ মিनिট সময় দিন। ।]

• তালিকার দলগুলোর থেকে কাদের কম সুবিধা আছে তা দেখ । এর যে কোনো দলের সাথে ব্যক্তিগতভাবেও মিল খুঁজে পাও কিনা তা দেখ। তুমি কি মনে কর, যে দল তুমি খুঁজে বের করেছ তাদের কম সুয়োগ-সুবিধা পাওয়ার প্রবণতা রয়েছে? তোমার নিজের অভিজ্ঞতা থেকে অথবা তোমার শোনা ক্ষমতার এমন পার্থক্য নিয়ে একটি অনুচ্ছেদ লেখ। [লেখার জন্য ৩-৫ মিনিট সময় দিन ।]

• यদি তুমি প্রত্যেক তালিকা হতে কমপক্ষে একটি দলকে শনাক্ত করতে পার তাহলে তোমার হাত তোলো । আমরা কি সবাই জানি বেশি সুযোগ-সুবিধা ভোগ করা এবং কম সুযোগ-সুবিধা থাকা বলতে কী বোঝায় ?

৬ কয়েকজন আগ্রহী শিক্ষার্থীকে ডাকুন যারা কম ক্ষমতা ও সুযোগ প্রাপ্ত দলের সদস্য হিলেবে নিজেদের বাস্তব কাহিনিগুলি পড়বে। (পড়ার জন্য কাউকে জোর করবেন না ।)

৭ নিম্মরূপে আলোচনা করুন (প্রত্যেক প্রশ্নের জন্য ১-২ টি উত্তর গ্রহণ করুন ।):

• কম সুয্োগের অধিকারী মানুষেরা যে আচরণ পায় তা নিয়ে তোমরা কী লক্ষ্য করেছ? [জানার চেম্টা করুন: যাদদর ক্ষমতা নেই তারা প্রায়ই বৈষম্যের শিকার ও নিপীড়িত হয়। ]

• কম ক্ষমতার মানুষদের মধ্যে এই আচরণ কী আবেগের জন্মা দেয়? [ আবেগগুনো বোর্ডে লিখুন। নিশ্চিত হোন যে, এগুলো
আবেগপূর্ণ শব্দ; কী ঘটেছে তার বর্ণনা নয়।]

• লিখিত এই অনুভূতি বা আবেগের মধ্যে তোমরা কী লক্ষ্য করেছ?

b- পূর্বের তালিকা দুইটি দেখুন এবং জিজ্ঞস করুন:

• সমতার ধারণা সম্পর্কে চিন্তা কর। উদাহরণস্বরূপ, প্রথম জোড়া নাও (ধনী ও গরীব মানুষ)। তোমরা এই বাক্যটি কীভাবে পূরণ করতে পার: “ধনী ও গরীবের মধ্যে সমতা .. ... .. ।" তোমাদের মনে কী আসছে? [ এখানে কোনো একক সঠিক জবাব নেই ।]

• অন্য কোনো দলের জন্য কে সমতার এই বাক্যটিকে সম্পূর্ণ করতে পারবে? অন্য কোনো শব্দ ব্যবহার করতে চেষ্টা কর অথবা অন্য কোনো ধারণা দাও।/যতক্ষণ সময় থাকে একাধিক দলের জন্য একই কাজ করুন ।]

• কে সর্বজনীন মানবাধিকার ঘোষণাপত্রের প্রথম অনুচ্ছেদটি মনে করতে পারে। /লক্ষণীয়: এটা সমতার অধিকার। প্রথম অনুচ্ছেদটি বোর্ডে লিখুন: “প্রত্যেক মানবশিশ্য স্বাধীনভাবে জন্ম নেয় এবং তারা প্রত্যেকেই মর্যাদা ও অধিকারের দিক থেকে সমান। তারা জন্মসুত্রে বুদ্ধি এবং বিবেকের অধিকারী এবং তাদের একে অপরের সাথে ভ্রাতৃসুলভ আচরণ করা উচিত "। ]

৯ নিম্নলিখিত প্রশ্ন দিয়ে আলোচনা শেষ করুন। (অথবা শিক্ষার্থীদের উদ্দুদ্ধ করুন যেন তারা শ্রেণিকক্ষের পাঠদানের পর প্রশ্নগুনো নিয়ে ভাবে): সমতা ও মর্যাদার অধিকার উপভোগ করতে গেলে সবাইকে অবশ্যই কিসের মুখোমুখি হতে হয়?

বাড়ির কাজ: প্রশ্নের লিখিত জবাব দাও : অধিকতর সমতাকে উৎসাহিত করতে ও প্রত্যেকের মর্যাদার প্রতি শ্রদ্ধাশীল হবার জন্য তোমরা তোমাদের দৈনন্দিন জীবনে কী করতে সক্ষম?
এটি নেয়া হয়েছে, Helping Teens Stop Violence: A Practical Guide for Counselors, Educators, and Parents (Allan Creighton and Paul Kivel. 1990, 1992. Alameda, CA: Hunter House Inc.) হরত ক্রয় করার জন্য <www.hunterhouse.com /shopexd.asp?id=308\&bc=no> এই ওয়েব সাইটে যান 


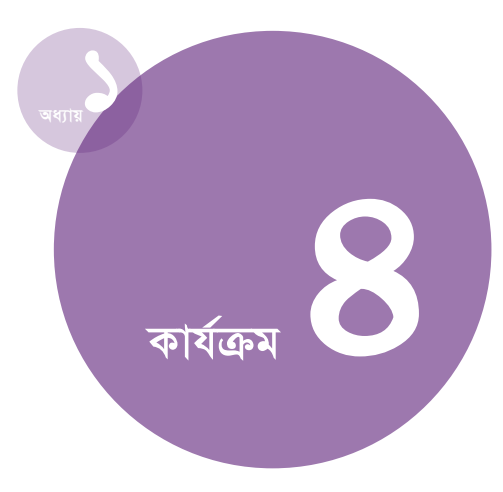

\section{সময়কাল :}

৪০-৬০ মিনিট, দুইটি নাকি তিনটি কেসস্টাডি বিশ্লেষণ করা হবে তার ওপর নির্ভর করবে।

\section{উপকরণ:}

বোর্ড ও চক; যে কেসস্টাডিগুলো বিশ্লেষণ করা হবে তাদের প্রত্যেকটির অনুলিপি। আপনি প্রথম অংশ কাগজের এক দিকে এবং অন্য অংশ আরেক দিকে রাখতে পারেন ।

\section{প्रস্তুতি:}

শিক্ষার্থীরা য্যৌন ও প্রজনন অধিকার সম্পর্কে জানার পরে এই অধিবেশনটি শুরু করা উচিত। পরবর্তী পৃষ্ঠা থেকে ৩টি কেসস্টাডি বাছাই করুন, অথবা আপনার দেশের কেস বাছাই করুন । যতক্ষণ সময় পান ২নং ধাপের প্রশ্নগুলো বোর্ডে লিখুন ।

\section{যৌন ও প্রজনন অধিকার সম্পর্কিত ঘটনাবলি}

অধিবেশন সম্পর্কিত ধারণা: শিক্ষার্থীরা যৌন বা প্রজনন অধিকার লজ্ঘন বিষয়ে সত্য ঘটনাবলি নিরীক্ষা ও আলোচনা করবে ।

উদ্দেশ্য: আমাদের মানবাধিকার চর্চার দক্ষতা কীভাবে ঘনিষ্ঠ সম্পর্ক এবং যৌন বিষয়কে প্রভাবিত করে সে বিষয়ে শিক্ষার্থীদেরকে বর্ণনা করতে সক্ষম করা; বিল্লেষণমূলক চিন্তার দক্ষতা শক্তিশালী করা।

\section{নির্দেশাবলি}

১ আজকে আমরা য্যৌন ও প্রজনন অধিকার সম্পর্কে বাস্তব ঘটনা আলোচনা করব। এলিকজার গল্পটির প্রথম অংশটি উচ্চ স্বরে পড়ার জন্য একজন আগ্রহী শিক্ষার্থীকে ডাকুন: গল্পটির প্রথম অংশই কেবল পড়ুন।

২ প্রথম অংশ পড়া শেষে শিক্ষার্থীদের তাদের খাতায় নিয়লিখিত প্রশ্নগুনোর উত্তর লিখতে বলুন । বোর্ডের প্রশ্নগুনো পড়ুন যেন সবাই শুনতে পারে :

• এই ঘটনা खুনে তোমাদের কী মনে হয়েছে?

• এই ঘটনার সাথে কোন্ কোন্ যৌন ও প্রজনন অধিকার সম্পর্কিত?

• তোমরা কি মনে কর এখানে মানবাধিকার লজ্ঘনের উলেখ আছে? কেন ?

• এজন্য কে দায়ী ? ঘটনার জন্য যাদের কোনো না কোনো দায় রয়েছে তাদের প্রত্যেকের নাম উলেখ কর।

৩ প্রত্যেক প্রক্নের উত্তরগুনো পড়ার জন্য এক অথবা দু’জন আগ্রহী শিক্ষার্থীকে ডাকুন এবং মতামত দিতে বলুন।

8 একই শিক্ষার্থীকে দ্বিতীয় অংশ পড়তে দিন (কী ঘটেছিল ?)। তারপর আলোচনা করুন:
- ফলাফল সম্পর্কে তোমাদের কী মনে হয়েছে?

• যদি ঘটনার শিকার ব্যক্তি ধনী ( বা পুরুষ্ব, বা বিপরীতলিঙ্গ ব্যক্তির প্রতি আসক্ত) হতো, তাহলে ঘটনাটি কেমন হতে পারত?

• এই যৌন অধিকার রক্ষা করার জন্য কোন্ কোন্ দৃষ্টিভঙ্গি অবশ্যই পরিবর্তন করা উচিত?

৫ এই ধাপগুনো আপনার অধিবেশনের প্রত্যেক কেসস্টাডির জন্য পুনরায় উলেখ করুন । (প্রত্যেক কেসস্টাডির জন্য ১০ মিনিট করে সময় দিন)।

৬ সবগুলো কেসস্টাডি আলোচনার পর নিম্নলিখিত প্রশ্ন জিজ্ঞাসার মাধ্যমে অধিবেশনের সার সংক্ষেপ করুন:

• এই ঘটনার মতো অন্য কোনো ঘটনা কি তোমরা জান ? কোন্ অধিকারগুলো ক্ষুণ্ন হয়েছিল ?

- তোমরা কি আমাদের দেশে বা অন্য কোন স্থানে যে কোন রকমের যৌন অধিকার ভঙ্গের কথা ওনেছছ?

• এ রকম অধিকার লজ্ঘন বন্ধ করার জন্য অবশ্যই কী করণীয়?

• আমরা আমাদের মানবাধিকার এবং আমাদের ঘনিষ্ঠ ও আবেগপূর্ণ সম্পর্কের এবং যৌন জীবনের মধ্যে সম্পর্ক নিয়ে কী সার সংক্ষেপ করতে পারি ? /মতামত জানতে চান এবং বোর্ডে সার সংক্ষেপ निখুন।] 


\section{যৌন ও প্রজনন অধিকার সম্পর্কিত ঘটনাবলি}

\section{এলিকজা}

প্রথম অংশ: এলিকজার গল্প: এলিকজা পোল্যান্ডের একজন মহিলা যার ছোটবেলা থেকেই চোখের সমস্যা। সে যখন গর্ভবতী হল তখন অনেক ডাক্তার তাকে উপদেশ দিল যে, গর্ভধারণ ও সন্তান প্রসবের জন্য তার চোখের দৃষ্টি চিরতরে নষ্ট হয়ে যেতে পারে। পোল্যান্ডের আইনে মহিলাদের স্বাস্থ্যগত ঝুঁকির ক্ষেত্রে গর্ভপাতের অধিকার দেয়া আছে। কিন্তু ডাক্তার এলিকজাকে গর্ভপাতের জন্য অনুমতি পত্র দিতে অস্বীকৃতি জানাল। তাই সন্তান ভূমিষ্ট হওয়া পর্যন্ত গর্ভধারণ করা ছাড়া এলিকজার কিছুই করার থাকল না ।

দ্বিতীয় অংশ: এলিকজার কী হয়েছিল ? বাচ্চার জন্ম এলিকজার চোখের দৃষ্টি আরও খারাপ করে দিল। সে কাজ করতে অক্ষম হয়ে পড়ল এবং দৈনন্দিন কাজকর্ম ও বাচ্চা প্রতিপালনের জন্য পরিপূর্ণভাবে অন্যের সাহায্যের ওপর নির্ভরশীল হয়ে পড়ল। এলিকজার ঘটনাটি ইউরোপিয়ান মানবাধিকার আদালতে নেয়া হলো। এখানে বলা হনো, মহিলাদের আইনগতভাবে স্বীকৃত গর্ভপাতের সুযোগ প্রদানের জন্য কার্যকরী ব্যবস্থা প্রতিষ্ঠা করা রাষ্ট্রের দায়িত্ব। তার যন্ত্রণা ও দুর্ভোগের কথা বিবেচনা করে আদালত তাকে অর্থনৈতিক ক্ষতিপূরণের ঘোষণা দিল।

\section{আমিনা}

প্রথম অংশ: আমিনার গল্প: আমিনা একজন নাইজেরিয়ান তালাকপ্রাপ্ত মহিলা যার তিন বছর বয়সের এক বাচ্চা আছে। মোহাম্মদের সাথে পরিচয়ের ১১ মাস পর সে তার সাথে যৌন মিলন করতে চায় এবং বিয়ে করতে প্রতিজ্ঞা করে। সে তাতে রাজী হয় এবং গর্ভবতী হয়ে পড়ে । কিন্তু মোহাম্মদ তাকে বিয়ে করল না এবং বিবাহবন্ধন ছাড়াই সে একটি মেয়ে সন্তানের জন্মা দেয়। সে ধর্মীয় আইনে ব্যভিচারের দায়ে অভিযুক্ত হলো। মোহাম্মদ কসম কেটে বলল যে, সে এই সন্তানের পিতা নয় এবং মুক্তি পেল। কিন্তু আমিনাকে ব্যভিচারের দায়ে পাথর নিক্ষেপ করে মৃত্যুদণ্ডের রায় দেয়া হলো । সে পুনর্বিচারের আবেদন করল কিন্তু পূর্বের রায় বহাল থাকল। বাচ্চা পরিচর্যার জন্য তার শাস্তি দুই বছর বিলম্বিত করা হল।

দ্বিতীয় অংশ: আমিনার কী হয়েছিল: অন্য একটি আবেদনে সে অপরাধ থেকে অব্যাহতি পেল এবং পাথর নিক্ষেপের মাধ্যনে মৃত্যুদণ্ডের রায় বাতিল করা হল। বিচারকগণ একমত হলেন যে, নিজেকে নির্দোষ প্রমাণের যথেষ্ট সুযোগ তার ছিল না । সরকার তাকে পাথর নিক্ষেপের মাধ্যমে মৃত্যুদণ্ড দেয়ার আদেশ প্রত্যাহার করল। তখন সে আবার বিয়ে করল। 


\section{যৌন ও প্রজনন অধিকার সম্পর্কিত ঘটনাবলী}

\section{ফাতিমা}

প্রথম অংশ: ফাতিমার গল্প: ফাতিমা ১১ বছর বয়সী পশ্চিম আফ্রিকান একটি মেয়ে। সে আড়িপেতে শুনে ফেলল যে, মা- বাবা তার খৎনা বিষয়ে কথা বলছে। সে ভীত হুয়ে গেল, কারণ তার মনে পড়ল তার বড় বোন কীভাবে সেই অনুষ্ঠান থেকে দুঃসহ ব্যথা ও যন্ত্রণা নিয়ে ফিরে এসেছিল। সে তার সবচেয়ে কাছের বন্ধুর কথাও মনে করল, যে খৎনার জন্য মারাত্মক সংক্রমণ নিয়ে তাদের এলাকার ক্লিনিকে ছিল । সে তার চলার পথে অন্য মেয়েদের যা দেখেছে সেটা করতে চায় না এবং সে তার মা-বাবাকে তার খৎনা না করানোর জন্য অনুরোধ করল । তারা তাদের মেয়ের কথা ওনতত চাইল না, কারণ তারা বিশ্বাস করত যে তার খৎনা না করালে সে বিবাহের অযোগ্য থেকে যাবে। এবং তারা মেনে নিতে পারছিল না যে এত ছোট ও অনভিজ্ঞ কেউ কোন মতামত দিতে পারে। যাই হোক, ফাতিমার বোন শহরে একটি সংস্থার কথা শুনেছিল যারা মেয়েদের খৎনার বিপদজনক ও স্বাস্থ্য ঝুঁকির বিষয়ে স্থানীয় পরিবারগুলোকে শিক্ষাদানের জন্য কাজ করে। তার বোন ঐ সংস্থার একজন কর্মীকে ফাতেমার অবস্থা নিয়ে মা-বারার সাথে কথা বলার জন্য তাদের বাড়িতে (কুটিরে) আসতে বলল ।

দ্বিতীয় অংশ: ফাতিমার কী হয়েছিল: সাহায্যকর্মী ফাতিমার মাবাবাকে বোঝাতে সক্ষম হলো যে, খৎনা তাদের মেয়ের স্বাস্থ্যের জন্য বিপজ্জনক এবং তার নারী জীবনে প্রবেশ করা বিষয়টির আরও অনেক বিকল্প পথ আছে। এখন ফাতিমা সুখী বিবাহিত জীবন যাপন করছে এবং সে তার মা-বাবার উদার মানসিকতার প্রতি কৃতজ্ঞ। সে এখন ঐ সংস্থার হয়ে কাজ করে যারা তার খৎনা ঠেকাতে সাহায্য করেছিল এবং কীভাবে খৎনা নিয়ে মা-বাবার সাথে কথা বলতে হয় তা নিয়ে স্কুলে মেয়েদের শিক্ষা দেয় ।

\section{ম্যাথিউ}

প্রথম অংশ: ম্যাথিউ’র গল্প: ম্যাথিউ যুক্তরাষ্ট্রের বিশ্ববিদ্যালয় পডুয়া একজন সমকামী ছাত্র। এক রাতে দু’জন যুবক তার সাথে সমকামী হওয়ার জন্য ছলনা করল এবং তাকে বার থেকে গাড়িতে করে বাসায় পৌঁছে দিতে চাইল। ম্যাথিউ তাদের সাথে গেল এবং তারা তাকে একটি প্রত্যন্ত অঞ্চলে নিয়ে গিয়ে তার কাছ থেকে সব কিছু ছিনিয়ে নিয়ে তাকে একটি বেড়ার সাথে বেঁধে বন্দুক দিয়ে নির্দয়ভাবে আঘাত করল ও অত্যাচার করল। তারা তাকে সেখানে মৃতপ্রায় অবস্থায় রেখে চলে গেল । ১৮- ঘন্টা পর একজন সাইকেল আরোহী ম্যাথিউকে বাঁধা অবস্থায় উদ্ধার করল। সে প্রথনে তাকে একটি কাকতাড়ুয়া ভেবেছিল। ম্যাথিউ তখনও অচেতন অবস্থায় ব্রেচে ছিল।

দ্বিতীয় অংশ: ম্যাথিউ’র কী হয়েছিল? ম্যাথিউ’র মাথার খুলি ভেঙ্গে গিয়েছিল এবং তার মস্তিক্ষের মারাত্যক ক্ষতি হয়েছিল। তার ক্ষত এতই মারাত্মক ছিল যে, ডাক্তাররা এটা ঠিক করতে পারছিল না । তার আর জ্ঞান ফিরল না এবং পাঁচ দিন পর মারা গেল । খুনিরা গ্রেফতার হল এবং পরিণানে একই সাথে দুইটি ফাঁসির আদেশ হল। ম্যাথিউ’র ঘটনা অপরাধকে ঘৃণা করার জন্য জাতীয়ভাবে আলোচিত হুলো । ম্যাথিউ’র নামে একটি আইন পাশ হল, যেটা মহিলা অথবা পুরুষ সমকামী, নারী এবং প্রতিবন্ধীদের প্রতি করা অপরাধকে ঘৃণা করার জন্য প্রণয়ন হল। ম্যাথিউ’র মা ম্যাথিউ শেপার্ড ফাউন্ডেশন প্রতিষ্ঠা করল যা শিক্ষা, প্রচার ও জনমত বৃদ্ধি কার্যক্রমের মাধ্যমে “ঘৃণার পরিবর্তে উপলধ্ধি, সমবেদনা ও গ্রহণযোগ্যতা” তৈরির জন্য কাজ করে। 


\section{যৌন ও প্রজনन অধিকার সম্পর্কিত ঘটনাবলি}

\section{लক্ষ্মী}

প্রথম অংশ: লক্ষীর গল্প: লক্ষী নেপালের একজন কিশোরী । তাকে ১২ বছরে বিয়ে করতে বাধ্য করা হল এবং সে স্বামীর বাড়ি নির্যাতিত হল। সে তা সহ্য করতে না পেরে পালালো এবং বাবার বাড়িতে ফিরে এল । কিন্ত্ত তার মা-বাবা আবার তাকে শ্বশ্রর বাড়িতে ফিরে যেতে বাধ্য করল। লশ্মী বলল, "পথথের মধ্যে আমি মুক্ত হতে পারলাম এবং একজন দয়ালু মহিলা আমাকে সাহায্য করল”। “সে বলল তার বোন নেপালের অন্য এক প্রান্তে কারখানায় কাজ করে, এবং আমি তার সাথে কাজে যোগ দিতে পারি ও শ্রু কারখানা থেকে কাপড় বিক্রি করতে হুবে ।" পথের মধ্যে লক্ষীকে অঞ্ঞান করে ইন্ডিয়ায় নেয়া হল। লক্ষী বলল, “আমি শুনলাম আমাকে ১৫,০০০ ইন্ডিয়ান রুপিতে বিক্রি করা হয়েছে। যখন আমি যৌনকর্মী হিসেবে কাজ করতে চাইতাম না তখন আমাকে মারা হতো । এক বছর আমাকে পতিতালয়ে আটকে রাখা হয়েছিল । পরে পুলিশ পতিতালয় ঘেরাও করল এবং আমি মুক্ত হরয় নেপালে ফিরে গেলাম। এর মধ্যে আমার বয়স হয়েছিল ১৪ বছর।"

দ্বিতীয় অংশ: লক্ষীর কী হয়েছিল? লক্ষ্মী ফিরে আসার পর তার মাবাবা তাকে গ্রহণ করতে অস্বীকার করল । সে আবার বিয়ে করল, কিন্তু তার এইচআইভি ধরা পড়ল। সে কখন জীবাণু দ্বারা আক্রান্ত হয়েছে -

জোর করে যৌন কাজ করানোর সময় নাকি বিয়ের পর সেটা নিশিচ নয়। 


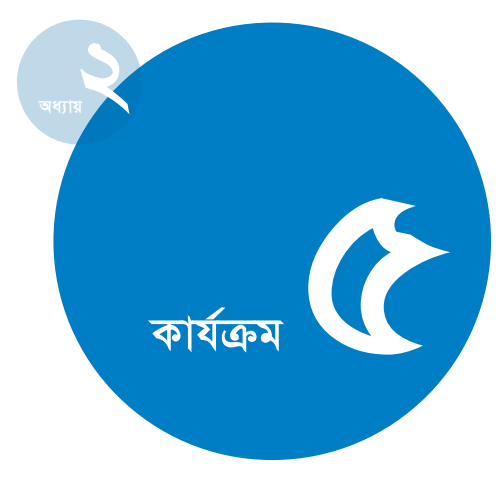

সময়কাল :

8৫ मिनिট

\section{উপকরণ:}

বোর্ড ও চক।

প্রস্তুতি:

আপনি আপনার এলাকার উপয্যাগী আরও কোন গুনাবলি যোগ করতে চান কিনা সেটা নির্ধারণ করুন ।

\section{পুরুষ এবং মহিলা বিষয়ক শব্দমালা}

অধিবেশন সম্পর্কিত ধারণা: সমাজ ‘পুরুত্য’ কিংবা ‘মহিলা’ বলতে কী বোঝায়, এই সম্বন্ধে শিক্ষার্থীরা দলবদ্ধ হয়ে শব্দমালা তৈরি করবে এবং এসব ধারণা কোথা থেকে এসেছে তা আলোচনা করবে। তাদের জেন্ডারের ধারণার সাথে পরিচয় করানো হবে।

উদ্দেশ্য: শিক্ষার্থীদেরকে ‘জেন্ডারের’ সংজ্ঞা দিতে শেখানো; পুরুষ এবং মহিলাদের ওপর আরোপিত কোন কোন গুনাবলি শারীরিক এবং কোনগুলো সমাজ দ্বারা নির্ধারিত সেগুলোর পার্থক্য করতে শেখানো; সমালোচনামূলক চিন্তা ও সৃজনশীল লেখার ক্ষমতা শক্তিশালী করা।

\section{निर्দেশাবলি}

১ শিক্ষার্থীদেরকে চার বা পাঁচ জন করে বিভিন্ন দলে বিভক্ত করুন (একই লিঙ্গের বা তা না হনেও চলবে)।

২ ব্যাখ্যা করুন:

• আজকে আমরা জেন্ডার নিয়ে আলোচনা করব (যা দ্বারা সমাজ পুরুষ্ব অথবা মহিলা হওয়া বোঝায়)। প্রত্যেক দল পুরুত্য এবং মহিলা হওয়ার সাথে সম্পর্কিত শব্দমালা তৈরি করবে।

‘শব্দমালা'র ধারণাটি পরিষ্কার করার জন্য আপনি বোর্ডে অন্য একটি বিষয়ের শব্দমালার উদাহরণ দিতে পারেন। নিচে ‘'ুুকুর’ শব্দের উদাহরণটি দেখ।

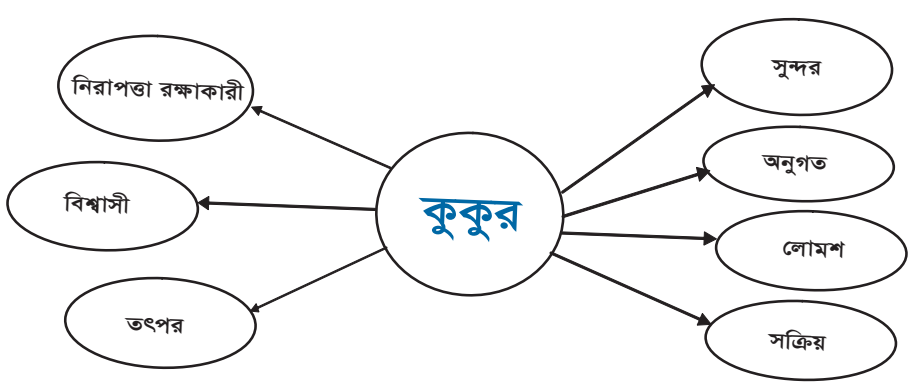

৩ প্রত্যেক দলকে ‘পুরুষ্য’ শব্দমালা টৈরি করতে দুই থেকে তিন মিনিট এবং আরও দুই থেকে তিন মিনিট ‘মহিলা’ শব্দমালা তৈরি করতে সময় দিন।

8 বোর্ডে ‘পুরুষ্য এবং ‘মহিলা’ লিখে আলাদা দুইটি কলাম তৈরি করুন । একটির নাম দিন ‘শারীরিক’ এবং অন্যটির ‘সামাজিক’। একটি দলের শিক্ষার্থীদের কাছে প্রশ্ন দিয়ে শুরু করুন:

• তোমাদের শব্দমালার একটি গুণ কী যেটা পুরুষ্য হওয়ার সাথে সম্পর্কিত?

• এই গুণ কি শারীরিকভাবে (‘শারীরিক’) নাকি সামাজিকভাবে নির্ধারিত ('সামাজিক')?

• যদি শিক্ষার্থীরা সামাজিক কোন গুণাবলিকে শারীরিক বলে নির্ধারণ করে, তাদেরকে প্রক্নের মাধ্যমে সঠিক করে দিন: যদি কোন ছেলে বা পুরুযের এই গুণ না থাকে তাহলেও কি সে পুরুষ থাকবে ? 
৫ পুরুষ্য হওয়ার জন্য সবগুলো গুণ না পাওয়া পর্যন্ত প্রত্যেক দল থেকে একটা করে নতুন গুণ যোগ করুন । নিশ্চিত হোন যে, এখানকার অনেকগুলো শব্দই শিক্ষার্থীদের শব্দমালায় আছে। (আপনাকে নির্দিষ্ট উত্তরের জন্য অনুসন্ধানী প্রশ্ন করতে হতে পারে ।) সাধারণত ‘পুরুষ হওয়ার’ বিষয়ে এই উদাহরণগুলির সাথে আর কী কী সম্পর্কিত?

• শারীরিকভাবে শক্তিশালী

• আবেগ প্রকাশ করেনা

- যৌনাকাজ্ক্ষী

• বিপরীত লিঙ্গের প্রতি যৌনকামী

• অর্থনৈতিকভাবে সফল

• পরিবার প্রধান

• শান্ত

• পিতা

- গর্বিত

• ক্ষমতাশালী

• ख্রীড়াবিদ

- माহमी

• সহিংস/সহিংসতা করতে ভয় পায় না

• রসিক

• বন্ধুর প্রতি বিশ্বাসী

৬ এ এই প্রক্রিয়া পুনরায় উল্লেখ করুন- ‘মেয়ে হওয়ার’ সাথে কী কী গুণাবলি সম্পর্কিত? সাধারণ গুণাবলি হল-

• সহানুভূতিপ্রবণ

- नম्य

• অনুগত

• আড্ডাপ্রিয়

• ভাল যোগাযোগকারী

• ভাল বউ

• ভীষণ আবেগপূর্ণ

• সুশৃংজ্খল/অনেক কাজে পারদর্শী
• বাস্তববাদী

- অহিংস

• বিनয়ী

- কুটিল

- পুরুষের চেয়ে শারীরিকভাবে দুর্বল

• যত্মবান/দায়িত্ববান

- मा

१ সব দলের আলোচনার জন্য ১০ মিনিট সময় রাখুন ।

• পুরুষ্ব ও মহিলাদের অল্প কিছু গুণাবলি শারীরিক। যেমনশধুমাত্র পুরুষরাই বাবা হতে পারে। শুবুমাত্র মহিলারাই সন্তান জন্ম দিতে বা দুধ পান করাতে পারে।

• কিন্তু পুরুষ্ব ও মহিলা হওয়ার বেশির ভাগ গুণাবলিই সামাজিকভাবে নির্ধারিত, শারীরিকভাবে নয়।

• পুরুষষ ও মহিলার ভূমিকা যা সামাজিকভাবে নির্ধারিত তাকে বলে ‘জেন্ডার ভূমিকা’। কেউ কি এই শব্দটি আগে শুনেছ?

• আমাদের সমাজে ‘জেন্ডার ভূমিকা’ বলতে তোমরা কী বোঝ ? মহিলাদের যেভাবে কাজ করতে বা জীবন যাপন করতে হয় সকল বিষয় কি তোমরা সমর্থন কর? পুরুষরা কীভাবে জীবন যাপন করে ?

• জেন্ডার সমতা বলতে তোমরা কী বোঝ?

• প্রত্যেক সম্প্রদায় এবং সমাজের কিছু মানুষ জেন্ডার এবং সমতা বলতে কিছু দৃষ্টিভঙ্গি লালন করে যেগুনো গতানুগতিক নয় । সমাজ যেমন সময় বা অঞ্চল ভেদে পরিবর্তিত হয়, জেন্ডার ভূমিকার দৃষ্টিভঙ্গিও তেমনি পরিবর্তিত হয়।

What's the Real Deal About Masculinity? (2008. Scenarios USA.) থেকে নেয়া হয়েছে। এটি পাবার জন্য www.scenariosusa.org/ge involved/MasculinityCurriculu উক্তিটিকে শেষ বা বিস্তারিত আলোচনা কর:

“একজন ছেলে /মেয়ে হুওয়া সহজ নয়, কারণ . . . . . ” 


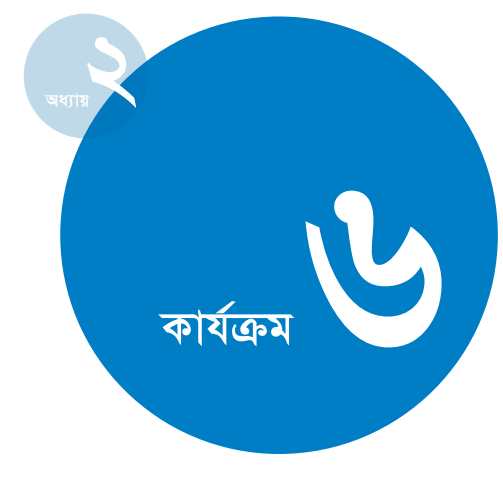

সময়কাল :

8 ৪িनिট (ধাপ নং ৫ বাড়ির কাজ হিলেবে দেয়া যেতে পারে।)

\section{উপকরণ:}

বোর্ড ও চক।

\section{প্রস্তুতি:}

অধিবেশন করুর পূর্বে এই বিষয়ে আপনার নিজের স্মৃতিচারণ করুন । স্মৃতি বিষয়ক কার্যক্র্ কিছু শিক্ষার্থীর জন্য কঠিন অনুভূতির সূত্রপাত করতে পারে। চিন্তা করুন্, কীভাবে সেগুলো সবচেয়ে ভালভাবে মোকাবেলা করা যায়- এবং আপনি কাকে পরিবর্তন করতে পারেন- পুনরায় কি কোন শিক্ষার্থীর সাহায্য করা উচিত।

\section{স্মৃত্চিারণ: শৈশবে জেন্ডার শিক্ষা}

অধিবেশন সম্পর্কিত ধারণা: শিক্ষার্থীরা এমন একটি সময়ের স্মৃতি চারণ করবে যখন তাদের সাথে লিঙ্গগত পার্থক্যের কারণে একটি নির্দিষ্টভাবে আচরণ করা হতো। সেই সকল অভিত্ঞতা সম্পর্কে তারা তাদের অনুভূতিগুনো মনে করবে।

উদ্দেশ্য: কীভাবে বাচ্চারা জেন্ডার ভূমিকা সম্পর্কে বার্তাগুলো গ্রহণ করে লে সম্পর্কে শিক্ষার্থীদেরকে উদাহরণ দিতে সক্ষম করা; এই বার্তাগুলো ব্যক্তিগত ও মানবাধিকারের দৃষ্টিকোণ থেকে বিবেচনা করা; বিশ্লেষণমূলক চিন্তার দক্ষতা শক্তিশালী করা ।

\section{নির্দেশাবলি}

১ একই লিঙ্গের শিক্ষার্থীদের চার বা পাঁচজনকে নিয়ে এক-একটি দল তৈরি করুন । তাদেরকে কলম ও খাতা দিন ।

২ ব্যাখ্যা করুন:

• আজকে আমরা জানার চেষ্টা করব ছেনে বা মেয়ে হিসেবে বেড়ে ওঠা বলতে কী বোঝায়। প্রথনে আমরা আমাদের স্মৃতিতে ছোট একটি পরিক্রমণ করব। তাই আরামদায়ক অবস্থান কর, কলম রেখে দাও এবং মন শিথিল কর।

• পুরনো কোনো সময়ের কথা মনে কর যখন তোমাদের মনে হয়েছিল যে, তোমাদের লিঙ্গগত পার্থক্যের কারণে তোমাদের সাথে ভিন্নরকম আচরণ করা হচ্ছে। যদি কিছু মনে করতে পার তাহলে লেখ।

• এখন মনে কর ঐ রকম আচরণ পাওয়ায় কী মনে হয়েছিল। তোমাদের ঐ অভিজ্ঞতার কিছু আবেগ বা অনুভূতির কথা লেখ । তোমাদের নিজ দলে তোমাদের স্মৃতি ভাগাভাগি করার সুযোগ থাকবে।

৩ কয়েক মিনিট পরে জিজ্ঞেস করুন:

- তোমাদের অভিজ্ঞতা অথবা অনুভূতি সম্পর্কে যেটুকু তোমাদের ইচ্ছা হয়, তোমাদের দলের সাথে আলোচনা করতে কয়েক মিনিট সময় নাও। यদি তোমরা না চাও তাহলে আদৌ আলোচনা করতে হবে না।
8 আরও পাঁচ বা দশ মিনিট পর জিজ্ঞস করুন:

- এই অভিজ্ঞতাগুনো মেয়ে বা মহিলাদের ভূমিকা ও মূল্যবোধ সম্পর্কে সামাজিক দৃষ্টিভঙ্গি ও রীতিনীতি নিয়ে আমাদের কী বনে? ছেলে বা পুরুষ সম্পর্কে?

• মানবাধিকার সম্পর্কে আমরা কী শিখেছিলাম তা মনে কর, এই দৃষ্টিতঙ্গি এবং রীতিনীতি কি তোমার কাছে সঠিক মনে হয়? কেন অথবা কেন নয় ?

- কোন পরিবর্তনগুলো পুরুষ এবং মহিলার সমতা অর্জনে প্রয়োজন হতে পারে?

৫ [সময় না থাকলে এই ধাপটি বাড়ির কাজ হিসাবে দেওয়া যেতে পারে।] শিক্ষার্থীদের মনে করিয়ে দিন যে, কোনো অবস্থা বা ঘটনার কিছু পরিণতি থাকতে পারে। গল্পের পরিণতি পরিবর্তন করার জন্য তাদের একটা সুযোগ দিন । ব্যাখ্যা করুন:

• এমন একটি স্মৃতিতে ফিরে যাও যেটা তুমি মনে করেছিলে ঠিক নয় বা অনৈতিক ছিল- এটা তোমার নিজের লেখাও হতে পারে বা তোমার দল থেকে শোনাও হুতে পারে।

- গল্পটির একটি নতুন সমাপ্তি লেখ যেটা তোমার কাছে সঠিক বা নৈতিক মনে হয়। 


\section{পিতৃত্বের প্রতিচ্ছবি}

অধিবেশন সম্পর্কিত ধারণা: শিক্ষার্থীরা তাদের পিতা বা পুরুষ্ব অভিভাবক সম্পর্কে লিখবে। তারা আলোচনা করবে, জেন্ডার ভূমিকা কীভাবে অভিভাবক হিসেবে পুরুষের ভূমিকা সীমিত করে দেয়, এবং তারা কেমন বাবা হতে চায় বা তাদদর সন্তানদের ( বা তাদদর পরিচিত বাচ্চাদের) জন্য চায় তা লিখবে ।

উদ্দেশ্য: শিক্ষার্থীদেরকে পুরুষ অভিভাবকত্ব সম্পর্কে অভিজ্ঞতা এবং মূল্যবোধ বিশেযণ করতে সক্ষম করা; নিজস্ব চিন্তা ও লেখার দক্ষতা শক্তিশালী করা।

\section{निर्দেশাবলি}

১ নিয়লিখিত ব্যাখ্যার মাধ্যমে বিষয়টির ধারণা দিন:

• আজকে আমরা পিতৃত্ব এবং অনুকরণীয় আদর্শ পুরুষ নিয়ে আলোচনা করব।

• আমি চাই তোমরা তোমাদের বাবাকে বা পুরুষ অভিভাবক বা তোমার জীবনে বাবার মত তাৎপর্যপূর্ণ অন্য কোন ব্যক্তিকে একটি ছোট্ট চিঠি লেখার মাধ্যনে শুরু কর। তোমার চিঠি অন্যদের কাছে প্রকাশ করতে হবে না । নিগ্নোক্ত যে কোন ভূমিকা দিয়ে তোমার চিঠি শুরু কর।

প্রিয় বাবা, তুমি ভেবেছ আমি তোমাকে ভুলে গেছি, কিন্তু আমি মনে রেখেছি--.

প্রিয় বাবা, তোমাকে আমার প্রয়োজন . .1

২ আনুমানিক ৫ মিনিট পর জিজ্ঞেস করুন :

• তোমার বাবা বা পুরুষ অভিভাবক বা অন্য যে কোন বিশেষ ব্যক্তি কেমন মানুষ ছিলেন? তাকে বর্ণনা করার জন্য কমপক্ষে ১০টি বিশ্লেষণ লেখ । আবার বলুন, তোমরা যা লিখেছ তা ব্যক্তিগত এবং প্রকাশ করার প্রয়োজন নেই।

• তোমার মতে, চমৎকার বাবা হওয়ার অংশ হিসেবে তুমি তোমার বাবার মট্যে দেখতে চাও এখন এমন ১০টি বিশ্লেষণ লেখ।
• তোমার বিশ্লেষণের দ্বিতীয় তালিকাটি দেখ এবং বেশিরভাগ বাবার মধ্যে যে বিশ্লেষণগুলো দেখা যায় সেগুনো উনেখ কর। যেগুলো অসাধারণ বা কিছু কিছু বাবার মধ্যে আছে ঐ বিশ্লেষণগুলোতে বৃত্ত পূরণ কর ।

• সন্তান যেভাবে চায় সেভাবে আচরণ করতে পুরুষদের ক্ষেত্রে প্রচলিত পুরুষ ভূমিকাগুলো কঠিন নাকি সহজ ভূমিকা পালন করে ?

• তোমরা কি এমন কোন বাবা বা বাবার মত কারও উদাহরণ দিতে পারবে যে প্রচলিত জেন্ডার ভূমিকাকে উপেক্ষা করে চলে ?

৩ পরিলেষে জিজ্ঞেস করুন্ন- তোমরা ভবিষ্যতে পিতৃত্বকে কীরূপ পরিবর্তনে দেখতে চাও?

বাড়ির কাজ: একজন পরিচিত প্রাপ্তবয়স্ক ব্যক্তির সাক্ষাৎকার নেবে এবং একজন ভাল বাবা হওয়ার জন্য কী প্রয়োজন সে বিষয়ে কথা বলতে বলবে।

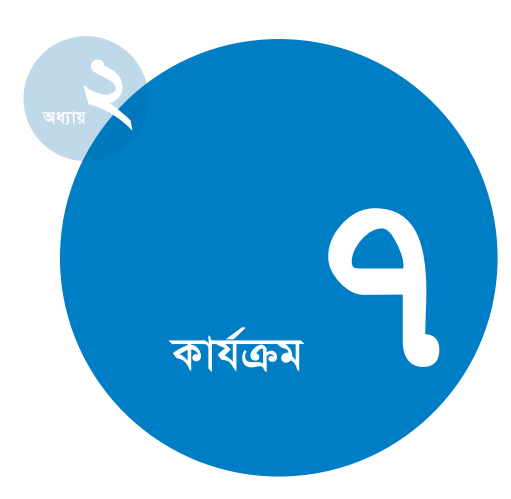

সময়কাল :

৪৫ মিनिট

\section{উপকরণ:}

বোর্ড ও চক।

\section{প्रস্তুতি:}

ধাপ ১-এর জন্য প্রশ্ন ও বাক্যগুনো বোর্ডে লিখুন অথবা এগুলো নিয়ে কর্মপরিকল্পনা টৈরি করতে পারেন । এই অধিবেশনে কিছু শিক্ষার্থীর (বিশেষ করে যাদের বাবার প্রতি বিরোধ বা নেতিবাচক ধারণা রয়েছে অথবা যাদের বাবা নেই) ক্ষেত্রে থাকতে পারে এমন আবেগের বিষয় নিয়ে ভাবুন । আপনি তাদেরকে কীভাবে সংবেদনশীলতার সাথে সাহায্য করবেন তা চিন্তা করুন । 


\section{কग्यंज्य}

\section{সময়কাল :}

ধাপ ১-৫: ৪০মিনিট

ধাপ ৬: ৪-৫ ঘন্টা

ধাপ ৭: ৯০-১২০ মিনিট

\section{উপকরণ:}

বোর্ড ও চক ; শিক্ষার্থীদের

হ্যান্ডআউটের কপি; টেপ।

\section{প्रস্তুতি:}

শিক্ষার্থীদের দিয়ে এই গবেষণা করানোর জন্য কর্তৃপক্ষের অনুমতি নিন। ধাপ ২-এর প্রত্যেকটি বিষয় প"থক কাগজে লিখুন। (যদি প্রয়োজন হয় বিষয়গুলোকে আপনার বিদ্যালয়ের উপযুক্ত ও সামঞ্জস্যপূর্ণ করে পরিবর্তন করতে পারেন । ধাপ ৩-এ ঠিক করে নিন কীভাবে শিক্ষার্থীদেরকে বিষয়বস্তুর সাথে পরিচিত করাবেন । সম্ভব হনে প্রত্যেক ছাত্রের জন্য হ্যান্ডআউটের কপি তৈরি করুন ।

\section{গবেষণা প্রকল্প : বিদ্যালয়ের পরিবেশে জেন্ডার}

অধিবেশন সম্পর্কিত ধারণা: শিক্ষার্থীরা বিদ্যালয়ে পড়ানোর ব্যাপারে জেন্ডারের ভূমিকা নিয়ে গবেষণা এবং তাদদর ফলাফল প্রকাশ করবে। এই প্রকল্পের জন্য শ্রেণিকক্ষের বাইরে গবেষণা ও সময় লাগবে।

উদ্দেশ্য: বিদ্যালয়ের পরিবেশ কীভাবে দৃঢ়তরভাবে গতানুগতিক জেন্ডার রীতিনীতিকে চ্যালেঞ্ঞ করতে পারে সে বিষয়ে শিক্ষার্থীদেরকে আলোচনায় উদ্দুদ্ধ করা; গবেষণার দক্ষতা শক্তিশালী করা।

\section{निर्দেশাবলি}

১ কক্ষের পৃথক পৃথক কোণে কাগজগুনো (গবেষণার বিষয়সহ) আটকে দিन।

২ ব্যাখ্যা করুন:

• পরবর্তী সপ্তাহে তোমরা গবেষণা পরিচালনা করতে সক্ষম হবে। গবেষণায় তোমাদেরকে যে প্রশ্নের উত্তর খুঁজতে হবে তা হলো “বিদ্যালয় কীভাবে গতানুগতিক জেন্ডার ভূমিকাকে আরো জোরদার অথবা চ্যালেঞ করতে পারে ?"

• চারটি গবেষণা দলের প্রত্যেকে নিম্নল্লিখিত বিষয়গুলি থেকে একটি করে বিষয়ে গবেষণা করবে: শ্রেণিকক্ষে আচার-আচরণ, পাঠ্যক্রম বহির্ভূত বিদ্যালয়ের অতিরিক্ত অন্যান্য কর্মকাণ্ড, পাঠ্যক্রম ও পাঠ্যবই, এবং বিদ্যালয় ও শিক্ষার পরিসংখ্যান।

• তোমাদের ভাল লাগে এমন দুইটি বিষয় খুঁজে বের করতে চেষ্টা কর।

৩ গবেষণা দল গঠনের জন্য শিক্ষার্থীদের পছন্দের বিষয়সহ কাগজে সই করতে বলুন । (অথবা তাদেরকে দলে ভাগ করে দিন।)

8 গবেষণা নির্দেশনা বিলি করুন এবং পুনরায় উলেখ করুন । নিশ্চিত হোন যে, কীভাবে কাজ করতে হরে তা সকলে বুঝতে পেরেছে ।

৫ প্রত্যেক দলকে তাদের হ্যান্ডআউট দিন। কীভাবে গবেষণা পরিচালনা করবে সে ব্যাপারে দলের মধ্যে আলোচনার সুযোগ দিন । ঘুরে ঘুরে দেখুন এবং আরও কোন পরামর্শের প্রয়োজন হলে তা বুঝিয়ে দিন ।
৬ গবেষণার জন্য শিক্ষার্থীদের দুই সপ্তাহ পর্যন্ত সময় দিন । প্রয়োজন অনুयায়ী সাহায্য করুন ।

१ গবেষণা শেষ হনে শিক্ষার্থীরা তাদের গবেষণা ও এর ফলাফল উপস্থাপন করবে। প্রত্যেক উপস্থাপনার জন্য ১৫ মিনিট সময় দিন। প্রতিটি উপস্থাপনার পর অন্য শিক্ষার্থীদের জিজ্ঞস করুন:

• এই দলের কাছে তোমাদের কোনো প্রশ্ন আছে?

• তোমাদের কাছে বিশেষ করে কোন্ বিষয়টি আকর্ষণীয় বা বিস্ময়ের মনে হয়েছে?

• তোমরা কি তাদের উপসংহারের সাথে একমত ? না হলে কেন একমত নও?

• তোমরা অন্য কী পরামর্শ বা সুপারিশ দেবে ?

৮ যখন সবগুলো দলের উপস্থাপন শেষ হবে তখন শ্রেণিকক্ষের সবাইকে জিজ্ঞস করুন:

• কীভাবে বিদ্যালয়ের পরিবেশ গতানুগতিক জেন্ডার রীতিনীতিকে আরো জোরদার করতে পারে ?

• কীভাবে এই পরিবেশ ঐসকল রীতিনীতিকে চ্যানেঞ্জ করতে পারে?

- পাঁচটি পরিবর্তন কী কী যা জেন্ডারের সাপেক্ষে বিদ্যালয়গুলিতে আরও ভাল পরিবেশ তৈরি করবে এবং সকলে সাদরে গ্রহণ করবে, সম্মান করবে এবং সবার জন্য নিরাপদ হবে ?

• তোমরা এটি হওয়ার জন্য কী করতে পার ? 


\section{সকল গবেষণা দলের জন্য নির্দেশাবলি}

শ্রেণিকক্ষ গবেষণা প্রকল্পের বিষয় হনেো: “বিদ্যালয়ের পরিবেশে জেন্ডারের ভূমিকা ।” প্রত্যেক দলই এ বিষয়ের বিভিন্ন দিক নিয়ে গবেষণা করবে। এখানে ধাপগুলি উলেখ করা হল:

১ দলের সকলে মিলে সতর্কতার সাথে প্রশ্নগুলি পড়।

২ কীভাবে প্রশ্নগুলির সর্বোত্তম উত্তর বের করা যায় তা আলোচনা কর। প্রয়োজন হলে শিক্ষকের সাহায্য নাও।

৩ দলের প্রত্যেক সদস্য আলাদা আলাদা প্রশ্নের দায়িত্ব নিবে নাকি দলের সকলে মিলে নির্দিষ্ট প্রশ্ন নিয়ে গবেষণা করবে তার সিদ্ধান্ত নাও।

8 তোমাদের প্রক্নের উত্তরের জন্য তথ্য সংগ্রহ কর। প্রয়োজন হলে শিক্ষকের সাহায্য নাও।

৫ যখন তোমরা তোমাদের উপাত্ত সংগ্রহ করে ফেলবে তখন একটি প্রতিবেদন লিখবে যেখানে নিয়ল্লিখিত ভাগগুলি থাকবে।

ক যে প্রশ্ন(গুলির) উত্তর তোমরা খুঁজতে চেষ্টা করেছ ।

খ গবেষণা পদ্ধতি (যেভাবে তোমরা গবেষণা করেছ)।

গ ফলাফল (তোমরা যা শিখেছ )।

ঘ উপসংহার (ফলাফল নিয়ে তোমরা যা ভাবছ/ তোমরা যে উপসংহার লিখেছ)।

ঙ পরবর্তীত করণীয় (বিদ্যালয়ের পরিবেশ আরও ভাল করতে পারে এবং যেগুলি সকলে সাদরে গ্রহণ করবে ও সকলের জন্য নিরাপদ এমন পরিবর্তন)।

তোমাদের দল প্রতিবেদনের ওপর ভিত্তি করে ১৫ মিনিটের একটি উপস্থাপনাপত্র তৈরি করবে। উপস্থাপনের পরিকল্পনা কর এবং এতে দনের সকল সদস্যের অন্তর্ভুক্তি নিশ্চিত কর। 


\section{১ নং দলের হ্যান্ডআউট:}

তোমরা কি মনে কর আমাদের পাঠ্যবইয়ে জেন্ডার বিভেদ প্রতিফলিত হয়? অথবা এগুলি কি জেন্ডার সমতা আনতে সাহায্য করে ?

নমুনা প্রশ্নগুলি:

• আমাদের পাঠ্য বইয়ে ছেলে এবং মেয়ে অথবা পুরুষ এবং মহিলার ছবি কীভাবে আঁকा আছে?

• কতগুলি মহিলা এবং কতগুলি পুরুষের ছবি আছে?

- মেয়েদের ঐতিহাসিক, মেধাগত, বৈজ্ঞানিক, সাহিত্য এবং শিল্পকলার অর্জনগুলিকে কি সমপূর্ণ তুলে ধরা হয়েছে?

• গল্পে ও রচনায় কে প্রধান ভূমিকা পালন করে এবং কে অধীনস্থ?

• বইয়ে কার মতামত প্রতিফলিত হয়? তোমাদের বইয়ের লেখক পুরুষ্য नाকি মহিলা?

• অন্য আর কী কী উপায়ে জেন্ডারের প্রথাগতরীতি-নীতি প্রতিফলিত ও জোরদার অথবা চ্যালেঞ করা হয়েছে?

• পাঠ্যবইয়ে অন্যান্য আর কোন গোষ্ঠীকে প্রথাগতভাবে উপস্থাপন করা হয়েছে?

গবেষণার পদ্ধতি:

প্রাথমিক বা মাধ্যমিকে ব্যবহৃত সমাজ, ইতিহাস বা সাহিত্যের কমপক্ষে দুইটি পাঠ্যবই নাও। উপরের নমুনা প্রশ্নের উত্তরের জন্য বইয়ের লেখা ও ছবি বিশেষণ কর।

\section{২ নং দলের হ্যান্ডআউট:}

শ্রেণিকক্ষের পরিবেশ কীভাবে প্রচলিত জেন্ডার ভূমিকাকে আরো জোরদার অথবা চ্যালেঞ করে ?

নমুনা প্রশ্নগুলি:

- শ্রেণিকক্ষের বেশির ভাগ সময়ে কারা কথা বলে ছেলেরা না কি মেয়েরা ? কে বেশি সময়ের জন্য কথা বনে ? কে বেশির ভাগ সময়ে ব্যাঘাত সৃষ্টি করে ? যদি শ্রেণিকক্ষে দলনেতা থাকে, তাহনে তারা ছেনে নাকি মেয়ে?

• কীভাবে শিক্ষক নিয়ম মাফিক জেন্ডার আচরণকে চ্যালেঞ অথবা আরো জোরদার করে ?

• প্রথাগতরীতি-নীতি যেমন তাদের ণৃ-তাত্ত্বিকগোষ্ঠী অথবা লিঙ্গ পরিচয়ের জন্য কি কিছু ছাত্র বেশি সুবিধা অথবা কম সুবিধা পায়?

গবেষণার পদ্ধতি:

বিভিন্ন শ্রেণিকক্ষ পর্যবেক্ষণ কর, বিশেষ করে পৃথক পৃথক শিক্ষকের সাথে। তোমরা যা দেখবে তা কীভাবে ‘পরিমাপ’ এবং নথিভূক্ত করবে তা নিয়ে পর্যবেক্ষণের পূর্বে দলের সাথে আলোচনা কর। তোমরা একটি সহজ ফরম তৈরি করতে পার যেটি তোমরা পর্যবেক্ষণের সময় পূরণ করবে। যদি তোমাদের ঘড়ি থাকে তাহলে ছাত্র এবং ছাত্রীরা কত সময় কথা বলে তা তোমরা দেখতে পার। 


\section{৩ নং দলের হ্যান্ডআউট:}

বিদ্যালয়ের পাঠ্যক্রম বহির্ভূত বিষয়ে শিক্ষার্থীদের অংশগ্রহণ এবং বিদ্যালয়ের সুযোগ-সুবিধার ক্ষেত্রে জেন্ডার রীতি-নীতি কীভাবে প্রভাব ফেলে ?

\section{নমুনা প্রশ্নগুলি:}

- ছেলে এবং মেয়েরা কি পাঠক্রম বহির্ভূত কর্মকাণ্ড সমানভাবে অংশগ্রহণ করে? যদি তাদের পৃথক কর্মকাত্ড অংশগ্রহণের প্রবণতা থাকে তাহলে কোনগুলিতে বেশি ছেলে এবং কোনগুলিতে বেশি মেয়ে থাকে?

• যদি সার্বিকভাবে তারা বিভিন্ন কর্মকাণ্ড অংশগ্রহণ করে তাহুেে ছেলে এবং মেয়ে কি একইভাবে অংশগ্রহণ করতে পারে ?

- যে সকল কর্মকাণ্ড ছেনেদের অন্তর্ভুক্ত করে এবং যে সকল কর্মকাণ্ত মেয়েদের অন্তর্ভুক্ত করে তার জন্য কি বিদ্যালয়ে সমান সুযোগ-সুবিধা (যেমন- টয়লেট, ব্যায়ামাগার, খেলার মাঠ ও এরূপ অন্যান্য), সরঞ্জাম, একইরূপ পোশাক ও ব্যবহারের জন্য আনা জিনিসপত্রের বরাদ্ল আছে?

- যে সকল কর্মকাণ্ড ছেলে-নেয়ে উভয়কে অন্তর্ভুক্ত করে সেখানে কে নেতৃত্বের ভূমিকা পালন করে ?

\section{গবেষণার পদ্ধতি:}

বিদ্যালয়ের সমস্ত পাঠক্রম বহির্ভূত কর্মকাণ্ত যেমন- খেলাধুলা, ছাত্র সংসদ, বিদ্যালয় ক্লাব ইত্যাদির একটি তালিকা তৈরি কর। কর্মকাণ্ডগুলি পর্যবেক্ষণ কর, যে সকল ছাত্র ও ছাত্রী এগুলির অন্তর্ভুক্ত তাদের সবার সাক্ষাৎকার গ্রহণ কর এবং ঐ দলগুলির শিক্ষক-উপদেষ্টার সাক্ষাৎকার গ্রহণ কর ।

\section{8 নং দলের হ্যান্ডআউট:}

বিদ্যালয়ে সার্বিক জেন্ডার সমতা কেমন ? এ বিষয়ে

পরিসংখ্যান থেকে আমরা কী দেখতে পাই?

নমুনা প্রশ্নগুলি:

• শিক্ষকদের বেশির ভাগই পুরুষ নাকি মহিলা? অধ্যক্ষ পুরুষ্ব নাকি মহিলা ? শিক্ষক-শিক্ষিকাদের মধ্যে কাদের অধ্যক্ষ হিসেবে পদোন্নতির সম্ভাবনা বেশি?

• পুরুষ্ব এবং মহিলারা কি সমানভাবে গণিত, সাহিত্য ও শিল্পকলা পড়ায়?

• সবচেয়ে ছোট শ্রেণিতে কে পড়ায় ? সবচেয়ে বড় শ্রেণিতে ?

• শিক্ষার্থীদের কী অবস্থা? ছেলে এবং মেয়েরা কি সমান সংখ্যায় ভর্ত হয়? यদি না হয়, তাহলে কেন নয়?

• সব শ্রেণিতেই কি ছেলে-পেয়েরা সমানভ।বে ভর্তি হতে পারে ? তারা কি প্রত্যেক শ্রেণিতে একইভাবে সাদরে গৃহিত হয়েছে বলে অনুভব করে?

গবেষণার পদ্ধতি:

তোমাদের বিদ্যালয়ের অধ্যক্ষ হয়ত তোমার প্রয়োজনীয় জেন্ডার বিষয়ক তথ্থের বেশির ভাগই দিতে পারবেন । তা না হলে যত বেশি সম্ভব শিক্ষকের সাথে তাদের শ্রেণি এবং বিভাগের বিভিন্ন অনুষদ যেখানে তারা পড়ান লে সম্পর্কে কথা বল । যদি সম্ভব হয় জাতীয় পর্যায়ের তথ্যও সং্রহ কর কীভাবে জেন্ডার রীতিনীতি গ্রেড লেভেল সমাপ্ত করাসহ শিক্ষাগত অর্জনে প্রভাব ফেলে । 


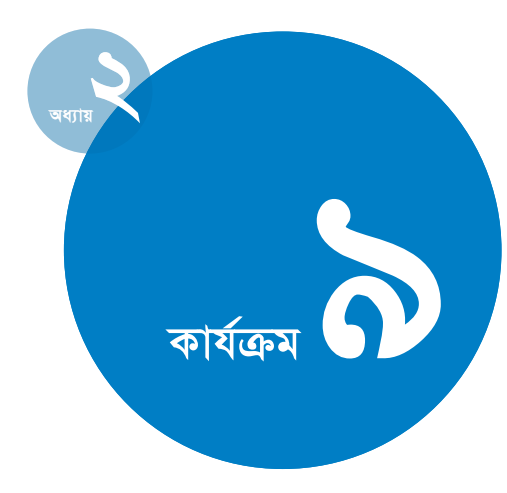

সময়কাল :

80 মিनिট

\section{উপকরণ:}

বোর্ড, চক, পত্রিকা, বিজ্ঞাপনসহ খবরের কাগজ, কাঁচি, বড় কাগজ, আঠা বা টেপ।

\section{প্রস্তুতি:}

দলের সকলের জন্য পর্যাপ্ত পরিমাণে বিজ্ঞাপনসহ পত্রিকা এবং সংবাদপত্র সংগ্রহ করুন অথবা শিক্ষার্থীদের এ ধরনের পত্রিকা আনতে বলুন।

\section{প্রথাগত ধারণার বিপনণ: বিজ্ঞাপন বিশেষণ}

অধিবেশন সম্পর্কিত ধারণা: শিক্ষার্থীরা বিজ্ঞাপনের পুরুষ্ব এবং মহিলাদের ছবি নিয়ে একটি চিত্রকর্ম তৈরি করবে, জেন্ডার বিষয়ে বিজ্ঞাপনের বার্তাগুলি বিশেযণ করবে, এবং প্রথাগত ধারণাকে ভেঙ্গে দেয় এমন বিজ্ঞাপন তৈরি করবে।

উদ্দেশ্য: শিক্ষার্থীদেরকে জেন্ডার বিষয়ে প্রথাগত ধারণা তৈরি ও স্থায়ী করাতে প্রিন্ট মিডিয়ার ভূমিকা শনাক্ত করতে ও আলোচনা করতে উদ্দুদ্ধ করা; সমালোচনামূলক চিন্তার দক্ষতা শক্তিশালী করা ।

\section{निর্দেশাবলি}

১ পত্রিকা বিলি করুন এবং ব্যাখ্যা করুন:

• আজকে আমরা কাগজের টুকরো জোড়া দিয়ে বিশেষ ধরনের চিত্র তৈরি করবো- পত্রিকা থেকে কাটা ছবিগুলি একটা কাগজে টেপ বা আঠা দিয়ে আটকিয়ে চিত্রকর্মটি তৈরি হবে ।

• তোমরা প্রত্যেকে দুইটি করে বিশেষ ধরনের চিত্র তৈরি করবে। একটিতে মহিলাদের ছবি এবং অন্যটিতে পুরুষদের ছবি থাকবে।

২ দুইটি বিশেষ ধরনের চিত্র শেষ করতে শিক্ষার্থীদের ১০-১৫ মিনিট সময় দিন। তারপর প্রকল্পের বাকি অংশ আলোচনা করুন:

• পুরুষদের ছবি দিয়ে বিশেষ ধরনের চিত্র টৈরি ওুরু করার জন্য বিজ্ঞাপনে তোমার দেখা পুরুষদের বৈশিষ্ট্যের একটি তালিকা তোমাদের খাতায় লিখ।

- মহিলাদের ছবি নিয়ে বিশেষ ধরনের চিত্র তৈরি করতে একই রকম কাজ কর।

৩ পরস্পরকে ছেদ করে এমন দুইটি বৃত্ত আঁকুন এবং একটি ‘পুরুষ্ব’ ও একটি ‘মহিলা’ এবং ছেদকৃত অংশে ‘উভয়’ নামকরণ করুন এবং

জিজ্ঞেস করুন:

• কোন বৈশিষ্ট্যগুনো শুধুমাত্র পুরুষ্দের বিজ্ঞাপনে আছে? এগুনোর তালিকা 'পুরুষ্য' বৃত্তে লেখ ।

• কোন বৈশিষ্ট্যগুলো শুধুমাত্র মহিলাদের বিজ্ঞাপনে আছে? এগুলোর তালিকা “মহিলা” বৃত্তে লেখ।

- কিছু বৈশিষ্ট্য কি পুরুষ্ব এবং মহিলা উভয় বিজ্ঞাপনে আছে? এগুলোর তালিকা উভয় বৃত্তের ছেদকৃত স্থানে লেখ ।
• তোমরা এই রেখাচিত্রে কী লক্ষ্য করেছ? এই বিজ্ঞাপনগুলো মহিলারা কীভাবে তাকাবে বা আচরণ করবে সে সম্পর্কে কী ধারণা প্রবর্তন করতে চায়? পুরুষ্টদের সম্পর্কেই বা কী ধারণা প্রবর্তন করতে চায় ?

• কীভাবে এই ছবিগুলো জেন্ডারের প্রথাগত ধারণা তৈরি ও স্থায়ী করে ? মহিলাদের কোন মূল্যবোধগুনো তারা বিপণন করে ? পুরুষদের কোনগুলো?

• কীভাবে এই ছবিগুলো বাস্তবতার সাথে মেলানো যায় ?

- কোনদিক থেকে মিডিয়ার ছবিগুলো প্রথাগত হয়ে যায়? মিডিয়া কি নতুন প্রথাগত ধারণা তৈরি করে নাকি সমাজের বিদ্যমান প্রথাগত ধারণাগুলো পুনরায় উলেখ এবং আরো জোরদার করে ? কেন ?

• মিডিয়ার ছবিগুলো দেখে যুব সমাজ কীভাবে প্রভাবিত হচ্ছে? পত্রিকা বা বাণিজ্যিক টেলিভিশন দেখে কি কখনও নিজেদের সম্বন্ধে খারাপ অনুভব করেছ? মানুষ কী করতে পারে যদি তারা দেখে বিজ্ঞাপনগুলো অশোভন বা ছবিগুলো প্রথাগত ধারণা চিত্রায়িত করে ?

বাড়ির কাজ: একটা ব্যতিক্রমধর্মী বিজ্ঞাপন তৈরি কর যেটি জেন্ডারের প্রথাগত ধারণাকে ভেঙ্গে ফেলে।/यদি সম্ভব হয় ছাত্রদেরকে তাদের সম্ন্ন কাজ দেওয়ালে ঝোলাতে বলুন বা সহপাঠীদের দেখাতে বলুন ।] 


\section{জেন্ডার ভিক্তিক স্থান}

অধিবেশন সম্পর্কিত ধারণা: শিক্ষার্থীরা কোন্ জায়গাগুনো ছেলে এবং মেয়েদের মধ্যে কাদের জন্য নিরাপদ এবং প্রবেশযোগ্য সেটা দেখিয়ে তাদদর এলাকার ম্যাপ তৈরি করবে। কীভাবে জেন্ডার ভূমিকা দ্বারা সমাজ জীবনে বসবাস প্রভাবিত হয় তা তারা বিল্লেষণ করবে। (এই অধিবেশনটি তার সমাজের জন্য প্রযোজ্য কিনা তা শিক্ষককে অবশ্যই সিদ্ধান্ত নিতে হবে।)

উদ্দেশ্য: জেন্ডার কীভাবে চলাচল, নিরাপত্তা এবং সমাজের বিভিন্ন স্থ|নে প্রবেশাধিকারকে প্রভাবিত করে সে বিষয়ে শিক্ষার্থীদেরকে বিশ্লেষণ করতে সক্ষম করা; জেন্ডার রীতিনীতি জনজীবনে মেয়ে ও মহিলাদের অংশগ্রহণকে প্রভাবিত করতে পারে এমন কমপক্ষে তিনটি উপায় বলা; বিমূর্ত চিন্তা এবং উপাত্তের ভৌগলিক চিত্রায়নের দক্ষতা শক্তিশালী করা।

\section{निर्দ্শশাবলি}

১ শিক্ষার্থীদের বলুন যে, তারা তাদের সমাজের ‘জেন্ডার ম্যাপ’ তৈরি করতে যাচ্ছে। এই ম্যাপে দেখানো হবে বাড়ির বাইরে কোন্ জায়গাগুনো প্রধানত ছেলে বা পুরুষদের (কোথায় তারা নিরাপদে দেখা-সাক্ষাৎ করে, খেলা করে অথবা অন্যান্য কাজে ব্যস্ত থাকে) এবং কোন্ জায়গাগুলো মেয়ে বা মহিলাদের, এবং কোন্ জায়গাগুলো উভয়ের জন্য।

২ শিক্ষার্থীদেরকে ছোট দলে বিভক্ত করুন (একই লিঙ্গের বা একই লিঙ্গের নয় ) এবং প্রত্যেককে আপনার সমাজের একটি সাধারণ ম্যাপ দিন । (ভাল দলের জন্য বাড়তি সময় দিন এবং নিজেদের ম্যাপ নিজেদেরকেই তৈরি করতে বলুন।)

৩ শিক্ষার্থীদের তিন রঙের একসেট রঙিন পেন্গিল (বা মার্কারপেন), এবং তাদের সমাজের নিম্মলিখিত জায়গাগুনোর একটা তালিকা দিন। ভাল দলকে তাদের জায়গাগুলোর তালিকা নিজেদেরকে টৈরি করতে বলুন ।

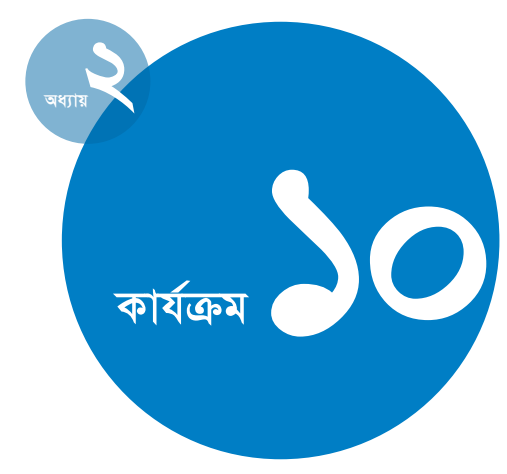

\section{সময়কাল :}

ধাপ ১-৫: ৪৫ মিনিট

\section{উপকরণ:}

বোর্ড ও চক; টেপ; এলাকার ৫ অথবা ৬টি সাধারণ ম্যাপের অনুলিপি যাতে তারা সময়ের আগে আঁকতে পারে (অথবা ভাল ছাত্রদের নিজেদের মানচিত্র আঁকার জন্য বড় কাগজ); রঙ পেন্সিল বা কলম।

श्रস্তুতি:

এলাকাগুলোর নামের তালিকা যাচাই করুন এবং আপনার সমাজকে বোঝানোর জন্য এগুনো পরিবর্তন করুন । 
8 প্রকল্পটি বর্ণনা করুন:

• প্রত্যেকটি জায়গায় ছেলেরা ও পুরুষরা, মেয়েরা ও মহিলারা বা সবাই সমানভাবে যেতে পারে কিনা তা তোমার দলের সাথে সংক্ষিপ্তভাবে আলোচনা কর। লক্ষ্য রেখ জায়গাগুনো আনন্দময় এবং নিরাপদ কিনা ।

• পুরুষ বান্ধব জায়গার জন্য এক রঙের পেন্সিল বেছে নাও , আরেকটি মেয়ে বান্ধব জায়গার জন্য এবং তৃতীয়টি জেন্ডার নিরপেক্ষ জায়গাগুলোর জন্য যা ছেলে-মেয়ে উভয়ের জন্য আনন্দময় এবং নিরাপদ । রং দিয়ে ম্যাপের ওপর জায়গার পরিচিতি দাও যা সেই জায়গার ‘জেন্ডারভিত্তিক’ বৈশিষ্ট্য নির্দেশ করে।

• কাজ শেষ হনে তোমার ম্যাপ এলাকার সাধারণ জায়গায় চলাফেরা, নিরাপত্তা এবং প্রবেশাধিকার সম্পর্কে কী নির্দেশ করে তা আলোচনা কর

৫ যখন তারা ম্যাপগুলি তৈরি শেষ করে তখন দলগুলির মধ্যে ঘুরুন এবং তাদের আলোচনা করতে সহায়তা করুন ।

৬ শিক্ষার্থীদেরকে তাদের ম্যাপগুলো দেওয়ালে টাঙ্গাতে বলুন। হয় প্রত্যেক দল তাদের ম্যাপ উপস্থাপন করবে অথবা ছাত্ররা পর্যায়ক্রমে ঘুরে ঘুরে প্রত্যেকের ম্যাপ দেখবে ।

৭ দলের সবাইকে জিজ্ঞস করুন (এবং তাদের উত্তরগুলো বোর্ডে লिখুন):
- তোমাদের পর্যবেক্ষণগুলো কী ছিল ? মেয়েদের কি ছেলেদের মত চলাফেরার স্বাধীনতা আছে?

• সাধারণ জায়গায় প্রবেশাধিকারের ক্ষেত্রে ছেলে এবং মেয়েদেরকে কীভাবে তুলনা করা হয়?

- জনসমাবেশ ছেলে এবং মেয়েদের জন্য কি একই রকম নিরাপদ?

• যখন ছেলেরা বড় হয় তখন কি এই অবস্থাগুলোর পরিবর্তন হয় ? যখন মেয়েরা বড় হয় তখন কি এই অবস্থাগুলোর পরিবর্তন হয়?

• এই পাথর্ক্যের সাধারণ কারণগুলো কী কী?

• জনসাধারণের জায়গার জেন্ডারভিত্তিক বৈশিষ্ট্য সম্পর্কে তোমরা কী অনুভব করো ?

• পরিপূর্ণ নাগরিক হওয়ার ক্ষেত্রে মেয়ে ও মহিলাদের নিরাপদ জায়গায় সীমিত প্রবেশাধিকার কীভাবে প্রভাব ফেলে ? [শিক্ষার্থীদের জিজ্ঞস করুন তারা কতজন নারীকে চেনে যাদের সরকারি সিদ্ধান্ত গ্রহণে ভূমিকা আছে? পুরুষদের তুলনায় কতজন নারীর সরকারি ক্ষমতা আছে? স্থানীয় সরকার পুরুষদের দ্বারা পরিচালিত হয় নাকি নারীদের দ্বারা?]

• ছেলে বা পুরুষরাও কি একই ভাবে প্রভাবিত হয়? কেন অথবা কেন নয়?

বাড়ির কাজ: এমন একটি জায়গা পছন্দ কর যেখানে মেয়ে বা মহিলারা নিরাপদ এবং স্বাচ্ছন্দবোধ করে না । এই অবস্থা পরিবর্তনের জন্য কী করতে হবে তা আলোচনা কর। কীভাবে এমন পরিবর্তন হতে পারে? 


\section{সক্রিয় নারীরা: একটি বই তৈরির প্রকল্প}

অধিবেশন সম্পর্কিত ধারণা: শিক্ষার্থীরা একজন নারীনেত্রী সম্পর্কে বাচ্চাদের বই তৈরি করবে। (লক্ষণীয়: এই অধিবেশনের জন্য পাঠাগার এবং ইন্টারনেট ব্যবহার সুবিধা থাকা প্রয়োজন, তাই আপনাকে অবশ্যই সেভাবে পরিকল্পনা করতে হবে।)

উদ্দেশ্য: শিক্ষার্থীদেরকে নারীনেত্রী সম্পর্কে জানতে সাহায্য করা এবং তাদের নিজেদের জীবনে নারীদের সাহস ও অর্জনের সাথে সম্পৃক্ত করতে সক্ষম করা; গবেষণা দক্ষতা শক্তিশালী করা।

\section{নির্দেশাবলি}

১ হ্যান্ডআউটগুনো বিতরণ করুন (অথবা এগুলো বোর্ডে লিখুন)।

২ শ্রেণিকক্ষের সবাইকে তিন বা চার জন করে দলে ভাগ করুন এবং ব্যাখ্যা করুন:

• আজকে আমরা এমন একটি প্রকল্প শুরু করব যেখানে আমরা ঐ সকল নারীদের সম্পর্কে জানব যারা সামাজিক ন্যায় বিচারের জন্য নেতৃত্ব দিয়েছিলেন বা দিচ্ছেন ।

- ্রত্যেক দলই পৃথক পৃথক নারীনেত্রীর জীবন ও কাজ নিয়ে গবেষণা করবে।

- এরপর প্রত্যেক দলই বাচ্চাদের জন্য ঐ নেত্রীকে নিয়ে একটি গল্প তৈরি করবে, এবং তা দিয়ে বাচ্চাদের জন্য একটি বাঁধাই করা বই তৈরি করবে। [শিক্ষার্থীদের জন্য এমন কোন আয়োজনের কথা বলুন যেখানে তারা নিকটবর্তী প্রাথমিক বিদ্যালয়ে বাচ্চাদের বই ধার বা পড়ার জন্য দিতে পারবে]

৩ সক্রিয় নারীদের তালিকাটি পুনরায় পডুন (হ্যান্ডআউট দেখুন)। [পুনরাবৃত্তি এড়ানোর জন্য কীভাবে তারা ‘সক্রিয় নারী’ পছন্দ বা নিয়োজিত করবে তা ব্যাখ্যা করুন]

- কেউ কি কখনও এই নারীদের সম্পর্কে কিছু সুনেছ?

• তোমরা অন্য কোন নারীনেত্রীর কথা বলতে পার, কিন্তু আমার কাছ থেকে জেনে নাও সে এই পাঠের উপযুক্ত কিনা ।

8 নির্দেশিত প্রশ্নগুলি পুনরায় পড্ডুন (হ্যাল্ডআউট দেখ বা বোর্ড থেকে শিক্ষার্থীদের প্রশ্নগুলো দেখে নিন)।
(४ ব্যাখ্যা করুন: ১) দলগুনো শ্রেণিকক্ষে বা বাড়িতে তাদের কাজ শেষ করতে চায় কিনা; ২) তাদের কাজ শেষ করতে ও জমা দিতে কত দিন লাগবে; ৩) কোথায় তারা তাদের সক্রিয় নারী সম্পর্কে তথ্য পেতে পারে ?

৬ ব্যাখ্যা করুন যে, যখন গবেষণা শেষ হয়ে যাবে তখন দলের প্রত্যেক সদস্য বই তৈরির অন্তত একটা অংশের দায়িত্ব নিবে: গল্পলেখা; সম্পাদনা ও লেখা সংশোধনের জন্য পড়া; কম্পিউটার চিত্রণ তৈরি করা; প্রচ্ছদ অলংকরণ ও তৈরি করা; বই বাঁধাই করা (লেলাই করা, টেপ লাগান, স্টাপল করা ইত্যাদি)।

৭ প্রত্যেক দলের জন্য তাদের ‘সক্রিয় নারীদের’ বাছাই করার জন্য এবং দলের মধ্যে বই তৈরির প্রতিনিধি বাছাই করতে বাকি সময় ব্যবহার করুন (হ্যান্ডআউট দেখুন)। এ প্রক্রিয়ায় সাহায্য করতে ঘুরে ঘুরে দেখুন। বইটি বন্ধুদের সামনে পড়তে এবং যদি সম্ভব হয় ছোটদের সামনে পড়তে। তারপর জিজ্ঞেস করুন:

• কোন্ কোন্ নারীকে তোমরা বিশেষভাবে প্রশংসা করেছ?

• তোমরা কি মনে কর যে, তোমরাও একসময় এমন কিছু করতে পারবে যা এদের যে কোন একজন নারীনেত্রী করেছে?

• সেটা করার জন্য কী প্রয়োজন হবে?
৮ সম্পূর্ণ বইটা উপস্থাপন করার জন্য : প্রত্যেক দলকে বলুন তাদের

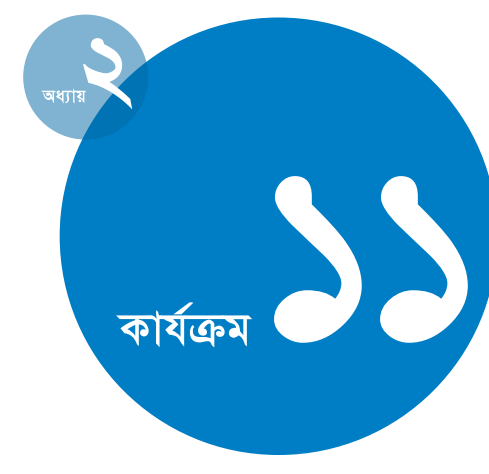

সময়কাল :

ধাপ-১-৭ :৪৫ মিনিট

বাস্তবায়ন: ২-৩ ঘন্টা শ্রেণিকক্ষে বা বাড়ি কাজ হিসেবে

ধাপ $b: ~ 8 ৫$ মিনিট

উপকরণ:

কাগজ; মার্কার; সুঁই/সুতা; টেপ, স্টাপলার বা বই তৈরির অন্যান্য উপকরণ; 'একটি বই তৈরি প্রকল্পের নির্দেশাবলি’ হ্যান্ডআউট।

\section{প्रস্তুতি:}

শিক্ষার্থীদের হ্যান্ডআউটে অন্তর্ভুক্ত সকল নারী আপনার অধিবেশনের জন্য উপযুক্ত হবে কিনা সেটা নিশিচ হওয়ার জন্য সম্পূর্ণ তালিকাটি যাচাই করুন । বড় একটি তালিকা নিন যেন প্রত্যেক তিন-চার জনের ছোট দল পৃথক একজন নারী নিয়ে শিখতে পারে। সম্ভব হলে শিক্ষার্থীদের জন্য হ্যান্ডআউটগুলো ফটোকপি করুন্ন। যদি আপনার শিক্ষার্থীরা পরিদর্শন করতে চায় এবং তাদের তৈরি বই ছোট বাচ্চাদের পড়াতে চায় তাহলে একটা প্রাথমিক বিদ্যালয়ের সাথে যোগাযোগ করুন । 


\section{সক্রিয় নারীরা: একটি-বই-তৈরি প্রকল্পের নির্দেশাবলি}

সক্রিয় নারীরা

\section{রাইগোবারতা মেনচু}

গুয়াতামালার মানবাধিকার নেত্রী

\section{রোসা পার্কস}

আনেরিকার নাগরিক অধিকার নেত্রী

মুকতারান বিবি

যৌন নিপীড়ণের বিরুদ্ধে সোচ্চার

পাকিস্থানি নেত্রী

আং সান সূচি

মায়ানমারের রাজটৈতিক নেত্টী

ওয়াংগারি মাথাই

কেনিয়ার পরিবেশবাদী নেত্রী

মিসেইল বাসেলেট

চিলির রাজটৈতিক নেত্রী

\section{অরূक্ধুতি রায়}

ভারতীয় লেখিকা

সোফি স্কল বা মার্থা ওয়ার্থেইমার

নাৎসিবাদের বিরুদ্ধে প্রতিবাদীদ্বয়

\section{হিলারী ক্লিনটন}

আমেরিকান রাজনৈতিক নেত্রী

ভারা নগাসা বা বেট্রিস নটুবা

ক্যামেরুনের বিচারকদ্বয়

\section{লুইস আরবর}

জাতিসংঘের মানবাধিকার বিষয়ক সাবেক

হাইকমিশনার
নারীনেত্রী সম্পর্কে বইয়ের জন্য নির্দেশিত গবেষণা প্রশ্ন:

১ারীটির নাম কী?

২ তার ছোটবেলা কী রকম ছিল?

৩ ে কী ধরনের সামাজিক সমস্যা দেখেছে? সে কীভাবে এটা দেখেছে?

8 এই সমস্যা সমাধানের জন্য সে কী করেছে বলে তোমাদের জানা আছে?

৫) এে এ বিষয়ে কী করেছিল?

৬ কেউ কি তাকে সাহায্য করেছিল ?

৭ প্রশংসা করতে পার এমন সময়ের একটা গল্প বল যখন তার ব্যক্তিগত গুণাবলি দেখানোর প্রয়োজন হয়। এই গুণ হতে পারে সাহস, দৃঢ়তা, সততা, ধৈর্য, সৃজনশীলতা, বুদ্ধিমত্তা, এবং অন্যদের সাথে মেশার ক্ষমতা, ভবিষ্যৎ সম্পর্কে ধারণা, বা অন্যান্য যে কোন গুণাবলি ।

৮ তার কাজের জন্য কীভাবে পৃথিবী আলাদা কিছু পেয়েছে তা আলোচনা কর ।

৯ যদি পার তার একটি উক্তি উল্লেখ কর ।

১০ তোমাদের বইয়ের একটা নাম দাও যা তার নামের চেয়ে বেশি কিছু, এমন কিছু যা সে করেছে অথবা তার ব্যক্তিগত মজার কোন গুণাবলি যা তুমি জেনেছ।

তোমার দলের সদস্যদের নাম পূরণ কর যারা . . .

গল্প লিখতে সাহায্য করবে (একাধিক ব্যক্তি হতে পারে):

গল্প সম্পাদনা করবে:

বইয়ের লেখা সংশোধন দেখবে:

গল্প বর্ণনা/ব্যাখ্যা করবে:

বইয়ের প্রচ্ছদ তৈরি ও অলংকরণ করবে:

বই বাঁধাই করবে এবং এটা সমন্বয় করবে: 


\section{শরীরের পরিবর্তন}

অধিবেশন সম্পর্কিত ধারণা: শিক্ষার্থীরা তাদদর সমাজে পুরুষ এবং মহিলাদের আদর্শ বাহ্যিক চেহারা চিহ্নিত করবে এবং এই আদর্শকে ধরে রাখতে তারা কী ধরনের কাজ করে তা পরীক্ষা করবে।

উদ্দেশ্য: শিক্ষার্থীদেরকে সামাজিকভাবে আদর্শ চেহারা নিশ্চিত করার জন্য প্রশ্নবিদ্ধ করা; মানুষ (বিশেষ করে মহিলা এবং কিশোরীরা) ঐ সকল বাহ্যিক চেহারা অর্জনের জন্য যে সকল কাজ করে, এবং এই কাজগুলোর জন্য যে ক্ষতিগুলো হয় সেগুলোর বর্ণনা করা; শিক্ষার্থীদের বিশ্লেষণমূলক চিন্তাশক্তি শক্তিশালী করা।

\section{নির্দেশাবলি}

১ নিচের প্রশ্নগুনোর মাধ্যমে বিষয়টি ওরু করুন:

• ি কিছু চাপ কী কী যেগুনো ছেলে ও মেয়েরা নিজেদেরকে একটা নির্দিষ্ট রকম দেখানোর জন্য অনুভব করে ?

- আমাদের অধিকাংশ মানুেুের জন্য এই কাজ্ষিত চেহারাগুলি কি বাস্তবসম্মত বা আদর্শ?

• যদি মানুষ এই আদর্শ চেহারা না পায় তবে কেমন অনুভব করে ?

- ি কিছু জিনিস কী যেগুলো মানুষ আকর্ষণীয় আদর্শ হওয়ার জন্য করে (বা তাদের বাচ্চাদের করতে হয়)? যেমন- চুলে? ত্বকে? দেহের গড়নে? [উত্তরগুলো বোর্ডে লিখুন।]

২ নিচের তালিকা ছাড়াও তোমরা আলোচনা করতে চাও এমন যে কোন অতিরিক্ত কাজ যোগ কর:

অতিরিক্ত মোটা/খাওয়া

অতিরিক্ত শরীর গঠন

চর্মসংস্কার/ ত্বক ফর্সা করা

রূপসজ্জা, মেহেদি বা নখ পালিশ লাগানো

ছিদ্র করা, আঁচড়ানো বা উলকি দেয়া

সেভ করা বা চুল ফেলানো

চুল সোজা করা, কোকড়ানো বা রং করা

রূপবর্ধক শল্যচিকিৎসা

মহিলাদের ভঙ্গাংকুর ছেদ বা পুরুষের খৎনা*

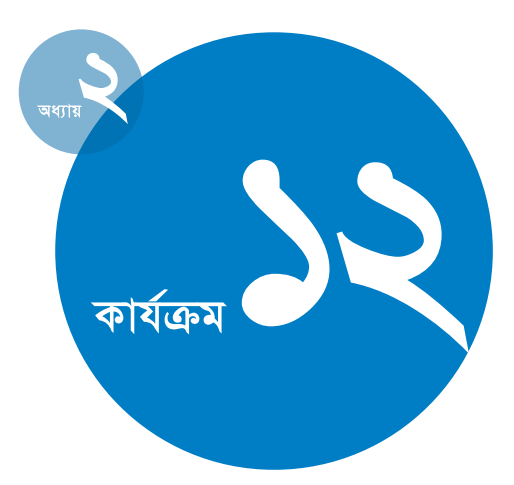

সময়কাল :

8৫ मिनिট

৩ প্রশ্ন করুন:

• তোমরা এই তালিকার কাজগুনো সম্পর্কে কী মনে কর? কোনগুলি শ্রুই দেখানোর জন্য এবং কোনগুলি ক্ষতি করতে পারে ?

- অন্য সমাজের/সংস্কৃতির মানুযেরা এই কাজগুলো নিয়ে কী ভাবে?

• এই আদর্শগুনো কোথা হতে আসে?

• একটি আদর্শ দেহ গড়ন তৈরির জন্য মহিলাদের ওপর কি পুরুষের চেয়ে বেশি সামাজিক চাপ থাকে? এই প্রক্রিয়ার জন্য কারা লাভবান হয় এবং কাদের ক্ষতি হয়? এই চাপ কীভাবে আত্নমর্যাদার ওপর খারাপ প্রভাব ফেলে ?

• শরীর পরিবর্তনসহ এই কাজগুলো বন্ধ করা কেন গুরুত্বপূর্ণ? এমন কোন কাজ আছে যা তোমরা বন্ধ হয়েছে দেখতে চাও?

• যখন তোমরা বড় হচ্ছিলে, কেউ কি তোমাদের বলেছিল যে এই কাজগুনো বিপদজনক এবং বন্ধ করা উচিত?

• তোমরা কি এ ব্যাপারে তোমাদের নিজেদের জ্ঞান এবং অনুভূতি নিয়ে কারো সাথে আলোচনা করেছ?

• কীভাবে তোমরা তা করতে পার?

\section{উপকরণ:}

বোর্ড ও চক

\section{প्रস্তুতি:}

যেভাবে আপনার সমাজের মানুষ তাদের চেহারা পরিবর্তন করতে পারে সেই পদ্ধতিগুলো ভাবুন। দেখুন কোন কাজগুলো শরীরের ওপর প্রভাব ফেলে। নিচের তালিকাটি পড়ন এবং দেখুন কোন কাজগুলো আপনি আপনার শ্রেণিকক্ষে আলোচনা করতে চান ।

* পুরুষের খৎনাও একটি সাধারণ ব্যাপার যা শরীর পরিবর্তনের অন্তর্ভুক্ত। যাই হোক, এই পদ্ধতিটিকে স্বাস্থ্যের উপকার হিসেবে দেখা হয়। ইহা এইচআইভিসহ কিছু নির্দিষ্ট যৌন সংক্রমণ থেকে আংশিকভাবে রক্ষা করে। 


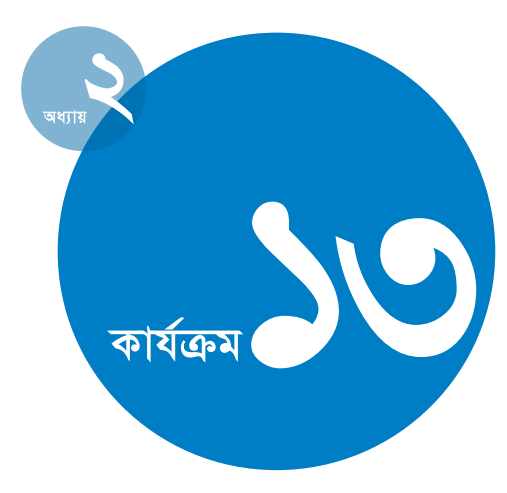

সময়কাল :

৯০ মিনিট (দুটি সেশনে

ভাগ করে হতে পারে)।

\section{উপকরণ:}

বোর্ড ও চক।

প্রস্তুতি:

আপনার নিজের সমাজের নির্যাতন সম্পর্কে ভাবুন বা পড়ন। সচেতন থাকুন, অনেক মানুষের ব্যক্তিগত অভিজ্ঞতার কারণে বা এটি একটি ব্যক্তিগত বিষয় এরকম বিশ্বাসের কারণে বিভিন্ন মানুষের সাথে পারিবারিক নির্যাতন নিয়ে কথা বলা একট কঠিন। কখনও

শিক্ষার্থীদেরকে নির্যাতন ঘটার স্থানে নিয়ে যাবেন না।

\section{ঘনিষ্ঠ সম্পর্কের ক্ষেত্রে নির্যাতন ও মর্যাদা}

অধিবেশন সম্পর্কিত ধারণা: শিক্ষার্থীরা ঘনিষ্ঠ সম্পর্কের মধ্যকার মর্যাদা এবং নির্যাতন নিয়ে ছোট নাটিকা তৈরি করবে। কীভাবে নিজেদের সম্পর্কের মধ্যে শ্রদ্ধাবোধ বাড়াতে হয় তা তারা আলোচনা করবে।

উদ্লেশ্য: শিক্ষার্থীদেরকে ঘনিষ্ঠ সম্পর্কের মধ্যে নির্যাতনমূলক আচরণ সম্পর্কে আলোচনায় উদুদ্ধ করা । কীভাবে মর্যাদাবোধ নিয়ে সম্পর্ক টৈরি করা যায় সে সম্পর্কে আলোচনা করা; সৃজনশীল লিখন দক্ষতা এবং কর্ম দক্ষতা বৃদ্ধি করা।

\section{নির্দেশাবলি}

১ এ এটিকে একটি গুরুত্বপূর্ণ বিষয় হিসেবে পরিচয় করিয়ে দিন । ব্যাখ্যা করুন:

• এই অধिবেশনে আমরা বিভিন্ন রকম নির্যাতন নিয়ে আলোচনা ও বিক্লেষণ করব যা মানুয মাঝে মাঝে ঘনিষ্ঠ সম্পর্কের মধ্যে করে থাকে।

• একটি ঘনিষ্ঠ সম্পর্কের- বিবাহিত, অবিবাহিত নারী-পুরুষ বা সমকামী যুগলদের নিয়ে ছোট নাটিকা তৈরি করতে তোমরা ১৫ মিনিট সময় পাবে।

• তোমাদের নাটিকাগুলো এমন হতে হবে যেন তা আমাদের বুঝতে সাহায্য করে যে, সম্মানের ওপর ভিত্তি করে সম্পর্ক তৈরি হয়।

২ শিক্ষার্থীদেরকে ৪টি দনে ভাগ করুন (অথবা শিক্ষার্থীদের সংখ্যার ওপর ভিত্তি করে আরো কম করতে পারেন), প্রত্যেক দলে ৫ থেকে ৬ জন থাকবে।

দল দুটিকে বলুন: ৫ মিনিটে একটি নাটিকা তৈরি কর যেখানে নির্যাতনের চিত্র আছে। এই নির্যাতন শারীরিক বা মানসিক হতে পারে। বাস্তবসম্মত ঘটনার উদাহরণ দেয়ার চেষ্টা কর যা তোমরা তোমাদের সমাজে দেখেছ বা শুনেছ।
অন্য দুই দলকে বলুন: পারস্পরিক সম্মানের সাথে তৈরি হয়েছে এমন সম্পর্কের ওপর ভিত্তি করে একটি নাটিকা (পাঁচ মিনিটের মধ্যে) টৈরি কর। কীভাবে দু’জন মানুষ নির্যাতনকে পাশ কাটিয়ে দ্বন্দ্ব ও মতামতের ভিন্নতাকে সমঝোতায় আনে তা দেখাও।

৩ ঘুরে ঘুরে দলগুলোর মধ্যে দেখুন এবং সাহায্যের প্রয়োজন আছে

8 নির্যাতনের ঘটনা নিয়ে তৈরি নাটিকা দিয়ে শরু করুন। প্রত্যেক দলকে তাদের নাটিকা শ্রেণিকক্ষে উপস্থাপন করতে বলুন। প্রত্যেক নাটিকার পর কারো কোন প্রশ্ন আছে কিনা জানতে চান। তারপর জানতে চান একজন ব্যক্তি বা সম্পর্কের কোন বিষয়গুলো নির্যাতন ঘটাতে সাহায্য করে।

৫ মর্যাদা নিয়ে তৈরি নাটিকাতে একই পদ্ধতি অনুসরণ করুন । কিন্তু প্রশ্ন করুন, একজন ব্যক্তি বা সম্পর্কের কোন্ বৈশিষ্ট্যগুলি এটিকে সুন্দর ও অহিংস করতে সাহায্য করে। কিনা জানতে চান। 
৬ নিচের প্রশ্নগুলো নিয়ে একটি আলোচনা করুন:

• ঘনিষ্ঠ সম্পর্কের মধ্যে কী কী ধরনের নির্যাতন হয়ে থাকে? /উত্তর জানার চেষ্টা করুন: নিয়ন্ত্রণ, শাসন, চিৎকার, হ্মকি বা শাসানো, এবং শারীরিক নির্যাতন।

• একটা নির্যাতনমূলক সম্পর্কের বৈশিষ্ট্যগুলি কী কী? [উত্তরগুলো বোর্ডে ‘নির্যাতনমূলক সম্পর্ক’ এই শিরোনামের নিচে লিখুন ।]

• কেন অনেক মানুষ, বিশেষ করে যুবসমাজ, পারিবারিক নির্যাতনের ব্যাপারে কিছু করতে অসহায় বোধ করে ? সত্যিই কি তাদের কিছই করার থাকে না ?

• কোন বিষয়গুলো একটা সুসম্পর্ক তৈরি করে ? মর্যাদার ওপর ভিত্তি করে সম্পর্ক গড়ার জন্য কোন বিষয়গুলো জরুরী ? [উত্তরগুলো ‘মর্যাদাপূর্ণ সম্পর্ক’ শিরোনামের নিচে লিখুন।
• নাটিকাগুলোতে যে ঘটনাগুলোর উল্লেখ রয়েছে সেগুলো কি বাস্তবসম্মত ? তোমরা কি এই ঘটনাগুলো তোমাদের দৈনন্দিন জীবনে দেখ? সুসম্পকের্র মধ্যে যে ঘটনাগুলোর উল্লেখ আছে সেগুলো কি বাস্তবসম্মত ? তোমরা কি এই ধরনের সম্পর্ক তোমাদের দৈনন্দিন জীবনে দেখ?

- শারীরিক নির্যাতন কি প্রথমে পুরুষরা মহিলাদের উপরে করে নাকি মহিলারাও একইভাবে পুরুষদের উপর করে ? যেকোন সম্পর্কের মধ্যে নির্যাতনের কারণগুলো কী কী বলে তোমরা মনে কর ? যখন এই ধরনের নির্যাতন দেখ তখন তোমরা স্বাভাবিকভাবে কী কর? তোমরা কী করতে পারতে ?

• তোমরা কি মনে কর সম্মানের সাথে কোন সম্পর্ক তৈরি করা সম্ভব ? আমরা ব্যক্তিগতভাবে ঘনিষ্ঠ সম্পর্ক গড়ে তোলার জন্য কী করতে পারি? 


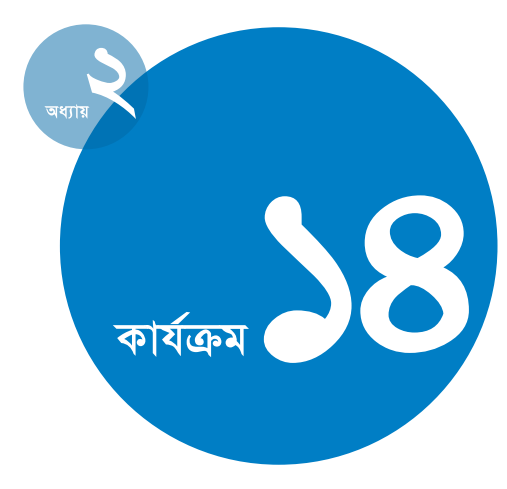

\section{সময়কাল :}

ধাপ ১-৪: ১ ঘণ্টা

ধাপ ৫-৭: ১ ঘণ্টা

\section{উপকরণ:}

বোর্ড ও চক।

\section{প्रস্তুতি:}

শিক্ষার্থীদের অবশ্যই নারী নির্যাতন সম্পর্কে পূর্বধারণা থাকতে হবে।

Empowering Young Women to Lead Change: A Training Manual (World YWCA. 2006. Geneva.) www.worldywca.org থেকে নেওয়া হয়েছে

\section{জেন্ডার ভিত্তিক নির্যাতনের বিরুদ্ধে পদক্ষেপ}

অধিবেশন সম্পর্কিত ধারণা: ছোট ছোট দলে বিভক্ত হয়ে শিক্ষার্থীরা মহিলাদ্রর বিরুদ্ধে নির্যাতন বন্ধের জন্য বিভিন্ন কৌশল তৈরি করবে এবং তাদদর কৌশলগুলি টেলিভিশনের সংবাদদ সমপ্রচার করবে।

উদ্দেশ্য: শিক্ষার্থীদেরকে মহিলাদের বিরুদ্ধে নির্যাতন বন্ধের পদক্ষেপ নিয়ে কমপক্ষে দুইটি পদক্ষেপ বলতে সক্ষম করা; সমস্যা সমাধান ও সাধারণ মানুমের সামনে কথা বলার দক্ষতা শক্তিশালী করা।

\section{निর্দেশাবলি}

১ শিক্ষার্থীদের নিয়ে চার বা পাঁচ জন করে দল তৈরি করুন । ব্যাখ্যা করুন যে, অর্ধেক শিক্ষার্থী টেলিভিশনে সমপ্রচারের জন্য একটি সংবাদ তৈরি করবে এবং বাকি অর্ধেক সরকারের কাছে বর্ণনার জন্য একটি উপস্থাপনা তৈরি করবে।

২ জিজ্ঞেস করুন, "মেয়ে ও নারীদের বিরুদ্ধে নির্যাতন বন্ধ করা কার দায়িত্ব ?"

৩ ব্যাখ্যা করুন:

• কিছু কৌশল বা কর্মসূচির কথা চিন্তা কর যেগুলো নারী निর্যাতন কমাতে পারে।

• তোমরা ৫ থেকে ১০ মিনিটের একটা উপস্থাপনা তৈরির জন্য ৪० মিনিট সময় পাবে ( তোমাদের দল অনুযায়ী তা হবে টেলিভিশন সংবাদ অথবা স্থানীয় ও জাতীয় সরকারের জন্য উপস্থাপন)।

• তোমাদের উপস্থাপনা তৈরির ধারণায় এটা থাকবে যে, তোমাদের দর্শক/শ্রোতা নির্যাতন সমস্যা বিষয়ে জানে। তোমাদের কাজ হচ্ছে সমস্যা সমাধানের জন্য কোন্ ইতিবাচক পদক্ষেপগুলি নেয়া হচ্ছে তা দেখানো
• যুবসমাজের সাথে সাথে প্রাপ্তবয়স্করাও প্রতিদিনের জীবনयাত্রায়; স্থানীয় বিভিন্ন সংস্থা এবং মানবগোষ্ঠী; সরকার বা জাতিসংঘের সংস্থাগুলি কী করেছে বা করতে পারে তা অন্তর্ভুক্ত করার চেষ্টা কর ।

• যুবক এবং পুরুষদেরকে এই কাজে কিভাবে অন্তর্ভুক্ত করা যায় তোমাদেরকে সে দিকেও লক্ষ্য রাখতে হবে।

স্থানীয়/ জাতীয় সরকারের জন্য উপস্থাপনা তৈরি বিষয়ে ব্যাখ্যা করুন: দলগত এবং এককভাবে যা করা হয়েছে সেটি তোমরা সরকারের উর্ধব্বতন ব্যক্তিদের জানাবে। কীভাবে সরকারের দায়িত্ব পালন করা উচিত তা স্পষ্ট কর। কিছু শিক্ষার্থী সরকারি কর্মকর্তার ভূমিকা পালন করতে পারে, এবং অন্যরা বিশেষজ্ঞ অথবা নাগরিকের ভূমিকা পালন করতে পারে।

সংবাদ সমপ্রচারের জন্য উপস্থাপনা তৈরি বিষয়ে ব্যাখ্যা করুন: লক্ষ্য রাখবে যে, তোমাদেরকে জনগণের মনোযোগ আকর্ষণ করতে হবে এবং যথাসম্ভব সংক্ষিপ্ত ও স্পষ্ট হতে হবে। ব্যক্তিগত সম্পর্কের মধ্যে নির্যাতনের সমস্যা সমাধানের জন্য করা হয়েছে এমন মানব-স্বার্থ সংশ্লিষ্ট গল্প ব্যবহার কর। তোমাদের উপস্থাপনকে ব্যক্তিগত ও আকর্ষণীয় কর। 
8 নিশ্চিত হোন যে, প্রত্যেকে কাজটি বুঝতে পেরেছে । দলগুলোকে বলুন যে, তারা প্রস্তুতির জন্য ৪৫ মিনিট সময় পাবে। তারপর তাদের কাজ ঘুরে ঘুরে দেখুন।

৫ উপস্থাপনা শুরুর আগে দলগুলোকে পুনরায় তাদের উপস্থাপনা দেখার জন্য এবং পরিকল্পনা ভালভাবে সাজানোর জন্য ৫ মিনিট সময় দিন

৬ প্রত্যেক দলকে তাদের উপস্থাপনাগুলি উপস্থাপন করতে বলুন তাদের কর্মসূচির ধারণা থেকে বিশেষ অংশ লিখে নিন ।

৭ সবগুলো উপস্থাপনা শোনার পর জিজ্ঞস করুন:

• নারী নির্যাতন কমানোর উপায় চিন্তা করা কি কঠিন ছিল?

• নারীদের নিয়ে কাজ করা বিভিন্ন দলের মধ্যে কোন্ বিষয়গুলো একই রকম?

- ছেলে বা পুরুষদের সাথে কাজ করতে যেয়ে কী মনে হয়েছে?

• তোমরা কোন ধারণাটি সবচেয়ে ভাল মনে কর, এবং কেন ?

• নারী নির্যাতন বন্ধের পদক্ষেপ নেয়া কি প্রত্যেকের দায়িত্ব ?

• যুবক হিসেবে মেয়ে ও মহিলাদের ওপর নির্যাতন বন্ধের জন্য পরিবারে, বিদ্যালয়ে এবং সমাজে এখন তোমরা কী (যদি থাকে) করতে পার ? 


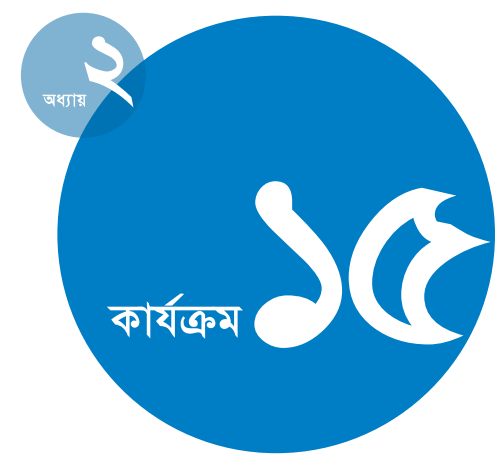

সময়কাল :

ধাপ ১-৩: ১৫ মিনিট

বাড়ির কাজ: ১ ঘণ্টা

ধাপ 8: ৩০ মিনিট

\section{উপকরণ:}

বোর্ড ও চক (ধাপ ৩-এর জন্য);

সাক্ষাৎকার তালিকার হ্যান্ডআউট।

প্রস্তুতি:

यদি কিছু শিক্ষার্থীর সাক্ষাৎকার নেয়ার মত বয়স্ক ব্যক্তি না থাকে তাহলে সাক্ষাৎকার দিতে ইচ্ছুক এমন কয়েকজন বয়স্ক ব্যক্তি খুঁজে বের করুন । সম্ভব হলে সব শিক্ষার্থীর জন্য হ্যান্ডআউটের অনুলিপি তৈরি করুন ।

Gendering Prevention Practices: A Practical Guide to Working with Gender in Sexual Safety and

HIV/AIDS Awareness Education. (Jill Lewis. 2003. NIKK- The Nordic Institute for women's Studies and Gender Research.)

<www.nikk.uio.no> থেকে নেয়া হয়েছে ।

\section{জেন্ডার ভূমিকা ও রীতিনীতি পরিবর্তনের চিত্র}

অধিবেশন সম্পর্কিত ধারণা: শিক্ষার্থীরা একজন দাদা/দাদীর (অথবা ঐ বয়সের যেকোন একজনের) যুবক বয়সের জেন্ডার ভূমিকা এবং রীতিনীতি জানার জন্য সাক্ষাৎকার নেবে, নিজেদের অভিজ্ঞতার সাথে তাদের তুলনা করবে এবং কীভাবে জেন্ডার ভূমিকা পরিবর্তন হয় তা আলোচনা করবে।

উদ্দেশ্য: শিক্ষার্থীদেরকে যেভাবে সময়ের সাথে সাথে জেন্ডার ভূমিকা পরিবর্তন হয়েছে কমপক্ষে এমন তিনটি মাধ্যম উল্লেখ করতে সাহায্য করা; গবেষণা দক্ষতা শক্তিশালী করা।

\section{नির্দেশাবলি}

১ ব্যাখ্যা করুন, এই অধিবেশনে দেখা যাবে সময়ের সাথে সাথে জেন্ডার রীতিনীতি কীভাবে পরিবর্তিত হয়েছে।

• তোমরা আলাদা আলাদা ভাবে দাদা/ দাদীর বয়সের দুইজনের সাক্ষাৎকার নেবে (তোমাদের দাদা/দাদী বা কেউ হতে পারে)। যদি সম্ভব হয় তাহলে তোমাদের সমলিঙ্গের কাউকে নেবে। যদি তোমরা এমন বয়স্ক কাউকে না জান বা পরিবারে না থাকে তাহলে (আমি) তোমাদেরকে সাক্ষাৎকারের জন্য বয়স্ক ব্যক্তি খুঁজে দিতে সাহায্য করব ।

• প্রত্যেক বয়স্ক ব্যক্তিকে তাদের কৈশোরে ও যৌববনে নারী এবং পুরুষের ভূমিকাগুলি কেমন ছিল তা জিজ্ঞস কর। তখন থেকে কী পরিবর্তন হয়েছে বলে তারা মনে করে তা খুঁজে বের কর।

২ সাক্ষাৎকারের তালিকা বোর্ডে আঁকুন, অথবা যদি আপনি তালিকার অনুলিপি করে থাকেন তাহলে তাদের মধ্যে বিলি করুন । তালিকাটি পুনরায় পড়ুন এবং কীভাবে এটা ব্যবহার করতে হবে ব্যাখ্যা করুন:

• বামপাশের কলামে ঐ ব্যক্তির উত্তরগুনো লেখ।

• সাক্ষাৎকারের পরে ডানপাশের কলামে আজকালকার দিনে ঐ ভূমিকাগুলো কেমন তা লেখ।
৩ নিশ্চিত হোন যে, সবাই কাজটি বুঝেছে এবং সাক্ষাৎকার নেয়ার মত কেউ আছে। কখন সাক্ষাৎকার এবং তালিকাটি অবশ্যই শেষ করতে হবে তা শিক্ষার্থীদেরকে বলুন ।

8 শিক্ষার্থীরা সাক্ষাৎকার শেষ করলে আলোচনা করুন:

- তুমি কার সাক্ষাৎকার নিয়েছ?

- জেন্ডার ভূমিকা নিয়ে বয়স্ক লোকের সাক্ষাৎকার নিতে কেমন লেগেছে? এমন কিছু কি ছিল যার জন্য তোমার বা তার অস্বস্তি অথবা আলোচনা করতে সমস্যা হয়েছে?

• তারা কি এমন কিছু বলেছে যাতে তোমরা অবাক হয়েছ? এই কাজের অভিজ্ঞতায় তোমাদের কী মনে হয়েছে, জেন্ডার ভূমিকা অপরিবর্তনীয় নাকি পরিবর্তন হতে পারে ?

- জীবনের কোন দিকটাতে জেন্ডার ভূমিকা এবং রীতিনীতি সবতেয়ে বেশি পরিবর্তন হয়েছে? কোন পরিবর্তনগুলো তোমার মনে হয়েছে ভালোর জন্য? কোনগুলো খারাপের জন্য? এবং কেন?

• অনেকগুলি বিষয়ই পরিবর্তন আনে। মিডিয়ার বিশ্বায়ন কীভাবে জেন্ডার ভূমিকার ওপর প্রভাব ফেলতে পারে ? প্রযুক্তি এবং ইন্টারনেটের ভূমিকা কী? বিভিন্ন নেতার কাজকর্ম কী ভূমিকা পালন করছে? সামাজিক ন্যায় বিচার প্রতিষ্ঠায় বিভিন্ন তৃণমূল আন্দোলনের কী ভূমিকা?

• তোমরা কি মনে কর জেন্ডার ভূমিকা ভবিষ্যৎ প্রজন্মেও পরিবর্তন হতে থাকবে, কীভাবে? 
সাক্ষাৎকারের তালিকা

\begin{tabular}{|c|c|c|}
\hline $\begin{array}{l}\text { বিষয় } \\
\text { এই বিষয়গুলো কেমন ছিল ? }\end{array}$ & দাদা/দাদীর সময়ে & এখন তোমাদের সময়ে \\
\hline বিদ্যালয়ে লেখা পড়া? & & \\
\hline গৃহস্থালির কাজ? & & \\
\hline বক্ধুদের সাথে মজা করা? & & \\
\hline থ্রেমের সম্পর্ক? & & \\
\hline মা-বাবা হওয়া? & & \\
\hline চাকরি করা ? & & \\
\hline অন্যান্য প্রশ্ন: & & \\
\hline
\end{tabular}




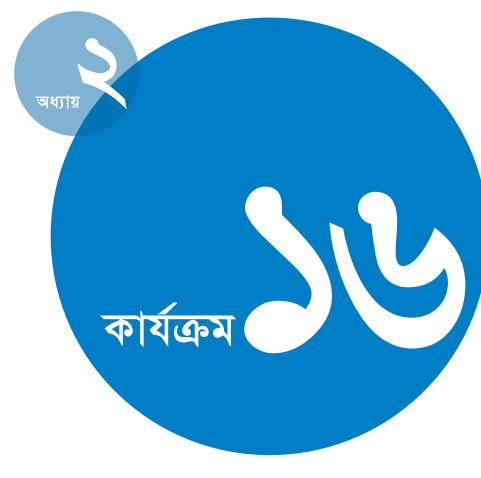

সময়কাল :

ধাপ ১-৭: ৪৫ মিনিট

ধাপ ৮: $8 ৫$ মিনিট

\section{উপকরণ:}

আপনি শিক্ষার্থীদেরকে কিছু গান, কবিতা বা গীতি কবিতার উদাহরণ দেখাতে পারেন।

\section{প्रস্তুতি:}

শিক্ষার্থীদের মধ্যে জনপ্রিয় এমন সংগীত সম্পর্কে নিজে পরিচিত হয়ে নিন। ধাপ 8-র জন্য আপনার অধিবেশনের সাথে মানানসই অন্য কোন বিষয় যোগ করার প্রয়োজন কিনা তা বিবেচনায় নিন । শিক্ষার্থীদেরকে তাদের নিজেদের অবস্থা সম্পর্কে ভাবতে উদ্পুদ্ধ করুন এবং আপনার সাথে বর্ণনা করতে বলুন। কিছু শিক্ষার্থীকে আপনি বিচারক হিসেবে অংশ নিতে বলবেন কিনা তা সিদ্বান্ত নিন (ধাপ ৬ দেখুন)।

\section{ক্ষমতার কাছে সত্য বলা}

অধিবেশন সম্পর্কিত ধারণা: গান এবং কবিতা লেখার মাধ্যমে শিক্ষার্থীরা সম্পর্কের মধ্যকার জেন্ডার সমতা নিয়ে কথা বলার চর্চা করবে। এই অধিবেশন ফরুর পূর্বে প্রত্যেক শিক্ষার্থীকে এই বইয়ের নিম্নলিখিত অধিবেশনগুনো থেকে কমপক্ষে একটি শেষ করতে হবে: ১, ৩, ৫ অথবা ৬।

উদ্দেশ্য: শিক্ষার্থীদেরকে নারী-পুরুষ্ব সম্পর্কের মধ্য্য অসম ক্ষমতাকে খুঁজে দেখা ও বিশ্লেষণ করতে সাহায্য করা।

\section{निর্দেশাবলি}

১ ব্যাখ্যা করুন যে, আমাদের সম্পর্কের মধ্যকার জেন্ডার ভূমিকা কীভাবে অসম ক্ষমতার জন্ম দেয় তা এই অধিবেশনে আলোচনা করা হবে। জিজ্ঞেস করুন:

• জেন্ডার ভূমিকা এবং সমতা সম্পর্কে আমরা কী কী বিষয় শিখেছি?

• যেখানে মানুষের পারস্পরিক সম্পর্কের মধ্যে ক্ষমতার সমবন্টন থাকে সেখানে জেন্ডার ভূমিকা কীভাবে প্রভাব ফেনতে পারে ? ( উত্তরের জন্য ৫-১০ মিনিট সময় দিন।)

২ শিক্ষার্থীদেরকে বলুন যে, তারা কবিতা বা গান লেখার মাধ্যমে এই বিষয়টি জানার চেষ্টা করবে। তারা হিপ-হপ (Hip-hop) কথিত শব্দ, র্যাপ (Rap) অথবা অন্য যে কোনভাবে করতে পারে ।

৩ শিক্ষার্থীদেরকে দু’জন করে জোড়া তৈরি করতে বলুন (সমলিঙ্গ বা ছেলে-মেয়ে ) এবং কীভাবে তারা তাদের কাজকে ভাগ করে নেবে তা নিয়ে সঙ্গীর সাথে আলোচনা করতে বলুন। যেমন- একই সাথে দু’জন লিখবে এবং উপস্থাপন করবে নাকি একজন লিখবে অন্যজন উপস্থাপন করবে।
8 বিভিন্ন দৃশ্য/ঘটনা/পরিবেশের উদাহরণ দিন যেন শিক্ষার্থীরা তা তাদের গান বা কবিতার জন্য ব্যবহার করতে পারে।

• একজন বালক তার সমকামী সঙ্গীকে রক্ষা করছে।

• একজন ব্যক্তি তার বন্ধুকে বলছে যে, তার কোন অধিকার নেই তার স্ত্রীকে যৌন মিলনে বাধ্য করা।

• একজন ব্যাংকার একজন প্রতিবাদী স্বামীকে ব্যাখ্যা করছে কেন সে তার স্ত্রীর নামে ঋণ দিল। ( অথবা তার স্ত্রী বলছে কেন তার নিজের টাকা থাকার প্রয়োজন বা যোগ্যতা আছে)

• একজন কিশোরী বয়স্ক লোকদের দ্বারা যৌন হয়রানির স্বীকার रচ্ছে।

• যদিও তারা দুজনই সারাদিন চাকরি করে তারপরও একজন স্বামী চাপ সৃষ্টি করছে যে গৃহস্থলির সকল কাজই এবং রান্না ও বাচ্চাদের যত্ম তার স্ত্রীর করা উচিত।

• একটি মেয়ে পতিতা হিসেবে বিক্রি হওয়ার পর যখন তার এলাকায় ফিরে এল তখন তাকে অপবাদ সইতে হচ্ছে।

- কম যৌতুক দেয়ার জন্য একজন তরুণী বধুকে তার স্বামী শাস্তি দিচ্ছে।

• একটি ছেলেকে অন্য ছেলেরা ব্যঙ্গ করে, কারণ তার আচরণ পুরুষের মত নয়। 
• এ একজন স্বামী বা থ্রেমিক যখন কনডম ব্যবহার করতে চাচ্ছে না তখন তার স্ত্রী বা প্রেমিকা তাকে বোঝানোর চেষ্টা করছে।

• এ একটি ছেলে তার মাকে রক্ষা করছে যখন তার বাবা তাকে অত্যাচার করছে।

- একটি ভিন্ন ধরনের মত: জাতিসংঘের প্রাক্তন মহাসচিব কফি আনানের একটি উক্তি “আমরা জানি এইডসের বিরুদ্ধে জোয়ার আনতে কী প্রয়োজন... ... ... এর জন্য প্রয়োজন ইতিবাচক পরিবর্তন যা মহিলা ও মেয়েদের অধিক ক্ষমতা দিবে ও আত্নবিশ্বাসী করবে, এবং সমাজের সব স্তরে নারী-পুরুষ সম্পর্কের রূপান্তর করবে।"

- অন্য কোন ধারণা যা তোমরা নিজেরা ভাব কিন্তু যা তোমার শিক্ষকের দ্বারা অনুমোদিত হওয়ার প্রয়োজন ।

৫ কাজটি শেষ করার জন্য শিক্ষার্থীদেরকে নির্দেশনা দিন:

- কোন বিষয়টি নিয়ে লিখতে চাও তা তোমার সঙ্গীর সাথে মিলে সিদ্ধান্ত নিতে কয়েক মিনিট সময় নাও।

- শ্রেণিতে পড়ালেখার বাকি সময় গান বা কবিতা লেখার জন্য ব্যয় কর।

• এই ঘটনাগুলোতে বিভিন্ন মানুষ বলতে পারে এমন বিভিন্ন যুক্তি সম্পর্কে ভাব এবং তারা তাদের কথা প্রমাণের জন্য কী বলতে পারে তা ভাব।

• এই গল্পের একজন ব্যক্তি হনে কেমন লাগতো এবং সে (নারী বা পুরুষ) কীভাবে তার অনুভূতি প্রকাশ করতে চায় তাও ভাব ।

• মনে রেখ, এটি ঠাট্টা বা নির্যাতনকে হালকা করে দেখার সময় नয়।
৬ যখন শিক্ষার্থীরা তাদের কাজের ফলাফল উপস্থাপন করতে থাকবে তখন ব্যাখ্যা করুন । যদি আপনি বিচারক দল রাখতে চান তাহলে তিন বা চারজন শিক্ষার্থীকে বলুন তাদের তিনটি পছন্দের উপস্থাপনা বাছাই করতে। আপনি এমন শিক্ষার্থীদেরকে বাছাই করতে পারেন যারা উপস্থাপন করতে অস্বস্তিবোধ করে।

१ শিক্ষার্থীদেরকে তাদের গান বা কবিতা লেখার জন্য ২৫-৩০ মিনিট সময় দিন । यদি প্রয়োজন হয় তাহুলে এটা নিয়ে বাড়ির কাজ হিলেবেও দিতে পারেন।

৮. যখন উপস্থাপনা কুরু হবে (এটি একটা আলাদা অধিবেশন হতে পারে) তখন শিক্ষার্থী-বিচারকদের সমবেত করুন এবং কিসের ওপর ভিত্তি করে বিজয়ী নির্বাচন করা হবে তা বুঝিয়ে বলুন (যেমন- সেরা বার্তা, সেরা উপস্থাপনী, সেরা গান, সেরা কবিতা)। শিক্ষার্থীদের উপস্থাপন করতে বলুন।

বাড়ির কাজ: নিচের যেকোন দুটি প্রশ্ন বেছে নাও এবং প্রত্যেকটি সম্পর্কেই তোমাদের পত্রিকা বা খাতায় একটি অনুচ্ছেদ লেখ।

- নারী পুরুষের একটি ঘনিষ্ঠ সম্পর্কের মধ্যে সাধারণত কার বেশি ক্ষমতা থাকে?

- অসম ক্ষমতার জন্য সম্পর্ককে কী মূল্য দিতে হয় ?

• একই লিঙ্গের মানুষের মধ্যকার সম্পর্কেও কি অসম ক্ষমতার বন্টন থাকে?

- কী কী উপায়ে মানুষ তাদের ঘনিষ্ঠ সম্পর্কের মধ্যে আরো সমতা আনার চেষ্টা করতে পারে ? 


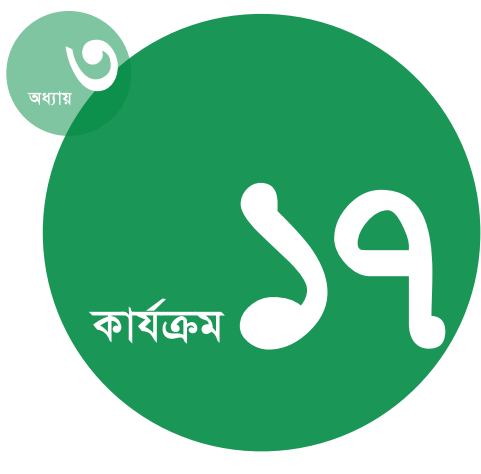

সময়কাল :

8৫ মिनिট

উপকরণ:

বোর্ড ও চক।

\section{প্রস্তুতি:}

কীভাবে বিতর্ক পরিচালিত হবে তা ভাবুন। পরবর্তী পৃষ্ঠার শিক্ষক-

নির্দেশিকা পুনরায় পড়ন । যদি সম্ভব হয়, অন্য শিক্ষকদেরকে বিতর্ক দেখার জন্য এবং বিচারকের দায়িত্ব পালন (কোন পক্ষ বিতর্কে জয়ী তা নির্ধারণ) করার জন্য ডাকুন । যদি অন্য কোন শিক্ষককে না পাওয়া যায় তাহলে দুইজন শিক্ষার্থীকে আপনার সাথে বিচারকের কাজ করার জন্য ডাকুন । বিজয়ী দলের জন্য পুরস্কারের ব্যবস্থা করুন ।

\section{যৌন শিক্ষা বিষয়ে বিতর্ক}

অধিবেশন সম্পর্কিত ধারণা: শিক্ষার্থীরা যৌনতা, জেন্ডার এবং যৌনস্বাস্থ্য বিষয়ে যুবক-যুবতীদের শেখা উচিত অথবা শুধুমাত্র বিয়ে করার সময় পর্যন্ত যৌনমিলন থেকে বিরত থাকতে হয় তা শেখা উচিত সে বিষয়ে বিতর্ক করবে।

উদ্দেশ্য: শিক্ষার্থীদেরকে যৌন বিষয়ক শিক্ষানীতি নিয়ে আলোচনা করতে শেখানো এবং তাদের নিজেদের মতামতের গুরুত্ব দেয়া; বিশ্লেষণ শক্তি ও সাধারণ জনগণের সাথে কথা বলার দক্ষতা শক্তিশালী করা।

\section{निर्দেশাবলি}

১ নিম্নলিখিত বিষয়গুনো দিয়ে অধিবেশনটি শুরু করুন:

• আমাদের মধ্যে যৌনতা বিষয়টিকে খুবই ব্যক্তিগত এবং গোপনীয় মনে করার প্রবণতা রয়েছে। কিন্তু যৌন বিষয়ের অনেক কিছুই সরকারি নীতির অংশ হয়ে গেছে। যেমন- অনেক আইন প্রণেতা, রাজনীতিবিদ এবং নাগরিকবৃন্দ যৌন ও এইচআইভি শিক্ষা বিষয়ে তাদের মতামত ব্যক্ত করেন।

• আজকে আমরা নিজেরাই যৌন ও এইচআইভি শিক্ষা নিয়ে বিতর্ক করব। আমরা একটি আনুষ্ঠনিক বিতর্ক করব যার অর্থ আমরা এই প্রক্নের উভয় দিক নিয়েই যুক্তিতর্ক তৈরি এবং উপস্থাপন করব।

২ শ্রেণির সবাইকে দুইভাগে বিভক্ত করুন । যদি আপনার বাইরের কোন বিচারক না থাকে তাহনে দু'জন আগ্রহী শিক্ষার্থীকে বিতর্কে অংশগ্রহনের বদলে আপনার সাথে বিচার কাজে সাহায্য করতে বলুন ।

৩ বিতর্কের বিষয়টি উপস্থাপন করুন: “যৌন শিক্ষা: বিদ্যালয়ে ছেলেমেয়েদের শেখানো উচিত যেন তারা বিয়ের আগে অবশ্যই যৌনমিলন থেকে বিরত থাকে; তাদেরকে কনডম, জনম বিরতিকরণ পদ্ধতি ও জেন্ডার সম্পর্ক বিষয়ে শিক্ষা দেয়া উচিত নয়।"

8 বিতর্কের পদ্ধতি সম্পর্কে আলোচনা করুন:

- একদল এই উক্তির পক্ষে যুক্তি তৈরি করবে এবং অন্যদল এর বিপক্ষে যুক্তি তৈরি করবে। তোমাদেরকে যে দনের অন্তর্ভুক্ত করা হয়েছে সেই দলের যুক্তির সাথে তোমরা একমত নাও হতে পার
কিন্তু নিজেকে অবশ্যই এই দলের কারও না কারও স্থানে কল্পনা করতে হবে। সমস্ত কারণ সম্পর্কে ভাব যেগুনো কেউ কেউ এই দলের যুক্তি হিলেবে মেনে নেবে। অন্যদলকে বোঝানোর জন্য যুক্তি এবং ঘটনা সম্পর্কে ভাব। বিতর্কের বিষয়বস্তুর সবদিকগুলো আলোচনা কর।

- সম্পূর্ণ দলের হয়ে যুক্তি উপস্থাপনের জন্য দু’জন বক্তা নির্বাচন কর [ছেলে-মেয়ে মিলানো দলে একজন ছেলে এবং একজন মেয়ে নেয়া উচিত ।]

- তোমাদের মধ্যে যারা প্রথম বক্তা নির্বাচিত হবে: তোমরা প্রত্যেকে তিন মিনিটের উদ্বোধনী যুক্তি উপস্থাপন করবে। তোমার দলের সদস্যরা যুক্তি তৈরি করতে সাহায্য করবে। দ্বিতীয় বক্তারা: তোমরা বিরোধী দলের উদ্বোধনী বক্তব্যের উত্তর দেবে। তাই ভালভাবে শুনবে এবং লিখে রাখবে যা তোমার তিন মিনিটের যুক্তি খণ্ডনে সাহায্য করবে।

• এরপর যারা উপস্থাপন করেনি তাদের মধ্যে কয়েক মিনিট প্রশ্নোত্তরের সুযোগ আছে: দলের সদস্যরা বিরোধী দলের জন্য কোন উক্তি বা প্রশ্ন করতে পারে। এবং বিরোধী দলের যারা বক্তব্য উপস্থাপন করেনি তাদের যে কেউ উত্তর দিতে পারে । 
• প্রত্যেক দল বিষয়বস্তু নিয়ে আলোচনা এবং যুক্তি তৈরির জন্য ১০ মিনিট করে সময় পাবে। বিতর্কের পর এক পক্ষকে বিজয়ী ঘোষণা করা হবে। বিচারকরা কোন পক্ষ সমর্থন করে তার ওপর ভিত্তি করে বিজয়ী দল ঘোষণা করা হবে না বরং কোন পক্ষ বেশি কার্যকরী যুক্তি তৈরি করেছে তার ওপর ভিত্তি করে ঘোষণা করা হবে।

(৫) বিতর্কের পক্ষ এবং বিপক্ষ দল ঘোষণা করুন । দলগুলোকে বক্তব্য তৈরির জন্য দশ মিনিট সময় দিন । ঘুরে ঘুরে তাদের সাহায্য করুন এবং দলের কাজে সকলের অংশগ্রহণ নিশ্চিত করুন । যদি আপনার ছাত্র-বিচারক থাকে তাহলে এই সময়ে তাদেরকে বক্তব্য উপস্থাপনের সময় দেখতে এবং সময় শেষ হলে বক্তাদেরকে জানাতে বলুন । ছাত্রবিচারকদেরকে বিতর্ক বিচার করার জন্য নির্দেশিকা দিন ( যেমনবিতর্ক থেকে বাস্তব উদাহরণগুলো শোনা)।

৬ প্রত্যেক দলের প্রথম বক্তাকে উদ্বোধনী যুক্তির জন্য তিন মিনিট করে সময় দিন (প্রত্যেককে তিন মিনিট করে মোট ছয় মিনিট)। এরপর প্রত্যেক দলের দ্বিতীয় বক্তাকে বিরোধী দলের যুক্তি খন্ডানোর জন্য সময় দিন (প্রত্যেককে তিন মিনিট করে মোট ছয় মিনিট)। সর্বশেষে দলের অন্য সদস্যদেরকে প্রশ্ন এবং মতামতের জন্য (ছয় মিনিট) সময় দিন । কখন সমাপনী বক্তব্য দিতে হবে তা প্রত্যেক দলকে জানিয়ে দিন ।

१ বিচারকদের সাথে পরামর্শ করে কোন দল বিজয়ী হয়েছে তা সিদ্ধান্ত নিন । ছাত্র-বিচারকদের সাথে একত্রে দলের ভাল দিক নিয়ে বস্তুনিষ্ঠ প্রশংসা করুন ।
৮ বিজয়ী দল ঘোষণার পর আলেচনা করুন্ন:

• তোমাদের মধ্যে নির্ধারিত দলের কতজন দলের সাথে একমত ছিলে [হাত তোল] এবং যারা একমত ছিলে না [হাত তোল]। তোমরা যা বিশ্বাস কর তার বিপরীত যুক্তি দেয়া সহজ নাকি কঠिन ছিল ?

• যৌনশিক্ষাই কি যৌন বিষয়ের একমাত্র দিক যা সরকারি নীতির অংশ হয়েছে? নাকি যৌনতার অনেক বিষয়ে সমাজ কিছুটা নিয়ন্ত্রণ আরোপ করে। যৌনতার সাথে সম্পর্কিত অন্যান্য কোন্ বিষয়গুলো সম্পর্কে আইন, প্রথা এবং সামাজিক রীতিনীতি আছে যেগুলো মানুষ চিন্তা করে ও স্বাধীনভাবে করতে পারে? /উত্তর জানার চেষ্টা করুন: জোরপূর্বক যৌনমিলন; ছেলে এবং মেয়েদের অনুমিত যৌন আচরণের পৃথক মান ('দ্বৈত-মান"); মেয়েদেরকে তাদের শরীর সবসময় জনসম্মুখের আড়ালে রাখতে হয় (কিছু জায়গায়); ছেলেদেরকে যৌনভাবে সক্রিয় হতে চাপ দেয়া; এবং সমকামিতাকে অপবাদ (অথবা অপরাধ) হিসেবে দেখা।

- সকলেই কি যৌনতা সম্পর্কে প্রচলিত সাংস্কৃতিক ভাবধারাকে সমর্থন করে? কিছু মানুষ কি অন্যরকম আচরণ এবং বিশ্বাস নিয়ে থাকতে পছন্দ করে? যৌনতা সম্পর্কে ধারণা কি সবখানে একই রকম নাকি বিভিন্ন রকম? চিন্তাধারা কি চিরদিন একই রকম থাকে নাকি পরিবর্তন হয়? তাহলে যৌনতা কি শুধুই ব্যক্তিগত বিষয়? নাকি তা এমন একটি বিষয় যা সম্পর্কে পুরো সমাজই উদ্বিগ্ন?
শিক্ষকদের জন্য তথ্য

বিদ্যালয়ে ছেলে-মেয়েদের শিক্ষা দেয়া উচিত যেন তারা অবশ্যই বিয়ের আগে যৌনমিলন থেকে বিরত থাকে; তাদেরকে কনডম, জন্মবিরতিকরণ পদ্ধতি এবং জেন্ডার সম্পর্ক নিয়ে পড়ানো উচিত নয়। হ্যাঁ অথবা না ?

অনেক দেশে যৌন শিক্ষা নিয়ে বিতর্ক চলছে । যারা বিশ্বাস করে যে যুবকযুবতীদের শুধুমাত্র যৌনমিলন থেকে বিরত থাকার পরামর্শ দেয়া উচিত তারা প্রায়ই মনে করে যে ছেলেমেয়েদের নিরাপদ যৌন সম্পর্ক নিয়ে পড়ালে তারা এই তথ্য না জেনে যা করত তার আগেই যৌন সম্পর্কে জড়িয়ে পড়বে। কিন্তু গবেষণায় দেখা গেছে যে, সমন্বিত যৌন শিক্ষা প্রথম যৌন সম্পর্কের বয়স কমিয়ে দেয় না। যারা সমন্বিত যৌন শিক্ষা সম্পর্কে বিশ্বাস করে (নিরাপদ যৌনমিলন নিয়ে তথ্যসহ) তাদের যুক্তি হল এগুনো ছেলেমেয়েদের যৌন জীবন নিয়ে স্বাস্থ্যকর, নিরাপদ সিদ্ধান্ত নেয়ার পদ্ধতি বলে দেয়। 


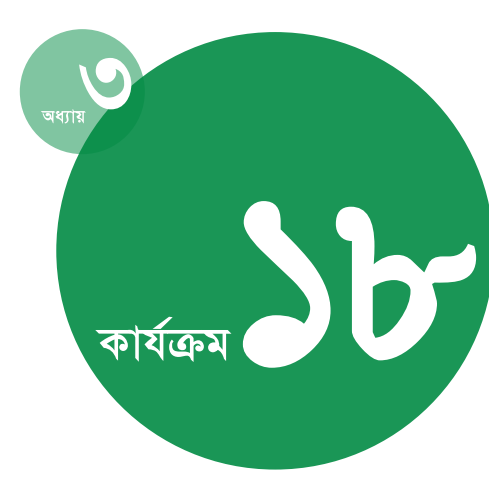

সময়কাল :

80 মিनिট

উপকরণ:

বোর্ড ও চক; অর্ধেক শিক্ষার্থীর জন্য "মায়ার গল্পের অনুলিপি " এবং বাকি অর্ধেকের জন্য “ রসুলের গল্পের অনুলিপি”; দুইটি দলের জন্য পর্যাপ্ত জায়গা যেন তারা অন্য দলের কথা না শুনে নিজেদের মধ্যে কথা বলতে পারে।

\section{প্রস্ত্ততি:}

বিশেষণ কী তা আলোচনার জন্য প্রস্তুত থাকুন । আলোচনা সম্মানজনক করার জন্য পরিবেশ তৈরি করুন । অসম্মানজনক স্থানীয় অকথ্য ভাষার, বিলেষ করে যৌনসক্রিয় মেয়েদের জন্য ব্যবহৃত, মোকাবেলা করার জন্য প্রস্তুত থাকুন। যৌন আচরণের দ্বৈতমান সম্পর্কে নির্দেশিকা বইয়ের

(<www.popcouncil.org/publi cations/books/2011_ItsAllOn e. asp> এই ঠিকানায় পাওয়া যাবে) ৩ নং অধ্যায় অথবা আপনার নিজস্ব শিক্ষাক্রদের তথ্যগুলি পুনরায় পড়ন।

\section{প্রথম অভিব্যক্তি}

অধিবেশন সম্পর্কিত ধারণা: গল্পের একটা চরিত্র ছেনে নাকি মেয়ে সে সম্পর্কে শিক্ষার্থীরা তাদের অনুমানের ওপর ভিত্তি করে দেয়া নিজেদের উত্তরগুলো পরীক্ষা করবে।

উদ্দেশ্য: আমরা প্রায়ই একটা মানুষকে তার লিঙ্গের ওপর ভিত্তি করে তার সম্পর্কে যে ধারণা বা বিচার করি সে বিষয়ে শিক্ষার্থীদের সচেতনতা বৃদ্ধি করা; বিশ্লেষণমূলক চিন্তার দক্ষতা শক্তিশালী করা।

\section{নির্দেশাবলি}

১ ব্যাখ্যা করুন:

• আজকে আমরা কিশোর-কিশোরীরা যেসকল সমস্যা, অনুভূতি এবং অভিজ্ঞতার মুখোমুখি হয় সেগুলো নিয়ে একটি গল্প পড়ব এবং আলোচনা করব।

• আমরা দু’টি দলে বিভক্ত হব। প্রত্যেক দলের সদস্যরা একটি গল্প পড়বে ও নিজেদের মতামত আলোচনা করবে, এবং তোমাদের উত্তরগুলি কাগজে লিখবে।

২ শ্রেণিকক্ষের সকল ছেলে-মেয়েদের দুই ভাগে ভাগ করুন। এক দলকে ‘মায়ার গল্প’ এবং অন্য দলকে ‘রসুনের গল্প’ বিলি করুন । (গল্প সম্পর্কে কোন কিছু ব্যাখ্যা করবেন না ।) দলগুনোকে গল্প পড়তে, প্রক্নের উত্তর দিতে এবং প্রত্যেকের উত্তর দলের উত্তরের সাথে মিলিয়ে দেখতে সময় দিন। দুই দলের মধ্যে যথেষ্ট দূরত্ব নিশ্চিত করুন, যেন তারা একে অন্যের কথা শুনতে না পায়।

৩ ১৫ মিনিট পর কারণ দেখানো ও ব্যাখ্যা ছাড়াই যে দল রসুলের গল্প পড়েছে তাদেরকে গল্পের শেষের প্রত্যেকটি প্রশ্নের উত্তর সংক্ষিপ্তভাবে দিতে বলুন । কিছু বিশেষ উত্তর ( বিশেষণ, বিশেষ্য নামকরণ) বোর্ডে লिখুন।
8 যারা মায়ার গল্প পড়েছে তাদের জন্য একই কাজ করুন ।

৫ রসুলের গল্প পড়া দল থেকে যে শব্দগুলি এসেছে সেগুলির ওপরে “রসুল” লিখুন। মায়ার গল্প পড়া দল থেকে যে শব্দগুলি এসেছে লেগুলির ওপরে “মায়া” লিখুন।

৬ জানিয়ে দিন যে, শ্যুমাত্র ব্যক্তির লিঙ্গ ছাড়া দুটি গল্প একই রকম এরপর জিজ্ঞেস করুন:

• আমরা মায়ার উত্তরের সাথে রসুলের উত্তরগুলি তুলনা করে কী লক্ষ্য করেছি?

• এই গল্পের মাধ্যন্ ছেলেদের মানের তুলনায় মেয়েদের মান সম্পর্কে আমরা কী বুঝতে পারি ?

• এরকম দ্বৈত-মান কি আমাদের সমাজের মেয়েদের ওপর খারাপ প্রভাব ফেলে ?

• এই দ্বৈত-মান নিয়ে তোমরা কী ভাব? 


\section{মায়ার গল্প}

যোল বছর বয়সে মায়া প্রথম যৌনমিলন করেছিল । তার দুইজন বন্ধু মাঝে মাঝে তার কখনও প্রেমিক হবে না বলে ঠাটা করত, এবং তারা তাকে কোন নির্দিষ্ট একটা ছেলেকে বেছে নিত এবং তার সাথে যৌনমিলন করতে উৎসাহিত করত। সে উৎসুক ছিল, কিন্ত প্রধানত সে তার বন্ধুদের দ্বারা প্রভাবিত হওয়া অনুভব করতো সুতরাং সে একজনকে বেছে নিল ও তারা যৌনসম্পর্ক তৈরি করল। বিদ্যালয়ের প্রায় প্রত্যেকেই বুঝতে পারল যে, মায়া এবং ছেলেটি যৌনমিলন করেছিল।

পরবর্তী দুই বছরে মায়া আরও চারটি ছেলের সাথে যৌন সম্পর্ক স্থাপন করল। তাদের একজন ছিল বিদ্যালয়ের বন্ধু, একজন ছিল প্রতিবেশী, একজনের সাথে চাকরির সময়ে পরিচয় হয়েছিল, আর একজনের সাথে বন্ধুর মাধ্যন্ পরিচয় হয়েছিল। লে যৌনমিলন উপভোগ করত। মায়া জানত যে, সে এই ছেলেদের কাউকেই বিয়ে করতে চায় না, কিন্তু সেই সময়ে প্রত্যেকটি ছেলের কাছেই সে সুন্দর ছিল । এবং লে তাদেরকে তার অনুভূতি সম্পর্কে কখনই মিথ্যা বলত না । যৌনসঙ্গী থাকাতে মায়া নিজেকে আকর্ষণীয় ও গুরুত্বপূর্ণ মনে করত।

বেশিরভাগ সময়ই মায়া কনডম ব্যবহারের প্রতি সতর্ক থাকত, কিন্তু একসময় সে গর্ভধারণ করে ফেলল ও গর্ভপাত ঘটালো । আরেক সময় একজন সঙ্গীর কাছ থেকে সে যৌনবাহিত সংক্রমণের শিকার হল, কিন্তু খুব দ্রুত এটির চিকিৎসা করালো ।

এখন লে একজন পুরুষের সাথে সম্পৃক্ত যার সাথে মায়ার পরিবার তাকে বিয়ে দিতে চায়। মায়া এই লোকটিকে পছন্দ করে কিন্তু তার সাথে বিয়ের আগের দুই বছর পর্যন্ত যৌনমিলন থেকে বিরত থাকতে চায়। অন্য একটি শহরে তার চাচাত বোনের সাথে দেখা করতে যেয়ে সে তার পুরনো এক থ্রেমিকের কাছে ছুটে গেল যে তার সাথে আবারও য্যেনমিলন করতে চায় এবং মায়া সিদ্ধান্ত নিল যে, বিয়ের আগে এই একবার যৌনমিলন করলে কাউকে কষ্ট দেয়া হবে না । সে মনে করল, “সর্বোপরি য্যেনমিলন ছাড়া দুই বছর অনেক সময় ।” শেষে সে তার খুব কাছের বন্ধু যাকে সে বুঝত, তার ওপর নির্ভর করল।

\section{গল্পটি পড়ার পর, এই প্রশ্নগুলো নিয়ে ভাব এবং এগুলো নিয়ে তোমার দলের সাথে আলোচনা কর:}

$১$ মায়ার বন্ধুরা মায়ার সম্পর্কে কী মনে করে বলে তোমরা মনে কর? তারা মায়ার সম্পর্কে বলার জন্য কী ‘নামকরণ’ করতে পারে?

\section{২ মায়ার সম্পর্কে তোমরা কী মনে কর?}

৩ মায়ার সম্পর্কে বলার জন্য তিনটা বা চারটা বিশেষণ নিয়ে একটা তালিকা তৈরি কর। যেমন- সে সুখী না অসুখী? আত্নবিশ্বাসী বা অনিরাপদ অনুভব করে? সৎ বা অসৎ? বাস্তববাদী বা বাস্তববাদী নয়? আকর্ষণীয় বা আকর্ষণীয় নয় ? সম্মান করার যোগ্য বা সম্মান করার যোগ্য নয় ? একজন মেয়ের জন্য আদর্শ বা আদর্শ নয়? উচ্ছৃজ্খল চরিত্রের বা উচ্ছৃজ্থল চরিত্রের নয়? 


\section{রসুলের গল্প}

যোল বছর বয়সে রসুলের প্রথম যৌন সম্পর্ক হয়েছিল। তার দুই বন্ধু মাঝে মাঝে তার কখনও প্রেমিকা হবে না বলে ঠাট্টা করত, এবং তারা তাকে একটা নির্দিষ্ট মেয়েকে বেছে নিতে ও তার সাথে যৌন সম্পর্ক করতে উৎসাহিত করত। সে উৎসুক ছিল, কিন্তু প্রধানত সে তার বন্ধুদের দ্বারা প্রভাবিত হওয়া অনুভব করতো সুতরাং সে একজনকে বেছে নিল ও তারা যৌনসম্পর্ক তৈরি করল । বিদ্যালয়ের প্রায় প্রত্যেকেই বুঝতে পারল যে, রসুল এবং মেয়েটি যৌনমিলন করেছিল ।

পরবর্তী দুই বছরে রসুল আরও চারটি মেয়ের সাথে যৌনমিলন করল। তাদের একজন ছিল বিদ্যালয়ের বন্ধু, একজন প্রতিবেশী, একজনের সাথে চাকরির সময়ে পরিচয় হয়েছিল, আর একজনের সাথে বন্ধুর মাধ্যমে পরিচয় হয়েছিল। সে যৌনমিলন উপভোগ করত। রসুল জানত যে, সে এই মেয়েদের কাউকেই বিয়ে করতে চায় না । কিন্তু সেই সময়ে প্রত্যেকটি মেয়ের কাছেই সে সুন্দর ছিল। সে তাদেরকে কখনই তার অনুভূতি সম্পর্কে মিথ্যা বলত না । যৌনসঙ্গী থাকাতে রসুল নিজেকে আকর্ষণীয় ও গুরুত্বপূর্ণ মনে করত।

বেশিরভাগ সময়ই রসুল কনডম ব্যবহারের প্রতি সতর্ক থাকত। কিন্তু একটি মেয়ে গর্ভবতী হয়ে গেল এবং গর্ভপাত ঘটালো । আরেকসময় একজন সঙ্গীর কাছ থেকে লে যৌনবাহিত সংক্রমণের শিকার হল । কিন্তু খুব দ্রংত এটির চিকিৎসা করালো।

এখন সে একজন যুবতী মহিলার সাথে সম্পৃক্ত যার সাথে তার পরিবার তাকে বিয়ে দিতে চায়। সে এই মহিলাকে পছন্দ করে, কিন্তু রসুল তার সাথে বিয়ের আগে দুই বছর পর্যন্ত যৌনমিলন থেকে বিরত থাকতে চায়। অন্য একটি শহরে তার চাচাত ভাইয়ের সাথে দেখা করতে যেয়ে সে তার পুরনো এক প্রেমিকার কাছে ছুটে গেল যে তার সাথে আবারও যৌনমিলন করতে চায়, এবং রসুল সিদ্ধান্ত নিল যে, বিয়ের আগে এই একবার যৌনমিলন করলে কাউকে কষ্ট দেয়া হবে না । সে মনে করল, “সর্বোপরি যৌনমিলন ছাড়া দুই বছর অনেক সময় ।" শেষে সে তার খুব কাছের বন্ধু যাকে সে বুঝত, তার ওপর নির্ভর করল ।

গল্পটি পড়ার পর, এই প্রশ্নগুলো নিয়ে ভাব এবং এগুলো নিয়ে তোমার দলের সাথে আলোচনা কর:

১ রসুলের বন্ধুরা রসুলের সম্পর্কে কী মনে করে বলে তোমরা মনে কর? তারা রসুলের সম্পর্কে বলার জন্য কী নামকরণ করতে পারে ?

\section{২ রসুল সম্পর্কে তোমরা কী মনে কর?}

৩ রসুল সম্পর্কে বলার জন্য তিনটি বা চারটি বিশেষণ নিয়ে একটি তালিকা তৈরি কর। যেমন- সে সুখী না অসুখী ? আত্নবিশ্বাসী বা অনিরাপদ অনুভব করে? সৎ বা অসৎ? বাস্তবাদী বা বাস্তববাদী নয়? আকর্ষণীয় বা অকর্ষণীয় নয়? সম্মান করার যোগ্য বা সম্মান করার যোগ্য নয় ? একজন ছেলের জন্য আদর্শ বা আদর্শ নয়? উচ্ছৃজ্খল চরিত্রের বা উচ্ছৃজ্খল চরিত্রের নয়? 


\section{निर्্দশাবলि}

১ শিক্ষার্থীদেরকে বলুন:

• আজকে আমরা যৌন আকাক্ষ্ণা বিষয়ে আলোচনা করব। এই বিষয়ের আমরা কিছু সাধারণ প্রচলিত ধারণা দূর করব।

• প্রথমে আমরা একটি সত্য বা মিথ্যা অনুশীলন শেষ করব।

২ অনুশীলনপত্রগুলো বিলি করুন এবং এটি দ্রংত পূরণ করার জন্য निर्দ̆শ দিন।

৩ একজন আগ্রহী শিক্ষার্থীকে প্রথম উক্তিটি পড়ার জন্য ডাকুন । এবং এরপর প্রশ্ন করে আলোচনায় উদ্বুদ্ধ করুন:

• তোমাদের মধ্যে কতজন মনে কর যে উক্তিটি সত্য?

- কে মনে করে এটি মিথ্যা ?

যদি কেউ সঠিক উত্তর না দিতে পারে তাহলে প্রশ্নের মাধ্যমে দলটিকে উত্তরে পৌঁছাতে সাহায্য করুন । যদি কেউই সঠিক উত্তরটি না দিতে পারে শুধুমাত্র তখনই আপনি তা জানিয়ে দিন। এরপরও কারো কোন প্রশ্ন বা মন্তব্য আছে কিনা তা নিশ্চিত হোন ।
8 প্রত্যেক উক্তির জন্যই একই প্রক্রিয়া অনুসরণ করুন ।

৫ শেষ দশ মিনিট প্রশ্নের জন্য রাখুন।

- অধিবেশনের শুরুতে কোন্ বিষয়গুলো তোমরা সত্য ভেবেছিলে যেগুলো মিথ্যায় পরিণত হয়েছে?

- আমাদের নিজেদের অনুভূতিতে এবং যৌনজীবনে এ ধরনের ভুল তথ্য কী প্রভাব ফেলতে পারে ?

• মহিলাদের আকাজ্ষার তুলনায় পুরুষদের আকাজ্ষা নিয়ে সামাজিক বার্তা সম্পর্কে তোমরা কী লক্ষ্য করেছ?

• এমন ব্যাপকভাবে প্রচলিত ধারণাগুলি যৌন সম্পর্কের ওপর কী প্রভাব ফেলতে পারে বলে তোমরা মনে কর ?

• কেন তোমরা মনে কর যে, পুরুষ ও মহিলাদের আকাক্ষা সম্পর্কে আমরা ভিন্ন বার্তা পাই? আমাদের কি এমন পাওয়া
সময়কাল :

8৫ মिनिট

\section{উপকরণ:}

বোর্ড ও চক; যৌন আকাক্ষার অনুশীলনপত্রের যথেষ্ট পরিমান অনুলিপি । অথবা সত্য/মিথ্যার প্রশ্নগুনো বোর্ডে লেখা; উত্তরমালা।

প्रস্তুতি:

অনুশীলনপত্র পড়ন ও প্রয়োজনমত খাপ খাইয়ে নিন । নিশ্চিত হোন যে, উত্তরগুলো স্পষ্টভাবে বোঝানোর জন্য আপনার কাছে প্রয়োজনীয় তথ্য আছে। 


\section{শিক্ষার্থীদের জন্য হ্যাভজাউট}

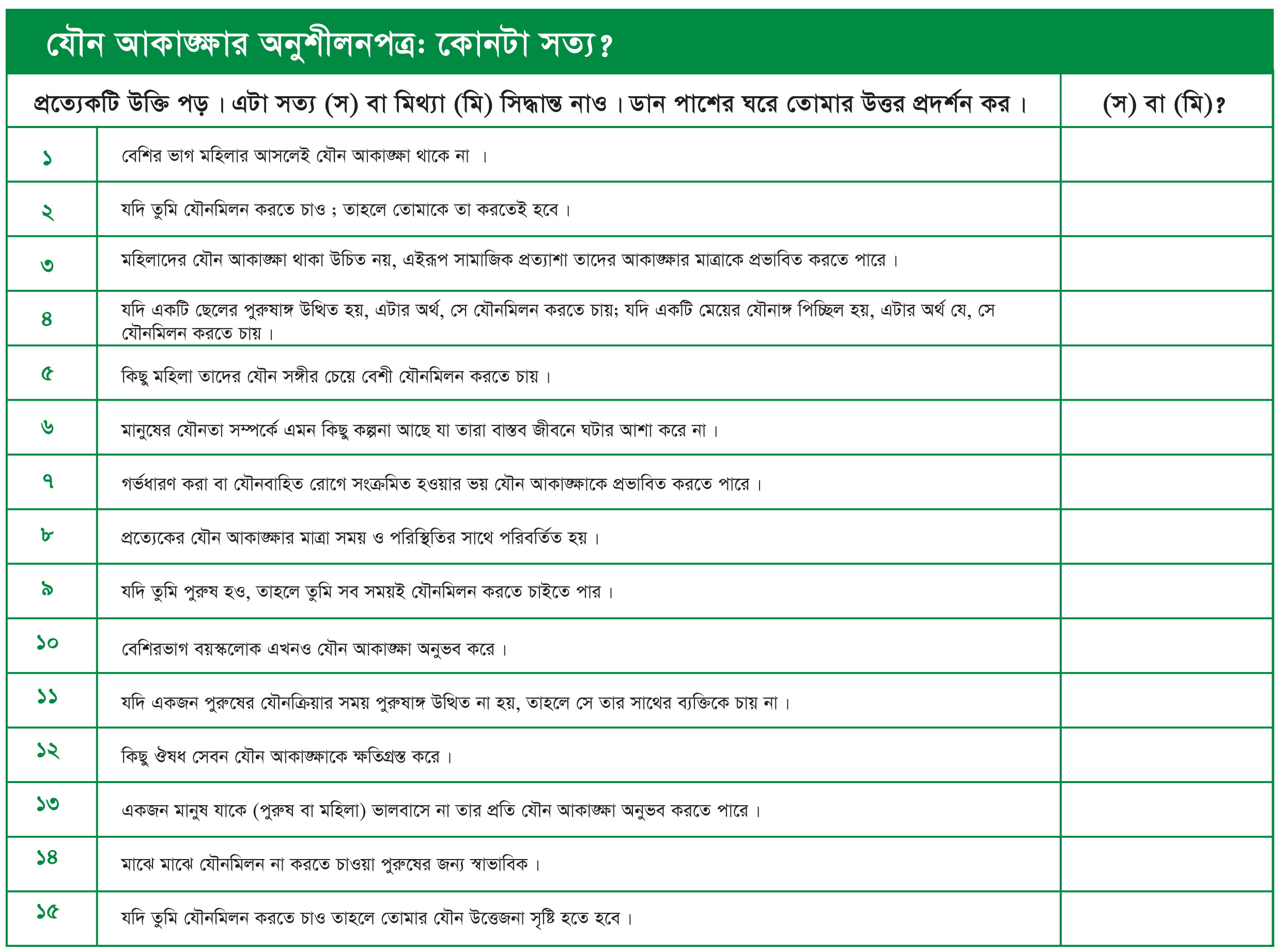




\section{যৌন আকাজ্ষার ওপর অনুশীলনের উত্তর: কোনটা সত্য?}

১ মিথ্যা বেশিরভাগ মহিলারই থৌন আকাজ্ষা আছে। যদি একজন মহিলা যৌনমিলন থেকে আনন্দ না পায় তাহলে সে আগ্রহ হারাতে পারে ।

২ মিথ্যা যৌন আকাক্ষা যৌনক্রিয়া উদুদ্ধ করে না । বস্তুত, মানুষ যৌনমিলন ছাড়াও যৌন আকাজ্ষার অনুভূতিটা উপভোগ করতে পারে।

৩ সত্য সামাজিক প্রত্যাশার দ্বারা যৌন আকাজক্ষা প্রভাবিত হয় । যদি একজন মহিলা মনে করে যে, তার যৌনমিলন করতে চাওয়া উচিত নয়, তাহুলে সে তার আকাক্ষাকে দমিয়ে রাখতে বা অস্বীকার করতে পারে।

8 মিথ্যা পুরুষাঙ্গ উথিত ও পিচ্ছিল কোন কারণ ছাড়াই হতে পারে, অথবা আকাজ্ষার সাথে সম্পর্কিত নয় এমন কোন কারণেও হতে পারে। যেমনবেশিরভাগ পুরুষেরই সকালে পুরুষাঙ্গ উথিত অবস্থায় ঘুম ভান্গে, কারণ তাদের মূত্রথলি পূর্ণ থাকে। কিশোর বয়সি ছেলেদের প্রায়ই স্বতঃস্টূর্তভাবে পুরুষাঙ্গ উথিত হয়, যা যৌন আকাজ্ষা বা উত্তেজনার সাথে সম্পর্কিত নয়।

৫ সত্য যৌন আকাক্ষার মাত্রা ব্যক্তি ও অবস্থান ভেদে বিভিন্ন রকম হয় । কিছু দম্পতির ক্ষেত্রে বা জীবনের কিছু সময়ে একজন মহিলা তার সঙ্গীর চেয়ে বেশী যৌনমিলন আশা করতে পারে।

৬ সত্য কল্পনা প্রায়ই শুধুই কল্পনা । একজন ব্যক্তি কল্পনাকে বাস্তবে নাও চেতে পারে ।

१ সত্য আবেগের মুহূর্তে, যেমন ভয়, একজন ব্যক্তির যৌন আকাজ্ষার ওপর প্রভাব ফেলতে পারে।

b- সত্য যৌন আকাজ্ষার ‘স্বাভাবিক’ পর্যায় বিভিন্ন রকম ও পরিবর্তন হয়। যৌন আকাজ্ষা শারীরিক, আবেগজনিত ও সামাজিক কারণ দ্বারা প্রভাবিত হয়। যদি একজন ব্যক্তির আকাজ্ষ্মার পর্যায় তার সমস্যার কারণ হয় তাহলে সে একজন যৌন স্বাস্থ্যকর্মীর সাথে কথা বলতে পারে।

৯ মিথ্যা পুরুষরা সব সময় যৌনমিলন করতে চায় না । তাদের আকাক্ষার বিভিন্ন পর্যায় আছে যেমন সব মানুমেরই থাকে।

১০ সত্য যৌন আকাজ্ক্ষা বয়সের সাথে সাথে কমে যেতে পারে । যদি একটি সমাজ বয়স্কদের যৌনতাকে ভ্রুকুটি করে বা বরদাস্ত না করে, তাহলে তাদের আকাক্ষার মাত্রা অপবাদ দ্বারা প্রভাবিত হতে পারে। অনেক মানুযের সারা জীবন ধরেই কিছুটা যৌন আকাক্ষকা থাকে।

১১ মিথ্যা একজন পুরুষের এৌনক্রিয়ার সময় স্বাস্থ্যগত অবস্থাসহ (যেমন- ডায়বেটিস বা হৃদরোগ), কোন ঔষুধের জন্য, এবং আবেগজনিত কারণ (যেমন-যৌন সামর্থ নিয়ে উদ্বিগ্ন হওয়া, অন্য কোন ব্যক্তির ওপর রাগ বা অন্য যে কোন কারণ, বা সে পূর্বে সম্পন্ন করেছে), বিভিন্ন কারণে পুরুষ্যাঙ্গ নাও উথিত হতে পারে।

১২ সত্য যৌন আকাজ্ষা কমে যাওয়া কিছু চিকিৎসার একটা পার্শ্ব প্রতিক্রিয়া । কিছু চিকিৎসা যেমন-ভায়াগ্রা, পুরুযাঙ্গ উথিত হওয়াকে বৃদ্ধি করে এবং শ্যুমাত্র ঐ কারণেই ব্যবহার করা হয়। প্রায়ই ডাক্তাররা রোগীদের সাথে চিকিৎসার যৌন প্রভাব নিয়ে আলোচনা করে না । যদি পার্শ্ব প্রতিক্রিয়া বেশি গুরুতর হয় তাহলে একজন ডাক্তার অথবা ফার্মাসিস্টের সাথে পরামর্শ করুন।

১৩ সত্য মানুয প্রায়ই তৌনন আকাজ্ষা ও ভালবাসার মধ্যে পার্থক্য করতে পারে না । তুমি কাউকে ভালবাসতত পার কিন্তু তার সাথে থ্যেনমিলন করতে না চেতে পার, এবং তুমি এমন কারো সাথে যৌনমিলন করতে চেতে পার যাকে তুমি ভালবালো না ।

১8 সত্য পুরুষ বা মহিলা যেই হোক না কেন কেউই সব সময় যৌনমিলন করতে চাইতে পারে না ।

১৫ মিথ্যা মাঝে মাঝে মানুষ যৌনমিলন করতে চায় কিন্তু যৌনভাবে উত্তেজিত হয় না । এমন অবস্থা বেশিরভাগ মানুমেরই কিছু সময়ে ঘটে। এটি চিন্তিত হবার মত কিছু নয় । 


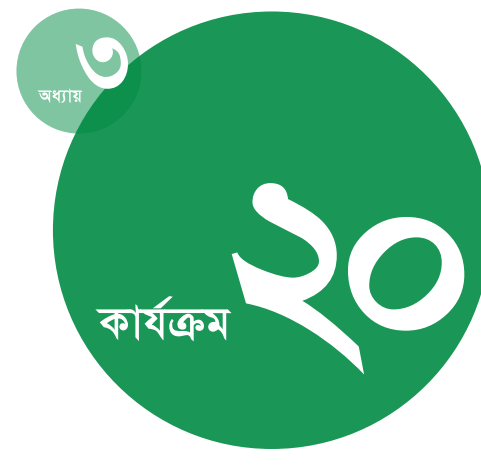

সময়কাল:

৩৫-8० মিनिট

\section{উপকরণ:}

বোর্ড ও চক।

প্রস্তুতি:

সম্পূর্ণ অধিবেশনটি ভালভাবে পড়ন; সংবেদনশীল এবং মর্যাদাপূর্ণভাবে সমাজে কীভাবে সংখ্যালঘুদের বিষয়ে আলোচনা করা যায় তা ভাবুন।

\section{ভিন্নতা অনুভব}

অধিবেশন সম্পর্কিত ধারণা: অন্যদের মতো একই রকম বা ভিন্ন রকম হলে কেমন মনে হয় তা শিক্ষার্থীরা খুঁজে দেখবে। যৌন সংখ্যালঘুসহ অন্যান্য সংখ্যালঘুদের মানবাধিকারকে দৃঢ়ভাবে পুনর্ব্যক্ত করবে।

উদ্দেশ্য: শিক্ষার্থীদের অন্যদের থেকে আলাদা হওয়ার অনুভূত্গুলো বুঝতে সক্ষম করা; যৌন সংখ্যালঘুসহ সকল মানুমের মানবাধিকারগুলো দৃঢ়ভাবে ব্যক্ত করা; আবেগের প্রতিফলন ও কথোপকথন দক্ষতা শক্তিশালী করা।

\section{নির্দেশাবলি}

১ নিম্মলিখিত ধাপ অনুসারে বিষয়টি উপস্থাপন করুন:

• তোমার নিজের সম্পর্কে এমন দুটি বিষয় ভাব (তোমার বয়স ছাড়া) যার দ্বারা তুমি এখানে তোমার সাথে যারা বসে আছে তাদের মত হতে পারো ।

- এ এখন এমন দুটি বিষয়ের কথা ভাব যার জন্য তুমি নিজেকে অন্যদের থেকে আলাদা মনে করো । তুমি এগুলি অন্যদের সাথে বলবে না । তারা তোমার সম্পর্কে যা- ইচ্ছা সত্য ভাবতে পারে।

- বিষয় তোমাকে অন্যদের মত করেছে তা নিয়ে তুমি নিজে কেমন অনুভব করো তা নিয়ে এক মিনিট ভাব। এই অনুভূতি প্রকাশ করে এমন চারটি আবেগপূর্ণ শব্দ তোমার নোট বইয়ে লিখ।

- এ এরপর, যে বিষয়গুনো তোমাকে অন্যদের থেকে পৃথক করেছে সেগুলো নিয়ে আবার ভাব। এটির অনুভূতি কেমন ? চারটি অনুভুতি বা আবেগপূর্ণ শব্দ লিখ।

২ নিয়লিখিত প্রশ্নগুনো নিয়ে একটি আলোচনা পরিচালনা করুন:

- এ একরকম বা আলাদা হওয়ার অনুভূতিগুলো না বলে, অন্যদের মত একরকম হওয়ার কিছু অনুভূতি কী? [উত্তরগুলো বোর্ডে এক সারিতে লিখুন।]

- আমাদের অন্যদের থেকে আলাদা হওয়ার অনুভূতিগুলো কেমন ? [উত্তরগুলো অন্য সারিতে লিখুন ।]
৩ দলটিকে মনে করিয়ে দিন প্রত্যেকে আলাদা অনুভব করে বা কিছুক্ষেত্রে “খাপ খাইয়ে নাও” নিতে পারে, এবং মাঝে মাঝে এভাবে অনুভব করা অস্বাভাবিক নয়। শেষ ১৫ মিনিট নিম্নলিখিত প্রশ্নগুলো আলোচনার জন্য বরাদ্দ করুন ।

- কখন অন্যদের থেকে আলাদা হওয়া ভাল ? স্বতন্ত্র হওয়ার সুবিধাগুলো को?

• কখন অন্যদের থেকে আলাদা হওয়া খারাপ?

• যে মানুষদেরকে অন্যদের থেকে আলাদা করা হয়, তারা অন্যদের থেকে কী আচরণ পেতে পারে ? এটা সম্পর্কে তোমরা কী ভাব? [জানার চেষ্টা করুনঃ এ ধরনের আচরণ কি সঠিক?]

• তোমরা যখন আলাদা অনুভব কর তখন কী আচরণ আশা কর? যে সকল মানুষ আলাদা (এবং কারও জন্য ক্ষতিকর নয়) তাদের কি অন্য সবার মত একই অধিকার আছে? তারা যেভাবে আলাদা সেটা কি কোন বিষয়?

বাড়ির কাজ: সমাজে একটি সংখ্যালঘু দলের কথা ভাব।/জানার চেষ্টা করুন: প্রতিবন্ধী, নিচু সামাজিক সম প্রদায়, সংখ্যালঘু জাতি-গোষ্ঠী বা নৃগোষ্ঠী, যৌন সংখ্যালঘু।] তোমাদের সমাজে তাদেরকে কীভাবে দেখা হয়? কখনও কখনও কি তাদের অধিকার ক্ষুন্ন হয়? কখনও কি ঐ সমপ্রদায় থেকে কেউ তাদের অধিকার রক্ষার জন্য কথা বলেছিল? 


\section{সমলিঙ্গের কারো প্রতি যৌন আকর্ষণ অনুভব করা: ব্যক্তিগত গল্প}

অধিবেশন সম্পর্কিত ধারণা: যে সকল কিশোর-কিশোরীরা নিজেদের সমকামী মনে করে শিক্ষার্থীরা তাদের গল্প পড়বে এবং তাদের অনুভূতি, অভিজ্ঞতা ও প্রতিক্রিয়া নিয়ে আলোচনা করবে । ভুল তথ্য সঠিক করা হবে।

উদ্দেশ্য: শিক্ষার্থীদেরকে যে সকল কিশোর-কিশোরীরা সমলিঙ্গের প্রতি আকর্ষণ অনুভব করে তাদের দুটি সাধারণ অভিজ্ঞতা বর্ণনা করতে শেখানো; তাদের অনুভূতির সাথে একাত্য করা; ভুল তথ্য সঠিক করা; সমালোচনামূলক চিন্তা এবং নিজের ভাষায় বর্ণনা করার দক্ষতা শক্তিশালী করা।

\section{নির্দেশাবলি}

১ শ্রেণিকক্ষের সকল শিক্ষার্থীকে তিনটি দলে ভাগ করুন:

• আজকে আমরা অল্পবয়সী ছেলে যারা সমকামী, তার অর্থ যারা বিশেষত বা প্রধানত সমলিঙ্গের মানুষের প্রতি আকর্ষণবোধ করে, তাদের সামাজিক অভিজ্ঞতা এবং অনুভূতি সম্পর্কে জানব। তোমরা অল্পবয়সী সমকামী ছেলেদের নিয়ে সত্য কেসস্টাডি পড়বে।

- প্রত্যেক দলের জন্য একটি আলাদা কেসস্টাডি আছে। তোমরা তিনটি প্রশ্ন আলোচনা করবে এবং তোমাদের উত্তর লিখবে।

২ নিম্নলিখিত প্রশ্নগুনো বোর্ডে লিখুন এবং শিক্ষার্থীরা যেন শুনতে পায় এমনভাবে এগুলো পড়ন:

গল্পের ব্যক্তিটি को কী অনুভূতির কথা বলেছে? যতগুলো অনুভূতি তোমরা লক্ষ্য করেছ সেগুলো লেখ।

গল্পের মানুষটি সমকামীদের সম্পর্কে কোন্ বিষয়গুলো বিশ্বাস করে?

৩ হ্যান্ড আউটগুলো বিতরণ করুন । দলগুলিকে এগুলো পড়তে দশ মিনিট সময় দিন, আলোচনা করুন এবং প্রশ্নগুলোর উত্তর লিখুন। শিক্ষার্থীদেরকে তাদের লিখিত উত্তরগুলো সবার সামনে পড়তে বলুন ।

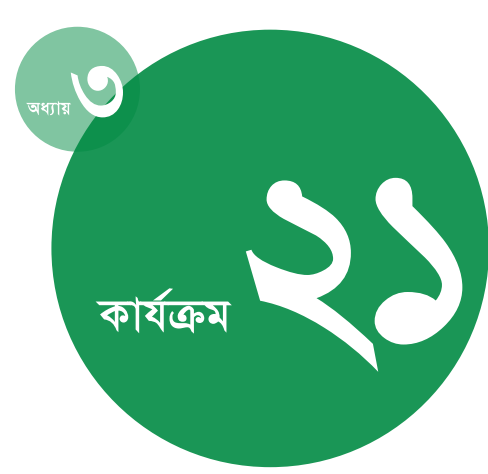

\section{সময়কাল :}

৪৫-৫० মিनिট

\section{উপকরণ:}

বোর্ড ও চক; সমকামী হওয়া সম্পর্কে কেসস্টাডির অনুলিপি।

৫ শ্রেণিকক্ষের সবার সাথে নিম্নলিখিত প্রশ্নগুলো আলোচনা করার জন্য ১৫ মিনিট সময় রাখুন:

• সর্বোপরি, নিয়া, লী এবং মৌ-এর সমকামী হওয়ার বিষয়ে কী রকম অনুভূতি ছিল। ঐ অনুভূতিগুনোর প্রধান কারণ কী ছিল? তারা কীভাবে বুঝতে পারল যে তারা পুরুষ্ অথবা মহিলা সমকামী? তাদের কি এমন কিছু ঘটেছিল যা তাদের 'সমকামী’ বানিয়েছিল ?

- এই গল্প পড়ে তোমাদের কী মনে হয়েছে? তোমাদের কোন বিষয়টি বিস্মিত করেছে? সমকামী মানুষ সম্পর্কে তোমরা কী শিখেছ?

- যৌন বিষয়ের বিভিন্নতা নিয়ে সমাজের দৃষ্টিভঙ্গি এবং ধারণার কি পরিবর্তন হচ্ছে?

\section{প্রস্তুতি:}

গল্প বা বিষয়বস্তু সম্পর্কে প্রশ্নের উত্তর দিতে নিজেকে সম্পূর্ণভাবে তৈরি করুন। আপনার শিক্ষার্থীরা বিষয়টিকে গুরুত্ব সহকারে নেবে এবং আপনি বিষয়টি পড়াতে স্বাচ্ছন্দ্যবোধ করবেন তা নিশ্চিত হোন । সম্ভব হুলে যৌন বিষয়ের বিভিন্নতা নিয়ে অভিজ্ঞ প্রতিষ্ঠান বা অন্য নির্ভরযোগ্য স্থান থেকে তথ্য সংগ্রহ করুন । যদি এখানে ব্যবহৃত শব্দ ('পুরুষ সমকামী’ ও 'মহিলা সমকামী') আপনার সমাজে খারাপ অর্থ বহন করে তাহলে এমন শব্দ ব্যবহার করুন যা খারাপ অর্থ বহন করে না । 


\section{সমকামী হয়ে ওঠা নিয়ে কেসষ্টাডি}

\section{১নং দলের হ্যান্ডআউট: নিয়ার গল্প:}

আমি পঁচিশ বছর বয়সের একজন মহিলা সমকামী। ছোটবেলায় আমি ভাল ছাত্রী ছিলাম এবং আমার অনেক বন্ধু ছিল। যখন আমি বয়ঃসঞ্ধিকালে পৌঁছলাম তখন আমার অন্যরকম অনুভব হতে থাকল। আমি কাউকে চুম্বন করছি এমন স্বপ্ন দেখতাম। আমি মানুষটির মুখ দেখতে পারতাম না, কিন্তু তার শরীরটা অনুভব করতে পারতাম, একটি মহিলার শরীর। আমি অনিশ্যতায় ভুগতাম ও নিজেকে ঘৃণা করতে শুরু করলাম। পনের বছর বয়সে হঠাৎ আমি দেখলাম যে, আমি সত্যিই আমার পরিচিত এবং আমার চেয়ে বড় একজন মেয়ের প্রতি আকর্ষণ বোধ করি। এই অনুভূতি ছিল সত্যিই পরাজয়ের। এগুনোকে আমার স্বপ্নের সাথে একত্রিত করে আমি ধীরে ধীরে বুঝতে পারলাম ও নিজের মধ্যে মেনে নিলাম যে, আমি মেয়েদের প্রতি আকর্ষণবোধ করি। কিন্তু প্রথমবার আমি 'সমকামী' শদ্দটি একটি পত্রিকার গল্পতে দেখি যেখানে একজন মহিলা পুলিশ অফিসার একটি সতের বছরের মেয়েকে উপর্যুপরি ধর্যণ করেছে। আমার মনে আছে পত্রিকাটি সমকামিতাকে বিকৃত, অস্বাভাবিক এবং একটি অসুখ হিসেবে দেখিয়েছিল। সেখানে সমকামী পুরুষ এবং এইড্স নিয়ে আরও একটি অনুচ্ছেদ ছিল।

উভয় গল্পেই সমকামীদেরকে বিকৃত এবং অসুস্থ হিসেবে দেখানো হয়েছিল । আমি বুঝলাম যে ‘ওদের একজন’ হলে আমি সমাজচ্যুত হব। সবাই ভালবাসা পেতে ও সমাজের অংশ হতে চায়- কেউ সমাজচ্যুত হতে চায় না । আমি মনে করলাম আমার মধ্যে কোন সমস্যা আছে, তাই আমি অসুস্থ। 'মহিলা সমকামী’ শব্দটি আমার মনে অনেক ভয়ের সৃষ্টি করল। এটা নিষিদ্ধ ছিল; আমি নিষিদ্ধ ছিলাম। আমি ভীত ছিলাম । আমি নিজেকে উদ্ভট এবং অস্বাভাবিক ভাবতাম। সামলে নেয়ার জন্য আমি লেখাপড়ায় আত্ননিয়োগ করলাম ও বইয়ের পোকা হিসেবে পরিচিতি লাভ করলাম ।

\section{২নং দলের হ্যান্ডআউট: লী’র গল্পः}

আমি তেইশ বছরের একজন পুরুষ সমকামী; আমি গ্রামে বড় হয়েছি। যখন আমার এগারো বছরের মত বয়স, তখন আমি লক্ষ্য করলাম যে, আমি পুরুষ দেহ দেখতে পছন্দ করি। আমি বিস্মিত হলাম এবং আমি এর কারণ জানতাম না । যেখানে আমি বসবাস করতাম সেখানে একজন লোক ছিল যার চেহারা ও আচরণ ছিল মহিলাদের মত; তার ডাকনাম ছিল ‘গার্ল'। আমি তার সম্পর্কে ভীত ছিলাম এবং আমি জানতাম যে, আমি তার মত হতে চাই না । আমার আরও মনে পড়ে যে, একসময় আমাদের শহর থেকে একটা যৌন বিষয়ক পত্রিকা পেয়েছিলাম এবং সেখানে সমকামী পুরুষদের নিয়ে কিছু তথ্য ছিল যেটা আমি পড়েছিলাম। এটি পড়ে আমি ভয় পেয়েছিলাম। তারপর আমরা শহরে চলে আসলাম যেখানে আমার বন্ধুরা মেয়েদের সাথে যৌনমিলন করতে চাওয়া নিয়ে কথা বলত। আমি তাদের সাথে মিথ্যা বলতাম যে, আমি একটি বিশেষ মেয়ের প্রতি আকৃষ্ট, কিন্তু সবসময়ই আমি পুরুষদের নিয়ে ভাবতাম। এটা সামলানোর জন্য আমি অন্যদের থেকে কঠিন আচরণ করতাম, ও খেলাধূলার সাথে সম্পৃক্ত হয়ে গেলাম । আমি আশা করেছিলাম, কেউ বুঝতে পারবে না যে, আমি সমকামী এবং কেউ বুঝতে পারল না । কিন্তু আমি নিজেকে ঘৃণা করতাম, কারণ আমি বুঝতাম না কেন আমি এমন ।

আমি ভাবতাম, “কেন আমি এটা করতে চেষ্টা করি না ?” আমি আলাদা আলাদা পাঁচটি মেয়ের সাথে মিশলাম । আমি তাদেরকে উপহার দিতাম, আমরা বাইরে ঘুরতে যেতাম, মাঝে মাঝে সিনেমাও দেখতাম। আমি চেষ্টা করছিলাম, কিন্তু এগুলো কোন কাজেই আসছিল না । এমনকি আমি একটি মেয়ের সাথে চারবার যৌনমিলন করেছিলাম, কিন্তু এটা আমার জন্য আনন্দদায়ক হয়নি। 


\section{সমকামী হয়ে ওঠা নিয়ে কেসষ্টাডি}

\section{৩নং দলের হ্যান্ড আউট: মো-এর গল্প:}

আমি বাইশ বছর বয়সের একজন সমকামী পুরুষ। যখন আমি বড় হচ্ছিলাম, তখন আমাদের কয়েকজন সমকামী প্রতিবেশী ছিল এবং আমার বাবা-মায়েরও সমকামী বন্ধু-বান্ধব ছিল। তাই আমি এটা বুঝে বড় হলাম যে, সমকামী মানুষরা অন্য সকলের মত একই রকম । একেবারেই আমার বাবা-মার মতো তাদের বাচ্চা ও চাকরি এবং পোষা কুকুর আছে। যখন আমার বয়স পনের বছর তখন আমি বুঝতে পারলাম যে, আমি আমার সবচেয়ে ভাল বন্ধু বেনোকে বন্ধুর চেয়েও বেশি পছন্দ করি। আমি জানতাম না এটা নিয়ে তার সাথে কীভাবে কথা বলব। তাই আমি আমার মায়ের সমকামী পুরুষ বন্ধুর সাথে দেখা করলাম; সে তার সঙ্গীর সাথে বিশ বছর ধরে বাস করছে। প্রথমে সে বলল যে, সে জানে আমরা কে তা জানার জন্য আমাদের অনেক সৎ মানসিকতার প্রয়োজন। সে তখন বেনোর আচরণ সম্পর্কে আমার কাছে জানতে চাইল এবং বেনোর কাছে বিশ্বস্ত হওয়ার জন্য উৎসাহিত করল। সে ব্যাখ্যা করল যে, বেনো আমার প্রতি একই রকম নাও অনুভব করতে পারে, এবং যদি আসলেই এমন হয় তাহনে বেনো আমার কাছ থেকে নিজেকে দূরে সরিয়ে নিতে পারে । পরের দিন ফুটবল খেলার পরে আমি বেনোকে তার প্রতি আমার অনুভূতির কথা বললাম। প্রথনে সে হতাশ হল এবং আমাকে বলল যে, তার মনে হয় না যে আমরা আর বন্ধু থাকতে পারব। আমি বিধ্বস্ত হয়ে পড়লাম। কিন্তু কয়েক দিন পরে বেনো আমার বাসায় আসল। সে আমাকে বলল যে, তার আমার বা অন্য কোন পুরুষের প্রতি কোন যৌন আকর্ষণ নেই, কিন্তু এখনও সে আমার বন্ধু থাকতে চায়। আমি আমার বন্ধুকে ফিরে পেয়ে খুব খুশি হলাম যদিও আমরা কখনও প্রেমের সম্পর্কে আবদ্ধ হলাম না । কিশোর বয়সে সমকামী হওয়া সবসময় সহজ নয়, এবং আমার প্রায়ই এমন মানুষের সাথে দেখা হত যারা আমার জীবন-যাত্রা নিয়ে অস্বস্তি বোধ করত। এমন একটি সহায়ক পরিবারের একজন বলে আমি জানতাম যে আমি ভাগ্যবান; অনেক কিশোরই যা পায় না। এই জন্য এখন আমি যেসব যুবকদের ফিরে যাওয়ার কোন জায়গা নেই তাদের জন্য একটি হট-লাইনে (সরাসরি যোগাযোগ করা যায় এমন) কাজ করি যেখানে তারা তাদের যৌন বিষয়ে প্রশ্ন করে । 


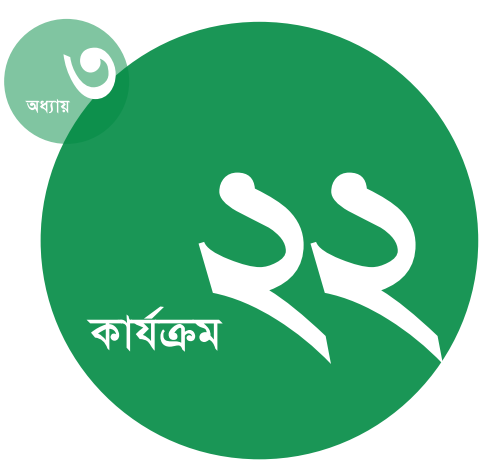

\section{সময়কাল :}

ধাপ-১-৪: ৪৫-৫০ মিনিট ধাপ ৫-৬ ( যদি আপনি একটি ফলোআপ প্রশ্নোত্তর পর্ব পরিকল্পনা করে তা শেষ করতে চান): ১০ মিনিট

\section{উপকরণ:}

বোর্ড ও চক, প্রত্যেক দলের জন্য শিক্ষার্থীদের হ্যান্ড আউটের একটি অনুলিপি; খালি কাগজের টুকরো (যদি আপনি ধাপ ৬ অন্তর্ভুক্ত করতে চান) এবং একটি ব্যাগ বা বড় খাম;

উত্তরপত্র।

\section{প्रস্তুতি:}

উক্তিগুলি নিয়ে তৈরি হ্যান্ডআউট ভালভাবে পড়ন এবং নিশ্চত হোন যে, কেন এগুলো সত্য বা মিথ্যা তা আপনি ব্যাখ্যা করতে পারবেন। আপনি ধাপ ৫ এবং ধাপ ৬ অন্তর্ভুক্ত করবেন কিনা তা সিন্ধান্ত নিন ? यদি করেন তাহলে লিখিত প্রশ্নগুলোর উত্তর দেয়ার জন্য একটি নির্ভরযোগ্য তথ্যের উৎস নিশ্চিত করুন ।

\section{যৌন আচরণ- প্রচলিত ধারণা এবং সত্য ঘটনা}

অধিবেশন সম্পর্কিত ধারণা: শিক্ষার্থীরা একটি খেলা করবে সেখানে তারা সিদ্ধান্ত নেবে যৌন আচরণ বিষয়ে উক্তিগুলি সত্য নাকি মিথ্যা । আলোচনার মাধ্যমে ভুল ধারণা শুদ্ধ করা হবে ।

উদ্দেশ্য: শিক্ষার্থীদেরকে যৌন আচরণ সম্পর্কে সঠিক তথ্য শনাক্ত করতে সক্ষম করা।

\section{निर्দেশাবলি}

১ শিক্ষার্থীদদরকে বলুন যে, তারা ব্যৌন আচরণ সম্পর্কে সাধারণ কিছু ভুল ধারণা নিয়ে আলোচনা করবে এবং সত্য থেকে মিথ্যাকে পৃথক করবে।

২ শিক্ষার্থীদদরকে দু'টি দলে ভাগ করুন। বোর্ডে প্রত্যেক দলের স্কোরশীটের জন্য আলাদা ঘর টৈরি করুন । হ্যাড্ডআউটগুলো বিতরণণ করুন এবং ব্যাখ্যা করুন:

• তোমরা এই অনুশীলনপজ্রের প্রত্যেকটি উক্তি নিয়ে দলীয়ভাবে আলোচনা কর এবং এগুলো সত্য নাকি মিথ্যা তা সিদ্ধান্ত নাও। অনুশীলনটি শেষ করার জন্য তোমাদদর দল সাত মিনিট সময় পাবে।

• সরর্বশ্বে আমরা সবগুলো প্রশ্ন পড়ব এবং শ্রত্যেক দল এগুলোর উত্তর দেবে। কোন দল যখন সঠিক উত্তর দেবে তখন আমি এক পয়েন্ট করে লিখে রাখব।/তাদেরকে কাজটি ফরু করতে দিন; 9 মिनिট সময় দিन।

৩ পর্যালোচনার জন্য প্রথম উক্তিটি সবাই ঔনতে পারে এভাবে পড়তে একজন আগ্রহী শিক্ষার্থীকে ডাকুন। এবার এটি সত্য না মিথ্যা তা মিলিয়ে দেখতে প্রত্যেক দলকে বলুন। সঠিক উত্তরটি বুঝিয়ে বলুন; যে দল বা দলগুলো সঠিক উত্তর দিযেছে তাদেরকে এক পয়েন্ট করে দেন, এবং কোন প্রশ্ন অথবা বিল্রান্তি থাকলে আলোচনা করুন। প্রত্যেকটি উক্তি পুনরায় উল্লেখ করুন। প্রত্যেকটির জন্য এক মিনিট করে সময় দিন।

8 বিশ মিনিট পর (সতেরটি উক্তি শেষ হওয়ার পর) কোন্ দল বিজয়ী হয়েছে তা ঘোষণা করুন । কেন যৌনতা এবং যৌন আচরণ সম্পর্কে এত বেশি প্রচলিত ভুল ধারণা রয়েছে তা জানতে চান?
যদি আপনি একটি ফলোআপ প্রু্নোত্তর পর্ব পরিকল্পনা করেন:

৫ সাদা কাগজের টুকরো বিতরণ করুন, তারপর আলোচনা করুন:

- বেশিরভাগ মানুযেরই যৌনতা এবং যৌন আচরণ নিয়ে প্রশ্ন বা সংশয় আছে। স্ব স্ব কাগজের টুকরোতে এ বিষয়ে তোমাদের নিজেদের যে কোন প্রশ্ন লেখ। তোমাদের নাম লিখ না। কিন্তু গুরুত্বপূর্ণ প্রশ্ন করার ব্যাপারে যথেষ্ট সতর্ক থাকবে। যদি তোমার কোন প্রশ্ন না থাকে তাহলে লেখ “আমার এই মুহূর্তে কোন প্রশ্ন নেই ।"

• শেষ হলে কাগজটি অর্ধেক করে ভাঁজ কর।

• আমি পরবর্তীতে তোমাদের সাথে গুরুত্বপূর্ণ প্রশ্নগুলোর উত্তর নিয়ে কথা বলব।

• আমি তোমাদের ওপর আস্থা রাখি যে, ঠাট্টা বা রসিকতা বোঝায় এমন অপরিণত বা ভুল যেগুলো প্রশ্ন করা থেকে তোমরা বিরত থাকবে; এমন প্রশ্নের উত্তর প্রদান করা হবে না ।

৬ কয়েক মিনিট পর, শিক্ষার্থীদেরকে কাগজগুলো ভাঁজ করতে দিন, এরপর রুমের মধ্যে ঘুরে কাগজগুনো একটি বড় খাম/বাক্সে সংগ্রহ করুন । শিক্ষার্থীরা চলে যাওয়ার পর প্রশ্লগুলো পড়ন। যে প্রশ্নগুনোর উত্তর দিতে পারবেন বলে আপনার নিজের ভেতর আত্নবিশ্বাস নেই, নির্ভরযোগ্য উৎস থেকে সেগুনোর উত্তর জেনে নিন, যেন আপনি উত্তর পর্বে সঠিক তথ্য দিতে পারেন ।

শিক্ষার্থীদের প্রশ্নের উত্তর দেয়ার জন্য পরবর্তী একটি পর্বের সময় রাখুন । 


\section{যৌন আচরণ- সত্য বা মিথ্যা ?}

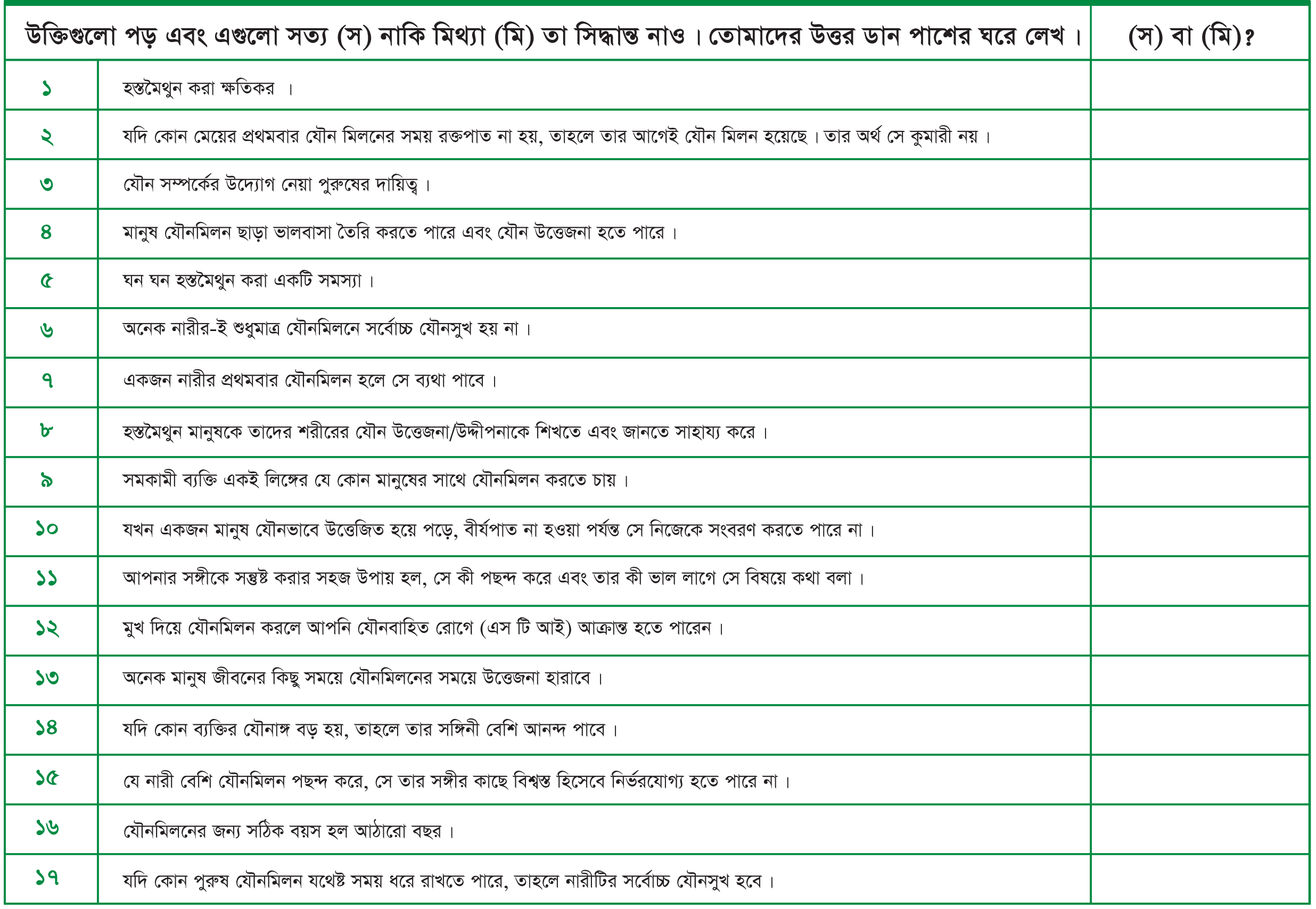




\section{যৌন আচরণ- সত্য বা মিথ্যা ?-এর উত্তরপত্র}

১ মিথ্যা হস্তমৈথুন ক্ষতিকর নয়; বরং এটা কারো নিজের শরীর সম্পর্কে জানার নিরাপদ এবং ভাল উপায়। যাই দোক, এটা একটি ব্যক্তিগত পছন্দ। বেশির ভাগ মানুষই হস্তনৈথুন করে, কিন্ত কিছু মানুষ এটা করতে পছন্দ করে না, এবং কিছু মানুষ এই ধারণায় আস্বস্ত হতে পারে না বা স্বস্তি পায় না ।

২ মিথ্যা কারও কারও সতীচ্ছেদের জন্য রক্তপাত হয়। কিন্ত সতীপর্দা যেকোন শারীরিক কাজকর্ম বা খেলার সময় সহজেই প্রসারিত বা ছিড়ে যেতে পারে , এবং আঙ্গুল বা ঋতুকালীন ব্যবহৃত কাপড়ের দ্বারা প্রসারিত হয়ে খুলে যেতে পারে। সতীত্ছেদ বা রক্তপাত না হওয়া মানে এই নয় যে, মেয়েটির আগেই যৌনমিলন হু়েছিল ।

৩ মিথ্যা অনেক সমাজে পুরুষদের যৌনমিলনে উদ্যোগী ভূমিকা নেয়ার রীতি আছে এবং ধরে নেয়া হয় নারীরা যৌনমিলনে বেশি অনুগত ভূমিকা পালন করবে/ নিস্ক্রিয় থাকবে । যাই হোক, এই রীতি জেন্ডার ভূমিকার কিছু সামাজিক ধারণাকে প্রতিফলিত করে; বাস্তবে নারীরাও যৌন মিলনে উদ্যোগী ভূমিকা নিতে পারে। এমনকি যেসকল নারী গতানুগতিক জেন্ডার ভূমিকাকে মেনে চলে, তারা প্রায়ই তাদের সঙ্গীকে যৌন বাসনা জানানোর জন্য পরোক্ষ উপায় তৈরি করে।

8 সত্য অনেক আচরণের দ্বারাই ভালবাসা টৈরি হতে পারে (আদর, চুম্বন, শরীর টেপা, পারস্পরিক উত্তেজনা)। যৌন মিলন ছাড়াও মানুষের পরস্পরের সাথে ছেঁয়া বা ঘষা লাগলেও যৌন সুখ হতে পারে।

৫ মিথ্যা ঘন ঘন হস্তনৈথুন করলে কোন সমস্যা হয় না । ফুমাত্র ঐ সময়ই হস্তনৈথুন সমস্যা হতে পারে যদি কোন ব্যক্তি এটা তার যেভাবে করা উচিত তা না করে অন্যভাবে করে বা যদি ঐ ব্যক্তি অন্যদেরকে বিরক্ত করে কিংবা এটা তাদের নিজেদের ক্ষতির কারণ হয়।

৬ সত্য বেশিরভাগ নারীই শধুমাত্র যৌনমিলনে সর্বোচ্চ যৌনসুখ পায় না । বরং তার ভগাঙ্কুরের উদ্দীপনার মাধ্যমে সবোচ্চ যৌনসুখ পায়। নারীরা বেশির ভাগ সময়ই সর্বোচ্চ যৌনসুখ পায় যদি তারা (অথবা তাদের সঙ্গী) যৌন মিলনের আগে, যৌনমিলনের সময়ে অথবা পরে সরাসরি ভগাঙ্কুরকে উত্তেজিত করে ।

৭ মিথ্যা কোন নারীর প্রথম য্যেনমিলনে ব্যথা হতেও পারে, নাও হতে পারে। অস্বস্তি অথবা ব্যথা কমানোর জন্য সঙ্গীদের একে অপরের শরীরকে অনুসন্ধান এবং পুরুষাঙ্গ প্রবেশের পূর্বে সম্পূর্ণ কানোত্তেজিত হতে সময় নেওয়া উচিত, যেন নারীর যোনিপথ ভালভাবে পিচ্ছিল হয় । যদি নেয়েটি বিচলিত বা ভীত হয়ে পড়ে তাহনে ঐ দম্পতি অপেক্ষা করতে পারে।

৮ সত্য কারো শরীর যৌন উদ্দীপনায় কীভাবে সাড়া দেয় তা শেখার বা জানার সবচেয়ে ভাল উপায় হল হস্তমৈথুন । কীভাবে সর্বোচ্চ যৌন উত্তেজনায় যাওয়া যায় এটা নারী এবং মেয়েদেরকে তা শিখতে সাহায্য করে।

৯ সত্য যে কোন ব্যক্তি সে বিষমকামী, সমকামী অথবা উভলিঙ্গ যা হোক না কেন, ত্ুমাত্র নির্দিষ্ট লোকের প্রতিই আকৃষ্ট হয় । যৌন আকর্ষণ অনেক কিছুর ওপর নির্ভর করে।

১০ মিথ্যা কিছু মানুষ বিশ্বাস করে যে, যদি তারা সত্যিই যৌনভাবে উত্তেজিত হয়ে পড়ে তাহলে তাদের সর্বোচ্চ যৌনসুখ পেতে হবে, কিন্তু এটা সত্য নয় । থামিয়ে দেয়ার জন্য কিছু অস্বস্তি পেতে পারে, কিন্তু অস্বস্তি নিজে-নিজেই কমে যাবে। যে কেউ -পুরুষ বা নারী- যৌন অভিজ্ঞতার যেকোন পর্যায়ে থেমে যেতে পারে । 


\section{যৌন আচরণ- সত্য বা মিথ্যা ?-এর উত্তরপত্র}

১১ সত্য কোনটি কামোত্তেনা জাগায় সে বিষয়ে প্রত্যেকের নিজস্ব পছন্দ আছে। কারো সঙ্গী কী পছন্দ বা আনন্দদায়ক মনে করে তা অনুমান করার চেয়ে বরং তাকে জিজ্ঞেস করা দ্রংততর এবং নির্ভরযোগ্য। যোগাযোগ হচ্ছে ইতিবাচক যৌন সম্পর্কের একটি প্রধান বিষয় যা উভয় সঙ্গীর জন্য আনন্দদায়ক।

১২ সত্য মুখের মাধ্যমে যৌনমিলন অনেক রকম যৌনবাহিত সংক্রমণের (এস টি আই) জন্ম দিতে পারে। যৌনবাহিত সংক্রমণগুলির তালিকায় আছে হারপিস, গনোরিয়া, হিউমান প্যাপিলোমা ভাইরাস (এইচ টি ভি অথবা জেনিটাল ওয়াট) সিফিলিস, ক্লেমিডিয়া, হেপাটাইটিস বি, ও শ্যানক্রোয়েড এবং এইচআইভি।

১৩ সত্য অনেক মানুষের জীবনের কিছু সময়ে এই অভিজ্ঞতা হবে। এটা স্বাভাবিক এবং ভীত হওয়ার কিছু নেই। এটি নিয়ে উদ্বেগ এটিকে আরও বেশি করে ঘটাতে পারে ।

১8 মিথ্যা বড় পুরুষাঙ্গ নারীদেরকে যৌনমিলনের সময় বেশি আনন্দ দেয় না । যদিও নারীরা ভিন্নমত পোষণ করে তারপরও বেশিরভাগ নারীরাই বলে পুরুষেরা কী করল সেটাই আসল, তার পুরুষান্গের আকার নয়। আসলে বেশি বড় পুরুষাঙ্গ কোন নারীর জন্য অস্বস্তিকর অথবা ব্যথাদায়ক হতে পারে।

১৫ মিথ্যা ৌনমিলন উপভোগ করা পুরুষ এবং নারীদের জন্য স্বাভাবিক। বিশ্বাসযোগ্যতার সাথে একজন মানুযের যৌনমিলন উপভোগ করার কোন হাত নেই। নারীদের যৌনমিলন পছন্দ করা উচিত নয় এই উক্তির পেছনের ধারণা দ্বৈত-মানকে প্রতিফলিত করে যে, পুরুষ্যরা যৌনমিলন পছন্দ করে এটি গ্রহণযোগ্য এবং প্রত্যাশিত, নারীরা নয়। এই ধারণা অনৈতিক, ভুল এবং গতানুগতিক।

১৬ মিথ্যা ৌনমিলনের কোন সঠিক বয়স নেই। প্রত্যেক ব্যক্তিকেই নির্ধারণ করতে হবে কখন লে যৌনমিলনের জন্য প্রস্তুত। কোন মানুষ প্রস্তুত কিনা তা তার পরিপক্কতার পর্যায়, সম্পর্ক, মূল্যবোধ এবং অনুভূতির মাত্রার ওপর নির্ভর করে। একজন মানুষ কখন যৌনমিলন সম্পর্কে জ্ঞাত হওয়ার জন্য যথেষ্ট যোগ্য হয় সেই বয়স সম্পর্কে প্রত্যেক দেশের আইন আছে।

১৭ মিথ্যা পুরুষটি কত দীর্ঘ সময় স্থায়ী হল তা ব্যতিরেকে শধুমাত্র যৌনমিলন অনেক নারীকে সর্বোচ্চ যৌনসুখ দেয় না; বেশির ভাগ ক্ষেত্রে নারীরা ভগাঙ্কুরের প্রত্যক্ষ উদ্দীপনার ফলে সর্বোচ্চ যৌনসুখ পায়। উভয় সঙ্গীর মধ্যে অকৃত্রিম যোগাযোগ যৌনক্রিয়া আনন্দদায়ক করতে সাহায্য করে । 


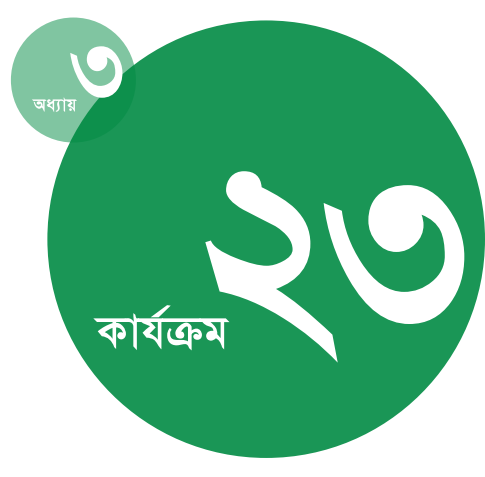

সময়কাল :

৩৫-৪० মিनिট

\section{উপকরণ:}

বোর্ড ও চক; ‘জোরপূর্বক পছন্দের উক্তি’ তালিকা

\section{প्रস্তুতি:}

জোরপূর্বক পছন্দের তালিকা থেকে যেকোন অসামঞ্যপ্র্ণ উক্তি বাদ দিন, এবং অর্থপূর্ণ হতে পারে এমন উক্তি যোগ করুন । বোর্ডের এক দিকে লিখুন ‘একমত’, অন্যদিকে লিখুন ‘একমত নই’।

\section{কেন আমরা এটি করি (বা করি না): যৌন সিদ্ধান্ত গ্রহণে জোরপূর্বক পছন্দের চর্চা}

অধিবেশন সম্পর্কিত ধারণা: কেন মানুষ যৌনমিলন করতে পছন্দ করে বা করে না এ বিষয়ের উক্তি সম্বলিত একটি তালিকা থেকে শিক্ষার্থীদেরকে বলতে বলা হবে যে তারা এগুলো সম্বন্ধে একমত নাকি দ্বিমত পোষণ করে।

উদ্দেশ্য: শিক্ষার্থীদেরকে তাদের নিজেদের ও অন্যদের যৌনসম্পর্কিত সিদ্ধান্ত গ্রহণের বিষয়ে সমালোচনামূলক প্রতিক্রিয়া জানাতে সক্ষম করা; ব্যক্তিত প্রতিক্রিয়া ও লেখার দক্ষতাকে শক্তিশালী করা।

\section{निर्দেশাবলি}

১ অধিবেশনটি সম্পর্কে বলুন:

• আজকে আমরা মানুযের যৌনমিলন করতে চাওয়া এবং না চাওয়ার কিছু কারণ নিয়ে আলোচনা করব। বিভিন্ন ধরনের পরিস্থিতি ও অনুভূতি মানুষ যৌনমিলন করবে কিনা সে বিষয়ে সিদ্ধান্ত নিতে প্রভাবিত করে।

- এই বিষয়টি পুঞ্খানুপুজ্খভাবে পরীক্ষা করার জন্য আমি কিছু উক্তি পড়ব। এগুলো মূল্যবোধমূলক উক্তি, এবং এগুলোর উত্তর প্রদানে কোন সঠিক বা ভুল নেই। প্রত্যেক উক্তির জন্য যদি তোমরা একমত হও তাহলে যেদিকে ‘একমত’ লেখা আছে সেদিকে যাও। यদি একমত না হও তাহলে যেদিকে ‘একমত নই’ লেখা আছে সেদিকে যাও। যদি তোমার সিদ্ধান্ত এই দুইয়ের মাঝে হয় তাহলে যে কোন একদিক পছন্দ করতে নিজেকে ‘বলপ্রয়োগ’ কর। তারপর কিছু শিক্ষার্থী তাদের মতামত আলোচনা করতে পারে। [নিশ্চিত হোন সবাই নিয়মগুলো বুঝতে পেরেছে ।]

২ প্রত্যেকটি উক্তি পড়ন। প্রত্যেক উক্তির পর শিক্ষার্থীদেরকে তাদের নির্বাচিত স্থানে যাওয়ার জন্য সময় দিন। আলোচনা শেষ না হলেও পরবর্তী উক্তিতে চলে যান। যতগুলো উক্তি পড়তে সময় পান ততগুলো পডুন ।
৩ সার সংক্ষেপ করার জন্য পাঁচ থেকে দশ মিনিট সময় রাখুন, জিজ্ঞস করুন:

- যৌনমিলন করতে চাওয়া বা না চাওয়া বিষয়ে সুস্পষ্টভাবে চিন্তা করা একজন যুবক-যুবতীর জন্য কেন গুরুতত্বপূর্ণ? [জানার চেষ্টা করুন: স্বাস্থ্য সুরক্ষার সাথে সাথে স্বস্তি, নিরাপত্তা, আগ্রহ, এবং আনন্দের অনুভূতি।

• যুবক-যুবতীদের যৌনমিলন করা বা না করার জন্য অনেক ধরনের কারণ থাকে। এই কারণের ভিন্নতার জন্য কোন ধরনের ভুল বোঝাবুঝি বা সমস্যার সৃষ্টি হয় ?

• আমরা দেখি যে, মানুষ প্রায়ই তাদের সকল প্রবৃত্তি এবং অনুভূতি সম্পর্কে সচেতন নয়, অথবা তাদের পরিস্থিতি/অবস্থা বিশ্লেষণ করে না । যা হচ্ছে তা সম্পর্কে আরও সচেতন হওয়ার কিছু উপায় কী, কীভাবে আমরা অনুভব করি, কী আমরা করতে চাই বা চাই না ? [জানার চেষ্টা করুন: একটি পত্রিকায় লেখা, বিশ্বস্ত কারো সাথে কথা বলা, সততার সাথে চিন্তা করা ।]

বাড়ির কাজ: তোমাদের মিশ্র অনুভূতি বা তোমাদের সিদ্ধান্ত নিতে সমস্যা হচ্ছে এমন (যৌন বিষয় হতে হবে এমন নয়) যেকোন বিষয়ে দুই বা তিনটি অনুচ্ছেদ লেখ। 


\section{‘জোরপূর্বক পছন্দের’ উক্তিগুলি}

১ ছেলেদের জন্য আমার খারাপ লাগে কারণ তাদেরকে সবসময় এমন আচরণ করতে হয় যেন তারা সব সময় যৌনমিলন করতে চায়, এমনকি যখন তারা এটি করতে না চায়।

২ যদি একটি মেয়ে তার থ্রেমিককে ভালবালে, তার সাথে থৌন সম্পর্কের মাধ্যমে মেয়েটির তা বোঝানো উচিত।

৩ আমার মনে হয় বেশির ভাগ কিলোর-কিশোরীর যৌন বিষয়ে দ্বান্দ্বিক অনুভূতি আছে; তারা একই সাথে যৌনমিলন করতে চায় এবং চায় না ।

8 আমি মনে করি, কাউকে যৌনমিলনের জন্য টাকা বা উপহার দেয়া সঠিক কাজ।

৫ আমি মনে করি যদি তোমার টাকার প্রয়োজন হয়, তাহলে যৌনমিলনের জন্য টাকা নেয়া সঠিক কাজ।

৬ আমি মনে করি একজন প্রকৃত মানুষ ঝুঁকি গ্রহণ করে এবং যৌনভাবে আক্রমণাত্নক হয়।

१ টেলিভিশন এবং পত্রিকার ছবি যুবক-যুবতীদের মধ্যে যৌনমিলন করা উচিত এমন অনুভূতির সৃষ্টি করে ।

৮ যদিও তুমি শারীরিক বল প্রয়োগ করছ না, তারপরও কারও ইচ্ছের বিরুদ্ধে যৌনমিলন করতে চাপ দেয়া ধর্ষণের সমান ।

৯ িছু মেয়ে এমন আচরণ করে যে, তারা আবেগপূর্ণ সম্পৃক্ততা ছাড়াই শুধুমাত্র যৌন আনন্দ চায়, কিন্তু গভীরে গিয়ে দেখ এটি আসলে তারা যা চায় তা নয়; তারা আসলে আবেগপূর্ণ সম্পৃক্ততা চায়।

১০ আমি এমন অনেক মেয়েকে জানি যারা যৌনমিলন করে কারণ তারা তা করতে বাধ্যবাধকতা অনুভব করে ।

১১ যে সকল মানুষ সমলিজ্গের মানুষের প্রতি আকর্ষিত তাদেরকে যৌন অভিজ্ঞতার জন্য তাদের বিষমকামী বন্ধুদের চেয়ে বেশিদিন (বেশি বয়স হওয়া পর্যন্ত) অপেক্ষা করা উচিত।

১২ অনেক যুবক-যুবতী একেবারেই যৌনমিলন করতে চায় না । এইডস বা গর্ভধারণ বা বড়রা যা বলে তাতে তারা যৌনমিলন করার দরকার নেই বলে অনুভব করে। এমনকি তাদের প্রেমিক বা প্রেমিকা থাকা সত্ত্বেও তারা থৌনমিলন করতেই চায় না।

১৩ ৌননমিলনে যে দুইজন মানুষ সম্পৃক্ত তাদের জন্য সবসময়ই এটি অতি আন্তরিক এবং ব্যক্তিগত অভিজ্ঞতা ।

১8 এমন অনেক মানুষ আছে যারা যৌনমিলন করার সিদ্ধান্ত নিয়ে পরবর্তীতে তা না করে।

১৫ এমন অনেক মানুষ আছে যারা য্যেন মিলন না করার সিদ্ধান্ত নিয়ে পরে তা না করে।

১৬ ৌৗনমিলন করার আগে বেশিরভাগ কিশোর-কিলোরী কীভাবে সংক্রমণ এবং গর্ভধারণ রোধ করা যায়, তার সাথে সাথে তাদের ভাল লাগবে কিনা বা তারা যৌনমিলন করতে চায় কিনা এ বিষয়ে সঙ্গীদের সাথে খোলামেলাভাবে আলোচনা করে। 


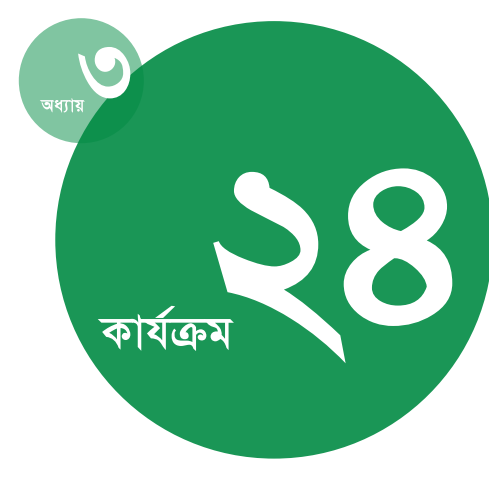

সময়কাল :

8৫ মिनिট

\section{উপকরণ:}

বোর্ড ও চক; নির্বাচিত কেসস্টাডির অনুলিপি, একটি লম্বা দড়ি বা শক্ত সুতা; পেপার ক্লিপ (অথবা টেপ); রঙীন মার্কার, যদি পাওয়া যায়।

\section{প्रস্তুতি:}

নির্দেশনা অনুযায়ী হ্যান্ডআউটগুলি পরিবর্তন করুন(‘যৌন হয়রানি এবং যৌন পছন্দের কেসস্টাডিগুলি দেখুন')। আপনার নির্বাচিত কেসস্টাডিগুলির চারটি অনুলিপি তৈরি করুন ।

\section{কোথায় অবস্থান করছি? যৌন পছন্দ এবং যৌন নিপীড়নের ধারাবাহিকতা}

অধিবেশন সম্পর্কিত ধারণা: শিক্ষার্থীরা জোরপূর্বক যৌনমিলন এবং ইচ্ছাকৃত বা কাজ্ষিত যৌনমিলনের ধারাবাহিকতার মধ্যে কেসস্টাডিগুনো স্থাপন করবে এবং ‘না’ বলার অধিকার নিড়ে আলোচনা করবে। (লক্ষণীয়: আপনি এই অধিবেশন শুরুর পূর্বে শিক্ষার্থীদের যৌন নির্যাতন ও যৌন পছন্দ বিষয়ে কিছু জ্ঞান থাকা উচিত।)

উদ্দেশ্য: শিক্ষার্থীদেরকে যৌন পছন্দের ধারাবাহিকতা নিয়ে আলোচনা করতে সক্ষম করা; যৌনমিলন চাওয়া হয়নি কিন্তু ইচ্ছাকৃত এমন কেস শনাক্ত করা; যৌনমিলনে না বলার অধিকার স্পষ্টভাবে বোঝা; বিমূর্ত চিন্তার দক্ষতা শক্তিশালী করা।

\section{निর্দেশাবলি}

১ কক্ষের একদিক থেকে অন্যদিকে আড়াআড়ি ভাবে সূতাটি লাগিয়ে দিন । এক প্রান্তের নাম দিন ‘সম্পূর্ণ জোরপূর্বক, কাজ্ষিত নয়’ এবং অন্য প্রান্তের নাম দিন ‘সম্পূর্ণ ইচ্ছাকৃত, কাজ্ষিক্ত'।

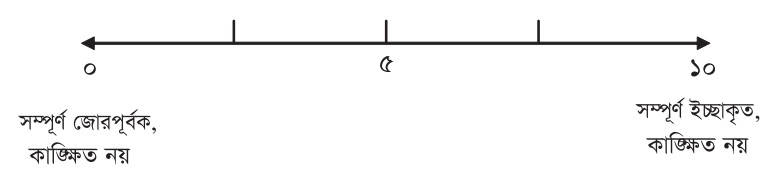

২ বিষয়বস্তুর ধারণা দিন এবং ব্যাখ্যা করুন যে, এখানে জোরপূর্বক ও সম্পূর্ণ অনাকাজ্ঞিত এবং সম্পূর্ণ ইচ্ছাকৃত ও কাজ্ষিত যৌন সম্পর্কের মধ্যকার ধারাবাহিক রেখা দেয়া আছে। জিজ্ঞেস করুন:

- আমরা জোরপূর্বক যৌনমিলনকে কী বলি? তারা উত্তর দেয়ার পর ধারাবাহিক রেখার ঐ প্রান্তে ‘ধর্ষণ’ শব্দটি যোগ করুন ।

• যদি একজন ব্যক্তি যৌনমিলনে রাজি হয়, সর্বদায় কি তার অর্থ যে সে সত্যিই যৌনমিলন করতে চায়? তারা উত্তর দেওয়ার পর, জিজ্ঞস করুন্ যখন যৌনমিলন ইচ্ছাকৃত কিন্তু সত্যিই কাজ্ষিত নয়, তা কি সূতার একপ্রান্তে হবে, নাকি দুইপ্রান্তের মাঝে যে কোন স্থানে।
৩ শিক্ষার্থীদেরকে চারটি দলে ভাগ করুন। প্রত্যেক দলকে হ্যান্ডআউট এবং একজোড়া পেপারক্লিপ দিন । ব্যাখ্যা করুন:

• তোমরা দলবদ্ধ হয়ে প্রত্যেকটি দৃশ্য/ঘটনা পড়। তারপর তা নিয়ে আলোচনা কর এবং সিদ্ধান্ত নাও যে কেসস্টাডিটি সুতার ০-১০ ধারাবাহিক রেখার কোন স্থানে পড়ে।

• তোমাদের নামসহ কেসটি চিহ্নিত কর।[রঙ্গিন মার্কার দিয়ে, যদি সম্ভব হয়]। তারপর তোমার মনে হয় সুতার যেখানে এটি উপযুক্ত, ঐ স্থানে পেপার ক্লিপ দিয়ে লাগিয়ে দাও।

8 দলগুলোর মধ্যে ঘুরে ঘুরে দেখুন এবং সাহায্য লাগবে কিনা জানতে চান । যদি তারা সবগুলো কেসস্টাডি শেষ করতে নাও পারে তাহলেও তাদেরকে মতামত আলোচনা করার জন্য বাড়তি সময় দিন।

৫ পনের মিনিট পর এক দল থেকে যেকোন একজনকে থ্রথম কেস স্টাডিটি পড়ার জন্য ডাকুন এবং ব্যাখ্যা করুন তার দল ০-১০ এর ধারাবাহিক রেখার কোথায় এটি রেখেছে এবং কেন । অন্য দলকে দুই থেকে তিন মিনিট সময় দিন তারা এটিকে ধারাবাহিক রেখার কোথায় রেখেছে তা চিহ্নিত করতে; তাদেরকে যেকোন ভিন্ন মতামত আলোচনার জন্য উৎসাহ দিন। প্রত্যেক কেস স্টাডির জন্য এই প্রক্রিয়ার পুনরাবৃত্তি করুন্ন । 
৬ শেষ দশ মিনিট সময় নিম্নলিখিত প্রশ্নগুলো আলোচনার জন্য রাখুন:

• কাউকে অনিচ্ছাকৃত যৌনমিলনে চাপ দেয়া হচ্ছে ঐ ব্যক্তির মানবাধিকার লজ্ঘন করা। তারা হাত তুলে বলো কে বিশ্বাস করে যে যুবক-যুবতীদের মধ্যে জোরপূর্বক যৌনমমিলন স্বাভাবিক ঘটনা ? কে মনে করে এটি সচরাচর ঘটে না?

• আমরা আলোচনা করেছিলাম যে, একজন ব্যক্তি যে যৌনমিলন করতে চায় না এবং যাকে তা করার জন্য বাধ্য করা হয়নি, সেও ইচ্ছাকৃতভাবে এই যৌনমিলনে জড়িত হতে পারে। কে বিশ্বাস করে এটি যুবক-যুবতীদের মধ্যে মোটামুটি স্বাভাবিক ভাবে ঘটে? কে মনে করে এটি সচরাচর ঘটে না? [মেয়েরা যেভাবে এটির উত্তর দেয় সেভাবেই ছেলেরা এই প্রশ্লের একই উত্তর দেয় কিনা তা লিখে রাখুন ।]

- জোরপূর্বক নয় এমনকি কাজ্ষিতও নয় এমন পরিস্থিতিতে কি ছেলে এবং মেয়েরা সমভাবে যৌনমিলন করতে চায়? [উত্তর জানার চেষ্টা করুন: সাধারণত ছেলে এবং মেয়েরা কি যৌনসম্পর্কের মধ্যে একই রকম ক্ষমতা পায়? প্রাপ্ত বয়স্ক নারী-পুরুষদের কী অবস্থা?] [লক্ষণীয়: গুরুত্বারোপ করুন যে, যদিও একটি ঘটনা ধারাবাহিক রেখার মারাত্নক ‘জোরপূর্বক'এর মধ্যে পড়ে না, তবুও তা অনাকাজ্ষিত হতে পারে।]

• একজন মানুষ কি সব সময় জানতে পারে যে তার সঙ্গী (মহিলা বা পুরুষ) সত্যিই যৌনমিলন করতে চায় কিনা ? নিশ্চিত হওয়ার কিছু উপায় কী? [উত্তর জানার চেষ্টা করুন: লোকটির কাছে জানতে চান! পূর্বেই একত্রে আলোচনা করা সর্বোত্ম । যদি তুমি জানতে চাও এবং তোমার সঙ্ কী চায় তা সম্পর্কে সে মহিলা বা পুরুষ নিশ্চিত না হয় তাহলে কী হবে?] 


\section{যৌন পছন্দ এবং যৌন নিপীড়ন সম্পর্কে কেসস্টাডি}

শিক্ষকের জন্য নির্দেশাবলি: নিম্মলিখিত কেসস্টাডি থেকে ছয়টি কেসস্টাডি বাছাই করুন (অথবা আপনি নিজের কেসস্টাডি লিখুন)। নিশ্চিত হোন যে, আপনার চূড়ান্ত বাছাইয়ের মধ্যে কমপক্ষে এমন একটি কেস আছে যেখানে একটি ছেলে যৌনমিলন করার জন্য বাধ্য হয়েছে। এগুনোকে আপনার শিক্ষার্থীদের জন্য উপযুক্ত এবং অর্থপূর্ণ করে পরিবর্তন করুন।

মোহাম্মদ ও অম্মা: মোহাম্মদ যৌনমিলন করতে চায়, কিন্তু তার স্ত্রী অম্মার আজকে রাতে এই ইচ্ছা হচ্ছে না । কিন্তু তাকে শেখানো হয়েছে যে, অসুস্থ বা মাসিক না হওয়া পর্যন্ত স্বামী যখনই চায় তখনই যৌনমিলন করা স্ত্রীর কর্তব্য, তাই সে মোহাম্মদের সাথে যৌনমিলন করে ।

নিনা ও কার্ল: নিনা, যার বয়স বাইশ বছর, কার্লের সাথে প্রায় ছয় মালের জন্য বাইরে যাচ্ছে। কার্ল অনেকবার তাকে বলেছে যে, সত্যিই সে তার সাথে যৌনমিলন করতে চায়, কিন্ত শ্রুমাত্র যদি নিনা তা চায়। নিনা অনিশ্চতাতার মধ্যে পড়ে, কিন্ত সে মনে করে তার প্রেমিক যা চায় তা তার করা উচিত। সে অন্য যুবতীদেরকে চেনে যারা তার প্রেমিকের সাথে যৌনমিলন করে এবং সে অবগত যে তা না করলে কার্ল তাকে ছেড়ে যেতে পারে, যদিও কার্ল কখনই তা করতে হুমকি দেয় না । পরবর্তীতে তারা অন্তরঙ্গ হল, যৌনমিলন করল ।

\section{জেকব ও গ্রেস: জেকব ও তার প্রেমিকা তার বাবা-মায়ের বাসায়} একা । জেকব নেশা করল ও তার কথা অস্পষ্ট হয়ে গেল, সে মেঝেতে শুয়ে পড়ল। গ্রেসও সামান্য নেশা করেছিল এবং জেকবের পাশে শয়ে পড়ল। নিজেকে দমন করতে না পেরে সে উদ্যোগ নিল ও জেকবের সাথে মুখ দিয়ে যৌনমিলন শুরু করল। জেকব দ্বিধান্বিত হয়ে পড়ল ও কী বলবে বুঝতে পারল না । পরের দিন সে কিছুই মনে করতে পারল ना।
হেনরি ও মারিয়া: হেনরি ও মারিয়া আবেগাপুত হয়ে চুম্বন করল । হেনরি যখন মারিয়ার কাপড় খুলতে শুরু করল, সে তাকে থামাতে চেষ্টা করল এবং 'না’ বলল। হেনরি মনে করল, মারিয়া আরও কিছু চাচ্ছে কিন্তু সে স্বীকার করতে ভয় পাচ্ছে। তাই সে চেষ্টা করতে থাকল। সে পাঁচ মিনিট ধরে 'না’ বলতে বলতত হেনরিকে ঠেলে ফেলে দেয়ার চেষ্টা করার পরে অবশেষে সে ধ্বস্তাধ্বস্তি করা ছেড়ে দিল এবং সেখানে শুয়ে পড়ল। হেনরি অগ্রসর হল এবং তার সাথে যৌনমিলন করল।

অজিত ও অনিলা: অজিত ও অনিলার মাত্র দুইবার সাক্ষাৎ হয়েছে। সব সময়ই অন্যরা উপস্থিত ছিল। অজিতকে একজন ভাল মানুষ মনে হয় বলে অনিলার বাবা-মা যখন বলল অজিত তাকে বিয়ে করতে চায় তখন সে রাজি হয়ে গেল । অনিলাকে শেখানো হয়েছে যে, যৌন সম্পর্কিত সবকিছুই লজ্জার। সে গেনেছে যে, প্রথমবার যৌনমিলনে ব্যথা লাণে ও তাকে রক্তাক্ত করবে। এটি তার মনে সত্যিই দাগ কাটল । সে অজিতকে সামান্যই জানত, এবং সে তার শরীর স্পর্শ করছে ভেবে লজ্জা পেত। সে যৌনমিলন করতে আগ্রহী ছিল না ও উত্তেজিত অনুভব করত না । কিন্তু সে জানত যে যখন তোমার বিয়ে হবে তোমাকে অবশ্যই বিয়ের রাতে য্যেনমিলন করতে হবে। সে অজিতকে তার সাথে যৌনমিলন করতে দিল । 


\section{যৌন পছন্দ এবং যৌন নিপীড়ন সম্পর্কে কেসস্টাডি}

সেলাহ ও জেমস: আগের বার সেলাহ্ তার স্বামী জেমসকে যৌনমিলনে অসম্মিতি জানিয়েছিল, জেমস তাকে শাসিয়েছিল ও খারাপভাবে মেরে চোখের নিচে কালি ফেলে দিয়েছিল। সে আজকে জেমসের সাথে যৌনমিলন করতে চায় না, কিন্তু জেমস তাকে আবার মারতে পারে, তাই সে অসম্মত হল না।

লিন ও জং: লিনের প্রেমিক জং সত্যিই যৌনমিলন করার জন্য চাপ দিচ্ছে। লিন জানে যে সে সত্যিই তা করতে চায় না, কিন্তু সে জং-এর সাথে এই নির্জন স্থানে আসলো। সে ভাবল এটি অনিবার্য এবং জং যা করতে চায় তা কিছু না বলেই করতে দিল ।

টিও ও হেলেনা: টিও ও হেলেনা বিশ্ববিদ্যালয়ের সহপাঠী। তারা কয়েক মাস ধরে মেলামেশা করছে এবং একে অপরের প্রতি অনেক আকর্ষণবোধ করছে। তাদের ভালবাসা গভীর নয়, কিন্তু তারা ঠিক করল যে তারা যৌনমিলন করতে চায়। কনডম ব্যবহার করতে সম্মত হওয়ার পর তারা যৌনমিলন করল।

গাইনা ও আহম্মেদ: গাইনা দরিদ্র পরিবারের মেয়ে। সে আহম্মেদের একটি দোকানে কাজ করে এবং তার বেতনই তার পরিবারের প্রধান আয়। একদিন আহম্মেদ তাকে স্পর্শ করতে শুরু করল এবং দোকান বন্ধ হওয়ার পর পেছনের দিকে গুদাম ঘরে টেনে নিয়ে গেল ও তার কাপড় উঁচু করল। গাইনা দ্বিধান্বিত ছিল এবং তার চাকরি হারানোর ভয় পাচ্ছিল । সে প্রতিরোধ করতে শুরু করল, কিন্তু যখন আহম্মেদ বলল, “কী? তুমি এখানে কাজ করতে চাওনা?”, সে আত্নসমর্পন করল।
জুলিয়া ও অস্কার: অস্কার জুলিয়ার সাথে যৌনমিলন করতে চায় কিনা সে নিশ্চিত নয়, কিন্তু জুলিয়া তা করতে বলল, এবং অস্কার ভীত ছিল যে, সে না বললে তার বন্ধুরা বুঝে ফেলবে ও তাকে নিয়ে তামাশা করবে। সে রাজি হল।

পিটার ও লরা: পিটার সব সময় লরাকে উপহার ও তার অন্যান্য প্রয়োজনীয় জিনিস কিনে দেয়। আজকে রাতে তারা খেতে গেল ও পিটার টাকা দিল । যদিও লরা যৌনমিলন করতে প্রস্তুত ছিল না, কিন্তু তার মনে হল যে, সে এগুলোর জন্য পিটারের কাছে ঋণী। লরা তাকে প্রত্যাখ্যান করল না ।

ভিরা এবং উইলিয়ামঃ ভিরা ও উইলিয়াম আগে একবার য্যেনমিলন করেছিল । আজকে রাতে উইলিয়াম ভিরাকে বলল যে, আজ সারাদিন সে ভিরাকে চেয়েছে। অন্যকিছু নয়, ভিরা ফ্যুমাত্র তার সাথে থাকবে এবং কথা বলবে, কিন্তু ভিরা ভাবল যেহেহু তারা একবার যৌনমিলন করেছে আর এটি করবে না । যখন উইলিয়াম তার পোষাক খুলতে শুরু করল, ভিরা তাকে কিছুই বলল না এবং তারা আবার যৌনমিলন করল।

ইমা ও রবার্ট: এক সন্ধ্যায় ইমা অনেক নেশা করল । রবার্ট তাকে বিছানায় অচেতন হয়ে শুয়ে থাকতে দেখল। রবার্ট তার পোষাক খুলে ফেলল ও তার সাথে যৌনমিলন করল। ইমা জাগল না। 


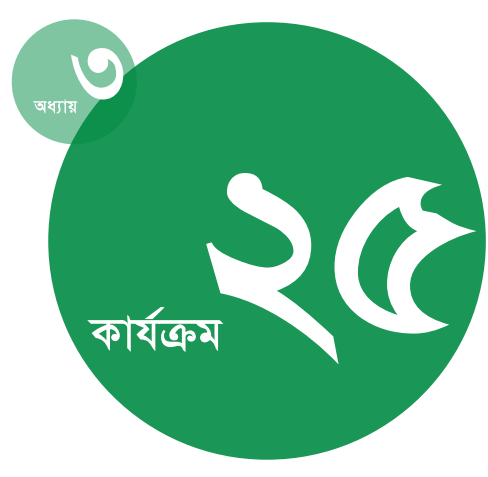

সময়কাল :

ধাপ ১-৬: ৪৫ মিনিট ধাপ ৭-৮: $8 ৫$ মিনিট

\section{উপকরণ:}

বোর্ড ও চক; বাছাইকৃত কেসস্টাডি সমূহ; শিক্ষকের তালিকার জন্য তথ্য; উত্তরপত্র।

\section{প्रস্তুতি:}

আপনার সমাজের স্বাভাবিক অবস্থা কেসস্টাডি বাছাই করুন এবং

সেখানকার নাম ও অন্যান্য খুঁটিনাটি বিষয় পরিবর্তন করে খাপ খাইয়ে নিন । অথবা, অন্যকোন কেসস্টাডি তৈরি করুন। এমন কিছু কেসস্টাডি ব্যবহার করুন যেখানকার চরিত্রগুলো জ্ঞাত সম্মতি দিতে সক্ষম এবং কিছ কেসস্টাডিতে সক্ষম না । यদি প্রয়োজন হয়, শিক্ষকের নির্দেশিকা থেকে তথ্যগুনো পুনরায় পড়ন । যতক্ষণ সময় থাকে নির্দেশনাগুলো বোর্ডে লিখুন। আপনার দেশে যৌন ক্রিয়াকলাপে সম্মতির বয়স সংক্রান্ত আইন খুঁজে দেখুন। ‘একমত নই’।

\section{সম্মতির বিষয়}

অধিবেশন সম্পর্কিত ধারণা: শিক্ষার্থীরা যৌন সম্মতি সম্পর্কে শিখবে। তারা এই ধারণাটি কেসস্টাডি, নাটিকা এবং আলোচনার মধ্যে অনুসন্ধান করবে।

উদ্দেশ্য: শিক্ষার্থীদেরকে যেকোন যৌন পরিস্থিতিতে সম্মতির গুরুত্বকে বুঝতে এবং মূল্যায়ন করতে সাহায্য করা; বিমূর্ত চিন্তার দক্ষতা শক্তিশালী করা।

\section{নির্দেশাবলি}

১ নিম্নলিখিত ব্যাখ্যা এবং প্রশ্নের মাধ্যনে অধিবেশনটির ধারণা দিন:

• আমাদের প্রত্যেকেরই দায়িত্ব আছে, যেমন- বাড়িতে, বিদ্যালয়ে, এবং আমাদের কর্মক্ষেত্রে। যদিও মাঝে মাঝে আমাদেরকে কিছু করতে বলা হয় (সঙ্গী বা এমনকি বড়দের দ্বারা) যেগুলো আমাদের দায়িত্বের অংশ নয়, সেগুলো বিভ্রান্তিকর বা বিরক্তিকর হতে পারে।

বিকল্প: বিষয়টির বিস্তারিত ধারণা দেয়ার জন্য নিম্মলিখিতভাবে আলোচনাটি সমপ্রসারিত করুন:

কোন কিছুর প্রতি সম্মতির অর্থ কী? [জানার চেষ্টা করুন: রাজি হওয়া, কোন ইচ্ছাকে নির্দেশ বা প্রকাশ করা ।]

আমরা বিভিন্ন কারণে কোনকিছু করতে সম্মতি দেই: মাঝে মাঝে আমরা কিছু করতে সম্মতি দেই, কারণ আমরা তা করতে চাই, যেমন- যদি একজন বন্ধু তোমাকে ফুটবল খেলতে আমন্ত্রণ জানায় এবং তুমি ফুটবল খেলতে চাও। অথবা আমরা কোন কিছু করতে সম্মতি দিতে পারি, যা আমরা সত্যিই করতে চাই না কিন্তু আমরা রাজি হই, কারণ তা আমাদের জন্য অন্যকোন কারণে গুরুত্বপূর্ণ, যেমন- যখন তোমাদের শিক্ষক তোমাদেরকে বাড়ির কাজ দেন, যা তোমরা করতে চাওনা, কিন্তু তোমরা তা কর, কারণ তোমরা বিদ্যালয়ে সফল হতে চাও
শধুমাত্র আমাদের জন্য করা কঠিন এ কারণে আমরা কিছু কাজ করতে চাই না যদিও মাঝে মাঝে আমরা তা করি, বা আমরা রূঢ় হতে চাই না। এই পরিস্থিতি একটা ঘটনার সাথে সংশ্লিষ্ট হতে পারে যা খুব গুরুতত্বপূর্ণ নয়, যেমন- তুমি যা পছন্দ কর না তা খাওয়া কারণ তা তোমার জন্য কেউ রান্না করেছে। অন্য সময়, এটি গুরুতর পরিণতিসহ আরো গুরুতর ঘটনার সাথে সংশ্লিষ্ট হতে পারে। গুরুতর বিষয়গুলো কী কী যেগুলো একজন যুবক-যুবতী করতে বা না করতে সম্মতি দিতেও পারে নাও পারে ? /यদি শিক্ষার্থীরা যৌন বিষয়টি উল্লেখ না করে, তাহলে তাদেরকে তা তালিকায় যোগ করতে বলুন।]

• যদি তুমি কখনও এমন কিছু করতে রাজী হও যা তোমার দায়িত্ব ছিল না এবং তুমি সত্যিই করতে চাওনি। তাহনে হাত তোল। কে একটি উদাহরণ আলোচনা করতে পারবে ?

- কিছু কারণ কী কী যার জন্য একজন মানুষ যা সত্যিই করতে চায়নি তা করতে রাজি হয়? উত্তরগুনো বোর্ডে লিখুন।

• আজকে আমরা খুঁজতে শুরু করব যৌন সংক্রান্ত পরিস্থিতিতে সত্যিকারের স্বাধীন ও জ্ঞাত সম্মতি বলতে কী বোঝায় ? 
২ জিজ্ঞেস করুন (এবং উত্তরগুনো বোর্ডে লিখুন):

- 'সम্মতি' শব্দটির অর্থ की?

• 'স্বাধীন ও জ্ঞাত সম্মতি' শব্দটির অর্থ कী?

৩ শিক্ষার্থীদেরকে যৌন পরিস্থিতিতে স্বাধীন ও জ্ঞাত সম্মতি প্রদানের জন্য নির্দেশনাগুলি সকলে ফুনতে পারে এভাবে পড়তে দিন।

8 প্রত্যেক দলই যেন পৃথক কেসস্টাডি পায় এজন্য ছোট ছোট দল তৈরি করুন। প্রত্যেক দলকে তাদের কেসস্টাডি দিন এবং ব্যাখ্যা করুন:

• তোমাদের দশ মিনিট সময় আছে। তোমাদের কেসস্টাডি আলোচনা কর, একটি ছোট (দুই থেকে তিন মিনিটের) নাটিকা তৈরি কর, এবং এই পরিস্থিতিতে সম্মতির জন্য নির্দেশাবলির কোনটি এখানে পূরণ হয়েছে বা হয়নি তা দেখ।

• একজন কেসস্টাডিটি শ্রেণিকক্ষের সবার সামনে পড়বে, অন্য দুইজন এটি অভিনয় করতে পারে এবং সম্মতির জন্য নির্দেশাবলির কোনটি এখানে পূরণ হয়েছে অথবা হয়নি তা একজন বা দুইজন ব্যাখ্যা করবে।/কাজটি বোঝার জন্য এবং এরপর প্রস্তুত হওয়ার জন্য দশ-বিশ মিনিট সময় দিন। প্রয়োজন অনুযায়ী সাহায্য করার জন্য দলগুলোর মধ্যে ঘুরে দেখুন।]

৫ দলগুলোকে (ক) তাদের কেসস্টাডি; (খ) তাদের নাটিকা; এবং (গ) এই কেসস্টাডিটিতে লোকটি তার স্বাধীন এবং জ্ঞাত সম্মতি দিতে পেরেছে কিনা এই বিষয়ে তাদের সম্মিলিত মতামত উপস্থাপন করতে বলুন।

৬ সম্মতির নির্দেশাবলি পূরণ হয়েছে কিনা এ ব্যাপারে দলের মতামতের সাথে কারা একমত অথবা একমত নয়। সম্মতি সঠিক উত্তরে পৌঁছানোর পূর্ব পর্যন্ত আলোচনা করুন

৭ প্রত্যেক দল/কেসস্টাডির জন্য ধাপ ৫ ও ধাপ ৬ এর পুনরাবৃত্তি করুন । প্রত্যেক কেসের জন্য দশ-বিশ মিনিট সময় দিন ।
৮. একটি আলোচনার মাধ্যমে শেষ করুন:

আমরা জানি এমন অনেক পরিস্থিতি আছে যখন একজন মানুষের না বলার নিয়ন্ত্রণ বা ক্ষমতা থাকে না। এটি মানবাধিকার লজ্থন ।

• অনেক ক্ষেত্রে একজন মানুষের যে নিয়ন্ত্রণ বা ক্ষমতার মাত্রা আছে তা অস্পষ্ট অথবা দরকষাকষির জন্য উনুক্ত হতে পারে। কিছু কিছু ক্ষেত্রে, তোমার নিজের মন থেকে বুঝার জন্য এটি একটি স্বতন্ত্র পার্থক্য তৈরি করতে পারে যে তোমার যৌনমিলন করতে সম্মত হওয়া বা না হওয়ার অধিকার আছে। এটি ক্ষমতা হিসেবে জ্ঞানের একটি উদাহরণ।

• একজন মানুষ কি তার সঙ্গীর সাথে কথা না বলে নিশ্চিতভাবে জানতে পারে যে তার সম্মতি আছে কিনা ? কেন বা কেন নয় ? একজন মানুষ যে যৌনমিলন করতে 'সম্মত', সে যে তা স্বাধীন ও জ্ঞাত হয়ে করছে তা নিশ্চিত হওয়ার জন্য কেউ কেউ কী করতে পারে?

বাড়ির কাজ: নিজেকে এমন কারো অবস্থানে রাখ যে চাপের সম্মুখীন হয়ে অনাকাঙ্ষিত যৌনমিলন করেছে। ব্যক্তিটি তোমার পড়া একটি কেসস্টাডি থেকে নেয়া হতে পারে বা আজকে শুনেছ বা এটি তোমার পরিচিত এমন কেউ হতে পারে, যে এমন পরিস্থিতিতে ছিল যেখানে যৌনমিলনে যথার্থ সম্মতি ছিল না (তাদের নাম উল্লেখ করো না)। ঐ ব্যক্তির একটি বক্তব্য তার কথাতে লেখ। যৌনতায় কী ঘটেছিল তা সংক্ষিপ্তভাবে না বলে তাদের অনুভূতিগুলোকে গুরুত্ব দাও। সম্পর্কটির মধ্যে কোন ক্ষমতার পার্থক্য আছে কিনা ভাব, যার জন্য ব্যক্তিটির ওপর চাপ ছিল। তোমার বক্তব্যটি লেখার উদ্লেশ্য হওয়া উচিত লোকটিকে তার নিয়ন্ত্রণ এবং সমান ক্ষমতার ধারণাকে ‘পুনরদদ্ধার’ করতে সাহায্য করা । যদি তোমার ভাল লাগে, তুমি বক্তব্যটি কবিতা রূপে লিখতে পার। 


\section{যৌনতা অন্তর্ভুক্ত এমন পরিস্থিতিতে অর্থপূর্ণ সম্মতি দেয়ার জন্য নির্দেশাবলি}

তুমি একটি নির্দিষ্ট যৌন কর্মকাণ্ত জড়িত হতে চাও কিনা তার সিদ্ধান্ত নেয়ার অধিকার তোমার নিজেরই আছে এটা বিশ্বাস কর। যোগাযোগ করা এবং তোমার সিদ্ধান্ত কাজে লাগাতে সক্ষম হওয়ার জন্য ক্ষমতার যথেষ্ট বুদ্ধি ও নিজের জীবনের ওপর নিয়ন্ত্রণ তোমার আছে।

একটা কাজ কী পরিণতি এবং ফলাফল বয়ে আনতে পারে তা বোঝার মতো এবং তোমার সঙ্গীর সাথে এ বিষয়ে কথা বলার মতো পরিপক্কতা তোমার আছে।

এমন সম্পর্ক বা পরিস্থিতিতে সমপপৃক্ত হও যেখানে তোমার সঙ্গী তোমার সিদ্ধান্ত বুঝবে এবং সম্মান করবে।

কাজটির সাথে কী অন্তর্ভুক্ত এবং এ সম্বন্ধে তোমার অনুভূতি কী; ঝুঁকিগুলো কী কী; এবং কীভাবে নিজেকে বা তোমার সঙ্গীকে অনাকাজ্ষিত গর্ভধারণ থেকে রক্ষা করবে তা জান ।

সুস্থ মনে থাক, সিদ্ধান্ত গ্রহনের সময় মদ বা নেশার দ্বারা বাধাগ্রস্ত হবে না।

তোমার সঙ্গীর বর্তমান যৌনস্বাস্থ্য সম্পর্কে সঠিক তথ্য নাও (যেকোন পূর্ববর্তী যৌন সম্পর্কিত ও মাদক গ্রহণের ঘটনাসহ যা তোমাকে ঝুঁকির মধ্যে ফেলতে পারে)

যেখানে বৈষয়িক বা আর্থিক কারণে যৌনমিলনের জন্য তোমার ওপর চাপ থাকার অভিজ্ঞতার সম্ভবনা আছে এমন ঘটনা যতদূর সম্ভব পরিহার কর। 


\section{কেস স্টাডিগুলি: এই ব্যক্তি কি অর্থপূর্ণ সম্মতি দিতে পারে ?}

ক এ এনার বয়স উনিশ বছর এবং তার প্রেমিক অল-এর বয়স বাইশ বছর। তারা ছয় মালের জন্য বাইরে গিয়েছে এবং প্রায় একমাস ধরে যৌনমিলন করছে। এনা সাধারণত যৌনমিলন উপভোগ করে, কিন্তু মাঝে মাঝে তার এটি ভাল লাগে না । ঐ সময়গুলোয় প্রায়ই অল এ ধরনের কথা বলে “যদি তুমি সত্যিই আমাকে ভালবাসতে, তাহলে তুমি এটি করতে চাইতে” অথবা “यদি তুমি আমার সাথে যৌনমিলন না করো, আমার হয়তবা এমন কাউকে খুঁজতে হবে যে তা করবে ।” এনা তাকে হারাতে চায় না, তাই প্রায়ই সে যৌনমিলন করতে রাজি হয় । এনা কি স্বাধীনভাবে মতামত দিতে পারে?

খ বেনি জানে যে লে এইচআইভিতে আক্রান্ত কিন্তু লে কাউকে বলেনি । সে বিয়ার সাথে প্রেম করছে, এবং সমপ্রতি তারা যৌনমিলন করা সম্পর্কে কথা বলছে। বেনি বিয়াকে তার এইচআইভি আক্রান্তের কথা না বলে কনডম ব্যবহার করার পরিকল্পনা করছে। বিয়া কি স্বাধীন এবং জ্ঞাত সম্মতি দিতে পারে ?

গ কার্লো ও ক্লিয়ার বয়স বিশ বছর। তারা এক বছর ধরে প্রেম করছে এবং তাদের পূর্ব সম্পর্ক নিয়ে সততার সাথে কথা বলেছে। তারা একে অপরের সাথে যৌনমিলন করতে চায়। তারা এটি নিয়ে আলোচনা করল এবং তথ্য পাওয়ার জন্য স্বাস্থ্য কেন্দ্রে যেতে একমত হল এবং তারপর তারা একসাথে সিদ্ধান্ত নেবে কীভাবে নিরাপদ থাকা যায় এবং যেকোন অনাকাজ্ষিত ঘটনা পরিহার করা যায়। ক্লিয়া কি স্বাধীন এবং জ্ঞাত সম্মতি দিতে পারে ?

ঘ ডম ও ডিনাহ্র বয়স আঠারো বছর এবং তারা যৌনমিলন করার সিদ্ধান্ত নিয়েছে। ডিনাহ্ বলে, লে গর্ভবতী হয়ে যাওয়ার ভয় পায়, কিন্তু ডম তাকে নিশ্চিত করে যে, প্রথমবার যৌনমিলন করলে তুমি গর্ভবতী হরে না । ডিনাহ্, কি স্বাধীন এবং জ্ঞাত সম্মতি দিতে পারে ?

ঙ ইভ’র বয়স চৌদ্দ বছর এবং সে মাধ্যমিক বিদ্যালয়ে পড়ে। ইডোর বয়স আঠারো বছর এবং ইভ’র বাবার সাথে কাজ করে। ইডো যখন ইভের বাসার এসেছিল তখন তার সাথে পরিচিত হয়েছিল। ইডো ও ইভ তার বাড়ি থেকে বের হয়ে অন্য কোথাও দেখা করতে শুরু করল। মাঝে মাঝে ইভের প্রয়োজন হনে ইডো তাকে টাকা ও উপহার দেয়। সম্রত্রতি ইডো তাকে বলতে কুরু করেছে সে তাকে কত ভালভালে এবং আসলে তার সাথে যৌনমিলন করতে চায় । ইভ কি স্বাধীন এবং জ্ঞাত সম্মতি দিতে পারে ?

ত ফিনের বয়স আঠারো বছর এবং সে তার সতের বছর বয়সের প্রেমিকা ফাতিমার সাথে থৌনমিলন করতে চায়। তারা এ ব্যাপারটি নিয়ে আলোচনা করেছে যে তাদের আগে কখনও যৌনমিলন হয়নি । ফিন একটি বই পড়ার সিদ্ধান্ত নিল যে, সে বড় হয়ে ওঠা, যৌনতা, পরিবার পরিকল্পনা এবং

এসটিআই সম্পর্কে তার মা, যিনি একজন ডাক্তার, তার লেখা । সে তার প্রেমিকার কাছে জানতে চাইল সে কী মনে করে এবং সে তাকে বইটি ধার দিতে চাইল। ফাতিমা বইটি পড়ল, এবং তারা আবার এটি নিয়ে কথা বলল । ফাতিমা বলল, সে বরং অপেক্ষা করতে চায়, তাতে ফিন রাজি হল । ফাতিমা কি স্বাধীন ও জ্ঞান সম্মতি দিতে পারে ? 


\section{কেস স্টাডিগুলি: এই ব্যক্তি কি অর্থপূর্ণ সম্মতি দিতে পারে?}

ছ বব্বিশ বছর বয়সের গিয়া একটি বাড়িতে কিছু ছাত্রদের একটি পার্টিতে রয়েছে এবং সে অনেক মদপান করেছে। তার সঙ্গী যার সাথে লে আগে যৌনমিলন করেছে, সে গিয়াকে টানতে টানতে একটি শোয়ার ঘরে নিয়ে গেল এবং তার কাপড় খুলতত শুরু করল । সে মলিন এবং অজ্ঞান হয়ে গেছে। গিয়া কি স্বাধীন ও জ্ঞাত সম্মতি দিতে পারে ?

জ ্যালি তার মায়ের কাছ থেকে শিখেছিল যে যদি একজন মহিলা তার স্বামীকে হারাতে না চায়, তাহলে তার স্বামী যা চায় তা করা খুব গুরুত্বপূর্ণ। তার হুগের সাথে বিয়ের জন্য বাগদান হয়েছে, এবং হুগ বলে যে, তারা এখন বাগদত্ত, এখন তারা যৌনমিলন করতে পারে। হ্যালি দৃঢ়ভাবে বিশ্বাস করে যে তার অপেক্ষা করা উচিত এবং বিয়ে হওয়া পর্যন্ত সে কুমারী থাকতে চায়, কিন্তু সে চিন্তা করে, “সে আমার স্বামী হবে এবং আমার মা বলেছে তাকে আমার মেনে চলা উচিত ।" হ্যালি কি স্বাধীন এবং জ্ঞাত সম্মতি দিতে পারে ?

ঝ ইসাকের অনেক সঙ্গী ছিল কিন্তু ইভানের কথা আলাদা, সে তার নতুন প্রেমিক এবং ইসাক বিশ্বাস করে যে ইভানের এখনও তার মতো যৌনমিলনের অভিজ্ঞতা হয়নি । যখন তারা যৌনমিলন করে তখন সংক্রমণের কোন ঝুঁকি নেই বলে মনে করার কারণে ইভান কনডম ব্যবহার করতে রাজি হয় না । ইভান কি স্বাধীন এবং জ্ঞাত সম্মতি দিতে পারে?

এ জিনের বয়স বিশ বছর এবং জোলেফের বয়স উনিশ বছর। প্রায় ছয় মাস আগে তার থ্রেমিক জিন জোসেফকে প্রশ্ন করল সে তার সাথে যৌনমিলন করতে চায় কিনা । তারা একে অপরের সাথে সৎ এবং খোলামেলা ছিল । তাই জিন তাকে বলল সে এর ফলাফলের কথা চিন্তা করে ভীত এবং এখনও প্রস্তুত নয়। সে বলল সে এখন শ্রু চুম্বন এবং একে অপরকে স্পর্শ করতে চায়। জিন সত্যিই য্যেনমিলন করার চেষ্টা করেছিল, কিন্তু সে জোলেফের সাথে একমত হল । এখন জোলেফ প্রস্তুত বলে মনে করে। সে জিনের কাছে জানতে চাইল তারা ঝুঁকি এড়ানোর জন্য কোথায় আরও তথ্য পাবে? জোসেফ কি স্বাধীন এবং জ্ঞাত সম্মতি দিতে পারে ? 


\section{“কেস স্টাডিগুলি: এই ব্যক্তি কি অর্থপূর্ণ সম্মতি দিতে পারে ?”-এর উত্তর}

লক্ষণীয়: আপনি অবশ্যই জানেন, যদি আপনার দেশের আইনে য্যেন কর্মকাত্তে সম্মতি প্রদানে কোন ন্যূনতম বয়স ঠিক করা থাকে, তবে এখানকার প্রত্যেক ঘটনার ব্যক্তিরা যৌনমিলনে সম্মতি দেয়ার জন্য যথেষ্ট বড় কিনা তা নির্রপণ করতে পারবেন। আপনার ঘটনাগুলোতে বা ঐ তথ্যের ভিত্তিত দেয়া উত্তরুগোঢে পরিবর্তনের প্রট্যোজন হতে পারে।

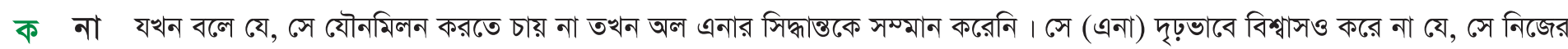
সিদ্ধান্ত নিতে পারে এবং তার পছন্দকে কার্যকরী করার মতো সম্পর্কের মধ্যে যথেষ্ট পরিমাণে ক্ষমতার সঢেতনতা নেই।

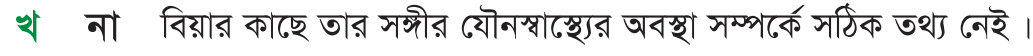

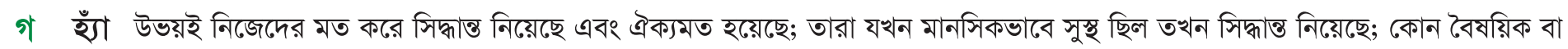
আর্থিক প্রবৃত্তি তাদেরকে প্রভাবিত করেনি; তারা তাদের সিদ্ধান্ত নিয়ে আলোচনা করতে সক্ষম; তারা জানে এর সাথে কী বিষয় সম্পৃক্ত আছে এবং তাদের প্রতিরোধ ব্যবস্থার প্রটয়াজন; তারা তাদদর অতীত আচরণ নিয়ে সততার সাথে কথা বলেছে; এবং আইনের কাছে ১ৌনমমিলনে সম্মতি দেয়ার মঢো यথেষ্ট বড় হয়েছে।

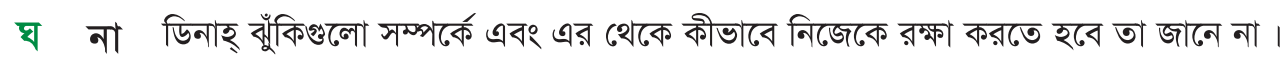

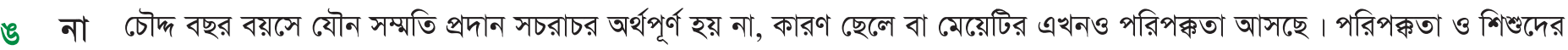

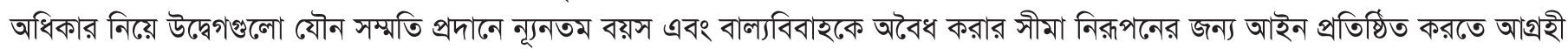
ভূমিকা পালন করেছে।

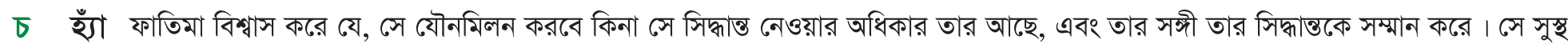
মনে সিদ্ধান্ত গ্রহণ করছে; কোন বৈবয়িক বা আর্থিক প্রবৃত্তির চাপ নেই; তার সিদ্ধান্ত নিয়ে আলোচনা এবং কাজে পরিণত করতেত সক্ষম; এবং সে জানে এর সাথে কী সম্পৃক্ত, ঝूँকিগুলো কী, এবং নিজেকে কীভাবে সুরক্ষা করতে হয়। তার কাছে তার সঙ্গীর পূর্ব্বর্তী ভ্যেনাচরণ সম্পর্ক সঠিক তথ্য আছে। বেশির ভাগ দেশেই, যদি তার ভাল লাগে তাহলে যৌন মিলনে সম্মতি দেয়ার জন্য সে যথেষ্ট বয়স্ক বরে বিবেচিত হবে, কিন্ত্ত আপনি যেখানে বাস করেন সেখানকার আইন পরীক্ষা করে দেখুন।

ছ না গিয়া মাতাল এবং প্রায় অঞ্ঞান ছিল। সে নিজের সম্পর্ক্ক সিদ্ধান্ত নিতে অক্ষম ছিল, এবং তার সঙ্গ তার সাথে আলোচনা করেনি ।

জ না হ্যালি জানে যে সে কী চায়, কিন্ত তার নিজের সিদ্ধান্ত নেওয়ার অধিকার আছে বলে তার বিশ্বাস হয় না, অথবা ক্ষমতা সম্পর্কে যথেষ্ট সটেতনতা এবং তার নিজের সিদ্ধান্তকে থ্রয়োগ করার মত নিয়ন্ত্রণ নিজের ওপর নেই।

ঝ ना ইजाনের কাছে তার সঙ্গীর পূর্ববর্তী ভ্যীনাচরণ সম্পর্ক সঠিক তথ্য নেই।

এ হ্যা জোলেফ বিশ্বাস করে যে তার নিজের সিদ্ধান্ত নেয়ার অধিকার আছে; সে আলোচনা এবং তার সিদ্ধান্ত থ্রয়োগ করতে সক্ষম, এবং তার সঙ্গী এটিকে সম্মান করে; সে মানসিক ভাবে সুস্থ; তার নিজের মতামত অগ্রাহ্য করার মত কোন আর্থিক বা ববৈয়িক প্রবৃত্তি নেই; সে সঢেতন এবং বুঁকি ও

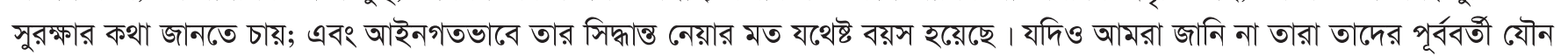
আচরণ এবং মাদক গ্রহণের সম্পর্কে আলোচনা করেছে কিনা, আমরা জানি যে তারা খোলামেলা এবং একে অপররর প্রতি বিশ্বস্ত। 


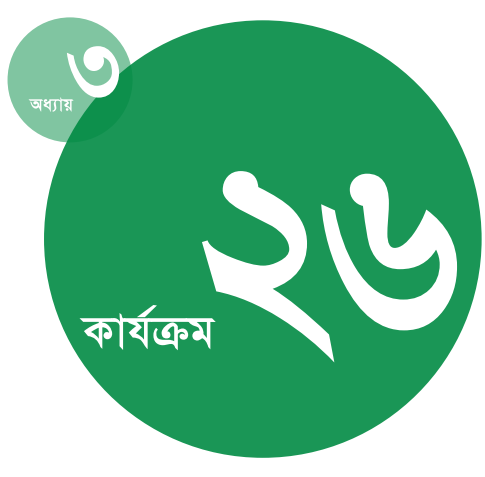

সময়কাল :

8৫ মিनिট

\section{উপকরণ:}

বোর্ড ও চক; "যৌনতা বিষয়ক নীতির

শিরোনামগুলো ।"

\section{প্রস্তুতি:}

প্রয়োজন হলে যেকোন শিরোনাম মুছে ফেলুন। শিরোনামগুলো বোর্ডে

লिখুন ।

\section{আলোচিত নীতি এবং ব্যক্তিগত মতামত}

অধিবেশন সম্পর্কিত ধারণা: শিক্ষার্থীরা এমন একটি সংবাদ শিরোনাম বাছাই করবে যা যৌনতার পরিবর্তনশীল রীতি-নীতিকে ব্যক্ত করে, এবং একটি ব্যক্তিগত মতামত লিখবে।

উদ্দেশ্য: শিক্ষার্থীদেরকে বুঝতে সাহায্য করা যে, যৌন রীতি-নীতি সময়ের সাথে সাথে পরিবর্তন হয়; একটি নির্দিষ্ট বিষয় সম্পর্কে তাদের নিজস্ব মূল্যবোধ স্পষ্ট করা; সমালোচনামূলক চিন্তা এবং লেখার দক্ষতা শক্তিশালী করা।

\section{नির্দেশাবলি}

১ নিয্নলিখিত বিষয়াবলি ব্যাখ্যার মাধ্যমে অধিবেশনটির ধারণা দিন:

- যৌনতা সম্পর্কে দৃষ্টিভঙ্গি এক সমাজ থেকে অন্য সমাজে বিভিন্ন রকম হয়। এগুলো য্যেকোন একটি সমাজের মধ্যেও পরিবর্তিত হচ্ছে। এই দৃষ্টিভঙ্গিগুনো যেভাবে পরিবর্তিত হচ্ছে তার কিছু মাধ্যম আজকে আমরা শনাক্ত করব এবং চিন্তা করব।

• বোর্ডের লেখা শিরোনামের প্রথম তালিকাটি পড়; এগুলোর বেশিরভাগ সারাবিশ্বের বাস্তব ঘটনা থেকে নেয়া হয়েছে। একটি বাছাই কর এবং এক পৃষ্ঠার ব্যক্তিগত মতামত লেখ। তোমরা এই বিষয়টি নিয়ে তোমাদের ব্যক্তিগত চিন্তা বা অনুভূতি লিখতে পার, কিন্তু এটি নিয়ে গভীরভাবে চিন্তা করতে চেষ্টা কর। দশ বা পনের বছর পরে বিষয়টির অবস্থা কেমন হবে তা বলার মাধ্যমে লেখা শেষ কর। তালিকাটি পড়তে দশ মিনিট এবং লেখার জন্য পনের মিনিট সময় নাও। তারপর তোমাদের মধ্যে কেউ কেউ তোমাদের লিখিত মতামত সবাইকে পড়ে শোনাতে পার।

২ প্রত্যেক শিরোনাম নিয়ে, তারা যা লিখেছে তা পড়ার জন্য একজন আগ্রহী শিক্ষার্থীকে ডাকুন। প্রত্যেকবার পড়ার পর ঐ শিক্ষার্থীকে ধন্যবাদ দিন। যদি প্রয়োজন হয়, বিষয়টি কী ছিল তা স্পষ্ট করুন এবং দৃষ্টিকটু কোন ভুল তথ্য থাকলে তা সঠিক করে দিন। নিম্নলিখিত যেকোন একটি প্রশ্নের মাধ্যমে আলোচনায় উৎসাহিত করুন:

• এই আলোচনা তোমাদের মধ্যে কী অনুভূতির জন্ম দিয়েছে?" • তোমাদের কী বলার আছে?
• এই বিষয়টি কী আমাদের দেশের সাথে সামঞ্জস্যপূর?

• অন্য কোন মতামত?

[প্রত্যেক শিরোনাম নিয়ে দুই থেকে তিন মিনিট পরিকল্পনা করুন। কিছু শিরোনাম বাছাই নাও হতে পারে? এক্ষেত্রে যে শিরোনামগুনো বাছাই হয়েছে সেগুনোকে আরো সময় দেবে।]

৩ রীতি-নীতি সবসময়ই পরিবর্তন হয় এটি শিক্ষার্থীদেরকে মনে করিয়ে অধিবেশন শেষ করুন । জিজ্ঞ্ঞস করুন:

• পরিবর্তন কি সবসময় সহজতর রীতি-নীতির দিকে হয়, নাকি এগুলো আরো কঠোর বা রক্ষণশীল রীতি-নীতির দিকে যেতে পারে?

- যৌনতা বা অন্য যেকোন বিষয়ে সামাজিক রীতি-নীতি কি নিজেরাই জাদুর মত পরিবর্তন হয়ে যায়? কী বা কারা য্যেন রীতিনীতি পরিবর্তনে অগ্রণী ভূমিকা পালন করে? [উত্তর জানার চেষ্টা করুন: বৃহৎ সামাজিক ঘটনা (যেমন উচ্চশিক্ষা এবং বিশ্বব্যাপী গণমাধ্যম) এবং ব্যক্তি বিশেষের ভূমিকা (জননেতা এবং সমাজের সদস্য উভয়েরই)।]

- মানুষ অনুকরণীয় আদর্শ হয়ে অন্যদেরকে শিক্ষিত করে, এবং নীতি পরিবর্তনের জন্য উপদেশ দিয়ে এই পরিবর্তন আনতে পারে। 


\section{যৌনতা বিষয়ক নীতির ‘শিরোনামগুলি’}

যে সকল নারীরা যৌন হয়রানির জন্য মামলা করে তারা বিজয়ী হয়; আদালতে প্রথম এমন একটি বিজয়।

কিশোর-কিশোরীদের জন্য ক্ষিনিক খোলা হলো ; দেশে এই থ্রথম ।

স্থানীয় মানুষ যৌন নির্যাতনের বিরুদ্ধে একটি প্যারেড প্রদর্শন করে; এটা প্রচার করে যে প্রকৃত মানুষ ধর্ষণ করে না।

সমকামী বিয়ের আইনগত অনুমতি কার্যকরী হচ্ছে; প্রথম জুটি সকাল b- টায় বিয়ে করে।

জোর করে বিয়ে দেওয়ার সময় চৌদ্দ বছরের মেয়ে পালিয়েছে। মেয়েদেরকে সব সময় সারা শরীর ঢেকে রাখতে হবে এমন পোশাক নীতি সম্পর্কে তারা অসন্তোষ প্রকাশ করেছে।

যাটজন যুবক একটি অঙ্গীকারনামায় স্বাক্ষর করেছে যে তারা শ্যুমাত্র ঐ সকল মেয়েদেরকে বিয়ে করবে যাদের খাৎনা করানো হয়নি। পাঁচজন কুমারী মেয়ের সাথে যৌনমিলন করার মাধ্যমে ‘সুস্থ’ হতে চাচ্ছিল এমন একজন এইচআইভি আক্রান্ত ব্যক্তিকে পুলিশ গ্রেফতার করেছে ।

নতুন গবেষণায় দেখা গেছে যে, বাবা-মায়েরা এখনও তাদের মেয়েদেরকে যৌন ব্যবসায়ীদের কাছে বিক্রি করে।

রাষ্ট্রপতি বলেছেন যে, তার মেয়ে একজন সমকামী তা জানার পর সমকামীদের প্রতি তার দৃষ্টিভঙির পরিবর্তন হয়েছে।

গর্ভপাত আইন আরও কঠোর করা হয়েছে; গর্ভপাত সেবাদানকারী গ্রেফতার হয়েছে। 


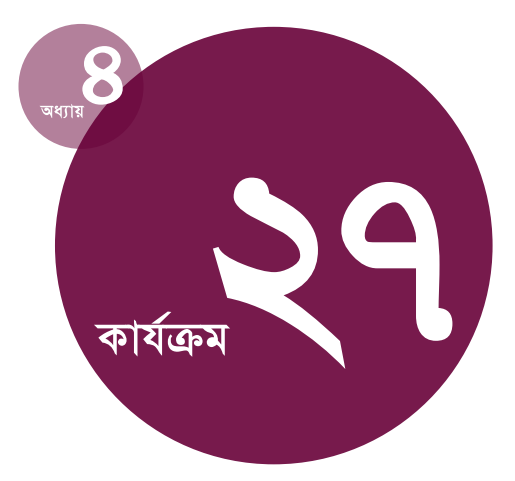

সময়কাল :

8৫ मिनिট

\section{উপকরণ:}

বোর্ড ও চক।

श्रत्্তুতি:

শফু নির্দেশাবলি পুনরায় পড়ুন ।

\section{আমার সম্পর্কগুলি}

অধিবেশন সম্পর্কিত ধারণা: শিক্ষার্থীরা তাদের সম্পর্কগুলি নিয়ে একটি চিত্রকর্ম উপস্থাপন করবে।

উদ্দেশ্য: শিক্ষার্থীদেরকে তাদের জীবনের বিভিন্ন সম্পর্কগুলি খুঁজে বের করতে এবং যেসকল গুণাবলিকে তারা তাদের জীবনে মূল্যায়ন করে ও ঘনিষ্ঠ সম্পর্কের মধ্যে চায় সেগুলোর নাম উল্লেখ করতে সাহায্য করা; শিক্ষার্থীদের সমালোচনামূলক চিন্তা শক্তিশালী করা।

\section{निর্দেশাবলি}

১ শিক্ষার্থীদের উদ্দেশ্যে ব্যাখ্যা করুন যে, তারা এখন মানুযের সাথে তাদের বিভিন্ন রকম সম্পর্ক শনাক্ত করবে। শিক্ষার্থীদেরকে তাদের সম্পর্কগুলির এবং অন্যান্য মানুযের সাথে যোগাযোগের একটি ব্যক্তিগত তালিকা তৈরি করতে বলুন।

২ স্বেচ্ছায় তাদের তালিকা অন্যদেরকে বলার জন্য কিছু শিক্ষার্থীকে ডাকুন । তাদের উল্লিখিত বিভিন্ন রকম সম্পর্ক বোর্ডে লিখে রাখুন, যেমন- আত্মীয়, বন্ধু বা প্রতিবেশীর সাথে সম্পর্ক।

৩ বোর্ডে সমকেন্দ্র বিশিষ্ট চারটি বৃত্তের একটি রেখাচিত্র আঁকুন (নিচেরটির মত)।

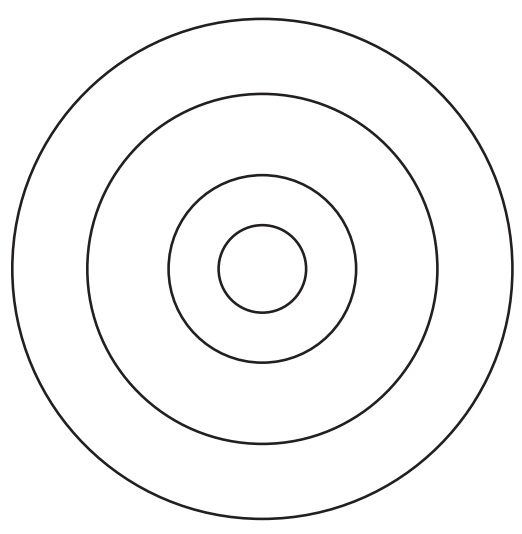

8 শিক্ষার্থীদেরকে একটি করে সাদা কাগজ নিতে বলুন । ব্যাখ্যা করুন:

• আমি যেমন এঁকেছি, এমন ছোট থেকে বড় চারটি বৃত্ত আঁক। সবচেয়ে বড় বৃত্তটির জন্য সম্পূর্ণ কাগজটি ব্যবহার কর।

• সবচেয়ে ছোট বৃত্তে তোমাদের নাম লেখ (বা তোমাদের একটি ছবি আঁক)।

• তোমার জীবনের সাথে জড়িত বিভিন্ন মানুষকে মনে কর।

• ঠিক এর পরের বৃত্তটিতে যারা তোমার সবচেয়ে ঘনিষ্ঠ তাদের নামগুলি লেখ (বা সাধারণ ছবি আঁক)।

• বাইরের দুটি বৃত্তে যারা বেশি কাছের নয় তাদের নামগুলি (বা ছবि) লেখ।

৫ শিক্ষার্থীদেরকে তাদের সবচেয়ে কাছে বসা তিন বা চারজনকে নিয়ে দল তৈরি করতে বলুন, এবং তাদের আঁকা ছবিগুলিকে দলের অন্য সদস্যদের সাথে ব্যাখ্যা করার জন্য প্রত্যেককে দুই মিনিটের মত সময় দিन। 
৬ নিয়লিখিত প্রশ্নগুলি নিয়ে আলোচনা পরিচালনা করুন:

• তোমাদের দলের প্রত্যেকে কি একই রকমের সম্পর্কের কথা লিখেছিল না-কি অন্যরকম লিখেছিল ? প্রত্যেকে কি তাদের পরিবারের সদস্য, বন্ধু, প্রতিবেশী, শিক্ষক, ধর্মীয় নেতা বা অন্যান্যদেরকে একই বৃত্তে নাকি আলাদা বৃত্তে রেখেছিল ?

- এ এমনি কিছু মূল্যবোধের কথা বল যেগুলো তোমরা তোমাদের নিকটতম মানুষের জন্য অনুভব কর।/উত্তর খুঁজুন: সৎ, সম্মানিত, আলোচনা, যত্মবান, বিশ্বস্ত, মজার, নিরাপদ, বোধগম্য, নির্ভরযোগ্য, আগ্রহী, স্নেহপরায়ণ। এই শব্দগুলো বোর্ডে লিখুন।]
৭ শিক্ষার্থীরা তাদের সম্পর্কগুলির মধ্যে যে গুণাবলির মূল্যায়ন করে লেগুলো বিশ্লেষণ করতে তাদের জন্য দশ মিনিট সময় রাখুন।

- তোমাদের লেখা একজন ব্যক্তির সম্পর্কে ভাব যাকে তোমরা ভেতরের দিকের বৃত্তে নিয়ে যেতে চাও। একটি শব্দ বেছে নাও যা ঐ ব্যক্তিকে যেভাবে তোমরা মূল্যায়ন কর তা সবচেয়ে ভালভাবে প্রকাশ করতে পারে ।

- তোমাকে বর্ণনা করার জন্য লোকে বলতে পারে এমন এক বা দুইটি শব্দ বোর্ডে লেখার জন্য এখন ভাব।

- যে গুণকে তুমি সবচেয়ে বেশি মূল্যায়ন কর (তোমার বা অন্যদের) সেটিকে আর্কষণীয় বর্ণে বা সৃজনশীলভাবে তোমার খাতায় লিখে উদযাপন কর।

- যা লিখেছ বা এঁকেছ, ঐ শব্দটির অর্থ তোমার কাছে কী তা ভাব। 


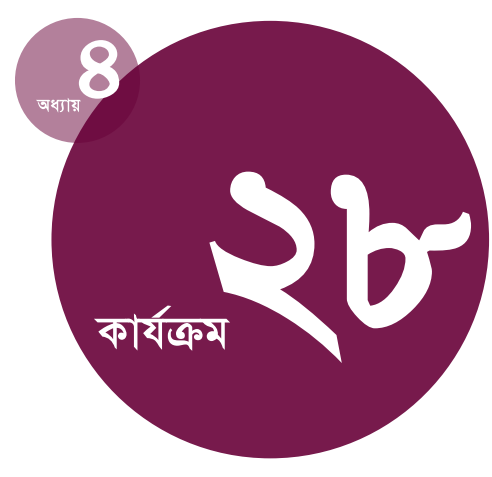

সময়কাল :

8৫ মিनिট

\section{উপকরণ:}

বোর্ড ও চক; প্রত্যেক ছোট দলের জন্য একটি করে কেসস্টাডি।

\section{প्रস্তুতি:}

শিক্ষার্থীদের হ্যান্ডআউট থেকে কেসস্টাডিগুলো পডুন এবং যেভাবে এগুনো আপনার সমাজের স্বাভাবিক অসমতার সাধারণ চিত্র তুলে ধরতে পারে সেভাবে পরিবর্তন অথবা স্থানান্তরিত করে নিন। প্রত্যেকটি কেসস্টাডি পৃথক পৃথক কাগজে ফটোকপি করুন

\section{এই দুইজনের মধ্যে কী হচ্ছে?}

অধিবেশন সম্পর্কিত ধারণা: শিক্ষার্থীরা সামাজিক অসমতার ওপর ভিত্তি করে গড়ে ওঠা কিছু সম্পর্ক নিয়ে কেসস্টাডি পড়বে; তারা অসমতাগুলো শনাক্ত করবে এবং সম্পর্কটির ওপর এগুলোর প্রভাব আলোচনা করবে।

উদ্দেশ্য: কীভাবে সামাজিক সমতা ঘনিষ্ঠ সম্পর্ককে প্রভাবিত করতে পারে শিক্ষার্থীদেরকে তা শনাক্ত করতে সাহায্য করা; বিশ্লেষণমূলক চিন্তার দক্ষতা শক্তিশালী করা ।

\section{निर्দেশাবলি}

১ ব্যাখ্যা করুন্ন:

• মানুষের মধ্যকার অসমতা এবং সমতার পার্থক্য কীভাবে আমাদের সম্পর্ককে প্রভাবিত করে আজকে আমরা সে বিষয়ে আলোচনা করব। আমাদের সমাজের বিভিন্ন মানবগোষ্ঠীর মধ্যে কী ধরনের অসমতা আছে? [জেন্ডার, বয়স, শ্রেণি, নৃতাত্ত্বিকগোষ্ঠী, যৌন পচ্ছন্দ, এবং শারীরিক সমতার ওপর ভিত্তি করে বর্ণবাদ, শ্রেণিভেদ ও অসমতা সম্পর্কে জানার চেষ্টা করুন ।]

• তোমরা চারটি দল গঠন করবে এবং প্রত্যেক দল আলোচ্য প্রশ্নসহ একটি কেসস্টাডি পড়বে। তারপর তোমরা তোমাদের প্রশ্নটির উত্তর দেবে।

২ প্রত্যেক দলকে একটি কেসস্টাডি এবং একটি আলোচ্য প্রক্নের অনলিপি দিন। তাদেরকে দশ মিনিট সময় দিন।

৩ একটি দলকে পাঁচ থেকে সাত মিনিটের মধ্যে তাদের কেসস্টাডিটি পড়তে এবং আলোচ্য প্রশ্নের উত্তর দিতে বলুন । তারপর মন্তব্য করার জন্য অন্যদেরকে কয়েক মিনিট সময় দিন
8 অন্য তিনটি কেসস্টাডির জন্য একই পদ্ধতি অনুসরণ করুন ।

৫ শিক্ষার্থীদেরকে তাদের নিজেদের ব্যক্তিগত সম্পর্কের মধ্যে সামাজিক অসমতা এবং এগুনোর প্রভাব নিয়ে চিন্তায় উদ্পুদ্ধ করে অধিবেশন শেষ করুন ।

বাড়ির কাজ : তোমার কেসস্টাডির যে কোন একজনকে একটি পোস্টকার্ড লেখ। তার সম্পর্কের মধ্যে অসমতার প্রভাব কমানোর জন্য করণীয় কী সে বিষয়ে তাকে উপদেশ দাও। 


\section{১নং দলের হ্যান্ডআউট:}

আমি আমার প্রেমিকের প্রতি আকৃষ্ট হয়েছি কারণ সে আমার চেয়ে বয়সে বড় ছিল এবং কেউ তাকে চিনত না । তাকে রহস্যজনক এবং উত্তেজিত মনে হত। আমরা প্রেমে পড়ি এবং এখন বেশির ভাগ সময়ই আমরা একসাথে কাটাই। এটা কীভাবে হয় তা তুমি জান। তার অভিজ্ঞতার সমস্ত গল্প শুনতে আমি সত্যিই পছন্দ করি। যখন আমরা বাইরে যাই, বেশির ভাগ ক্ষেত্রেই সে সিদ্ধান্ত নেয়- আমরা কোথায় যাব। কারণ সে টাকা খরচ করে এবং সমস্ত বিখ্যাত স্থান চেনে। সে আমাকে অনেক প্রভাবিত করে। আমার কখনই খেলাধূলার প্রতি আকর্ষণ ছিল না । কিন্তু সে ফুটবল ভালবাসে। তাই আমি আমার অবসর সময়ের অনেকটা তার সাথে খেলা করে অথবা টিভিতে খেলা দেখে ব্যয় করি ।

আমরা একে অপরের সাথে পরিচিত হওয়ার কয়েক মাস পরে সে আমাকে বলল যে, সে আমার সাথে প্রেম করতে চায়। আমি দ্বিধাদ্বন্দ্বে ছিলাম । কিন্তু আমি ভেবেছিলাম, যদি আমি এটা না করি তাহলে সে আমাকে ছেড়ে যেতে পারে। সর্বোপরি তার বয়স ২৩ বছর এবং সে অভিজ্ঞ। এখন আমার বন্ধুদের সাথে খুব কম দেখা হয়। তারা অভিযোগ করে এবং বলে যে, আমি পরিবর্তন হয়ে গেছি । তাদের কথা আমার খুব মনে পড়ে এবং মাঝে মাঝে আমি ভাবি আমি কত বেশি পড়তাম এবং পড়তে কত পছন্দ করতাম। কিন্তু তুমি জান, আমি ু্বু এটাই ভাবি- যখন তোমরা প্রেমে পড় তখন কী হয়।

\section{প্রশ্নাবলি:}

১ সমাজে বিভিন্ন গোষ্ঠীর মধ্যে নানা রকমের অসমতা রয়েছে (যেমন- মানুমের নৃতাত্ত্বিক অথবা নৃগোষ্ঠী, অর্থনৈতিক শ্রেণি, লিঙ, বয়স, অথবা অন্যান্য বৈশিষ্ট্যের ওপর ভিত্তি করে অপেক্ষাকৃত বেশি অথবা কম সামাজিক মর্যাদা থাকতে পারে)। এই সম্পর্কটির মধ্যে কোন্ ধরনের অসমতা বিদ্যমান?

২ সমাজে তার মর্যাদার কারণে এই সম্পর্কের মধ্যে কে বেশি ক্ষমতাশীল অবস্থানে আছে? এই অসমতার ভিত্তি কী ? কীভাবে এই বেশি ক্ষমতাশীল অবস্থান প্রদর্শিত হয়?

৩ োন্ লোকটি তার সামাজিক অবস্থানের কারণে এই সম্পর্কর মধ্যে ইীন অবস্থানে আছে? কীভাবে এই হীন অবস্থান প্রদর্শিত হয়?

8 এটির প্রভাব কী? यদি থাকে, এই অসমতা কি প্রত্যেকের মধ্যে আছে? এটি কীভাবে সম্পর্কটির ওপর প্রভাব ফেলে ?

৫ তোমরা কি মনে কর এই ধরনের ঘটনা আমাদের সমাজে বিদ্যমান? অনুগ্রহপূর্বক এগুলো নিয়ে মন্তব্য কর। 


\section{২নং দলের হ্যান্ডআউট:}

গত বছর থেকে আমি আমাদের গ্রামের রাগবি দলের একজনের সাথে বাইরে যেতে শরু করি। আমাদের মধ্যে সবকিছু গভীর হতে ফরু করল। একদিন রাতে গ্রামের সভাকক্ষে একটি সভার সময়ে সে আমাকে সভাকক্ষের পেছনে দেখা করার জন্য ইশারা করল । আমার মা এবং বাবা সেখানে ছিল । কিন্তু আমি জানতাম যে, তারা মনে করবে আমি অন্য নেয়েদের সাথে আছি। তাই সে এবং আমি পালিয়ে গেলাম এবং সভাকরে পেছনে জঙ্গলে যাওয়ার জন্য হঁঁটতে ওরু করলাম। যখন আমরা জঙলে পৌঁছলাম, আমরা চুম্বন শুরু করলাম এবং তখন সে আমার স্তন ছেঁঁয়া শুরু করল। আমি জানতাম যে, আমরা যৌনমিলন করতে যাচ্ছি । আমি আসলে তা চাইনি । আমি শুধুমাত্র তার সাথে কথা বলতে এবং চুম্বন করতে চেয়েছি, আর কিছু নয়। কিন্ত আমি তার সাথে গেলাম এবং সে চেয়েছে বলে আমরা যৌনমিলনের পর্ব শেষ করলাম।

পরে আমি কেঁদেছি । কারণ আমি জানতাম যে, আমি আমার মা-বাবাকে অসম্মান করেছি এবং আমি আমার কুমারিত্ব হারিয়েছি । আমি কনডম সম্পর্কে জানতাম এবং জানতাম যে, এগুলো একটি মেয়েকে গর্ভবতী হওয়া থেকে এবং রোগ থেকে রক্ষা করতে পারে। কিন্তু ঐ সময় আমি অন্য কিছু নিয়ে ভাবছিলাম যেমন- আমি এটি করছি জানলে আমার মা-বাবার কেমন লাগবে। আমি বেশি চিন্তিত ছিলাম এ বিষয়ে যে, আমি আমার কুমারিত্ব হারিয়েছি । যখন আমি গর্ভবতী হলাম, আমার মা-বাবা ক্ষিপ্ত হল এবং আমাকে আমার প্রেমিকের সাথে আর দেখা করতে নিযেধ করল ।

\section{প্রশ্নাবলি:}

১ সমাজে বিভিন্ন গোষ্ঠীর মধ্যে নানা রকমের অসমতা রয়েছে (যেমন- মানুষের নৃতাত্ত্বিক অথবা নৃগোষ্ঠী, অর্থনৈতিক শ্রেণি, লিঞ, বয়স, অথবা অন্যান্য বৈশিষ্ট্যের ওপর ভিত্তি করে অপেক্ষাকৃত বেশি অথবা কম সামাজিক মর্যাদা থাকতে পারে)। এই সম্পর্কটির মধ্যে কোন ধরনের অসমতা বিদ্যমান?

২ সমাজে তার মর্যাদার কারণে এই সম্পর্কের মধ্যে কে বেশি ক্ষমতাশীল অবস্থানে আছে? এই অসমতার ভিত্তি কী? কীভাবে এই বেশি ক্ষমতাশীল অবস্থান প্রদর্শিত হয়?

৩ োন্ লোকটি তার সামাজিক অবস্থানের কারণে এই সম্পর্কের মধ্যে ইীন অবস্থানে আছে? কীভাবে এই ইীন অবস্থান প্রদর্শিত হয়?

8 এটির প্রভাব কী? যদি থাকে, এই অসমতা কি প্রত্যেকের মধ্যে আছে? এটি কীভাবে সম্পর্কটির ওপর প্রভাব ফেলে ?

৫ তোমরা কি মনে কর এই ধরনের ঘটনা আমাদের সমাজে বিদ্যমান? অনুগ্রহপূর্বক এগুলো নিয়ে মন্তব্য কর। 


\section{৩নং দলের হ্যান্ডআউট:}

যখন আমার বয়স ৩৫ বছর হল, তখন আমি ভাবলাম আমার এখন বিয়ে করা উচিত। পরিশেষে আমি একটি চাকরি পেয়েছি এবং একটি পরিবার চালাতে সমর্থ। আমি বিশ্ববিদ্যালয়ে থেকে স্নাতক ডিগ্রি নিয়েছি । কিন্তু দেশে খুব বেশি বেকারত্ব ছিল। সেখানে চাকরি খুঁজে পাওয়া খুব কঠিন ছিল। সে জন্য আমি চাকরি খুঁজতে অন্যদেলে গেলাম । যখন আমি আমার মাকে দেখতে দেশে ফিরলাম, আমি আমাদের গ্রানে একজন বউ খেঁজার সিদ্ধান্ত নিলাম । আমি অনেকগুলো যুবতী মেয়েকে দেখলাম এবং একজনকে খুব পছন্দ করলাম। তার বয়স তখন ২০ বছর এবং সে শান্ত স্বভাবের ছিল। সে প্রাথমিক বিদ্যালয়ের লেখাপড়া শেষ করেছে, কিন্ত কখনই গ্রাম থেকে দুরে যায়নি । যদিও আমি তাকে বললাম যে, আমার সাথে নিয়ে যাওয়ার পূর্ব পর্যন্ত তাকে আমার মায়ের সাথে থাকতে হবে। সে আমাকে বিয়ে করতে রাজি হল।

আমাদের প্রথম বাচ্চা হওয়ার পরে আমি তাকে জন্ম নিয়ন্ত্রণ পদ্ধতি ব্যবহার করতে দিতে চাইলাম না । তাই আমি প্রত্যাহার পদ্ধতি ব্যবহার করতাম। আমি তাকে এবং আমাদের বাচ্চাকে প্রয়োজনীয় সবকিছু দিতে সর্বোচ্চ চেষ্টা করতাম। সে এখনও আমার মায়ের সাথে থাকে কিন্তু কখনও সমস্যা সৃষ্টি করে না বা বলে না যে, তার কিছু প্রয়োজন । আমি তাকে ভালবাসি কিন্তু কিছু বিষয় আছে যেগুলো নিয়ে আমি তার সাথে আলোচনা করতে পারি না। কিছু বিষয় সে কিছুই বুঝতে পারবে না । তুমি জান যে, সে আসলেই পৃথিবী সম্পর্কে বেশি কিছু জানে না ।

\section{প্রশ্নাবলি:}

১ সমাজে বিভিন্ন গোষ্ঠীর মধ্যে নানা রকমের অসমতা রয়েছে (যেমন- মানুভের নৃতাত্ত্বিক অথবা নৃগোষ্ঠী, অর্থনৈতিক শ্রেণি, লিঙ্গ, বয়স, অথবা অন্যান্য বৈশিষ্ট্যের ওপর ভিত্তি করে অপেক্ষাকৃত বেশি অথবা কম সামাজিক মর্যাদা থাকতে পারে)। এই সম্পর্কটির মধ্যে কোন ধরনের অসমতা বিদ্যমান?

২ সমাজে তার মর্যাদার কারণে এই সম্পর্কের মধ্যে কে বেশি ক্ষমতাশীল অবস্থানে আছে? এই অসমতার ভিত্তি কী ? কীভাবে এই বেশি ক্ষমতাশীল অবস্থান প্রদর্শিত হয় ?

৩ োন্ লোকটি তার সামাজিক অবস্থানের কারণে এই সম্পর্কের মধ্যে হীন অবস্থানে আছে? কীভাবে এই হীন অবস্থান প্রদর্শিত হয়?

8 এটির প্রভাব কী? যদি থাকে, এই অসমতা কি প্রত্যেকের মধ্যে আছে? এটি কীভাবে সম্পর্কটির ওপর প্রভাব ফেলে ?

৫ তোমরা কি মনে কর এই ধরনের ঘটনা আমাদের সমাজে বিদ্যমান? অনুগ্রহপূর্বক এগুনো নিয়ে মন্তব্য কর। 


\section{8নং দলের হ্যান্ডআউট:}

আমি বিদেশে কিছু দিনের জন্য চাকরি করতে গেলাম এবং সেখানে আমার সাথে যারা কাজ করে তাদের একজন পুরুষের প্রেমে পড়লাম । যখন আমি গর্ভবতী হয়ে গেলাম, আমরা বিয়ে করার সিদ্ধান্ত নিলাম । আমার মা-বাবা এই বিয়ের বিরুদ্ধে ছিল । কারণ সে দরিদ্র পরিবারের ছিল । তারা বলল, “আমরা মনে করি না তোমার সমকক্ষ নয় এমন কাউকে বিয়ে করার ফলাফল তুমি জান । কেন তুমি এখনই দেশে ফিরে এসে বাচ্চাটির জন্ম দিচ্ছে না ? আমরা তোমাকে সাহায্য করব । কিন্ত যাই ছোক আমরা বিয়ে করলাম । যখন আমি চাকরি করব তখন যেন লে বিশ্ববিদ্যালয়ের একটি ডিগ্রী নিতে পারে এজন্য আমরা আমার দেশে ফিরে আসার সিদ্ধান্ত নিলাম । যদিও লেখাপড়া করার পূর্বে তাকে ভাষা শিখতে হবে।

দুর্ভাগ্যবশত, তার কাছে এটা কঠিন মনে হল । সে বাচ্চাটির সাথে বাড়ি থাকছিল এবং ভাষা শেখার চেষ্টা করছিল। কিন্তু সে খুব বিচ্ছিন্নতা অনুভব করতো । সে আরো বেশি হতাশাগ্রস্থ হয়ে পড়ল এবং তার মর্যাদাবোধ অনেক নিটে নেমে গেল । টাকার জন্য তাকে আমার ওপর নির্ভর করতে হত এবং অপরাধী বোধ করত। কারণ আমাদের নিয়মিত তার পরিবারের জন্য টাকা পাঠাতত হত। সে লেখাপড়া করছে না, অথবা চাকরি খুঁজছে না, অথবা কাজ করছে না এটি আমি পছন্দ করতাম না । কিন্ত কিছু বলার ব্যাপারে আমি নিজে সচেতন থাকতাম। এটি এমনই স্পর্শকাতর বিষয় ছিল, এবং আমি তার অনুভূতিকে আঘাত করতে চাইতাম না । অবশেবে সে তার দেশ থেকে আসা কিছু মানুষের বন্ধু হতে শুরু করল এবং তাদের সাথে সময় কাটাতে শুরু করল, বারে যেয়ে মদ পান করত। একদিন এমনকি সে আমার ওপর সহিংস হয়ে পড়ল। যা আমাদের জন্য একটি সত্যিকারের জাগরণ বার্তা ছিল।

\section{প্রশ্নাবলি:}

১ সমাজে বিভিন্ন গোষ্ঠীর মধ্য্য নানা রকমের অসমতা রয়েছে (যেমন- মানুষের নৃতাত্ত্বিক অথবা নৃগোষ্ঠী, অর্থনৈতিক শ্রেণি, লিঙ্গ, বয়স, অথবা অন্যান্য বৈশিষ্ট্যের ওপর ভিত্তি করে অপেক্ষাকৃত বেশি অথবা কম সামাজিক মর্যাদা থাকতে পারে)। এই সম্পর্কটির মট্যে কোন ধরনের অসমতা বিদ্যমান?

২ সমাজে তার মর্যাদার কারণে এই সম্পর্কের মধ্যে কে বেশি ক্ষমতাশীল অবস্থানে আছে? এই অসমতার ভিত্তি কী? কীভাবে এই বেশি ক্ষমতাশীল অবস্থান প্রদর্শিত হয় ?

৩ োন্ লোকটি তার সামাজিক অবস্থানের কারণে এই সম্পর্কের মধ্যে হীন অবস্থানে আছে? কীভাবে এই হীন অবস্থান প্রদর্শিত হয়?

8 এটির প্রভাব কী? যদি থাকে, এই অসমতা কি প্রত্যেকের মধ্যে আছে? এটি কীভাবে সম্পর্কটির ওপর প্রভাব ফেলে ?

৫ তোমরা কি মনে কর এই ধরনের ঘটনা আমাদের সমাজে বিদ্যমান? অনুগ্রহপূর্বক এগুনো নিয়ে মন্তব্য কর। 


\section{এই কী ভালবাসা?}

অধিবেশন সম্পর্কিত ধারণা: শিক্ষার্থীরা ভালবাসার সাথে সম্পর্কিত অন্যান্য আবেগগুলি থেকে ভালবাসার পার্থক্য করবে।

উদ্দেশ্য: শিক্ষার্থীদেরকে ভালবাসা, মোহ এবং ঈর্ষা সম্পর্কে ব্যক্ত করতে সক্ষম করা; বিশ্লেষণমূলক চিন্তার দক্ষতা শক্তশালী করা।

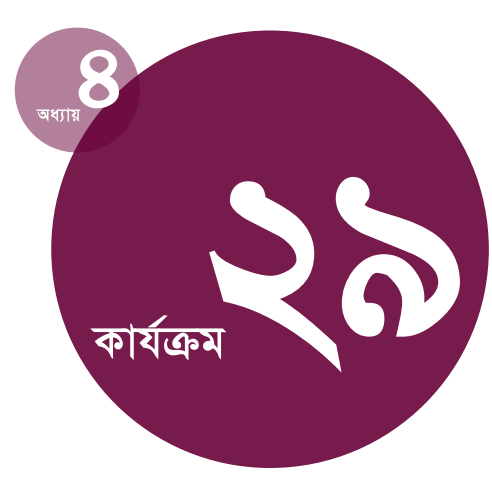

\section{निर्দেশাবলি}

১ শিক্ষার্থীদেরকে পাঁচটি দলে ভাগ করুন । প্রত্যেক দলকে বলুন আপনার প্রস্তুতকৃত কাগজ থেকে একটি করে কাগজ তুলে নিতে বোর্ডে লিখুন "ভালবাসা এবং এর মধ্যে পार्थक्य की?"

২ দলগুলোকে একটি কাগজের টুকরো নিতে এবং বাক্যটি বোর্ডে লিখে তাদের কাগজে লেখা শব্দটি দিয়ে বাক্য সম্পূর্ণ করতে বলুন। তাদের প্রশ্ন নিয়ে আলোচনা করে উত্তর লিখতে বলুন।

৩ প্রায় পাঁচ মিনিট পর, একটি দলকে তাদের প্রশ্ন পড়তে এবং উত্তর উপস্থাপন করতে বলুন।

নিয়লিখিত প্রশ্নগুনো একটি সংক্ষিপ্ত আলোচনার নির্দেশনা দিতে পারে

• কেউ কি ভিন্নমত পোষণ কর অথবা মন্তব্য করতে চাও?

• সমাজ কি মেয়ে এবং ছেলেদেরকে এই অনুভূতি প্রকাশের সমান সুযোগ দেয় ?

• তোমরা কি এমন একটি উদাহরণ দিতে পার যখন কেউ এই অনুভূতির সাথে ভালবাসাকে মিলিয়ে ফেলেছে? এই অনুভূতির সাথে যদি ভালবাসা মিলিয়ে যায়, তাহনে কী ঘটে ?

• এই অনুভূতি কি ভালবাসার একটি অংশ হতে পারে ?
8 বাকি চারটি দলের জন্য ধাপ ৩ অনুসরণ করুন ।

৫ নিম্নলিখিত প্রশ্নগুনো আলোচনার জন্য দশ মিনিট সময় রাখুন:

• যুবক-যুবতীদের ভালবাসা এবং শিহরণের অভিব্যক্তি কোথা থেকে সৃষ্টি হয় ?

- চলচ্চিত্র এবং প্রেমের উপন্যাস কি এই অনুভূতিগুনোর বাস্তব চিত্র তুলে ধরে?

• যদি না হয় তাহলে এগুলো যুবক-যুবতীদের প্রত্যাশার ওপর কী প্রভাব ফেলে বলে তোমরা মনে কর?

• ভালবাসা এবং এই অন্যান্য অনুভূতির পার্থক্য সম্পর্কে তোমাদের নিজস্ব বিশ্বাসকে সচেতন করা কেন গুরুত্বপূর্ণ?

\section{সময়কাল :}

8৫ मिनिট

\section{উপকরণ:}

বোর্ড ও চক; কয়েকটি শব্দ লেখা কাগজের টুকরা ।

\section{প्रস্তুতি:}

অধिবেশনটি সমাজ, স্থান এবং শিক্ষার্থীদের ভাষার উপযুক্ত করে পরিবর্তন করুন । পাঁচটি কাগজের টুকরো নিন এবং প্রত্যেক কাগজে নিয়লিখিত যে কোন একটি শব্দ

लिখুন।

• মোহ

• ভালবাসার অস্তিত্ব

• শিহরণ

• যৌন আकর্ষণ

• ঈর্যাকাতরতা 


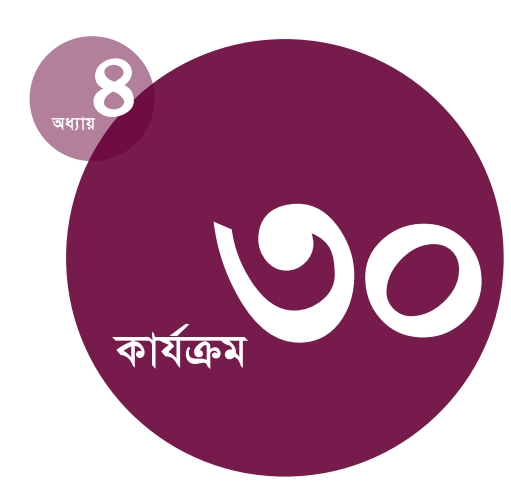

সময়কাল :

8৫ মিनिট

\section{উপকরণ:}

বোর্ড ও চক; অনুশীলনপত্রের অনুলিপি "আমি কি প্রস্তুত? আমি কীভাবে সিদ্ধান্ত নেব ?"; শিক্ষার্থীদের অনুশীলনপত্র “যৌনমিলন করতে প্রস্তুত মনে হওয়া”।

\section{প্রস্তুতি:}

হ্যান্ডআউটে বিভিন্ন অবস্থার তালিকাটি পুনরায় পড়ন এবং নিশ্চিত হোন যে, আপনি সম্ভাব্য যে কোন প্রশ্নের উত্তর দিতে সক্ষম ।

\section{যৌনমিলনের সিদ্ধান্ত নেয়া}

অধিবেশন সম্পর্কিত ধারণা: শিক্ষার্থীরা পরিপক্কতা, আত্ন-সচেতনতা, ফলপ্রসূতা এবং যোগাযোগ দক্ষতার সূচকগুলো পরীক্ষা করবে, যেগুলো একজন যুবক বা যুবতীর নিরাপদ ও আরামদায়ক যৌন সম্পর্কের দাবি আদায়ে কথা বলার জন্য প্রয়োজন। (লক্ষণীয়: এই অধিবেশনের পূর্বে আপনার শিক্ষার্থীদদর যৌন আচরণ এবং যৌন নিপীড়ন সম্পর্কে জানা থাকতে হবে।)

উদ্দেশ্য: নিরাপদ এবং আরামদায়ক যৌন সম্পর্কের দাবি আদায়ের কথা বলতে সক্ষম হওয়ার জন্য যে দক্ষতা প্রয়োজন শিক্ষার্থীদেরকে সেই দক্ষতাগুনো শনাক্ত করতে সাহায্য করা; সমালোচনামূলক চিন্তাশক্তির দক্ষতা শক্তিশালী করা।

\section{निर्দেশাবলি}

১ নিম্নলিখিত ব্যাখ্যার মাধ্যমে অধিবেশনটি শুরু করুন:

• যৌন সক্রিয় হবে কিনা অথবা কখন হবে সে সিদ্ধান্ত নেওয়ার জন্য যুবক-যুবতীরা বিভিন্ন মাপকাঠি ব্যবহার করে। কেউ কেউ একটি নির্দিষ্ট বয়স পর্যন্ত অপেক্ষা করে; অন্যরা বিয়ে অথবা বাগদান পর্যন্ত অপেক্ষা করতে পছন্দ করে। অন্যরা যৌনমিলন করতে শুরু করে কারণ কেউ তাদের জন্য কম বা বেশি সিদ্ধান্তটি নেয়। অনেক যুবক-যুবতী যখন যৌনমিলন করবে কিনা এই সিদ্ধান্ত যাচাই করে তখন তারা তাদের সম্পর্ককে কীভাবে অনুভব করে তা ভাবে।

- যৌনক্রিয়ার জন্য প্রস্তুত কিনা তা নিরূপণ করা অনেক যুবকযুবতীর জন্য কঠিন। এই অধিবেশনটি তোমাদেরকে পরিপক্কতা, আত্নসচেতনতা এবং যোগায্যাগ দক্ষতা সম্পর্কে ভাবতে সাহায্য করবে যা যৌনক্রিয়ায় প্রস্তুত হওয়ার জন্য ভূমিকা পালন করে। তোমাদের কাছে কোনটি সবচেয়ে গুরুত্বপূর্ণ তাও শনাক্ত করতে সাহায্য করবে।
২ শিক্ষার্থীদেরকে জোড়া গঠন করতে বলুন; প্রত্যেক জোড়াকে একটি করে অনুশীলনপত্র দিন (নির্দেশনাসহ)। ব্যাখ্যা করুন:

• হ্যান্ডআউটে দেয়া তালিকাটি দেখ। এগুলো হল কিছু অনুভূতি এবং অবস্থার তালিকা যেগুলো একটি যৌন সম্পর্কের মধ্যে স্বস্তি এবং নিরাপত্তার অনুভূতি থাকার ব্যাপারে গুরুত্বপূর্ণ হতে পারে। যদি একজন ব্যক্তি যৌনমিলন করতে চায় এবং তার সম্পর্কের মধ্যে বেশির ভাগ অথবা সকল অবস্থা বিদ্যমান থাকে, তাহলে ঐ ব্যক্তি যৌনমিলনে অনেক বেশি স্বস্তি পাবে এবং নিরাপদ থাকবে ।

• যদি এগুলোর বেশির ভাগ অবস্থা কোন সম্পর্কের মধ্যে অনুপস্থিত থাকে, তাহলে ঐ ব্যক্তি অস্বস্তিতে এবং নিরাপদ নয় এমন যৌনমিলনের ঝুঁকির মধ্যে থাকবে। 
৩ অনুশীলনীর নির্দেশনাগুলি সকলে ফননতে পারে এভাবে পড়ন। সকলে অধিবেশনটি বুঝতে পেরেছে সে বিষয়ে নিশ্চিত হোন । তাদেরকে বলুন যে, তারা কিছু প্রশ্নের আলোকে বিভিন্ন ঘটনা পর্যালোচনা করবে। তাদের নিজেদের উত্তর নিয়ে মতৈক্যে পৌঁছানোর চেষ্টা করা উচিত, কিন্তু যদি তারা সেটি না পারে তাহলে এর অতিরিক্ত আরো ঘটনা পর্যালোচনা করতে পারে। প্রশ্নগুলো আলোচনা করতে এবং উত্তরগুলো লিখতে তাদের ১৫ মিনিট সময় দিন।

8 দলগুলোকে পুনরায় একত্রিত করুন । এবং অনুশীলনপত্রের ১-৫নং প্রশ্নগুনো পড়ন ও শিক্ষার্থীদেরকে উত্তর দিতে বলুন। শিক্ষার্থীরা বেশির ভাগ ক্ষেত্রে তাদের সঙ্গীদের সাথে একমত নাকি দ্বিমত পোষণ করে তা জিজ্ঞেস করুন ।

৫ অনুশীলনপত্রের ৬নং প্রশ্নের ক্ষেত্রে শিক্ষার্থীদৈর উত্তর পর্যালোচনা করার জন্য বেশি সময় নিন । তাদের উত্তরগুলো বোর্ডে লিখুন। নিয়লিখিত প্রশ্নগুলো আলোচনার জন্য নির্দেশনা দিতে পারে:

• কোন অবস্থাগুলো ছেলেদের কাছে অগ্রাধিকার পায়?

- কোনটি মেয়েদের কাছে বেশি গুরুত্বপূর্ণ?

• ছেলে এবং মেয়েদের মধ্যে অগ্রাধিকারের যেকোন পার্থক্যকে আপনি কীভাবে ব্যাখ্যা এবং অনুভব করেন ?
৬ অনুশীলনপত্রের ৭ নং এবং ৮- নং প্রশ্ন আলোচনার মাধ্যমে অধিবেশনটি শেষ করুন । এরপর জিজ্ঞেস করুন:

- এগুলোর মধ্যে কিছু অবস্থা কি তোমাদের কাছে গুরুত্বপূর্ণ নয়, এমন কিছু কী আছে যা যৌনমিলন করবে কিনা সে ব্যাপারে সিদ্ধান্ত নেয়ার জন্য তোমাদের কাছে কোনভাবেই গুরুত্বপূপর্ণ नয়?

• তুমি কিশোর বয়সে যৌনভাবে সক্রিয়, অথবা তুমি বড় হওয়া বা বিয়ে করা পর্যন্ত অপেক্ষা করছ, যাই হোক না কেন, এই অবস্থাগুলো প্রতিষ্ঠিত হলে তোমাদের যৌন জীবনকে আরো নিরাপদ এবং আরো আরামদায়ক করতে পারে।/যদি আপনি শিক্ষাক্রমের অংশ হিসেবে অন্য কোন ঘটনা যোগ করার পরিকল্পনা করেন তাহলে শিক্ষার্থীদেরকে তা জানান। যেমনসকল বিষয় সন্নিবেশিত একক শিক্ষাক্রমের প্রথম খণ্ড যোগাযোগ, শরীর এবং শরীর বিদ্যা, যৌন স্বাস্থ্য / এইচআইভি এবং জেন্ডার বিষয়গুলো অন্তর্ভুক্ত করে।] 


\section{আমি কি প্রস্তুত? আমি কীভাবে সিদ্ধান্ত নেব ?}

এই অনুশীলনের জন্য, তোমাদের বয়সী একজন যুবক বা যুবতী ভ্যীনকাজের জন্য সক্রিয় হবে কিনা সেই সিদ্ধান্ত নিতে চেষ্টা করছে। ঐ যুবক বা যুবতী সঠিক সিদ্ধান্ত নিতে চায় এবং একটি প্রশ্ন করছে: “আমি যৌন মিলনের জন্য টৈরি কিনা এই সিদ্ধান্ত নেয়ার জন্য আমার ভাবা উচিত এমন দুইটি সবচেয়ে গুরুত্বপূপ্ণ অনুভূতি অথবা অবস্থা কী কী ?” ঐ যুবক বা যুবতী এ প্রশ্নটি তিনজন ব্যক্তির কাছে করেছে: একজন ভাল বন্ধু, একজন কাউন্গিলর (অথবা মনোবিজ্ঞানী), এবং একজন এইডস রোগী।

\section{এখন এই ধাপগুলো অনুসরণ কর:}

১ তোমাদের অনুশীলনপত্রের তালিকাটি পড়।

২ এ এখানকার প্রত্যেকটি ব্যক্তির উপরের প্রশ্নটির কী উত্তর দেয়া উচিত তা নিয়ে আলোচনা কর এবং সিদ্ধান্ত নাও।

৩ ভাল বন্ধু: ভাল বন্ধু সবচেয়ে গুরুত্বপূর্ণ বলতে পারে বলে মনে কর এমন দুইটি অনুভূতি বা অবস্থার পাশের বক্সে একটি স্বাভাবিক হাসিমুখ আঁক (:) )

8 কাউন্সিলর: একজন কাউন্সিলর বা মনোবিজ্ঞানী সবচেয়ে গুরুত্বপূর্ণ বলতে পারে বনে মনে কর এমন দুইটি অনুভূতি বা অবস্থার পাকের বক্সে একটি টিক চিহ্ন দাও $(\checkmark) ।$

৫ একজন এইডস আক্রান্ত ব্যক্তি সবচেয়ে গুরুত্বপূর্ণ বলতে পারে বলে মনে কর এমন দুইটি অনুভূতি বা অবস্থার পাকের বক্সে একটি বিশ্ব এইডস সচেতনতার লোগো আঁক ( $\mathrm{X})$ ।

৬ আলোচনা: তোমরা কি মনে কর এগুলোর মধ্যে কোন একটি অনুভূতি বা অবস্থা মেয়েদের চেয়ে ছেলেদের কাছে বেশি গুরুত্বপূর্ণ? তোমরা যে সকল অবস্থা মেয়েদের চেয়ে ছেলেদের কাছে বেশি গুরুত্বপূর্ণ বনে মনে কর তার পাণে একটি ছেলের শরীরের রেখাছবি আঁক। তোমরা যেসকল অবস্থা ছেলেদের চেয়ে মেয়েদের কাছে বেশি গুরুত্বপূর্ণ বলে মনে কর তার পাশে একটি মেয়ের শরীরের রেখাছবি আঁক।

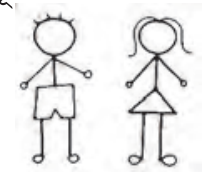

৭ এখন এমন দুইটি অনুভূতি বা অবস্থার কথা ভাব যেগুনো যুবক-যুবতীরা যখন প্রথমবার যৌনমিলন করার সিদ্ধান্ত নেয় তখন প্রায়ই এড়িয়ে যায় অথবা কঠিন মনে করে। এমন দুইটি বিষয়ের পরবর্তী বক্সের একটি দুঃখী মুখ আঁক (i)

৮ এমন আরও কি কোন অতিরিক্ত অনুভূতি বা অবস্থা আছে যেগুলো কারও প্রথমবার যৌনমিলন করার পূর্ব্বে ভাবা গুরুত্বপূর্ণ বনে তোমরা মনে কর? 


\section{যৌনমিলন করতে ‘প্রস্তুত’ মনে হওয়া}

\begin{tabular}{|c|c|}
\hline যৌনমিলন করার জন্য একটি অনুভূতি বা অবস্থা & এখানে আাক \\
\hline $\begin{array}{l}\text { তুমি তোমার নিজের ও সঙ্গীর মূল্যবোধকে সম্মান করছ বলে } \\
\text { মনে করা। }\end{array}$ & \\
\hline অন্য ব্যক্তির সাথে আন্তরিকতা অনুভব করা । & \\
\hline $\begin{array}{l}\text { তুমি এবং অন্য ব্যক্তি একে অপরকে সম্মান করছ বলে মনে } \\
\text { করা । }\end{array}$ & \\
\hline $\begin{array}{l}\text { তুমি এবং অন্য ব্যক্তি একে অপরকে বিশ্বাস করো বলে মনে } \\
\text { করা । }\end{array}$ & \\
\hline $\begin{array}{l}\text { তুমি এবং ঐ ব্যক্তি একত্রে মিলে সিদ্ধান্ত নিয়েছ এবং উভয়ে } \\
\text { যৌনমিলন করতে চাও এই অনুভূতি। }\end{array}$ & \\
\hline তোমার নিজের শরীরে আরামদায়ক অনুভব করা । & \\
\hline $\begin{array}{l}\text { যৌনভাবে কী তোমাকে ভাল অনুভূতি দেয় তা তুমি জান এ } \\
\text { বিষয়ে আত্রবিশ্বাসী হওয়া । }\end{array}$ & \\
\hline ব্যক্তিটির প্রতি যৌন আকর্ষণ অনুভব করা । & \\
\hline $\begin{array}{l}\text { ভাল যৌন অনুভূতি কী, তা নিয়ে অন্য ব্যক্তির সাথে আলোচনা } \\
\text { করতে স্বস্তিবোধ করা। }\end{array}$ & \\
\hline
\end{tabular}

যৌনমিলন করার জন্য একটি অনুভূতি বা অবস্থা এখানে আঁক

ছেলেটির বা মেয়েটির কোনটি ভাল লাগে তা জিজ্ঞস করতে স্বস্তিবোধ করা ।

ব্যক্তিটির সাথে কনডম ব্যবহার নিয়ে আলোচনা করতে

স্বস্তিবোধ করা ।

কনডম (অথবা অন্য যে কোন জন্মবিরতিকরণ পদ্ধতি) থাকা এবং কীভাবে এগুলো ব্যবহার করতে হয় তা জানা ।

তোমার এইচআইভি আছে কিনা তা জানা।

তোমার এবং তোমার সঙ্গীয় এইচআইভি আছে কিনা তা নিয়ে কথা বলতে স্বস্তিবোধ করা ।

यদি তোমাদের মধ্যে যে কোন একজন যৌনমিলন বাদ দিতে চাও তাহলে এটি বলতে নিরাপদ বোধ করা।

গোপনীয়তা থাকা।

পরবর্তীতে তোমার উপহাস বা কলঙ্কের ঝুঁকি থাকবে না তা অনুভব্ব করা।

অन्यान्य? 


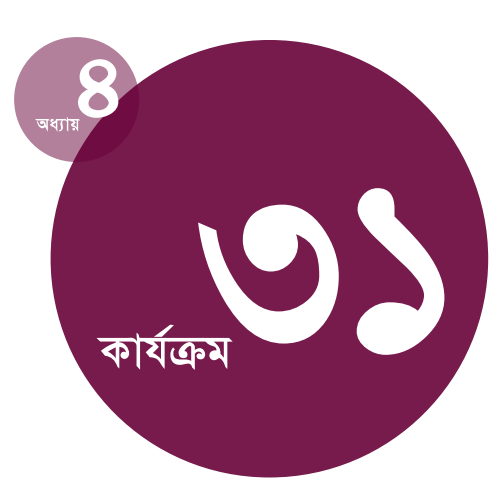

সময়কাল :

8৫ मिनिট

\section{উপকরণ:}

বোর্ড ও চক।

\section{প्रস্তুতি:}

ডান পাণে দেয়া আচরণের তালিকাটি শিক্ষার্থীদের সাথে সংক্ষিত্ত আলোচনার উপযুক্ত কিনা তা নিশ্চিত হবার জন্য পুনরায় পড়ুন । আপনি প্রত্যেকটির একটি সহজ ব্যাখ্যা দিতে পারবেন লে ব্যাপারেও নিশ্চিত হোন। এগুনোর মধ্যে কিছু আচরণ আপনার সমাজে স্থানীয়ভাবে খুব কম দেখা যেতে পারে, কিন্তু শিক্ষার্থীরা এগুলো সম্পর্কে অন্য কোন শ্রেণিকক্ষের পাঠদান, চলচ্চিত্র অথবা ইন্টারনেট থেকে জেনে থাকতে পারে।

\section{বিভিন্ন সম্পর্কের মধ্যে আমরা কীভাবে একত্রে বসবাস করি}

অধিবেশন সম্পর্কিত ধারণা: বিয়ে এবং পরিবার গঠনের সাথে জড়িত রীতিনীতি পরিবর্তন হচ্ছে কিনা এবং কীভাবে হচ্ছে শিক্ষার্থীরা সে বিষয়ে জানবে।

উদ্দেশ্য: শিক্ষার্থীদেরকে তাদের নিজস্ব আকাজ্কা এবং দীর্ঘস্থায়ী সম্পর্কের মূল্যবোধ সম্পর্কে স্পষ্ট ধারণা পেতে সাহায্য করা; সামাজিক মূল্যবোধ সময়ের সাথে সাথে পরিবর্তন হয় তা বোঝানো; বিশ্লেষণমূলক চিন্তা এবং লেখার দক্ষতা শক্তিশালী করা।

\section{निर्্দশাবলि}

১ নিচের তালিকাটি বোর্ডে লিখুন:

বাল্য বিবাহ

সমকামী সম্পর্ক অথবা বিয়ে

বিয়ের আগে যে সকল দম্পতিরা যৌনমিলন অথবা একত্রে

বসবাস করছে

পারিবারিকভাবে বিয়ে

স্ত্রী, স্বামীকে অমান্য করায় স্বামী তাকে পেটায়

বিয়ে বিষয়ে পরামর্শ

যৌথ পরিবারে একসাথে বসবাস করা

বাবা হিলেবে পুরুষরা বেশি সম্পৃক্ত হচ্ছে

একটি পুরুষের একাধিক বউ আছে

বিবাহ বিচ্ছেদ

কনেপণ / যৌতুক
২ ব্যাখ্যা করুন:

- এগুলো ভালবাসা এবং বিয়ে সংক্রান্ত কিছু রীতিনীতির তালিকা যা বিভিন্ন সংস্কৃতিতে চর্চা করা হয়। এগুলোর মধ্যে কিছু- যেমন বিবাহ-বিচ্ছেদ, সমকামী বিয়ে, অথবা বিয়ের ন্যুনতম বয়স হলো আইনগত বিষয়। অন্য রীতিগুলো- যেমন যৌথ পরিবারে একসাথে বসবাস, বিয়ের আগে দম্পত্রের একসাথে বসবাস, অথবা পিতৃত্বের ধরন- সাধারণত যথাযথভাবে অপেক্ষাকৃত কম প্রতিষ্ঠিত। এগুলোর মট্যে কিছু রীতি তোমরা সম্ভবত অনুমোদন কর এবং অন্যগুলো সম্ভবত অনুমোদন কর না।

• তালিকাটি পড় এবং এমন একটি চর্চা বাছাই কর যেটি পরবর্তী প্রজন্মে পরিবর্তন হবে বলে মনে কর। এটি এমন একটি পরিবর্তন যেটি ভাল বা খারাপের জন্য হতে পারে বলে মনে কর। 
৩ শিক্ষার্থীদেরকে তাদের খাতা খুলতে বলুন । তাদেরকে এই পরিবর্তন নিয়ে কমপক্ষে আধাপাতা লিখতে বলুন। তাদের চিন্তা শক্তিকে জাগানোর জন্য নিম্নলিখিত প্রশ্নগুনো জিজ্ঞস করুন ।

• তালিকার কোন রীতিগুলো পরবর্তী প্রজন্মে পরিবর্তন হবে বলে তোমাদের মনে হয়? এই পরিবর্তন আমাদের সংস্কৃতিতে ঘটবে নাকি অন্য কোথাও ঘটবে বলে তোমরা মনে কর?

• তোমরা কি মানুষের মনোভাব পরিবর্তন প্রত্যাশা কর ? চর্চার মধ্যে পরিবর্তন কতটা স্বাভাবিক? এই চর্চাটি যে উপায়ে ঘটে তার কি পরিবর্তন হয়? আর কী? চর্চাকে নিয়ন্ত্রণ করে এমন আইন পরিবর্তন হবে বলে কি তোমরা মনে কর?

• তোমরা যে পরিবর্তনগুলি দেখতে চাও সে বিষয়ে একমত কিনা, তা আলোচনা করার জন্য নিশ্চিত হও তোমরা কি তোমাদের মতামত ব্যক্তিগত অভিজ্ঞতার ওপর ভিত্তি করে নাকি তোমরা যা পড়েছ বা ক্তেছ তার ওপর ভিত্তি করে গড়ে তুলেছ?

8 তালিকাটি পডুন। প্রত্যেক আচরণের জন্য, একজন আগ্রহী শিক্ষার্থীকে ডাকুন যে ঐ আচরণের ওপর লিখেছে এবং তার লেখাটি পড়বে। তারপর একটি অথবা দুইটি সংক্ষিপ্ত মন্তব্য করতে অনুমতি দিন; প্রত্যেক বিষয়ের জন্য একজন আগ্রহী শিক্ষার্থী আছে কিনা তার ওপর ভিত্তি করে বিভিন্ন রকম সময় বরাদ্ল দিন
৫ আলোচনার জন্য শেষ পাঁচ মিনিট হাতে রাখুন:

- সকল সমাজ অথবা এমনকি সম্পূর্ণ দেশে ভালবাসা এবং বিয়ের প্রেক্ষাপট পরিবর্তন করার কী কী কারণ আছে বলে তোমরা মনে কর?

• মানুষ কোথা হতে নতুন ধারণা পায় এবং কীভাবে ঐ ধারণাগুলো জনপ্রিয় হয়? [জানার চেষ্টা করুন: যে সকল ব্যক্তি অন্যরকমভাবে থাকতে পছন্দ করে; গণমাধ্যম, বিভিন্ন সংস্কৃতির পারস্পরিক বিনিময়; অর্থনৈতিক পরিবর্তন যা পরিবারের সদস্যরা যেখানে থাকে তার ওপর প্রভাব ফেলে; রাজনৈতিক আন্দোলন ।]

• কল্পনা কর তোমার একজন কলমীবন্ধু আছে, যে তোমার চেয়ে আলাদা সংস্কৃতিতে বসবাস করে। আমাদের থেকে তোমার কলমীবন্ধু যেখানে বাস করে সেখানকার আচরণ/রীতি অনেক আলাদা । যখন তুমি শ্রেণিকক্ষ ত্যাগ করবে, তখন এখানে মানুষ দীর্ঘস্থায়ী সঙ্গীকে কীভাবে খোঁজে, একসাথে বসবালের জন্য কী ব্যবস্থা আছে, এবং বিবাহ শুরু ও বিচ্ছেদের জন্য সাধারণ আচরণ/রীতিগুলি কী কী তা নিয়ে ভাব যা তুমি তোমার কলমীবন্ধুকে বর্ণনা করবে ? 


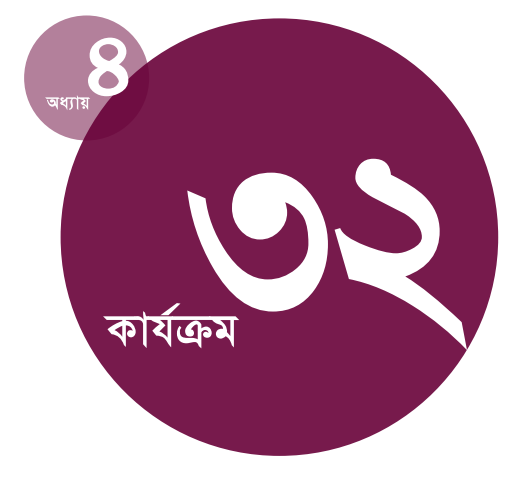

\section{সময়কাল :}

ধাপ ১-৩: ৪০ মিনিট

ধাপ 8: ৫ মিনিট (ধাপ ৫-৬ এর আগে অবশ্যই কমপক্ষে একদিন সময় দিতে হবে)

ধাপ ৫-৬: ৪০ মিনিট

\section{উপকরণ:}

বোর্ড ও চক।

\section{প्रস্তুতি:}

এটি দুইটি অংশের একটি অধিবেশন (ধাপ ১-৩ এবং ধাপ 8-৬); আপনি যেকোন একটি অংশ অথবা উভয় অংশ উপস্থাপন করতে পারেন ।

সিদ্ধান্ত নিন আপনি একটি নাকি উভয় অংশ পরিচালনা করবেন। আপনি যদি ধাপ 8-৬ পরিচালনা করেন, তাহলে নিশ্চিত হোন যে, ধাপ ৫-৬ এর কমপক্ষে একদিন পূর্বে ধাপ 8 শেষ করবেন ।

\section{আমি কী খোঁজ করছি}

অধিবেশন সম্পর্কিত ধারণা: শিক্ষার্থীরা একজন দীর্ঘস্থায়ী সঙ্গীর মধ্যে কী গুণাবলি চায় সেগুনোর তালিকা করবে এবং তাদের উত্তরগুলো জেন্ডারের দৃষ্টিডশি থেকে বিশ্লেষণ করবে। শিক্ষার্থীরা যা শিখেছে তা একজন কাল্পনিক ভবিষ্যৎ সঙ্গীর জন্য একটি চিঠি অথবা কবিতায় সংকলন করবে।

উদ্দেশ্য: শিক্ষার্থীরা কোন বৈশিষ্যুগুনো একজন দীর্ঘস্থায়ী সঙ্গীর ক্ষেত্রে সবচেয়ে মূল্যবান মনে করে সেগুলোকে তাদের শনাক্ত করতে এবং ব্যক্ত করতে সক্ষম করা; তাদের মূল্যবোধ স্পষ্ট করা এবং বিমূর্ত চিন্তা ও উদ্ভাবনী লেখার দক্ষতা শক্তিশালী করা ।

\section{निर्দেশাবলি}

১ ব্যাখ্যা করুন:

• আজকে আমরা একজন দীর্ঘস্থায়ী বা জীবনসঙ্গীর ক্ষেত্রে কোন গুণ বা বৈশিষ্ট্যগুলো খুঁজব তা মনোযোগের সাথে ভাবব।

• তোমাদের খাতা নাও এবং দম্পতি বা দীর্ঘস্থায়ী সঙ্গী হবার ক্ষেত্রে তোমরা গুরুত্বপূর্ণ মনে কর এমন কমপক্ষে পাঁচটি গুণ অথবা বৈশিষ্ট্যের কথা লেখ।

- গুরুত্বের ভিত্তিতে বৈশিষ্ট্যুগুলোকে সাজাও, সবচেয়ে গুরুতত্বপূর্ণ্টিকে ১নং দিয়ে শুরু কর।

২ শিক্ষার্থীদেরকে দুইটি দল গঠন করতে বলুন। একদলে সব ছেলে এবং একদলে সব মেয়ে (যদি শ্রেণিকক্ষের সবাই ছেলে অথবা সবাই মেয়ে না হয়)। यদি আপনার শিক্ষার্থীর সংখ্যা বেশি হয়, আপনার দুইটি মেয়েদের এবং দুইটি ছেলেদের দল গঠন করার প্রয়োজন হতে পারে। ব্যাখ্যা করুন:

• তোমার তালিকা তোমাদের দলের অন্যদের সাথে মিলিয়ে দেখ । কোন গুণাবলি অথবা বৈশিষ্ট্যুুলো তোমার দলে বেশিরভাগ ক্ষেত্রেই '১ নং' এবং '২ নং' এ আছে (সেগুলো হল খুবই গুরুত্বপূর্ণ) তা শনাক্ত কর। [তোমরা দেখতে পার যে, বিভিন্ন মানুষ একই গুণের জন্য বিভিন্ন শব্দ ব্যবহার করেছে, যেমন- উদার/দাতা; মজার/রসিক। ঘুরে ঘুরে দেখুন এবং প্রয়োজন অনুযায়ী সাহায্য করুন।।]
• প্রত্যেক দল থেকে একজনকে ডাকুন এবং তাদের দলের শনাক্তকৃত সবচেয়ে গুরুত্বপূর্ণ তিন অথবা চারটি গুণ বোর্ডে লিখতে বলুন। তালিকাটি ছেলেদের নাকি মেয়েদের দলের তা লিখে রাখুন।

৩ প্রত্যেকটি তালিকা ভালভাবে পড়ুন এবং নিয়লিখিত প্রশ্নগুনো নিয়ে একটি আলোচনা পরিচালনা করুন:

• এই দুইটি তালিকা থেকে তোমরা কী লক্ষ্য করেছ?

• এগুলোর মধ্যে कী মিল আছে?

• এগুলোর মধ্যে কী পার্থক্য আছে?

• কেন এগুলোর মধ্যে পার্থক্য আছে বনে তোমরা মনে কর?

- মেয়েদের কি ছেলেদের কাছে তাদের তালিকা নিয়ে কোন প্রশ্ন আছে?

- ছেলেদের কি মেয়েদের কাছে কোন প্রশ্ন আছে?

• তোমরা কি তালিকার মধ্যে কোন জেন্ডারের প্রথাগত ধারণা লক্ষ্য করেছ? যদি তা হয়, তাহনে কোনগুনো? 
8 (এই ধাপটি অবশ্যই ৫-৬ এর কমপক্ষে একদিন আগে শেষ করতে হবে। সম্ভব হলে নির্দিষ্ট কাজটি বোর্ডে লিখুন।) উদ্ভাবনীমূলক নির্দিষ্ট কাজটি ব্যাখ্যা করুন । এটি শ্রেণিকক্ষে শেষ করা হবে নাকি বাড়ির কাজ হিসেবে দেয়া হবে তা পরিষ্কার করে বলুন ।

- কেমন মানুষ তুমি তোমার বাকি জীবনে অথবা দীর্ঘস্থায়ী সঙ্গী হিলেবে পছন্দ করবে তা কল্পনা কর ।

- কল্পনার ঐ মানুষটির জন্য একটি চিঠি, কবিতা অথবা গান লেখ ।

- ঐ মানুষটির কাছ থেকে এবং দীর্ঘস্থায়ী সম্পর্কের মধ্যে তুমি কী খুঁজছো সে সম্পর্কে লেখ। তোমার প্রত্যাশা কী কী, তুমি কী রকম আচরণ আশা কর, এবং তুমি কী মেনে নেবে না সেগুলো অন্তর্ভুক্ত কর।

• অন্য দিকে, তুমি যদি তোমার জীবনে কোন দীর্ঘস্থায়ী সম্পর্ক রাখতে না চাও তাহনে তুমি তা নিয়ে লিখতে অথবা গাইতে পার।

• আগামীকাল (অথবা পরবর্তী সেশনে), তোমরা যা লিখেছ তা পড়ার অথবা উপস্থাপন করার একটি সুযোগ পাবে।
৫ উপস্থাপনার জন্য একটি দিন রাখুন, যখন শিক্ষার্থীরা তাদের চিঠি, কবিতা, এবং গান অন্যদের সামনে উপস্থাপন করবে। যদি আপনার প্রত্যেককে পড়তে অথবা উপস্থাপনা করতে দেয়ার সময় না থাকে তাহনে শুধুমাত্র আগ্রহী শিক্ষার্থীদের নিন। আপনি এ ছাড়াও শিক্ষার্থীদেরকে লেখকের নাম ছাড়া পড়ার জন্য তাদের লেখা, আপনার কাছে দিতে বলতে পারেন।

৬ নিম্নলিখিত প্রশ্নগুলো নিয়ে আলোচনা করার জন্য দশ মিনিট সময় হাতে রাখুন:

- তোমরা যা শুনেছ তার মধ্যে নির্দিষভাবে কী তোমাদেরকে স্পর্শ করেছে?

- ছেলে এবং মেয়েরা তাদের সঙ্গীদের মধ্যে যা চায় তার পার্থক্য কীভাবে সম্পর্কগুলোকে প্রভাবিত করে?

• তোমরা কীভাবে এই পার্থক্যগুলোকে ব্যাখ্যা করবে? তার মানে, কীভাবে মানুষ কিছু বৈশিষ্ট্যকে বেশি গুরুত্ব দেয় ?

- সুখী হওয়ার জন্য প্রত্যেকের কি একটি দীর্ঘস্থায়ী সম্পর্ক গড়ার প্রয়োজন আছে?

• কী ধরনের সম্পর্ক তোমাদের চাওয়া (অথবা না চাওয়া) উচিত এখন তা নিয়ে ভাবা কেন গুরুত্বপূর্ণ বলে মনে কর ? 


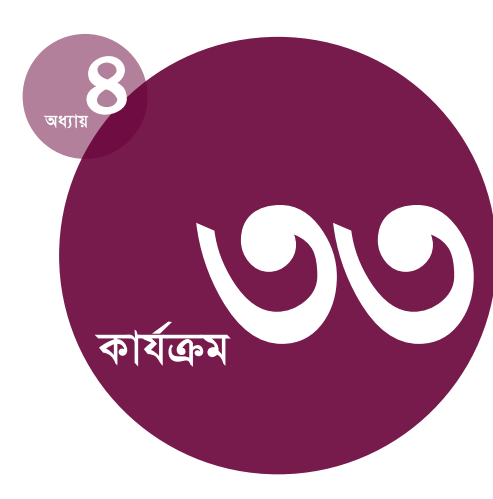

\section{সময়কাল :}

ধাপ ১-৩: ৩০ মিনিট

ধাপ 8-৬: ৫০ মিনিট

\section{উপকরণ:}

বোর্ড ও চক; চারটি সম্পর্কের গল্প (“সমস্যাযুক্ত সম্পর্কের কেসস্টাডি”), প্রত্যেকটি পৃথক কাগজে লেখা।

\section{প्रস্তুতি:}

কেসস্টাডিগুলো থেকে আপনার শিক্ষার্থীদের জন্য সবচেয়ে উপযুক্ত চারটি সম্পর্কের চিত্র বেছে নিন । এগুলোকে প্রয়োজন অনুযায়ী পরিবর্তন করুন এবং প্রত্যেকটি আলাদা আলাদা কাগজে অনুলিপি করুন । সমস্যাযুক্ত সম্পর্কের জন্য কারো নির্দেশনার প্রয়োজন আছে কিনা এবং কে সম্পর্কের মধ্যে নির্যাতনের ঝুঁকিতে থেকে সাহায্য চাইতে পারে তা খুঁজে বের করুন । (৬ নং ধাপের শেষ প্রশ্নটি দেখুন)।

\section{ভালবাসায় সমস্যাগুলো}

অধিবেশন সম্পর্কিত ধারণা: শিক্ষার্থীরা সম্পর্কের মধ্যে যে সমস্যাগুলি আছে তা দেখিয়ে এবং কিসের জন্য একটি সম্পর্ক শেষ হতে পারে তা ব্যক্ত করে গল্পগুলি শেষ করবে। (লক্ষণীয়: এই অধিবেশনের আগে শিক্ষার্থীদের ঘনিষ্ঠ সম্পর্করর মধ্য্য নিপীড়ন থেকে মুক্ত হবার অধিকার বিষয়ে জানা থাকতে হবে।)

উদ্দেশ্য: শিক্ষার্থীদদরকে সম্পর্কের মধ্যকার সমস্যাগুলি সমাধানের চেষ্টা করার এবং সম্পর্ক শেষ করার কৌশল শনাক্ত করতে সক্ষম করা; তারা একটি সম্পর্কের মধ্যে কোন আচরণগুলো নেনে নেবে না সেগুলো শনাক্ত করা; সম্পর্কের মধ্যকার মর্যাদা নিয়ে তাদের মূল্যবোধকে স্পষ্ট করা; সহযোগিতামূলক শিক্ষা পদ্ধতি চর্চা করা এবং মানুষের সাথে কথা বলার স্বাচ্ছন্দ্য বৃদ্ধি করা।

\section{निर्দেশাবলি}

১ ব্যাখ্যা করুন:

• আজকে আমরা সম্পর্কের মধ্যকার সমস্যা নিয়ে আলোচনা করব ।

- মানুষ তাদের প্রেমের সম্পর্কের মধ্যে স্বাভাবিকভাবে কী কী সমস্যার মুখোমুখি হয়? [উত্তরগুলো বোর্ডে লিখুন। উদাহরণগুলোর হতে পারে: পরিবার সম্পর্কিত, যোগাযোগ, অসামঞ্জস্যপূর্ণ আকাজ্ষা অথবা অঙ্গীকার, ঈর্যা এবং অর্থ বা সিদ্ধান্ত গ্রহণের ক্ষমতা নিয়ে দ্বন্দ্ব।]

২ শ্রেণিকক্ষের সবাইকে চারটি দলে ভাগ করুন । ব্যাখ্যা করুন:

• আমি প্রত্যেক দলকে সম্পর্কের সূচনা নিয়ে একটি গল্প পড়তে দেব। প্রত্যেক দলকে অবশ্যই সিদ্ধান্ত নিতে হবে কীভাবে গল্পটি শেষ করবে। যা করা সম্ভব হতো তার আলোকে এই দম্পতির কী করা উচিত তা সিদ্ধান্ত নাও।

• পাঁচ মিনিটের কম সময়ে তোমাদের সমাপ্ত করা গল্পটি নিয়ে একটি নাটিকার মাধ্যমে অভিনয় করার প্রস্তুতি নাও। যখন তোমরা কাজ করবে তখন দম্পতিটির কথোপকথন কল্পনা এবং অভিনয় কর।
• প্রত্যেক দল থেকে দুইজনকে দুইজন সঙ্গীর ভূমিকা নিতে হবে, এবং অন্য শিক্ষার্থীরা নতুন কিছু যোগ করবে অথবা অন্য ভূমিকা নিতে পারে, যেমন- পরিবারের সদস্যদের ভূমিকা।

৩ প্রত্যেক দলকে একটি করে সম্পর্কের চিত্র ও প্রস্তুতি নেয়ার জন্য সময় দিন ।

8 একটি দলকে তাদের গল্প অভিনয় করে দেখাতে বলুন। শিক্ষার্থীদের আলোচনার জন্য কয়েক মিনিট সময় নিন:

• এই দম্পতির মধ্যে কী সমস্যা অথবা দ্বন্দ্ব আছে?

• তারা তাদের সমস্যা কীভাবে সমাধানের চেষ্টা করেছে?

- দলটি যেভাবে সমস্যার সমাধান করেছে তা নিয়ে তোমরা কী ভাব? এই ফলাফলটি কতটা বাস্তবসম্মত ?

• পরামর্শ হিসেবে কারো কি অন্য কোন মতামত আছে? 
৫ অন্য নাটিকাগুলো একইভাবে করুন । প্রত্যেক দলকে দশ মিনিট করে সময় দিন (নাটিকা এবং আলোচনার জন্য)।

৬ নিয়লিখিত প্রশ্নগুলো নিয়ে একটি আলোচনা পরিচালনার মাধ্যমে শেষ করুন:

- কী কী লক্ষণ একটি সম্পর্কের মধ্যে সমস্যা তৈরির বিষয়ে সতর্ক করতে পারে ?

• কোন পরিস্থিতিগুলো একজন মানুষকে সমস্যা অথবা নির্যাতনমূলক সম্পর্কের মধ্যে রাখতে পারে ? (যেমনবাচ্চা, অর্থনৈতিক প্রয়োজন, বিবাহ বিচ্ছেদের লজ্জা।)

• কখনও কখনও মানুষ বিয়েসহ বিভিন্ন সম্পর্কের সমাপ্তি ঘটায়। মানুষ কি তালাকপ্রাপ্ত পুরুষ এবং মহিলাদেরকে বিবাহিত দম্পতিদের মতো একইভাবে অথবা আলাদাভাবে মূল্যায়ন করে? [যদি পার্থক্য থাকে, তাহলে জিজ্ঞেস করুন: কেন তাদের আলাদা মনে করা হয়? তোমাদের কাছে এগুনো কেমন লাগে?]

• কোন পরিস্থিতি অথবা আচার-আচরণগুলো তোমাদের সম্পর্কের সমাপ্তি ঘটানোর কারণ হতে পারে ?

• যে ব্যক্তি সম্পর্কের মধ্যে নির্যাতনের ঝুঁকিতে রয়েছে, সাহায্যের জন্য সে কোথায় যেতে পারে ? একজন মানুষ সমস্যাযুক্ত সম্পর্কের বিষয়ে সাহায্য অথবা অধি পরামর্শের জন্য কোথায় যেতে পারে ? 


\section{সমস্যাযুক্ত সম্পর্ক নিয়ে কেসস্টাডি}

এ্যাডে এবং বেটো মাধ্যমিক বিদ্যালয় থেকে সম্পর্ক ফরু করেছিল এবং এখন তারা পৃথক বিশ্ববিদ্যালয়ে লেখাপড়া করছে। তারা উভয়ে ছুটির দিনে বাড়িতে যায়। গতরাতে তারা অন্য বন্ধুদের সাথে বাইরে গিয়েছিল, এবং বেটো এ্যাডেকে নিয়ে কিছু মন্তব্য করেছিল যেগুনো তার অনুভূতিতে আঘাত করেছিল। যখন এ্যাডেকে বিমর্ষ দেখাচ্ছিল, তখন বেটো তাকে বলেছিল লে শুধু ঠাট্টা করেছিল। পরবর্তীতে, এ্যাডে বেটোকে এমন একটি মহিলার বিষয়ে জানতে চেয়েছিল যাকে নিয়ে বেটো আলোচনা করেছিল। বেটো স্বীকার করেছিল যে, লে তার সাথে যৌনমিলন করেছিল-

চালা এবং দাউদের এক বছর বিয়ে হয়েছে। তারা প্রায়ই উত্তপ্ত বাক্য বিনিময় করত কিন্তু তারা প্রায় এক মাসের আগে কখনই ঠাণ্ডা হত না। কালার পরিবার নিয়ে তাদের অনেক ঝগড়া হয়েছিল এবং দাউদ তাকে কয়েকবার চড় মেরেছিল। পরের দিন দাউদ খুব দুঃখিত ছিল, এবং চালার জন্য উপহার নিয়ে এসেছিল। এবং প্রতিজ্ঞা করেছিল এমন আর কখনও ঘটবে না। কিন্তু গতরাতে এমন হয়েছে। আজকে সকালে যখন চালার ঘুম ভেঙ্গেছে, তখন তার চোখ কালো এবং ফুলে গিয়ে বন্ধ হয়ে ছিল

এলিনা এবং ফিন্টজ কয়েক বছর থেকে থ্রেম করছে। তারা অনেক সময় একসাথে থেকেছে- তারা অনেক হালে, সবকিছু নিয়ে আলোচনা করে এবং তাদদর আবেগপূর্ণ শারীরিক সম্পর্ক ছিল। এখন মনে হয় তারা একে অপরের সাথে খুব বেশি অভ্যস্ত হয়ে গেছে। তারা বেশিরভাগ সময় টিভি দেখে এবং খুব কম কথা বলে। এমনকি তাদের যৌনজীবনও গৎবাধা এবং অনিয়মিত হয়ে গেছে। এলিনা বিস্মিত “আমার ভালবাসার আগের অনুভূতির কী হয়েছে?"

গ্রেটি এবং হেনরি প্রায় এক বছর আগে থেকে বাইরে যাওয়া শুরু করেছে। গ্রেটির বয়স ১৮ বছর এবং মাত্র বিশ্ববিদ্যালয়ে লেখাপড়া শুরু করেছে। হেনরির বয়স ২৫ বছর এবং সে চাকরিজীবি। গ্রেটি বিশ্ববিদ্যালয়ে যাওয়ার ব্যাপারে সত্যিই উৎসাহিত এবং নতুন বন্ধু তৈরির ব্যাপারে অগ্রগামী। সে অনেক লেখাপড়া করে, এবং ক্যাম্পালের জীবন উপভোগ করে। হেনরি মনে করে সে এখন বিয়ে এবং পরিবার তৈরির জন্য প্রস্তুত। সে মনে করে সে গ্রেটিকে বিয়ে করতে চায়। সে সমপ্রতি বিয়ের কথা বলেছে এবং গ্রেটির প্রতিক্রিয়ায় হতভম্ব হয়ে গেছে। গ্রেটি হেনরির দিকে তাকাল যেন সে (হেনরি) পাগল এবং বলল, "আমি মাত্র আমার লেখাপড়া শুরু করেছি । আমি এখন জীবনকে উপভোগ করতে চাই এবং ভাল কিছু করার ওপর গুরুত্ব দিতে চাই, বিয়ে করতে চাই না!" 


\section{সমস্যাযুক্ত সম্পর্ক নিয়ে কেসস্টাডি}

ইভান এবং জোসেফা এক বছরেরও বেশি সময় ধরে সঙ্গী । তারা দুইজনই কাজ করে এবং তাদের বন্ধুদের সাথে বাইরে যেতে পছন্দ করে। গতরাতে তারা একটি ক্লাবে গিয়েছিল। জোসেফা তার মেয়ে বন্ধুদের সাথে আলাপ করছিল, হাসাহাসি করছিল এবং নাচ করছিল। ইভান অনেকের সাথে নাচ করছিল এবং সদ্য পরিচিত এক মহিলার সাথে অনেক মজার কাথোপকথন হয়েছিল। সে মনে করে তার এবং জোলেফোর উভয়েরই একটি ভাল সময় পার হয়েছে। কিন্তু যখন তারা বাড়ি ফিরল, জোলেফা রাগান্বিত ছিল। “আমি দেখেছি তুমি ঐ মহিলার দিকে কীভাবে তাকাচ্ছিলে। আমাকে বলো না যে তুমি তার প্রতি আকর্ষিত হওনি! এবং তুমি যেভাবে নাচ করছিনে! ঐখানকার সকলের মনোযোগ আকর্ষণ করতে চেষ্টা করছিলে!”

কিকি, বিশ্ববিদ্যালয়ের একজন শিক্ষার্থী, খত্তকালীন কাজ করে, কিন্তু খুব বেশি আয় করে না । লিসা একজন পেশাজীবী এবং ভাল বেতন পায়, তাই সে তাদের বেশির ভাগ ব্যয় বহন করে। কিকি এবং লিসা ঠিক করল যে যখন সম্ভব হয় কিকি মুদি জিনিসপত্র কিনবে এবং ভাড়া দেবে। কিন্তু এটি খুব কম সময়ই হয়। যখন কিকি তার নিজের জন্য পোশাক কেনে, তার দামী জিনিস কেনার প্রবণতা থাকে। এতে লিসা বিরক্ত হয় কারণ সে মনে করে এই ব্যয় অপ্রয়োজনীয়। কয়েকদিন আগে, কিকি ছুটির দিনে যে কাজ করেছিল তা থেকে কিছু টাকা পেয়েছিল। গতকাল কিকি বাইরে যায় এবং লিসার সাথে আলোচনা না করে ঐ টাকার অর্ধেকের বেশি একটি কম্পিউটার কিনে খরচ করে ফেলে । যখন সে এটি নিয়ে বাড়ি আসল, লিসা খুব রেগে গেল ।

মিকা এবং নবু বিবাহিত এবং এক বছর, তিন বছর ও ছয় বছর বয়সের তিনটি সন্তান আছে। যখন মিকার মাসিক হতে দেরি হচ্ছিল, তখন সে স্বাস্থ্যকেন্দ্রে গেল এবং জানল যে, সে পাঁচ সপ্তাহের গর্ভবতী । যখন মিকা এটি শ্নল, তার উন্মাত্ত লাগছিল। নবু এবং সে কোনমতে জীবন যাপন করে এবং তাদের অর্থ সীমিত। সে আরো রাগান্বিত ছিল। সে সন্দেহ করল যে, যে রাতে নবু মাতাল হয়ে বাড়ি ফিরেছিল এবং কনডম ব্যবহার করেনি সেই রাতে সে গর্ভবতী হয়েছে। সে নবুকে বলল যে, সে গর্ভপাত করাতে চায়। নবু হতভম্ব হয়ে যায়। সে বলল “আমি জানি এটা কষ্টের, কিন্তু আমি তোমাকে তা করতে দিতে চাই না----" 


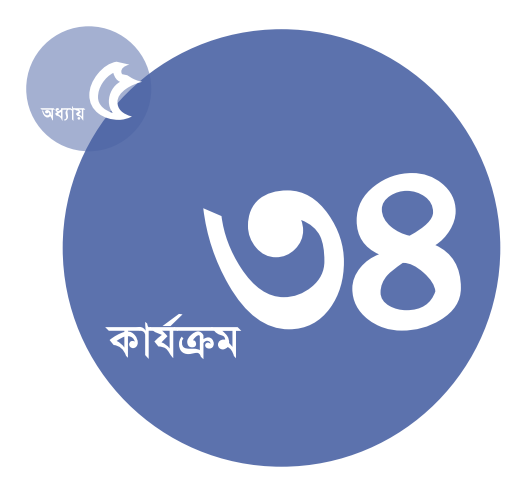

\section{আমি কি তা বলেছিলাম ? যোগাযোগের বিভিন্ন ধরন}

অধিবেশন সম্পর্কিত ধারণা: শিক্ষার্থীরা অস্বস্তিকর পরিস্থিতিতে মৌখিকভাবে প্রতিক্রিয়া জানানোর বিকল্প পদ্ধতি শনাক্ত করবে এবং উগ্রতা, দৃঢ়তা ও আনুগত্যের মধ্যে পার্থক্য নির্ণয় করবে। তারা অস্বস্তিকর পরিস্থিতিতে প্রতিক্রিয়া জানানোর নিজস্ব ধরন নিয়ে একটি প্রশ্নপত্র পূরণ করবে ।

উদ্দেশ্য: শিক্ষার্থীদেরকে বিভিন্ন রকম যোগাযোগের ধরন সম্পর্কে উপলক্ধি বৃদ্ধি করা এবং তাদের দৃঢ প্রতিক্রিয়া জানানোর সক্ষমতা বৃদ্ধি করা; তাদের বিল্লেষণ এবং কথোপপকথনের দক্ষতা বৃদ্ধি করা।

\section{সময়কাল:}

8৫-৬০ মিনিট

\section{উপকরণ:}

যদি সম্ভব হয় প্রত্যেক ছাত্রের জন্য উভয় অনুশীলনপত্রের অনুলিপি (“আত্ন-মূল্যায়ন” এবং “এই উত্তরগুনো বিশ্লেষণ কর”)।

\section{প्रস্তুতি:}

যদি সম্ভব হয় হ্যান্ডআউটগুলোর অনুলিপি তৈরি করুন । অন্যথায়, তাদেরকে পড়ানোর প্রস্তুতি নিন ।

\section{नির্দেশাবলি}

১ অধিবেশনটির ধারণা দিন:

• আজকে আমরা যোগাযোগের বিভিন্ন ধরন সম্পর্কে শিখব।

• বিশেষ করে কোন পরিস্থিতিতে যদি আমাদের কাছে সবকিছুই অস্বস্তিকর লাগে, তাহলে আমরা স্পষ্টভাবে অথবা যেভাবে আমরা চাই সেভাবে যোগাভোগ করতে পারিনা।

- কী কী কারণে আমাদের ইচ্ছাকে সবচেয়ে ভালভাবে প্রকাশ করার ক্ষেত্রে সমস্যা হতে পারে ? [নিম্নলিখিত কারণগুলো জানার চেষ্টা করুন এবং সেগুনো বোর্ডে লিখুন:] আমি জানিনা কীভাবে নিজেকে স্পষ্টভাবে প্রকাশ করতে হয়। আমি ভীত হই যে, যদি আমি একমত না হই, তাহলে অন্যরা আমার সম্পর্কে খারাপ ধারণা করবে।

আমি ভীত হই যে, আমি কোনভাবেই আমি শুনতে পারবনা যে আমি যা বলি তার কোন অর্থ নেই।

আমি ভীত হই যে, আমার মেজাজ বিগড়ে যেতে পারে।

মধ্যস্থতার জন্য অন্য মানুমের ওপর আস্থা রাখা আমার কাছে সহজ মনে হয়।
• প্রথনে, আমরা উগ্র, অনুগত এবং দৃঢ় যোগাযোগের মধ্যে পার্থক্য দেখব। তারপর তোমরা একটি অস্বস্তিকর পরিস্থিতিতে তোমাদের নিজস্ব প্রতিক্রিয়ার ধরন নিয়ে একটি প্রশ্নপত্র পুরণ করবে।

- অনুগত যোগাযোগ এবং উগ্র যোগাযোগের মধ্যে কী পার্থক্য আছে বলে তোমাদের মনে হয়? আমরা দৃঢ় যোগাযোগ বলতে কী বুঝি? [উত্তর জানার চেষ্টা করুন: সৎ হওয়া; স্পষ্ট হওয়া; তোমাদের অনুভূতি এবং প্রয়োজন সম্পর্কে কথা বলা; দৃঢ়তা সূচক উগ্রতা ছাড়া অঙ্গঙ্গি ব্যবহার করা; নিজের জন্য কথা বলা; স্পষ্টভাবে কথা বলা।

অস্পষ্ট যোগাযোগ বলতে কী বোঝায় ?

[উত্তর জানার চেষ্টা করুন: সম্পূর্ণ অনুগত না হওয়া, কিন্ত স্পষ্ট হতে অপারগ।] 
২ শিক্ষার্থীদেরকে জোড়া গঠন করতে বলুন এবং “এই উত্তরগুলো বিশ্লেষণ কর” অনুশীলনীপত্র বিতরণ করুন (অথবা সকলে শুনতে পায় এমনভাবে পড়ার প্রস্তুতি নিন)

• তোমাদের সঙ্গীদেরকে নিয়ে বাম দিকের সারির দ্বন্দ্যময় পরিস্থিতির বর্ণনা পড়। তারপর পরবর্তী সারির বিভিন্ন উত্তরগুলো পড়। প্রত্যেক উত্তরের ক্ষেত্রে দেখ যে ঐ উত্তরটি উগ্র, অনুগত নাকি দৃঢ়। দ্বন্দ্ব পরিস্থিতি ৫ এর জন্য একটি উক্তি এবং বিভিন্ন রকমের উত্তর তৈরি কর।

৩ ্রত্যেকটি দ্বনन্ব পড়ন এবং শিক্ষার্থীদেরকে সঠিক উত্তর দিতে বলুন এবং দৃঢ় উত্তরের তুলনায় উগ্র উত্তর শুনতে কেমন মনে হয়েছে মন্তব্য করতে বলুন। (উত্তরপত্রঃ উত্তর ক, চ, ঝ ও ট হলে উগ্র। উত্তর গ, ঘ ও জ হলে অনুগত এবং উত্তর খ, ঙ, ছ ও ঞ হলে দৃঢ়।
8 “আত্মমূল্যায়ন” এর অনুশীলনপত্র বিতরণ করুন এবং শিক্ষার্থীদেরকে বলুন নিজেদের জন্য এটি পূরণ করতে। (বিকল্পভাবে বোর্ডে উক্তিগুনো এবং সম্ভাব্য উত্তরগুনো লিখুন, এগুলো সকনে শুনতে পারে এমনভাবে পড়ন এবং শিক্ষার্থীদের তাদের নিজেদের উত্তরগুলো লিখতে বলুন।)

৫ নিম্মলিখিত প্রশ্নগুনো নিয়ে আলোচনা শেষ করুন:

• তোমাদের নিজেদের নিজস্ব যোগাযোগের ধরন বিষয়ে তোমরা को শিখেছে?

• কীভাবে দৃঢ়তার সাথে যোগাযোগ করতে হয় তা শিখতে কি এগুলো কাজে লাগবে ? 
আত্নমূল্যায়ন: যখন আমি অস্বস্তিবোধ করব তখন আমি কীভাবে যোগাযোগ করব?

যখন আমি একজন ছেলের সাথে অস্বস্তিকর পরিস্থিতিতে থাকব, তখন আমার হওয়ার প্রবণতা থাকবে:

$\square$ অনুগত $\quad \square$ দৃঢ़ প্রত্যয়ী, সম্মানজনক $\quad \square$ আক্রমণাত্নক $\quad \square$ পরোক্ষ অথবা মেকি/বানানো।

যখন আমি একজন মেয়ের সাথে অস্বস্তিকর পরিস্থিতিতে থাকব, আমার হওয়ার প্রবণতা থাকবে:

$\square$ অনুগত $\quad \square$ দৃঢ़ প্রত্যয়ী, সম্মানজনক $\quad \square$ আক্রমণাত্মক $\quad \square$ পরোক্ষ অথবা মেকি/বানানো ।

যখন আমি একজন বয়স্ক পুরুষের সাথে অস্বস্তিকর পরিস্থিতিতে থাকব, আমার হওয়ার প্রবণতা থাকবে:

$\square$ অনুগত $\quad \square$ দৃঢ প্রত্যয়ী, সম্মানজনক $\quad \square$ আক্রমণাত্নক $\quad \square$ পরোক্ষ অথবা মেকি/বানানো।

যখন আমি একজন বয়স্ক মহিলার সাথে অস্বস্তিকর পরিস্থিতিতে থাকব, আমার হওয়ার প্রবণতা থাকবে:

$\square$ অনুগত $\square$ দৃঢ़ প্রত্যয়ী, সম্মানজনক $\quad \square$ আক্রমণাত্নক $\square$ পরোক্ষ অথবা মেকি/বানানো।

যখন আমি এমন একজনের সাথে অস্বস্তিকর পরিস্থিতিতে থাকব যাকে আমি আমার সমকক্ষ মনে করি না, আমার হওয়ার প্রবণতা থাকবে:

$\square$ অনুগত $\quad \square$ দৃঢ़ প্রত্যয়, সম্মানজনক $\quad \square$ আক্রমণাত্নক $\quad \square$ পরোক্ষ অথবা মেকি/বানানো।

$\square$ আমি এটির উত্তর দিতে পারি না, কারণ আমি সবাইকে আমার সমকক্ষ মনে করি।

আমার কাছে রাগের অনুভূতি প্রকাশ করা হল:

$\square$ একেবারে সহজ (কিন্তু আমি মাঝে মাঝে উগ্রভাবে উত্তর দেই)।

$\square$ একেবারে সহজ (এবং আমি কখনই উগ্রভাবে অথবা নিপীড়নমূলক উত্তর করি না)।

$\square$ সহজও নয় কঠিনও নয়

$\square$ কিছুটা অথবা অনেক কঠিন

যখন আমি নিজেকে দুর্বল অথবা ঝুঁকিপূর্ণ মনে করি তার প্রকাশ করা হল:

$\square$ একেবারে সহজ (কিন্তু আমি মাঝে মাঝে উগ্রভাবে উত্তর দেই)।

$\square$ একেবারে সহজ (এবং আমি কখনই উগ্রভাবে অথবা নিপীড়নমূলক উত্তর করি না)।

$\square$ সহজও নয় কঠিনও নয়

$\square$ কিছুটা অথবা অনেক কঠিন 


\section{এই উত্তরগুলো বিপ্লেষণ কর}

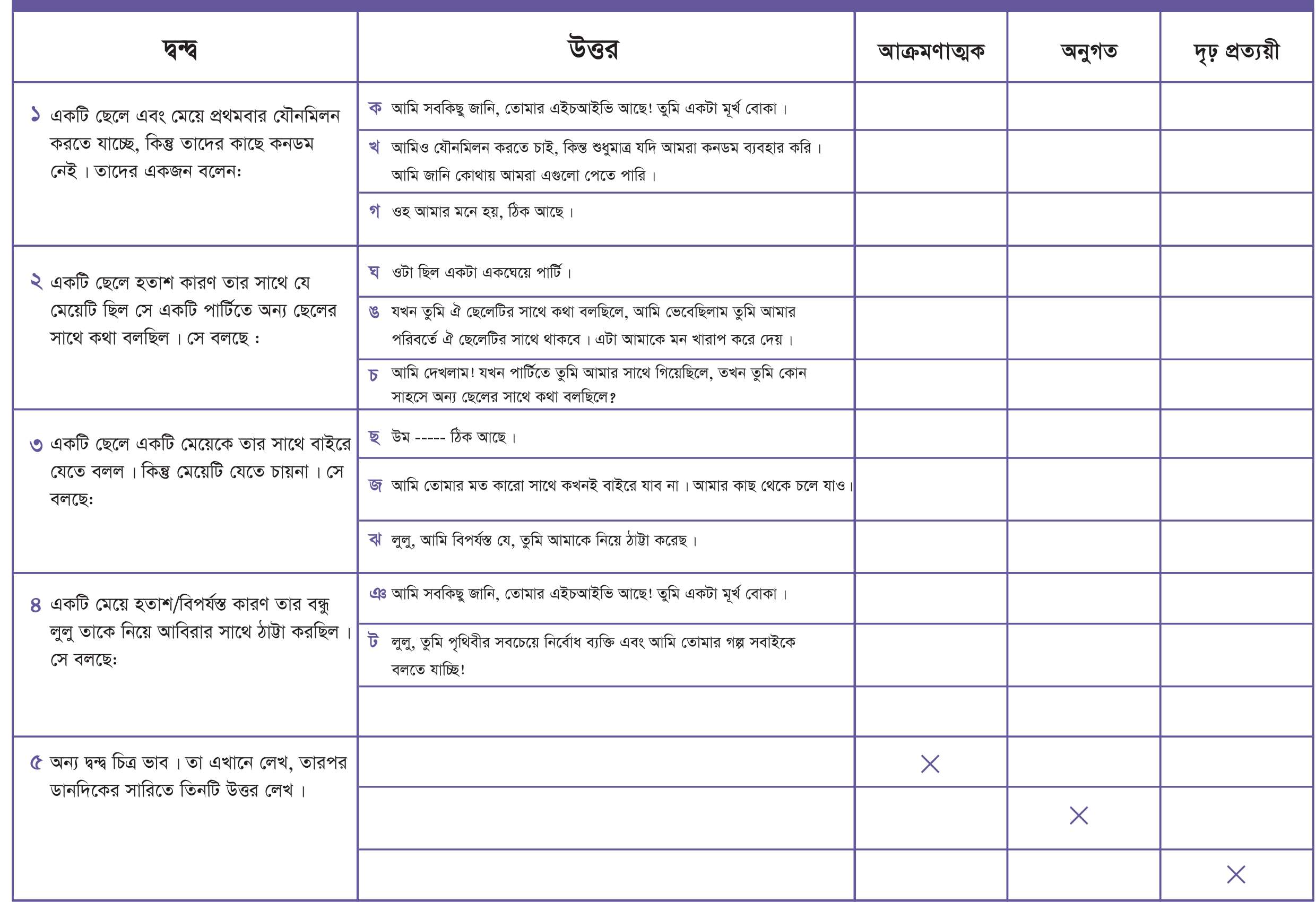




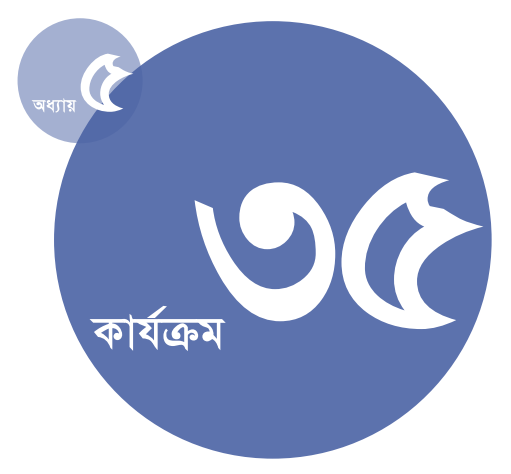

সময়কাল:

৫০-৬০ মিনিট

\section{উপকরণ:}

বোর্ড ও চক; কিউ কার্ড হ্যান্ডআউট।

\section{প্রস্তুতি:}

নির্দেশিকা (অথবা আপনার নিজের শিক্ষাক্রম) এর অধ্যায়-৫ থেকে

কার্যকরী যোগায্যাগ বিষয়ের

তথ্যগুনো পুনরায় পড়ন। ধাপ ৬ এর

নির্দিষ্ট আচরণের তালিকাটি পুনরায়

পড়ন এবং আপনার সমাজের

গ্রহণযোগ্য আচরণ যদি ভিন্ন হয়ে

থাকে তাহুলে এগুনো পরিবর্তন করুন

(যেমন- কিছু কিছু স্থানে চোখের

ইশারাকে ভদ্রভাবে দেখা হয় না; না সুচক মাথা নাড়ার ভিন্ন অর্থ থাকতে

পারে)। এক (অথবা দুই) জোড়া

আগ্রহী শিক্ষার্থীকে খুঁজুন যারা কার্যকরী

ভূমিকা পালন করতে পারবে এবং

তাদের জন্য কিউ কার্ড তৈরি করতে

পারবে (নমুনা কিউ কার্ড দেখুন)।

আগেরদিন আগ্রহী শিক্ষার্থীদেরকে

তৈরি করলে ভাল হয়, তাহলে তারা

মোহড়া করতে পারবে অভিনয়ের

আগে। সর্বশেষে, আপনার নিজেব

শ্রবণ দক্ষতা সম্পর্কে সচেতন হোন-

বিশেষ করে এই সেশন-এর সময়ে!

\section{সক্রিয় শ্রবণ}

অধিবেশন সম্পর্কিত ধারণা: শিক্ষার্থীরা এমন আচরণ শিখবে, যেগুলো যোগাযোগ বৃদ্ধি (অথবা হানি) করে।

উদ্লেশ্য: যোগাযোগ বৃদ্ধি করে এমন আচরণে শিক্ষার্থীদেরকে অভ্যন্ত হতে সক্ষম করা।

\section{निर्দেশাবলি}

১ থ্রত্যেক নাটিকার জন্য আগ্রহী ভূমিকা পালনকারী শিক্ষার্থীদেরকে একটি করে কিউ কার্ড দিন। यদি প্রয়োজন হয়, যখন আপনি ২নং ধাপ করবেন তখন প্রস্তুত হওওয়ার জন্য অথবা মোহড়ার জন্য কয়েক মিনিট সময় দিन।

২ ব্যাখ্যার মাধ্যমে শ্রেণিকক্ষের বাকিদের কাছে বিষয়টির ধারণা দিন:

• আমরা বলি যে, যোগাযোগ হচ্ছে দ্বিমুখি প্রক্রিয়া । যখন একজন মানুষ কথা বলছে, তখন অন্যজন কি করছে? [ফুনছে ।] তাই শ্রবণ কি যোগায্যাদের অংশ? [ঁ্যা ।] আজকে আমরা দেখব যখন আমরা শুনছি তখন কী যোগাযোগ করছি। আমরা শিখব যে, 'সক্রিয় শ্রবণ’ মানুমের মধ্যে যোগাযোগে কত বেশি পার্থক্য করে।

• আমরা একটি প্রত্যক্ষ প্রমাণের মাধ্যমে শুরু করব। তোমাদের খাতা নাও। শ্রোতাদেরকে পর্যবেণ কর, তোমরা পর্যবেক্ষণ করেছ এমন প্রত্যেকটি আচরণ লেখ, যেগুলো তোমরা মনে কর যে, কার্যকরী যোগাযোগের মাধ্যমে পেয়েছ।

৩ আগ্রহী শিক্ষার্থীদেরকে ‘নেতিবাচক’ যোগায্যাগের নাটিকাটি উপস্থাপন করতে বলুন (নাটিকা \#১)।
8 প্রশ্নের মাধ্যমে নাটিকাটি ব্যক্ত করুন:

• তোমরা কীভাবে এই কথোপকথনটি মূল্যায়ন করবে? এটা কি সন্তুষ্টিকর এবং কার্যকরী ছিল?

• ‘শ্রোতারা’ কি ভাষাগত এবং ভাষাহীন আচরণ উপস্থাপন করেছিল ? শিক্ষার্থীরা যে নেতিবাচক আচরণগুলো উল্লেখ করেছে তার প্রত্যকটি বোর্ডে লিখুন। নিম্ললিখিত উত্তরগুনো জানার চেষ্টা করুনঃ]

\section{ভাষাহীন আচরণ:}

ভ্রুটিটি করা

চিন্তিত দেখানো, আকাশের দিকে তাকিয়ে চোখ ঘোরানো 'না' সূচকভাবে মাথা ঘোরানো এদিক ওদিক তাকানো অথবা ঘোরাঘুরি করা, বিরক্তি ভাব দেখানো অথবা

অনুৎসাহিত, হাই তোলা

\section{ভাষাগত আচরণ:}

বক্তার কথায় বিঘ্ন ঘটানো, বক্তা খুব বেশি কথা বলছে তা নির্দেশ করে অধর্য্য দেখানো,

বক্তাকে নেতিবাচকভাবে মূল্যায়ন করা, যেমন ‘তুমি ভুল বলছ’ অথবা 'এটি একটি অকার্যকর ধারণা !

• ‘বক্তাকে’ জিজ্ঞস করুন শ্রোতারা না ওনলে কেমন অনূভূত হয় 
৫ আগ্রহী শিক্ষার্থীদেরকে ‘ইতিবাচক’ যোগায্যাগের নাটিকাটি উপস্থাপন করতে বলুন (নাটিকা \# ২)

৬ প্রশ্নের মাধ্যমে নাটিকাটি ব্যক্ত করুন:

• এই কথোপকথনটি তোমরা কীভাবে মূল্যায়ন করবে? এটা কি আরো বেশি সন্তুষ্টিকর ছিল ?

• কোন ভাষাগত এবং ভাযাহীন আচরণ এই কথোপকথনটি পূর্বেরটির চেয়ে বেশি সার্থক করেছে? [বোর্ডে, শিক্ষার্থীদের উল্লিখিত প্রত্যেকটি ইতিবাচক আচরণ লিখুন। নিম্মলিখিত উত্তরগুনো জানার চেষ্টা করুন]

\section{ভাষাহীন আচরণ:}

চোখের মাধ্যমে যোগাযোগ রক্ষা করা

সম্মতিসূচক মাথা নাড়ানো (যেন ‘ছ্যা’ বলছ)

তুমি শুনছ এটা দেখানোর জন্য বক্তার দিকে একটু হেনে থাকা হাসি বিনিময় করা অথবা পিঠ প্রশংসা সূচক স্পর্শ করা

\section{ভাষাগত আচরণ:}

তুমি বক্তার কথা বুঝেছ তা নিশ্চত হতে স্প/ষ্টভ/বে জানতে চাওয়া, আরো বেশি শোনার জন্য আগ্রহ দেখানো (যেমন, এটি সম্পর্কে আমাকে আরো কিছু বলুন...........") বক্তার অনুভূতির মূল্যায়ন করা (যেমন, “আমি বুঝতে পারি তোমার কেমন মনে হয় ”)

বক্তার চেতনার মূল্যায়ন করা (যেমন, “ভালবিষয় ”) বক্তাকে অসম্পূর্ণ মনে করা হচ্ছে এমন কোন উক্তি থেকে বিরত থাকा

বক্তার মনে যা ছিল তা আপনার সাথে আলোচনা করার জন্য তাকে ধন্যবাদ দেয়া
৭ সক্রিয় শ্রবণ চর্চা করার জন্য শিক্ষার্থীদেরকে তিনজন করে দল তৈরি করতে বলুন । ব্যাখ্যা করুন:

• প্রত্যেক দল থেকে, একজন দুই মিনিটের জন্য কথা বলবে। যখন তোমার কথা বলার সময় আসবে, তখন তুমি এমন বিষয়ে কথা বলবে যা নিয়ে তুমি উদ্বিগ্ন অথবা হতাশ। এই অনুশীলনীর জন্য, এমন একটি বিষয় বেছে নাও যা গোপনীয় নয় এবং সহজে তোমার সহপাঠীদের সাথে আলোচনা করতে পার। তোমার আশাবাদী, রাগান্বিত, দুঃখিত, ব্যথিত অথবা উদ্বিগ্ন লাগতে পারে । যদি তুমি বলার জন্য কোন কিছু চিন্তা করতে না পার, যুব পরিষদের প্রধান হওয়ার জন্য তোমাকে ভোট দিতে বল ।

• দ্বিতীয় ব্যক্তি সক্রিয় শ্রবণের চর্চা করবে এবং তৃতীয়জন পর্যবেক্ষণ করবে ও মতামত দেবে। প্রধানত তোমরা শ্রোতার জন্য মতামত দেবে, কিন্তু তোমরা বক্তার জন্যও মতামত দিতে পার। দুই মিনিট পর, তোমাদের ভূমিকা চক্রাকারে আবর্তিত হবে। তারপর আমরা তৃতীয় রাউন্ড শুরু করব। এইভাবে, তোমাদের প্রত্যেকে প্রত্যেকটি ভূমিকা পালন করবে।

৮ প্রথমবার শুরু করুন্ন:

• বক্তা, তোমার জীবনের এমন কোন বিষয় যা নিয়ে তুমি হতাশ তা শ্রোতাকে বলতে শুরু কর।

• [২ মিনিট পর:] থাম। পর্যবেক্ষক, ৬০ সেকেন্ডে মতামত দাও। 
৯ এক মিনিট পর: দ্বিতীয়বার ফুরু করুন:

• বক্তা, তুমি শ্রোতা হয়ে যাও। শ্রোতা, পর্যবেক্ষকের ভূমিকা নাও। পর্যবেক্ষক, তুমি বক্তা হবে

• [দুই মিনিট পর: ] থাম। পর্যবেক্ষক, এক মিনিটে মতামত দাও।

১০ এক মিনিট পর: রাউন্ড ৩ শুরু করুন । যখন শিক্ষার্থীরা এই রাউন্ড শেষ করছে, আপনি শ্যুমাত্র তাদের ভাবনাকে উদুদ্ধ করার জন্য চিকিৎসক লেখক কার্ল মিনিংজার-এর এই উক্তিটি বোর্ডে লিখতে পারেন “শ্রবণ একটি চৌম্বকীয় এবং অদ্ভুত বিষয়, একটি সৃষ্টিশীল শক্তি। যে বন্ধুরা আমাদের কথা শোনে, তাদেরকে আমরা এগিয়ে নিতে পারি। যখন আমাদেরকে শোনা হয়, তা আমাদেরকে সৃষ্টি করে, আমাদেরকে উন্মোচন এবং প্রসারিত করে।"

• প্রথম অংশ চক্রাকারে একাধিকবার করুন এবং আবার শুরু করুন ।

• [দুই মিনিট পর:] থাম। পর্যবেক্ষক, মতামত দাও।

১১ এই প্রশ্নগুনোর যেকোন একটি নিয়ে আলোচনার জন্য দশ মিনিট সময় রাখুন:

• যখন তুমি বক্তা এবং অন্য ব্যক্তি তোমার কথা না শোনে তখন তোমার কেমন লাগে?

• যখন দুইজন ব্যক্তির মধ্যে সত্যিকারের দ্বন্দ্ব থাকে এবং যখন উভয়ই হতাশ থাকে তখন কী ঘটে? তখন ভালভাবে যোগাযোগ করা সহজ নাকি কঠিন?
- কিছু গবেষক পর্যবেক্ষণ করেছেন যে, পুরুষ এবং মহিলাদের যোগাযোগের ভিন্ন ভিন্ন ধরন রয়েছে। যখন মেয়ে এবং নারীরা হতাশ থাকে তখন নিজেদেরকে কীভাবে প্রকাশ করতে হবে, এটা নিয়ে তারা কী শিখেছে?

• যখন ছেলে এবং পুরুষরা হতাশ থাকে তখন নিজেদেরকে কীভাবে প্রকাশ করতে হয় এটা নিয়ে তারা কি শিখেছে? মানুষ কি এমনভাবে যোগাযোগ করতে শিখতে পারে যেটা সহিংস অথবা উগ্গ নয়?

• যুবক-যুবতীদের ঘনিষ্ঠ সম্পর্কের মধ্যকার যোগাযোগের ক্ষেত্রে সহিংসতা এবং উগ্রতা কী প্রভাব ফেলতে পারে বলে তোমরা মনে কর?

• মানুষ পুরুষ এবং মহিলাদের মধ্যে যোগাযোগের উন্নয়ন করার জন্য কী করতে পারে, বিশেষ করে যখন তাদের দ্বন্দ্ব হয় ? যখন তোমরা পর্যবেক্ষক ছিলে তখন তোমাদের মধ্যে কতজন গঠনমূলক মতামত দিতে সক্ষম হয়েছিলে ?

• [यদি সময় থাকে:] দেয়ালে লেখা উক্তিটি সম্পর্কে তোমরা কী মনে কর ? মতামত দাও?

• এ এমন একটি বিষয় ভাব যা তুমি তোমার যোগাযোগের ধরন উন্নয়নের জন্য করতে চাও। আজকে রাতে এটি চর্চা কর এবং সপ্তাহ জুড়ে অন্যদের সাথে চর্চা কর। যখন তুমি তোমার নিজেকে প্রকাশ করতে চেষ্টা কর তখন অন্যরা কীভাবে শোনে সেটাও লক্ষ্য কর। 


\section{“দুর্বল শ্রোতা" নাটিকার জন্য সংলাপ কার্ড: (\# ১)}

তোমাদের মধ্যে একজন “বক্তা” হবে এবং একজন “শ্রোতা” হবে।

বক্তা: একটি কাল্পনিক ঘটনার ওপর ভিত্তি করে একটি নাটিকা টৈরি কর। যেমন ধর, ১. তোমার এমন কিছু ঘটেছিল (মিছা মিছি) যেটি তুমি মনে কর সঠিক ছিল না; অথবা ২. একটি খারাপ পরিকল্পনা অথবা ধারণা (মিছা মিছি) তুমি করেছিনে এবং যা তুমি করার জন্য উদগ্রীব ছিলে । নাটিকায় তোমার এমন কিছু অভিজ্ঞতা অথবা ধারণা এবং অনুভূতি তুলে ধরবে যা তোমার নিজের কাছে স্পষ্ট। তুমি তোমার সঙ্গীর সাথে এটি নিয়ে কথা বলতে কয়েক মিনিট সময় নাও, শোনাতে চেষ্টা কর । দুই অথবা তিন মিনিট বলার পরিকল্পনা কর।

তোমার সঙ্গী যেভাবে শ্নছে সেভাবে কথা বলার চেষ্টা কর। যদি মনে হয় সে তোমার কথা ওনছে না, তোমার হতাশা দেখাও, জোরে বল, অথবা এই অবস্থায় যা করলে স্বাভাবিক মনে হয়, সেভাবে সাড়া দাও। অপর ব্যক্তিটি যদি মনোযোগ এবং শ্রদ্ধার সাথে তোমার কথা শোনে তাহলে অনুরূপ আচরণ কর।

শ্রোতা: তুমি একজন দুর্বল শ্রোতা । যখন বক্তা তোমার সাথে কথা বলার চেষ্টা করছিল, দুর্বল শ্রবণ দক্ষতা দেখাও। তোমার কাজ হচ্ছে নিচের তালিকা থেকে যতবেশি সম্ভব নেতিবাচক ভাষাগত এবং ভাষাহীন আচরণগুলো করা:

ভাষাহীন আচরণ:

• ভ্রুকুটি করা

• চিন্তিত দেখানো, আকাশের দিকে তাকিয়ে চোখ ঘোরানো

• ‘না’ সূচকভাবে মাথা ঘোরানো

• ধৈর্যহীন ভাব দেখানো, এদিক ওদিক তাকানো অথবা ঘোরাঘুরি করা, বিরক্তি ভাব দেখানো অথবা হাই তোলা

ভাষাগত আচরণ:

• বক্তার কথায় বিঘ্ন ঘটানো, নিজেদের মধ্যে কথা বলা বা প্রসঙ্গ বদল করা

• বক্তাকে নেতিবাচকভাবে মূল্যায়ন করা, যেমন ‘তুমি ভুল বলছ’ অথবা ‘এটি একটি নির্বোধ ধারণা ।’ 


\title{
“ভাল শ্রোতা” নাটিকার (\#২) জন্য সংলাপের কার্ড :
}

\author{
তোমাদের মধ্যে একজন ‘বক্তা’ হবে এবং একজন ‘শ্রোতা’ হবে ।
}

বক্তা: (নাটিকা \# ১ এর মত একই নির্দেশনা): একটি কাল্পনিক ঘটনার ওপর ভিত্তি করে একটি নাটিকা টৈরি কর। যেমন ধর, ১. তোমার এমন কিছু ঘটেছিল (মিছা মিছি) যেটি তুমি মনে কর সঠিক ছিল না; অথবা ২. একটি খারাপ পরিকল্পনা অথবা ধারণা (মিছা মিছি) তুমি করেছিলে এবং যা তুমি করার জন্য উদগ্রীব ছিনে। নাটিকায় তোমার এমন কিছু অভিজ্ঞতা অথবা ধারণা এবং অনুভূতি তুলে ধরবে যে ধারণাটি তোমার নিজের কাছেও স্পষ্ট। তুমি তোমার সঙ্গীর সাথে এটি নিয়ে কথা বলতে কয়েক মিনিট সময় নাও, শোনাতে চেষ্টা কর। দুই অথবা তিন মিনিটে বলার পরিকল্পনা কর।

তোমার সঙ্গী যেভাবে শনছে সেভাবে কথা বলার চেষ্টা কর । যদি মনে হয় সে তোমার কথা শুনছে না, তোমার হতাশা দেখাও, জোরে বল, অথবা এই অবস্থায় যা করলে স্বাভাবিক মনে হয়, সেভাবে সাড়া দাও। অপর ব্যক্তিটি যদি মনোযোগ এবং শ্রদ্ধার সাথে তোমার কথা শোনে তাহনে অনুরূপ আচরণ কর।

শ্রোতা: তুমি এমন একজন শ্রোতা যে সক্রিয়ভাবে শুনছ । যখন বক্তা তোমার সাথে কথা বলার চেষ্টা করবে, সক্রিয় শ্রবণ দক্ষতা দেখাও। তোমার কাজ হচ্ছে নিচের তালিকা থেকে যতবেশি সম্ভব ইতিবাচকভাবে ভাষাগত এবং ভাষাইীন আচরণগুলো করা:

ভাযাহীন আচরণ:

• চোখের মাধ্যমে যোগায্যাগ রক্ষা করা

• সম্মতিসূচক মাথা নাড়ানো (যেন ‘হ্যা’ বলছ)

• তুমি অনছ এটা দেখানোর জন্য বক্তার দিকে একটু হেনে থাকা

• হাসি বিনিময় করা অথবা পিঠ চাপড়ে দেয়া

ভাষাগত আচরণ:

• তুমি বক্তার কথা বুঝতে পারছ, তা বুঝনোর জন্য আরো বেশি শোনার আগ্রহ দেখানো (যেমন, এটি সম্পর্কে আমাকে আরো কিছু বলুন..........”)

• বক্তার অনুভূতির মূল্যায়ন করা (যেমন, “আমি বুঝতে পারছি তোমার কেমন লাগছে।”)

• বক্তার চেতনার মূল্যায়ন করা (যেমন, “ভাল বিষয় ।")

• বক্তা নিজেকে অসম্পূর্ণ মনে করে, এমন কোন উক্তি থেকে বিরত থাকা

• বক্তার মনে যা ছিল তা আপনার সাথে আলোচনা করার/তুলে ধরার জন্য তাকে ধন্যবাদ দেয়া 


\section{দৃঢ়প্রত্যয়ী যোগাযোগের চর্চা}

অধিবেশন সম্পর্কিত ধারণা: শিক্ষার্থীরা দৃঢৃপ্রত্যয়ী আচরণগুলো পুনরায় পড়বে, সংস্কৃতিতে বিষয়টি কোনভাবে জড়িত তা আলোচনা করবে, এবং যেখানে অধিকার ক্ষুপ্নহয়, সেখানে দৃঢ়প্রত্যয়ী যোগাযোগের চর্চা করবে।

উদ্দেশ্য: শিক্ষার্থীদেরকে তাদের প্রয়োজন অনুযায়ী আরো দৃঢ়ভাবে যোগাযোগ করতে সক্ষম করা; তাদের কথোপকথন দক্ষতা শক্তিশালী করা।

\section{নির্দেশাবলি}

১ এই নির্দেশনামূলক প্রশ্নগুলো দিয়ে শুরু করুন:

• আজকে আমরা দৃঢ় যোগাযোগ সম্পর্কে আরো কিছু শিখব। কখনও কখনও তোমরা যা চাও তা স্পষ্টভাবে বলা কঠিন হয়ে যায় (যেমন, তুমি বিদ্যালয়ের একজন নেতা হিলেবে গণ্য হতে চাও)। তুমি যা চাওনা তা বলা কঠিন হয়ে যেতে পারে (যেমন, কেউ তোমার জন্য যা তৈরি করেছে, তা তুমি খেতে চাও না)।

- কেউ কি এমন কোন সময়ের কথা মনে করতে পারবে যখন কীভাবে তোমার আকাজ্ষা অথবা ইচ্ছা স্পষ্টভাবে প্রকাশ করবে তা নিয়ে সংশয়ে ছিলে ? [ছেলে এবং মেয়েরা এই প্রশ্নের একই উত্তর দিচ্ছে কিনা তা লক্ষ্য করুন ।]

• এমনকি যখন প্রকৃতপক্ষেই বলা গুরুত্বপূর্ণ হয়, অথবা না বলার ফলে যখন শারীরিক এবং মানসিক কোন ঘটনার জন্মা হয়, তখনো কথা বলা কঠিন হতে পারে। বিনয়ের সাথে কিন্তু স্পষ্টভাবে কথা বলার চর্চা করা গুরুত্বপূর্ণ ।

• তোমাদের খাতা নাও। আমরা একটি প্রশ্ন অথবা উক্তিমালা পড়ব যেগুলো হল 'সংলাপ’। প্রত্যেকটির জন্য একটি করে উত্তর লেখ যেগুনো দৃঢ, কিন্তু উগ্র নয়। সম্পূর্ণ বাক্য ব্যবহার কর।

প্রথম সংলাপ হল: 'শ্রেণিপ্রধান হিসেবে তোমাকে কেন আমার

• ভোট দেওয়া উচিত তা আমাকে বল’।

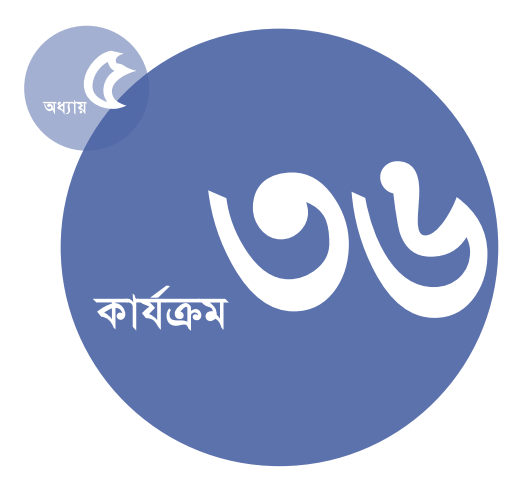

• কে এটির দৃঢ় উত্তর দিতে পারবে? [উপযুক্ত উত্তরে পৌঁছানোর জন্য শিক্ষার্থীদেরকে সাহায্য করুন। যেমন: "আমি বিশ্বাস করি শ্রেণিপ্রধানের জন্য আমি সবচেয়ে যোগ্য ব্যক্তি। আমি বিশ্বাস করি আমি তোমার সমর্থন নিতে পারব । উত্তরগুনো সম্পূর্ণ বাক্যে দেয়া হয়েছে কিনা তা নিশ্চিত হোন।]

২ প্রত্যেক সংলাপের জন্য এই প্রক্রিয়ার পুনরাবৃত্তি করুন (তালিকা দেখুন)।

৩ নিয়লিখিত প্রশ্নগুনোর মাধ্যমে আলোচনা শেয করুন:

• কিছু মানুষ ভাবতে পারে যে দৃঢ় হওয়া তাদের সমাজবিরোধী অথবা এটা অভদ্রতা । কোন অবস্থায় একজন মানুষের দৃঢ় হওয়া উচিত, যদিও এমন উত্তর রূঢ়/ অভদ্র হিসেবে গণ্য হয়? [জানার চেষ্টা করুন: যখন তোমাদের মনে হয় তোমাদের অধিকার ক্ষুন্ন হচ্ছে।]

• যেসকল মেয়েরা দৃঢ় তাদেরকে কি যে সকল ছেলেরা দৃঢ় তাদের মত করে দেখা হয়? যদি সংখ্যালঘু জাতিগোষ্ঠী অথবা নৃগোষ্ঠীর কোন ব্যক্তি দৃঢ় হয় তাহলে কি তাকে ভিন্নভাবে দেখা হয় ? এমন প্রতিবন্ধকতা কীভাবে মানুষের নিজস্ব মতামত নির্দ্বিধায় ব্যক্ত করার ক্ষমতাকে খর্ব করে ?

বাড়ির কাজ: আরো বেশি দৃঢ় হওয়ার জন্য পরবর্তী দুইদিন কমপক্ষে একবার করে চর্চা কর । কী ঘটেছে এবং তোমার কী মনে হয়েছে তা লেখ।
৩০-৪০ মিनिট

\section{উপকরণ:}

আপনার জন্য সংলাপ এবং নমুনা উত্তরের অনুলিপি।

প্রস্তুতি:

সংলাপ এবং নমুনা উত্তরগুলো পড়ন; সমাজের সাথে আরো উপযুক্ত করার জন্য এগুলোর কিছু কিছু পরিবর্তন করতে পারেন।
সময়কাল : 


\section{সংলাপ এবং নমুনা উত্তরের তালিকা:}

নিম্নলিখিত উক্তিগুলো দৃঢ় উত্তর খেঁাজার জন্য ব্যবহার করুন । আপনার শিক্ষার্থীরা উপযুক্ত দৃঢ় উত্তরে পৌঁছাতে সমস্যায় পড়তে পারে, তাই নমুনা উত্তরগুলো নিচে দেয়া হল ।

১ স সংলাপ: “তুমি কি তোমার পরীক্ষার উত্তরগুলো আমাকে দেখে লিখতে দেবে ? শিক্ষক জানবে না ।" নমুনা উত্তর: “দুঃখিত। আমি পরীক্ষার উত্তর দেখানো পছন্দ করিনা ।"

২ সংলাপ: তুমি তোমার তত্ত্বাবধায়ককে কাজের সময় দেখাও এবং উৎপাদন বৃদ্ধির জন্য বলতে চাও। নমুনা উত্তর: “ তত্ত্বাবধায়ক, আমি আপনার সাথে উৎপাদন বৃদ্ধির বিষয়ে কথা বলতে চাই যা আমি মনে করি আমি অর্জন করেছি ।”

৩ সংলাপ: তোমার বাবাকে বল, তার ইচ্ছার পরিবর্তে, তুমি আগামী বছর বিদ্যালয়ে লেখাপড়া চালিয়ে যেতে চাও। নমুনা উত্তর: "বাবা, আমি আপনার মতামতকে সম্মান করি, কিন্তু বিদ্যালয়ের লেখাপড়া চালিয়ে যাওয়া আমার জন্য খুবই গুরুত্বপূর্ণ। আমি আমার যুক্তিগুলো আপনার সাথে আলোচনা করতে চাই।"

8 সংলাপ: “প্রত্যেকের মত কেন তুমি খেলা পছন্দ কর না? তোমার সমস্যা কী ?" নমুনা উত্তর: “প্রত্যেকেই পৃথক। আমি পড়া এবং গানশোনা পছন্দ করি।"

৫ সংলাপ: “আমাদের সাথে ঝুলে পুরানো কারখানার পেছনে এসো; পুলিশ কখনও সেখানে যাবেনা ।" নমুনা উত্তর: “না, ধন্যবাদ। আমার যাওয়ার আগ্রহ নেই ।"

৬ সংলাপ: “আমি হলাম মেয়র। আমি শনছি যে, আপনারা নগরভবনের একটি ভবন, বিদ্যালয় পরবর্তী ক্লাবের জন্য ব্যবহার করার অনুরোধ করছেন ।"

নমুনা উত্তর: "হ্যাঁ, সম্মানিত মেয়র। আমরা দায়িত্ববান এবং জায়গাটির ভাল ব্যবহার করব। আপনি কি আমাদের পরিকল্পনা সম্পর্কে আরো কোন তথ্য চান?"

१ সংলাপ: “তোমার সাথে দেয়াল চিত্রে কাজ করতে যদি দুইজন সহপাঠীকে রাজি করাতে পার, আমরা তোমাদেরকে দেয়ালটি রং করতে দেব।” (সহপাঠীদের সাথে কথা বল।)"

নমুনা উত্তর: “যদি আরো দুইজন শিক্ষার্থী আমাদের সাথে যোগ দেয়, তাহনে আমরা একটি দেয়াল চিত্রাঙ্কনের অনুমতি পাব। তোমরা কি আমার সাথে যোগ দিতে চাও?"

৮ সংলাপ: “আমরা একটি আনন্দ অ্রমণে যাচ্ছি। আমাদের কোথায় যাওয়া উচিত বলে তুমি মনে কর ?” নমুনা উত্তর: “আমি ------- যেতে চাই এবং মনে করি এটি অন্যদের জন্যও আনন্দদায়ক হবে ।”

৯ সংলাপ: “অন্য শ্রেণির ঐ বাচ্চাটি এই পথে হাঁটছে। চল তাকে সরিয়ে দেই।" নমুনা উত্তর: “তাকে একা থাকতে দাও। সে আমাদের কিছু করতে পারবে না ।”

১০ স সংলাপ: এখন তোমরা অধিবেশনের শুরুতে যে পরিস্থিতির কথা মনে করেছিলে তা নিয়ে ভাব, যখন তোমাকে একটা সময়ের কথা বলতে বলা হয়েছিল যে, তোমার ইচ্ছা হয় তুমি আরো সরাসরি তোমার ইচ্ছাগুলো প্রকাশ করতে পারতে। ঐ পরিস্থিতিতে তোমার কী বলার ছিল তা লেখ । তোমাকে এই উত্তর সবাইকে দেখাতে বলা হবে না। 


\section{বিরোধ মধ্যস্থতার পাঁচটি ধাপ}

অধিবেশন সম্পর্কিত ধারণা: শিক্ষার্থীরা পারস্পরিক বিরোধ নিয়ে সরাসরি ও মর্যাদার সাথে কথা বলার পাঁচটি ধাপের একটি মডেল শিখবে এবং চর্চা করবে। (নোট: এই অধিবেশনটি হচ্ছে আরো অগ্রসর যোগাব্যাগ দক্ষতা টৈরি করার জন্য।)

উদ্দেশ্য: শিক্ষার্থীদেরকে যে কোন ঘনিষ্ঠ সম্পর্কের মধ্যকার দ্বন্দ্রের মধ্যস্থতা করার কথোপকথনে দক্ষতা উন্নয়নে শিক্ষার্থীদদর মনোলোগী করা ; তাদের বিশ্লেষণ ক্ষমতা বৃদ্ধি করা।

\section{निর্দেশাবলি}

১ ব্যাখ্যা করুন:

• আজকে আমরা দ্বন্দ্ব নিয়ে মধ্যস্থতা করার পদ্ধতি শিখব এবং চর্চা করব। আমরা একত্রে একটি ঘটনা পড়ব। এরপর আমরা জোড়া গঠন করব এবং প্রত্যেকে চর্চা করব।

• এখানে আমরা সবাই মিলে একটি কাল্পনিক ঘটনা চর্চা করব। যেমন: রণ মনে করে কিরণ তাকে নিয়ে অন্য বন্ধুদের সাথে মজা করে। এবং এতে রণ খুব বিপর্যস্ত ।

২ বোর্ডে প্রথম শিরোনামটি ('যখন তুমি ।) লিখুন এবং আলোচনা করুন:

• কোন নির্দিষ্ট আচরণ রণকে বিপর্যস্ত করছে সে সম্পর্কে ভাব। সাধারণ দোষারোপ না করে, যেমন “তুমি একজন বন্ধু খারাপ..," নির্দিষ্ট আচরণ নিয়ে আলোচনা কর যা এই ঘটনায় বিপর্যস্ত হওয়ার কারণ ছিল।/উত্তর খুঁজুন: “যখন তুমি আমাকে নিয়ে মজা করছিলে . . ." অথবা “যখন আমি শুনলাম, তুমি আমাকে নিয়ে মজা করেছ ........." দলটির দেয়া আচরণমূলক শব্দগুলো বোর্ডে লিখে শিরোনামটির বাকি অংশ পূরণ করুন ।]

• ঐ ব্যক্তির সাথে বিশেষ করে বিপর্যস্ত হওয়ার আচরণটি নিয়ে যোগাভোগ করা কেন গুরুত্বপূর?

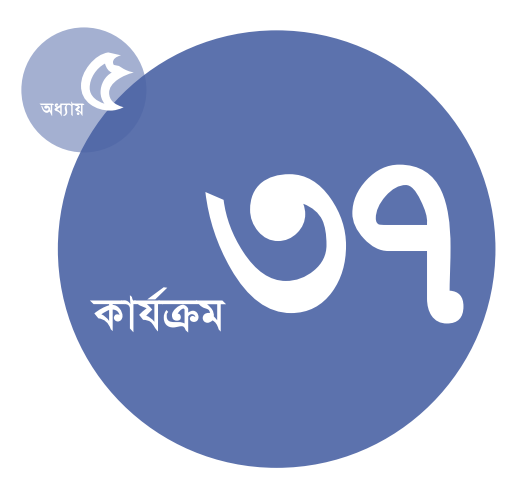

- যদি আচরণটি সম্পর্কে তোমার উপলধ্ধি সঠিক কি-না তা নিশিত না হও তাহলে তা মনে কর। যেমন তুমি বলতে পার, “আমার ভুল হতে পারে কিন্তু আমি শুনেছি তুমি আমাকে নিয়ে অন্যদের সাথে মজা করছিলে ।" ঘটনার সাথে তোমার ধারণা মিলে যাওয়ার সম্ভাবনাকে গ্রহণ করা কেন গুরুত্বপূর্ণ হতে পারে ?

৩ দ্বিতীয় শিরোনামটি লিখুন: “আমি মনে করি যে ?” এবং আলোচনা করুন:

- অনেক মানুষ এই ধাপটি বাদ দিয়ে যেতে পারে কিন্তু এটি অত্যন্ত গুরুত্বপূর্ণ। এটি মনে করিয়ে দেয় যে আমরা নিজেদের উত্তর ও প্রতিক্রিয়া ভেবে দেখি ।

• রণ কী মনে করতে পারে অথবা ভাবতে শুরু করতে পারে? " যখন তুমি আমাকে নিয়ে অন্যদের সাথে মজা কর, আমি মনে করি (ভাবতে শুরু কর). . . ."

তুমি আমার অনুভূতির গুরুত্ব দাও না । অথবা:

তুমি অতীতেও অন্যদের সাথে আমার সম্বন্ধে মজা করতে পার। অথবা:

তুমি নিজে তোমার সম্বন্ধে মোটেও ভাল চিন্তা কর না। অথবা, সবাই আমাকে নিয়ে হাসাহাসি করে।

অন্যান্য উত্তর?

\section{সময়কাল :}

৪৫-৬০ মিনিট, শিক্ষার্থীদের বিষয়বস্তু ভালোভাবে বোঝার ওপর নির্ভর করে।

\section{উপকরণ :}

বোড ও চক।

\section{প्रস্তুতি:}

দ্বন্দ্ব সমাধানের পাঁচটি ধাপ আপনার নিজের জীবনের কোন পরিস্থিতিতে কীভাবে প্রয়োগ করবেন তা ভাবার মাধ্যনে এগুলোর সাথে পরিচিত হোন । দ্বন্দ্বের উদাহরণগুনো আপনার দলের জন্য বেশি উপযুক্ত হবে এমন ঘটনায় পরিবর্তন করতে পরেন । যদি এমন করেন, একটি সাধারণ উদাহরণ বেছে নেয়ার ব্যাপারে সতর্ক থাকুন, এবং এই উদাহরণটির সাথে প্রত্যেকটি ধাপ কীভাবে উপস্থাপন করবেন তা ভাবুন। এই ধাপগুলোর ব্যবহার প্রথমবার

শিক্ষার্থীদের কাছে বেমানান মনে হতে পারে, কিন্তু সাধারণ উদাহরণ ব্যবহার এবং চর্চা এটিকে সহজ করে দেবে। (এমন কি ছোট বাচ্চাদের এই ধাপগুলোর দ্বারা নির্দেশ দেয়া যেতে পারে, কিন্তু যদি আপনার কাছে অধিবেশনটি খুব বেশি ব্যয়বহুল মনে হয়, লেক্ষেত্রে শুধুমাত্র ধাপ ২ ও 8 এর ওপর জোর দিয়ে এটি পরিবর্তন করুন ।) 
[ শিক্ষার্থীদের বলুন যে, অনেক উত্তর সঠিক হতে পারে, এবং দ্বিতীয় শিরোনামটি সম্পন্ন করতে একজন শিক্ষার্থীর উত্তর निখুন ।]

“তুমি আমার অনুভূতির গুরুত্ব দাও না” এটি বলা থেকে “আমি মনে করি (অথবা চিন্তিত যে) তুমি আমার অনুভূতির গুরুত্ব দাও না" বলাটা কতটা ভিন্ন ?

8 একজন শিক্ষার্থীকে শব্দপূরণসহ প্রথম দুইটি শিরোনাম পড়তে দিন । এরপর তৃতীয় শিরোনামটি লিখুন: "এবং এতে আমি অনুভব করি যে ?" নিম্নরূপে আলোচনা করুন:

• রণের ঠিক কী রকম আবেগানুভূতি হতে পারে তা ভাব। তার চিন্তা নয় বরং তার অনুভূতি।

• তোমার কী অনুভূতি হতে পারে যদি তোমার মনে হয় যে তোমার বন্ধু তোমাকে নিয়ে অন্যদের সাথে মজা করে? [উত্তর জানার চেষ্টা করুন: কষ্ট পাওয়া, প্রতারিত মনে হওয়া, রাগান্বিত হওয়া, অপ্রস্তুত হওয়া, একাকী, লজ্জিত।] [আলোচিত অনুভূতিগুলো থেকে ২টি অথবা ৩টি শব্দ যা অনুভূতি বর্ণনা করে তা বোর্ডে লিখে তৃতীয় শিরোনামটি সম্পন্ন করুন । ]

৫ একজন শিক্ষার্থীকে বোর্ডে যতটুকু কথোপকথন লেখা হয়েছে তা পড়তে বলুন। চতুর্থ শিরোনামটি লিখুন: “এবং এতে আমি করতে চেয়েছি ?" নিয়রূপে আলোচনা করুন্ন:

• রণ কী কাজ করতে চাইতে পারে [ যে কোন সম্ভাব্য উত্তর জানার চেষ্টা করুন, যেমন “এতে আমি করতে চেয়েছি:
মানুষের কাছে তোমার সম্পর্কে খারাপ বলতে;

চিৎকার করতে;

তোমার সাথে বন্ধুত্ব নষ্ট করতে; অথবা

ঘরে বলে থাকতে ও কারো মুখোমুখি না হতে ’]

[প্রস্তাবিত এক অথবা দুইটি উত্তর লিখে চতুর্থ শিরোনামটি সম্পন্ন করুন। শিক্ষার্থীদের মনে করিয়ে দিন যে, আমরা একটা নির্দিষ্ট সময়ে যা করতে চাই তা প্রায়ই আমাদের পরিকল্পনার সাথে মেলে না ।]

৬ একজন শিক্ষার্থীকে বোর্ডে লেখা সম্পূর্ণ কথোপকথনটি পড়তে বলুন, এরপর চূড়ান্ত শিরোনামটি লিখুন: “কিন্তু আমি এখনও ?" নিম্নরূপে আলোচনা করুুন:

- কিছু কিছু পরিস্থিতিতে কেউ এতো বিপর্যস্ত হতে পারে যে, তার আর ঐ ব্যক্তিটির সাথে কখনও মিশতে ইচ্ছা হয় না । কিন্তু বেশিরভাগ ক্ষেত্রেই, বক্তা ঐ ব্যক্তিটির সাথে সম্পর্কটি টিকিয়ে রাখতে চায়। এগুলোর সব কিছু শোনার পর ‘শ্রোতার’ কেমন অনুভূতি रয়?

• কীভাবে রণ তার সার্বিক অনুভূতিগুলো সহজজ ও সততার/স্বাভাবিকভাবে ব্যক্ত করতে পারে ? [এমন উত্তর জানার চেষ্টা করুন: “আমি এখনও. . . . তোমাকে গুরুত্ব দেই”; অথবা বন্ধু থাকতে চাই "’]

[সঠিক উত্তর দিয়ে চূড়ান্ত ধাপটি বোর্ডে লিখুন।]

৭ সম্পূর্ণ কথোপকথনটি পড়ুন । কোন মতামত আছে কিনা তা জানতে চান। 
৮ শিক্ষার্থীদের মধ্যে থেকে ২জন করে জোড়া গঠন করতে বলুন, এরপর ব্যাখ্যা করুন:

• এখন তোমাদের নিজেদের মধ্যে এই ধাপগুলো চর্চা করার একটি সুযোগ আছে। তুমি সহজভাবে সামলাতে পারনি এমন একটা দ্বন্দ্বের কথা ভাব।/यদি কেউ এরকম পরিস্থিতির কথা চিন্তা করতে না পারে, আপনি নিচের ঘটনাগুলোর একটা তুলে ধরতে পারেন: তোমার বাবা খুব অসুস্থ হলো কিন্তু তোমার সবচেয়ে থ্রিয় বন্ধু তেমন কোন সমবেদনা জানালো না ।

তোমার চাচাত ভাই তোমার বন্ধুকে জানিয়ে দিল যে, তোমার বাবা জেলখানায়।

তুমি তোমার বই হারিয়ে কিনতে পারছ না, কিন্তু তোমার বন্ধু কখনই তার বইটা তোমার সাথে ভাগাভাগি করে পড়ে না।

যখন তোমরা একসাথে কিছু করতে চাও, তোমার বন্ধু সবসময় দেরী করে।

একটি পার্টিতে তোমার প্রেমিকা বেশিরভাপ সময়ই অন্য ছেলের সাথে কথা বলছিল।

তোমার প্রেমিক বলেছে যে যদি সত্যিই তুমি তাকে ভালবাসতে, তাহলে তুমি যৌন মিলন করতে।

তোমার বন্ধু তোমাকে ‘তোমার কুমারীত্ হারাতে’ চাপ দিচ্ছে। তুমি কনডম ব্যবহার করতে চাইলে তোমার প্রেমিকা বিরক্ত হয়।]

• যে প্রথম আসবে তার সঙ্গীকে বলুন কোন দ্বন্দটি তারা সমাধান করবে।

• দ্বনদদটি সমাধান করার জন্য শিরোনাম পাঁচটি ব্যবহার করতে বলুন । পাঁচটি ধাপের সবগুনো সম্পন্ন করতে এক সাথে পাঁচ মিনিটের
মত সময় দিন, প্রথম দিকে আত্মবিশ্বাসের অভাব মনে হলে চিন্তিত হবেন না। দেখুন শেষটা কেমন হয়।

• যখন তোমরা শ্রোতা, প্রত্যেক ধাপে আমরা যা আলোচনা করেছি তোমার সঙ্গীকে তা স্মরণ করতে সাহায্য কর।

৯ শিক্ষার্থীরা ধাপগুলো যেন সহজভাবে ব্যবহার করতে পারে তা নিশ্চিত হওয়ার জন্য ধাপ পাঁচটি পূনরায় পর্যালোচনা করুন:

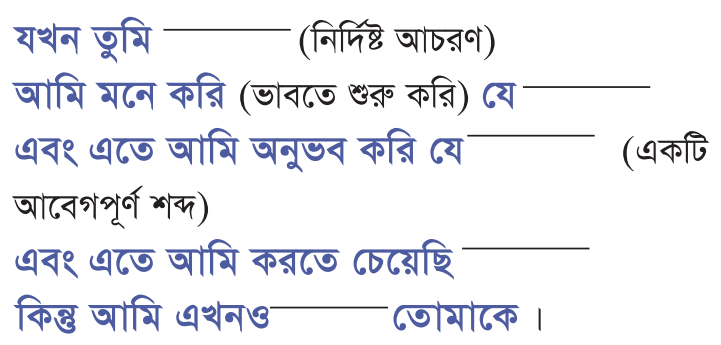

১০ সময় রাখুন যেন প্রত্যেক সঙ্গী পাঁচ মিনিট করে সময় পায়।

১১ আলোচনায় উৎসাহিত করুন:

- তোমার পূর্ব্বের যে কোন দ্বন্দ্বের চেয়ে তুমি কি এখন নিজেকে ভিন্নভাবে উপস্থাপন করতে পেরেছ?

• তোমার প্রকৃত অনুভূতি প্রকাশ করতে এই শব্দগুলি কি সাহায্য করে?

- তোমার নিজের অনুভূতি জানতে ও প্রকাশ করতে কেমন লाগে?

- মনে রেখ তোমার সঠিকভাবে মূল্যায়িত হওয়ার অধিকার আছে। তুমি যখন মনে করছ সঠিক মর্যাদা পাচ্ছ না, তখন নিজের জন্য কথা বলা উচিত। তুমি যদি না বল তাহলে কে বলবে? 


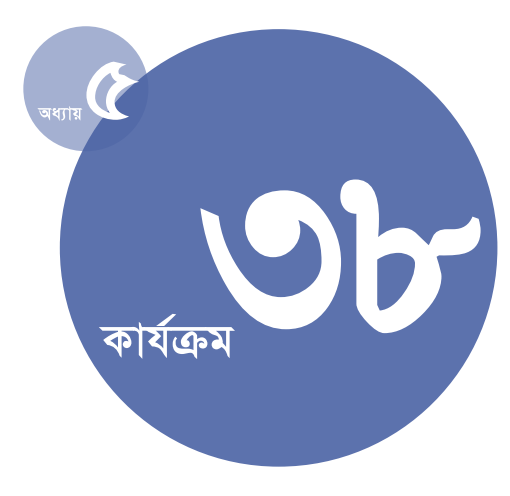

\section{যৌনতা ও যৌনস্বাস্থ্য নিয়ে আলোচনা}

অধিবেশন সম্পর্কিত ধারণা: শিক্ষার্থীরা আলোচনা এবং অভিনয়ের মাধ্যন্ যৌন আচরণ, সিদ্ধান্ত এবং স্বাস্থ্য বিষয়ে যোগাযোগ করার জন্য প্রয়োজনীয় ভাষাগত এবং ভাষাহীন দক্ষতার চর্চা করবে।

উদ্দেশ্য: যৌন বিষয়ের সিদ্ধান্ত গ্রহণ এবং স্বাস্থ্য বিষয়ক যোগাযোগের জন্য প্রয়োজনীয় ভাষাগত এবং ভাযাহীন দক্ষতা শক্তিশালী করা; বিশ্লেষণ এবং কতোপকথন দক্ষতা বৃদ্ধি করা ।

\section{সময়কাল:}

ধাপ ১- 8: 80 মিনিট ধাপ ৫ - ৬: ৩০ মিনিট

\section{উপকরণ:}

বোর্ড ও চক; টেপ ও মার্কার; পোষ্টার বোর্ড বা লম্বা কাগজ; নাটিকা ও পোষ্টার দলের কাজ।

\section{প্রস্তুতি :}

নাটিকা এবং পোস্টারের কাজগুনো পর্যালোচনা করুন এবং এগুলো আপনার অধিবেশনের উপযুক্ত করে পরিবর্তন করুন । আপনি যদি এই অধिবেশনটি দুইটি সেশনে ভাগ করতে চান তাহলে সিদ্ধান্ত নিন প্রথম লেশনটি কোথা থেকে আলাদা করবেন । সেশনের সময়ে বিলি করার জন্য প্রত্যেক নাটিকা এবং পোস্টার পৃথক পৃথক কাগজে লিখুন। কীভাবে আপনি এই নাটিকার প্রথম অংশের বিরতি দিয়ে নাটিকাটি লম্বা করবেন কিনা তা ভাবুন, যেমন: হাত তালি দিয়ে, এরপর “আবার এটি চেষ্টা কর” বলে।

\section{निर्দেশাবলি}

১ প্রশ্নের মাধ্যমে অধিবেশনটির পরিচয় ধারণা দিন:

• যুবক-যুবতীরা তাদের বন্ধুদের সাথে এবং যৌন বিষয়ক গণমাধ্যমে যৌন বিষয়ে কথা বলতে পারে। কিন্তু বেশির ভাগ যুবক-যুবতীদের জন্য তাদের প্রেমিক অথবা প্রেমিকার সাথে করা যৌনকাজ নিয়ে কথা বলা সহজ নাকি কঠিন?

- যে ব্যক্তি যৌনতার দিকে অগ্রসর হচ্ছে তার প্রশ্নের উত্তর দেয়া সহজ নাকি কঠিন?

• যৌন আচরণ এবং যৌনস্বাস্থ্য বিষয়ের সাথে সংযুক্ত ব্যক্তির সাথে যোগাযোগ করতে সক্ষম হওয়ার গুরুত্বপূর্ণ কারণগুলো की কী? [জানার চেষ্টা করুন: তুমি যৌনবিষয়ক যোগাযোগ করতে চাও কিনা না তা নিশ্চিত হওয়া; কী ধরনের যোগাযোগের সাথে তুমি স্বস্তিবোধ করো, এবং অপর ব্যক্তিটি কিসের সাথে স্বস্তিবোধ করো; (এইচআইভিসহ) এসটিআই এর বিরুদ্ধে প্রতিরোধ ব্যবস্থা নেয়া; গর্ভধারণের সাথে সম্পর্কিত মনোভাব স্পব্ট করা; এবং অনাকাজ্ষিত গর্ভধারণের প্রতিরোধক ব্যবস্থা নেয়া।]
• আমরা জোড়া তৈরি করব এবং ছোট দলে বিভক্ত হব। আটজন শিক্ষার্থী (চার জোড়ায়) দৃঢ় যোগাযোগ পদ্ধতি ব্যবহার করে কীভাবে বিভিন্ন ধরনের বিষয় মোকাবেলা করা যায় এক মিনিটের নাটকের মাধ্যমে তা চর্চা করবে। বাকি শিক্ষার্থীরা চারটি দল তৈরি করবে এবং পোস্টার তৈরি ও উপস্থাপন করবে; প্রত্যেকটি পোস্টারে বিশেষ ধরনের জটিল কথোপোকথন শুরু করার তিনটি পদ্ধতি থাকবে।

২ জোড়া ও ছোট দল তৈরি করুন এবং নাটিকা ও পোস্টার বিলি করুন:

৩ প্রত্যেক জোড়াকে তাদের এক মিনিটের নাটিকাটি উপস্থাপন করতে বলুন। প্রতি জোড়ার ক্ষেত্রে, প্রথম ব্যক্তি যত দ্র্ত ‘শেষ করে’, বলুন “থাম! এটি আবার চেষ্টা কর। এটি হচ্ছে অভিনেতার জন্য নাটিকার দ্বিতীয় অংশ (দৃঢ়) শুরু করার 
8 নাটিকা চারটি শেষ হওয়ার পর, আলোচনায় উদ্ুুদ্ধ করুন । শিক্ষার্থীদের জিজ্ঞস করুন:

• কোন অংশ (অনুগতমূলক অথবা দৃঢ়) বেশি ঘটে বলে তোমরা মনে কর ?

- তোমরা এমন কী দেখেছিলে যা সহায়ক?

• এই কথোপকথন চালানোর জন্য তোমাদের আর কী পরামর্শ আছে?

• এই কথোপকথন কতদূর যেতে পারে বলে আমরা আশা করি?

৫ প্রত্যেক পোস্টারের দলকে তাদের পোস্টার উপস্থাপন করতে বলুন দলের একজনকে পোস্টার উপস্থাপন করতে বলুন এবং দ্বিতীয়জনকে প্রশ্নের উত্তর দিতে বলুন, এবং মন্তব্য ও পরামর্শ দিতে অনুরোধ করুন । (তোমরা আরো কিছু দিনের জন্য পোস্টারগুলো দেয়ালে রাখতে পার।)

৬ নিম্মলিখিত প্রশ্নগুনো আলোচনার মাধ্যমে শেষ করুন:

- আমরা দেখেছি যে, যৌন পরিস্থিতিতে অপর ব্যক্তির যদি তোমার চেয়ে বেশি মর্যাদা অথবা ক্ষমতা থাকে তাহনে তোমাদের নিজেদের জন্য কথা বলা তোমাদের জন্য কঠিন হয়ে যায়। মনে রেখ যে, তোমাদের সর্বদাই যৌন পরিস্থিতিতে সম্মতি দেয়ার অথবা সম্মতি প্রত্যাহার করার অধিকার আছে।
- ী কাভব আমরা যৌনতার সাথে সম্পর্কিত জটিল কথোপথন শুরু তৈরি করতে পারি অথবা অনুশীলন করতে পারি? /জানার চেষ্টা করুন: আয়নার সামনে অনুশীলন করতে পারি; ফুরু করার আগে কী চাই তা লিখতে পারি।]

বাড়ির কাজ: এমন একটি কথোপকথন সম্পর্কে (যে কোন বিষয়ে) ভাব, যা তোমার পক্ষে শুরু করা কঠিন ছিল। ঐ কথোপথনটি শুরু করার জন্য একটি পাঞ্ডুলিপি লেখ। মনে রেখ কেউ একজন এই পাঞুলিপিটি পড়ে ফেলতে পারে এবং ভাবতে পারে যে, তুমি সত্যিই এই কথোপকথনটি করেছিলে। তাই তোমার কোন গোপনীয় বিষয় থাকলে যখন পাঞুুলিপিটি লেখা শেষ করবে, তখন তা নষ্ট করে ফেলবে। প্রথমে এই কাজটি নিজে নিজে কর; এটি লেখার জন্য তোমার একটু ভাল লাগতে পারে। 


\section{নাটিকার দায়িত্ব}

জোড়া-১ (পুরুষ/ মহিলা জোড়া):

যৌনবাহিত সংক্রমণ প্রতিরোধ নিয়ে এক মিনিটের একটি কথোপকথন দেখাও। ‘এ’ ব্যক্তি বিষয়টির শুরু করবে। 'বি’ ব্যক্তি তুলনামূলকভাবে বয়স্ক এবং সে অসহযোগিতা করবে। তুমি সিদ্ধান্ত নাও কোন চরিত্রটি পুরুষ এবং কোন চরিত্রটি মহিলা । তুমি এই দৃশ্যটি দুই মিনিটে অভিনয় করতে পারবে। প্রথম বার, ‘এ’ ব্যক্তিকে কনডম ব্যবহার না করতে চেয়ে আত্মপ সমর্থন করতে বলো । শিক্ষক বিরতি দেয়ার পরে, নাটিকাটি শেষ কর এবং কীভাবে ‘এ’ ব্যক্তি কথোপকথন চালিয়ে যেতে পারে তা দেখিয়ে দ্বিতীয়বার এটি অভিনয় কর।

জোড়া-২ (পুরুষ/ মহিলা জোড়া):

ব্যক্তি ‘এ’ গর্ভধারণ প্রতিরোধ করার জন্য কথোপকথন ফরু করে। ব্যক্তি ‘বি’ একটি বাচ্চা চায় এবং জন্ম নিয়ন্ত্রণ পদ্ধতির চিন্তাকে বিরোধিতা করছে। তুমি সিদ্ধান্ত নিতে পার কোন চরিত্রটি পুরুষ এবং কোন চরিত্রটি মহিলা । তুমি এই দৃশ্যটি দুই মিনিটে অভিনয় করবে। প্রথমবার, ব্যক্তি ‘এ’ কে জন্ম নিয়ন্ত্রণ পদ্ধতি ব্যবহার না করতে রাজি হওয়ার জন্য আত্নপক্ষ সমর্থন করতে বলুন । শিক্ষক থামিয়ে দেয়ার পর, নাটিকা বন্ধ কর এবং কীভাবে ব্যক্তি ‘এ’ কথোপকথন চালিয়ে যেতে পারে তা দেখিয়ে দ্বিতীয়বার অভিনয় কর।

জোড়া-৩ (পুরুষ/ মহিলা জোড়া):

ব্যক্তি ‘এ’ এবং ব্যক্তি ‘বি’ সহপাঠী; তোমরা সিদ্ধান্ত নিতে পার কোন চরিত্রটি পুরুষ এবং কোন চরিত্রটি মহিলা । যখন তারা বিদ্যালয়ে একসাথে হাটছিল, তখন ব্যক্তি ‘এ’ বারবার ব্যক্তি ‘বি’ এর শরীরে হাত দিচ্ছিল। ব্যক্তি ‘বি’ এটি পছন্দ করেনা । তুমি এই দৃশ্যটি দুই মিনিটে অভিনয় করবে। প্রথমবার, ব্যক্তি ‘বি’ অখুশি, কিন্তু কিছু বলল না । শিক্ষক থামিয়ে দেয়ার পরে, কীভাবে ব্যক্তি ‘বি’ দৃঢ়ভাবে উত্তর দিতে পারে তা দেখিয়ে দৃশ্যটি আবার কর।

জোড়া-8 (পুরুষ/ মহিলা জোড়া):

একটি মেয়ে যখন কমিউনিটি সেন্টারের ভেতর হঁঁটছে, তখন ব্যক্তি ‘এ’ (কমিউনিটি সেন্টারের একজন বয়স্ক পুরুষ নেতা) তার হাত মেয়েটির (ব্যক্তি‘বি’) শরীরে রাখছে। তুমি এই দৃশ্যটি দুই মিনিটে অভিনয় করবে। প্রথমবার, ব্যক্তি ‘বি’ অখুশি, কিন্তু কিছু বলেনা । শিক্ষক থামিয়ে দেয়ার পর, ব্যক্তি ‘বি’ কীভাবে দৃঢ় উত্তর দিতে পারে তা দেখিয়ে দৃশ্যটি আবার কর। 


\section{পোস্টার-দলীয় দায়িত্ব}

পোস্টার দল- ১ :

‘তোমার যৌন সংক্রমণ থাকতে পারে’ বিষয়টি নিয়ে ঘনিষ্ঠ সঙ্গীর সাথে কথা বলার কী উপায় তা আলোচনা কর । কথোপকথন শুরু করার তিনটি মাধ্যম দেখিয়ে একটি পোস্টার টৈরি কর। পোস্টারে একটি ছবি থাকতে পারে।

পোস্টার দল- ২ :

তোমার থ্রেমিক অথবা থ্রেমিকার সাথে তুমি যৌনমিলন করতে চাও এবং তারও ঐ বিষয়ে একই রকম মনে হচ্ছে কিনা তা জানতে চাওয়ার কী উপায় তা আলোচনা কর। কথোকপথন ফুরু করার তিনটি পদ্ধতি দেখিয়ে একটি পোস্টার তৈরি কর। পোস্টারে এই বিষয় নিয়ে দুইজন কথা বলছে তার বর্ণনা

থাকতে পারে।

পোস্টার দল- ৩ :

কীভাবে একজন ব্যক্তি যৌন নিপীড়ন নিয়ে একজন সঙ্গী অথবা বিশ্বস্ত পূর্ণবয়স্ক ব্যক্তির সাথে কথোপকথন শুরু করতে পারে তা আলোচনা কর । কথোপকথন শুরু করার জন্য তিনটি পদ্ধতি দেখিয়ে একটি পোস্টার তৈরি কর। পোষ্টারে এই পরিস্থিতি নিয়ে দুইজন ব্যক্তি কথা বলছে তার বর্ণনা থাকতে পারে।

পোস্টার দল- 8 :

এক ব্যক্তি যে তার সঙ্গীর সাথে য্যেন মিলন করেছিল কিন্তু যৌন মিলনে স্বস্তিবোধ করে না, সে তার সঙ্গীর সাথে সম্পর্ক রাখতে চাওয়ার বিষয়ে কীভাবে বলতে পারে তা আলোচনা কর । কথোপকথন শরু করার তিনটি পদ্ধতি দেখিয়ে একটি পোস্টার তৈরি কর। পোস্টারে এই পরিস্থিতি নিয়ে দুইজন কথা বলছে তার বর্ণনা থাকতে পারে। 


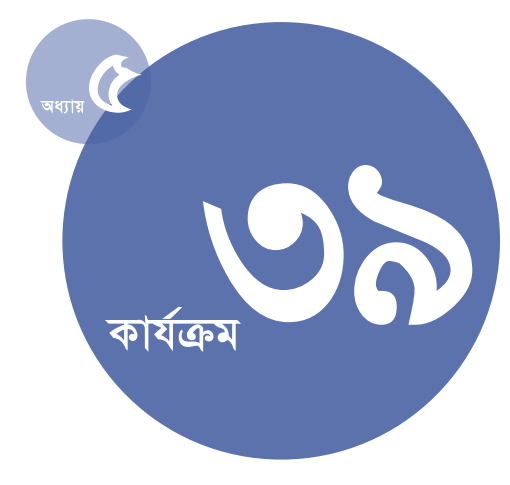

\section{কঠিন সিদ্ধান্তগুলো বাস্তবায়ন করা}

অধিবেশন সম্পর্কিত ধারণা: শিক্ষার্থীরা একটি সিদ্ধান্ত বাস্তবায়ন করার মূল ধাপগুলো শিখবে এবং একজন মানুয জটিল সিদ্ধান্ত বাস্তবায়ন করছে তা বর্ননা করে রম্য নাটিকা তৈরি করবে।

উদ্দেশ্য: শিক্ষার্থীদের জটিল সিদ্ধান্ত সহজ ও সুচিন্তিতভাবে বাস্তবায়ন করার ক্ষমতা শক্তিশালী করা; বিশ্লেযণ এবং কথোপকথন দক্ষতা শক্তিশালী করা।

\section{সময়কাল:}

ধাপ ১-৫: 80 মিनिট

ধাপ ৬: ৮০০ মিনিট (বাড়ির কাজ

হিলেবে দেয়া যেতে পারে অথবা

শ্রেণিকক্ষে করা যেতে পারে)

\section{উপকরণ:}

বোর্ড ও চক; উভয় হ্যান্ড আউট; কাগজ এবং ছবি আঁকার সকল উপকরণ (রং পেন্সিল, মার্কার, কলম)।

\section{প্রস্তুতি:}

নির্দেশিকার বইয়ের (এই বইয়ের সহগামী খণ্ড) অথবা আপনার শিক্ষাক্রমের অধ্যায়-৫ থেকে সিদ্ধান্ত বাস্তবায়নের অংশটকু পর্যালোচনা করুন । 'জটিল সিদ্ধান্তের নমুনা’ এর হ্যান্ডআউট পড়ন এবং যেগুনো আপনি নিতে চান লেগুলো বাছাই করুন, অথবা আপনার শ্রেণিকক্ষের উপযুক্ত করে অন্যকিছু তৈরি করুন । যদি সম্ভব হয়, প্রত্যেক শিক্ষার্থীর জন্য "জটিল সিদ্ধান্ত বাস্তবায়নের আটটি ধাপ” এবং 'জটিল সিদ্ধান্তের নমুনা’ এর অনুলিপি তৈরি করুন (এগুলো একটি হ্যান্ড আউটে সংয়ুক্ত হতে পারে)।

\section{নির্দেশাবলি}

১ বিষয়টি ধারণা দেয়ার জন্য জিজ্ঞেস করুন:

• এমন একটি সময়ের কথা ভাব যখন তুমি একটি সিদ্ধান্ত নিয়েছিলে কিন্তু তা কার্যকরভাবে বাস্তবায়ন করতে সমস্যা হয়েছিল।/উদাহরণ দিন, যেমন: সিদ্ধান্ত নেয়া: তুমি বাড়ি ফিরে যত দ্রুত সম্ভব বাড়ির কাজ শেষ করে ফেলবে; কনডম ব্যবহার করা; একটি অসুখি সম্পর্ক শেষ করে দেয়া; কাউকে থ্রেমের জন্য বলা; একটি স্পর্শকাতর বিষয়ে মা-বাবার সাথে কথা বলা; তোমার অনুভূতিতে আঘাত লেগেছিল তা একজন বন্ধুকে জানানো, অথবা এইচআইভি পরীক্ষা করানো]

• সিদ্ধান্তটি কী ছিল তা লেখ। তারপর যেগুনোর জন্য তোমার সিদ্ধান্তটি বাস্তবায়ন করতে কঠিন হয়েছিল সেগুলোর সকল বিষয় নিয়ে ভাব । সেগুলো লেখ ।

• একটি সিদ্ধান্ত বাস্তবায়নের পথে কী কী বিষয় থাকতে পারে?

২ “আটটি ধাপ এর .................” হ্যান্ডআউট বিলি করুন এবং দলটিকে বলুন ( নিচে প্রদত্ত বৃত্তির উদাহরণের বিকল্প উদাহরণ ব্যবহার করুন যদি সেগুলো আরো বেশি উপযুক্ত মনে হয়):

• এখন একটি কাল্পনিক সিদ্ধান্ত নাও।

• কল্পনা কর যে তুমি অন্য কোন দেশের বিশ্ববিদ্যালয়ে বৃত্তির জন্য আবেদন করার সিদ্ধান্ত নিয়েছ। এটি তোমার স্বপ্ন।
৩ বৃত্তি অথবা (অন্য কোন) উদাহরণ ব্যবহার করে শিক্ষার্থীদেরকে হ্যান্ডআউটের আটটি ধাপ পড়তে সাহায্য করুন ।

ধাপ এক: কী তোমার সিদ্ধান্ত বাস্তবায়ন সহজ করতে পারে ? [জানার চেষ্টা করুন: কোন বৃত্তি পর্যাপ্ত তা খুঁজে বের করা, ভাল গ্রেড পাওয়া, আবেদন করার জন্য নির্দেশিকাগুলো দুইবার যাচাই করা, আবেদন করার জন্য কম্পিউটার অথবা টাইপরাইটার এর ব্যবস্থা রাখা।]

ধাপ দুই: এই সিদ্ধান্ত বাস্তবায়নের ক্ষেত্রে মুখোমুখি হতে পার এমন সাধারণ বাধাগুলো কী? [জানার চেষ্টা করুন: যেগুলো পর্যাপ্ত আছে সেগুলো খুঁজে পাওয়ার কোন পদ্ধতি না থাকা, মাবাবার বিরোধিতা, আবেদনের ক্ষেত্রে ভাষাগত সমস্যা, আবেদনপত্র সঠিকভাবে পূরণ করার সময় না থাকা, তোমার শিক্ষকের কাছ থেকে সুপারিশপত্র চাইতে ভয় পাওয়া, তোমার গ্রেড যথেষ্ট ভাল না হতে পারে, যাতায়াতের খরচ নিয়ে উদ্বিগ্ন থাকা।

ধাপ তিন: এ তুমি কীভাবে তোমার সিদ্ধান্ত গোপনীয় এবং নিরাপদভাবে অনুশীলন করতে পার? [জানার চেষ্টা করুন: একজন শিক্ষকের কাছে একটি খসড়া অনুরোধ পাঠাও, তোমার মা-বাবার কাছে অনুরোধ জানানোর চর্চা কর, বৃত্তি নির্দেশিকাটি সম্পূর্ণভাবে পড় এবং ভাব কেন তুমি একজন শক্তিশালী প্রার্থী।] 
ধাপ চার: সিদ্ধান্তটি আলোচনা কর এবং একজন সহায়ক ব্যক্তির সাথে পরিকল্পনা কর। এটি কে হতে পারে ? [জানার চেষ্টা করুন: একজন বন্ধু, শিক্ষক, আত্যীয়, মা-বাবা, বৃত্তি প্রদান অফিসের কেউ, এমন কেউ যে বিশ্ববিদ্যালয়ের শিক্ষাকে মূল্যায়ন করে, এমন কেউ যার একটি বৃত্তি আছে।]

ধাপ পাঁচ : ভাব, কীভাবে তোমার সিদ্ধান্ত সবচেয়ে নিরাপদভাবে বাস্তবায়ন করবে? এই উদাহরণে কী কী সম্ভাব্য ঝুঁকি থাকতে পারে? [জানার চেষ্টা করুন: মা-বাবাকে এই বিষয়টি জানালে একটি মারাত্নক দ্বন্দ্ব হতে পারে; আবেদন পত্রে যে কোন প্রকার অসততা মারাত্নক পরিণতি বয়ে আনতে পারে; (যদি বৃত্তিটি পাওয়া যায়) তাহলে অন্য দেশে যাওয়ার সাথে কিছু ঝুঁকি থাকতে পারে।

ধাপ ছয়: তোমার পরিকল্পনা অনুসারে সিদ্ধান্ত বাস্তবায়ন কর, তুমি আসলে কী করবে? /জানার চেষ্টা করুন: একজন সাহায্যকারী বন্ধুর সাথে কথা বলব, একজন শিক্ষকের সাথে কথা বলার জন্য ভালভাবে প্রস্তুতি নেব, যে মা-বাবা অনিচ্ছুক হতে পারে তাদেরকে সবচেয়ে ভাল কীভাবে বলা যেতে পারে তা ভাব, তোমার গ্রেড ধরে রাখার জন্য কঠোর পরিশ্রম কর-তারপর তোমার আবেদনপত্র পূরণ কর।]
ধাপ সাত: এই সিদ্ধান্ত বাস্তবায়ন করতে যদি বাধা সৃষ্টি হয়, আলাদা একটি কর্মপরিকল্পনা তৈরি কর অথবা তোমার সিদ্ধান্ত পুণর্বিবেচনা কর। যেমন: যদি তোমার অন্যদেশে লেখাপড়ার বিষয়ে তোমার মা-বাবা স্বস্তিবোধ না করে, তুমি কী করতে পারতে? [তোমার নিজের দেশে বৃত্তির জন্য আবেদন কর।] যদি তোমার গ্রেড এই নির্দিষ্ট বৃত্তির জন্য যথেষ্ট ভাল না হয়, তুমি কী করতে পার? [অন্য বৃত্তির সুযোগ খেঁ|জ।]

ধাপ আট: সচেতন হও যে, যদি তুমি কোন বাধার সম্মুখীন নাও হও, তাহলেও তোমার সিদ্ধান্ত পূণর্মূল্যায়ন করার ও তোমার মন পরিবর্তন করার অধিকার আছে। এই মুহুর্তে বৃত্তির জন্য আবেদন করার ক্ষেত্রে কোন বিষয়গুলো তোমাকে তোমার সিদ্ধান্ত পুনরায় ভাবার জন্য প্রভাবিত করতে পারে, এমনকি যদি তুমি কোন বাধার সম্মুখীন নাও হও? [জানার চেষ্টা করুন: খুঁজে দেখ যে, দেশী বিশ্ববিদ্যালয় তোমাকে একই প্রোগ্রামের সুযোগ দিতে পারে, তোমার পরিবারের কাছাকাছি বাস করা গুরুত্বপূর এই সিদ্ধান্ত নেয়া, বাইরে যাওয়ার বিষয়ে ভীত হওয়া, বিশ্ববিদ্যালয়ে পড়ার সময় বিশ্ববিদ্যালয়ে কাজ করার প্রয়োজনীয়তা, এক বছরের জন্য তোমার পরিকল্পনা পরিবর্তনের সিদ্ধান্ত নেয়া।

[তুমি এমন কিছু বলে শিক্ষাকে কম গুরুত্ব দিতে পার যে, "যদি তোমাদের মধ্যে কেউ কখনও শিক্ষার জন্য এমন কোন সুযোগ পাও, আমি আশা করি তোমরা তা গ্রহণ করবে ॥] 
8 “ কঠিন সিদ্ধান্তের নমুনা” এর হ্যান্ডআউট বিলি করুন । ব্যাখ্যা করুন:

• এই ধাপগুলো ব্যবহার করে চর্চা করার জন্য তোমাদের প্রত্যেকে এই তালিকা থেকে একটি করে সিদ্ধান্ত বেছে নেবে। তোমাদের কাজ হচ্ছে, যে এই সিদ্ধান্তটি নিয়েছে তার চরিত্র নিয়ে একটি রম্য নাটিকা (ধারাবাহিক কার্টুন) লেখা। চরিত্রটি সিদ্ধান্ত বাস্তবায়নের আটটি ধাপ পার করছে তা রম্য নাটিকায় দেখাবে। তোমরা কিছু ধাপ দ্রংত শেষ করতে পার; যেমন, চরিত্রটি শুধু যা করছে তা বলতে পারে। তোমাদের রম্য নাটিকার কমপক্ষে এক অথবা দুইটি ধাপ বিস্তারিত বর্ণনা

• করবে।

সেশনের বাকি সময়ে আপনি রম্য নাটিকাগুলো শুরু করতে পারেন। এই সেশন শেষ করতে আপনার কমপক্ষে এক ঘণ্টা ব্যয় করা উচিত। [লক্ষণীয়: দ্বিতীয় ঘণ্টাটি বাড়ির কাজের জন্য হতে পারে, অথবা দ্বিতীয় সেশনের জন্য হতে পারে।

শিক্ষার্থীরা কাজটি বুঝতে পেরেছে তা নিশ্চিত হওয়ার জন্য
৫ শিক্ষার্থীদের তাদের রম্য নাটিকা শুরু করতে বলুন। তারা সবাই কাজটি বুঝতে পেরেছে তা নিশ্চিত হওয়ার জন্য এবং তাদেরকে আটটি ধাপের মডেলটি বাস্তবায়নে সাহায্য করার জন্য কক্ষের মধ্যে ঘুরে ঘুরে দেখুন ।

৬ (বাড়ির কাজ হিসেবে দেয়া যেতে পারে): কাজটি শেষ করার জন্য শিক্ষার্থীদেরকে বেশি সময় দিন । যখন তারা কাজ করবে তখন তাদের মতামত একে অপরের সাথে আলোচনা করতে দিন । যখন সবগুলো রম্য নাটিকা শেষ হয়ে যাবে, তাদেরকে তা দেওয়ালে ঝোলাতে বলুন অথবা বই তৈরি করতে বলুন। 


\section{একটি কঠিন সিদ্ধান্ত বাস্তবায়নের জন্য আটটি ধাপ}

ধাপ এক: कী করলে সিদ্ধান্তটি বাস্তবায়ন সবচেয়ে সহজ হবে তা ভাব।

ধাপ দুই: একটি সিদ্ধান্ত বাস্তবায়নে কী কী সম্ভাব্য বাধা আসতে পারে তা ভাব।

ধাপ তিন: তোমার সিদ্ধান্ত নিরাপদ বা গোপনীয়ভাবে পূর্বাভিনয় কর।

ধাপ চার: সিদ্ধান্ত এবং পরিকল্পনা একজন সাহায্যকারী ব্যক্তির সাথে আলোচনা কর।

ধাপ পাঁচ: তোমার সিদ্ধান্ত সবচেয়ে নিরাপদে কীভাবে বাস্তবায়ন করবে তা ভাব।

ধাপ ছয়: তোমার পরিকল্পনা অনুযায়ী তোমার সিদ্ধান্তটি কার্যকর কর।

ধাপ সাত: একটি সিদ্ধান্ত বাস্তবায়নে যদি বাধা আলে, তাহলে অন্য একটি কর্মপরিকল্পনা টৈরি কর অথবা সিদ্ধান্তটি পুনর্বিবেচনা কর।

ধাপ আট: যদি মানুষ কোন বাধার সম্মুখীন নাও, তাহলেও তাদের সিদ্ধান্ত পুণর্মূল্যায়ন করার ও তাদের মন পরিবর্তন করার অধিকার আছে লে বিষয়ে সচেতন হও। 


\section{কঠিন সিদ্ধান্তের নমুনা}

তুমি অন্যদেশের একটি বিশ্ববিদ্যালয়ে ভর্তি হওয়ার জন্য বৃত্তির চেষ্টা করার সিদ্ধান্ত নিয়েছ।

তোমার মা-বাবার পছন্দের কাউকে বিয়ে না করার সিদ্ধান্ত নিয়েছ। তুমি তোমার মা-বাবার অপছন্দের কাউকে বিয়ে করার সিদ্ধান্ত নিয়েছ।

তুমি প্রত্যেকবার যৌনমিলনের সময় কনডম ব্যবহারের সিদ্ধান্ত নিয়েছ।

যদিও তোমার সঙ্গীর সাথে যৌনমিলন না করলে সে তোমাকে ত্যাগ করবে বলে শাসিয়েছে, তারপরও তুমি তোমার সঙ্গীর সাথে যৌনমিলন না করার সিদ্ধান্ত নিয়েছ।

তুমি তোমার ঘনিষ্ঠ সঙ্গীর (প্রেমিক অথবা প্রেমিকার) সাথে সম্পর্ক ছিন্ন করার সিদ্ধান্ত নিয়েছ।

তুমি তোমার মা-বাবাকে বলার সিদ্ধান্ত নিয়েছ যে, তুমি (অথবা তোমার সঙ্গী) গর্ভবতী।

তুমি ধুমপান (অথবা মদ্যপান) ছেড়ে দেয়ার সিদ্ধান্ত নিয়েছ।

তুমি একজন সহিংস সঙ্গীকে ত্যাগ করার সিদ্ধান্ত নিয়েছ।

তুমি তোমার মা-বাবাকে বলার সিদ্ধান্ত নিয়েছ যে, তুমি একজন সমকামী।

তুমি এইচআইভি পরীক্ষা করানোর সিদ্ধান্ত নিয়েছ।

তুমি তোমার সঙ্গীকে বলার সিদ্ধান্ত নিয়েছ যে, তুমি যৌনমিলনের সময় আনন্দ (অথবা চরম উত্তেজনা) পাও না ।

তুমি তোমার স্বামী/স্ত্রী, যৌন সঙ্গী অথবা মা-বাবাকে বলার সিদ্ধান্ত নিয়েছ যে, তুমি এইচআইভিতে আক্রান্ত।

তুমি সিদ্ধান্ত নিয়েছ যে, তোমার সমাজের প্রচলিত বিষয়গুলো পালন করবে না (যেমন, নারীদের যৌনাঞছেদ করতে চাও না, একটি নির্দিষ্ট বয়লে বিদ্যালয় ছাড়তত চাওনা, অথবা কোন দলের সাথে যোগ দিতে চাওনা।)

তুমি তোমার সঙ্গী অথবা স্বামী/স্ত্রীকে বলার সিদ্ধান্ত নিয়েছ যে, তার অন্য কারো সাথে যৌন সম্পর্ক আছে এটা তুমি জান ।

তুমি তোমার সঙ্গী অথবা স্বামী/স্ত্রীকে বলার সিদ্ধান্ত নিয়েছ যে, তুমি অবিশ্বাসী ছিলে ।

गे४ 


\section{তুমি আমার মধ্যে कী দেখ}

অধিবেশন সম্পর্কিত ধারণা: শিক্ষার্থীরা শারীরিক গঠন সম্পর্কিত সামাজিক ও মানষিক চাপ নিয়ে আলোচনা করবে এবং তারা নিজেদের ভাল লাগে এমন একটি শারীরিক বৈশিষ্য নিয়ে কথা বলবে। ছোট দলে বিভক্ত হয়ে শিক্ষার্থীরা শারীরিক গঠনের সাথে সম্পর্কহীন বৈশিষ্ট্য যার জন্য অন্যরা তার প্রশংসা করে সে বিষয়ে ঙনবে ।

উদ্লেশ্য: শারীরিক গঠন নিয়ে সামাজিক চাপ কীভাবে আত্মসম্মানকে প্রভাবিত করে-শিক্ষার্থীদেরকে তা বুঝিয়ে বলতে সক্ষম করা । নিজেদের ভাল গুণ (শারীরিক গঠনের সাথে সম্পর্কিত এবং সম্পর্কিত নয় উভয়ই) সম্পর্কে তাদের সচেতনতা বাড়ানো; বন্ধুদের প্রতি সহানুভূতি বৃদ্ধি করা ।

\section{निর্দেশাবলি}

১ নিচের নির্দেশিত শ্রশ্নগুনো নিয়ে মুক্ত আলোচনা করুন:

• আমরা আমাদের শরীর নিয়ে কেমন অনুভব করি তা অনেকগুলো বিষয়ের ওপর নির্ভর করে। আমাদের শরীর কি শক্তিশালী এবং সুস্থ? সমাজ এবং গণমাধ্যম কি সব ধরনের শরীরকে আকর্ষণীয়ভাবে তুলে ধরে? তোমার কেমন লাগে যখন খুব অল্প সময়ের মধ্যে তোমার শরীরের বড় পরিবর্তনের সাথে নিজেকে মানিয়ে নিতে হয়? চেহারার ওপর কি অনেক বেশি গুরুত্ব দেয়া হয় এবং অন্যান্য গুণের ওপর যথেষ্ট গুরুত্ব দেয়া হয়না? এমন কিছু প্রশ্ন আজকে আমরা জানার চেষ্টা করব।

• তোমরা নিজেদের নয় বছর বয়সের কথা ভাব। বেশিরভাগ নয়-বছর বয়সীরা তাদের শরীর নিয়ে এবং তারা দেখতে কেমন তা নিয়ে কেমন অনুভব করে ? তারা কি তাদের চেহারা নিয়ে খুব চিন্তিত থাকে? [শিক্ষার্থীদেরকে ১ থেকে ১০ এর মধ্যে সংখ্যা আকারে উত্তর দিতে বলতে পারেন, যেমন ১=তারা মোটেই চিন্তিত নয়, এবং ১০= তারা এটি নিয়ে খুবই চিন্তিত/ বেশির ভাগ সময়।]
• বয়ঃসঞ্ধিকালে কী হয়? বেশিরভাগ কিশোর-কিণোরী কি তাদের চেহারা নিয়ে নিশ্চিন্ত এবং স্বস্তিতে থাকে, নাকি তারা দেখতে কেমন তা নিয়ে দুশ্চিন্তায় থাকে? [আবারও ১-১০ এর মধ্যে উত্তর দেয়া যেতে পারে।]

- বিজ্ঞাপন এবং চলচ্চিত্র থেকে কিশোর কিশোরীরা তাদের চেহারা ও শরীর দেখতে কেমন হওয়া উচিত এ সম্পর্কে কী ধরনের বার্তা এবং ছবি পেয়ে থাকে? এই চাপ কি মেয়েদের উপর বেশী নাকি ছেলেদের ওপর বেশী?

• কিশোর-কিশোরীরা কি প্রায়ই তাদের চেহারা দ্বারা বিবেচিত হয়? বেশিরভাগ মানুষই কি চেহারার ভিত্তিতে বিবেচিত হতে চায়? অন্যান্য কোন গুণাবলির জন্য মানুষ অন্যের প্রশংসা আশা করে ? [মেয়েদের সাথে সাথে ছেলেদেরকেও ডাকুন । জানার চেষ্টা করুন: মেধাবী, সৎ, রসিকতা জ্ঞান সম্পন্ন, পরিশ্রমী, সাহসী, দয়ালু, শিল্পী, গায়ক, দৌড়বিদ, উদার, নীতিবান, ভাল শ্রোতা, বিশ্বাসী এবং এরকম অন্যান্য গুণাবলি। কমপক্ষে আট থেকে দশটি বৈশিষ্ট্য তৈরি করুন এবং বোর্ডে লিখুন। উল্লেখ করুন যে, মেয়েদের সাথে সাথে ছেলেরাও এই গুণাবলির জন্য প্রশংসা পেতে চায়।]

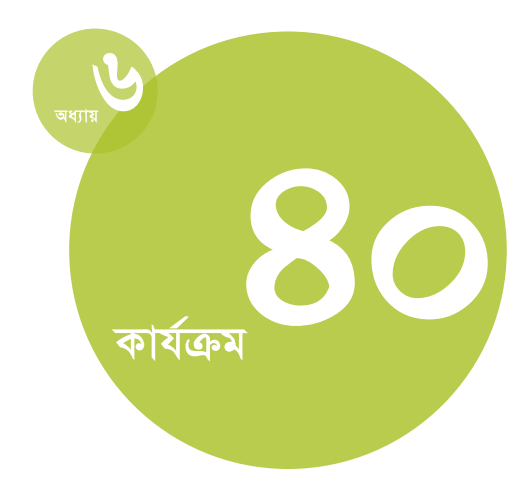

সময়কাল : ৪৫ মিনিট

উপকরণ:

বোর্ড ও চক; প্রত্যেক শিক্ষার্থীর জন্য একটি করে সাদা কাগজ (সম্ভব হলে, রঙীন কাগজ, পোষ্টার-ওয়েট, আকারে বড় অথবা বিলেষ কাগজ ব্যবহার করুন)।

প্রস্তুতি:

যদি আপনি জানেন যে শিক্ষার্থীদের মধ্য্য বৈরী সম্পর্ক আছে, তাহুলে (ধাপ-৩) এ ছোট দলটি এমনভাবে গঠন করুন যেন ঐ শিক্ষার্থীরা এক দলে না পড়ে। ধাপ ৩ সহ আপনার সময়ের প্রতি খেয়াল রাখা গুরুত্বপূর্ণ, যেন প্রত্যেক শিক্ষার্থী সেশন শেষ হওয়ার আগে প্রশংসিত হওয়ার সুযোগ পায়। যদি আপনি শরীরের গঠঠনের ওপর বিজ্ঞাপনের প্রভাবও ব্যাখ্যা করতে চান তাহুলে অধিবেশন ৯ দেখুন 
২ শিক্ষার্থীদেরকে কাগজ এবং কলম নিতে বলুন । ব্যাখ্যা করুন্ন:

- তোমরা নিজেদের চেহারার বা শরীরের এমন কিছু নিয়ে ভাব যা তোমাদের ভাল লাগে। এটি তোমাদের হাসি, চোখ, হাঁটার ধরন, পেশী, চুল, অথবা উচ্চতা হতে পারে। আবার দেহের গড়ন, নাক, নাচের অথবা চলাচলের ধরন, মুখের গড়ন, বাহু অথবা পা, হাত, ত্বক, গালের টোল, ঠোঁট। অথবা অন্য যে কোন কিছু হতে পারে ।

• এ এটি শুধু তোমাদের নিজেদের জন্য-অন্য কারো সামনে করতে বলা হবে না। ঐসব গুণাবলি বর্ণনা করার জন্য একটা কাব্যিক বাক্য লেখ, যেমন “আমার হাসি পুরো ঘরকে আলোয় ভরে দেয়” অথবা “আমার চোখ সমুদ্রের মতো গভীর।"

• সময় দশ মিনিট। যখন লেখা শেষ হবে, তোমাদের লেখাগুনো একটি গোপন স্থানে রেখে দেবে।

৩ লেখাটি রাখা হয়ে গেলে শিক্ষার্থীদের পাঁচটি দলে ভাগ করুন । প্রত্যেক দলকে পাঁচটি করে কাগজ ও একটি করে মার্কার দিন । ব্যাখ্যা করুন:

• মনে রেখ, আমরা সবাই নিজেদের চেহারার বাইরেও অন্যান্য গুণাবলির জন্য প্রশংসা পেতে চাই।

• তোমার দলের একজনকে নিয়ে শুরু কর, কেউ একজন একটি সাদা কাগজে তার নাম লিখবে। তারপর, দলের প্রত্যেকে একে একে তাকে নিয়ে প্রশংসা করে এমনকিছু বলবে যা তার শারীরিক বৈশিট্ট্যের বাইরে। এটি উপরে লেখা যোগ্যতার যে কোন একটি [বোর্ডে দেখতে বলুন।] অথবা তার অন্য কোন ভাল গুণ হতে পারে। এটা ঐ শিক্ষার্থীর জন্য
চোখে পড়ার মতন কোন বৈশিষ্ট্য অথবা এমন কোন বৈশিষ্ট্য হতে পারে যার প্রতি আজকের আগে তোমরা তেমন কোন মনোযোগ দাওনি ।

• যখন এই গুণের কথা বলবে, তার নামসহ তা কাগজে লিখবে।

- তোমার লেখার পর কাগজটি দলের অন্যদের কাছে দিবে। দলের প্রত্যেকের কাছে যাওয়া পর্যন্ত এটি করতে থাকবে।

• দনের প্রত্যেক সদস্যের জন্য এই প্রক্রিয়ার পুনরাবৃত্তি করবে। মৌখিক এবং লিখিত উভয়ভাবে প্রশংসা করবে । দলের প্রত্যেকের কাছে পালাক্রমে কাগজ ঘুরে যেতে দুই মিনিটের বেশি নিও না । আমি সময়ের হিসাব রাখব যেন সবাই লিখতে সুযোগ পায়। /বাকি সময়টুকু নিশ্চিতভাবে সমান পাঁচ ভাগে ভাগ করুন । ঘুরে ঘুরে দেখুন দলগুলো সময়মত এবং সঠিকভাবে কাজটি করছে কিনা। $]$

• ুরু করার পূর্বে, এই কার্যক্রমটি পরিচালনা করার জন্য কী কী নিয়ম আমাদের সবার মেনে চলা উচিত? [ জানার চেষ্টা করুন: শ্রদ্ধাশীল হওয়া; অন্যরা যা বলেছে তা আরেকবার না বলে নতুন কিছু ভাবা; নিজের পালা/সুযোগ ছেড়ে না দেয়া। এমনকি দলে यদি এমন কেউ থাকে যাকে তুমি খুব একটা পছন্দ করনা, মনে রেখ সবার মধ্যেই কিছু ভাল গুণ আছে। অন্যদের সাথে এমন আচরণ কর যেমনটি তুমি অন্যদের কাছ থেকে পেতে চাও ।]

• যখন তোমাকে নিয়ে “প্রশংসা পাতা” লেখা শেষ হনে, তুমি তোমারটি রেখে দিতে পারো। এটা তোমার অর্জন! 


\section{শারীরিক পরিবর্তন}

অধিবেশন সম্পর্কিত ধারণাঃ শিক্ষার্থীরা বয়ঃসন্ধিকালের যেসব শারীরিক পরিবর্তন হয় সেগুলো নিয়ে বই এবং গান তৈরি করবে।

উদ্দেশ্য: শিক্ষার্থীদেরকে বয়ঃসন্ধিকালের মৌলিক শারীরিক পরিবর্তনগুলোর বর্ণনা দিতে সক্ষম করা; দলীয়ভাবে কাজ করা এবং উপস্থাপন করার দক্ষতা বৃদ্ধি করা।

\section{निर्द्यकाবলि}

১ এই পাঠটি যে বয়ঃসঙ্ধিকালীন শারীরিক পরিবর্তন নিয়ে তা ব্যাখ্যা করুন । জিজ্ঞেস করুন:

• শরীরের ওপর বয়ঃসঞ্ধিকালের প্রতাব নিয়ে আলোচনা করার সঠিক বয়স কোনটি-বয়ঃসঞ্ধিকালে পৌছানোর পরে নাকি আগে? বেশির ভাগ বাচ্চারা কি 'বয়ঃসঙ্ধিকালে কী হয়’ তা যথেষ্ট পরিমাণে শিখে থাকে?

- তোমরা ছোটদের জন্য বয়ঃসন্ধিকাল নিয়ে কিছু উপকরণ টৈরি করবে, যারা পরবর্তী এক অথবা দুই বছরে বয়ঃসন্ধিকালে পৌছাবে। তিনজনের একটি দল গঠন করবে। একই দলের সবাই ছেলে অথবা সবাই মেয়ে অন্তর্ভুক্ত করবে। মেয়েদের দল মেয়েদের বয়ঃসন্ধিকালের পরিবর্তন এবং ছেলেদের দল ছেলেদের বয়ঃসন্ধিকালের পরিবর্তন উপস্থাপন করবে।

• তোমাদের পছন্দমত পদ্ধতিতে তথ্য উপস্থাপন করবে। যেমন, তোমরা হিপ-হপ কবিতা বা গান তৈরি করতে পার, বাচ্চাদের বই অথবা একটি পোস্টার তৈরি করতে পার অথবা সংবাদ পত্র কলামে একটি বাচ্চার ‘প্রিয় ডাক্তার’ কে লেখা একটি চিঠি ও জবাব লিখতে পার। সৃজনশীল হও! তোমরা কৌতুক, ছন্দ, নাচ অথবা শুধু বর্ণনা পদ্ধতি ব্যবহার করতে পার। যাই করবে, যে তথ্যগুলো উপস্থাপন করবে সেগুলো অবশ্যই সঠিক এবং সহায়ক হতে হবে।/यদি উপস্থাপন করার ব্যবস্থা করা হয়, শিক্ষার্থীদেরকে বুঝিয়ে বলুন যে, তারা তাদের জিনিসগুলো আরো ছোট বাচ্চাদের কাছে উপস্থাপন করবে।]
• সাহায্যের জন্য, আমি প্রত্যেক দলকে একটি বয়ঃসন্ধিকালীন তথ্যমালা (ছেলে এবং মেয়েদের) বিলি করবো।

২ তিনজনের দল এবং তথ্যমালার হ্যান্ডআউট তৈরি করুন । যথেষ্ট পরিমাণে ছবি আঁকার উপকরণ বিতরণ করুন । দলগুলোকে বলুন যে তাদের সময় ২৫ মিনিট।

৩ যদি ছোটদের জন্য উপস্থাপনের ব্যবস্থা করে থাকেন: সেশনের বাকি সময় রুমের মধ্যে ঘুরে ঘুরে দলগুলোকে হ্যান্ডআউট বিতরণ ও আলোচনা করুন এবং উপস্থাপনের আগে তাদের কাজ সমাপ্ত করতে বলুন। ভুল এবং বাদ পড়া তথ্য সঠিক করতে অথবা পূরণ করতে সাহায্য করুন ।

যদি উপস্থাপনগুলি নিজের শ্রেণির বন্ধুদের মধ্যে হয়: দলগুলোকে ডাকুন এবং যতক্ষণ সময় থাকে উপস্থাপন করতে বলুন, এবং বাকি দলগুনোকে পরবর্তী পাঠদানের সময়ে এ উপস্থাপন করতে বলুন। অন্য শিক্ষার্থীদেরকে ভুল তথ্য সঠিক করতে এবং বাদ পড়া তথ্য পূরণ করতে অংশগ্রহণ করান।

8 সেশনের শেযে, বয়ঃসন্ধিকালীন তথ্যশীটগুলো বিতরণ করুন যেন প্রত্যেক শিক্ষার্থী “বয়ঃসন্ধিকাল এবং মেয়েরা” এর একটি এবং “বয়ঃসন্ধিকাল এবং ছেলেরা” এর একটি অনুলিপি পায়।

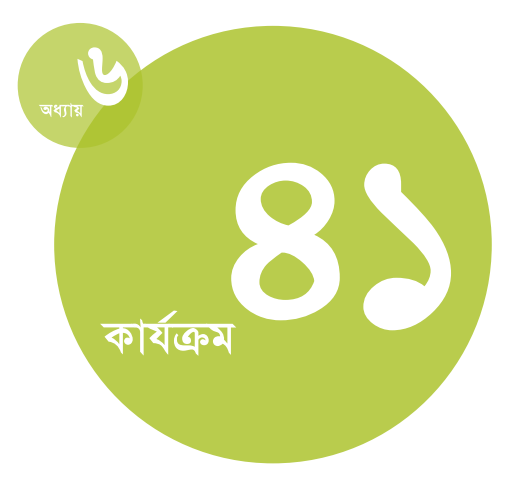

সময়কাল :

৬০ মিনিট (এক অথবা দুইটি লেশন)

\section{উপকরণ:}

একটি বই তৈরি ও ‘বাঁধাই’ করার জন্য ছবি আঁকার উপকরণ (যেমন- কাগজ, মার্কার, পোষ্টার বোর্ড, বড় কাগজ, সুঁই । সূতা অথবা স্ট্যাপলার)। ছেলেলেয়েদের বয়ঃসন্ধিকাল নিয়ে নির্ভরযোগ্য তথ্যমালা (ফ্যাক্টশীট), যেমন ধরা যাক নির্দেশিকা (এই বইয়ের সহ-গামী খণ্); <www. popcouncil.org/publications/ books/2011_ItsAllOne. asp> এই ঠিকানায় পাওয়া যাবে।

প্রস্তুতি:

ফ্যাক্টশীটগুলো ভালোভাবে পড়ন এবং আত্মস্থ করুন যেন শিক্ষার্থীরদের টৈরি করা জিনিসগুলোতে সহায়তা করতে পারেন এবং ভূলগুলো শুধরে দিতে পারেন। ছেলেমেয়েদের বয়ঃসন্ধির ওপর তৈরি তথ্যমালাগুলো ফটোকপি করুন । সম্ভব হলে, আপনার শিক্ষার্থীদের তৈরি জিনিসগুনো প্রাক-বয়ঃসন্ধিকালীন শিক্ষার্থীদের সামনে উপস্থাপন করার ব্যবস্থা করুন । 


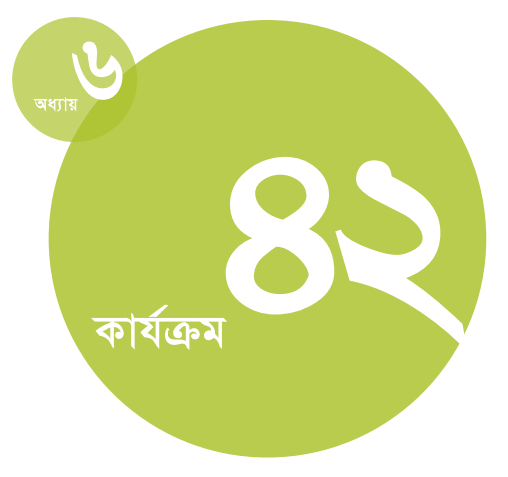

সময়কাল :

8৫ मिनिট

\section{উপকরণ:}

বোর্ড ও চক; কাগজের টকরো;

শিক্ষকদের জন্য তথ্য ও উত্তরপত্র

("বয়ঃসন্ধিকাল ও কিলোর-কিশোরী সম্পর্কিত পরিবর্তন")।

\section{প্রস্তুতি:}

তথ্য ও শিকদের জন্য উত্তরপত্রে বর্ণিত প্রত্যেকটি ‘পরিবর্তন’ পথথক কাগজে অনুলিপি করুন: ভাঁজ করুন এবং একটি

খামে রাখুন। ধাপ-৩ এ বর্ণিত

‘পরিবর্তনগুনো’ পুনরায় পড়ন এবং

আপনার সমাজে বয়ঃসঞ্ধিকালে কিলোর-

কিশোরীদের জীবন কিভাবে পরিবর্তিত

হয় তা বর্ণনা করার জন্য বর্ণিত

‘পরিবর্তনগুলো' তে পরিমার্জন আনুন

অথবা কিছু সংযুক্ত করুন ।

\section{বয়ঃসন্ধিকাল: কী আশা করা হয়}

অধিবেশন সম্পর্কিত ধারণা: শিক্ষার্থীরা ছেলে এবং মেয়েদের বয়ঃসন্ধিকালে ঘটে এমন সামাজিক পরিবর্তনগুনো বিশ্লেষণ করবে। (লক্ষণীয়: এই অধিবেশন শেষ করার পূর্বে শিক্ষার্থীদের অধিবেশন ৪১ অথবা বয়ঃসন্ধিকালের শারীরিক পরিবর্তন নিয়ে অন্যান্য পাঠ শেষ করবে।)

উদ্লেশ্য: শিক্ষার্থীদেরকে বয়ঃসন্ধিকালে জেন্ডার ভূমিকা পরিবর্তনের তিনটি উপায় বলতে এবং ছেলে, মেয়ে ও জেন্ডার সম্পর্কের ওপর এই পরিবর্তনের প্রভাব নিবিড়ভাবে নিরূপন করতে সক্ষম করা; সমালোচনামূলক চিন্তার দক্ষতা বৃদ্ধি করা ।

\section{নির্দেশাবলি}

১১ নিয়লিখিত ব্যাখ্যার মাধ্যমে বিষয়টি ঞুরু করুন্ন:

• সকল যুবক-যুবতীর জীবনে বয়ঃসন্ধিকাল ও কৈশোরের পরিবর্তনগুলো ঘটে। এদের মধ্যে কিছু পরিবর্তন হচ্ছে স্বাভাবিক শারীরিক বৃদ্ধি। অন্যগুলো শারীরিক বৃদ্ধি নয়; এগুলো মানুষ তোমাদের সাথে যেভাবে আচরণ করে সেই পরিবর্তন।

• আমি তোমাদের কাছে যাব এবং তোমাদের কাউকে একটি কাগজের টুকরো খুলতে বলব। এটি জোরে জোরে পড়বে এবং আমাকে বলবে এটা কি শারীরিক পরিবর্তন যা পৃথিবীর সকল যুবক-যুবতীর হয়, নাকি বয়ঃসন্ধিকাল অথবা কৈশোরে সমাজ যুবদের সাথে যে আচরণ করে সেই পরিবর্তন ।/यদি এই পরিবর্তনগুলো নিয়ে তোমার কাগজের টুকরো তৈরি করে না থাকে, তাহলে এলোমেলোভাবে বাছাই করা তালিকা দুইটি থেকে “বয়ঃসন্ধিকাল ও কৈশোর পরিবর্তন”এ বর্ণিত পরিবর্তনগুলো নিজে পড়।]

• যুবরা যখন বয়ঃসন্ধিকালে পৌঁছায়, সমাজ তাদের প্রতি আচরণে প্রায়ই কী পরিবর্তন করে তা এই অধিবেশনের বাকি সময় বিস্তারিভাবে দেখব। আমি তোমাদের শারীরিক পরিবর্তনের কথা বলছি না।
২ বোর্ডের ওপরের দিকে লিখুন মানুষ তোমাদের সাথে আচরণে কী পরিবর্তন আনতে পারে। তার নিচে, বোর্ডের একদিকে লিখুন ছেলে এবং অন্যদিকে লিখুন মেয়ে। ব্যাখ্যা করুন:

- ছোট ছেলেমেয়েরা যখন বয়ঃসন্ধিকালে পৌঁছায় তখন তাদের কী হয়? মানুষ কি তাদেরকে আলাদাভাবে দেখে? যখন আমরা এই পরিবর্তনগুলো বিস্তরিতভাবে দেখব, আমি চাই যে তোমরা আমাকে বল, এগুলো কি মেয়েদের ক্ষেত্রে প্রযোজ্য নাকি ছেলেদের ক্ষেত্রে প্রযোজ্য? যদি এগুনো উভয়ের ক্ষেত্রে প্রযোজ্য হয়, তাহলে আমাকে বল এগুলো কি মেয়েদের এবং ছেলেদের একইভাবে প্রভাবিত করে নাকি ভিন্নভাবে (এবং কীভাবে) প্রভাবিত করে?

৩ পরবর্তী পৃষ্ঠার তালিকা (আপনি পুনরায় পড়া এবং পরিবর্তন করার পরে) থেকে প্রত্যেকটি বিষয় পড়ন এবং শিক্ষার্থীদেরকে জিজ্ঞস করুন এগুলো ছেলেদের, মেয়েদের নাকি উভয়ের ক্ষেত্রে প্রযোজ্য। यদি এখানে ভিন্নমত থাকে তবে থামুন, আলোচনা করুন এবং ঐ বিষয়টি ছেলে, মেয়ে অথবা উভয়ের নিঢে লিখুন। 
বয়ঃসন্ধিকাল: মানুষ তোমার সাথে কীভাবে আচরণ পরিবর্তন করতে পারে:

- জনসমাগম হয় এমন স্থানে চলাচলের জন্য বেশি স্বাধীনতা (রাস্তা, পার্ক, কমিউনিটি সেন্টার, কেনাকাটার স্থান)।

• জনসমাগম হয় এমন স্থানে চলাচলের জন্য কম স্বাধীনতা।

• আরো বেশি গৃহস্থালির দায়িত্ব (যেমন গৃহস্থালির টুকিটাকি কাজ, অথবা বাচ্চার দেখাশোনা)।

• অর্থ উপার্জন শুরু করার জন্য আরো বেশি দায়িত্ব।

• এমনভাবে পোশাক পরার জন্য চাপ যেন শরীর আরো ঢেকে থাকে অথবা উনুক্ত থাকে।

- ছেলে এবং মেয়েদের সাথে আরো বেশি সমাজিক মেলামেশা অথবা আরো কম সামাজিক মেলামেলা ।

• ক্ষতিকর কাজের মাধ্যমে বয়স্ক রীতি-নীতির সাথে সম্পৃক্ত হওয়া/করা (যেমন-মেয়েদের যৌনাঙ্গছেদ)।

• ক্ষতিকর নয় এমন কাজের মাধ্যনে বয়স্ক রীতি-নীতির সাথে সম্পৃক্ত হওয়া।

- যৌন অভিজ্ঞতা অর্জনের জন্য সামাজিক চাপ বৃদ্ধি।

• বিয়ের জন্য প্রস্তুত হতে সামাজিক চাপ বৃদ্ধি।

- বিদ্যালয় এবং সমাজে নেতৃত্ব দেয়ার সুযোগ বৃদ্ধি।

• খেলাধূলায় সফল হওয়ার জন্য সামাজিক চাপ।

- নিজের পরিবার দ্বারা বিদ্যালয়ের লেখাপড়া ছাড়িয়ে দেয়ার সম্ভাবনা বৃদ্ধি।

• যৌন হয়রানির শিকার ।

• খারাপ দলের সাথে মেশার জন্য চাপ।

• বিপদজনক ঝুঁকি গ্রহণের মাধ্যমে জেন্ডার ভূমিকা মেনে নেয়ার চাপ।

• উপহার, টাকা অথবা বিদ্যালয়ের বেতন দেয়ার বিনিময়ে যৌনমিলন করতে চাপ।
8 শিক্ষার্থীদেরকে ছেলে ও মেয়েদের আলাদা করে ছোট ছোট দলে ভাগ করুন । বয়ঃসন্ধিকাল ও কৈশোরের সাথে সাথে ছেলেমেয়েদের জেন্ডার ভূমিকা ও জীবনযাত্রার পরিবর্তন নিয়ে আলোচনা করতে বলুন; সুনির্দিষ্টভাবে জিজ্ঞেস করুন:

- বয়ঃসন্ধিকালে ছেলেমেয়েরা যে সামাজিক আকাজ্ষার পরিবর্তন ও অভিজ্ঞতার সম্মুখীন হয় সেগুলো কি খুব চমকপ্রদ নাকি চমকপ্রদ নয়?

- বয়ঃসন্ধিকালে ছেলেরা কোন কোন বিষয়ের সম্মুখীন হয় বলে তোমরা লক্ষ্য করেছ? তাদের স্বাধীনতা কি বৃদ্ধি পাচ্ছে নাকি কমে যাচ্ছে? এই অভিজ্ঞতার গুরুত্ব কি বয়ঃসন্ধিকালের পরে হারিয়ে যায় নাকি প্রাপ্তবয়স্ক হওয়া পর্যন্ত ঐ ব্যক্তির জীবনে প্রভাব ফেলে ?

• বয়ঃসন্ধিকালে মেয়েরা কোন কোন বিষয়ের সম্মুখীন হয় হয় বলে তোমরা লক্ষ্য করেছ? তাদের স্বাধীনতা কি বৃদ্ধি পাচ্ছে নাকি কমে যাচ্ছে? এই অভিজ্ঞতার গুরুত্ব কি বয়ঃসন্ধিকালের পরে হারিয়ে যায় নাকি প্রাপ্তবয়স্ক হওয়া পর্যন্ত ঐ ব্যক্তির জীবনে প্রভাব ফেলে ?

৫ সম্পূর্ণ দলকে আবার একত্রিত করুন এবং জিজ্ঞেস করুন:

- বিষয়গুলো কি অন্যরকম হতে পারত? আরো ভালভাবে বা সৎভাবে কি জীবনযাপন করা সম্ভব? তোমাদের লক্ষ্য কী?

• এমন একটি পদ্ধতির কথা বল যাতে করে সমাজ ছেলে-মেয়েদের বয়ঃসন্ধিকাল ও কৈশোরের ভাল অভিজ্ঞতার ব্যবস্থা করতে পারে।

বাড়ির কাজ: ছোট দুইটি চিঠি লেখ: “আমার ভবিষ্যৎ মেয়ের কাছে প্রতিজ্ঞা” এবং “আমার ভবিষ্যৎ ছেলের কাছে প্রতিজ্ঞা ।” তোমাদের লেখাটি হবে আজকের আলোচনার ভিত্তিতে। 


\section{বয়ঃসন্ধিকাল ও কৈশোর সর্প্পকিত পরিবর্তন}

শিক্ষকের জন্য নির্দেশাবলি: বুলেট করা প্রতিটি বিষয় পৃথক কাগজে অনুলিপি করুন এবং ভাঁজ করে একটি খামে রাখুন। শিক্ষার্থীরা শারীরিক (শরীরের মধ্যে) এবং সামাজিক (মানুঃ্ের সাথে কীভাবে জেন্ডারের ভিত্তিতে আচরণ করা হয়) পরিবর্তনের মধ্যে সঠিকভাবে পার্থক্য করতে পারছে কি না তা নিশ্চিত হওয়ার জন্য প্রয়োজনে কাগজটি উত্তরপত্র হিসেবে ব্যবহার করুন।

\section{শারীরিক পরিবর্তন}

দেহের বিভিন্ন অংশে লোম হওয়া

ঘाম বেশি হওয়া

স্তন ভারী হওয়া (মেয়েদের)

স্বপ্নদোষ (ছেলেদের)

কন্ঠস্বরের পরিবর্তন (ছেলেদের)

শরীরের উচ্চতা ও ওজন বৃদ্ধি; বাড়তি পুষ্টির প্রয়োজন

যৌনানুভূতি বৃদ্ধি

মাসিকের রক্ত্রাব/পিচ্ছিল পদার্থ নিঃসরণ (মেয়েদের)

\section{মানুষ তোমার সাথে কীভাবে আচরণ করে তার}

\section{পরিবর্তন:}

বিদ্যালয় এবং সমাজে নেতৃত্ব দেয়ার সুযোগ টৈরি হয়

বড়দদর রীতি-নীতির সাথে সম্পৃক্ততা ৃতরি হয়

দায়িত্বৈর পরিবর্তন হয়

যৌনকরর্ম সংক্রান্ত মানসিক চাপ টৈরি হয়

পোশাক পরিচ্ছদদ নতুন নিয়ম

ছেলে ও মেয়েদের সামাজিক মেলামেশার নতুন নিয়ম

অনুমোদিত স্বাধীনতার মাত্রার পরিবর্তন 


\section{শরীরের মজাদার বিষয়গুলো}

অধিবেশন সম্পর্কিত ধারণা: শিক্ষার্থীরা শব্দজট ও অনুশীলনীর মাধ্যন্মে শরীর ও প্রজননের ‘মজাদার বিষয়গুলো’ পড়বে এবং শিখবে। (লক্ষণীয়: এই অধিবেশনটি প্রজননের মৌলিক বিষয় অথবা প্রজনন পাঠের পরিপূরক, এটি বিষয়বস্তুর সম্পূর্ণ ধারণা দেয় ना ।)

উল্লেশ্য: শিক্ষার্থীদের প্রজনন সংক্রান্ত জ্ঞান বৃদ্ধি করা; প্রজননবিদ্যায় আগ্রহী করা।

\section{নির্দেশাবলি}

১ শব্দজটের অনুলিপিগুলো বিতরণ করুন এবং ব্যাখ্যা করুন্ন:

• আমরা প্রজনন স্বান্থ্যের নৌলিক বিষয়গুলো শিখছি। পর্যালোচনার জন্য প্রত্যেকটি ইঙ্দিত পড় এবং সঠিক উত্তর দিয়ে শব্দজট পূরণ কর।

• প্রতিটি উত্তর সঠিক ঘরে বসাতে হবে।

- পাশাপাশি ঘরগুলো যেখানে উপর-নিচের ঘরের সাথে মিলবে সেখানে একটাই বর্ণ বসবে।

২ ১০-১৫ মিনিট পরে শ্রেণিকক্ষ ঘুরে সঠিক উত্তর পর্যবেক্ষণ করুন । আকর্ষণীয় বিষয়ের কপিগুলো বিতরণ করুন এবং আলোচনা করুন

৩ ‘মজাদার বিষয়ের’ হ্যান্ডআউটগুলো বিতরণ করুন । ব্যাখ্যা করুন:

• আমরা এখন শরীর সম্পর্কে আরও অনেক বিষয় জানব। এগুলোর বেশিরভাগই প্রজনন সম্পর্কে, কিন্তু কিছু আছে নিছকই শরীর সম্পর্কে মজার বিষয়।

• আকর্ষণীয় বিষয়গুলোর তালিকা পড়তে পাঁচ মিনিটের মতো সময় নাও। এরপর তোমার কাছে সবচেয়ে বেশি মজার মনে. হয় এমন একটি বিষয়ে বৃত্ত পূরণ কর।

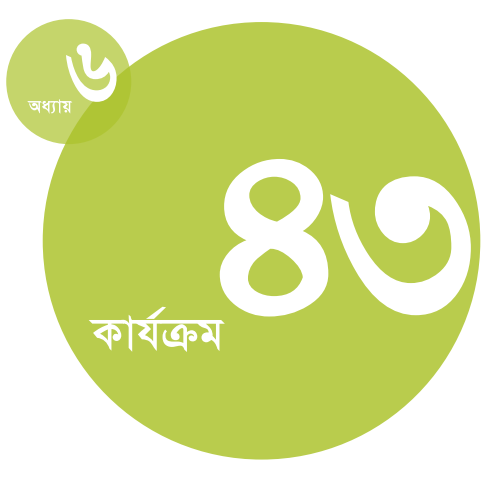

সময়কল :

ধাপ ১-২: ২০ মিনিট

ধাপ ৩-৫: ২৫ মিনিট

উপকরণ:

প্রত্যেক শিক্ষার্থীর জন্য শব্দজট;

• দুইজনকে তোমার পাশে বসতে দাও এবং দেখ তুমি যে বিষয়টিতে বৃত্ত পূরণ করেছ সেটি কি তাদের সাথে মিলে যায় নাকি পৃথক।

• তোমাদের মধ্যে কতজন একই বিষয়ে বৃত্ত পূরণ করেছ? কতজন পৃথক বিষয়ে বৃত্ত পূরণ করেছ? [হাত তোল]

• তোমাদের মধ্যে কতজন কমপক্ষে একটি নতুন তথ্য শিখেছ?

8 শিক্ষার্থীদেরকে বলুন যে বিষয়টিতে তারা বিশেষ ভাবে আগ্রহী, সেটা স্বেচ্ছায় তুলে নিতে। এরপর শিক্ষকের উত্তরপত্র থেকে বিষয়টির ওপর বাড়তি তথ্য তুলে ধরুন। সম্ভব হলে, শিক্ষার্থীদের কাছ থেকে তথ্য পাওয়ার জন্য প্রশ্ন করুন ।

৫ যদি সময় থাকে: যখন ১৫ নাম্বার বিষয়ে পৌঁছাবেন, তখন বাহু দৈর্ঘ্যের সাথে উচ্চতার তুলনা পর্বে সাহায্য করার জন্য শিক্ষার্থীদেরকে জোড়া গঠন করতে বলুন । ব্যাখ্যা করুন যে, এই বিষয়গুলি প্রজননের সাথে সরাসরি সম্পৃক্ত নয়, বরং নিজের শরীর সম্পর্কে জানার অংশ।
‘মজাদার বিষয়ের’ হ্যান্ডআউট; শিক্ষকের উত্তরপত্র; প্রজননবিদ্যা সম্পর্কে একট। নির্ভরয়োগ্য তথ্যের উৎস, যেমন নির্দেশিকা (এই বইয়ের সহ-গামী খণ্ড) বইয়ের অধ্যায় ৬ ও সহয়োগী তথ্যমালা (ফ্যাক্টশীট)।

প্রস্তুতি:

উপকরণগুলো বিলেষ করে মাসিক চক্র, পুরুষ ও মহিলাদের যৌন ও থ্রজনন তন্ত্র, প্রজনন ও গর্ভধারণ, এবং বন্ধাত্ত্য ও সহায়ক প্রজনন সম্পর্কে সতর্কতার সাথে পড়ন। হ্যান্ডআউটে শব্দজটের উত্তর রাখবেন কিনা সিদ্ধান্ত নিন। সিদ্ধান্ত নিন সবগুলো ‘মজাদার বিষয়’ রাখবেন নাকি বিষয়বস্তুর ভিন্নতা ও সময়ের জন্য কিছু বিষয় বাদ দেবেন 


\section{প্রজননতন্ত্রের শব্দজট}

প্রতিটি যোগসূত্র পড় ও সঠিক উত্তর পূরণ কর। উত্তর অবশ্যই সঠিক সংখ্যার ঘরগুনোর সাথে মিলতে হবে। যেই ঘরে ওপর-নিচের উত্তরগুনো পাশাপাশি উত্তরগুলোকে ছেদ করবে সেই ঘরে একই বর্ণ বসবে।

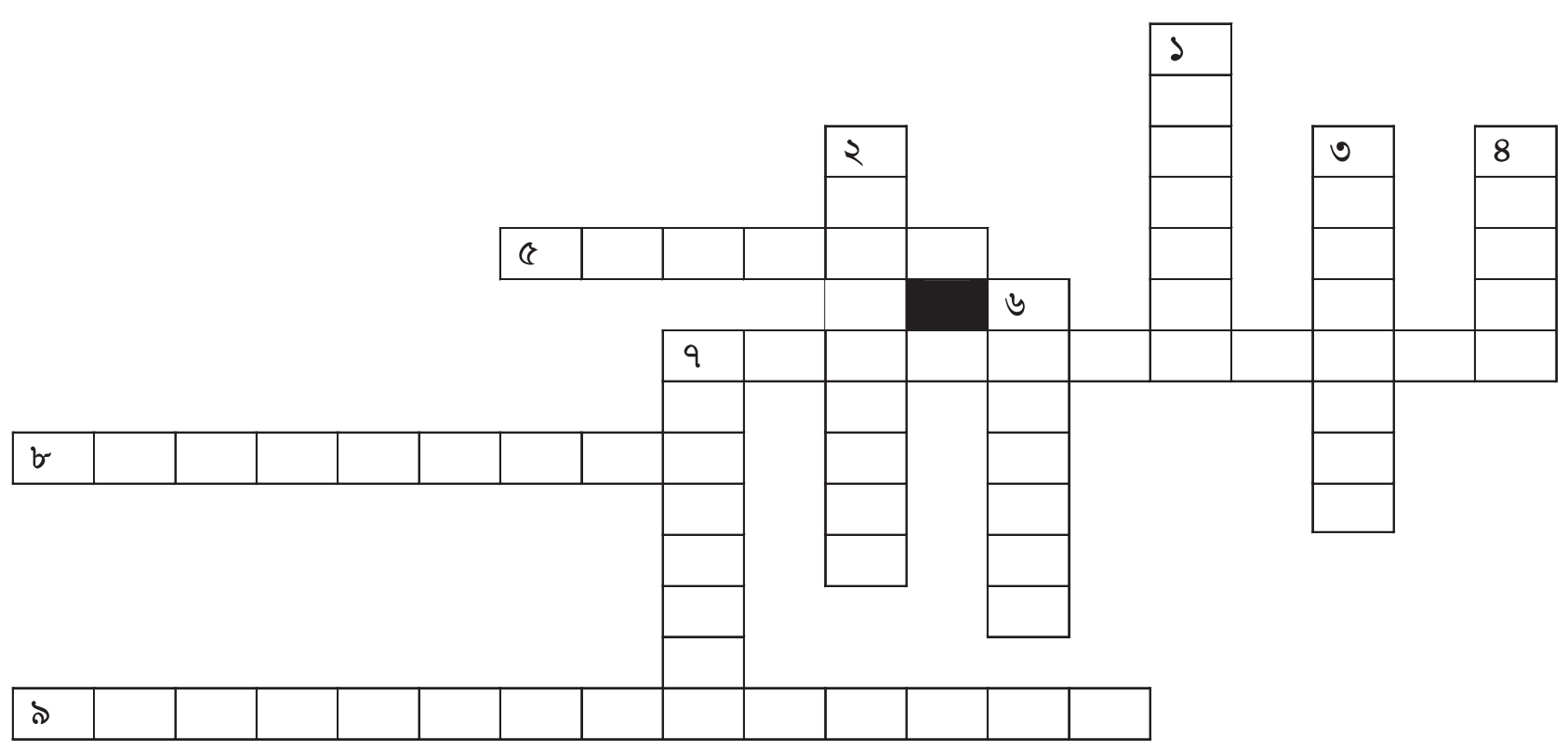

\section{পাশাপাশি}

৫. যেখানে শিল বেড়ে ওঠঠ

৭. পুরুষাঙ্গ হতে দ্রুত বেরে বীর্য বের হওয়া

৮. মহিলাদের ঋতুস্রাব বন্ধ হওয়ার সময়

৯. গর্ভাশয় ও জরায়ুর সংত্যাগকারী নালি; এখানে ডিম্বাণু ও কক্রাণু মিলিত হয় (দूটি শব্দ)

ejaculation; 8. menopause; 9.fallopion

tubes. ওপর-নিচে: 1 .wet dream;

2.ovulation; 3 .testicle; 4 .semen;

6.puberty; 7.erection

\section{ওপর-নিচে}

১. ঘুনের মধ্যে বীর্য বের হওয়া (দুটি শব্দ)

২. ডিম্বাশয় হতে ডিম্বাণু মুক্ত হওয়া

৩. ওক্রাণু তৈরি হওয়ার জায়গা

8. বীর্যপাতের সময় পুরুষাঙ্গ হতে যে ঘন তরল পদার্থ বের হয়

৬. যে প্রক্রিয়ায় একটি শিফ্যর শরীর দ্রুত পূর্ণবয়ক্ণ মানুভের শরীরে পরিণত হয় এবং প্রজনनক্ষম হয় 


\section{তোমাদের শরীরের মজাদার বিষয়গুলো}

কোন বিষয়টি তোমার কাছে সবচেয়ে অব্ভুত ও আকর্যণীয় মনে হয়েছে? মজাদার বিষয়ের তলিকাটি পড়। যে বিষয়টি পড়ে তোমার মনে হবে অবশ্যই জানা উচিত তার পাকের সংখ্যাটিতে বৃত্ত দ্বারা গোল কর।

১ অনেক নারী ঋতুচ(্রের উর্বর দিনগুলোতেত ব্যেনকর্জে বেশি আগ্রহী হয়ে থাকে।

২ যে সকল দম্পতিরা বাচ্চা দিতে অক্ষম; সমস্যাটি নারীর জন্যও হতে পারে আবার পুরুষের জন্যও হতে পারে ।

৩ মানবদেহের ক্ষুদ্রান্ত্র প্রায় ৭ মিটার লম্বা ।

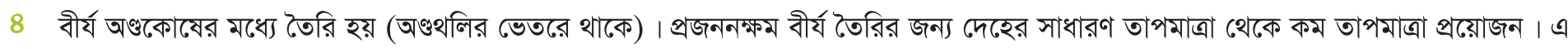
কারণে অণ্থলি দেহের বাইরের দিকে অবস্থিত, এটা শীতল তাপমাত্রা নিয়ন্ত্রণ করে।

৫ যদিও নারী ওখুমাত্র ঋতুচট্রের বিশেষ কয়েকদিন সন্তান উৎপাদনে সক্ষম থাকে, কিন্ত পুরুষয়া বয়ঃসন্ধি থেকে ওরু করে বিরতিহীনভাবে সন্তন উৎপাদনে সক্ষম থাকে।

৬ একবার বীর্যপাতত ১ কোটিরও বেশি ফক্রাণু নিগ্গত হয়।

१ মানবদদদ প্রতি ২8 ঘন্টায় প্রায় ১ লিটার লালা টতরি করে।

৮. কোন মহিলা অথবা কিলোরী তার যোনিপথ থেকে বের হওয়া পিচ্ছিল পদার্থের সাধারণ পরিবর্তন লক্ষ্য করলে জানতে পারে কখন ডিম্বাণু নির্গত হয় এবং এভাবে সে বুঝঢত পারে কখন সে প্রজননক্ষম হয় (অথবা গর্ভধারণ করতে পারে) এবং খতুস্রাবের সম্ভাব্য দিন বলতে পারে।

৯ মানবদেহের শতকরা ৫০ ভাগেরও বেশি হচ্ছে পানি ।

১০ গর্ভবতী মহিলার পেটের আকারের সাথে তাঁর বাচ্চার লিজের (বাচ্চা ছেলে বা মেয়ে) কোন সম্পর্ক নেই।

১১ গর্ভধারণের শেষ দিকে, একজন মহিলার কোমরের হাড়ের জোড়াগুলো ঢিলা হয়ে যায়, এতে ঐ জায়গা প্রসারিত হয় এবং বাচ্চার নড়াচড়া করার জায়গা তৈরি হয়।

১২ খুব কম ক্ষেত্রেই একজন মহিলার একটার পরিবর্তে দুইটা ডিম্বাণু নির্গত হয়। দুইটিই যদি ফ্রাণু দ্বারা নিযিক্ত হয় তাহনে যমজ বাচ্চা হয় (চেহারা এবং লিঙ্গ আলাদা বা একই হতে পারে)। यদি একটি ডিম্বাণু নিষিক্ত হয়ে দুইভাগে ভাগ হয় তাহলে যমজ শিঞর চেহারা এবং লিঙ একই হয়।

১৩ বেশিরভাগ ছেলেদেরই বয়ঃসন্ধিকালে স্বপ্নদোষ হয়।

১৪ মানব ঙ্রণণের লিঙ্গ পিতার শ্রাণ্রাণু দ্বারা নির্ধারিত হয়, মায়ের ডিম্বাণু দ্বারা নয়।

১৫ তোমার পুরো শরীরের দৈর্ঘ্য তোমার প্রসারিত বাহুর দৈর্ঘ্যের সমান । 


\section{‘মজাদার বিষয়শ'-অতিরিক তথ্য}

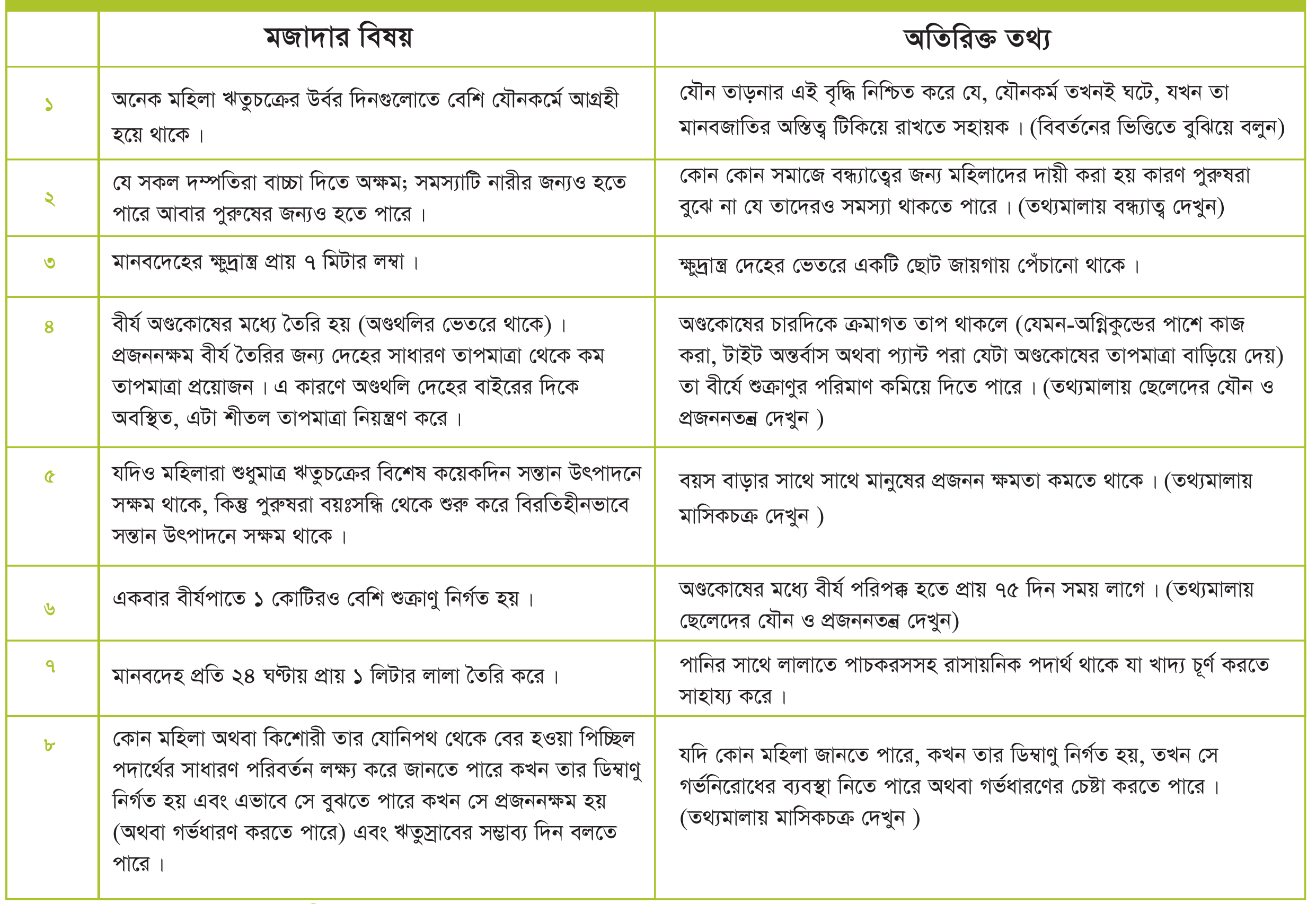




\section{‘মজাদার বিষয়’-অতিরিক তথ্য}

\begin{tabular}{|c|c|c|}
\hline & মজাদার বিষয় & অতিরিক্ত তথ্য \\
\hline ৯ & মানবদেছের শতকরা ৫০ ভাগেরও বেশি হচ্ছে পানি। & এবং তোমার সবচেয়ে বড় অঙ্গ হচ্ছে তোমার ত্বক। \\
\hline so & $\begin{array}{l}\text { গর্ভবতী মহিলার পেটের আকারের সাথে তাঁর বাচ্চার লিঙ্গের (বাচ্চা } \\
\text { ছেলে বা মেয়ো) কোন সম্পর্ক নেই । }\end{array}$ & $\begin{array}{l}\text { গর্ভবতী মহিলাদের ওপর চালানো এক গবেষণায় পেটের আকারের সাথে গর্ভের } \\
\text { বাচ্চা ছেলে না মেয়ে (লিঙ্গ) তার কোন সম্পর্ক পাওয়া যায়নি । }\end{array}$ \\
\hline ১১ & $\begin{array}{l}\text { গর্ভধারণের শেষ দিকে, একজন মহিলার কোমরের হাড়ের জোড়াগুনো } \\
\text { ঢিলা হয়ে যায়, এতে ঐ জায়গা প্রসারিত হয় এবং বাচ্চার নড়াচড়া } \\
\text { করার জায়গা টৈরি হয়। }\end{array}$ & $\begin{array}{l}\text { মহিলাদের গর্ভধারণের শেষ দিকে সাবধানে চলাচল করতে হয় । কারণ তার } \\
\text { মনে হতে পারে হাড়ের িলা জায়গাগুনো নড়াচড়া করছে (তথ্যমালায় সন্তান } \\
\text { জন্মদান ও বুকের দুধপান অংশ দেখুন )। }\end{array}$ \\
\hline गे & $\begin{array}{l}\text { খুব কম ক্ষেত্রেই একজন মহিলার একটার পরিবর্তে দুইটা ডিম্বাণু } \\
\text { নির্গত হয় । দুইটিই যদি ক্রাণু দ্বারা নিষিক্ত হয় তাহলে যমজ বাচ্চা } \\
\text { হয় (চেহারা এবং লিঙ্গ আলাদা বা একই হতে পারে) । যদি একটি } \\
\text { ডিম্বাণু নিষিক্ত হয়ে দুইভাগে ভাগ হয় তাহনে যমজ শিঞ্র চেহারা } \\
\text { এবং লিঙ্গ একই হয়। }\end{array}$ & $\begin{array}{l}\text { যমজ বাচ্চারা দুটি আলাদা ডিম্বাণু থেকে জন্ম নিলে তারা দেখতে অন্য যে কোন } \\
\text { আপন ভাই বা বোনের মত হবে, কিন্তু একই রকম দেখতে যমজরা একই } \\
\text { নিউক্লিয়াস থেকে জন্ম হয় । তাদের প্রায় একই ডিএনএ (জিন) থাকে । } \\
\text { (তথ্যমালায় প্রজনন ও গর্ভধারণ অংশ দেখুন ) }\end{array}$ \\
\hline ১৩ & বেশিরভাগ ছেলেদেরই বয়ঃসন্ধিকালে স্বপ্নদোষ হয়। & $\begin{array}{l}\text { ঘুনের মধ্যে ছেলেদের/পুরুম্দের বীর্যপাত (একে স্বপ্নদোষও বলে) হয়ে থাকে। } \\
\text { স্বপ্নদোষ একটি স্বাভাবিক ব্যাপার এবং এটা কোনভাবেই ক্ষতিকর নয় । }\end{array}$ \\
\hline 28 & $\begin{array}{l}\text { মানব ভ্রगণের লিঙ্গ পিতার ক্রক্রাণু দ্বারা নির্ধারিত হয়, মায়ের ডিম্বাণু } \\
\text { দ্বারা নয়। }\end{array}$ & 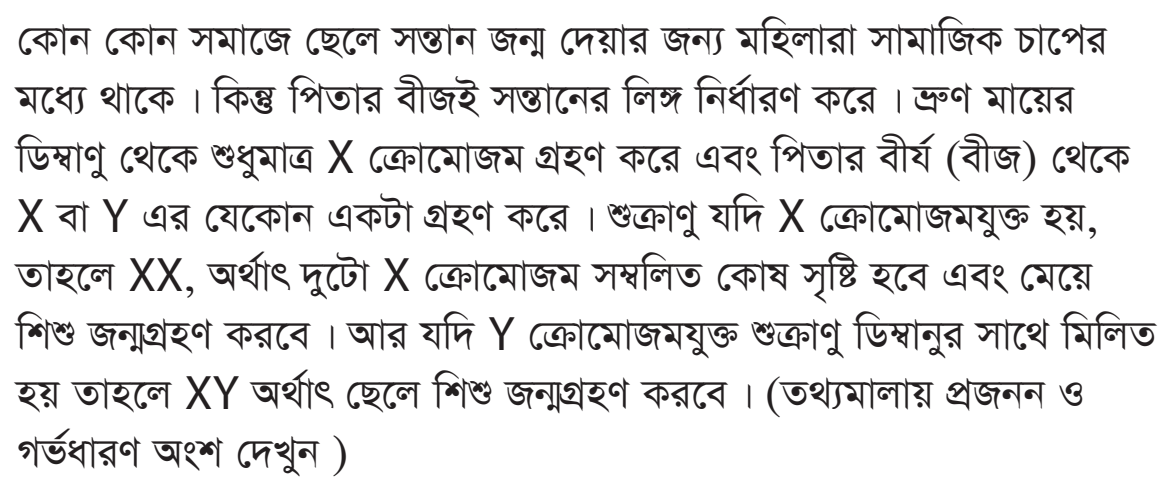 \\
\hline$د \notin$ & তোমার পুরো শরীরের দৈর্ঘ্য তোমার প্রসারিত বাহুর দৈর্ট্য্যের সমান । & এটি যাচাই করে দেখ! \\
\hline
\end{tabular}




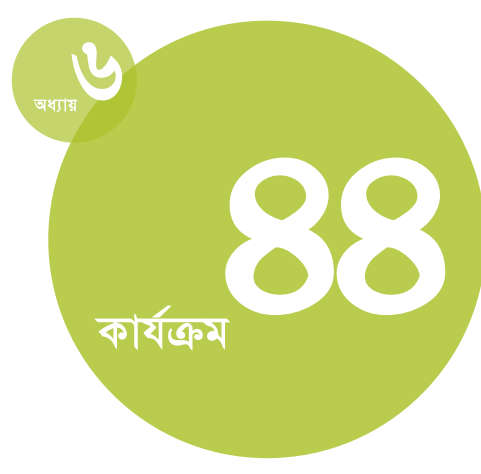

সময়কাল :

ধাপ ১- ৬: ৪৫ মিনিট

ধাপ ৭ (বাড়ির কাজ): ৪০ মিনিট

ধাপ ৮-১১ (ঐচ্ছিক): 80 মিনিট

\section{উপকরণ:}

শিক্ষার্থীদের জন্য হ্যান্ডআউটের অনলিপি। অ - ঋ এ উলিখিত

বিষয়গুলো শিক্ষার্থীদের পরিচিতির জন্য: নির্দেশিকা থেকে লিঙ্গ নির্ধারণ; বন্ধ্যাত্য; সহায়ক প্রজনন; শিশুর জন্ম ও বুকের দুধ পান: এবং গর্ভপাতের ওপর তথ্যমালার অনুলিপি। নির্দেশিকা হুলো এই বইয়ের মতন একটি বই যা <www.popcou ncil.org/publications/books/20 11_ItsAllOne. asp> এই ঠিকানায় পাওয়া যাবে।

\section{প্রস্তুতি:}

হ্যান্ডআউটের বিষয়গুলো পর্যালোচনা এবং পরিবর্তন করুন (প্রয়োজন অনুযায়ী)। হ্যান্ডআউটগুলো ফটোকপি করুন অথবা পাঠদানের পর্র্বে চড়ান্ত বিষয়ের তালিকাটি বোর্ডে লিখুন বাছাইকত তথ্যুুলো ফটোকপি করুন শিক্ষার্থীরা তাদের সম্পাদনা করা চড়ান্ত কাজটি কোথায় জমা দিতে পারবে তা ঠিক করুন (যেমন, স্থানীয় সংবাদপত্র, “এক মিনিটের মন্তব্য” নামে ধারাবাহিক রেডিও শো, বিদ্যালয়ের এ্যাসেম্বলী, অথবা একটি বই হিসেবে)

\section{প্রজনন: ব্যক্তিগত বিষয়ের চেয়েও বেশি কিছু}

অধিবেশন সম্পর্কিত ধারণা: শিক্ষার্থীরা প্রজনন বিষয়ক (কিশোর পিতৃত্ব থেকে লিঙ্গ নির্ধারণ পর্যন্ত) ১৫টি সমসাময়িক জনস্বাস্থ্য এবং নীতি শিখবে। তারা একটি পত্রিকায় ব্যক্তিগত মতামত লিখবে এবং সারসংক্ষেপ সংকলন/সম্পাদনা করে জনসাধারণের জন্য গল্পাকারে একটি খবর তৈরী করবে।

উদ্লেশ্য: প্রজনন বিষয়ক জনস্বাস্থ্য এবং নীতিতে শিক্ষার্থীদের জ্ঞান এবং সচেতনতা বৃদ্ধি করা; তাদের লেখার ও সম্পাদনার দক্ষতা বৃদ্ধি করা; সমাজে তারা মতামত তৈরী করতে পারে, তাদের মধ্যে এই বিশ্বাসকে দৃঢ় করা ।

\section{নির্দেশাবলি}

১ ব্যাখ্যা করুন:

• আমরা মানুষের প্রজনন সম্পর্কে পড়ছি। প্রজনন কি সবসময় গোপনীয় বিষয়, যারা সরাসরি যুক্ত শুধু তাদের আগ্রহের বিষয়? নাকি প্রজননের কিছু বিষয় জনসাধারণেরও আগ্রহের বিষয়, যেমন স্বাস্থ্যকর্মকর্তা, সমাজ, এবং অন্যান্যদের ? [উদাহরণ খুঁজুন, যেমন- স্বাস্থ্য সেবার জন্য অর্থায়ন, গর্ভপাত বিষয়ে আইন, হসপিটালের নিয়ম-কানুন, এবং এমন অন্যান্য বিষয় ।]

• আসলে, প্রজনন স্বাস্থ্য একটি জনগুরুত্বপূর্ণ বিষয়। এটি প্রায়ই বিতর্কের উৎস। আধুনিক পৃথিবীতত-নতুন প্রযুক্তি, এইচআইভি, পরিবর্তিত সাংস্কৃতিক রীতি-নীতি- প্রজননের সাথে যুক্ত বিষয়গুলো শেখা গুরুত্বপূর্ণ। এগুলো তোমাদের জীবনের ওপর প্রভাব ফেলতে পারে।

- আজকে আমরা একটি প্রকল্প শুরু করব। তোমরা প্রত্যেকে এমন একটি বিষয়ের নাম বলবে যাতে তোমাদের আগ্রহ আছে অথবা তোমাদের কাছে বিষয়টির একটি নিজস্ব অর্থ আছে। [কীভাবে করবেন তা তুলে ধরুন - যেমন, স্থানীয় সংবাদপত্রের জন্য একটি লেখা তৈরী করে।]
২ বিষয়গুনোর তালিকা ভালমত পড়ন । শিক্ষার্থীদেরকে নিজেদের মত বিষয়গুলো সবার সামনে তুলে ধরতে বলুন। বিষয়গুলোতে প্রত্যেকের একটি মৌলিক ধারণা আছে এর দ্বারা তা নিশ্চিত হবে। এরপর ব্যাখ্যা করুন:

• প্রত্যেকেই একটি বিষয় বেছে নেবে যে বিষয়টিতে তোমাদের কিছু ব্যক্তিগত অর্থ, গুরুত্ব, অথবা কৌতূহল আছে। এটি বড় কোন গবেষণা প্রকল্প নয়! দুইটি বড় অনুচ্ছেদের বেশি লিখবে না। প্রত্যেক অনুচ্ছেদের জন্য তোমাদের কাছে সাধারণ নির্দেশনামূলক প্রশ্ন থাকবে। এটা হতে হবে তোমাদের সর্বোত্তম লেখা ।

• আজকে তোমরা যে বিষয় নিয়ে লিখবে তার পাশে সই করবে এবং এই বিষয়ে অল্প কিছু শিখবে। কিছু বিষয়ের জন্য, আমি তোমাদেরকে একটি অনুচ্ছেদ অথবা এক পৃষ্ঠা পড়তে দেব। অন্য বিষয়গুলোর জন্য, তোমাদের শ্রেণিকক্ষের তিনজনের সাক্ষাতকার নেবে এবং তাদের মতামত লেখবে। 
৩ হ্যান্ডআউটে দেয়া বাড়ির কাজের নির্দেশনাগুলো পড়ন । যদি আপনার কাছে শিক্ষার্থীদের জন্য হ্যান্ডআউটের অনুলিপি করা না থাকে, নির্দেশনাগুলো “নিউজ ফ্লাশ” অনুচ্ছেদের জন্য বোর্ডে লিখুন।

8 [यদি আপনি ঐচ্ছিক সম্পাদনা করার কাজ করতে চান:] আগামীকাল তুমি আরেকজন শিক্ষার্থীর সাথে শুধুমাত্র তোমার দ্বিতীয় অনুচ্ছেদ (সংবাদ খণ্ণ) বিনিময় করবে। একে অপরের সাথে তোমাদের লেখা স্পষ্ট করা, ব্যাকরণ সঠিক করা, লেখা গোছানো এবং আকর্ষণীয় করবে। আমরা এই সংবাদ খণ্ডগুলো "গর্ভধারণ এবং প্রসবের সমসাময়িক বিষয়” শিরোনামে একসাথে করব এবং সবার সামনে তুলে ধরব-[যেমন, স্থানীয় সংবাদপত্র অথবা রেডিও।]

৫ নিশ্চিত হোন যে, কাজটি ভালভাবে হবে। শিক্ষার্থীদের একটি কাগজ দিন এবং সই করতে বলুন । যদি শিক্ষার্থীরা কোন বিষয় ভালভাবে বুঝতে চায়, তাহলে আপনার সাথে আলোচনা করতে উৎসাহিত করুন । যে বিষয়ে এখনো কেউ সই করেনি এমন একটি বিষয়ে তাদেরকে সই করতে বলুন।
৬ বাকী সময় শিক্ষার্থীদের নিচের কাজ কুরু করার জন্য রাখুন: ক - চ এর বিষয়গুলো শিক্ষার্থীরাদের বাছাইয়ের জন্য: তাদেরকে উপকরণের অনুলিপি দিন এবং পড়া ওরু করতে বলুন। (যদি বাড়ি নিয়ে যাওয়ার মত পর্যাপ্ত অনুলিপি থাকে, তারা বাড়িতে পড়া শেষ করতে পারে।)

ছ- ঢ এর বিষয়গুলো শিক্ষার্থীরাদের বাছাইয়ের জন্য: তাদেরকে একে অপরের সাক্ষাৎকার নিতে বলুন এবং মতামতগুলো লিখতে বলুন। শিক্ষার্থীরা যদি অন্যান্যদের মতামত নিতে পারে তাহলে সে ব্যাপারেও তাদেরকে উৎসাহ দিন। (সাক্ষাৎকার বাড়িতেও শেষ করা যেতে পারে, যেমন, পরিবারের সদস্যদের সাথে ।)

१ বাড়ির কাজ বোঝানোর জন্য পাঁচ মিনিট সময় রাখুন ।

\section{বাড়ির কাজ:}

यদি এখনও তোমাদের পড়া শেষ না হয়, তাহুে এটি বাড়িতে গিয়ে শেষ কর। তারপর তোমাদের দুইটি অনুচ্ছেদ লেখ । হ্যান্ডআউটের প্রশ্নগুলোর উত্তর দিবে এবং প্রত্যেক অনুচ্ছেদ আলাদা আলাদা কাগজে লেখার কথা মনে রাখবে।

ধাপ b-১১ এর জন্য পরবর্তী পাতায় দেখুন (ঐচ্ছিক সম্পাদনার কাজ)। 


\section{ঐচ্ছিক কার্যাবলি:}

\section{সম্পাদনা, আলোচনা এবং সৃষ্টি}

b শিক্ষার্থীদেরকে দ্বিতীয় অনুচ্ছেদ (সংবাদ সংক্ষেপ) সম্পাদনার জন্য নিজেদের মধ্যে জোড়া বেঁধে তৈরি হতে বলুন । যদি দুই বা বেশি শিক্ষার্থী একই বিষয়ে কাজ করে থাকে, তাদেরকে একসাথে রাখুন এবং সংবাদ টুকরোগুনো থেকে একটি সংবাদ তৈরি করতে উৎসাহ দিন যেন পাঠকরা পড়ে বিভ্রান্ত না হয় অথবা বিরক্ত না হয়।

৯ শিক্ষার্থীদেরকে জিজ্ঞস করুন:

• ভাল লেখার বৈশিষ্ট্যগুলো কী? [জানার চেষ্টা করুন: স্পস্টতা; একই কথা বারবার না বলা; গোছানো; শব্দের সুন্দর ব্যবহার; প্রয়োজনীয় আবেগের ব্যবহার; সঠিক ব্যাকরণ ও বানান। এই বিষয়গুলো বোর্ডে লিখুন।

• কারো লেখার মতামত দেওয়ার ভাল উপায় কী? [জানার চেষ্টা করুন: তোমার পছন্দ হয়েছে লেখার এমন কিছু দিক উল্লেখ কর; সমালোচনা করার সময় সংবেদনশীল এবং শ্রদ্ধাশীল হও; কিছু পরামর্শ দাও, কিন্তু ঐ ব্যক্তির লেখা নতুন করে লিখ না।]

• মতামত গ্রহণের ভাল উপায় কী? [জানার চেষ্টা করুন: ভাল মতামতের জন্য লোকটিকে ধন্যবাদ দাও; যেখানে তুমি থেমে যাবে অথবা সাহায্যের প্রয়োজন হবে তা প্রকাশ কর; তোমার পরের কাজের জন্য আরো বেশি মতামত চাও।]

১০ শিক্ষার্থীদেরকে একে অপরের মতামত দিতে বলুন, প্রথমজনের অনুচ্ছেদের জন্য দশ মিনিট, দ্বিতীয় ব্যক্তির অনুচ্ছেদের জন্য দশ মিনিট, এবং প্রত্যেকের সর্বশেষ আলোচনার জন্য দশ মিনিট সময় দিন। প্রত্যেক দশ মিনিট শেষ হনে জানিয়ে দিন।

১১ সংশোধিত অনুচ্ছেদগুলো (ব্যক্তিগত মতামতসহ) শিক্ষার্থীদের কাছ থেকে নিয়ে নিন এবং দলের সকলের পড়ার জন্য সংবাদ সংক্ষেপগুলো দেয়ালে লাগিয়ে দিন। यদি সময় থাকে (অথবা পরের দিন): কিছু শিক্ষার্থীকে তাদের অনুচ্ছেদ অথবা চূড়ান্ত লেখাটি নিয়ে আলোচনা করার জন্য ডাকুন ।

করণীয়: শিক্ষার্থীদের সংবাদ টুকরোগুলো একত্রিত করুন । তাদেরকে সংবাদ টুকরোগুলো একসাথে স্টাপল করতে অথবা একটি ডিজিটাল ফাইলে যুক্ত করতে বলুন, এবং এগুলো কোন সংবাদপত্র বা মানুষজনকে জানানোর জন্য পাঠান। সংবাদ টুকরোগুলো একত্রিত করার সময়ে একটি শিরোনাম দিন অথবা চূড়ান্ত জিনিসটি পাঠানোর পূর্বে একটি সূচনা চিঠি জেখার মাধ্যমে তাদের উদ্ভাবনী শক্তি কাজে লাগাতে উৎসাহ দিন। 


\section{গর্ভধারণ ও প্রসবের সমসাময়িক বিষয়}

তোমাদের বিষয়টি নিয়ে দেখার জন্য নির্দেশাবলি: তোমরা দুইটি আলাদা আলাদা কাগজে দুইটি অনুচ্ছেদ

লিখবে। প্রথম অনুচ্ছেদটি হচ্ছে তোমার ব্যক্তিগত মতামত। বিষয়টি সম্পর্কে তোমরা শুধুমাত্র নিজের অনুভূতি লিখবে। এই বিষয় তোমাদের কোন অভিজ্ঞতা থাকলে তাও লিখতে পার। দ্বিতীয় অনুচ্ছেদটি হুো “সংক্ষিপ্ত সংবাদ"। এই সংক্ষিপ্ত সংবাদের উদ্দেশ্য হলো অন্যদের শেখানো।

\section{তোমাদের সংক্ষিপু সংবাদ তৈরি করতে:}

১ তোমাদের বিষয়ের তথ্য সংগ্রহ কর । ক - চ বিষয়ের জন্য, নির্দেশিত উপকরণ থেকে তথ্য সংগ্রহ কর । ছ - ঢ বিষয়ের জন্য তিনজন মানুযের সংক্ষিপ্ত সাক্ষাৎকার থেকে তথ্য সংগ্রহ কর; তারা সহপাঠী হতে পারে, তবে সম্ভব হলে ভিন্ন মতামতের কাউকে নাও ।

২ বিষয়টি কী তা ব্যাখ্যা কর?

৩ কেন এটি “খবর” অথবা বিতর্কিত অথবা চিন্তা ভাবনার বিষয় তা বর্ণনা কর।

8 একটি উপসংহার দিয়ে অনুচ্ছেদটি শেষ কর অথবা পাঠকের উদ্দেশ্যে কোন প্রশ্ন রেখেও শেষ করা যেতে পারে যা নিয়ে লে ভাববে । যখন তোমরা লিখবে, লেখাটি যে জনসাধারণের জন্য তা অবশ্যই মনে রাখবে, যেমন সংবাদপত্রের পাঠক অথবা রেডিও শ্রোতা।

৫ সর্বলেশে, লেখার নিচে, তোমাদের উল্লিখিত তথ্যগুলো পড়ার মাধ্যমে পেয়েছ নাকি মানুযের সাক্ষাৎকার নিয়ে সংগ্রহ করেছে তা লেখ । 


\section{গর্ভধারণ এবং প্রসব: সমসাময়িক বিষয় এবং নির্দেশনামূলক প্রশ্ন}

পড় এবং বিষয়গুলো নিয়ে ভাব (ক - চ):

ক ছেলেদের প্রাধান্য দেয়া হয় এমনক্ষেত্রে লিঙ্গ নির্বাচন দেখ: লিঙ্গ নির্বাচনের ওপর তথ্যমালা। ভাব: ভবিষ্যতে এই সমস্যার সমাধান কী?

খ সহায়ক প্রজনন (সন্তান জন্মদানে অক্ষম দম্পত্তিদেরকে প্রযুক্তিগত সহায়তা দেয়া) দেখ: বন্ধ্যাত্ম এবং সহায়ক প্রজনন সম্পর্কে তথ্যমালা। ভাব: গর্ভধারণের জন্য কাউকে নিয়োগ ও মজুরি দিতে তোমার কেমন লাগবে (প্রতিনিধিত্বকারী মা)।

গ অয্রপচার: মাঝে মাঝে অয্রপচার যখন প্রয়োজন নয় তখন করা হয়; আবার মাঝে মাঝে প্রয়োজনের সময় এর সুযোগ থাকে না । কোনটি সম্পূর্ণ সঠিক? দেখ: শিশ্জন্ম ও বুকের দুধ পান করানোর ওপর তথ্যমালা (উদর ও জরায়ু কেটে সন্তানপ্রসব অংশ)। ভাব: অর্থনৈতিক বিষয়গুনো কীভাবে অস্রপচারের মাধ্যনে প্রসবকে প্রভাবিত করে ?

ঘ যখন গর্ভপাত সেচ্ছায় নয় ঃ জোর করে গর্ভপাত অথবা জোরপূর্বক মাতৃত্ব। দেখ: গর্ভপাতের ওপর তথ্যমালা ভাব: গর্ভপাতের জন্য কি কাউকে জোর করা উচিত? অনাকাজ্কিত গর্ভ রাখার জন্য কি কাউকে জোর করা উচিত?

is প্রসবকালীন ফিস্টুলা:

দেখ: শিশ্জজন্মা ও বুকের দুধ পান করানোর ওপর তথ্যমালা (প্রসবকালীন ফিস্টুলার অংশ)

ভাব: বেশিরভাগ মানুষ কেন এই সমস্যা সম্পর্কে জানে না? বিষয়টি নিয়ে কী করা যেতে পারে ?

ত গর্ভবতী হওয়া অথবা এইচআইভিতে আক্রান্ত হওয়া:

দেখ: প্রজনন ও গর্ভধারণের ওপর তথ্যমালা (গর্ভকালীন সুস্থতা নিশ্চিত করা অংশ)।

ভাব: এই বিষয়ে তোমার অনুভূতি কী? 


\section{গর্ভধারণ এবং প্রসব: সমসাময়িক বিষয় এবং নির্দেশনামূলক প্রশ্ন}

\section{সাক্ষাৎকার নাও এবং বিষয়গুলো নিয়ে ভাব (ছ-ণ):}

ছ কিশোর পিতৃত্ব

তিনজন মানুষের সাক্ষাৎকার নাও, জিজ্ঞেস কর: কিশোররা কি বাবা হওয়ার জন্য তৈরি থাকে? বেশিরভাগ কিশোর বাবারা কি তাদের সন্তানের প্রতি দায়িত্বশীল এবং সক্রিয়? ? অনাকাজ্ষিত গর্ভরোধ করার জন্য ছেনেদের দায়িত্ব কী ?

জ মেয়েদের জন্য মাসিক ব্যবস্থাপনা সহজ করা

তিনজন মেয়ের সাক্ষাৎকার নাও, জিজ্ঞেস কর: বেশিরভাগ মেয়েরা মাসিকের সময় পরিক্কার থাকতে এবং রক্ত শুযে নেয়ার জন্য কী করে? খরচ কি কোন বিষয় ? মাসিকের ব্যাঘাতের জন্য কতটা সমস্যা হয়?

ঝ দত্তক নেয়া: প্রকৃত মা-বাবা/সন্তানকে চেনা অথবা না চেনা

অনেক বাচ্চাকে কেউ না কেউ দত্তক নেয়, এইসব বাচ্চারা জানেনা তাদের প্রকৃত মা-বাবা কারা । কখনও কখনও বাচ্চা একজন অথবা মা-বাবা উভয়ের তথ্য পায় অথবা তাদের সাথে যোগাভোগও হয়। কখনও কখনও কোন তথ্য পাওয়া যায় না বা যোগাযোগ করাও সম্ভব হয় না । তিনজন মানুষের সাক্ষাৎকার নাও, জিজ্ঞেস কর: কোনটি সবচেয়ে ভাল বনে তোমার মনে হয়? একটি বাচ্চার কি তার প্রকৃত মা-বাবার সম্পর্কে জানা উচিত? তার কি প্রকৃত মা-বাবার সাথে যোগাযোগ করার সুযোগ থাকা উচিত ?

এ জন্ম বিষয়ক পাঠদান:

বাবা এবং মা উভয়কেক সন্তান জন্ম ও মা-বাবা হওয়ার জন্য প্রস্তুত করা ।

কোনো কোনো জায়গায় দম্পতিরা বাচ্চা হওয়ার প্রস্তুতি নিতে একসাথে পাঠ গ্রহণ করে। তারা বাচ্চার জন্ম ও যত্ন নেয়া সম্পর্কে শেখে। তিনজনের সাক্ষাৎকার নাও। জিজ্ঞেস কর: আমাদের সমাজে কি এ ধরনের পাঠ গ্রহনের প্রয়োজন আছে? বাবারা এইসব তথ্য জানলে কী ভাল হয়?

ট সন্তান জন্মের সময় বাবার উপস্থিতি

তিনজন মেয়ের সাক্ষাৎকার নাও, জিজ্ঞেস কর: যখন তাদদর সন্তানের জন্ম হয় তখন কি বাবার প্রসবকক্ষে উপস্থিত থাকা উচিত? এই অভিজ্ঞতা কি সন্তানের সাথে তাদের বন্ধনের ওপর কোন প্রভাব ফেলে ?

ঠ মাতৃ-কালীন ছুটি এবং পিতৃ-কালীন ছুটির নীতি

তিনজন মেয়ের সাক্ষাৎকার নাও, জিজ্ঞেস কর: সন্তান জন্মের পর একজন নতুন মায়ের কাজ থেকে কত দিনের জন্য ছুটি পাওয়া উচিত? কিছু কিছু দেশে বাবারাও এই ছুটি পায়; তোমার কী মনে হয়? বাবাদের কতদিনের ছুটি পাওয়া উচিত?

ড প্রজনন সম্পর্কে তোমাকে শিখিয়েছে কে এবং কখন?

তিনজন মেয়ের সাক্ষাৎকার নাও, জিজ্ঞেস কর: বাচ্চার জন্ম কোথা থেকে হয় এ সম্পর্কে তোমাকে কে শিখিয়েছে? তখন তোমার বয়স কত ছিল? মানুষের প্রজনন সম্বন্ধে জানার সবচেড়ে ভাল মাধ্যম কী বলে তুমি মনে কর?

ঢ পরিবারের আকারকে প্রভাবিত করতে প্রণোদনা দেওয়া সংক্রান্ত সরকারী নীতিমালা

কিছু কিছু সরকার জনসংখ্যা বাড়ানোর জন্য যে সকল দম্পতির বেশি বাচ্চা আছে তাদেরকে টাকা দেয় । আবার কিছু কিছু সরকার তাদের জনসংখ্যার দ্রুত বৃদ্ধি নিয়ে চিন্তিত এবং সন্তান জন্মদান পুরোপুরি বন্ধ করার জন্য অপারেশন করলে নগদ অর্থ অথবা উপহার দেয়। তিনজন মেয়ের সাক্ষাৎকার নাও, জিজ্ঞেস কর: তুমি এর কোন একটি নীতিকে কি সমর্থন কর? কেন কর অথবা কেন কর না ?

ণ গর্ভবতী এবং বিদ্যালয় ?

প্রত্যেক দিন, বিদ্যালয়গামী কিশোরী এখনও গর্ভবতী হচ্ছে। কিছু স্থানে, তারা বিদ্যালয়ের লেখা-পড়া চালিয়ে যাওয়ার অনুমতি পায়, আবার কোন কোন জায়গায় তাদেরকে জোরপূর্বক বিদ্যালয় ছাড়ানো হয়। তিনজন মেয়ের সাক্ষাৎকার নাও, জিজ্ঞেস কর: কিশোরী গর্ভবতী এবং তাদের বাচ্চাকে সাহায্য করার জন্য সবচেয়ে ভাল নীতি কী? বাবাদের ভূমিকা এবং দায়িত্ব কী? 


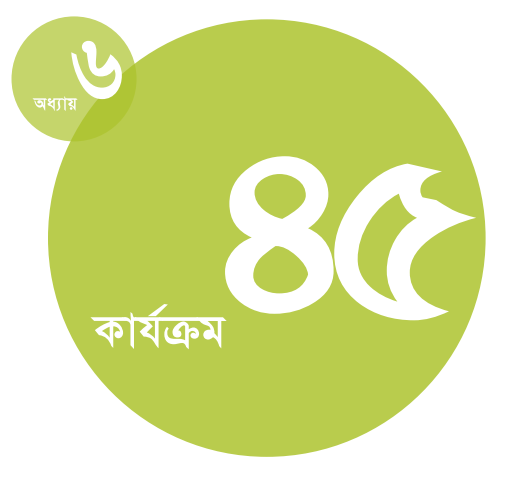

সময়কাল :

৬০-৮৫ মিনিট, ব্যবহৃত কেসস্টাডির

সংখ্যার ওপর নির্ভর করে

উপকরণ:

বোর্ড ও চক; প্রত্যেক কেসস্টাডির একটি করে অনুলিপি।

\section{প্রস্তুতি:}

প্রদত্ত আটটি কেসস্টাডি পর্যালোচনা

করুন । আপনার পরিবেশ উপযোগী এবং

বাস্তব সম্মত হয় এমনভাব্রে

কেসস্টাডিগুলোতে পরিবর্তন আনুন

অথবা কিছু যুক্ত করুন

$<$ www.popcouncil.org/publicat

ions/ books/2011_ItsAllOne. asp> এই ঠিকানায় পাওয়া সহায়ক বই

নির্দেশিকা থেকে প্রাসঙ্গিক বিষয়গুলো

(অধ্যায় ৬ “গর্ভাবস্থা, প্রজনন ও

গর্ভধারণ এবং শিশুজন্ম ও বুকের দুধপান

করানো” এর ওপর তথ্যমালা) পড়ন।

\section{প্রতি ঘণ্টার প্রতিটি মিনিট: গর্ভধারণ সংক্রান্ত মৃত্যুর গল্প}

অধিবেশন সম্পর্কিত ধারণাঃ শিক্ষার্থীরা মাতৃমৃত্যু সম্পর্কে কেস স্টাডি পড়বে। কীভাবে এই মৃত্যুগুনো রোধ করা যেত তা নাটিকা এবং আলোচনার মাধ্যমে তারা উপস্থাপন করবে।

উদ্দেশ্য: দারিদ্র্য এবং জেন্ডার অসমতা প্রধানত কীভাবে গর্ভবতী মহিলাদের মৃত্যুর (এবং মারাত্যক স্বাস্থ্য সমস্যার) কারণ হয় শিক্ষার্থীদদরকে তা বর্ণনা করতে শেখানো; কীভাবে এই পরিণতি রোধ করা যায় তা শনাক্ত করা; মাতৃমৃত্যুকে একটি গুরুত্বপূর্ণ বিষয় হিসেবে ভাবার জন্য তাদের মনোযোগ বাড়ানো; তাদের বিশ্লেষণ দক্ষতা বৃদ্ধি করা।

\section{নির্দেশাবলি}

১ বোর্ডে 'মাতৃমৃত্যু' শদ্দটি লিখুন। নিটের থ্রশনগুলো জিজ্ঞেস করুন এবং উত্তরগুলো বোর্ডে লিখুন:

• 'মাতৃমৃত্যু’ শব্দটি বলতে তোমরা কী বোঝ? [এর কাছাকাছি একটি সংজ্ঞা বের করুন: "গর্ভধারণ অথবা শিশুজন্মজনিত কারণে একজন মহিলার মৃত্যু”।]

• এখানে কিছু সমস্যা আছে: পৃথিবীতত গর্ভধারণ জনিত কারণে কি পরিমাণ মহিলা এবং মেয়েরা মৃত্যুবরণ করে বলে তোমরা মনে কর ? প্রত্যেক সপ্তাহে একজন ? প্রত্যেক দশ মিনিটে নাকি প্রত্যেক দিন? [কিছু অনুমান করার সুযোগ দিন।] উত্তর হল প্রত্যেক বছরের প্রত্যেক দিনের প্রত্যেক মিনিটে। যে এক ঘণ্তা আমরা মাতৃমৃত্যুর বিষয়টি নিয়ে শিখব, এই সময়ে যাটজন মহিলা এবং মেয়ে মারা যাবে। প্রত্যেক বছর, আধা-মিলিয়ন মৃত্য এই কারণে ঘটে। অনেক মহিলা এবং মেয়ে ঠিক মারা যায়না কিন্তু গর্ভধারণ সংক্রান্ত সমস্যায় ভোগে।

• তোমাদের কি মনে হয় এই মৃত্যুগুনো বিশ্বব্যাপি একই পরিমাণে ঘটে? কত শতাংশ মাতৃম্য়্য় ‘উন্নয়নশীল’ দেশে (৫ে দেশগুলো শিল্প সমৃদ্ধ এবং ধনী নয়) ঘটে বলে তোমরা মনে কর? দশভাগের একভাগ? অর্ধেক? দুই-তৃতীয়াংশ? [অনুমান
করতে কিছু সময় দিন ।] এর উত্তর হল ৯৯ শতাংশ। মাতৃমৃত্যু ‘উন্নত’ দেশে হয়না বললেই চলে।

• কিশোরী মেয়েরাও অনেক বেশি ঝুঁকির মধ্যে আছে। বয়স্ক মহিলাদের চেয়ে ১৫ বছরের কম বয়সি মেয়েরা প্রসবজনিত কারণে বেশি মারা যায়

• কত সংখ্যক মাতৃমৃত্যু প্রতিরোধযোগ্য বলে তোমাদের মনে হয়? অল্প কিছু? অর্ধেকের বেশি? এক তৃতীয়াংশ? [কিছু অনুমান করতে দিন ।] বিশেষজ্ঞদের রিপোর্ট অনুযায়ী “এই মৃত্যুর 'প্রায় সবই’ প্রতিরোধয়োগ্য।

• কয়েক দশক পূর্বের তুলনায় বর্তমানে মাতৃত্বজনিত কারণে মহিলা এবং মেয়েরা কম নাকি বেশি মারা যাচ্ছে বলে তোমাদের মনে হয়? [অনুমান করতে কিছু সময় দিন।] উত্তর হল একই রকম ।

• আজকে আমরা এই বিষয়টি নিয়ে আরো বেশি কিছু শিখব। তোমরা খুঁজে বের করবে তোমাদের নিজেদের জীবনের এবং অন্যদের জীবনের পরিবর্তন আনার জন্য তোমরা কীভাবে সাহায্য করতে পার। 
২ আপনি যতগুলো কেসস্টাডি ব্যবহার করতে চান শ্রেণিকক্ষের শিক্ষার্থীদেরকে ততগুলো দলে ভাগ করুন। প্রত্যেক দলকে একটি করে কেসস্টাডি পড়তে দিন, ভাঁজ করে দিন যেন তারা আগে পড়তে

না পারে। ব্যাখ্যা করুন:

• প্রত্যেক দলই মাতৃজনিত মৃত্যুর একটি করে কেসস্টাডি পেয়েছ। তোমরা তোমাদের কেসস্টাডিটি পড়ার পর নিজেদের দলে বিষয়টি নিয়ে আলোচনা কর, প্রশ্ন কর: কী ভুল হয়েছিল ? (মহিলা অথবা মেয়েটির মৃত্যুর কারণ হিসেবে প্রধানত কোন বিষয়টি কাজ করেছিল?) কী হওয়া উচিত ছিল? (কোন বিষয়টি অন্যভাবে ঘটতে পারত এবং তার জীবন রক্ষা করতে পারত?) (প্রধান দুইটি প্রশ্ন বোর্ডে লিখুন।

- তোমরা মহিলার জীবনে ঘটে যাওয়া গল্পটি নিয়ে একটি ছোট নাটিকা (দুই অথাব তিন মিনিটের) তৈরি করবে। প্রথমে তোমরা যেভাবে আছে সেভাবেই উপস্থাপন করবে। তারপর মহিলার জীবন রক্ষার জন্য কী পদক্ষেপ নেয়া যেতে পারত তার তুলনামূলক চিত্র দেখিয়ে এটি আবার উপস্থাপন করবে। দ্বিতীয়বার অভিনয়ের জন্য তোমরা একই মানুষকে নিতে পার, অথবা তোমাদের দলের অন্য সদস্যরা দ্বিতীয় নাটিকায় অভিনয় করতে পারে।
৩ প্রত্যেক দলকে তাদের নাটিকা দুইটি উপস্থাপন করতে দিন ।

8 নাটিকাগুলো উপস্থাপন শেষে আলোচনার জন্য ১৫ মিনিট সময় রাখুন:

- এই গল্পগুলো তোমাদের মধ্যে কি অনুভূতি তৈরি করেছে? কেসস্টাডির কোন বিষয়টি তোমাদের মধ্যে এমন অনুভূতি তৈরি করল ?

• ব্যক্তি, পরিবারের সদস্য, সমাজ, স্বাস্থ্যকর্মী এবং সরকার সকলেই এই মৃত্যু প্রতিরোধের জন্য কাজ করতে পারে । একজন মহিলা গর্ভবতী হওয়ার আগে তার ( এবং নবজাত শিশ্ডর) স্বাস্থ্য রক্ষার জন্য কী কী গুরুত্বপূর্ণ কাজ করা যেতে পারে ? [উত্তরগুলো বোর্ডে লিখুন; প্রয়োজন মতো পূরণ করুন ।]

• গর্ভধারণের সময় কী করা যেতে পারে ? /উত্তরগুলো বোর্ডে লিখুন; প্রয়োজন মতো পূরণ করুন ।]

• প্রসবের সময় কী করা যেতে পারে ? [উত্তরগুলো বোর্ডে লিখুন; প্রয়োজন অনুযায়ী পূরণ কর।]

• কীভাবে আমরা ১৫ বছরের কমবয়সী মেয়েদের মধ্যে মাতৃমৃত্যু প্রতিরোধ করতে পারি ?

• আজকে তোমরা যা শিখলে তা কীভাবে কাজে লাগাবে ? 


\section{মাতৃমৃত্যু নিয়ে কেসস্টাডি}

রানির গল্পः রানির বয়স যখন ১৩ বছর, তার মা সন্তান প্রসবের সময় মারা যায়। তার বাবা তাকে বলল যে, রান্নাবান্নায় সাহায্য করতে এবং ছোট ভাইবোনদের যত্ন নেয়ার জন্য তাকে বিদ্যালয়ে যাওয়া বন্ধ করতে হবে। তার বড়ভাই তখনও বিদ্যালয়ে পড়ত এবং রানিকে তার লেখা পড়া চালিয়ে যাওয়ার জন্য সাহায্য করতে চেষ্টা করল । কিন্তু রানি লেখাপড়া ছেড়ে দিল কারণ অন্যান্য কাজের পর লেখাপড়ার জন্য লে কোন সময় পেত না । ১৯ বছর বয়সে রানির সাথে রম্মেশের বিয়ে হল এবং রমেশ ও তার মা-বাবার সংসারে বসবাসের জন্য চলে গেল । রমেশের পরিবার রানির শিশ্য পালনের জ্ঞানকে মূল্যায়ন করত এবং তারা চাইত তখনই রানির বাচ্চা হোক।

রানি যখন গর্ভবতী, তার স্বামী তার ছেলে হওয়ার কথা বলত। রানী গোপনে কল্পনা করত যে, তার একটি মেয়ে হবে এবং সে তার নিজের কাছে প্রতিজ্ঞা করল যে, সে তার মেয়েকে বিদ্যালয়ের লেখাপড়া শেষ করতে দেবে, কারণ সে নিজে করতে পারেনি । আট মাস পর স্থানীয় রীতি অনুযায়ী রানি বাচ্চা প্রসবের জন্য তার বাবার বাড়ির গ্রামে ফিরে আসল। যখন তার প্রসব বেদনা শুরু হল, তাকে দেখতে একজন স্থানীয় ডাক্তার আসল। সারাদিন এবং সারারাত প্রসব বেদনার পরও যখন বাচ্চার জন্ম হল না, ডাক্তার তাকে সবচেয়ে কাছের হাসপাতালে নিয়ে যেতে বলল যা ছিল সেখান থেকে ১০০ কিলোমিটার দূরে। রাত অনেক বেশি হওয়ায় সকাল হওয়ার পূর্বে রানিকে নিয়ে যাওয়ার কেউ গাড়ির ব্যবস্থা করতে পারল না । অবশেষে রানি যখন জেলা হাসপাতালে পৌছাল, সে তার বাচ্চা দিল । ছেলে না হওয়ায় তার স্বামী হতাশ হল কিন্তু রানি তার সদ্যজাত মেয়ের কানে কানে নিজের প্রতিজ্ঞার কথা বলল। ইতিম্ধ্যে তার ভীষণ রক্তপাত শুরু হল। অত্র্রুত হাসপাতালে কর্মরত ব্যক্তিরা তাকে রক্ত দেয়ার ব্যবস্থা করল, রানি কোমায় চলে গেল । অনেক চেষ্টা সত্ত্বেও রানির অনেক রক্ত ক্ষরণ হল এবং সে তার মায়ের মত একইভাবে মারা গেল ।

চ্যারিটির গল্পঃ চ্যারিটির বয়স ছিল ১৫ বছর। বিদ্যালয়ে গিয়ে, বাড়িতে লেখাপড়া করে, এবং গৃহস্থালির কাজ করে তার সময় কেটে যায়। সে কয়েকবার একটি লোকের সাথে বেড়াতে গিয়েছে। লোকেটির সাথে লে তার বিদ্যালয়ের কাছ থেকে পরিচিত হয়েছে। মাঝে মাঝে চ্যারিটিকে লে উপহার এবং সামান্য টাকা-পয়সা দিত যেগুনো সে সাধারণত তার বিদ্যালয়ের বই কিনতে ব্যয় করত। সে তার সাথে যৌনমিলন করত এবং সে সময় চ্যারিটি তাকে কনডম ব্যবহার করতে বলত যা সে বিদ্যালয় থেকে শিখেছে। লোকটি তাকে দুশ্চিন্তা করতে নিযেধ করল । চ্যারিটি তাকে খুব বেশি পছন্দ করত না, তাই সে সিদ্ধান্ত নিল লোকটির সাথে আর দেখা করবে না । অবিলম্বে চ্যারিটি বুঝতে পারল সে গর্ভবতী । যখন সে তার মাবাবাকে বলল, তার বাবা তাকে মারল এবং বাড়ি থেকে বের হওয়া বন্ধ করে দিল। সে কয়েক মাস পর বিদ্যালয় যাওয়া ছেড়ে দিল এবং অন্য শহরে তার এক চাচীর সাথে থাকার জন্য চলে গেল । তার পরিবার একমত হল যে, তার চাচী বাচ্চাটাকে লালন-পালন করবে যেন কেউ চ্যারিটির গর্ভধারণের কথা জানতে না পারে । যখন তার প্রসব বেদনা শুরু হল, বাচ্চাটি যেন নিজে নিজেই বের হয়ে আসতে পারে এজন্য তার চাচী তাকে শক্ত থাকতে বলল। বাচ্চাটি জন্ম নালীতে আটকে গেল। চাচী একজন স্থানীয় ধাত্রীকে ডেকে আনল। ধাত্রী চ্যারিটির যৌনাঙ্গ রেজার দিয়ে প্রশস্ত করে খোলার জন্য কেটে ফেলল যেন বাচ্চাটি বেরিয়ে আসতে পারে । বাচ্চাটি দ্রংত বেরিয়ে আসল এবং চ্যারিটি ভাবল যে সে তার পুরোনো জীবনে ফিরে যাবে যে জীবন ছিল বিষণ্ন কিন্তু বুদ্ধিদীপ্ত। কয়েকদিনের মধ্যে সে দেখল তার যৌনাঙ্গ যেখানে কাটা হয়েছিল সেখানে সংক্রমণ শুরু হয়েছে। কয়েকদিন পর চ্যারিটির ভীষণ জ্বর আসল । তার চাচী তাকে হাসপাতালে নিয়ে যেতে চাইল, কিন্তু চ্যারিটি গেলনা । সে ভয় পেল যে হাসপাতালের কেউ হয়ত জেনে ফেলবে যে লে গর্ভবতী ছিল । পরদিন সকানে যখন তার জ্বর আরো খারাপ পর্যায়ে গেল, তার চাচী কোনভাবে তার জন্য একটি এ্যাম্বুলেন্স ডেকে আনল, কিন্তু ততক্ষণে অনেক দেরি হয়ে গিয়েছিল । হাসপাতালে পৌছানোর কয়েক ঘণ্টা পর চ্যারিটি মারা গেল । 


\section{মাতৃমৃত্যু নিয়ে কেসস্টাডি}

এ্যানার গল্পঃ এ্যানা তার স্বামীর সাথে বলিভিয়াতে এ্যনডিয়ার একটি প্রত্যন্ত গ্রামে বাস করত। সে কখনও পড়তে অথবা লিখতে শেখেনি। সে একবার জন্ম নিয়ন্ত্রণ পিল খেয়েছিল, কিন্তু তার স্বামী জন্মানিয়ন্ত্রণ করতে অস্বীকার করায় সে তা বন্ধ করে দিল । যখন এ্যানা গর্ভবতী হল, তখন একদিন সে প্রসবপূর্ব সেবার জন্য স্থানীয় স্বাস্থ্যকেন্দ্রে গেল । সে স্বাস্থ্যকেন্দ্রে গিয়ে লজ্জা পেল কারণ সে স্প্যানিশ ভাষা বলতে পারত না এবং হাসপাতালে কর্তব্যরত ব্যক্তিরা কিউচুয়া ভাযায় কোন কথা বলছিল না । তার মনে হল যে ডাক্তার তার প্রতি কোন আগ্রহ দেখায়নি এবং চাচ্ছিল যে, সে চটে যাক। গর্ভধারণের নয় মাসের সময়, এ্যানা তার তলপেটে ব্যথা অনুভব করতে লাগল। একজন প্রতিবেশী ধাত্রী এ্যানার পরিবারকে বলল যে, বাচ্চার অবস্থা নাজুক এবং এ্যানাকে স্বাস্থ্যকেন্দ্রে নিয়ে যেতে হবে।

প্রসবপূর্ব সেবার সময় তার সাথে যেভাবে আচরণ করা হয়েছিল, তা মনে করে সে স্বাস্থ্যকেন্দ্রে যেতত ভয় পাচ্ছিল । আবার তার স্বামী শুনেছিল যে, প্রসব পরবর্তী রোগীদেরকে হাসপাতালে কিছু টাকা দিতে হয় কিন্তু তার পরিবার এগুলো বহন করতে অক্ষম ছিল। এ্যানার অবস্থা আরও খারাপ দিকে গেল । ধাত্রী এ্যানাকে স্বাস্থ্যকেন্দ্রে নেয়ার জন্য চাপ দিল । তাদের কোন গাড়ি ছিল না, তাই তারা এ্যানাকে ঘোড়ার গাড়িতে করে নিয়ে গেল । স্বাস্থ্যকেন্দ্র ছিল গ্রাম থেকে ১৫ কিলোমিটার দূরে। ঘোড়ার গাড়ির জন্য এ ছিল এক দীর্ঘ পথ। অর্ধেক পথে এ্যানার ভীযণ রক্তপাত শুরু হল এবং সে মারা গেল।

ফাতেমার গল্পঃ ফাতেমা একজন শিক্ষিকা হতে চাইত। যাই হোক, যখন তার বয়স ১৫ বছর, তার মা-বাবা তাকে বিয়ে দিয়ে দিলেন এবং সে তার ট্রাকচালক স্বামীর সাথে বসবাস করার জন্য চলে গেল। আলীর মা-বাবা তাদেরকে বাচ্চা নেয়ার জন্য চাপ দিল, তাই ফাতেমা জন্ম নিয়ন্ত্রণ পদ্ধতি ব্যবহার করল না । সে পাঁচ বছরের মধ্যে তিনটি বাচ্চার জন্ম দিল। প্রত্যেক প্রসবের সময় একজন স্থানীয় ধাত্রী তাকে সাহায্য করত। সে যখন চতুর্থ বাচ্চার জন্য আটমাসের গর্ভবতী, আলী তাকে প্রসব কাজে সাহায্য করাচ্ছিল। ফাতিমা খুব অসুস্থ্যবোধ করছিল। ফাতিমার কাছে স্বাস্থ্য কেন্দ্রে যাতায়াতের মত টাকা ছিল না কারণ পরিবার খরচের টাকা তার স্বামীর কাছে থাকত। ফাতিমা তাই আলীর ঘরে ফেরার অপেক্ষা করছিল। আলী বাড়ি ফিরতে ফিরতে ফাতিমা ভীষণ জ্বরে কাঁপছিল এবং খুব দুর্বল হয়ে পড়ল। আলী তার হাসপাতালে যাওয়ার ব্যবস্থা করল, কিন্তু তারা পৌছাতে পৌছাতে, ফাতেমা মারা গেল ।

মারিয়া ক্লারার গল্পঃ মারিয়া ক্লারা তার স্বামী, পেড্রো, এবং তার ভাইয়ের ছেলের (তার যে ভাই দুর্ঘটনায় মারা গিয়েছিল তার ছেলে) সাথে বাস করত। তারা নিকটস্থ শহর থেকে অনেক উঁুচতে একটি পাহাড়ি গ্রামে বাস করত যা ছিল নিকটস্থ হাসপাতালে থেকে ঘোড়ার গাড়িতে নিচের দিকে সাত ঘণ্টার পথ। যখন মারিয়া ক্লারা গর্ভবতী হল, সে স্বাস্থ্যলেবার জন্য হাসপাতালে গেল । ডাক্তার বলল তার বাচ্চা প্রসবের দুই সপ্তাহ আগে হাসপাতালে আসা উচিত যেন শেষমুহূর্তে সে স্বাস্থ্যলেবা থেকে অনেক দূরে কোন বিপদজনক জটিলতার মুখোমুখি না হয়। যখন সে গর্ভাবস্থার শেষ পর্যায়ে পৌছাল, মারিয়া ক্লারা বাড়িতে থাকতে ভয় পাচ্ছিল। পেড্রো তার এতদূরে যাওয়ার বিষয়ে অভিযোগ করছিল কারণ সে রান্না করতে জানে না । ক্লারা এটাও জানত যে, বেশির ভাগ রাততই পেড্রো মদ্যপান করে এবং মাঝে মাঝে ভাঙচুর করে, তাই সে ভীত ছিল যে পেদ্রো তাকে পেটাবে। সে আরো উদ্বিগ্ন ছিল যে, পেদ্রো কাজ শেষে যে, বারে যায় সেখানকার এক মহিলা, যাকে সে পেড্রোর সাথে দেখেছিল, বেশিরভাগ সময় তার সাথে কাটাবে। মারিয়া ক্লারা সিদ্ধান্ত নিল যে, ডাক্তার তার বাচ্চা প্রসবের যে তারিখ বলেছে তার মাত্র এক সপ্তাহ পূর্বে সে হাসপাতালে যাবে। কিন্তু হাসপাতালে যাওয়ার আগের দিনই কোনভাবে তার বাচ্চাটি জন্ম নিল এবং মারিয়া ক্লারার রক্তক্ষরণ শুরু হল । পেড্রো তাকে বাঁচানোর মত সময়ে হাসপাতালে নিয়ে যেতে পারল না, এবং লে মারা গেল । 


\section{মাতৃমৃত্যু নিয়ে কেসস্টাডি}

ইলেরুতকনেলের গল্পঃ ইয়েরুকনেলের জন্ম ইথিওপিয়ার একটি প্রত্যন্ত অঞ্চলে । যখন তার বয়স ১৩ বছর, জ্বালানী কাঠ সং্রহ করার সময় সে অপহৃত হল এবং ৩২ বছর বয়সী বিপত্নীক ও দুই সন্তানসহ জেনা সাহেবের সাথে তার বিয়ে হল । তার কিছুদিন পরই, সে গর্ভবতী হয়ে গেল। উপদেশ পাওয়ার মত বা তার সমস্যা নিয়ে কথা বলার মত ইয়েরুকনেলের কেউ ছিলনা । যখন তার প্রসব বেদনা শুরু হল, সে আতঙ্কিত হয়ে গেল । বালিশ শক্ত করে আঁকড়ে ধরে সে তার মাকে ডাকতে লাগল, তার গাল বেয়ে অশ্রু গড়িয়ে পড়ল। তার ভীষণ জ্বর ও তীব্র যন্ত্রণা ঔরু হল; এবং প্রচুর ঘামতে থাকল । কয়েকজন প্রতিবেশী মহিলা ভেতরে আসল, মোমবাতি জ্বালাল, এবং ইয়েরুকনেসের জন্য প্রার্থনা শুরুত করল। জেনা সাহেব গ্রামের সনাতন ধাত্রীকে ডাকল কিন্তু এমন পরিস্থিতি সামলানোর মত প্রশিক্ষণ ধাত্রীর ছিল না । তাই জনাব জেনা গ্রামের লোকদেরকে তাকে হাসপাতালে নিয়ে যেতে বলল । হাসপাতালে পৌঁছতে পৌঁছতে একটি মৃত শিফ্ড জন্ম নিল এবং ইয়েররুকনেস ১৫ বছর বয়লে মারা গেল।

হুসানের গল্পः স সায়েদা বিবি পাকিস্তানের উত্তর-পশ্চিম সীমান্ত প্রদেশের মার্দানে বাস করত। সে বলল কীভাবে তার একমাত্র সন্তান হুসান পরি ১৩ বছর বয়লে সন্তান প্রসবের সময় মারা যায়। "যখন হ্সানের বয়স আট বছর, তার বাবা সড়ক দুর্ঘটনায় মারা যায়। আমার ভয় করত যদি আমিও মারা যাই, তাহলে আমার মেয়েকে কে দেখাশোনা করবে? সুখি এবং নিরাপদ থাকার জন্য আমি তার বিয়ে দিতে চাইলাম । তার জন্য বিয়ের পাত্র দেখলাম; কাছাকাছি গ্রামের এক কৃষক। হুসান পরি তার বিয়ে নিয়ে খুব আনন্দিত ছিল। তিন মালের মধ্যে সে গর্ভবতী হয়ে গেল, এবং আমি তাকে সনাতন ধাত্রীর কাছে নিয়ে গেলাম। সে তাকে বমির প্রতিযেধক/ঔষধ দিল। আমরা ধাত্রীকে সামান্য পয়সা দিতে পারতাম এবং আমরা তার দেয়া ভেষজ ঔষধ পছন্দ করতাম। হুসান কঠোর পরিশ্রম করত এবং সামান্য খাবার খেত- কুসংস্কারের জন্য সে ডিম এবং মাছ খেত না- এবং সে ভীষণ চিকন হয়ে গেল । আমি চিন্তিত ছিলাম কীভাবে সে প্রসব বেদনা সহ্য করবে। যখন আমরা তার চিৎকার ও্নলাম তখন তাকে সাহায্য করতে গেলাম কিন্তু বাচ্চাটি বেরিয়ে আসল না । ধাত্রী ভীত হয়ে পড়ল, সাহায্য করতে অস্বীকার করল এবং বলল আমাদের অবশ্যই তাকে হাসপাতালে নিতে হবে। রাস্তায়, হ্সান পরি নীল হয়ে গেল, বরফের মত ঠাণ্ণা হয়ে গেল তার শরীর এবং আমার বাহুর ওপর গে মারা গেল । আমিও তাকে অথবা তার বাচ্চাকে বাঁচাতে পারলাম না । আমি মনে করতে পারিনা কীভাবে আমরা বাড়ি পৌঁছলাম, অথবা কীভাবে শেষকৃত্য হল । সেই ব্যথা বয়ে বেড়ানোর ছিল না ।"

ফ্লোরেনেরের গল্পঃ ফ্লোরেন্স ২৪ বছর বয়সের একজন অবিবাহিত মহিলা । সে সবজি চাষ এবং বিক্রি করে। একদিন রাতে বাজার থেকে ফেরার পথে সে ধর্ষিত হল। সে কাউকে বলতত ভয় পেল, এবং যখন ঐ মাসে তার মাসিক হল না, সে বুঝতে পারল যে, ধর্ষণের কারণে সে গর্ভবতী হয়েছে। यদিও তার দেশে গর্ভপাত আইনত নিষিদ্ধ ছিল এবং সাধারণত অস্বাস্থ্যকর পরিবেশে করা হত, ফ্লোরেন্স দৃঢ়প্রতিজ্ঞ ছিল যে তার গর্ভের শিশ্ককে সে রাখবে না । সে একজনের কাছে গেল যে তার যোনিপথে কিছু একটা ঢুকিয়ে দিল এবং বলল যে, এক সপ্তাহের মধ্যে রক্তপাত বন্ধ হয়ে যাবে। সপ্তাহের শেষে, ফ্লোরেন্সের রক্তক্ষরণ চলতেই থাকল, এবং তার ভীষণ জ্রর আসল।

সে একটি হাসপাতালে গেল, একজন ডাক্তার তাকে ভর্তি করল এবং তাকে এন্টিবায়োটিক দিল। প্রথম ফোরেন্স শাস্তি পাওয়া অথবা গ্রেফতার হওয়ার ভয়ে প্রকাশ করতে ভয় পেল যে, সে গর্ভপাত করিয়েছিল। অবশেযে, সে ডাক্তারকে খুলে বলল। ডাক্তার মনে করত যে, গর্ভপাত নিরাপদ ও ঝুঁকিমুক্ত হওয়া উচিত, ফ্লোরেন্সের কষ্ট সে গভীরভাবে অনুভব করল এবং তাকে বাঁচানোর জন্য তার সর্বোচ্চ চেষ্টা করল । দশদিন পরে সে মারা গেল এবং ডাক্তার কাঁদল। 


\section{যৌনবাহিত সংক্রমণ (এসটিআই) সম্পর্কিত বিষয়াবলি}

অধিবেশন সম্পর্কিত ধারণা: শিক্ষার্থীরা এইচআইভি ও এইডসসহ এসটিআই সম্পর্কিত গুরুত্বপূর্ণ তথ্য সম্বলিত পোস্টার তৈরি করবে।

উদ্দেশ্য: কীভাবে এসটিআই সংক্রমিত হয়, এগুলোর সংক্রমণ কীভাবে প্রতিরোধ করা যায়, এবং এগুলোর পরীক্ষা ও চিকিৎসা কোথায় পাওয়া যায় তা বর্ণনা করতে শিক্ষার্থীদেরকে সক্ষম করা; পরীক্ষা ও চিকিৎসা নেয়ার গুরুত্ব সম্পর্কে তাদের সচেতনতা বৃদ্ধি করা।

\section{निर्দেশাবলি}

১ ব্যাখ্যা করুন যে, এই পাঠটি এইচআইভি ও এইডসসহ যৌনবাহিত সংক্রমণ সম্পর্কে- একে এসটিআইও বলে ।

২ ছেলে-মেয়ে মিলিয়ে শ্রেণিকক্ষের সবাইকে চারটি দলে ভাগ করুন । তালিকাটি থেকে প্রত্যেক দলকে একটি করে বিষয় দিন (অথবা তাদেরকে পছন্দ করতে দিন; সকল বিষয় যেন অন্তর্ভুক্ত হয় তার চেষ্টা করুন্ন।) ব্যাখ্যা করুন:

• তোমাদের বিষয়টি নিয়ে তোমরা দলগতভাবে একটি তথ্য সম্বলিত পোস্টার তৈরি করবে। তোমাদের কল্পনাশক্তি কাজে লাগাও।

• যতটা সম্ভব পুজ্খানুপুজ্খভাবে কর। পরীক্ষা, চিকিৎসা এবং প্রতিরোধ বিষয়ে তথ্য্য সংযুক্ত কর। তোমরা তথ্য সম্বলিত উপকরণ ব্যবহার করতে পার। কিছু কিছু ক্ষেত্রে (যেমন ‘প্রচলিত ধারণা' সম্পর্কিত (পাস্টারের জন্য) ধারণা পাওয়ার জন্য সহপাঠীদের সাক্ষাৎকার নিতে পার।

৩ ঘটনা সম্বলিত উপকরণ এবং পোস্টার বিলি করুন । শিক্ষার্থীদেরকে মনে করিয়ে দিন যে, তাদের তথ্য সংগ্রহ করার জন্য দশ মিনিট, এগুলো আলোচনা ও পোস্টারের জন্য পরিকল্পনা করতে দশ মিনিট, এবং পোস্টার তৈরি করতে পনের মিনিট সময় আছে। দলগুনো যখন গবেষণা, আলোচনা এবং তাদের পোস্টার টৈরি করবে তখন তাদেরকে সাহায্য করার জন্য ঘুরে ঘুরে দেখুন। যদি তারা পোস্টার সম্পূর্ণ করতে না পারে, তাহলে সময় বাড়ান অথবা তাদেরকে বলুন তারা এটি শেষ করতে কখন সময় নেবে।
ধারাবাহিক পরবর্তী একটি সেশনে

8 यদি প্রয়োজন হয়, দলগুলোকে তাদের পোস্টার শেষ করতে অল্প কিছু সময় দিন ।

৫ প্রত্যেক দলকে তাদের পোস্টার উপস্থাপন করতে এবং শ্রেণিকক্ষের শিক্ষার্থীদের থেকে প্রশ্ন নিতে বলুন ।

৬ যতক্ষণ সম্ভব শ্রেণিকক্ষে পোস্টারগুলো প্রদর্শন করুন ।

\section{পোস্টারের বিষয়বস্তু}

এসটিআই সম্পর্কে প্রচলিত ভুল ধারণা

এইচআইভি ও এইডস সম্পর্কে প্রচলিত ভুল ধারণা

দ্বৈত প্রতিরোধ

এইচআইভি ও এইডস সম্পর্কে পাঁচটি গুরুত্বপূর্ণ বিষয়

ক্লামেডিয়া को?

এসটিআই এবং জেন্ডার

ক্ল্যানেডিয়া ও গনোরিয়া কীভাবে ভবিষ্যৎ সন্তান জন্মদান ক্ষমতার

ওপর খারাপ প্রভাব ফেলতে পারে ?

এইচপিভি ও হার্পস: দুটি গুরুতত্বপূর্ণ এসটিআই

প्रজনनতন্ত্রের সংক্রমণ की?

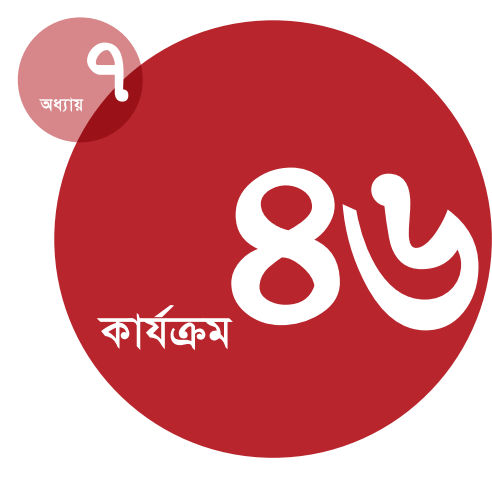

সময়কাল :

ধাপ ১-৩: ৪০ মিনিট

ধাপ 8-৬: 80 মিनिট

\section{উপকরণ:}

পোস্টার পেপার ও মার্কার; নির্দেশিকার (এই বইয়ের সহ-গামী খন্ড) একটি অনুলিপি অথবা ঐ বই থেকে প্রয়োজনীয় পৃষ্ঠাগুলোর অনুলিপি: যৌনবাহিত সংক্রমণ, এইচআইভি ও এইডস এবং প্রজননতন্ত্রের সংক্রমণ সম্পর্কিত ফ্যাক্টশীট; এবং অধ্যায়-৭ এর এসটিআই ও এইচআইভি অংশ। নির্দেশিকাটি<www.popcouncil.or g/publications/books/2011_Its AllOne. asp> এই ঠিকানায় পাওয়া যাবে। অথবা এসটিআই ও এইচআইভি সম্পর্কে অন্য নির্ভরযোগ্য তথ্য ব্যবহার করু॰ ।

\section{প्रস্তুতি:}

সম্ভব হলে ওপরে উল্লিখিত তালিকার অনেকগুলো অনুলিপি তৈরি করুন । আপনার সমাজের যুবক-যুবতীরা কোথায় পরীক্ষা এবং চিকিৎসা করাতে পারে সেই তথ্য খুঁজুন। পোস্টারের বিষয়গুলো বোর্ডে লিখুন। 


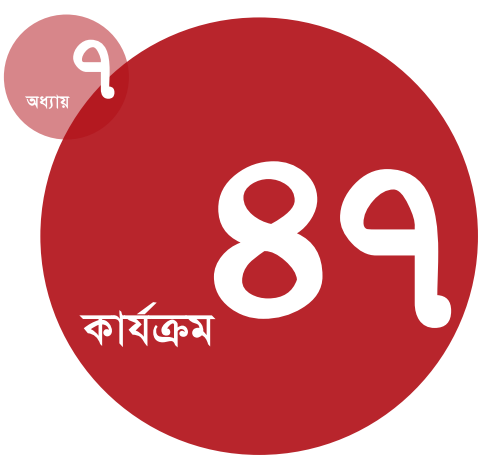

সময়কাল :

ধাপ ১-৪ (কিছু মানুষ কনডম ব্যবহার করে না কেন): 80 মিনিট

ধাপ ৫-৭ (কনডমের সঠিক ব্যবহার): ১৫ মিनिট

\section{উপকরণ:}

বোর্ড ও চক; শিক্ষকদের জন্য উত্তরপত্র; ধাপ ১-৪ এর জন্য অনুশীলনপত্রের অনুলিপি। ধাপ ৫-৭ এর জন্য ফ্লাশকার্ড। যদি মানানসই হয়, প্রদর্শনের জন্য নমুনা হিলেবে একটি করে পুরুষ এবং মহিলা কनডম।

\section{প্রস্তুতি:}

মহিলা কনডম স্থানীয়ভাবে পর্যাপ্ত কিনা ও কোথায় পাওয়া যায় তা খুঁজে বের করুন্ন। পুরুষ্ব ও মহিলা কনডম সম্পর্কে নির্দেশিকার (এই বইয়ের সহ-গামী খন্ড) শেষে অথবা অন্য কোন নির্ভরযোগ্য সূত্র থেকে জন্মনিরোধক ফ্যাক্টশীটের তথ্যগুলো পুনরায় পড়ুন। ধাপ ১-8 এর জন্য অনুশীলনপত্র ও উত্তরপত্র উভয়ই পুনরায় পড়ুন । ধাপ ৫-৭ এর জন্য ফ্লাশ কার্ডগুলো তৈরি করুন ("কনডমের ব্যবহার সঠিক ধাপগুনো” দেখুন)।

\section{জেন্ডার ও কনডম ব্যবহার}

অধিবেশন সম্পর্কিত ধারণা: শিক্ষার্থীরা কনডম ব্যবহারের বাধাগুলো (জেন্ডার রীতিনীতিসহ) ও এগুলো অতিক্রম করার উপায়গুলো বিশ্লেষণ করবে, এবং কীভাবে কনডম ব্যবহার করতে হয় তা শিখবে। এই অধিবেশনটি সেসব শিক্ষার্থীদেরকে উদ্দেশ্য করে যাদের এসটিআই, এইচআইভি এবং অনাকাজ্ষিত গর্ভধারণের প্রতিরোধ ব্যবস্থা হিসেবে কনডম সম্পর্কে মৌলিক ধারণা আছে।

উদ্দেশ্য: শিক্ষার্থীরা কনডম ব্যবহারের বাধাগুনো ও কীভাবে এগুনো সমাধানে ব্যবস্থা গ্রহণ করা যায় তা বর্ণনা করতে সক্ষম করা; কনডমের সঠিক ব্যবহার বর্ণনা করা; সমালোচনামূলক চিন্তার দক্ষতা শক্তিশালী করা।

\section{निर्দেশাবলি}

১ ব্যাখ্যা করুন (এবং মূল উত্তরগুলো লিখুন):

• আজকে আমরা কনডমের ব্যবহার নিয়ে আলোচনা করব। আমরা কনডম সম্পর্কে কী জানি? [জানার চেষ্টা করুন: কনডম এসটিআই, এইচআইভি এবং অনাকাজ্ষিত গর্ভধারণ প্রতিরোধ করে; এগুলো পুরুষরা ব্যবহার করে ।]

• কারা কনডম ব্যবহার করতে পারে ? মহিলাদের জন্য কি কোন কনডম আছে, নাকি শুধু পুরুষ্দের জন্য? [জানার চেষ্টা করুন্ন: মহিলাদের জন্যও কনডম আছে। এই পদ্ধতি সম্পর্কে মৌলিক ধারণা দিন।]

• কনডম সম্পর্কে জানা গুরুত্বপূর্ণ কেন ? [জানার চেষ্টা করুন: প্রতিদিন মানুষ এইডস বা অনিরাপদ গর্ভপাতের জটিলতার জন্য মারা যাচ্ছে; এই মৃত্যুগুনো প্রতিরোধ করা যেত, যদি মানুষ কনডম ব্যবহার করত। কনডম গর্ভধারণ, এইচআইভি, এবং অন্যান্য সংক্রমণ থেকে রক্ষা করে।]

• যদি মানুষ কনডম সম্পর্কে জানে, এর অর্থ কি তারা এটা ব্যবহার করবে?
২ শ্রেণিকক্ষের সবাইকে জোড়ায় জোড়ায় ভাগ করুন এবং অনুশীলনপত্র বিতরণ করুন । ব্যাখ্যা করুন:

• মানুষ কেন কনডম ব্যবহার করে না বা এগুলো সঠিকভাবে ব্যবহার করে না তার অনেক কারণ আছে। তোমাদের অনুশীলনপত্রে নয়টি দম্পতির বাস্তব অভিজ্ঞতা দেয়া আছে। কী এই দম্পত্দেরকে সাহায্য করতে পারত সে বিষয়ে এখানে পরামর্শও দেয়া আছে।

• তোমার সঙ্গীসহ প্রত্যেক দম্পতির পরিস্থিতি পড়। তারপর সিদ্ধান্ত নাও কোন সমাধানটি বা সমাধানগুলো তাদের পরিস্থিতিতে মানানসই: পরবর্তী সময়ে সঠিকভাবে কনডম ব্যবহার করতে কোন বিষয়টি তাদের সাহায্য করতে পারে ?

৩ শ্রেণিকক্ষের সবাইকে আবার একত্র করুন এবং অনুশীলনপত্রে বর্ণিত প্রত্যেক দম্পতির পরিস্থিতি আলোচনা করুন। গ.চ ও ঝ দম্পতিদের জন্য (যেগুলো জেন্ডার অসমতার সাথে সম্পৃক্ত) যতক্ষণ সময় থাকে আরো আলোচনা চালিয়ে যান ।

8 নয়টি দম্পতির সকলের পরিস্থিতি জানার পর, নিম্নলিখিত প্রশ্নগুনো নিয়ে আলোচনা করুন: 
• কনডম ব্যবহার করার দায়িত্ব কার ?

- কেন মেয়ে বা মহিলাদের মহিলা কনডম ব্যবহারের সুযোগ থাকা প্রয়োজন হতে পারে? [জানার চেষ্টা করুন: যদি তাদের পুরুষ সঙ্গী কনডম ব্যবহার না করে তাহলে তারা নিজেদেরকে নিজেরাই রক্ষা করত।]

• মানুষ কীভাবে নিশ্চিত হতে পারে যে, যখন তাদের কনডমের প্রয়োজন হবে তখন তারা তা ব্যবহার করতে প্রস্তুত ?

৫ শ্রেণিকক্ষের সকলকে দুই বা তিনটি দলে ভাগ করুন । (প্রত্যেক দলে প্রায় ১২ থেকে ১৬ জন করে) । বিষয়টি সম্পর্কে বলুন:

• মানুষ কনডম ব্যবহার করে না তার একটি কারণ হল তারা জানে না কীভাবে এটি ব্যবহার করতে হয়। মারাত্নক স্বাস্থ্য সমস্যার পরিণতির জন্য এটি একটি সামান্য কারণ। তাই আমরা এখন পুরুষ্ব কনডমের সঠিক ব্যবহার পদ্ধতি শিখব। কীভাবে কনডম ব্যবহার করতে হয় তার জানার জন্য যথেষ্ট বড় হওয়ার অর্থ এই নয় যে, তোমরা যৌনমিলন করার জন্য প্রস্তুত । কিন্তু কীভাবে কনডম ব্যবহার করতে হয় সেটা তোমার কনডম প্রয়োজন হওয়ার পূর্বেই জানা ভাল । তুমি যৌনমিলন করার পরে নয়, তাহলে খুব দেরি হয়ে যাবে। কনডম ব্যবহার করতে পূর্বে থেকেই তোমরা কিছু পদক্ষেপ নিতে পার। তার মানে, তোমরা যখন কোন অন্তরঙ্গ পরিস্থিতিতে আছ তার পূর্বে। যখন ইতিমধ্যে তোমরা অন্তরঙ্গ মুহূর্তে কিন্তু যৌনমিলনের ঠিক পূর্ব পরিস্থিতিতে তখন তোমরা কিছু পদক্ষেপ নিতে পার। যৌনমিলনের সময় কনডম ব্যবহারের ব্যাপারে কী করতে হয় তা তোমাদের জানা উচিত । এবং কনডম ব্যবহারের পর তোমাদের এটি কী করা উচিত তাও তোমাদের জানা উচিত [বোর্ডে লিখুন 'যৌনমিলনের অনেক পূর্বে,' ‘ঠিক যৌনমিলনের পূর্বে,' ‘যৌনমিলনের সময়ে,' এবং ‘যৌনমিলনের পর ।]
৬ অধিবেশনটি ব্যাখ্যা করুন:

- আমি পুরুষদের কনডম ব্যবহারের অন্তর্ভুক্ত সব ধাপগুলো আলাদা আলাদা করেছি এবং প্রত্যেকটি ধাপ আলাদা কার্ডে লিখেছি । আমি প্রত্যেকটি দলকে একসেট ফ্লাশকার্ড দেব। [কার্ডগুলো বিতরণ করার পূর্বে নিশ্চিত হোন যে, এগুলো এলোমেলোভাবে সাজানো আছে।] ধাপগুলো সঠিকভাবে সাজানোর জন্য প্রত্যেক দল অবশ্যই একসাথে কাজ করবে।

- সম্ভব হলে, প্রত্যেকটি ফ্লাশকার্ড দলের ভিন্ন ভিন্ন ব্যক্তির কাছে থাকা উচিত। তারপর আপনার দলকে চারটি গুচ্ছে ভাগ করুন । [বোর্ডে লেখা চারটি বিকল্পের দিকে।] যাদের কাছে প্রথম ধাপের, যৌনমিলনের অনেক পূর্বে ধাপটির, ফ্লাশকার্ডগুলো আছে তাদের প্রথম গুচ্ছে অন্তর্ভুক্ত হওওয়া উচিত। পরবর্তী দল যৌনমিলনের ঠিক পূর্বে ধাপের জন্য নেয়া ফ্লাশকার্ডগুলো দেখাবে। কার্ডের তৃতীয় গুচ্ছ যৌনমিলনের সময়ে ধাপটির অন্তর্ভুক্ত হওয়া উচিত। চতুর্থ দল যৌনমিলনের পরবর্তী ধাপটি প্রদর্শন করবে। প্রতিটি দলের মধ্যে প্রত্যেকটি ধাপ সুবিন্যস্তভাবে সাজানোর চেষ্টা কর। তারপর তোমাদের কার্ড অনুসারে পর্যায়ক্রমে সারিবদ্ধ হয়ে দাঁড়াও। [লক্ষণীয়: প্রথম গুচ্ছের জন্য এটি একটু বেশি কঠিন হতে পারে ।]

• তোমাদের সময় পাঁচ মিনিট। শুরু কর!

৭ পঁঁচ মিনিট পর প্রক্রিয়াটি বন্ধ করুন এবং সম্পূর্ণ দলটিকে একত্রিত করুন। শিক্ষার্থীদেরকে পর্যায়ক্রমে ধাপগুলোর ব্যাখ্যা করতে বলুন। পর্যায়ক্রম ঠিক নেই এমন যেকোন ধাপ সঠিক করতে প্রশ্ন করার জন্য থামিয়ে দিন (উত্তরপত্র দেখুন) । যেকোন প্রশ্ন উত্থাপিত হলে তার উত্তর দিন। 
কনডম ব্যবহার করতে মানুষকে ক্ষমতায়নের সমাধানগুলি

নিচে নয়জন দম্পতির গল্প দেয়া আছে যারা সঠিকভাবে কনডম ব্যবহার করেনি । প্রত্যেকের ক্ষেত্রে, কী কী এই দম্পতিকে সাহায্য করতে পারত তা ভাব। নিম্মলিখিত তালিকা থেকে এক বা একাধিক ‘সমাধান’ শনাক্ত কর এবং সেইসব সংখ্যাকে বৃত্ত দ্বারা পূরণ কর যা নিচের তালিকায় প্রদত্ত যেকোন একটি সমাধানের সাথে সংগতিপূর্ণ। যেমন, “মেয়ে ও ছেলের মধ্যে বেশি সমতা এবং মতার সমবন্টন” বোঝাতে বৃত্ত '২’ পূরণ কর। (যদি তোমরা মনে কর দম্পতিটির একের অধিক সমাধান প্রয়োজন তাহলে তোমরা একের অধিক নাম্বারে বৃত্ত পূরণ করতে পার।)

সমাধান-১ সঠিকভাবে কনডম ব্যবহার সম্পর্কে তথ্য

সমাধান-২ মেয়ে ও ছেলের মধ্যে বেশি সমতা এবং ক্ষমতার সমবণ্টন

সমাধান-৩ ভাল যোগাযোগ দক্ষতা

সমাধান-8 কনডম কোথায় পাওয়া যাবে সে বিষয়ে তথ্য

সমাধান-৫ এ এইচআইভি ও গর্ভধারণের ঝুঁকি সম্পর্কে আরো বেশি বাস্তব ধারণা

সমাধান-৬ যৌনমিলন সম্পর্কে আলোচনার জন্য একটি নিরাপদ জায়গা

দম্পতি ক : এই দুইজন যুবক-যুবতী কডনম ব্যবহার করে যৌনমিলন করেছে। ছেলেটির বীর্যপাত হবার পর সে একইভাবে পাঁচ মিনিট শুয়ে থাকল । তার পুরুষাा্গ নরম ও ছোট হয়ে গেল এবং যখন সে সামান্য একটু নড়ল, সে বুঝতে পেরে বিস্মিত হল যে তার বীর্যের সামান্য পরিমাণ কনডমের বাহিরে নির্গত হয়ে তার প্রেমিকার যোনিপথের মুখে পড়ছে।
দম্পতি ক-এর প্রয়োজন:
২

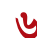

দম্পতি খ : এই দুইজন যুবক-যুবতী যৌনমিলন এবং কনডম ব্যবহার করার সিদ্ধান্ত নিয়েছে। সঙীদের একজন স্থানীয় বাজারে খুঁজে বিক্রির জন্য কোনোই পুরুষ বা মহিলা কনডম পেল না । অন্যজন অাবল যে, বিদ্যালয়় কনডম পর্যা|ত্ত থাকতে পারে কিন্তু নার্স বলল নেই। তাদের

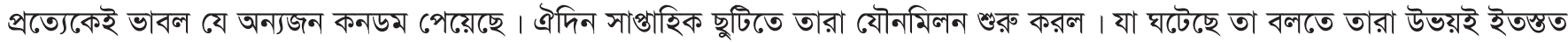
বোধ করল। তাই তারা বিষয়টি উপেক্ষা করল এবং য্যীনমিলনে এগিয়ে এলো ও কনডম ব্যবহার ছাড়া ব্যেনমিলন করল।
দম্পতি খ-এর প্রয়োজন: ১
২
8
৫

দম্পতি গ : এই দুইজন যুবক-যুবতী য্যীনমিলন করতে চায়। ম্মেয়েটি তার থ্রেমিককে বলল যে, সে কনডম এনেনে। কিন্ত ছেলেেটি বলল, ‘উপায় নেই ----- এগুলো বাজে লাগে!' মেয়েটি জোর করতে চেষ্টা করল কিন্তু ছেলেটি রেগে গেল। ছেলেটি মেয়েটিকে বলল যে, সে বাচ্চাদের মজতা আচরণ করছে। এবং তাকে জিজ্ঞস করল যে, সে সত্যিই তাকে ভালবালে কিনা । পরিশেঘে, মেয়েটি যুক্তি দেয়া ছেড়ে দিল এবং তারা কনডম ছাড়া যৌনমিলন করল।

দম্পতি গ-এর প্রয়োজন: । ১ 


\section{কনডম ব্যবহার করতে মানুষকে ক্ষমতায়নের সমাধানগুলি}

দম্পতি ঘ : এই দুইজন যুবক-যুবতী যৌনমিলন করতে চায় এবং কনডম ব্যবহার করতে একমত। কিন্তু যখন এগুলো ব্যবহারের সময় আলে ছেলেটি আনাড়ির মত কনডমের মোড়ক হাতড়াতত থাকে এবং তার উত্তেজনা হারায়। এই সময় তারা যৌনমিলন করা বাদ দেয়। এবং পরবর্তীতত যখন তারা একত্রিত হয়, ছেলেটি আবার তার উত্তেজনা হারিয়ে যাবে মনে করে উদ্বিগ্ন হয়ে পড়ে। সে তার প্রেমিকাকে বলে, কনডম পরিবেশটি নষ্ট করে দেয়। তাই তারা কনডম ছাড়া যৌনমিলনে একমত হয়ে অগ্রসর হয় এবং যৌনমিলন করে।

দম্পতি ঘ-এর প্রয়োজন: ১

দম্পতি ঙ : এই দুজন যুবক-যুবতী যৌন মিলনের সিদ্ধান্ত নিয়েছে। তারা উভয়ই মনে করে কনডম ব্যবহার একটি ভাল ধারণা । কিন্তু তারা ভীত যে, যদি তারা কনডম ব্যবহার করার পরামর্শ দেয় তাহলে তাদের সঙ্গী ভাববে যে তারা ‘নোংরা’। তাদের মধ্যে কেউই জানে না যে কীভাবে বিষয়টি আলোচনায় আনতে হয়। তাই অবশেষে তারা কনডম ছাড়া যৌনমিলন করল।

দম্পতি ঙ-এর প্রয়োজন: ১

দম্পতি চ : যোল বছর বয়সের এই বালক একটি খারাপ দলের সাথে জড়িত। সে দলটি ত্যাগ করতে চায়। কিন্ত্ত তা করা কঠিন এবং বিপদজনক হতে পারে । যাই হোক, তার দল তাকে এটি ত্যাগ করার অনুমতি দিবে, যদি সে বাবা হয়। ছেলেটি তার পনের বছর বয়সের প্রেমিকাকে বুঝিয়ে বলল যে, যদি তাদের একটি বাচ্চা হয় তাহলে এটি তার জীবন রক্ষা করতে পারে। এবং তাই সে একজন ভাল বাবা হবে। মেয়েটি কী করবে তা নিয়ে দ্বিধান্বিত ছিল । সে লেখাপড়া শেষ করতে চায় এবং মা হওয়ার জন্য প্রস্তুত বলে মনে করে না । কিন্তু সে এটাও মনে করে যে, তার প্রেমিককে তার সাহায্য করা উচিত। তারা কনডম ছাড়া য্যীনমিলন করল।
দম্পতি চ-এর প্রয়োজন:
8
४ $\quad$ s

দম্পতি ছ : এই দুইজন যুবক-যুবতী য্যেনমিলন করার সিদ্ধান্ত নিয়েছে। ছেলেটি জিজ্ঞস করল, তাদের প্রতিরোধক ব্যবস্থা নেওয়া উচিত কিনা । কিন্তু মেয়েটি বলল যে, তার মাত্র মাসিক হয়েছে তাই সে গর্ভবতী হবে না।
দম্পতি ছ-এর প্রয়োজন:
২
8
৫

দম্পতি জ : এই দুইজন যুবক-যুবতী যৌনমিলন করার সিদ্ধান্ত নিয়েছে। তারা এইচআইভি প্রতিরোধ করার জন্য কনডম ব্যবহার করবে কিনা তা নিয়ে আলোচনা করল। কিন্তু তারা একমত হল যে, যদি তারা অসুস্থ হত তাহনে তারা জানত। তাই তারা যৌনমিলনে অগ্রসর হল এবং কনডম ছাড়া যৌনমিলন করল।

দম্পতি জ-এর প্রয়োজন: ১

দম্পতি ঝ : একটি সতের বছর বয়সের মেয়ে পঁচিশ বছর বয়সের একটি লোকের সাথে যৌনমিলন করে, যে তাকে উপহার এবং মাঝে মাঝে তার খরচে সাহায্য করতে টাকা দেয়। মাঝে মাঝে লোকটি কনডম ব্যবহার করে কিন্তু এখন তার কাছে কোন কনডম নেই। মেয়েটি মনে করল যে, তাদের অপেক্ষা করা এবং অন্য সময়ে যৌনমিলন করা উচিত। কিন্তু লোকটি প্রতিজ্ঞা করল- কনডম ব্যবহার ছাড়া যৌনমিলন ঠিক হবে। মেয়েটি ইতিমধ্যে তার কাছ থেকে এই সপ্তাহে টাকা নিয়েছে। তাই তার মনে হল- সে না করতে পারে না । তারা কনডম ছাড়া যৌনমিলন করল।

দম্পতি ঝ-এর প্রয়োজন: ১ 


\section{“ কনডম ব্যবহার করতে মানুষকে মতায়নের সমাধানগুলি”-এর উত্তরপত্র}

শিক্ষকদের জন্য লক্ষণীয়: নিয়লিখিত তালিকাতত প্রত্যেক গল্পের দম্পতির জন্য প্রধান 'সমাধানগুলি' শনাক্ত করা আছে। যে কোনো গল্পের জন্য শিক্ষার্থীরা অতিরিক্ত সমাধান দিতে পারে। উত্তর দেয়ার ব্যাপারে নমনীয় হোন, কিন্ত কমপক্ষে নিশ্চিত হোন যে, নিচের তালিকায় দেয়া সমাধানগুলি চূড়ান্ত উত্তরে অন্তর্ভুক্ত হয়েছে।

দম্পতি ক : সমাধান ১ : (সঠিকভাবে কনডম ব্যবহার সম্পর্কে তথ্য)

দম্পতি খ : সমাধান 8 : (ভাল যোগাযোগ দক্ষতা); এবং

সমাধান ৩ : (কনডম কোথায় পাওয়া যাবে সে বিষয়ে তথ্য)

দম্পতি গ : সমাধান ২ : (ছেলে ও মেয়ের মধ্যে বেশি সমতা এবং ক্ষমতার সমবন্টন)

সমাধান ৩ : (ভাল যোগাযোগ দক্ষতা); এবং

দম্পতি ঘ : স সমাধান ৬ : (ছেলেদের জন্য য্যীনমিলন সম্পর্কে আলোচনার জন্য একটি নিরাপদ জায়গা)

দম্পতি : : সমাধান ৩ : (ভাল য্যাগাযোগ দক্ষতা); এবং

সমাধান ৫ : (এইচআইভি ও গর্ভধারণের ঝুঁকি সম্পর্কে আরো বেশি বাস্তব ধারণা)

দম্পতি চ : স সমাধান ২ : (ছেলে ও মেয়ের মধ্যে বেশি সমতা এবং ক্ষমতার সমবন্টন)

দম্পতি ছ : সমাধান ৫ : (এইচআইভি ও গর্ভধারণের ঝুঁকি সম্পর্কে আরো বেশি বাস্তব ধারণা)

দম্পতি জ : সমাধান ৫ : (এইচআইভি ও গর্ভধারণের ঝুঁকি সম্পর্কে আরো বেশি বাস্তব ধারণা)

দম্পতি ঝ : সমাধান ১ : (ছেলে ও মেয়ের মধ্যে বেশি সমতা এবং ক্ষমতার সমবট্টন) 


\section{কনডম ব্যবহারের সঠিক ধাপগুলি}

শিক্ষকদের জন্য লক্ষণীয়: ১৬টি ফ্লাশকার্ড তৈরি করুন । নিচের তালিকা থেকে প্রতিটি ফ্লাশকার্ডে একটি ধাপ লিখুন। 'ধাপ নাম্বার’ সংযুক্ত করবেন না। (নিচের নাম্বারগুলো আপনার উত্তরপত্র অনুসারে দেয়া হয়েছে।) মনে করে দেখুন যে , ১-৫ পর্যন্ত ধাপগুলো পরিবর্তনশীল।

\section{(যৌনমিলনের অনেক পূর্বে )}

১ তোমার সঙ্গীর সাথে নিরাপদ যৌনমিলন নিয়ে আলোচনা কর।

২ কনডম (এবং যদি চাও লুব্রিকেন্ট) ঐয় কর অথবা একটি ক্লিনিক বা অন্য সামাজিক কেন্দ্র থোঁজ করো যারা এগুনো বিনামূল্যে বিতরণ করে ।

৩ তোমাদের কনডম একটি ফক্ক, ঠাণ্ণা স্থানে (পকেটে নয়) রাখ।

8 কনডমের মেয়াদোত্তীর হওয়ার তারিখ দেখ এবং নিশ্চিত হও যে, তারিখ শেষ হয়ে যায়নি।

৫ কনডম পরার চর্চা কর যেন পরবর্তীতে এটি ব্যবহারের সময় স্বস্তিবোধ কর।

(যৌনমিলনের ঠিক পূর্বে)

৬ শৃঙ্গার কর। ভগাঙ্কুর স্পর্শসহ শৃঙ্গার যোনি পিচ্ছিল করতে সাহায্য করতে পারে।

৭ সতর্ক হয়ে ধীরে ধীরে কনডমটি খোল যেন এটি না ছিঁড়ে (দাঁত ব্যবহার করবে না)।

৮. যখন তোমার পুরুষাঙ্গ খাঁড়া হয়------ কনডমের সামনের অংশ টিপে ধর এবং কনডমটি পুরুষাঙ্গের মাথায় বসাও।

৯ কনডনের সামনের অংশ টিপে ধরে পুরুষাঙ্গ সম্পূর্ণ না ঢোকা পর্যন্ত মোড়ানো কনডমটি পর।

১০ যৌনাাঙ্ যদি এখনও শ্ক মনে হয়, আরো শৃঙ্গার কর, অথবা কনডমের বাইরের দিক পানি জাতীয় লুব্রিকেন্ট বা লালা দিয়ে ভিজিয়ে নাও। কখনও ভ্যাসলিন বা অন্য কোন তৈলাক্ত উপাদান ব্যবহার করবে না । কারণ এগুলো কনডমটি দুর্বল ও ছিঁড়ে ফেলতে পারে।

(যৌনমিলনের সময়)

১১ কনডমটি যদি ছিঁড়ে যায়, পুরুষটির সাথে সাথে পুরুষাঙ্গ বের করে ফেলা উচিত /যদি বীর্যপাত হয়ে যায় তাহলে জরুরি গর্ভনিরোধক সম্পর্কে আপনি শিক্ষার্থীদেরকে মনে করিয়ে দিতে পারেন ।]

১২ বীর্যপাতের পর পুরুষাঙ্গ উখিত থাকা অবস্থায়

১৩ পুরুষাঙ্গের গোড়ায় কনডমের খোলা মুখটি শক্ত করে ধর।

(যৌনমিলনের ঠিক পরে )

১৪ পুরুষাঙ্গের গোড়ায় কনডমটি ধরে ধীরে ধীরে পুরুষাঙ্গ বের করে আন।

১৫ কনডমের গোড়া সতর্কতার সাথে ধরে কনডমটি বের কর যেন বীর্য গড়িয়ে না পড়ে ।

১৬ কনডমটি বাঁধ বা টয়লেট পেপারে মোড়াও এবং সঠিকভাবে ফেলে দাও। 


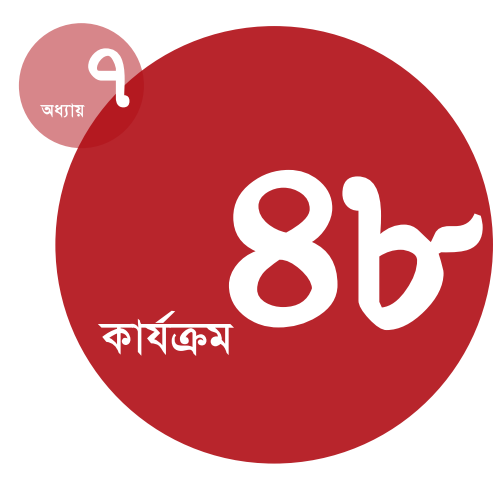

সময়কাল :

ধাপ ১ - ৩: 80 মিনিট

ধাপ 8 -১০: 8 মিনিট

\section{উপকরণ:}

বোর্ড ও চক।

\section{প্রস্তুতি:}

আলোচনার বিষয়গুলো প্রয়োজন মত পুনরায় পড়ন এবং পরিবর্তন করুন (যেমন, দম্পতিরা অন্তর্ভুক্ত হতে পারে)। সতর্কতার সাথে আপনার সময়সূচি ঠিক করুন এবং ভূমিকাঅভিনয় সংক্ষিপ্ত রাখুন।

\section{যৌনমিলন ও নিরাপত্তা নিয়ে একটি কথোপকথন শুরু}

অধিবেশন সম্পর্কিত ধারণা: শিক্ষার্থীরা গুরুত্বপূপূর যৌন নিরাপত্তা ও স্বাস্থ্য সম্পর্কিত যে বিষয়গুলো প্রায়ই উপেক্ষিত হয় সেগুলো নিয়ে কথোপকথনের চর্চা করু করবে। (লক্ষণীয়: এই অধিবেশনের সাথে অধিবেশন ৩৮-এর কিছু মিল আছে; শিক্ষক এই গুরুতত্বপূর্ণ দক্ষতাগুনো শেখানোর জন্য যে কোন একটি বা উভয় অধিবেশন আলোচনা করতে পারে।)

উদ্দেশ্য: শিক্ষার্থীদেরকে যৌনমিলন ও নিরাপত্তা সম্পর্কিত বিষয়ে চিন্তা করতে এবং এ সম্পর্কিত আলোচনা চর্চা ফুরুর উদ্যোগ নেয়া ও আলোচনা চালিয়ে যেতে সক্ষম করা; সমালোচনামূলক চিন্তার দক্ষতা শক্তিশালী করা।

\section{निर्্দশাবলি}

১ নিয়নিिিত নির্দেশনামূলক প্রশ্নগুলো দিয়ে অধिব্বেননটি ফরু করুন:

• আজকে আমরা যৌন নিরাপত্তা নিয়ে আলোচনা করার চর্চা বিষয়ে ভাবব। যৌন সম্পর্কিত বিষয়ে কথা বলা কতটা সহজ? কেন ?

• কোন বিষয়টি এটিকে আরো সহজ করতে পারে ?

২ শিক্ষার্থীদদর জোড়া টৈরি করতে বলুন (ছেলে-মেয়ে জোড়া হুলে ভাল হয়)। নিয়লিिিত বিষয়গুলো বোর্ড্ড লিখুন: য্যেনমিলন করবে কি করবে না भूर्य বযৗন অভिज्ञण যৌনবাহিত সংং্রমণ, এইচআইভি ও এইডস পূর্ব্ব মাদক গ্রহণ কনডম ব্যবহার

৩ ব্যাখ্যা করুন:

• তোমাদের জোড়ায় তোমরা জটিল কিন্তু গুরুত্বপূণর বিষয়ে আলোচনা শুরুর চর্চা করবে। বোর্ডের প্রত্যেকটি বিষয় নিয়ে সম্ভাব্য যৌনসঙ্গীর সাথে কীভাবে কথোপকথন শুরু করা যায় তা আলোচনা কর। কথোপকথন শুরু করার কমপক্ষে একটি নির্দিষ্ট উপায় লেখ।
• প্রথম কথোপকথন কখন ওরু হওয়া উচিত সে বিষয়েও সিদ্ধান্ত নাও- কখন তুমি দেখা কর? প্রথম চুম্বনের পর ? যখন তুমি ইতিমধ্যে য্যীনমিলনের পরিস্থিতিতে জড়িত ? মনে রেখ, মানুষের সবকিছু নিয়ে একবারে কথা বলার প্রয়োজন নেই।

8 প্রত্যেক বিষয়ের জন্য একটি দলকে তাদের ধারণা নিয়ে আলোচনা করতে বলুন; তাদের উত্তরগুলো বোর্ডে লিখুন। জিজ্ঞস করুন:

• কেউ কি অন্য কোন পরামর্শ দিতে চাও? (এগুলো তালিকায় যোগ করুন)।

• কোন্ ধারণাটি কার্যকর হতে পারে বলে তোমরা মনে কর এবং কেন? তোমাদের মতে ভাল প্রকাশ ভঙি হতে পারে না এমন কোন বিষয়ে পরামর্শ আছে কি ? কেন ?

• সম্পর্কের কোন অবস্থায় এই কথোপকথন ওরু হওয়া উচিত? কেন?

৫ পাঁচটি বিষয়ের সবগুলো পুনরায় পড়ার পর জিজ্ঞেস করুন:

• কী এই ধরনের কথোপকথনকে আরো সহজ করতে পারে?

• এখন আমরা বাস্তব জীবনের সাথে এই ধরনের কথোপকথন কীভাবে মেলানো যায় তা নিয়ে ভাবব। 
৬ ব্যাখ্যা করুন:

• প্রথম ব্যক্তি কথোপকথন শুরু করবে। পক্ষান্তরে দ্বিতীয় ব্যক্তি কথোপকথনটিতে জটিলতা তৈরি করবে। দ্বিতীয় ব্যক্তি অদক্ষতা দেখাতে পারে, অমত পোষণ করতে পারে, বা কথোপকথনটি এড়িয়ে যাওয়ার চেষ্টা করতে পারে ।

• প্রথম ব্যক্তির কাজ হচ্ছে কথোপকথনটি কমপক্ষে কিছুটা সামনে এগিয়ে নেয়ার চেষ্টা করা।

৭ দুইজন আগ্রহী শিক্ষার্থীকে প্রথম বিষয়টি (যৌনমিলন করবে কিনা) নিয়ে একটি কথোপকথন করতে বলুন । ব্যাখ্যা করুন:

• তোমাদের চরিত্রগুলির মতো নামসহ এসো এবং বাস্তবসম্মত হবার চেষ্টা কর।

• এখানে তোমাদের জন্য দৃশ্য আছে।/নিচের দৃশ্যটি পড়ার জন্য নতুন নামগুলো যুক্ত কর।]

• “আলি এবং লিয়া কিছুদিন থেকে অন্তরঙ্গভাবে মিশছে এবং শারীরিক আকর্ষণ অনুভব করতে শুরু করেছে । তারা যৌনমিলন করেনি। তারা অন্তরঙ্গভাবে মিশতে শুরু করার পূর্বে কেউ অন্যের যৌন আচরণ ও মাদক গ্রহণের বিষয়ে নিশ্চিত হয়নি । আলি বিশ্বাস করে তারা যৌনভাবে আরো সম্পৃক্ত হতে পারত এবং এইচআইভি নিয়ে সত্যিই উদ্বিগ্ন । ঠিক এই মুহুর্তে তারা হাঁটছে।"

- কীভাবে আগ্রহী দুইজন কথা বলছে প্রত্যেকেই সে বিষয়ে সংক্ষিপ্ত মন্তব্য লিখবে।

৮ নিম্নলিখিত প্রশ্নগুলো নিয়ে একটি সংক্ষিপ্ত আলোচনা করুন:

- কোনটি ভাল হচ্ছিল ? কী অন্যভাবে মোকাবেলা করা যেত ?

• কথোপথনটি কি বাস্তবসম্মত ছিল?

• তোমাদের কি আলী বা লিয়ার জন্য কোন উপদেশ আছে?
৯ নচের দৃশ্যগুলোর জন্য যতক্ষণ সময় থাকে ধাপ ৬-৮- এর পুনরাবৃত্তি করুন:

• যৌনমিলন করবে কি করবে না সে বিষয়ে অঞ্জলী মো -এর সাথে কথোপকথন শুরু করে। কী করবে সে বিষয়ে তারা একমত হতেও পারে, নাও হতে পারে।

- কার্লো তাদের পূর্ব যৌন অভিজ্ঞতা এবং মাদকগ্রহণ নিয়ে মোর -এর সাথে কথোপকথন শুরু করে;

- হেনরি এবং মিয়া কথা বলেছে এবং তারা যৌনমিলন করতে চায় বলে মনে করে। হেনরি কনডম ব্যবহার নিয়ে মিয়ার সাথে একটি কথোপকথন শুরু করে। [হেনরিকে গোপনে নির্দেশ দিন যে, সে কনডম ছাড়া যৌনমিলন করতে চায় না এবং মিয়াকে গোপনে নির্দেশ দিন যে, সে কনডম ব্যবহার জরুরি মনে করে না।

১০ নিম্নলিখিত প্রধান প্রধান উত্তরগুলো বোর্ডে লিখে শেষ করুন:

• এ রকম কথোপকথন শুরুর পূর্বে তোমার নিজের সম্পর্কে কী ভাবা প্রয়োজন ? [জানার চেষ্টা করুন: তুমি কেমন অনুভব কর, তুমি কী চাও, তুমি কী বলতে চাও।]

- সফল কথোপকথনের জন্য পরামর্শগুলো কী কী?

• সম্মানের সাথে 'না’ বলার জন্য পরমার্শগুলো কী কী?

- প্রত্যেক ব্যক্তির কোন কোন অধিকার আছে? [জানার চেষ্টা করুন: তোমার মত প্রকাশের অধিকার, না বলার অধিকার, তোমার নিজের স্বাস্থ্য রক্ষা করার অধিকার।]

• সম্পর্কের মধ্যে এ রকম কথোপকথন শুরু করা কার দায়িত্ব? কেন ? 


\section{এইডস হচ্ছে একটি নামসহ একজন ব্যক্তি}

অধিবেশন সম্পর্কিত ধারণা: শিক্ষার্থীরা এইচআইভি আক্রান্ত একজন মানুষের গল্প শুনবে ।

উদ্দেশ্য: শিক্ষার্থীদেরকে এইচআইভি ও এইডস নিয়ে বেঁচে থাকা মানুষকে বুঝতে ও তাদের জন্য সহানুভূতি তৈরিতে সক্ষম করা।

\section{সময়কাল :}

ধাপ ১: ১০ মিনিট (একদিন পূর্বে)

ধাপ ২-৪: ৪০ মিনিট (পরিবর্তনশীল)

\section{উপকরণ:}

বোর্ড ও চক; শিক্ষার্থীদের জন্য

হ্যান্ডআউট (যদি কোন বক্তা না থাকে)।

\section{প्रস্তুতি:}

এক বा দুইজন অতিথিকে আমন্ত্রণ জানান যারা এইচআইভিতে আক্রান্ত (একজন পুরুষ ও একজন মহিলা হললে ভাল হয়)। यদি তা সম্ভব না হয় তাহলে এইচআইভি ও এইডস নিয়ে কাজ করে এমন কোন দলের একজন সদস্যকে অথবা এমন কেউ যে এইচআইভি আক্রান্ত মানুমের সেবা করে তাকে আমন্ত্রণ করুন । বক্তাকে তার নিজের ব্যক্তিগত গল্প বলতে বলুন। বক্তাকে শিক্ষার্থীদদর বয়স ও এইডস সম্পর্কে তাদের পূর্বজ্ঞান এবং উপস্থাপন/থ্রশ্নের জন্য বরাদ্কত সময় জানিয়ে দিন । বক্তাকে নিম্নলিখিত বিষয়গুলো বিবেচনায় নিয়ে আলোচনা করতে বলুন:

কারো এইচআইভি আছে কিনা তা জানা কারো এইচআইভি আছে কিনা তা প্রকাশ করা অপবাদ/বৈষম্য

সহায়তা পাওয়া

স্বাস্থ্য পরিচর্যা ও চিকিৎসা

প্রতিদিনের জীবন যাপন

পূরুষ ও মহিলাদের জন্য এইচআইভি নিয়ে বেঁচে থাকা কতটা আলাদা

বক্তা আসার পূর্ব পর্যন্ত ধাপ-১ চালিয়ে যান ।

\section{নির্দেশাবলি}

\section{বক্তা আসার পূর্বে}

১ শিক্ষার্থীদেরকে বলুন পরবর্তী সেশনে এক বা একাধিক অতিথি বক্তা এইচআইভি নিয়ে তাদের ব্যক্তিগত অভিজ্ঞতা বলবে। জিজ্ঞস করুন: "তোমরা কী বিষয়ে শুনতে পছন্দ করবে?" “তোমাদের কীসের ভয় আছে?" যে কোন ভয়ের উত্তর দিতে প্রয়োজন মতো এইচআইভি সম্পর্কে তথ্যগুলো পুনরায় পড়ন। শিক্ষার্থীদেরকে মূল নিয়ম-কানুনগুনো মনে করিয়ে দিন; যদি আপনার কাছে নিয়ম-কানুন না থাকে, তাদেরকে চিন্তা করে একজন অতিথি বক্তার সাথে সঠিক আচরণের নিয়মকানুন বের করার জন্য বলুন ।

বিকল্প কার্যক্রম: যদি আপনার সমাজে অতিথি বক্তাকে আমন্ত্রণ করা সম্ভব না হয়, আপনি ‘সত্য গল্প'-এর হ্যান্ডআউট ব্যবহার করতে পারেন । শিক্ষার্থীদের বলুন, গল্পের মানুষের স্থানীয় নাম দিতে এবং গল্পগুলো সকলে শুনতে পারে এভাবে পড়তে। তাদেরকে প্রশ্ন করার অনুমতি দিন, তারপর ধাপ ৩ ও ধাপ 8 চালিয়ে যান ।

\section{বক্তা অবস্থানের সময়ে}

২ অতিথি বক্তার সংক্ষিপ্ত পরিচিতি ও ধন্যবাদ দিন । অতিথি কতক্ষণ কথা বলবে তা উল্লেখ করুন ।

৩ শিক্ষার্থীদের প্রশ্ন করার জন্য সময় রাখুন । তারপর বক্তাকে ধন্যবাদ ও বিদায় জানান ।

\section{বক্তা চলে যাওয়ার পরপরই}

8 নিচের প্রশ্নগুলো নিয়ে আলোচনা করুন:

• উপস্থাপনের সময়ে তোমাদের কেমন অনুভূতি ও প্রতিক্রিয়া रয়েছিল?

• উপস্থাপনের কোন অংশ তোমাদের কাছে বেশি অর্থপূর্ণ বা বিস্মায়ের ছিল?

• এইচআইভি হওয়া কিসের মত এ সম্পর্কে তোমরা কী শিখেছ?

- বক্তা তার কোন অনুভূতি হওয়ার কথা বলেছে?

- সে কী কী প্রচলিত ধারণা বা বিশ্বাসের মুখোমুখি হয়েছিল?

• তোমাদের কি আর কোন প্রশ্ন আছে?

- আজকে তোমরা সবচেয়ে গুরুত্বপূর্ণ কী বিষয় শিখলে বা অভিজ্ঞতা হল ? তোমরা যা শিখলে তা কীভাবে তোমাদেরকে আলাদাভাবে তৈরি করবে? [জানার চেষ্টা করুন: আমি এইচআইভি ও এইডস আক্রান্ত ব্যক্তির জন্য আলাদা রকম অনুভব করব এবং আলাদা রকম ব্যবহার করব। আমি এইচআইভি সংক্রমণ প্রতিরোধ সম্পর্কে আরো সতর্ক হব।] 


\section{সত্যগল্প (যদি কোন বক্তা না থাকে তাহলে ব্যবহার করুন)}

এমউনজি’র গল্পः আমি ২২ বছর বয়সী একজন জাম্বিয়ান নারী এবং এইচআাইভি ভাইরাস আক্রান্ত, যা এইডসের কারণ। ছোটবেলায় আমি আমার বাবা-মা উভয়কেক এইডসের জন্য হারিয়েছি এবং আমার বড় বোনের কাছে বেড়় উঠেছি। যখন আমার বয়স ১৯ বছর, আমি গর্ভবর্তী হলাম এবং স্থানীয় স্বাহ্থ্য কেন্দ্রে প্রসবপৃর্ব সেবা নিতে ফরু করলাম। সেখানে তারা আমার এইচআইভি পরীক্ষা করল। যখন আমাকে বলা হল আমার পরীকার্কর ফলাফল এইচআইভি পজেটিভ হয়েছে, আমি তা বিশ্বাস করতে পারলাম না । আমি সুস্থবোধ করতাম এবং স্বাস্থ্যবতী দেখাত। এটি সত্য মনে হয় না। আমি মারা থ্যেত চাইনা। যখন আমি আমার প্রেমিককে বললাম, সে হেলে এটি উড়িক়ে দিল। সে বলল, "ছাড়, তোমাকে অসুস্থ দেখায় না। তারা তোমাকে কেবল ঘাবড়ে দেয়ার চেষ্টা করছে।” কিন্তু তারপর সে পরীক্ষা করল এবং জানল, সেও আমার মত আক্রান্ত। সৌভাগ্যবশত, আমার বোন খুব স্লেহপ্রবণ ছিল। সে আমার চিকিৎসার টাকা দিয়ে সাহায্য করত এবং আরো অন্য অনেকভাবে সাহায্য করত। আমি সিদ্ধান্ত নিলাম যে, আমি এইচআইভিকে আমার জীবনের সম্পূর্ণ বাধা হতে দেব না । যদি আমি চাই, আমি এটির সাথে লড়াই করতে পারি। এটি সৌভাগ্যের যে, আমি পরীক্ষা করিয়েছিলাম, কারণ আমি এন্টিরেট্রোভাইরাল থেরাপি শুরু করতে পেরেছিলাম যা আমাকে সুস্থ রেখেছে এবং আমার বাচ্চাকে এইডলের ভাইরাস নিয়ে জন্ম নেয়া থেকে রক্ষা করেছে। কষ্টের হল, আমার অবস্থার জন্য যখন অন্য মানুষেরা আমার সাথে খারাপ আচরণ করে। আমি জানি না কতদিন আমি সুস্থ থাকব, কিন্তু আমি পরিপূর্ণ জীবন যাপন করছি । আমি একজন ভাল মা এবং আমি চাকরি করি: কীভাবে যুবক-যুবতীরা নিজেদেরকে এবং তাদের সঙ্গীদের এই রোগ হওয়া থেকে রক্ষা করবে সে বিষয়ে পড়াই।
ব্রেট’র গল্পः আমার বয়স যখন ১৮- বছর, আমি চটপটে ছিলাম এবং ফ্যাশন করে চুল কাটতাম। দিনের বেলায় কলেজের পাঠদান কক্ষে আর রাতে শহরের নামকরা কোন বারের নাইট ক্লাবে কিংবা মেট্রোতে যেতাম । মাত্র দুই বছর পর, আমি যখন একটি ফোনের দোকানের সুয়িং-ডোর (যে দরোজার কপাট ভেতর ও বাহির দু’দিকেই খোলা যায় এবং আপনা হতেই বন্ধ হয়ে যায়) -এর মধ্যে দাঁড়িয়ে তখন জানতে পারলাম যে আমার শরীরের সেই ভাইরাস আছে যাহা এইডসের কারণ। আমি হতবুদ্ধি হয়ে গেলাম। কিন্তু আমার স্বাস্থ্যে কিছু ঘাটতি ছিল যা আমি যুবক বয়লে তৈরি করেছি। আমি সাহসী এবং আদর্শবাদী ছিলাম। আমি বিশ্বাস করতাম যে, আমরা- রোগীরা, ডাক্তাররা, বিজ্ঞানীরা এবং গবেষকরা- আমাদের সত্যিই যদি ইচ্ছা থাকত আমরা এইডস সারাতে পারতাম। আমি জানি যে, ওটা ভাববাদী শোনায়। কিন্তু আমাদের সঠিক সময়ে অনুপ্রেরণার প্রয়োজন ছিল। ভাববাদ হচ্ছে তাই, যা সামান্য আশাতেও আমাকে সচল রাখে। আমি সব সময় লিখতে চাইতাম। আমার রোগ নির্ণয়ের পর আমি নির্দিষ্টভাবে জানলাম কী সম্বন্ধে আমার লিখতে হবে এবং বিষয়বস্তুর ওপর ঝাপিয়ে পড়লাম। আমি প্রাস ভয়েস নামের একটি পত্রিকা শুরু করার জন্য অনেক কট্টে যথেষ্ট টাকা জোগাড় করলাম। আমার লক্ষ্য ছিল এই ভাইরাসে আক্রান্ত মানুযের জীবন যাপন নিয়ে একটি ম্যাগাজিন প্রকাশ করা, যা এধরনের মধ্যে প্রথম । মাঝে মাঝে আমার পরিশ্রান্ত/নিঃশেষিত লাগত, কিন্ত লেখালিখি ও আমার বন্ধুরা আমাকে সচল রাখত। আমি আমার বয়সের সম্পূর্ণটাই এইচআইভি আক্রান্ত ছিলাম। যদিও আমাদের এখনও এইডসের প্রতিষেধক নেই। কিন্তু ২০০৮- সালে ভাইরাসটিকে আটকে রাখার জন্য আমরা একটি চমৎকার ঔষধ পেয়েছি। প্রতিবছর এটি উন্নত হচ্ছে। কিন্তু এই নতুন ঔষধ সহজ ও আরামপ্রদ নয়। এটি তোমার শরীরের ওপর কঠিন প্রভাব ফেলে এবং এটি অন্যভাবে তোমাকে অসুস্থ হওয়া থেকে হঠাৎ মুক্তি দেবে না। তোমার সিটবেল্ট বাধ। ধুমপান করো না। পরিমিত মদ্যপান কর। যখন তোমার প্রয়োজন তখন মানসিক সাহায্য নাও। শরীরচর্চা কর। সবসময় কনডম ব্যবহার কর এবং নিজের জীবনের প্রত্যেকটি দিকে কঠোরভাবে সৎ থাক।/অনুমতিসহ টেস্ট পজেটিভ অ্যাওয়ার নেটওয়ার্ক <www.tpan.com> থেকে নেয়া ।] 


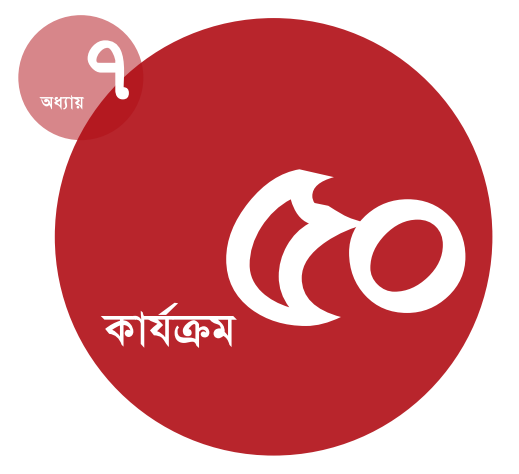

সময়কাল :

ধাপ ১-৪: 8৫ মিনিট

ধাপ ৫-৬: ৪৫ মিনিট

\section{উপকরণ:}

বোর্ড ও চক।

\section{প्रস্তুতি:}

যখন আপনি ধাপ-১ এর গভীর উত্তর জানতে চান তখন ‘খোলা প্রশ্ন’ নিয়ে ভাবুন (ছঁ্যা/ না প্রশ্ন নয়) । সম্ভব হলে আপনার সমাজে এইচআইভির ব্যাপকতা সম্পর্কে তথ্য সংগ্রহ করুন। এই অধিবেশন কিছু ছাত্রের মধ্য থেকে কঠিন অনুভূতি টেনে বের করতে পারে; আপনি কীভাবে যথোপযুক্তভাবে শিক্ষার্থীদেরকে সাহায্য করতে পারবেন তা ভাবুন। প্রত্যেকের গোপনীয়তাকে সম্মান করুন এবং কাউকে তার গল্প জোরে পড়তে বলার দরকার নাই।

\section{তোমার কী বলার আছে? তুমি কী জানতে চাও?}

অধিবেশন সম্পর্কিত ধারণা: মানুভের কতটা দায়িত্ব রয়েছে তার এইচআইভি সম্পর্কিত অবস্থা তার যৌন সঙ্গীর কাছে প্রকাশ করার সে বিষয়ে শিক্ষার্থীরা আলোচনা করবে। তারা গোপনীয়তার অধিকার এবং নিজেকে ক্ষতি থেকে রক্ষা করার অধিকারের মধ্যকার জটিল ভারসাম্য নিয়ে নিরীক্ষা করবে। তারা সৃজনশীল লেখার মাধ্যমে এই উভয় সংকট সম্পর্কে তাদের অনুভূতিগুলো জানার চেষ্টা করবে। (লক্ষণীয়: অধিবেশনের পূর্বে শিক্ষার্থীদের মানবাধিকার, সম্মতি, এবং এইচআইভি ও এইডস সম্পর্কে মৌলিক তথ্য জানা থাকতে হবে।)

উদ্লেশ্য: যৌনসঙ্গীর কাছে এইচআইভির অবস্থা প্রকাশ করতে মানুমের কতটা দায়িত্ব বা বাধ্যবাধকতা রয়েছে সে সম্পর্কে শিক্ষার্থীদেরকে গভীরভাবে ভাবতে সক্ষম করা।

\section{निर्দেশাবলি}

১ নিয়লিখিত প্রশ্নগুনোর মাধ্যমে অধিবেশনটি শুরু করুন। [শিক্ষার্থীদের উত্তরগুলো বোর্ডে লিখুন।]

- আজকে আমরা একটি সূক্ষ ও জটিল প্রশ্ন নিয়ে আলোচনা করব: মানুষ তার যৌনসঙ্গীর কাছে এইচআইভি আছে কিনা তা নিয়ে আলোচনা করতে বাধ্য হয় কিনা ।

- তোমরা কি মনে কর যেসকল মানুষের এইচআইভি আছে তারা সকলে সেটা তাদের যৌনসঙ্গীর কাছে বলে ?

- কী কী কারণে একজন মানুষ তার অবস্থা সঙ্গীকে না বলতে পারে? [জানার চেষ্টা করুন: তার গোপনীয়তা রক্ষা করা; অপবাদ, ছেড়ে যাওয়া এবং বৈষম্যের ভয়।]

- কী কী কারণে মানুষ এইচআইভি পরীক্ষা করাতে চেষ্টা নাও করতে পারে? [জানার চেষ্টা করুন: তারা এইচআইভি পরীক্ষা করাতে ভয় পায় (এইচআইভি আছে); পরীক্ষার জন্য কোনো ক্লিনিকে যেতে অস্বস্তিবোধ করে; বিনামূল্যে পরীক্ষার করানোর জায়গার ব্যাপারে জানে না; সুস্থবোধ করে ও সুস্থ দেখায় এবং বিশ্বাস করে না যে তারা আক্রান্ত হতে পারে; বিদ্যালয়ে যাওয়া বা কাজের জন্য পরীক্ষা করানোর সময় বের করতে পারে না; ফলাফল গোপন রাখা হবে তা বিশ্বাস করে না।]
- কী কী কারণে মানুফের এইচআইভি পরীক্ষা করানো উচিত? [জানার চেষ্টা করুন: যদি পরীক্ষার ফলাফল 'না' হয় তাহলে তারা স্বস্তি পাবে। যদি পরীক্ষার ফলাফল ‘ঁঁ্যা’ হয় তাহলে তারা চিকিৎসা শুরু করতে পারে; সঙ্গীদেরকে ভবিষ্যৎ সংক্রমণ থেকে নিরাপদ রাখে; বর্তমান এবং অতীত সঙ্গীকে জানাতে পারবে যেন তারা পরীক্ষা করাতে পারে।]

২ শিক্ষার্থীদেরকে তাদের লেখার খাতা বের করতে বলুন । ব্যাখ্যা করুন:

• মানুষ কেন তাদের এইচআইভির অবস্থা পরীক্ষা করতে এবং তাদের সঙ্গীকে বলতে পছন্দ করে না তা ভেবে দেখার একটি সুয্যাগ আমাদের আছে। তোমাদের কাজ হচ্ছে একটি গল্প লেখা যা এই বিষয়ের গভীরতর ভাব প্রকাশ করবে। তোমাদের গল্প তিন পৃষ্ঠার বেশি বড় হওয়া উচিত নয়।

- তোমাদের গল্প কী বিষয়ে হওয়া উচিত? তোমাদের গল্প এমন কারো বিষয়ে হতে পারে যে পরীক্ষা করাতে পছন্দ করে না । অথবা এটি এমন কারো বিষয়ে হতে পারে যে তার সঙ্গীর কাছ থেকে এইচআইভি সংক্রমিত হয়েছে যার এইচআইভি ছিল কিন্তু প্রকাশ করেনি । অথবা এটি এমন কারো সম্বন্ধে হতে পারে যে জানে সে এইচআইভিতে আক্রান্ত এবং তার সঙ্গীকে বলেছে। 
• তোমাদের গল্পে कী কী বিষয় এবং অনুভূতি থোঁজা উচিত? তোমাদের গল্পের মধ্যে কিছু উত্তেজনা থাকা উচিত। কীভাবে তোমাদের গল্পের চরিত্রটি সিদ্ধান্তে পৌছছায়, সে এ বিষয়ে কেমন অনুভব করে, এবং কী ঘটেছিল (সিদ্ধান্তটি কীভাবে তোমাদের গল্পের চরিত্রটির নিজের জীবনের এবং অন্য মানুষের জীবনের ওপর প্রভাব ফেলে) তা দেখাও । চরিত্রটি কেমন অনুভব করে (ভীত? সহানুভূতিপ্রাপ্ত? একাকী? স্বস্তিবোধ ? অনুপোচনাপূর্ণ? ভালবাসাপ্রাপ্ত? প্রশংসিত ? তিক্ত?) তা ভাব।

• গল্পটি কি বাস্তবসম্মত নাকি কাল্পনিক হওয়া উচিত? গল্পটি সম্পূর্ণভাবে উদ্ভাবনীমূলক বা তোমাদের নিজেদের অভিজ্ঞতার ওপর ভিত্তি করে বা তোমাদের জানা কারোর অবস্থা সম্পর্কে হতে পারে। কিন্তু কারো প্রকৃত নাম ব্যবহার না করা গুরুত্বপূর্ণ। শেষ কথা: এই গল্প বড় হওয়া উচিত নয়, কিন্তু অবশ্যই গুরুতত্বপূর্ণ হবে। এটি একটি চিন্তাশীল কাজ, হাস্যকর কিছু নয়। তোমাদের গল্প শ্রেণিকক্ষের সময়ের বাইরে শেষ করতে পার যদি শ্রেণিকক্ষে পাঠদানের সময় শেষ হয়ে যায়।

৩ শিক্ষার্থীদের ভাবতে ওরু করতে এবং তাদদর গল্প লিখতে বলুন । যদি আপনি শিক্ষার্থীদের গল্প পড়তে বলার পরিকল্পনা করেন, তাহলে তা তাদের লেখা শুরু করার আগে উল্লেখ করুন। তাদেরকে বলুন যে, যদি তারা গল্প তৈরি করতে সমস্যায় পড়ে তাহলে তারা আপনার সাথে কথা বলতে পারে। [আপনি পরামর্শ দিতে পারেন: একটি ছেলে পরীক্ষা করাতে ভয় পায় কারণ সে এইডসে তার মা-বাবা উভয়কে হারিয়েছে। একটি মেয়ে তার সঙ্গীকে বলতে ভয় পায় যে, সে ধর্ষণের ফলে এইচআইভি আক্রান্ত। কারণ তার প্রেমিক বিশ্বাস করত যে, তাদের মিলনের পূর্বে মেয়েটি কুমারী ছিল।]

8 গল্পগুলো সংগ্রহ করুন, অথবা শিক্ষার্থীদের সেগুলো বাড়ির কাজের সাথে শেষ করতে বলুন । যাই হোক, দ্বিতীয় সেশনের পূর্বে (যেখানে শিক্ষার্থীরা তাদের গল্প সবাইকে পড়ে শোনাবে) প্রত্যেকটি গল্প যে সঠিক ও নামহীন তা নিশিচ হতে নিজে পডুন ।
৫ শিক্ষার্থীদেরকে তাদের গল্প পড়ার জন্য ডাকুন । (তাদের সবাইকে পড়তে হবে না ।)

৬ নিয়লিখিত প্রশ্নগুলো আলোচনার মাধ্যমে অধিবেশন শেষ করুন:

- তোমাদের কেউ কি সহপাঠীর গল্প শ্নে বা নিজেদের গল্প লিখতে যেয়ে পৃথকভাবে কোন উভয় সংকটে পড়েছ? কিছু উপায় কী যেভাবে তোমরা তোমাদের বুঝতে পার তোমার মনোভাব বা অনুভূতি পরিবর্তিত হয়েছে? [জানার চেষ্টা করুন: তোমরা এইচআইভি পরীক্ষা করানো আগে যেমন ভাবতে তার চেয়ে কি এখন কঠিন বা সহজ ভাবছ? তুমি আগে সঙ্গীকে বলার বিষয়ে যেমন ভাবতে তার চেয়ে এখন এটি বেশি নাকি কম গুরুত্বপূর্ণ ভাবছ? তুমি কি এখন মনে কর যে, তুমি একজন ভালবাসার বা যৌনসঙ্গীর কাছে অন্যভাবে সাড়া দিতে পারতে যে তোমাকে বলেছে সে এইচআইভিতে আক্রান্ত ?]

• এইচআইভির অবস্থা প্রকাশ করা কি সবসময় সহজ-সরল বিষয়?

- িজেদের ও সঙ্দের সুস্বাদ্থ্যের জন্য যতটা সৎ হওয়া প্রয়োজন আমাদের সঙ্গীদের ও নিজেদের কাছে ততটা সৎ কিনা তা আমরা কীভাবে নিশ্চিত হতে পারি ?

- যখন কোন মানুষ ক্লিনিকে যায় তখন কী ঐচ্ছিক, নামহীন পরীক্ষা ও অধি-পরামর্শ কার্যকরভাবে দেয়া বা উৎসাহিত করা উচিত অথবা শ্রুমাত্র যখন কেউ স্বাস্থ্যকর্মীদের এইচআইভি পরীক্ষা করানোর অনুরোধ করবে, তখনই কি তাদের এই পরীক্ষা করানো উচিত ?

• কারো কি কোন শেষ মন্তব্য আছে? /সারা বিশ্বে ছড়ানো এইচআইভির জন্য আমাদের সকলের সৎ, অন্যের আবেগঅনুভূতির সাথে একাত্ন হওয়ার ক্ষমতা, আত্মশৃজ্খলা, সাহস, এবং আশাবাদী হবার প্রয়োজনীয়তা সম্পর্কে মন্তব্য দিয়ে সেশন শেষ করার চেষ্টা করুন ।] 


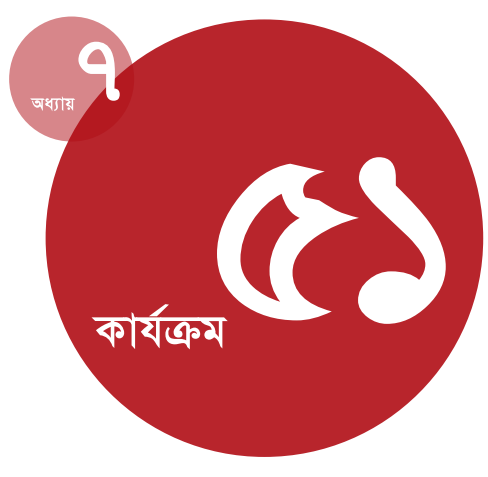

\section{জন্মবিরতিকরণ পদ্ধতির জ্ঞান নিয়ে খেলা}

অধিবেশন সম্পর্কিত ধারণা: শিক্ষার্থীরা শব্দের ধাঁধাঁ এবং খেলার মাধ্যমে জন্মবিরতিকরণ পদ্ধতি সম্পর্কে তথ্য পুনরায় আলোচনা করবে। তারা জন্মবিরতিকরণের যৌথ দায়িত্ব সম্পর্কে আলোচনা করবে। (লক্ষণীয়: এই অধিবেশনটি জন্মবিরতিকরণ পদ্ধতির একটি পরিচিতি বা একটি পুনরালোচনা হিলেবে কাজ করবে।)

উদ্দেশ্য: জন্মবিরতিকরণ পদ্ধতি সম্পর্কে শিক্ষার্থীদের জ্ঞান পুনরায় যাচাই এবং শক্তিশালী করা; লেখার দক্ষতা শক্তিশালী করা।

\section{সময়কাল :}

8৫ মিनिট

\section{উপকরণ:}

বোর্ড ও চক; শব্দজটের অনুলিপি; এবং ‘জনুবিরতিকরণ পদ্ধতির তালিকা'-এর হ্যান্ডআউট; জন্মবিরতিকরণ পদ্ধতির নামসহ কাগজের টুকরো; একটি ব্যাগ; নির্দেশিকার (এই বইয়ের সহ-গামী খন্ড) বা অন্য নির্ভরযোগ্য বই থেকে জন্মাবিরতিকরণ পদ্ধতি সম্পর্কে ফ্যাক্টশীটের চারটি অনুলিপি; সম্ভব হলে, একটি ঘড়ি (৩০ সেকেন্ড অন্তর সময় দেখার জন্য); বিজয়ী দলের জন্য ছোট উপহার।

\section{প्रস্তুতি:}

শব্দজেটের (উত্তরসহ বা ছাড়া) ফটোকপি করুন । জন্মাবিরতিকরণ পদ্ধতির তালিকা পুনরায় যাচাই করুন; আপনি যে পদ্ধতিগুলো আলোচনা করতে পারবেন না তা বাদ দিন। এই সেশনের পূর্বে শিক্ষার্থীদের আপনার তালিকার পদ্ধতিগুলো সম্পর্কে শেখান । আপনার তালিকার প্রত্যেকটি জন্মবিরতিকরণ পদ্ধতি পৃথক পৃথক কাগজের টুকরায় লিখুন । কাগজের টুকরোগুলো অর্ধেক করে ভাঁজ করুন এবং এগুলো একটি ব্যাগে রাখুন । ধাপ-৩-এর লক্ষণীয় বিষয় পড়ন এবং সেই অনুযায়ী পরিকল্পনা করুন । নিজে জন্মাবিরতিকরণ পদ্ধতির তথ্য পুনরায় পড়ন।

\section{নির্দেশাবলি}

বাড়ির কাজ (এই অধিবেশনের পূর্বে করতে দিতে হবে): ‘জন্মবিরতিকর' শব্দজট সম্পূর্ণ করুন । (প্রত্যেক শিক্ষার্থীকে একটি করে খালি শব্দজট বিতরণ করুন ।)

\section{পরবর্তী দিনে ধাপ-১ থেকে শুরু করুন ।}

১ দলবদ্ধ হয়ে শব্দজটের উত্তর পুনরায় পড়ুন । যে কোন প্রশ্নের উত্তর দিन।

২ শিক্ষার্থীদেরকে বলুন, তারা ‘পাঁচটি প্রশ্ন’ নামের একটি খেলা করতে যাচ্ছে, যা বিভিন্ন জন্মবিরতিকরণ পদ্ধতি সম্পর্কে তাদের জ্ঞান পরীক্ষা করবে। তিনটি দল গঠন করুন এবং ব্যাখ্যা করুন্

• এই ব্যাগে কাগজের টুকরো আছে, এবং প্রত্যেক কাগজের টুকরোতে পৃথক পৃথক জন্মবিরতিকরণ পদ্ধতির নাম আছে। ১ নং দল একটি কাগজের টুকরা বের করে খেলা ওুরু করবে।

\section{• ২ নং ও ৩ নং দল অনুমান করবে ১ নং দলের কাছে থাকা} কাগজে কোন জন্মবিরতিকরণ পদ্ধতির নাম আছে। জন্মবিরতিকরণ পদ্ধতির নাম খুঁজে বের করার জন্য তোমরা শফুমাত্র ‘হ্যা’ বা ‘না’ প্রশ্ন করতে পার। [ স্পষ্ট করুন যে, প্রত্যেকে ‘হঁ্যা’ এবং ‘না’ প্রশ্ন বলতে কী বোঝায় তা বুঝতে পেরেছে।] প্রথমে, ২নং দল ১নং দলকে একটি প্রশ্ন করবে।
৩ নিয়মগুলো ব্যাখ্যা করুন। ।লক্ষণীয়: যদি আপনার শিক্ষার্থীদের জন্মবিরতিকরণ পদ্ধতি সম্পর্কে কিছু পূর্ব জ্ঞান থাকে তাহলে সঠিক উত্তর (পদ্ধতি) খুঁজে বের করতে সাহায্য নেয়ার জন্য ‘ঁ্যা’ ও ‘না’ সূত্রগুলো ব্যবহার করতে পারেন। যদি তাদের জন্মবিরতিকরণ পদ্ধতির মৌলিক ধারণাও না থাকে, তাহলে সূত্রগুলো কার্যকরভাবে ব্যবহার করার জন্য প্রত্যেক দলের নিজস্ব ফ্যাক্টশীট প্রয়োজন হতে পারে । ]

- প্রশ্নগুলোর সঠিক উত্তর দেয়া হয়েছে কিনা তা নিশিচ হতে, যে দল উত্তর দিচ্ছে তারা জন্মবিরতিকরণ পদ্ধতির ফ্যাক্টশীট দেখে বলতে পারে। [ফ্যাক্টশীট নাও।] মনে রেখ, ‘'ছাঁ’ অথবা 'না’ উত্তর দেয়ার জন্য তোমাদের ৩০ সেকেন্ড সময় আছে। তোমরা উত্তর দেয়ার আগে নিজেদের মধ্যে উত্তরটি নিয়ে শান্তভাবে আলোচনা করতে পার। তোমাদের কাগজের টুকরোয় যা লেখা আছে তোমরা তা অবশ্যই আমাকে দেখাবে, যেন আমি যে কোন ভুল তথ্য সঠিক করে দিতে পারি । যদি তোমরা তোমাদের পদ্ধতি সম্পর্কে ভুল তথ্য দাও, তোমাদের দল অর্ধেক পয়েন্ট হারাবে এবং মনে রেখ, তোমরা শুধুমাত্র ‘হ্যা’ বা 'না’ বলে উত্তর দিতে পারবে। পরিশেষে, তোমরা কতগুনো প্রন্নের উত্তর দিলে তার হিসাব রাখার এবং সঠিকভাবে পদ্ধতি শনাক্ত না করে পাঁচটি প্রশ্নের উত্তর দেয়া হয়ে গেলে তা ঘোষণা করার জন্য তোমাদের দল থেকে কাউকে নিয়োগ কর। 
• ১ ১নং দল থেকে ২নং দলের প্রথম প্রশ্নের উত্তর শোনার পর, ২নং দল জন্মবিরতিকরণ পদ্ধতিটির নাম অনুমান করতে পারে । যদি ২নং দল ভুল উত্তর দেয় (অথবা অনুমান না করতে চায়), ৩নং দল একটি প্রশ্ন করতে পারে। যে কোন দল সঠিক উত্তর (এক পয়েন্ট জয়ী) না দেয়া পর্যন্ত, অথবা পাঁচটি প্রশ্ন জিজ্ঞেস না করা পর্যন্ত , ২নং ও ৩ নং দল পর্যায়ক্রমে প্রশ্ন করবে

• যদি পাঁচটি প্রশ্ন জিজ্ঞেস করা হয়ে যাওয়ার পর কোন দলই পদ্ধতিটি সঠিকভাবে অনুমান করতে না পারে, ১নং দল সঠিক উত্তরটি ঘোষণা করবে। পদ্ধতিটি সম্পর্কে গুরুত্বপূর্ণ তথ্য স্পষ্ট করে বলবে ও পয়েন্ট পাবে।

8 কীভাবে খেলাটি চলবে তা ব্যাখ্যা করুু ।

• এরপর ২নং দল একটি কাগজের টুকরো তুলে নেবে এবং ১নং ও ৩নং দল প্রশ্ন করবে। যতক্ষণ আমাদের সময় থাকে অথবা সবগুলো কাগজের টুকরো বের হওয়া পর্যন্ত আমরা এইভাবে চক্রাকারে চলবো।

প্রত্যেকে কি নিয়মগুলো বঝতে পেরেছে? [যদি প্রয়োজন হয় একটি কাগজের টুকরো (পদ্ধতি) দিয়ে খেলাটি করে দেখান।]

৫ প্রত্যেক প্রশ্নের উত্তরের জন্য ৩০ সেকেন্ড পর জানানোর জন্য একজন সময় নিয়ন্ত্রক নিয়োগ করুন । বোর্ডে পয়েন্ট লিখে স্কোর রাখার জন্য আরেকজন শিক্ষার্থী নিয়োগ করুন ।
৬ ১ ১নং দলকে একটি কাগজের টুকরো বেছে নিতে বলুন এবং তাদেরকে জন্মবিরতিকরণ পদ্ধতির ফ্যাক্টশীট দিন । কাগজের প্রত্যেক টুকরো বের করার পরই তা যাচাই করে নিশ্চিত হোন; দ্রংত তথ্যসুত্রের জন্য নিজের কাছে জন্মবিরতিকরণ পদ্ধতির ফ্যাক্টশীটের একটি অনুলিপি রাখুন, যেন আপনি যে কোন ভুল তথ্য সঠিক করতে পারেন ।

৭ সঠিক উত্তর দেয়ার পর অথবা পাঁচটি প্রশ্নের উত্তর দেয়া হয়ে যাবার পর থামুন। বিজয়ী দলকে পয়েন্ট দিন এবং ২নং দলকে একটি কাগজের টুকরো বেছে নিতে বলুন । দলগুলোকে এইভাবে ২০ মিনিটের মত অথবা সেশন শেষ হবার দশ মিনিট পূর্ব পর্যন্ত পর্যায়ক্রমে এইভাবে করতে বলুন ।

৮ বিজয়ী দল ঘোষণা করুন ও যদি আপনার কাছে পুরস্কার থাকে তাহলে এটি তাদেরকে দিন।

৯ আলোচনার জন্য পাঁচ থেকে দশ মিনিট সময় রাখুন।

• আজকে জনুবিরতিকরণ পদ্ধতি সম্পর্কে তোমরা আগে জানতে না এবং যা তোমাদের কাছে আকর্ষণীয় মনে হয়েছে এমন কী শিখলে?

- [পরবর্তী প্রশ্ন বাড়ির কাজ হতে আলাদা হতে পারে; নিচে দেখুন ।] প্রত্যেকের কি জন্মবিরতিকরণ সম্পর্কে জানার প্রয়োজন আছে? অনাকাজ্ষিত গর্ভধারণ প্রতিরোধ করা কার দায়িত্ব?

বাড়ির কাজ: একটি অনুচ্ছেদে এই প্রশ্নগুলোর উত্তর লেখ: প্রত্যেকের কি জন্মবিরতিকরণ সম্পর্কে জানার প্রয়োজন আছে? অনাকাজ্ষিত গর্ভধারণ প্রতিরোধ করা কার দায়িত্ব? তোমরা তোমাদের মতামত সরাসরি বা একটি গল্প আকারে লিখতে পার । 


\section{জন্মবিরতিকরণ পদ্ধতির শব্দজট}

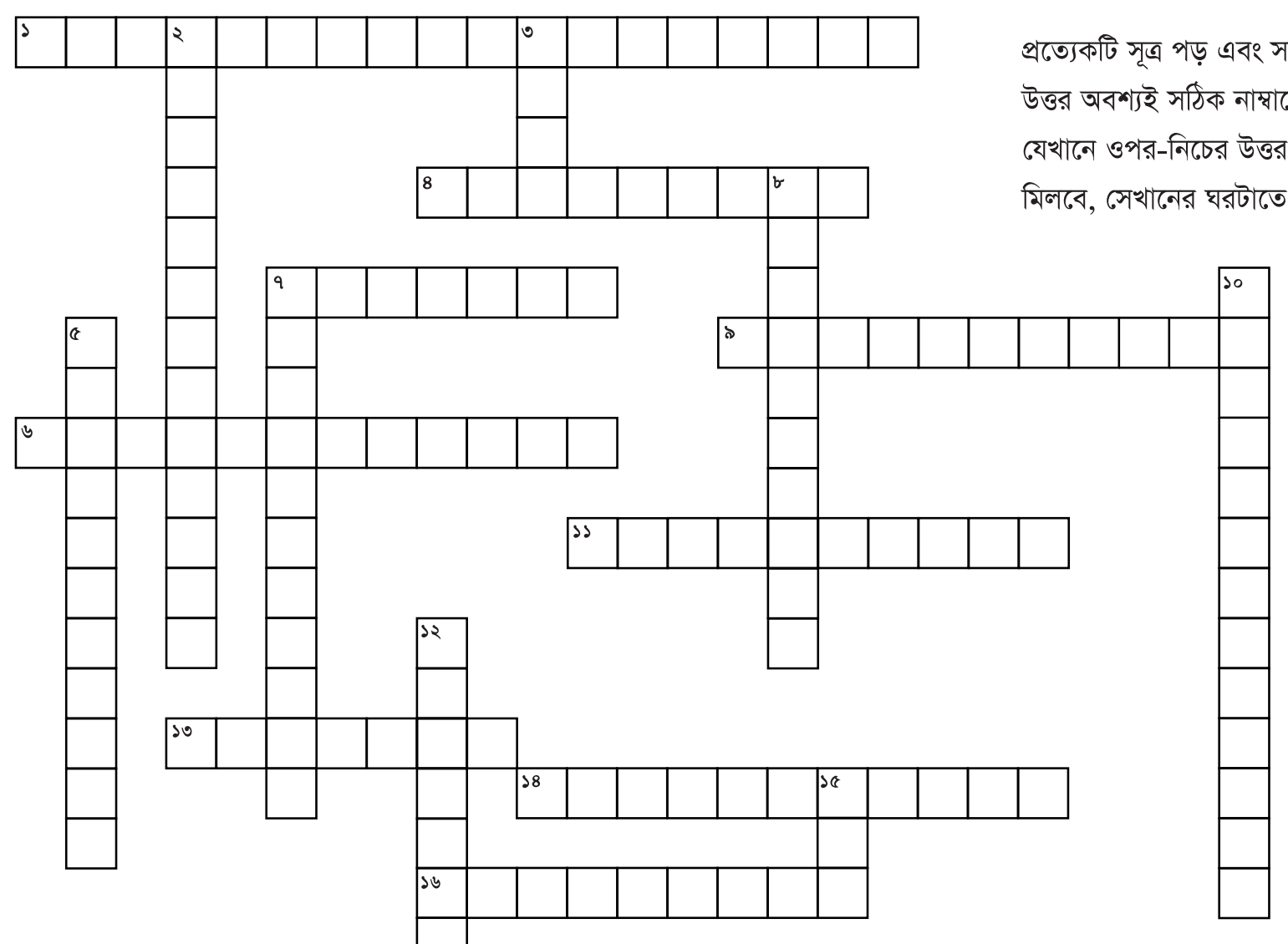

পাশাপাশি

১. একজন মহিলা বা মেয়ে তার ত্বকে ব্যান্ড-এইডের মত ব্যবহার করে, এটি এসটিআই/এইচআইভি প্রতিরোধ করে না (দুই শব্দের)

8. একটি শৈল্য চিকিৎসার পদ্ধতি যা পুরুম্দদের বীর্য নিঃসরণ প্রতিরোধ করে

৬. একটি পাতলা খাপ বা থলে যা একজন মহিলা বা মেয়ে তার নিজের শরীরে বীর্য ঢুকতে বাধা দেয়ার জন্য তার যৌনাঙ্গের ভেতর পরে

৭. একটি ছোট দণ যা একজন মহিলা বা মেয়ের বাহুতে ঢোকানো হয়

৯. একটি ---------পদ্ধতি একজন মহিনা বা মেয়ের জন্য একটি থার্মোমিটার ব্যবহার করে বলা হয় কখন সে জনমশীল নয়

১১. বীর্যপাত্র পূর্বে পুরুষাঙ্গ যৌনাঙ্গ থেকে বের করে আনা

১৩. একজন মহিনা বা মেয়ে গর্ভধারণ প্রতিরোধ করার জন্য প্রতিদিন এটা গ্রহণ করে (সাধারণ नाম, দুই শব্দের)

১৪. বলয়- আকৃতির বস্তু একজন মহিলা বা মেয়ে যৌনাঞ্জের ভেতর পরে, এটা এসটিআই/ এইচআইভি থেকে রক্ষা করে না (দুই শব্দের)

১৬. একটি রাবার কাপ, যা তৈলাক্ত পদার্থে পূর্ণ এবং সম্পূর্ণ যোনীপথসহ যৌনাঙ্গের ভেতর পরতে হয়

১৬৪
ওপর-নিচে

২. একটি অপারেশন যেখানে ডিম্বাণু ও ওক্রাণু মিলিত হওয়া থেকে রক্ষা করার জন্য ডিম্বনালী কেটে বা বেধে রাখা হয় (দূই শব্দের)

৩. যৌনাঞ্জের ভেতর পরতে হয় এবং প্রায় T এর মত আকার (শব্দসংক্ষেপ, বহুবচন)

৫. অক্রাণুকে মারার জন্য বিভিন্ন রকম পদার্থ যৌনাগ্ে ঢোকাতে হয় (বহুবচন)

৫. ওুক্রাণুকে মারার জন্য বিভিন্ন রকম পদার্থ যৌনাগ্রে ঢোকাতে হয় (বহুবচন) পিচ্ছিল পদার্থকে (বহুবচন) ঘন করতে যা প্রদান করা হয়

৮. যৌনমিলনের সময় এটি একজন পুরুষ বা ছেলে তার যৌনাঙ্গে পরে; এটি গর্ভধারণ প্রতিরোধ করে এবং এসটিআই/ এইচআইভি হতে রক্ষা করে (দুই শব্দের)

১০. একজন মহিলা বা মেয়ে তার. . . . . পরিমাণ এবং ঘনত্তের ওপর নির্ভর করে বলতে পারে

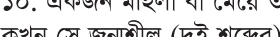

১২. একজন মহিলা বা মেয়ে তার মাসিকচক্র একটি. .... . . লিখে হিসাব করতে পারে কখন गে জন্মাশী ।

১৫. বুকের দুধ পান করানোর ফলে প্রাকৃতিকভাবে হয় (শব্দ সংক্ষেপ)।

s; 5. Spermicides; 7

Cervical mucus; 12. Calendar; 15. LAM 


\section{সারা বিশ্বে ব্যবহৃত জন্মবিরতিকরণ পদ্ধতির তালিকা}

অস্থায়ী, ব্যবহারকারীর দ্বারা নিয়ন্তিত পদ্ধতি (যা অক্রাণুকে ডিম্বাণুর কাছে পৌছছাতে বাধা দেয়)
পুরুষ কনডম
মহিলা কনডম
ডায়াফ্রাম
সার্ভিকাল ক্যাপ
স্পার্মিসাইডস

যে সকল পদ্ধতি শরীরের ভেতরে কাজ করে

স্বল্প মেয়াদী, ব্যবহারকারীর দ্বারা নিয়ন্তিত

$$
\text { খাবার বড়ি }
$$

জরুরি জন্মবিরতিকরণ পদ্ধতি

দীর্ঘ মেয়াদী

\section{ইনজেকশন}

হরমোনাল ভাজিনাল রিং

হরমোনাল পাচ

হরমোনাল ইমপ্লান্ট

ইন্ট্রাইউটেরাইন ডিভাইস (IUD)

প্রাকৃতিক পদ্ধতি (যেগুলোর জন্য নির্দিষ্ট আচরণ এবং কারোর নিজের শরীর সম্পর্কে বোঝার প্রয়োজন হয়)

ল্যাকটেশনাল এ্যনেনোরিয়া পদ্ধতি (LAM)

\section{প্রত্যাহার}

ফার্টিলিটি অ্যাওয়ারনেস-এর সার্ভিকাল মিউকাস পদ্ধতি

টেম্পারেচার মেথড অব ফার্টিলিটি অ্যাওয়্যারনেস

কালেন্ডার স্ট্যান্ডার্ড ডে বা ‘সাইকেল বিড্স’ মেথড অব ফার্টিলিটি অ্যাওয়ারনেস (রিদম মেথডও বলা হয়)

বিরত থাকা

আউটার কোর্স (এৌনমিলন ছাড়া যৌন ক্রিয়া)

স্থায়ী শৈল্য চিকিৎসা সম্বন্ধীয় পদ্ধতি

টিউবাল লাইগেশন (অথবা মহিলা বন্ধ্যাকরণের জন্য পদ্ধতি)

ভ্যাসেকটমি 


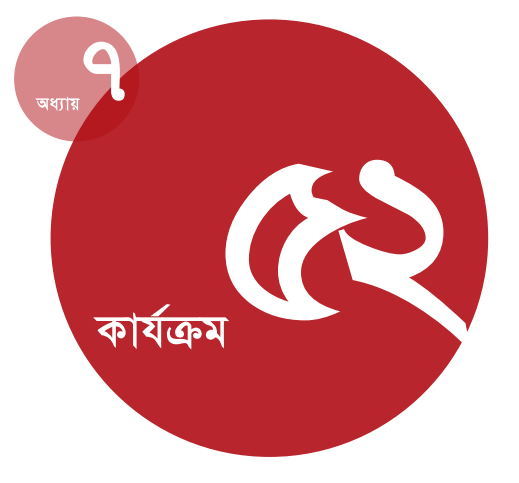

\section{সময়কাল :}

৬০ মিনিট (দুইটি অংশে ভাগ করা যেতে পারে)

\section{উপকরণ:}

বোর্ড ও চক; প্রত্যেক দলের জন্য 'গর্ভপাতের কেস' হ্যান্ডআউট থেকে একটি কেসস্টাডি (অথবা সবগুলো কেসের একটি অনুলিপি এবং প্রত্যেক দলকে একটি কেস দিতে হবে); নির্দেশিকা (এই বইয়ের সহ-গামী খন্ড)<www.popcouncil.org/publ ications/books/2011_ItsAllOne. asp> এই ঠিকানায় পাওয়া যাবে, অথবা ঐ খণ্ড থেকে অধ্যায় ৭-এর অনাকাজ্ষিত গর্ভধারণ ও গর্ভপাত অংশটি অনুলিপি করতে হবে

\section{প्रস্তুতি:}

কেন মহিলারা গর্ভপাত করাতে চায় তার একটি পূর্ণ তালিকা করার জন্য ওপরের উপকরণগুলো পুনরায় পড়ন ।

কেসস্টাডিগুলো পুনরায় পড়ুন এবং যদি প্রয়োজন হয় সামাজিকভাবে মানানসই করার জন্য এগুলো পরিবর্তন করুন । নিশ্চিত হোন যে, কেসগুলোতে গর্ভপাত করানোর বিভিন্ন কারণগুলো প্রতিফলিত হয়েছে। আপনি নিজে গর্ভপাত (বিশেষ করে কম বয়সী ছেলেমেয়েদের বিষয়ে) এবং শিশ্ড সহায়ক স্থানীয় আইনের সাথে পরিচিত হোন ।

\section{তার মতামতে চলা: গর্ভপাত করানোর সিদ্ধান্ত}

অধিবেশন সম্পর্কিত ধারণা: শিক্ষার্থীরা কেসস্টাডি পড়বে এবং কী কারণে মহিলারা গর্ভপাত করাতে চায় তার কারণগুনো আলোচনা করবে

উদ্দেশ্য: শিক্ষার্থীদেরকে মহিলা এবং মেয়েরা যে যে কারণে গর্ভপাত করাতে চায় তার তিনটি কারণ আলোচনা করতে সক্ষম করা; সমালোচনামূলক চিন্তাশক্তি এবং কথোপকথন দক্ষতা শক্তিশালী করা।

\section{নির্দেশাবলি}

১ নিচের প্রশ্নগুনো দিয়ে অধিবেশনটি শুরু করুন:

• আমরা জীবনের যে জটিল সিদ্ধান্তগুলো নিয়ে থাকি আজকে তা নিয়ে আলোচনা করব। এমন একটি সময়ের কথা ভাব যখন তোমাকে বা তোমার কাছের কাউকে- একটি কঠিন সিদ্ধান্ত নিতে হয়েছিল যার সাথে অন্যরা একমত নাও হতে পারত। [কয়েক মূহুর্ত সময় দিন]

• এ এটির অনুভূতি কেমন? তোমাকে (বা তুমি যার কথা ভাবছ) কি অন্যরা সমর্থন করেছিল ?

• না হলে, তা কীভাবে সিদ্ধান্তটিকে প্রভাবিত করেছিল, এবং তোমার কেমন অনুভূতি হয়েছিল ?

• মিলিয়ন মিলিয়ন মহিলা এবং মেয়েদেরকে নিজেদের অনাকাজ্ষিক গর্ভধারণের জন্য সিদ্ধান্ত নিতে হয়। কারো কারো জন্য এই সিদ্ধান্ত নেয়া সহজ এবং স্পষ্ট, যখন অন্যদের জন্য এটি কঠিন এবং জটিল ।

• আজকে আমরা গর্ভপাত করানোর সিদ্ধান্ত নিয়ে আলোচনা করব। গর্ভপাত করানো সঠিক নাকি ভুল আমরা তা আলোচনা করব না । তার পরিবর্ত কেন কেউ কেউ গর্ভপাত করায় তা বোঝানোর জন্য কোন বিষয়টি ঐ সিদ্ধান্তটি নিতে সাহায্য করেছিল তা বিবেচনা করব।
• যখন একজন মহিলা বা মেয়ে গর্ভবতী হয় তখন তার কী কী পছন্দ থাকে? [জানার চেষ্টা করুন: বাচ্চাটির জন্ম দেয়া এবং লালনপালন করা, বাচ্চাটিকে দত্তক দেয়া; অথবা গর্ভপাত করানো ।]

২ শিক্ষার্থীদেরকে চার বা পাঁচজন করে ছোট দলে ভাগ করুন । প্রত্যেক দলকে একটি করে কেসস্টাডি দিন এবং তাদেরকে এগুলো পড়তে, যে গল্পটি বলছে তার নাম পূরণ করতে, তারপর নিচের প্রশ্নগুলো আলোচনা করতে বলুন । [এগুনো বোর্ডে লিখুন]:

- কেন এই মেয়েটি গর্ভপাত করিয়েছে?

• তার সিদ্ধান্তে অন্য মানুষেরা কী ভূমিকা পালন করেছে? [যখন তারা কাজ করবে তখন বোর্ডে লিখুন “মেয়ে এবং মহিলারা গর্ভপাত পছন্দ করে কারণ:"।]

৩ দলগুলোকে আবার একত্রিত করুন । প্রথম দলটিকে তাদের কেসস্টাডি পড়তে বলুন এবং নিচের প্রশ্নগুনো আলোচনা করতে পাঁচ থেকে সাত মিনিট সময় দিন:

- কেন এই মেয়েটি গর্ভপাত করিয়েছিল ?

- প্রত্যেকে কি একমত যে এগুনো তার জন্য কারণ ছিল ? সেখানে কি আর কোন কারণ ছিল ? [্्্শ্ব করে কেসটির সাথে সম্পর্কিত অন্য কারণ জানার চেষ্টা করুন ।] 
• তার সিদ্ধান্তে অন্য মানুযেরা (একজন সঙ্গী, পরিবার, বন্ধু, বা অন্যরা) কী ভূমিকা পালন করেছিল, সরাসরি বা তাদের মনে?

• কেউ কি কোন মন্তব্য করতে চাও ?

8 প্রত্যেক কেলের জন্য এই প্রক্রিয়ার পুনরাবৃত্তি করুন্ন। প্রত্যেক কেসের জন্য সাত মিনিট সময় দিন

৫ এই প্রশ্নগুনো পুনরায় পড়ার জন্য ১০-১৫ মিনিট সময় রাখুন:

• তোমাদের মতে অল্পবয়সী মহিলারা যে গর্ভপাত করে এগুলো কি তার কারণ (বোর্ডে)? অন্য আর কী কারণ আছে বলে তোমরা মনে কর যার জন্য মহিলারা গর্ভপাত করে? [বোর্ডের তালিকায় সেগুলো যোগ করুন ।]

• বিশ্বব্যাপি, বেশিরভাগ মহিলা যারা গর্ভপাত করে তারা বিবাহিত। তোমরা কি কিছু পরিস্থিতির কথা মনে করতে পার, যার জন্য একজন বিবাহিত মহিলা গর্ভপাত করতে চাইতে পারে?

• কিছু কিছু দেশে ব্যাপকভাবে গর্ভপাত করা হয়, যেখানে অন্যান্য দেশে গর্ভপাত আইনত নিষিদ্ধ (অথবা শুধুমাত্র সামান্য কিছু পরিস্থিতিতে অনুমতি দেয়া হয়)।

• যেখানে পদ্ধতিটি এমনকি অনৈতিক এবং অনিরাপদও হতে পারে সেখানে কেন মহিলা এবং মেয়েরা গর্ভপাত করে ?
বাড়ির কাজ: নিচের যে কোন একটি উক্তি বেছে নাও

যখন তুমি অন্যদের বিচার করবে, তারা কী তা তুমি দেখবে না, তুমি দেখবে তুমি को? - ওয়েনি ডায়ার

আমরা কখনও অন্যের জীবনকে বিচার করতে পারিনা, কারণ প্রত্যেক ব্যক্তি শ্রুমাত্র তার নিজের কষ্ট এবং আত্নত্যাগের কথা জানে। তুমি সঠিক পথে আছো এটা জানা একটি বিষয়, কিন্ত্ত তোমারটাই একমাত্র পথ সেটা জানা অন্য বিষয়।

-পাওনো কোয়েল্হ

মনে রেখ যে আমি মানুষ। আমাকে বিচার করার পূর্বে বা আমার সাথে কীভাবে আচরণ করবে তা সিদ্ধান্ত নিতে আমার মতামতে কিছুক্ষণ চল। যদি তুমি কর, আমি মনে করি তুমি আরো বেশি বুঝবে আমরা মাঝামাঝি অবস্থায় থাকতে পারব এবং বাকিপথ একসাথে চলতে পারব - এরিক হার্ভে এবং স্টিভ ভেঞ্চরা

যদি আমার পক্ষে সম্ভব হয়, তাহলে সবাইকে খুশি করার জন্য ভালবাসা উচিত; কিন্তু সবাইকে খুশি করতে যেয়ে, আমি হয়ত কাউকেই খুশি করতে সক্ষম হবো না । তাই আমি একটি উপসংহারে পৌঁছেছি যে, সবচেয়ে ভাল কাজ হচ্ছে কারো নিজের বিবেককে খুশি করা এবং পৃথিবীকে তার নিজস্ব বিচার, সুবিধা এবং

এরপর, একটি করে উক্তির অনুলিপি কর এবং নিচের প্রশ্নগুনোর উত্তর দাও:

• এ এই উক্তিটি তোমার কাছে কেমন লেগেছে?

• তুমি লেখকের সাথে একমত নাকি দ্বিমত পোষণ কর?

• এই উক্তির অর্থ কীভাবে একটি মেয়ের অবস্থার সাথে সম্পর্কিত যে একটি অনাকাজ্ষিত গর্ভধারণ শেষ করতে চায়? 


\section{গর্ভপাতের কেসগুলো}

\section{১ নং দলের হ্যান্ডআউট}

আমার নাম ------- । আমার প্রেমিক লু এবং আমার উভয়েরই বয়স ২০ বছর এবং দুই বছর ধরে প্রেম করছি। আমি জন্মিনিয়ন্রণ পিল খাই, যদিও গতমালে দুইবার পিল খেতে ভুলে গেছি। তারপর আমি জানলাম যে আমি গর্ভবতী। আমি মাত্র একটি নতুন চাকরি শুরু করেছি যেটি আমি ভালবাসি । কিন্তু এখানে এখনও খুব ভাল বেতন দেয় না। আমি আসলে আমার জীবনটা এখন যেমন আছে তেমনটা পছন্দ করি এবং কোন বাচ্চা চাই না । আমি একটি পরিবার পরিকল্পনা ক্লিনিকে গিয়ে একজন সেবিকার কাছ থেকে পরামর্শ পেলাম যিনি কঠোরভাবে আমাকে বিয়ে করতে এবং বাচ্চাটি রাখতে উদ্দুদ্ধ করল। লু সেবিকার সাথে একমত হল কারণ সে মনে করেছিল অবশেষে আমিতো মা হতে চাইব, তাহলে এখন নয় কেন ? আমি হতাশ কারণ আমার মনে হল, যে বাচ্চাটি আমি চাইনা তাকে রাখতে আমাকে চাপ দেয়া হচ্ছে। আমি একটি জায়গায় যাবার সিদ্ধান্ত নিলাম, আমি শুনেছি সেখানে একজন ডাক্তার আছেন যিনি অনেক প্রশ্ন করা ছাড়াই গর্ভপাত করায়।

\section{২ নং দলের হ্যান্ডআউট}

আমার নাম --------- । আমার বয়স ২০ বছর এবং আমি আমার গ্রামের প্রথম ব্যক্তি ছিলাম যে রাজধানী শহরের বিশ্ববিদ্যালয়ে পড়ার সুযোগ পেয়েছি। আমার পরিবার, বন্ধুবান্ধব এবং প্রতিবেশীদের অনেক আশা ছিল যে, আমার সাফল্য আমাদের এলাকাতে সত্যিকার অর্থে পরিবর্তন শুরু করবে। পাঠ গ্রহণ (কাস) শুরু হবার অল্প কিছুদিন পর আমি অন্য একটি ছাত্রের সাথে প্রেম করতে ঞুরু করলাম এবং কয়েক মাস পর আমরা যৌনমিলন করতে। আমরা বেশির ভাগ সময়ে কনডম ব্যবহার করতাম, কিন্তু একদিন যখন আমরা পরিস্থিতির শিকার হলাম তখন কনডম ছিল না । যখন আমি আবিষ্কার করলাম, আমি গর্ভবতী তখন আমাদের কী করা উচিত তা নিয়ে কথা বলতে আমার প্রেমিকের কাছে গেলাম। কিন্তু সে হঠাৎ করে দূরবর্তী ও দুষ্প্রাপ্য হয়ে গেল। আমি আমাদের দুজনেরই বন্ধু এমন একজনের কাছ থেকে ফুনলাম যে, সে ভাবছে আমি বিয়ের জন্য তাকে ফাঁদদ ফেলার চেষ্ঠা করছি। আমি এখনই বিয়ে করতে চাইনা। কিন্তু আমি আমার বয়সে কুমারী মা-ও হতে চাইনা। আমি আমার লেখাপড়া শেষ করতে চাই এবং আমার স্বপ্নে পৌছছানোর সুযোগ পেতে চাই। আমি সাহায্যের জন্য আমার পরিবারের কাছে যেতে চাইলাম। কিন্তু আমার ভয় হল, তারা আমার ওপর অখুশি হবে এবং আমি তাদেরকে ছোট করতে চাইলাম না । তাই আমি কাউকে না বলার এবং আমার প্রতিদিনের খরচ থেকে বাঁচিয়ে গর্ভপাতের জন্য ব্যয় করার সিদ্ধান্ত নিলাম। 


\section{গর্ভপাতের কেসগুলো}

\section{৩ নং দলের হ্যান্ডআউট}

আমার নাম ------- । আমি একজন ১৭ বছর বয়সের ছেলে । যখন আমি জানলাম যে আমার প্রেমিকা গর্ভবতী আমি ভাবলাম কী? কীভাবে! না! আমার অনুভূতি ছিল আঘাত, ভয়, দুশ্চিন্তা, এবং বিস্ময়ের মিশ্রণ। কিন্ত্ত আমি জন্মশীল জানতে পেরে আমার ক্ষুদ্র একটি অংশে সামান্য গর্ববোধও হচ্ছিল। কিন্তু পরিশেবে, আমার এই প্রশ্নটি নিয়ে ভাবতে হল যে, “এখন কোনটি----- পিতৃত্ব, দত্তক, গর্ভপাত?” আমার প্রেমিকা এবং আমি উভয়ই বিদ্যালয়ে পড়ি। এবং আমরা জানি ভাল বাবা-মা হওয়ার জন্য আমরা অনেক ছোট। আমরা সিদ্ধান্ত নিলাম, গর্ভপাত আমাদের জন্য সবচেয়ে ভাল সিদ্ধান্ত। একজন বন্ধু একটি ক্লিনিকে যেতে বলল এবং আমরা একত্রে গেলাম। ক্লিনিকের কর্মী ব্যাখ্যা করল আসলে কী ঘটতে যাচ্ছে। আমরা আসার পূর্বে সে আমাদেরকে জন্মবিরতিকরণ সম্পর্কেও বলল এবং আমাদেরকে এক বক্স কনডম দিল। আমাকে আমার নিজস্ব অনেক অনুভূতির মোকাবেলা করতে হয়েছে। কিন্তু আমি গর্বিত যে, আমি এই কঠিন সিদ্ধান্তের মাধ্যমে আমার প্রেমিকাকে সাহায্য করেছি।

\section{8 নং দলের হ্যান্ডআউট}

আমার নাম --------। আমার বয়স ১৫ বছর এবং আমরা বড় যৌথ পরিবারে বাস করি। প্রত্যেক বছর, আমার চাচী ও চাচা এবং তাদের ছেলে, আমার চাচাত ভাই, যার বয়স ১৮ বছর আমাদের বাড়িতে বেড়াতে আসে। এই বছর একদিন যখন প্রত্যেকে বাড়ির বাইরে সে আমার কাজে জিজ্ঞেস করল- সে আমাকে স্পর্শ করবে কিনা এবং আমাকেও তার সাথে একই রকম করতে বলল। এটা অস্বাভাবিক মনে হল এবং আমি সত্যিই এটা পছন্দ করতাম না । কিন্তু সে ছিল আমার বড় চাচাত ভাই এবং আমি তাকে হতাশ করতে চাইলাম না । যখন সে আমার বন্ত্র অনাবৃত করতে শুরু করল এবং আমার ওপর উঠল আমি ঘাবড়ে গেলাম এবং তাকে ঠেলে ফেলে দেয়ার চেষ্টা করলাম। কিন্তু সে অনেক শক্তিশালী ছিল এবং সে আমাকে ধর্ষণ করল। যখন আমি বুঝলাম আমি গর্ভবতী, আমি খুব ঘাবড়ে গেলাম। আমি আমার মাকে সাহায্য করার জন্য বলতে চাইলাম কিন্তু যা ঘটেছিল তা বলতে খুব লজ্জা পাচ্ছিলাম । অবশেবে আমার সাহস হল এবং আমার মাকে বললাম। সে সাথে সাথে আমাকে গর্ভপাত করার জন্য নিয়ে গেল এবং বিষয়টি সবার সাথে আলোচনা করতে চাইল না । আমি আর গর্ভবতী অবস্থায় থেকে মুক্তি পেলাম । কিন্তু যখন আমি এই কঠিন অভিজ্ঞতার মধ্য দিয়ে যাচ্ছিলাম তখন আমার ইচ্ছা হত যদি আমি কারো সাথে কথা বলতে পারতাম। 


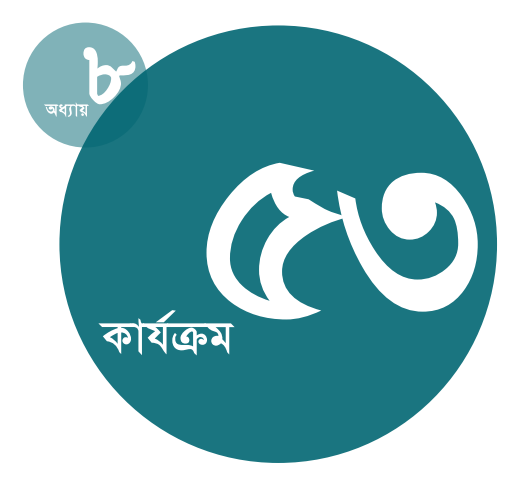

\section{সময়কাল :}

৬০-৯০ মিনিট: কতগুলো উক্তি আপনি বাছাই করবেন তার ওপর নির্ভর করবে।

\section{উপকরণ:}

বোর্ড ও চক; দুই পৃষ্ঠার হ্যাল্ডআউট "সামাজিক ন্যায়বিচার ও আন্দোলনের ওপর উক্তিসমূহ।"

\section{श्रস্তুতি:}

উক্তিগুলো আবার পড়ন এবং আপনার সমাজে উপযুক্ত নয় এমন যে কোন উক্তি বাদ দিন। আপনি আপনার নিজের দেশ বা সমাজ থেকে অন্যান্য উক্তি যোগ করতে পারবেন । উক্তির সংখ্যা (এবং শিক্ষার্থীদের আবৃত্তি) আপনার সময়ের সাথে সামঞ্জস্যপূর্ণ করুন । আপনি যে উক্তিগুলো ব্যবহার করবেন সেগুলোর কপি তৈরি করুন বা বোর্ডে লিখুন ।

\section{জাগো! পরিবর্তন হচ্ছে}

অধিবেশন সম্পর্কিত ধারণা: শিক্ষার্থীরা এমন একটি উক্তি ঠিক করবে যা তাদেরকে অনুপ্রাণিত করে এবং উক্তিটির বার্তা তাদের নিজেদের জীবনে প্রটয়াগ করার অর্থ কী হবে তা জানার চেষ্টা করবে।

উদ্দেশ্য: তারা যে পৃথিবীতে বাস করে তাকে পরিবর্তন করার জন্য শিক্ষার্থীদের প্রেরণা দেওয়া এবং তাদের আত্নবিশ্বাস বৃদ্ধি করা ।

\section{निर्দেশাবলি}

১ ব্যাখ্যা করুন:

• আজকে আমরা জানার চেষ্টা করবো- সামাজিক ন্যায় বিচারের জন্য কাজ করা এবং আমরা যে পৃথিবীতে বসবাস করি তাকে পরিবর্তন করার অর্থ কী, এমনকি খুব ছোট পরিসরে। যারা তাদের নিজদের প্রচেষ্টায় পরিবর্তন এনেছে তাদের ধারাবাহিক উক্তিগুলি পুনরায় পড়বো।

- সম্পূর্ণ তালিকাটি পড় এবং যে উক্তিটি তোমাকে সবচেয়ে বেশি অনুপ্রাণিত করে সেটি বাছাই কর। তারপর তোমার লেখার খাতা নাও এবং একটি পৃষ্ঠার উপরের দিকে তোমার উক্তিটি লেখ (যার নামের সাথে এটি সংযুক্ত তার নাম সহ)।

• ভাব, তোমার উক্তিটির অর্থ কী। কমপক্ষে তিনটি অনুচ্ছেদে একটি উত্তর লেখ । উক্তিটির বার্তা নিয়ে তুমি কী ভাবছ তা প্রথম অনুচ্ছেদে থাকা উচিত। কেন এই উক্তিটি তোমার কাছে অর্থপূর্ণ এবং কেন তুমি এটি বাছাই করেছ তা দ্বিতীয় অনুচ্ছেদে বলা উচিত। সর্বশেষ অনুচ্ছেদে, যদি উক্তিটির বার্তা তুমি তোমার নিজ জীবনে প্রয়োগ করতে চাও তার অর্থ কী হতে পারে তা আলোচনা কর। (অথবা যদি ইতিমধ্যে তুমি এটি প্রয়োগ করে থাক তাহলে তা আলোচনা কর) (লেখার জন্য শিক্ষার্থীদেরকে সময় দিन]
২ তালিকার প্রথম উক্তিটি কেউ বাছাই করেছে কিনা তা জিজ্ঞেস করুন । যদি করে, ঐ শিক্ষার্থীকে তার অনুচ্ছেদ পড়তে বলুন। ( শিক্ষার্থীরা যে অংশ গোপন রাখতে চায় তা না পড়ার অনুমতি দিন।) যদি একাধিক শিক্ষার্থী একটি উক্তি পছন্দ করে তাহলে তাদের যে কোন একটি উক্তি পড়ার অনুমতি দিন। এইভাবে আপনার তালিকার সবগুলো উক্তি শেষ করুন । ২ মিনিট করে প্রায় ১২ টি পাঠের জন্য পরিকল্পনা করুন ।

৩ নিম্নলিখিত নির্দেশনামূলক প্রশ্নগুলো দিয়ে শেষ করুন:

• এই উক্তিগুলো থেকে তোমরা কী কী গুরুত্বপূর্ণ বার্তা পেয়েছ?

• সামাজিক ন্যায় বিচারের আন্দোলনকারীরা কী কী ইতিবাচক ব্যক্তিগত সুবিধা ভোগ করে ?

- কিছু সমস্যা এবং ঝুঁকি কী कী ?

• যে বিষয়গুলো নিয়ে সাধারণ নাগরিকেরা উদ্বিগ্ন সে বিষয়ে পদক্ষেপ নেওয়া কি তাদের জন্য গুরত্বপূর্ণ ?

বাড়ির কাজ : তোমার উক্তিটি আলোচনা করার জন্য একটি পদ্ধতি বের কর। তুমি এটি বাড়িতে পরিবারের সদস্যদের সাথে আলোচনা করতে পার। এটি যত্লসহকারে লেখ এবং কোথাও পাঠাও। এটি তোমার ই-মেইল স্বারে রাখ, বা তোমার অনুপ্রেরণামূলক বার্তাটি আলোচনা করার অন্য উপায় বের কর। তুমি তোমার উক্তিটি কীভাবে আলোচনা করছ তা লেখ এবং আগামীকাল জমা দাও। 


\section{সামাজিক ন্যায়বিচার ও আন্দোলন বিষয়ক উক্তি}

তুমি পৃথিবীতে যে পরিবর্তন দেখতে চাও সেই পরিবর্তন কর।

-মোহনদাস গান্ধী, ভারতীয়দের জাতীয় পিতা হিসাবে স্বীকৃত। সে অহিংস নাগরিক অসহযোগ আন্দোলনে উদ্দুদ্ধ করেছিলেন, যা বিট্রেন থেকে ইন্ডিয়াকে স্বাধীন করতে নেতৃত্ব দিয়েছিল এবং সারা বিশ্বে নাগরিক অধিকার ও স্বাধীনতার জন্য আন্দোলনে উদুদ্ধ করেছিল।

শক্তিমান ও শক্তিহীনের বিবাদে কাউকে সমর্থন না করার মানে হলো শক্তিমানের পক্ষ অবলম্বন করা, নিরপেক্ষ থাকা নয়।

- পাওনো ফ্রেইরি, ব্রাজিলের একজন প্রভাবশালী শিক্ষাবিদ, যিনি গরীবদের বিশ্লেষণমূলক সচেতনতা জাগ্রত করার জন্য প্রথম শিক্ষাচর্চা শুরু করেছিলেন ।

দাবী ছাড়া ক্ষ্তাসীনরা কোনকিছু স্বীকার করে না। এটি অতীতেও ছিল না, ভবিষ্যতেও থাকবে না। খুঁজে বের কর জনগণ কী বনে, এবং তখনই তুমি দেখবে, ঠিক কতটা অবিচার ও অন্যায় তাদের ওপর করা হচ্ছে এবং এগুলো চলতেই থাকবে যদি না কথা কিংবা আঘাতের মাধ্যমে বা উভয়ের মাধ্যনে তা প্রতিহত করা হয়। যারা নির্যাতিত হয় তাদের সহুক্ষমতা দিয়ে নৃশংসতার সীমা নির্ধারিত হয়।

-ফ্রেডেরিক ডগলাস, একজন ক্রীতদাস হিলেবে জন্ম এবং যুক্তরাষ্ট্রের দাসপ্রথা বিরোধী আন্দোলনের নেতা হয়েছিল। সে ছিল একজন লেখক, বক্তা ও প্রেসিডেন্ট আব্রাহাম লিংকনের উপদেষ্টা এবং সার্বজনীন সমতায় বিশ্বাসী।

স্বাধীনতা এমন কিছু নয় যে কেউ কাউকে উপহার হিসেবে দিল । তারা এটিকে নিজস্ব বলে দাবী করে এবং কেউ তাদের কাছ থেকে আলাদা করতে পারে না।

-কেওয়ামি নখরুমাহু, আধুনিক ঘানার প্রতিষ্ঠাতা ও প্রথম রাষ্ট্রথথ্রতি এবং একজন প্রভাবশালী প্যান-আফ্রিকানিস্ট।

দায়িত্ব কেবল তাদের উপরই বর্তায় না যারা আমাদের দেশের নেতৃত্বে থাকেন কিংবা যারা নির্দিষ্ট কাজের জন্য নিয়োগ পান বা নির্বাচিত হন । এটি আলাদাভাবে আমাদের প্রত্যকের উপরই বর্তায়।

-চৌদ্দতম দালাই লামা, তিব্বতের বৌদ্ধদের ধর্মীয় নেতা এবং তিব্বতের একজন স্বাধীনতাকর্মী।

কখনো সংশয় প্রকাশ করো না যে, চিন্তাশীল ও প্রত্যয়ী নাগরিকদের ছোট একটি অংশ পৃথিবীকে পাল্টে দিতে পারে। বাস্তবিক অর্থে, এরাই এ কাজটি করেছে ।

- মারগারেট মীড, আরেরিকার একজন সাংস্কৃতিক নৃতাত্ত্বিক। 


\section{সামাজিক ন্যায়বিচার ও আন্দোলন বিষয়ক উক্তি}

পৃথিবীটা খুবই ভয়ানক জায়গা, তাদের কারণে নয় যারা মন্দ কাজ করে, বরং তাদের কারণে যারা এগুলো সহ্য করে এবং প্রতিকার করে না । - আলবার্ট আইনস্টাইন, একজন তত্ত্বীয় পদার্থবিদ, আপেক্ষিকতার তত্ত্বের জন্য সর্বাধিক পরিচিত। নাৎসিবাদদর বিরুদ্ধে সোচ্চার, তিনি পারমানবিক পরীক্ষা ও বর্ণবাদের বিরুদ্ধের নেতাও ছিলেন।

যখন নাৎসিরা কমিউনিস্টদের প্রতি হুমকি হয়ে এলো, আমি চুপ করে রইলাম কারণ আমি কমিউনিস্ট ছিলাম না । যখন তারা সোস্যাল ডেমোক্রেটদের স্তব্ধ করলো, আমি চুপ করে রইলাম কারণ আমি সোস্যাল ডেমোক্রেট ছিলাম না । যখন তারা ট্রেড ইউনিয়নপন্থিদের জন্য হুমকি হয়ে এনো, আমি তখনও চুপ ছিলাম কারণ আমি ট্রেড ইউনিয়নিস্ট নই। যখন তারা ইহুদিদের জন্য হুমকি হলো, আমি চুপ রইলাম কারণ আমি ইহুদি নই। যখন তারা আমার প্রতি তেড়ে এলো, আমার পক্ষে কথা বলার আর কেউ ছিলনা।

- মার্টিন নেইমোলার, একজন সুপরিচিত জার্মান ধর্মবিশেষজ্ঞ ও যাজক, যাকে বন্দিশালায় রাখা হয়েছিল কিন্তু তিনি ব্রেটে ছিলেন । তিনি সারা জীবন একজন যুদ্ধবিরোধী কর্মী ছিলেন ।

যে কোন এক জায়গার অন্যায় বিচার সর্বত্র ন্যায়বিচারের প্রতি হুমকি। আমরা সবাই একে অপরের সাথে নিয়তির বাঁধনে বাঁধা । যা একজনের প্রতি সরাসরি ঘটে, তা সবার কাছেই পরোক্ষভাবে ঘটে।

- মার্টিন লুথার কিং জুনিয়র, একজন মন্ত্রী, আনেরিকার নাগরিক অধিকার আন্দোলনের সর্বাধিক জনপ্রিয় নেতা। অহিংসা নীতির একজন প্রবর্তক এবং সকল বর্ণের মানুষকে সমমর্যাদা দেয়ার জন্য সক্রিয়, তিনি ছিলেন সর্ব কনিষ্ঠ নোবেল শান্তি পুরস্কার বিজয়ী ব্যক্তি।

এটা খববই সামান্য যা নাগরিকরা করে এবং এটাই তাদের পৃথক করে দেয়। আমার ছোট কাজ হলো গাছ লাগানো।

- ওয়াংগারি মাথাই, কেনিয়ার একজন পরিবেশ, জেন্ডার ও রাজনীতি বিষয়ক নেত্রী। তিনি সবুজায়ন আন্দোলন শুরু করেছিলেন এবং ২০০৪ সালে নোবেল শান্তি পুরস্কার লাভ করেন (প্রথম আফ্রিকান নারী হিলেবে যিনি এটি লাভ করেন)। তিনি কেনিয়ার একজন সংসদ সদস্যও निर्याচিত হন। 


\section{আমি একটি সমস্যা নিয়ে উদ্বিগ্ন}

অধিবেশন সম্পর্কিত ধারণা: শিক্ষার্থীরা সমাজের একটি বিষয় অথবা সমস্যা শনাক্ত করবে যা তাদের ওপর প্রভাব ফেলে। তারা তাদের নিজেদের জীবনে এ্যাডভোকেসি প্রক্রিয়া খুঁজে বের করবে।

উদ্দেশ্য: শিক্ষার্থীরা উদ্বিগ্ন এমন একটি বিষয় শনাক্ত করতে তাদের সাহায্য করা; যোগাযোগ এবং কথা বলার দতা চর্চা করা; তাদের নিজেদের জীবনে ইতিবাচক পরিবর্তন করার জন্য ভাবা।

\section{निর্দেশাবলি}

১ বিষয়টি সম্পর্কে বলুন:

• আমাদের জীবনে প্রভাব রয়েছে এমন বিভিন্ন বিষয়ে আমরা শিখেছি-বিশেষ করে জেন্ডার, স্বাস্থ্য, এবং অধিকার বিষয়ে। আমরা পড়েছি এমন কিছু নির্দিষ্ট বিষয় কোনগুলো? [কোন বিষয়গুলো পড়েছে তা জানার চেষ্টা করূন ]

- তোমরা প্রত্যেকে একটি করে সামাজিক সমস্যা শনাক্ত করবে যা নিয়ে তোমরা উদ্বিগ্ন । আমরা আলোচনা করেছি এমন একটি বিষয়ের সাথে এটি সম্পৃক্ত হওয়া উচিত।

২ প্রত্যেক ছাত্রকে “একটি সমস্যা যা নিড়ে আমি উদ্বিগ্ন” এর তালিকাটি বিতরণ করুন । ব্যাখ্যা করুন:

• এই কাগজের উভয় পাণে তোমার নাম লেখ । তারপর “কোন বিষয়গুলো নিয়ে আমি উদ্বিগ্ন ?”- এই প্রশ্নটি নিজেকে করে সামাজিক ন্যায়বিচারের তালিকাটি পুনরায় পড়।

• তোমরা দেখবে যে তালিকাটি তিন বিভাগে বিভক্ত (জেন্ডার, যৌনস্বাস্থ্য, এবং যৌন অধিকার)। তিনটি বিষয় বাছাই কর যেগুলোতে তোমাদের আগ্রহ আছে। এগুনো সব একটি বিভাগ থেকেও আসতে পারে অথবা এগুনোর সব ভিন্ন ভিন্ন বিভাগ থেকেও আসতে পারে।

- সামাজিক ন্যায়বিচারের এমন একটি বিষয় যেটা নিয়ে তোমরা উদ্বিগ্ন অথচ যা তালিকাটিতে নেই তাও তোমরা যোগ করতে পার।
৩ শিক্ষাথীদেরকে সমলিঙ্গবিশিষ্ট জোড়ায় ভাগ করুন। ব্যাখ্যা করুন

• জোড়া বদ্ধ হয়ে, এক বা দুইটি বিষয় যা তোমাদের কাছে উল্লেখযোগ্য তা নিয়ে আলোচনা করতে বিশ মিনিট সময় নাও।

• তোমাদের পছন্দগুলোর কোনটি জোর করে করেছ, অথবা এগুনো তোমরা কেন আরো বেশি করে শিখতে চাও তা আলোচনা কর। তোমাদের কোন গল্প আলোচনা করার থাকতে পারে।

• একে অপরের কথা মনোযোগের সাথে শোনার চর্চা কর এবং প্রয়োজনীয় কথোপকথনে জড়িত হও।

• মনে রেখ: তোমরা সকল সহপাঠিদেরকে সম্মান করবে। আমরা একে আপরের সাথে যেভাবে আচরণ করি তা দিয়ে এই শ্রেণিকক্ষ থেকেই সমান ন্যায়বিচার ও সম্মান শুরু ।

8 শ্রেণিকক্ষের সবাইকে আবার একত্রিত করুন এবং জিজ্ঞেস করুন কেউ বলতে চাই কিনা কোন সমস্যাটি বা সমস্যাগুলো তারা বাছাই করেছে এবং কেন । যে সকল শিক্ষার্থী স্বেচ্ছায় বলতে চায় তাদেরকে বলার সুযোগ দিন ।

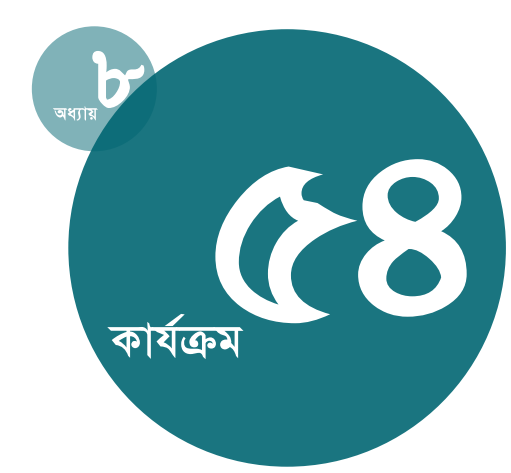

\section{সময়কাল :}

ধাপ ১-8: 8৫ মিনিট

ধাপ ৫-৮: 8৫ মিনিট

উপকরণ:

বোর্ড ও চক; একটি অনুশীলনপত্র "একটি সমস্যা যা নিয়ে আমি উদ্বিগ্ন"

এবং প্রত্যেক ছাত্রের জন্য ('হৃদয়

থেকে একটি চিঠি’) এর একটি করে হ্যান্ডআউট; গাইডলাইন (এই বইয়ের সহ-গামী খ-) থেকে অধ্যায় ৮-এর একটি অনুলিপি<www.popcoun cil.org/publications/books/20 11_ItsAllOne.asp> এই ঠিকানায় পাওয়া যাবে।

প्रস্তুতি:

‘আমি উদ্বিগুন এমন একটি বিষয়’-এর তালিকাটি পননরায় পড়ন। আপনার

শিক্ষার্থীদের সাথে সম্পু্তুক্ত করা নিরাপদ নয় এমন বিষয়গুলো বাদ দিন। স্থানীয় প্রাসঙ্গিক বিষয়গুনো যোগ করুন

'হুদয় থেকে একটি চিঠি' হ্যান্ডআউটটি পুনরায় পড়ন। যদি আপনি

শিক্ষার্থীদেরুকে (গাইডলাইনের অধ্যায় b- ব্যবহার করে ) একটি এ্যাডভোকেসি প্রকল্পে সম্পৃক্ত করার পরিকল্পনা করেন, তাহলে হ্যান্ডআউটের 'সাধারণ পদক্ষেপ'-এর ডান কলামটি রাখন । यদি তা না করেন তাহলে নিদ্দেশনা ও সাহায্য ছাড়া শিক্ষার্থীরা যে এ্যাডভোকেসি শরু করছিল তা এড়িয়ে যাবার জন্য ঐ কলামটি বাদ দিন। 
৫ সামাজিক পরিবর্তনের জন্য কাজ করা বিষয়ে(এ্যাডভোকেসি) শিক্ষার্থীদের ধারণা দিন। ব্যাখ্যা করুন:

• িছু মানুষ পৃথিবীটাকে পরিবর্তন করার জন্য বৃহৎ প্রচারাভিযানে নিজেদেরও জড়ায়। তোমাদের মধ্যে কেউ কি শুনেছ যে, অনুশীলনপত্রের কোন একটি সমস্যা যা নিয়ে তুমি বা তোমার সহপাঠীরা উদ্বিগ্ন তা সমাধান করার জন্য মানুষ পদক্ষেপ গ্রহণ করেছে?

• আমরা যে বিষয়গুনো শিখছি -জেন্ডার সমতা, এবং যৌন ও প্রজনন স্বান্য্য এবং অধিকার, এ ক্ষেত্রে গৃহীত কার্যক্রমের অবস্থা কী ? [কিছু উদাহরণ জেনে নিন । যদি প্রয়োজন হয়, গাইডলাইনের অধ্যায় bঅথবা আপনার নিজের অভিজ্ঞতা থেকে একটি উদাহরণ দিন]

• এ একটি পরিবর্তনের উদাহরণ কী যা এই কাজগুলো পৃথিবীতে এনেছে? [আপনি কিছু উদাহরণ দিতে পারবেন । যেমন: মেয়েদের যৌনাঙচ্ছেদের রীতি বাদ দেওয়া, বিদ্যালয়ে মেয়েদের সংখ্যা বৃদ্ধি, জেন্ডার ভিত্তিক নির্যাতনের বিরুদ্ধে প্রচারণায় পুরুষের অন্তর্ভুক্তি, গর্ভপাত বৈধকরণ, সমকামী যুবক-যুবতীদের জন্য বিদ্যালয় নিরাপদ করা এবং গর্ভবতী মেয়েদের বিদ্যালয়ে লেখাপড়া করার অনুমতি দেওয়া।

৬ ছোট ছোট পরিবর্তনের ধারণা দিন । নিয়লিখিত বিষয়গুলো নিয়ে একটি আলোচনা করুন:

- এ্যাডভোকেসি প্রায়ই বড় পরিবতনের নির্দেশ করে। কিন্তু একটি ছোট প্রচেষ্টাও তোমার নিজের জীবনে অথবা তোমাদের চারপাশের অন্য মানুমের জীবনে একটি অর্থপূর্ণ ইতিবাচক পরিবর্তন আনতে পারে।

• এ একটি ছোট পদণেরের কথা ভাব যা তুমি একটি পরিবর্তন আনার জন্য করতে পার। বিশেষ করে জেন্ডার সমতা উন্নয়নে জন্য অথবা নিজের ও অন্যদের যৌন বা প্রজনন অধিকার রক্ষার জন্য।
[ এই ধারণাগুনো জানার চেষ্টা করুন: অন্যের শ্রতি বৈষম্য না করা, জেন্ডার দ্বারা বিভক্ত দায়িত্বগুলো ভাগ করে নেওয়া, যৌনমিলন করার জন্য কখনই অন্যকে বাধ্য না করার সিদ্ধান্ত নেয়া, অথবা অতীতে কারো সাথে সম্মানজনক আচরণ করোনি তা নিয়ে দুঃখ প্রকাশ করে কিছু লেখা ।]

१ শিক্ষার্থীদেরকে মনে করিয়ে দিন যে, এ্যাডভোকেসিতে জড়িত হবার সাথে ব্যক্তিগত কিছু সুবিধা বা ঝুঁকি থাকে। জিজ্ঞস করুন:

- এ্যাডভেকেসি অথবা সামাজিক ন্যায়বিচারের কাজে জড়িত হয়ে একজন ব্যক্তির কী কী সুবিধা অথবা উন্নয়ন হতে পারে? [জানার চেষ্টা করুন: অন্যদের ওপর তোমার প্রভাব আছে জেনে ক্ষমতাবান অনুভব করা, নতুন নতুন সম্পর্ক তৈরি করা, ব্যক্তিগত দক্ষতা শক্তিশালী করা যেমন- কথা বলা বা লেখার দক্ষতা, অন্যদের কাছ থেকে প্রশংসা পাওয়া।]

• এ্যাডভোকেসি বা সামাজিক ন্যায়বিচারের কাজে জড়িত হয়ে একজন ব্যক্তি কী কী ঝুঁকির মুখোমুখি হতে পারে ? [জানার চেষ্টা কর"ন: সম্ভাব্য সামাজিক অসম্মতি; বিদ্যালয়ে কাজ অথবা জীবনের অন্যান্য গুরুত্বপূর্ণ বিষয় থেকে মনোযোগ হারিয়ে ফেলা; তোমার যে পরিবর্তন করার উদ্দেশ্য ছিল তা অর্জিত না হলে হতাশ হওয়া; এমনকি তোমার কার্যক্রম সম্পূর বৈধ এবং অহিংস হওয়া সত্ত্বেও গ্রেফতার হওয়া অথবা অন্যান্য বিপদের মুখোমুখি হওয়া; শিক্ষার্থীদেরকে মনে করিয়ে দিন যে, নিরাপদ থাকা গুরুত্বপূর্ণ ।]

৮ (বাড়ির কাজ হিলেবে দেয়া যেতে পারে।) হ্যান্ডআউট বিতরণ করুন, অথবা হ্যান্ডআউটের প্রশ্নগুলো বোর্ডে লিখুন। শিক্ষার্থীদেরকে বলুন:

- তোমাদের কাছে গুরুত্বপূর্ণ এমন একটি বিষয় এবং এই বিষয় নিয়ে তোমরা কী করতে পার তা নিয়ে মন থেকে একটি চিঠি লেখ ।

• চিঠিটি তোমার নিজের কাছে, মা-বাবার কাছে, বন্ধু বা ভালবাসার সঙ্গীর কাছে বা স্থানীয় নেতার কাছে লিখতে পারো। 


\section{আমি একটি সমস্যা নিয়ে উদ্বিগ্ন}

নিম্মলিখিত তালিকায় কিছু সামাজিক সমস্যা অন্তর্ভুক্ত আছে যেগুলো নিয়ে অনেক মানুষ উদ্বিগু, এবং সংস্কার সাধন করার চেষ্টা করছে। এই তালিকার ঘটনাগুলো তিন ধরনের বিষয়ের ওপর আলোকপাত করেছে: জেন্ডার, যৌন স্বাস্থ্য, এবং যৌন অধিকার। চিন্তা কর কোন বিষয়গুলো তোমার কাছে বেশি গুরুত্বপূর্ণ হতে পারে। তুমি সম্ভবত একটি সমস্যা নিট্যে উদ্বিগ্ন যা এই সমস্যাগুলোর যেকোন একটির মত অথবা এগুলোর সবগুনো থেকে আলাদা একটি বিষয়ের কথা ভেবে থাকতে পার।

তিনটি বিষয় বাছাই কর যেগুনো নিয়ে তুমি উদ্বিগ্ন। এগুনো সব একধরনের বিষয় থেকে হতে পারে, আবার সবগুনো আলাদা আলাদা ধরনের বিষয় থেকেও হতে পারে।

\section{জেন্ডার সম্পর্কিত বিষয়গুলি}

$\square$ আমাদের পাঠ্যবই প্রথাগত ধ্যানধারণা আরো শক্তিশালী করে।

$\square$ আমাদের বিদ্যালয়ের কিছু রীতিনীতি কিছু কিছু মানবগোষ্ঠীর সাথে বৈষম্য করে ।

$\square$ বিদ্যালয়ে খুব বেশি বিব্রত করা এবং ঠাট্টা চলে ।

$\square$ আমাদের সমাজের ছেলেরা ‘শক্তিশালী’ অথবা সাহসী আচরণ করার জন্য চাপ অনুভব করে।

$\square$ মেয়েরা বড় হলে অর্থ সম্পর্কে তাদের কী জানা দরকার সে সম্পর্কে মেয়েদের জানার সুযোগের অভাব আছে।

$\square$ মেয়েদের খেলাধুলা করা ও দলবদ্ধ হওয়ার জন্য যথেষ্ট সুযোগ নেই।

$\square$ মেয়েদের বন্ধু ও সহ-সঙ্গীদের সাথে মেলামেশা করার মত নিরাপদ জায়গা নেই।

$\square$ বিজ্ঞাপনে পুরুষ এবং মহিলাদের এমনভাবে চিত্রায়িত করা হয় যা আমাদের জন্য ক্ষতিকর।

$\square$ কীভাবে ভাল বাবা হতে হয় অনেক যুবকের সে সর্প্পকে জানার অভাব রয়েছে।

$\square$ জেন্ডারভিত্তিক নির্যাতন ( মহিলা ও মেয়েদের বিরুদ্ধে নির্যাতন) খুবই সাধারণ এবং এমনকি গ্রহণয়াগ্য।

$\square$ যে সকল পুরুষরা তাদের স্ত্রী ও সন্তানের প্রতি হিংস্র আচরণ করে তারা এ্যালকোহল গ্রহণের পর আরো বেশি হিংস্র হয়ে যায়।

$\square$ জেন্ডার রীতিনীতি বিশৃংখলা কমাতে অবদান রাখে।

$\square$ অনেক বেশি সংখ্যক মহিলা ও মেয়েরা একটি সুন্দর চেহারা পাওয়ার জন্য সৌন্দর্যবর্ধক অস্ত্রোপচার করতে চায়।

$\square$ মেয়েদের বিদ্যালয়ে যাওয়ার জন্য তাদের ভাইদের সমান সুযোগ সুবিধা নেই ।

$\square$ গর্ভবতী মেয়েদের বিদ্যালয়ে লেখাপড়া চালিয়ে যাবার অনুমতি নেই।

$\square$ বাড়িতে ছেলে ও মেয়েদের সাথে সম আচরণ করা হয় না ।

$\square$ সমাজের মানুষ জেন্ডার অসমতা সম্পর্কে যথেষ্ঠ বোঝে না ।

$\square$ খুব কম মানুষ যৌন হয়রানির সমস্যা সম্পর্কে সচেতন। 


\section{আমি একটি সমস্যা নিয়ে উদ্বিগ্ন}

\section{যৌন স্বাস্থ্য সম্পর্কিত বিষয়গুলি}

$\square$ কিশোর-কিশোরীদের যুব-বান্ধব যৌন ও প্রজনন স্বাস্থ্য সেবা পাবার সুয্যাগ নেই।

$\square$ কনডম পাওয়া কঠিন ।

$\square$ আমার অনেক সহ-সঙ্গী এইচআইভি সম্পর্কে জানে না ।

$\square$ আমাদের এলাকার অনেক বিদ্যালয় এইচআইভি সম্পর্কে পড়ায় না।

$\square$ অনেক মানুষ তাদের শরীরে এইচআইভি আছে কিনা তা জানে না ।

$\square$ যুবক-যুবতীরা তদের নিজেদের শরীর সম্পর্কে মৌলিক তথ্য জানে না ।

$\square$ যুবক-যুবতীরদের মধ্যে যৌনবাহিত সংক্রমণের হার অনেক বেশি ।

$\square$ পৃথিবীর কিছু অংশের অনেক মহিলা এবং মেয়ে যোনিপথের ফিস্টুলার ঝুঁকিতে আছে।

$\square$ মানুষ মাতৃমৃত্যু সম্পর্কে বেশি কিছু জানে না বা যত্মবান নয়।

$\square$ গর্ভপাত আইনগতভাবে নিষিদ্ধ, ফলে অনেক স্থানে এটি বিপদজনক।

$\square$ অन्্ान्্

\section{যৌন অধিকার সম্পর্কিত বিষয়গুলি}

$\square$ অনেক সমাজে নিকট সম্পর্কীয় নারী-পুরুষের অবৈধ যৌনমিলন সমস্যা ব্যাপকভাবে অবহেলিত।

$\square$ অনেক মেয়ের শিশু অবস্থায় বিয়ে হয়ে যায়।

$\square$ জনগণ এখনও মেয়েদের যৌনাঙ্গ ছেদ করে।

$\square$ অনেক মেয়েরা ‘সুগার ড্যাডিদের’ দ্বারা যৌন হয়রানির শিকার হচ্ছে।

$\square$ অনেক যুবক-যুবতী বিশেষ করে মেয়েরা যৌন ব্যবসার কারণে পাচারের শিকার হয় ।

$\square$ প্রায়ই ধর্ষণ হচ্ছে এবং এমন কি মেনে নেওয়াও হয়।

$\square$ জনগণ অনুধাবন করে না যে ছেলেদেরও যৌন হয়রানির ঝুঁকি আছে।

$\square$ সমকমীদের সাথে প্রায়ই সম্মানজনক আচরণ করা হয় না ।

$\square$ অনেক যুবক-যুবতী বিশেষ করে মেয়েরা অনুভব করে না যে, সত্যিকার অর্থে তাদের কনডম ব্যবহার করতে চাপ দেওয়ার অধিকার আছে।

$\square$ এইচআইভি ও এইডস আক্রান্ত মানুষ যথেষ্ট সহায়তা ও সম্মান পায় না ।

$\square$ অন্যান্য

নিচিত হও যে, এই অনুশীলনপত্রের উভয় পৃষ্ঠায় তোমার নাম লিখেছ ! 


\section{হৃদয় থেকে একটি চিঠি}

লক্ষণীয়: ইতিবাচক পরিবর্তনের চেষ্টা করার অনেক পদ্ধতি আছে। মনে রেখ: ছোট থেকে শুরু করা গুরত্বপূর্ণ। নিরাপদ এবং বৈধ কর্মসূচি পছন্দ করাটাও গুরত্বপূর্ণ। কিছু সাধারণ কর্মসূচি যা মানুষ গ্রহণ করে সেগুলো হল:

কারো সাথে বৈষম্য না করার সিদ্ধান্ত নেওয়া

যে বন্ধুর স্বাস্থ্যকেন্দ্র থেকে সেবা নেবার প্রয়োজন তাকে সাথে নিয়ে যাওয়া

অন্য মানুমের বিরুদ্ধে নির্যাতনে জড়িয়ে না পড়ার অঙ্গিকার করা

যার কথা বলার প্রয়োজন তার কথা শোনা

আমি যে বিষয় নিয়ে উদ্বিগ্ন সে বিষয়ে আরো বেশি শেখা

বিষয়টি নিয়ে আমার বন্ধু ও পরিবারের সাথে কথা বলা
একটি ছোট সামাজিক কর্মীদল গঠন করা

সরকারি কর্মকর্তাকে একটি চিঠি লেখা

আমার সহ-সঙ্গীদের জন্য পত্রিকায় একটি রচনা লেখা

আমি যে বিষয়ে উদ্বিগ্ন সে বিষয় নিয়ে কাজ করে এমন একটি সংস্থায় যোগদান করা আমার বিদ্যালয়কে একটি নীতি (উদ্বিগ্ন বিষয়ের সাথে সম্পর্কিত) পরিবর্তন করতে বলা সমাজে একটি ঘটনার পরিকল্পনা করা

প্রিয়

আমি জেন্ডার সমতা এবং যৌনস্বাস্থ্য ও অধিকার বিষয়ে শিখছি । আমি বুঝি যে, আমি একটি ইতিবাচক পরিবর্তনের জন্য একটি ছোট কিন্তু অর্থপূর্ণ পদক্ষেপ নিতে পারি। একটি বিষয় যা নিয়ে আমি উদ্বিগ্ন তা হল

একটি জিনিস যা আমি ইতিবাচক পরিবর্তনের জন্য করতে পারি তা হলো /ওপরের তালিকা থেকে একটি কর্মসূচি বাছাই কর, অথবা পৃথক একটি মতবাদ লেখ ] :

আমি যা আশা করছি তা হলো

আমি একজন মানুষ হতে চাই যে

স্বাক্ষর, 


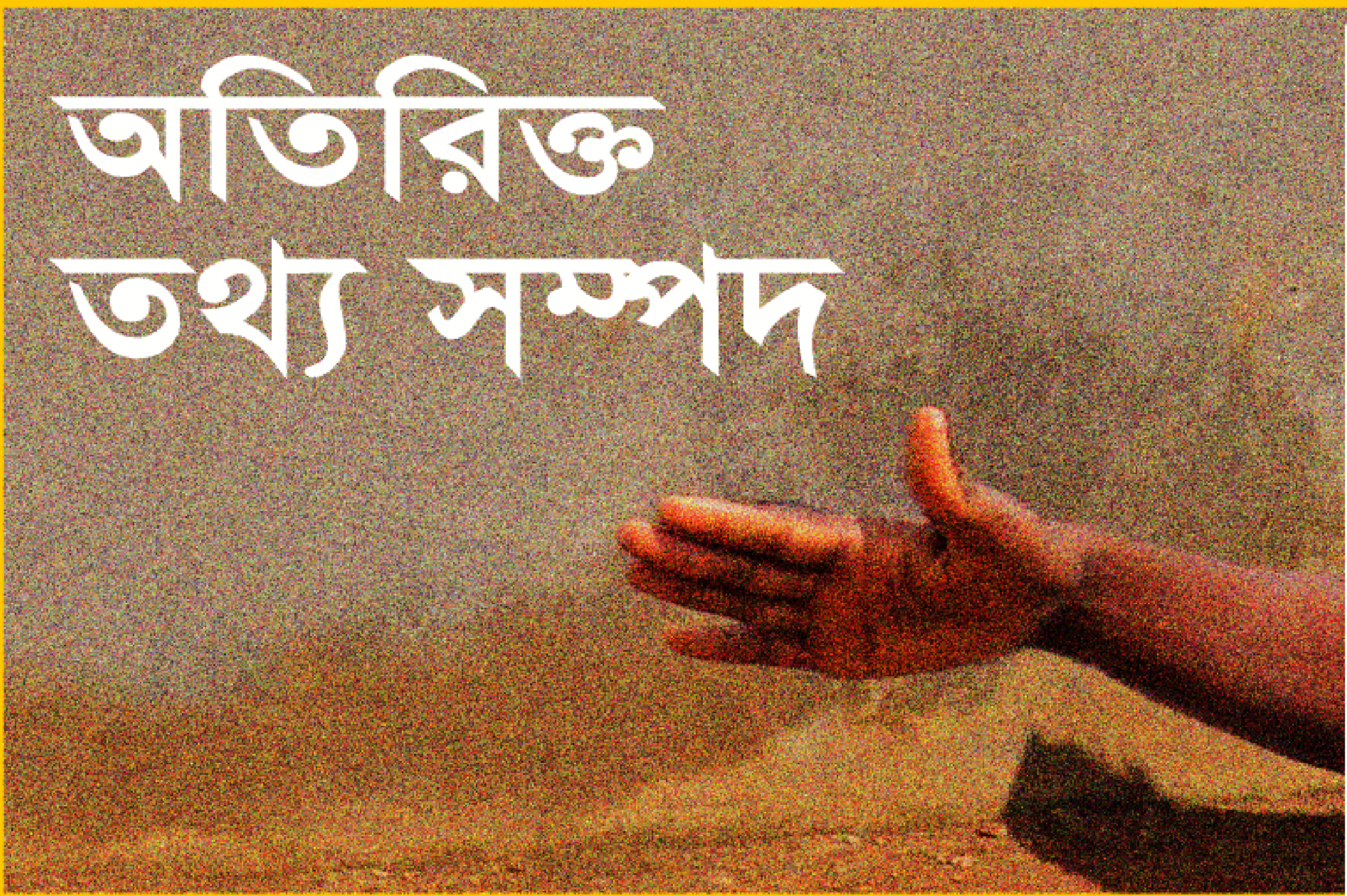




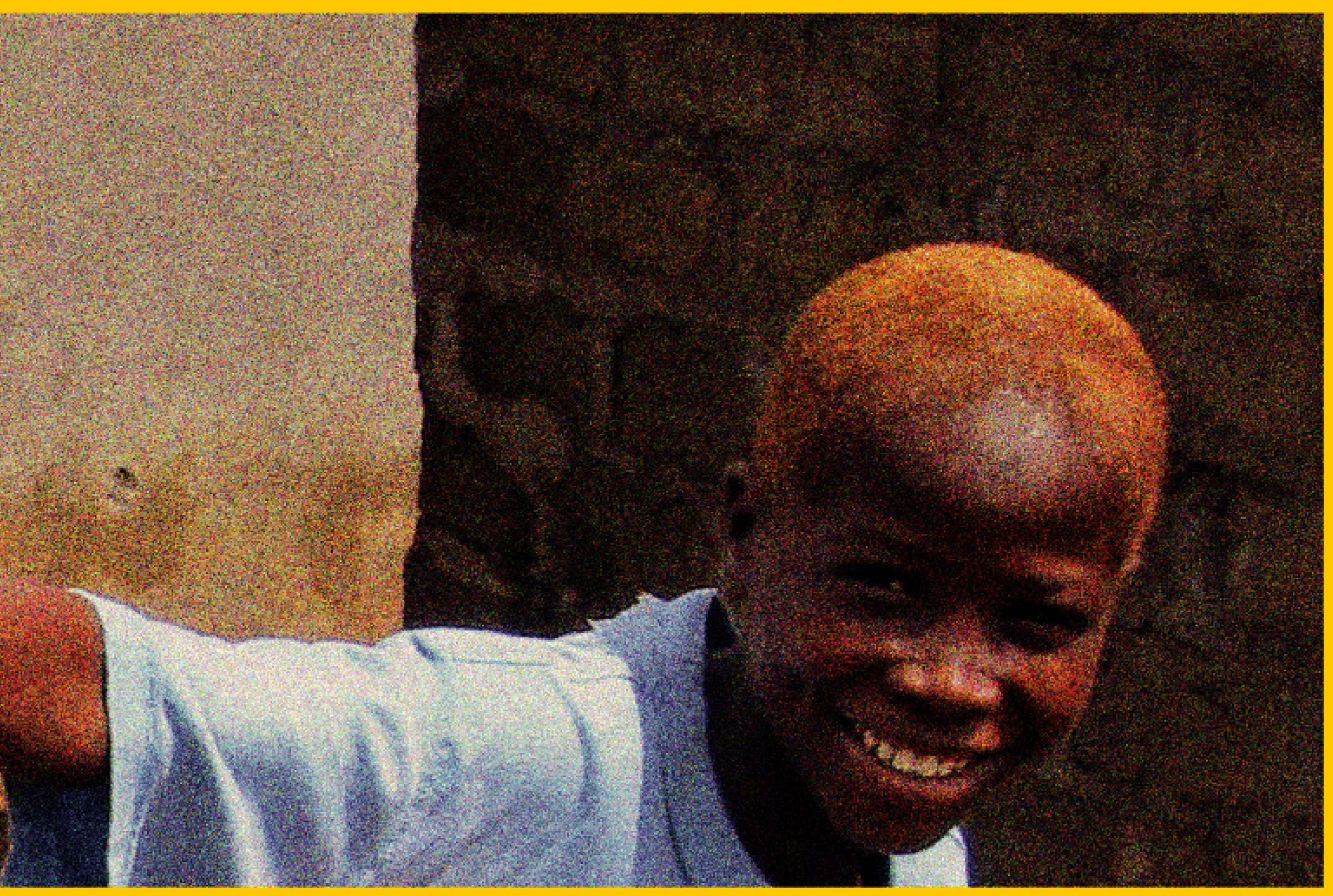


Aahung in Pakistan undertakes training, research, and advocacy to create an environment where every individual's sexual rights are respected, protected, and fulfilled, $<$ www.aahung.org $>$.

Conscientizing Male Adolescents in Nigeria aims to develop male adolescents' critical awareness of sexist prejudices and practices and the attitudes and skills to change them. For more information see $Q / C / Q$ issue 14:<www.popcouncil.org/ pdfs/qcq/qcq14.pdf $>$. Also available in French.

Dance4Life engages dancers, musicians, peer educators, and young people living with HIV to educate and empower young people in the fight against HIV and AIDS. $<$ www.dance4life.com $>$.

Democracia y Sexualidad (DEMYSEX) is a network of Mexican organizations that aim to strengthen sex education to ensure gender equity and the exercise of sexual rights in the development of a democratic culture, <www.demysex.org.mx $>$.

Girls Power Initiative in Nigeria aims to equip girls with the information, skills, and opportunities for action to grow into competent and confident young women. $<$ www.gpinigeria.org $>$.

Girls Incorporated in the United States provides educational programs for girls, particularly in high-risk, underserved areas, to enable them to challenge gender discrimination and lead successful, independent, and fulfilling lives. <www.girlsinc.org >.
International Centre for Reproductive Health and Sexual Rights (INCRESE)

in Nigeria works to expand access to sexual health and rights information and services, $<w w w . i n c r e s e-i n c r e s e . o r g>$.

MenEngage is a global alliance working to engage men and boys in reducing gender inequalities and in promoting the health and well-being of women, men, and children $<$ www.menengage.org $>$

Raising Voices in Uganda works to prevent violence against women by addressing root causes such as traditional gender roles and the imbalance of power $\wedge$ between women and men. $<$ www.raisingvoices.org $>$.

Scenarios USA (originally inspired by Scenarios from the Sahel) sponsors a curriculum and script-writing contest based on reflection about gender issues. Winning scripts are turned into short films. The "What's the Real Deal about Masculinity?" curriculum and films are available for purchase, but the films may also be viewed at no cost online, $<$ www.scenariosusa.org $>$.

Sonke Gender Justice Network works with men, women, young people, and children in Southern Africa to achieve gender equality, prevent gender-based violence, and reduce the spread of HIV and the impact of AIDS. <www.genderjustice.org.za>.

White Ribbon Campaign works to educate men and boys to end violence against women. <www.whiteribbon.ca>. 
ABC: Teaching Human Rights-Practical Activities for Primary and Secondary Schools, Office of the United Nations High Commissioner for Human Rights, 2003.124 pages. This manual helps educators foster human rights awareness and action. $<$ www.ohchr.org/EN/PublicationsResources/Pages/TrainingEducation.aspx >. Also available in Arabic, Chinese, French, Russian, and Spanish.

\section{African Transformation: The Way Forward, Facilitator's Workshop Guide, Health} Communication Partnership, USAID, and Communication for Development Foundation Uganda, 2005.118 pages. Tools for conducting community workshops about gender norms in our lives, <www.mmc.org/

mmc_search.php?sp=\&ref_crmb=Resources\&ref_id=resources\&step=results\& view=detail\&detail id=PL AFR 302\&adv=mat\&swater $>$.

Construyendo Derechos: Talleres de Conversation Para Adolescentes, FLACSO and UNFPA, 2006.90 pages. Instructions for guided discussions on eight aspects of sexuality and rights, <www.issuu.com/flacso.chile/docs/construyendo_derechos/15 $>$. Aavailable in Spanish only.

Empowering Young Women to Lead Change: A Training Manual, World Y WCA and UNFPA, 2006.124 pages. For facilitators who wish to empower young women to become leaders, and to catalyze positive change in their lives and communities. $<$ www.worldywca.org/world_ywca/communications/resources/empowering young_women_to_lead_change $>$. Also available in Spanish and French.

Engaging Boys and Men in Gender Transformation:The Group Education Manual, EngenderHealth and Promundo, 2008. 356 pages. Participatory exercises to reach men (and their partners), exploring gender socialization and its impact on HIV prevention and care, <www.acquireproject.org/archive/files/

7.0_engage_men_as_partners/7.2_resources/7.2.3_tools/Group_Education_ Manual_final.pdf $>$.

Filling the Gaps: Hard to Teach Topics in Sexuality Education, Sexuality Information and Education Council of the United States, 1998.193 pages. This manual provides lesson plans on such topics as abstinence, pregnancy options, sexual behavior, and sexual identity, <www.siecus.org/pubs/filling_the_gaps.pdf>.
Gendering Prevention Practices: A Practical Guide to Working with Gender in Sexual Safety and HIV/AIDS Awareness Education, Nordic Institute for Women's Studies and.'Gender Research, 2003.52 pages. This manual aims to develop young people's gender awareness as a means to promote safer sexual behavior. <hivaidsclearinghouse unesco.org/search/resources/HIV\%20AIDS\%20237 pdf>.

Gender or Sex: Who Cares?, Ipas and Health and Development Networks, 2001.96 pages. This training resource aims to increase adolescents' and youth workers' skills and understanding of gender and reproductive health.

<www.ipas.org/Publications/Gender_or_sex_Who_cares.aspx7ht>.

Human Rights Education Series, Human Rights Resource Center, University of Minnesota, 2000. The third of this six-book series, published with Amnesty International USA and the Gay, Lesbian and Straight Education Network, focuses on sexual diversity and rights. <www1.umn.edu/humanrts/edumat $>$.

International Programme on Sexuality Publications, Youth Incentives This website includes brochures, fact sheets, lesson plans, and advocacy issue briefs. $<$ www.youthincentives.org/rutgersnisso_groep/youthincentives/Downloads $>$. Also available in French.

\section{Keep the Best Change the Rest: Participatory Tools for Working with Communities} on Gender and Sexuality, International HIV/AIDS Alliance, 2007.96 pages. This kit contains activities to help community members explore how gender and sexuality affect their lives and identify ways to improve their relationships and protect their sexual health, <www.aidsalliance.org/

custom asp/publications/view.asp?publication $\_i d=257$ \&language $=e n>$.

MediaLitKit ${ }^{\mathrm{TM}}$ Framework for K-12 Media Literacy, Center for Media Literacy, 2005.137 pages. This kit includes an explanation of media-literacy teaching and provides strategies for implementing media literacy, $<w w w$.medialit.org $>$. Lesson-plan library available at $<w w w$.medialit.org/reading_room/rr4_lessonplan.php $>$.Also available in Spanish

The New 'My Changing Body.' Institute for Reproductive Health, forthcoming. This curriculum teaches young people about puberty and their maturing bodies, with an emphasis on menstruation and fertility awareness, <www.irh.org $>$.

Also available in Spanish and French. 
One Man Can Workshop Activities: Talking to Men about Gender, Domestic and Sexual Violence and HIV/AIDS, Sonke Gender Justice, 2006. 48 pages. This manual offers activities to encourage men and boys to reflect on their own attitudes and behavior regarding gender, women, domestic and sexual violence, HIV/AIDS, democracy, and human rights, <www.genderjustice.org.za/ onemancan/complete-oneman-can-toolkit/download-the-complete-to.html>. Also available in Afrikaans, French, isiXhosa, and isiZulu.

\section{Our Future: Sexuality and Life-skills Education for Young People, Grades 4-5,}

Grades 6-7, and Grades 8-9, International AIDS Alliance. 2006 and 2007.132 pages, 128 pages, and 150 pages. Three volumes provide information and learning activities on puberty, friendship, gender, sexuality, pregnancy, sexually transmitted infections, HIV, AIDS, and drug use. They also provide ideas about preparing parents and guardians to discuss sexuality with children, <www.aidsalliance.org/custom_asp/publications/ Iview.asp?publication_id=211\&language $=e n>$.

People's Movement for Human Rights Education (PDHRE-International) works to develop and advance human rights education. The organization publishes human rights training manuals and teaching materials, <www.pdhre.org $>$.

Rights and Desire: A Facilitator's Manual to Healthy Sexuality, Breakthrough, 2006.106 pages. The purpose of this manual is to generate positive dialogue about relationships, sex, and sexuality. <breakthrough.tv/download/rights-and-desire-afacilitator-s-manual-to-healthy-sexuality>.

Sakhi Saheli - Promoting Gender Equity and Empowering Young Women: A Training Manual, CORO for Literacy, Horizons/Population Council, and InstitutoPromundo, 2008.136 pages. The manual promotes reflection to enable young women to understand how gender norms affect their lives and increase their vulnerability to HIV and other reproductive health problems,

$<w w w . p o p c o u n c i l . o r g / p d f s / h o r i z o n s / I n d i a \_S a k h i S a h e l i$ Eng.pdf $>$.

Sexuality and Life-skills: Participatory Activities on Sexual and Reproductive Health with Young People, International AIDS Alliance. 2008.172 pages. Provides activities to help young people develop knowledge, positive attitudes, and skills to grow up and enjoy sexual and reproductive health and welleing.<www.aidsalliance.org/graphics/secretariat/publications/

Sexuality and lifeskills.pdf>.
Stepping Stones: A Training Package on HIV/AIDS, Communication and Relationship Skills, ACTIONAID, 1995. 240 pages. £11.25. This training manual contains instructions for a workshop for exploring social, sexual, and psychological needs and practicing different ways of behaving in relationships. <www.steppingstonesfeedback.org $>$. It can be purchased at $<w w w$.talcuk.org/books/bs-stepping-stones.htm $>$.

Tools for Change: An Educator's Resource Site, Centre for Research and Education on Violence Against Women and Children at the University of Western Ontario. This website provides a list of resources for grades 3-9 that promote healthy, equal relationships. <www.toolsforchange.ca $>$.

Working with Young Men Series, Project $\mathrm{H}$ of Instituto Promundo, with ECOS Comunicacao em Sexualidade, Programa de Apoio ao Pai (PAPAI), Salud Y Genero. 314 pages. Guides for educating young men aged 15-24 about gender roles, violence, and sexuality, <www.promundo.org.br/396>. Also available in

Portuguese and Spanish.

\section{Working with Young Women: Empowerment, Health, and Rights, Instituto} Promundo, Salud y Genero, ECOS, Instituto PAPAI and World Education. 2009. 143 pages. These manuals provide activities on gender equity and women's rights to be used for young women aged 15-24. <www.promundo.org.br/352>.

Also available in Portuguese.

Yaari Dosti - Young Men Redefine Masculinity, Population Council, CORO for Literacy, MAMTA, and Instituto Promundo, 2006.110 pages. This manual promotes gender equity as a strategy for the prevention of HIV infection.

$<w w w . p o p c o u n c i l . o r g / p d f s / h o r i z o n s / y a a r i d o s t i e n g . p d f>$. Also available in Hindi at $<$ www.popcouncil.org/pdfs/horizons/yaaridostihindi.pdf>

Young Men and HIV Prevention: A Toolkit for Action, Promundo and UNFPA 2007. Two documents, 115 pages and 38 pages. Provides conceptual and practical information on how to design, implement, and evaluate HIV- and AIDS-prevention activities that incorporate a gender perspective and engage young men. $<$ Error! Hyperlink reference not valid.>. Also available in Portuguese and Spanish. 
Doorways: School-Related Gender-Based Violence Prevention and Response,

USAID, 2009. This set of three manuals (one each for teachers, students, and community counselors) supports a community-school partnership to make schools safe,

$<$ www.usaid.gov/our_work/cross-cutting_programs/wid/doorways.html>.

Gender, HIV, and Human Rights: A Training Manual, UNIFEM, UNFPA, UNAIDS, 2000. 213 pages. Includes a one-day and a two-day training and aims to enhance educators' understanding of the gender and human rights dimensions of the HIV pandemic.

$<$ www.unifem.org/resources/item_detail.php?Product|D=5>.

Gender or Sex: Who Cares?: Notes for Training of Trainers, I pas, 2002. 68 pages. These resource notes enable experienced facilitators to conduct the Gender or Sex

Who Cares? training (see curricula section above), <www.ipas.org/

Publications/Gender_or_sex_Notes_for_Training_of_Trainers.aspx?ht>.

Also available in Spanish and Creole.

The Human Rights Education Handbook: Effective Practices for Learning, Action, and Change, the Human Rights Resource Center, University of Minnesota, 2000.This manual guides educators to teach effectively about human rights.

$<$ www1.umn edu/humanrts/edumat/hreduseries/hrhandbook/tochtml>.

\section{Our Future: Preparing to Teach Sexuality and Life Skills, An Awareness Training}

Manual for Teachers and Community Workers, International AIDS Alliance, 2008.94 pages. Prepares educators to teach the Our Future curriculum (see curricula and activities section above), <www.aidsalliance.org/custom_asp/publications/view. asp?publication_id=293>.

Training of Trainers: Designing and Delivering Effective Human Rights Education, Training Manual, Equitas - International Centre for Human Rights Education, 2007. 202 pages. Outline of a six-day experiential workshop on how to train trainers in human

rights education, <www.equitas.org/english/ed-manuals/ ed-manuals.php >. Also available in Russian (French edition forthcoming).

Training Trainers for Development, Centre for Development and Population Activities, 1995.93 pages. This manual offers a six-day workshop for teaching participatory training techniques, <www.cedpa.org/content/publication/ detail/757>. Also available in Spanish and French.

\section{reading on teaching and learning}

Experiential Learning Cycles: Overview of 9 Experiential Learning Cycle Models.

This website reviews nine models for experiential learning and their application. $<w w w . w i l d e r d o m . c o m / e x p e r i e n t i a l / e l c / E x p e r i e n t i a l L e a r n i n g C y c l e . h t m>$.

Gold Dust Resources, Quality Improvement Agency. This website provides resources, including information sheets and video activities, to strengthen skills and knowledge among new and experienced teachers, <www.goldust.org.uk $>$.

Hesperian Foundation. Publishes books and newsletters addressing the underlying social causes of poor health and suggesting ways groups can organize to improve health conditions in their communities, <www.hesperian.org/publications download.php>. Materials available in up to 152 languages.

The Learner-centered Teaching Series, Teaching Effectiveness Program, University of Oregon. This website offers a four-part series on learner-centered teaching, including an overview, syllabus development, teaching content, and student assessment, $<$ http://tep.uoregon.edu/workshops/teachertraining/ learnercentered/learnercentered.html>

Paulo Freire and Informal Education, the Encyclopaedia of Informal Education, 2002 This webpage provides a basic introduction to Paolo Freire's work and offers additional references and links, <www.infed.org/thinkers/et-freir.htm $>$.

Training for Transformation, Volumes 1-3, ITDG Publishing, 2002.462 pages. \$50.Thi three-volume set of innovative training techniques integrates various approaches and methodologies for participatory education, organizational development, and community self-reliance. They can be ordered from the Hesperian Foundation at: <www.hesperian.org/mm5/merchant.mvc?

Screen $=$ PROD\&Store_Code=HB\&Product_Code $=$ B803\&Category_Code $=$ HEB $>$. 
Canadian Guidelines for Sexual Health Education, Ministry of Health of Canada, 2003. An example of how a government addresses sex education. $<$ www.phacaspc.gc.ca/publicat/cgshe-Idnemss/cgshe_toc.htm>

Also available in French.

CARE. CARE has various relevant publications, including Addressing the Social Factors That Influence Sexual and Reproductive Health, and the ISOFI Toolkit:Tools for Action and Learning on Gender and Sexuality (to help program staff explore gender and sexuality issues), $<$ www.care.org/careswork/whatwedo/health/srh/ publications.asp $>$. Also available in Spanish and French.

Developing Guidelines for Comprehensive Sexuality Education, Sexuality Education and Information Council of the United States, 1999.36 pages. This handbook provides educators, policymakers, and activists with a step-by-step guide for developing guidelines for comprehensive sexuality education programs. $<$ www.siecus.org/pubs/guidelines/guideintl.pdf $>$.

\section{Dynamic Contextual Analysis of Young People's Sexual Health:}

A Context Specific Approach to Understanding Barriers to, and Opportunities

for, Change. Thomas Coram Research Unit, University of Southampton, 2006.

51 pages. Describes how to conduct a dynamic contextual analysis of factors

that affect young people's sexual lives as a basis for programming.

$<$ www.safepassages.soton.ac.uk/pdfs/DCA2.pdf $>$.

Facing the Challenges of HIV, AIDS, STDs: A Gender-based Response, Royal Tropical Institute, Southern Africa AIDS Information Dissemination Service and -World Health

Organization, Amsterdam, 1995.56 pages. Information and checklist for policymakers and program implementers regarding incorporating gender into policies and programs on HIV and AIDS and other STIs. < data.unaids.org/Topics/

Gender/FacingChallenges_en.pdf>. Also available in Hindi.
HIV/AIDS Education: A Gender Perspective, Tips and Tools, UNICEF, 200224 pages. Background information, checklists, and activities for training educators in formal and nonformal settings, <www.unicef.org/lifeskills/index_14927.html $>$ or $<$ www.ibe.unesco.org/AIDS/doc/UNICEF_Gender_Eng.pdf $>$.

Also available in Spanish and French.

IPPF Framework for Comprehensive Sexuality Education, International Planned Parenthood Federation, 2006.9 pages. An overview of comprehensive sexuality education and a basic planning framework for implementation.

$<$ www.ippf.org/en/Resources/Guides-toolkits/Framework+for+Comprehensive+ Sexuality+Education.htm>. Also available in Spanish and French.

\section{Key Issues in the Implementation of Programmes for Adolescent Sexual and}

Reproductive Health, Department of Child and Adolescent Health and Development, World Health Organization, 2004.51 pages. Reviews adolescent sexual and reproductive health programming issues, <www.who.int/

child adolescent health/documents/fch cah 04 3/en/index.html>.

RHIYA (The Reproductive Health Initiative for Youth in Asia), was a European Union and UNFPA initiative in South and Southeast Asian countries that sought to improve the sexual and reproductive health of 10-24-year-olds. The project produced a series of case studies and program implementation reports:

$<$ www.unfpa.org/eu_partnership/rhiya $>$.

Synergizing HIV/AIDS and Sexual and Reproductive Health and Rights - A Manua for NGOs, AIDSNET, 2006. 22 pages. Provides evidence and guidelines for integrating sexual/reproductive health, rights, and gender into HIV work. <www.aidsnet.dk/Defau It.aspx?|D=2366>

Toolkit for Mainstreaming HIV and AIDS in the Education Sector: Guidelines for Development Cooperation Agencies, Joint UN Programme on HIV/AIDS, 2008.75 pages. Training resources and materials, <unesdoc.unesco.org/ images/0015/001566/156673E.pdf>. 
Africa Regional Sexuality Resource Centre (ARSRC), based in Lagos, Nigeria, organizes sexuality institutes in the Africa region.

$<$ www.arsrc.org/training/asi/background.htm>.

Creating Resources for Empowerment in Action (CREA) works to empower women to articulate, demand, and access their human rights through technical assistance and training in women's human rights, gender, and sexuality. Based in India, CREA conducts a number of annual courses around the world. <web.crea world.org $>$.

Girls Power Initiative, based in Calabar, Nigeria, holds a Gender Development Institute to create awareness on gender, rights, and sexuality, <www.gpinigeria.org $>$.

Global Youth Coalition on HIV/AIDS is a youth-led network of 4,000 young leaders and adult allies in 150 countries worldwide. GYCA trains and empowers young leaders to scale up HIV and AIDS interventions among their peers.

$<$ www.youthaidscoalition.org $>$.

Instituto Promundo, based in Brazil, developed Project $\mathrm{H}$ and Project $\mathrm{M}$ to address gender, rights, and sexual health issues with young men and women. The projects provide technical assistance and training in gender and sexuality education. $<$ www. promundo.org.br>. Also available in Portuguese.

\section{International School for Humanities and Social Sciences, Universiteit van}

Amsterdam, conducts research and a Summer Institute on Sexuality, Culture, and

Society that explores the social dimensions of sexuality across cultures. Fellowships are available for participants from selected countries.

$<$ www.ishss.uva.nl/Summerlnstitute/index.html $>$.
National Sexuality Resource Center, San Francisco State University has a

Summer Institute on Sexuality and Culture. $<$ nsrc.sfsu.edu $>$.

The Pleasure Project, with offices in Oxford, UK, and India, provides training and technical assistance to trainers and counselors on taking a sex-positive approach to their work, <www.thepleasureproject.org $>$.

Reprolatina, based in Brazil, provides training in gender and reproductive and sexual health and rights in Bolivia, Brazil, Chile, and Paraguay.

<www.reprolatina.org.br/site/html/entrada/index.asp>.

Also available in Portuguese.

Swedish Association for Sexuality Education (RFSU) provides technical assistance and training on young people's sexual and reproductive health and rights and sexual diversity and human rights, <www.rfsu.se/default_en-us.asp $>$.

Also available in Swedish.

Talking about Reproductive and Sexual Health Issues (TARSHI), based in India, provides training in sexuality, gender, and rights in the South and Southeast Asian region, <www.tarshi.net>. Also available in Hindi. 
Action Canada for Population and Development (ACPD) promotes global

development focused on s exual and reproductive rights and health. $<$ www.acpd.ca $>$.

Advocates for Youth advocates for policies that enable young people to make informed, responsible decisions about their reproductive and sexual health in the United States and globally, <www.advocatesforyouth.org $>$.

Amnesty International, a human rights organization, promotes girls' safe access to education through its Safe Schools campaign. For a report and fact sheets, see $<$ www.amnesty.org/en/campaigns/stop-violence-against-women/issues/ empowerment-women/safe-schools>.

BRIDGE, Institute for Development Studies, undertakes gender advocacy by bridging the gaps among theory, policy, and practice with accessible and diverse gender information in print and online, $<w w w . b r i d g e . i d s . a c . u k>$.

Catholics for Choice (CFC) works to advance sexual and reproductive ethics based on justice, a commitment to women's well-being, and the moral capacity of people to make sound decisions about their lives, <www.catholicsforchoice.org $>$.

Center for Health and Gender Equity (CHANGE) works to ensure that United States international policies and programs promote sexual and reproductive rights and health, <www.genderhealth.org/index.php $>$.

Center for Reproductive Rights is a legal advocacy organization working worldwide. For publications on adolescent sexual and reproductive rights, see:

$<$ www.reproductiverights.org/pdf/adolescents\%20bp FINAL.pdf $>$.

$<$ www.reproductiverights.org/pdf/BRB_SexEd.pdf>.

$<w w w . r e p r o d u c t i v e r i g h t s . o r g / p d f / S e x u a l i t y E d u c a t i o n f o r A d o l e s c e n t s . p d f>$.

CHOICE for Youth and Sexuality is a Dutch-based youth organization that promotes sexual and reproductive health and rights for young people.

$<$ www.choiceforyouth.org $>$.

Family Care International works to prevent pregnancy- and childbirth-related injury and death and to ensure sexual and reproductive health and rights internationally, $<$ www.familycareintl.org/en/home $>$.

Family Violence Prevention Fund works to end violence against women and children within the home and community and to help those affected by violence in the United States and around the world, <www.endabuse.org >
Human Rights Watch works to protect the human rights of people around the world, including women's rights; lesbian, gay, bisexual and transgender rights; and rights related to health, including HIV. <www.hrw.org $>$.

International Gay and Lesbian Human Rights Commission (IGLHRC) works to secure full enjoyment of human rights by all people regardless of sexual orientation or expression, gender identity or expression, or HIV status, <www.iglhrc.org $>$. International Lesbian and Gay Association (ILGA) is a global network of organizations committed to achieving equal rights for lesbian, gay, bisexual, transgender, and intersexual (LGBTI) people, <www.ilga.org/index.asp >

International Planned Parenthood Federation (IPPF) is a global network of member associations that provide and campaign for sexual and reproductive health care and rights for all. <www.ippf.org/en>.

International Women's Health Coalition (IWHC) seeks to generate health and population policies, programs, and funding that promote and protect the rights and health of girls and women worldwide, <www.iwhc.org >

Ipas works globally to increase women's ability to exercise their sexual and reproductive rights and to reduce abortion-related deaths and injuries. $<$ www.ipas.org $>$.

Sexuality Information and Education Council of the United States (SIECUS) works for sexuality education and sexual health and rights, <www.siecus.org >. Women's Global Network for Reproductive Rights (WGNRR) brings together groups and individuals committed to advocating for women's reproductive rights. $<$ www.wgnrr.org $>$.

World Association for Sexual Health (WAS) works to promote sexual health for all through its international body, its regional continental federations, and national organizations, <www.worldsexology.org/index.asp>

Youth Coalition, an international organization of young people, works to promote young people's sexual and reproductive rights at national, regional, and international levels and to secure the meaningful participation of young people in decisionmaking that affects them, <www.youthcoalition.org $>$.

Many of the organizations on this page have materials available in multiple languages. 
Africa Regional Sexuality Resource Centre, hosted by Action Health Incorporated in Nigeria, seeks to contribute to the development of positive sexuality programs and policies in Africa through education, informed public dialogue, and advocacy. $<$ www.arsrc.org/index.htm >.

Amanitare: African Partnership for the Sexual and Reproductive Health and Rights of Women and Girls works on women's bodily integrity and sexual and reproductive rights in Africa, advocating for attention to women's rights in HIV/ AIDS, maternal mortality, and contraception. <www.amanitare.org.za $>$.

Asian-Pacific Resource and Research Centre for Women (ARROW) promotes and protects women's health rights and needs, focusing on sexuality and reproductive health.<www.arrow.org.my//index.php?option=com_content\&task= view\&id=12\&ltemid=29>.

\section{ASTRA - Central and Eastern European Women's Network for Sexual and}

Reproductive Health and Rights, a network of NGOs and individuals, advocates for sexual and reproductive health and rights, especially in Central and Eastern Europe. $<$ wWw.astra.org.pl $>$.

Council of Europe Youth Centre seeks to promote European unity and the dignity of its citizens by ensuring respect for human rights, pluralist democracy, and the rule of law. See the "All different, all equal" campaign:<alldifferent-allequal.info>.

\section{Latin American and Caribbean Women's Health Network (LACWHN) is a}

network of organizations and individuals promoting women's health, human rights,

and citizenship, <www.reddesalud.org/english/sitio/portada.htm >

Latin American Center on Sexuality and Human Rights disseminates knowledge about sexuality from a human rights perspective to help combat gender inequality and contribute to the struggle against the discrimination of sexual minorities in the region, $<w w w . c l a m . o r g . b r>$.
National Sexuality Resource Center, at San Francisco State University, develops content, provides information, and conducts trainings in the United States with a positive and social justice perspective on sexuality. $<$ nsrc.sfsu.edu $>$.

SIECCAN (Sex Information and Education Council of Canada) fosters public and professional education about human sexuality through information, consultation, research, and publishing, <www.sieccan.org/index.html>.

South and Southeast Asia Resource Centre on Sexuality, hosted by Talking about Reproductive and Sexual Health Issues (TARSHI) in India <www.tarshi.net>, aims to increase knowledge about sexuality, sexual health, and sexual well-being in South and Southeast Asia, <www.asiasrc.org/plspk/2007_1/at_resource_centre.asp >.

Women for Women's Human Rights (WWHR), based in Turkey, works nationally and internationally to promote women's rights. It supports the Coalition for Sexual and Bodily Rights in Muslim Societies, <www.wwhr.org/index.php >.

Women Living Under Muslim Laws (WLUML) is an international solidarity network that aims to strengthen the movement for equality and rights of women whose lives are shaped, conditioned, or governed by laws and customs said to derive from Islam, $<$ www.wluml.org/english/index.shtml $>$.

YouAct, European Youth Network for Sexual and Reproductive Health and Rights, a network of young people in Europe who are active in the field of sexual and reproductive rights, aims to empower young people to make their voices heard. $<$ www.youact.org $>$.

Most of the organizations on this page have materials available in languages specific to their region. 
Advocacy Kit for Growing Up Global: The Changing Transitions to Adulthood in Developing Countries, Advocates for Youth and Population Reference Bureau, 2005. This kit contains fact sheets summarizing findings from an expert review of young people's transition to adulthood; it also includes advocacy tips.

<www.advocatesforyouth.org/publications/growingupglobal/index.htm>.

Growing Up Global may be purchased, or read online:

$<$ www.nap.edu/catalog.php?record_id=11174>.

Deadly Inertia: A Cross-country Study of Educational Responses to HIV/ AIDS Global Campaign for Education, Brussels, Belgium, 2005. 52 pages. This publication reviews the education sector response to the HIV pandemic.

$<$ www.comminit.com/en/node/218512/38 and www.unesco.org/bpi/aids-iatt/ deadlyinertia.pdf $>$.

Gender and Sexuality Cutting Edge Pack, BRIDGE, 2006 and 2007. This pack contains three documents that examine the link between gender, sexuality, and sexual rights: Gender and Sexuality Overview Report (51 pages); Supporting Resources Collection (70 pages); and Gender and Development In Brief "Sexuality" (6 pages). <w ww.bridge, ids.ac.uk/reports gend_CEP.html\#Sexuality>. Also available in French and Spanish.

HIV/AIDS and Women: Resources to Support Policy and Advocacy, Women, Ink, the International Women's Tribune Centre. Approximately 50 action-oriented tools, reports, and case studies to support advocacy on women and HIV and AIDS.

$<$ www.womenink.org/resources/HIVAIDS.htm>.

HIV testing:The mutual rights and responsibilities of partners, Ruth DixonMueller and Adrienne Germain. 2007. Lancet 370(9602): 1808-1809. This commentary argues for recognizing mutual rights and responsibilities of both partners in a sexual relationship or exchange. <www.ph.ucla.edu/EPI/seaids/ . lancet370J808_1809 2007.pdf>.
International Guidelines on Sexuality Education: An evidence informed approach to effective sex, relationships, and HIV/STI education, UNESCO, June 2009 (draft version). This document is not a curriculum. Rather, it focuses on the 'why' and 'what' issues that require attention in strategies to introduce or strengthen sexuality education. It explains what sexuality education is and why it is important. <unesdoc unesco.org/images/0018/001832/183281e.pdf> Also available in French and Spanish.

Ministerial Declaration -"Educating To Prevent." 2008. This declaration reflects the shared commitment by Ministers of Health and Education in Latin America and the Caribbean to strengthen HIV prevention efforts by ensuring access to quality, comprehensive sexuality education and sexual and reproductive health services. It seeks to foster equity among all people and to combat discrimination. < data.unaids. org/pub/BaseDocument/2008/20080801_minsterdeclaration_en.pdf>.Also available in Spanish at data.unaids.org/pub/BaseDocument/2008/ 20080801_minsterdeclaration_es.pdf.

Sexuality and relationships education: Toward a social studies approach, Deborah Rogow and Nicole Haberland. 2005. Sex Education 5(4):333-344. This paper argues for grounding sexuality and relationships education within a social studies framework, with an emphasis on gender, social context, and human rights. $<w w w . p o p c o u n c i l . o r g / p d f s / S E \_5 \_4 . p d f>$.

Also available in French at <WwW.popcouncil.org/pdfs/SE_5_4_fr.pdf $>$.

Also available in Spanish at $<$ www.popcouncil.org/pdfs/SE 5 _ 4 esp.pdf $>$.

\section{Triple Jeopardy: Female Adolescence, Sexual Violence and HIV/AIDS} International Women's Health Coalition, 2008. 6 pages. This information brief provides relevant data, an explanation of the link between sexual violence and young girls' vulnerability to HIV and suggests policy and program responses. $<w w w$.iwhc.org/index. php?option=com_content\&task=view\&id=2693\& Itemid=824>. Also available in French, Portuguese and Spanish.

Yogyakarta Principles, This document addresses the application of human rights to issues of sexual orientation and gender identity, <www.yogyakartaprinciples.org/ index.html>. Also available in Arabic, Chinese, French, Russian, and Spanish. 

गर्बल बिख्ष

畣

है

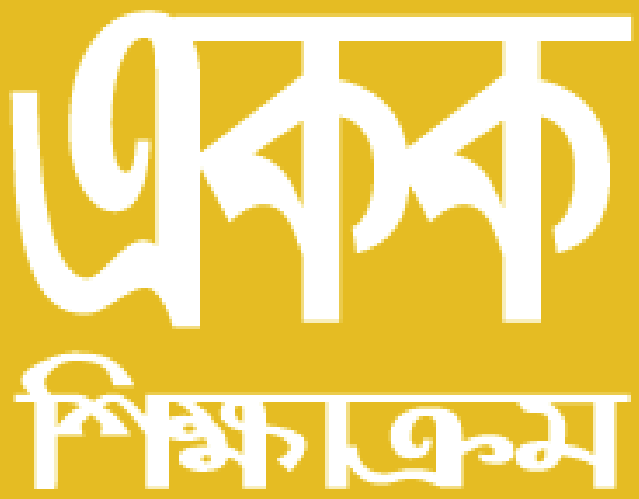

$\frac{4}{4}$ 